UNITED STATES DEPARTMENT OF THE INTERIOR

GEOLOGICAL SURVEY

OREGON

Basic data for thermal springs and wells

as recorded in GEOTHERM

By

James D. Bliss

Open-File Report 83-435

This report is preliminary and has not been reviewed for conformity with U.S. Geological Survey

editorial standards and stratigraphic nomenclature. Any use of trade names is for descriptive purposes only and does not imply endorsement by the USGS.

Menlo Park, California

May 1983 
INTRODUCTION

GEOTHERM, a computerized information system now off-line, was used to maintain data on the geology, geochemistry and hydrology of geothermal sites primarily within the United States. The system was proposed at the First Geothermal Implementation Conference in New Zealand in 1974 (Swanson, 1977) and was active until 1983. The primary mission was to provide a broad informational framework for the Geothermal Research Program (Duffield and Guffanti, 1981). GEOTHERM was used to support national geothermal assessments--in 1978 (Muffler, 1979) and 1982 (Reed, 1983). It was however a public system and provided data to both public and private sectors. A detailed discussion on databases in GEOTHERM and a general scheme of how the information system operated can be found in Bliss and Rapport (1983).

This report on Oregon is one of a series intended to preserve the data collected for GEOTHERM and make the data available to the public. States with significant geochemical data for geothermal fluids will be covered in individual reports such as this. A report will also be issued to cover miscellaneous data collected for sites in the central and eastern United States. The data presented in this series is also available as a data file on the internationally-available General Electric Mark III service, a timeshare network. Those interested in accessing that system should contact the Energy Resource Center, University of OKlahoma, Norman, OKlahoma 73070. It is anticipated that a portion of the data will also be available on magnetic tape from the National Technical Information Service, U. S. Department of Commerce, Springfield, VA 22161. It will not be available until after the completion of the open-file series.

\section{GEOTHERM INDEXES}

Three computer-generated indexes are found in appendices $A$, $B$, and $C$ of this report. The indexes give one line summaries of each GEOTHERM record describing the chemistry of geothermal springs and wells in the sample file for Oregon. Each index is sorted by different variables to assist the user in locating geothermal records describing specific sites.

Appendix $A(p, 218-224)$ is sorted by the county name and the name of the source. Al so given are latitude, longitude (both use decimal minutes), township, range, section, GEOTHERM record identifier, and temperature $\left({ }^{\circ} \mathrm{C}\right)$. In conducting a search of Appendix A, site names are quite useful for locating springs or wells for which a specific name is commonly used, but sites which do not have specific names are more difficult to locate. It is suggested that site titles which begin with words such as warm, hot, unnamed, pumped, well, or spring be checked. Descriptive text found as part of the site name and the site coordinates should be used to assist in determining location. 
Appendix $B(p .225-331)$ is sorted by township, range, and section. Also given are name of source, GEOTHERM record identifier, and temperature $\left({ }^{\circ} \mathrm{C}\right)$. Records missing items used for sorting will be listed first.

Appendix $C$ (p. 332-339) is first sorted into one-degree blocks by latitude, and longitude, and then by name of source. Adjacent one-degree blocks which are published as a 1:250,000 map are combined under the appropriate map name. Also given are GEOTHERM record identifier, and temperature $\left({ }^{\circ} \mathrm{C}\right)$. Records missing items used for sorting will be listed first. Numbers with a blank in the same position as zero will be given first.

\section{GEOTHERM SAMPLE FILE}

GEOTHERM sample file contains 346 records for Oregon (Table 1). Records may be present which are duplicates for the same analyses. A record may contain data on location, sample description, analysis type (water, condensate, or gas), collection condition, flow rates, and the chemical and physical properties of the fluid. Stable and radioactive isotopic data are occasionally available. Some records may contain only location and temperature. When sufficient chemical data was available, the charge balance (percentage of difference in anion- and cation-milliequivalents) was computed and added to the record. Many of the numeric fields in the sample file can be directly qualified. The qualifier code preceeds the number when appropriate. The codes and their meaning are given in Table 1.

Each thermal spring or well usually is represented by several records. This may document temporal changes in the geothermal fluids. Judgement on what constituted acceptable data was extremely complicated and the primary attempt was to insure that each GEOTHERM record faithfully reproduced the published data. On occasion, glaring inconsistencies or data clearly of poor quality were excluded. Regrettably, no database can be constructed or supported without the introduction of errors. The user, therefore, is advised to check with the published literature whenever possible. Users should carefully and critically evaluate the records they use.

This compilation should contain all of the chemical data for geothermal fluids in Oregon available as of December, 1981. However, no claim is made for completeness, and published sources have probably been missed. About $28 \%$ of the records in this list contains information which was unpublished at the time of data entry. A critically evaluated and corrected list of over 2000 records for the United States was extracted from the sample file and issued as a reference document for the national lowtemperature geothermal resource assessment (Reed and others, 1983). This, along with a list of geothermal springs by Berry, and others, 1980, may be useful to some users. 


\section{GEOTHERM BIBLIOGRAPHY}

A bibliography is given in Appendix D ( $p .340-342)$. The abbreviated form of the reference (called code) is identifed as the record source in the full record 1 ist and is used to sort the entries in this appendix. Codes with a leading " $\star$ " identify records based on information which was unpublished at the time the record was prepared. Codes with a trailing "*" in the full GEOTHERM record are also described in greater detail in Appendix $D$ and are listed ahead of published sources.

\section{ACKNOWLEDGMENTS}

Contributions and support to GEOTHERM have been made by many in both federal and state agencies. This includes the U.S. Department of Energy (and associated contractors), and U.S. National Oceanic and Atmospheric Administration. Data-entry forms for most sites in Oregon were prepared by the staff of either the Oregon Department of Geology and Mineral Industries (DOGAMI) or the U.S. Geological Survey.

\section{REFERENCES CITED}

Berry, G.W., Grim, P. J., and Ikelman, J. A., 1980, Thermal springs 1ist for the United States: National Oceanic and Amospheric Administration, Key to Geophysical Records Document No. 12,59 p.

Bliss, J. D., and Rapport, Amy, 1983, GEOTHERM: the U.S. Geological Survey geothermal information system: Computers \& Geosciences, v. 9, no. 1., p. 35-39.

Duffield, W. A., and Guffanti, Marianne, 1981, The geothermal research program of the U.S. Geological Survey: U.S. Geological Survey Open-File Report 81-564, $108 \mathrm{p}$.

Muffler, L. J. P., ed., 1979, Assessment of geothermal resources of the United States--1978: U.S. Geological Survey Circular $790,163 \mathrm{p}$.

Reed, M. J., ed., 1983, Assessment of 10w-temperature geothermal resources of the United States--1982: U.S. Geological Survey Circular 892 .

Reed, M. J., Mariner, R. H., Brook, C. A., and Sorey, M. L., 1983, Selected data for low-temperature (less than $90^{\circ} \mathrm{C}$ ) geothermal systems in the United States; reference data for U.S. Geological Survey Circular 892: U.S. Geological Survey Open-File Report 83-250, 129 p.

Swanson, J. R., 1977, GEOTHERM data file: Geothermal Resources Council Transactions, v. 1, p. 285. 
State of Oregon: computer-generated listing of records describing geothermal-fluid samples. [A few records may be for cold springs or wells--this was to provide ground-water references for some studies.]

ORGANIZATION: Records are sorted by county and then by the name of the spring or well. Order is the same in Appendix A.

UTM: The UTM Easting label was omitted. However, if the UTM Easting figure is present in the record, it will be found directly below the UTM Northing label.

QUALIFICATION CODES: AII numeric attributes may be qualified. The codes and their meaning:

$$
\begin{aligned}
& L=\text { less than } \\
& G=\text { greater than } \\
& E=\text { estimated } \\
& T=\text { trace (no numeric value reported) } \\
& N=\text { not detected (not followed by number) } \\
& Q=\text { qualified (other data in qualification field) } \\
& R=\text { midpoint of range (actual range in qualification field) }
\end{aligned}
$$

REFERENCE: An expanded citation of the reference is found in Appendix D. The abbreviated form used in this table is called "CODE" in the appendix. Unpublished sources are preceded with "*". Those which begin and end with a "*" are also found in Appendix $D$. 


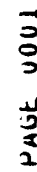
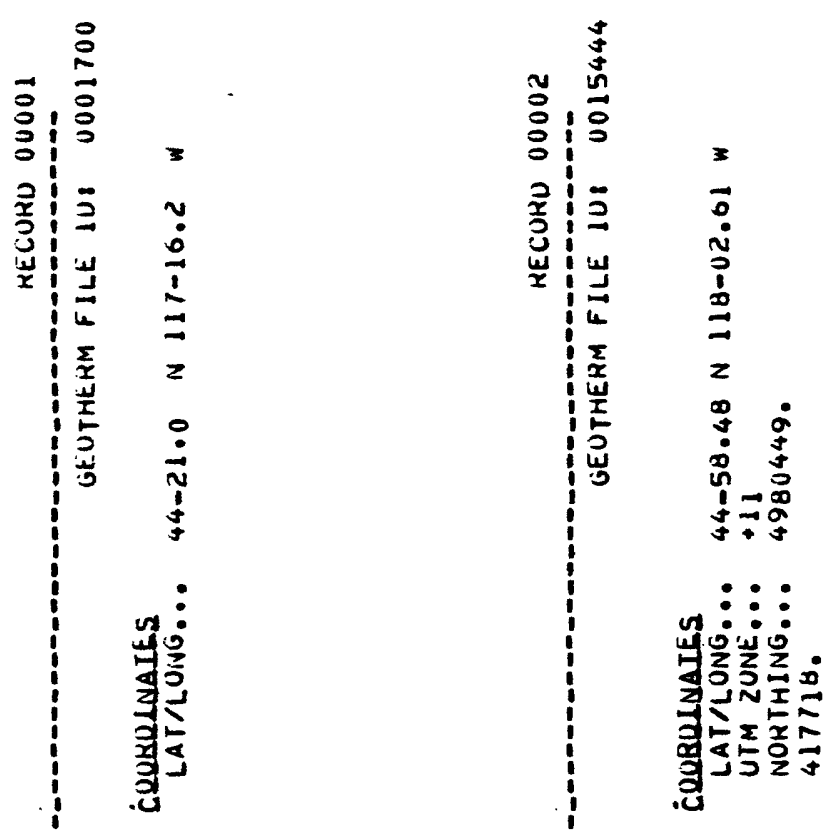

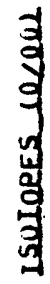
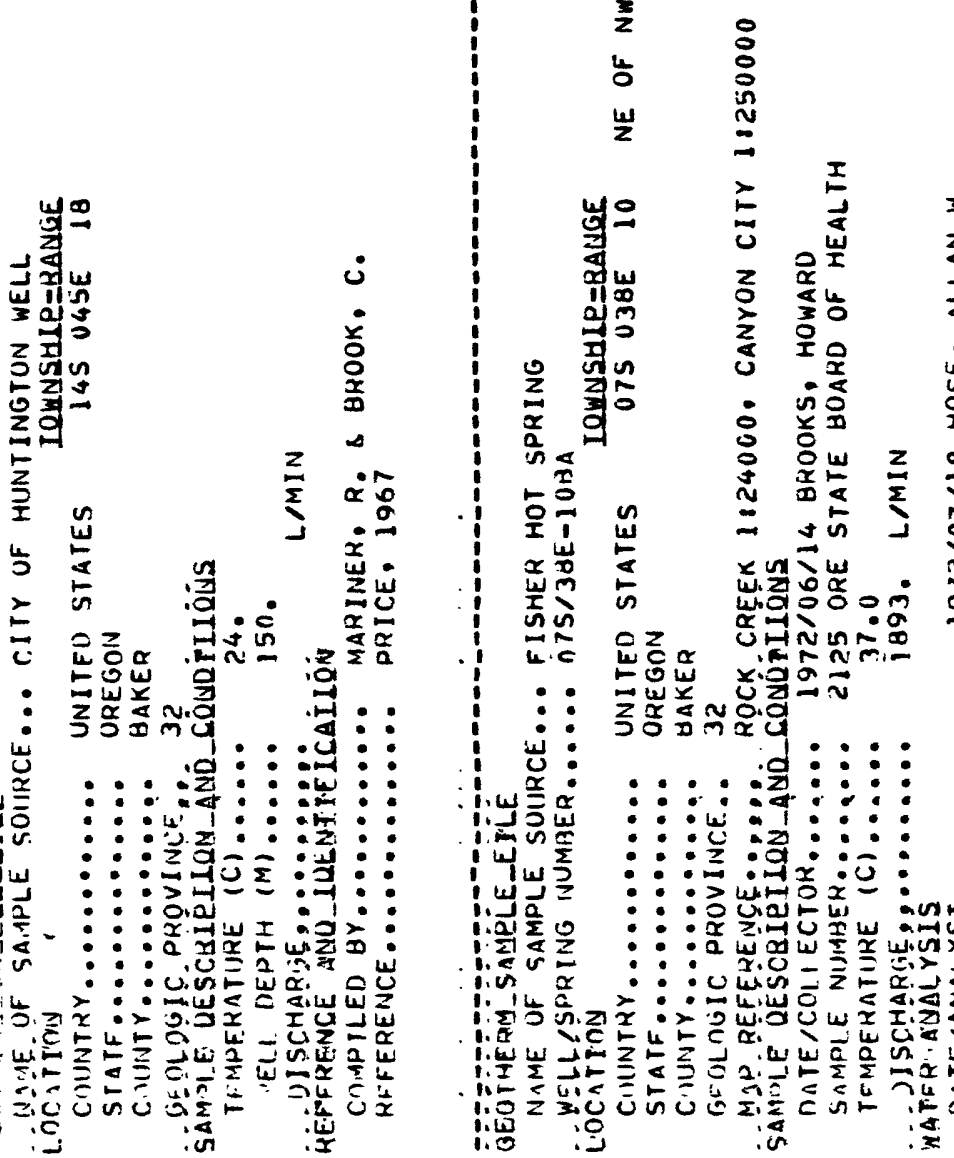

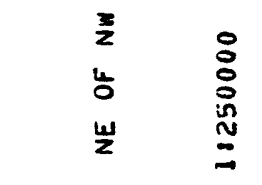

¿̊:

$\underset{\substack{n\\}}{\stackrel{m}{\underline{x}}}$

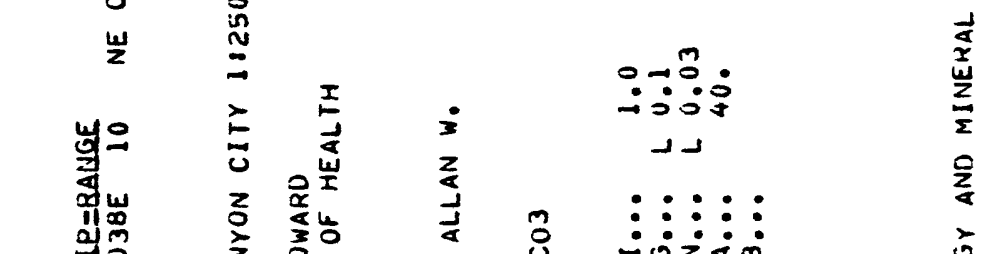

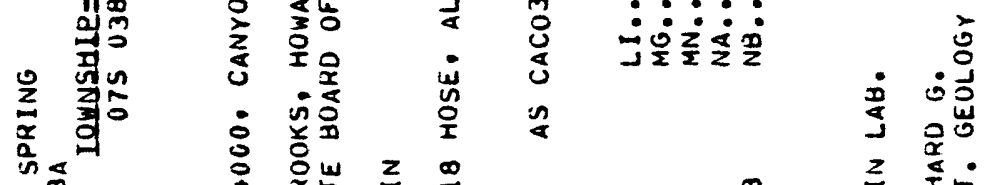

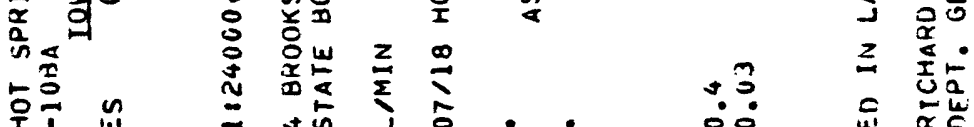

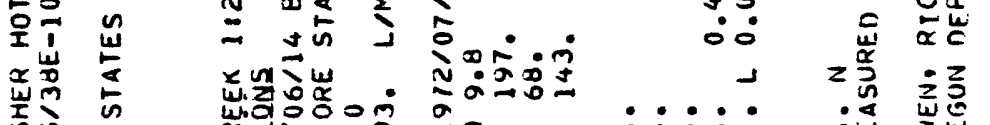

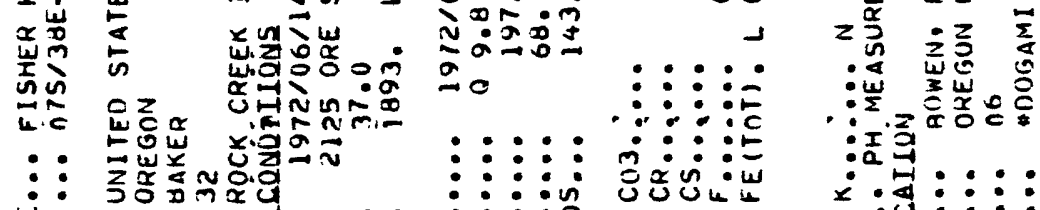

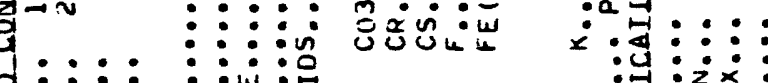

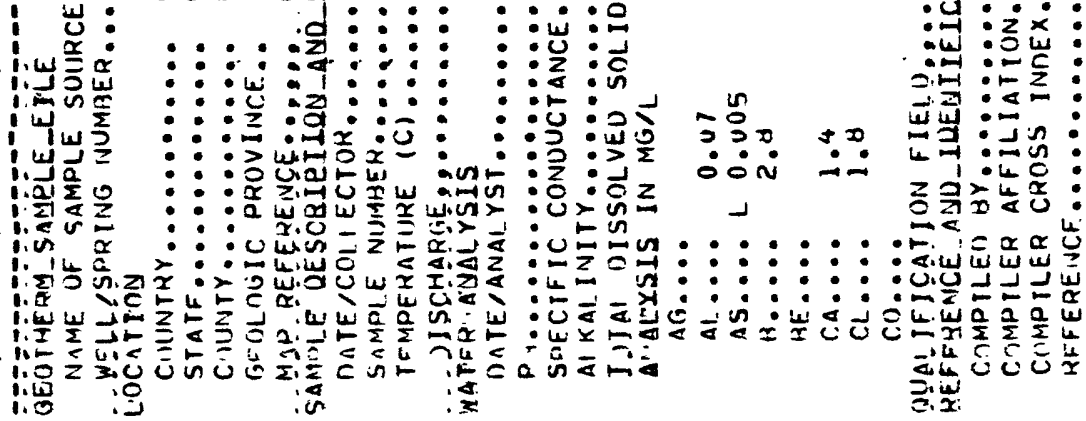



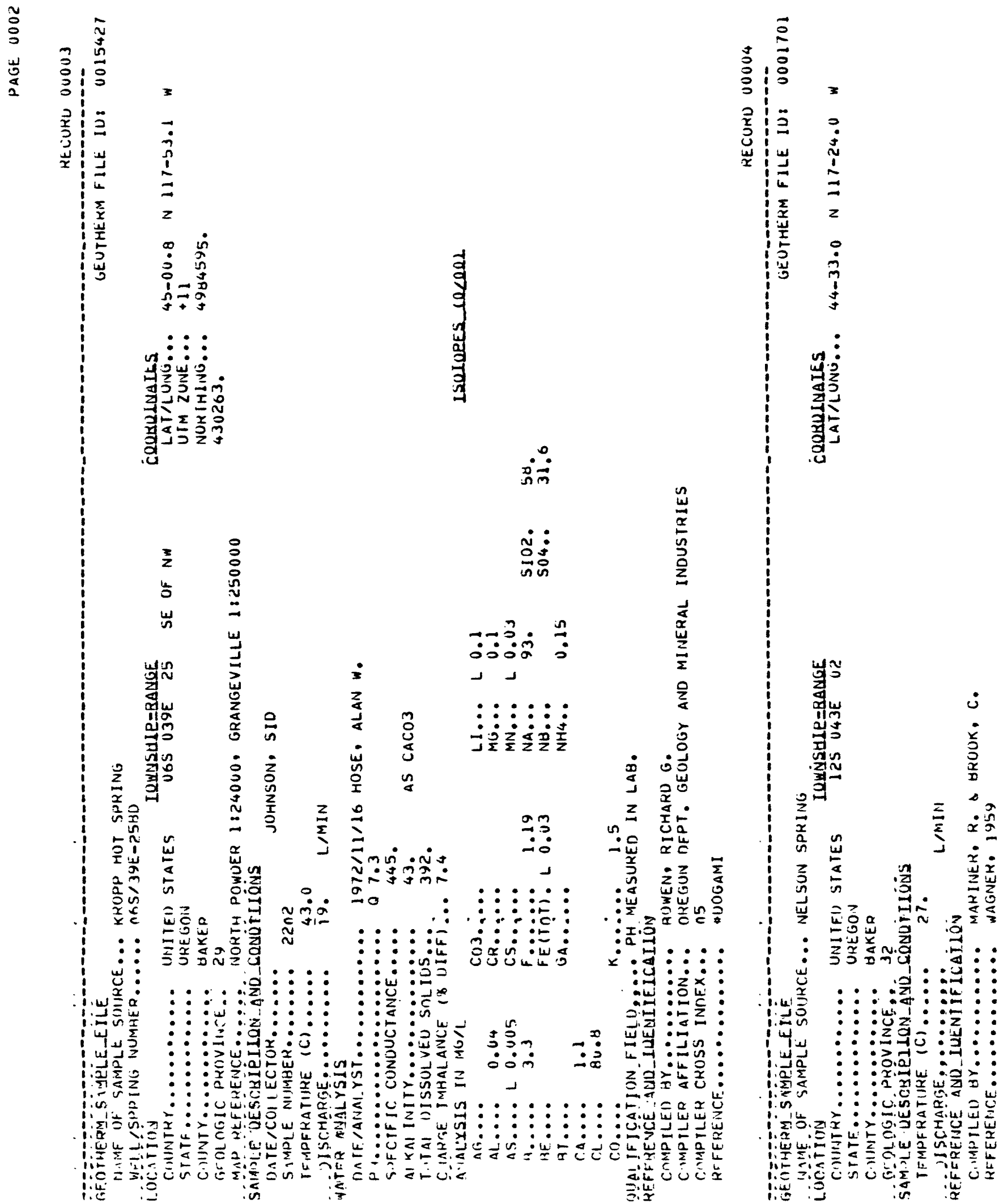


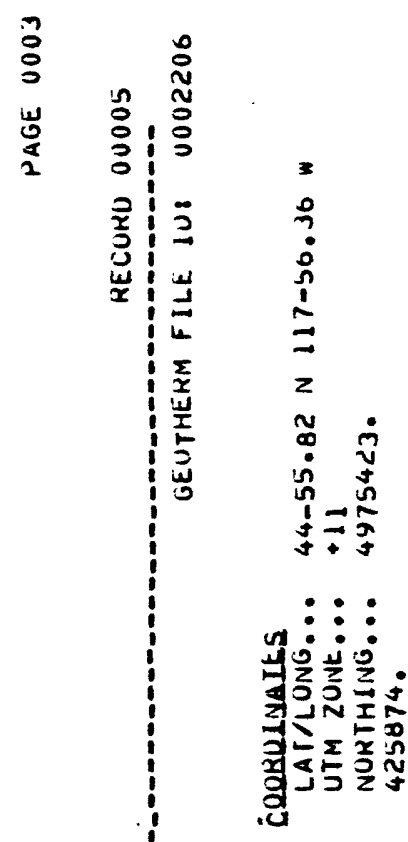

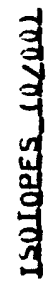

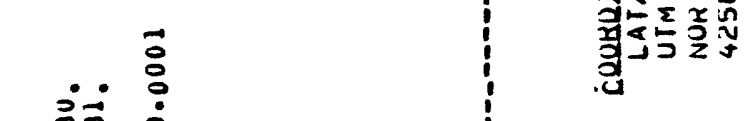

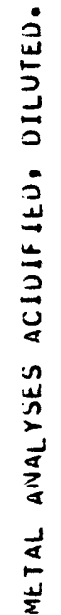

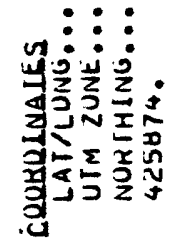

$\frac{j}{\sum_{0}}$

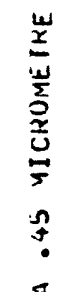

㟧

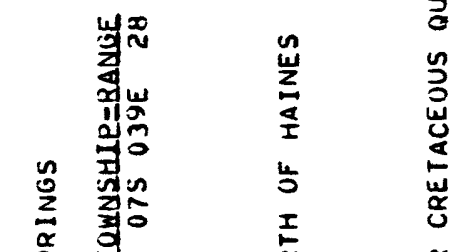

$z z \stackrel{\dot{0}}{\dot{0}}=$

$:::: \vdots:$

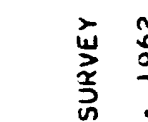

还究站

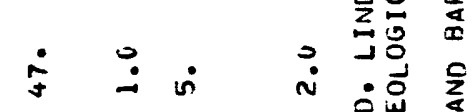

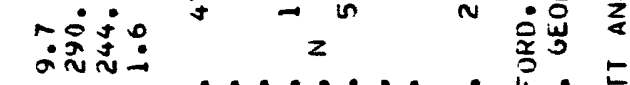

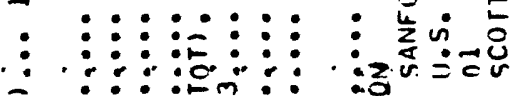

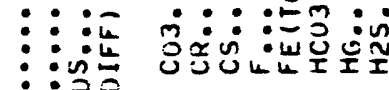
: 约光 :

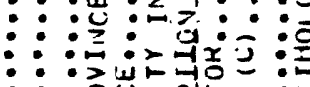

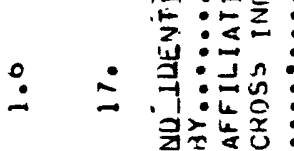

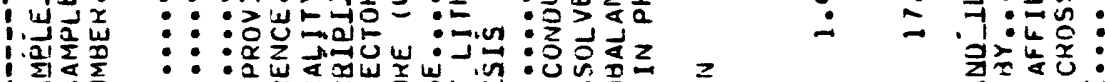
:

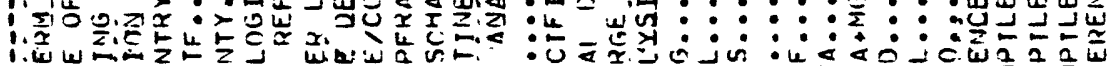

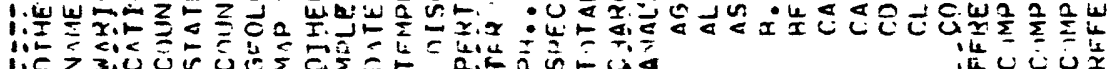

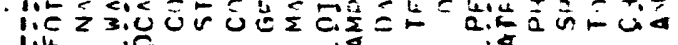
喵 

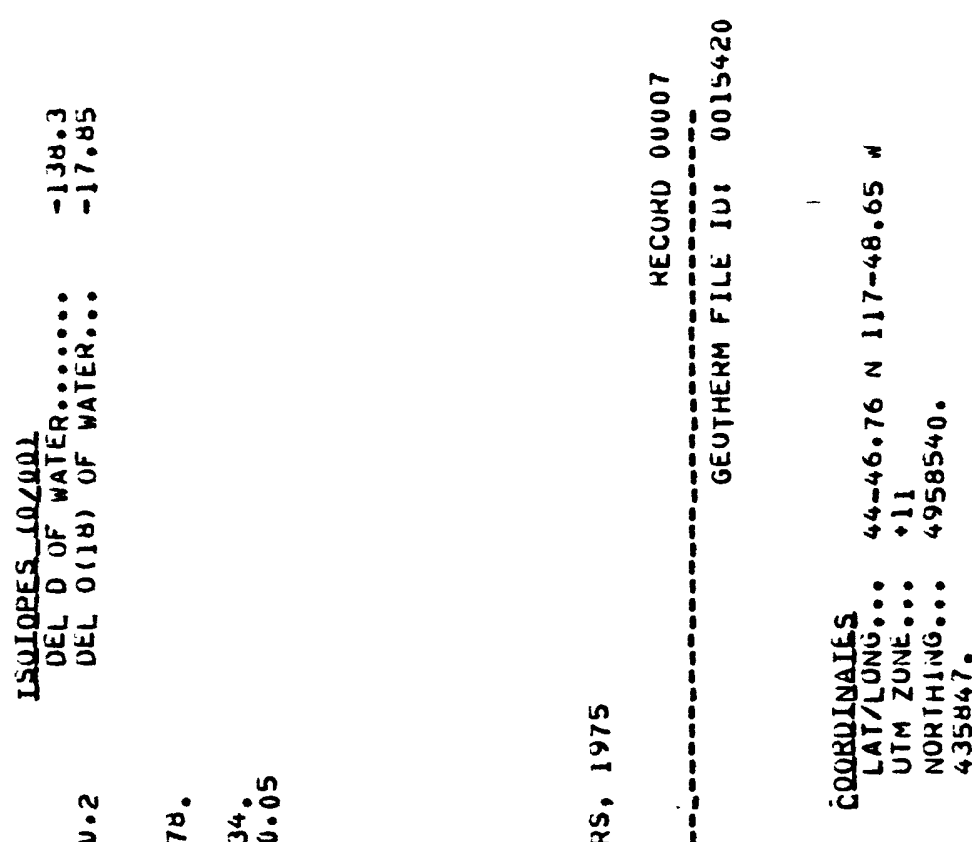

ב)

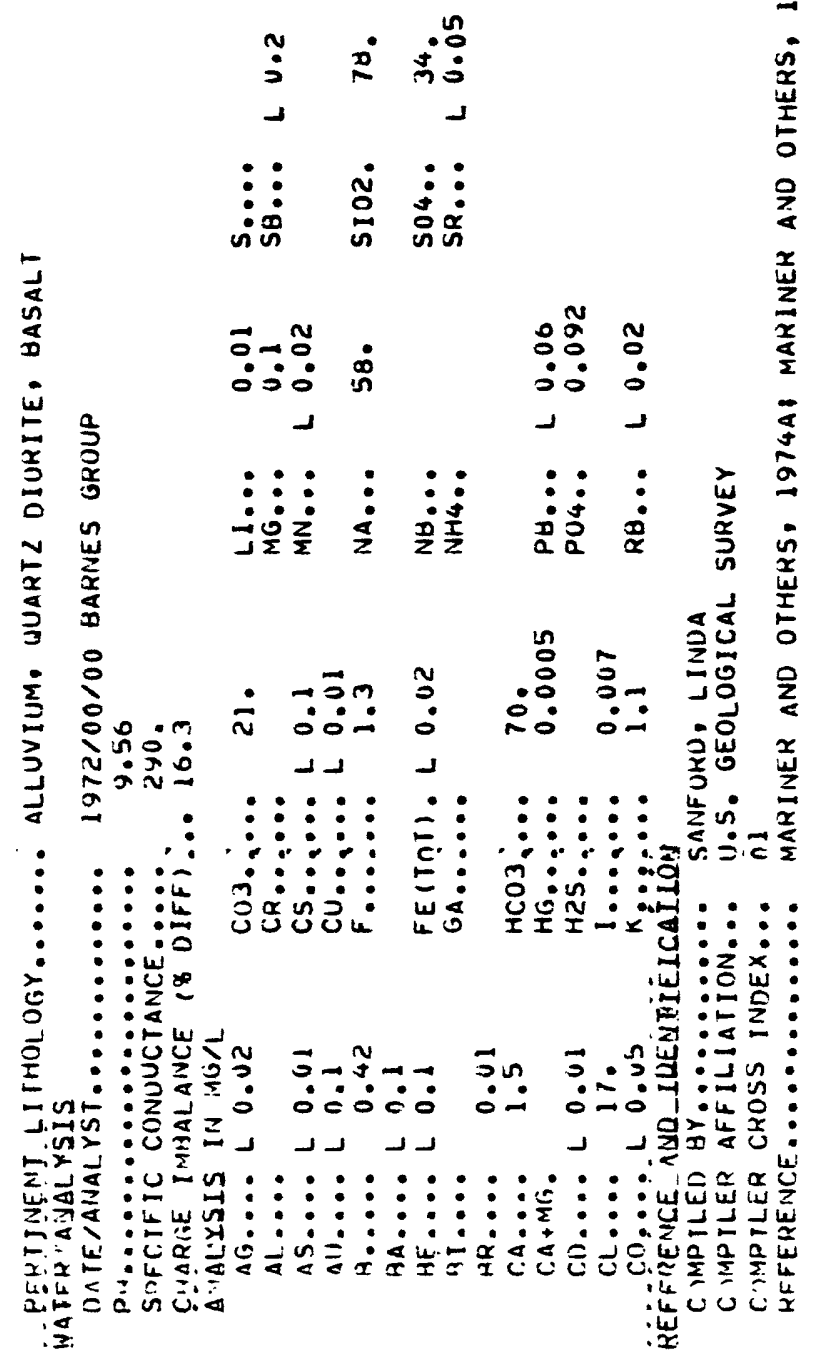

$\stackrel{0}{0}$ 苛蕰 $u$
w
5
w

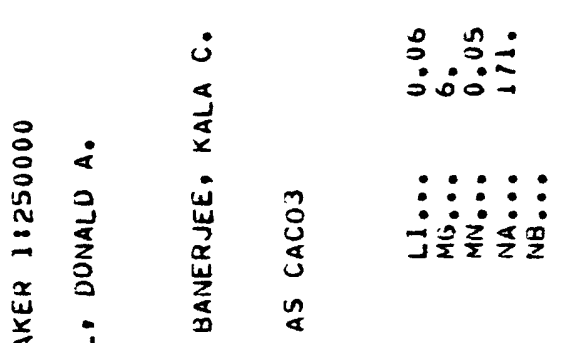
突造 w

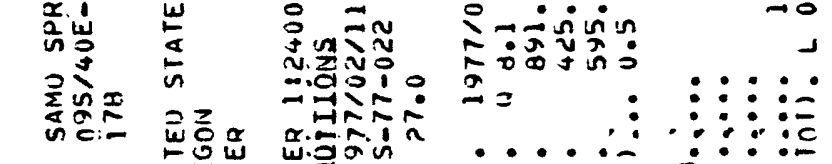

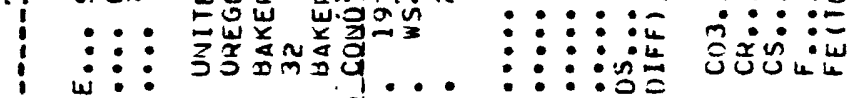

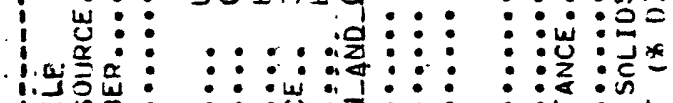
:

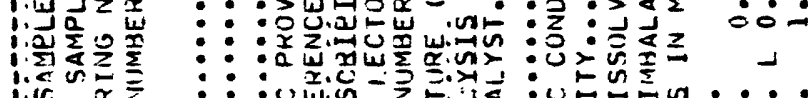

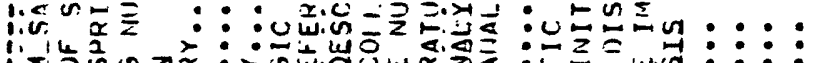

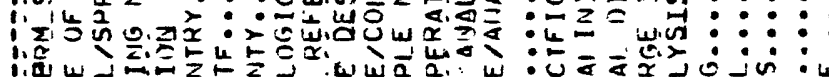

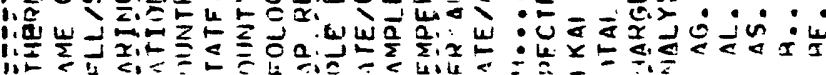

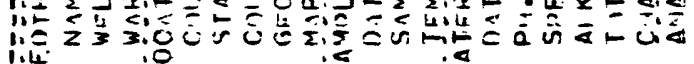


$n$
0
0
$\vdots$
0

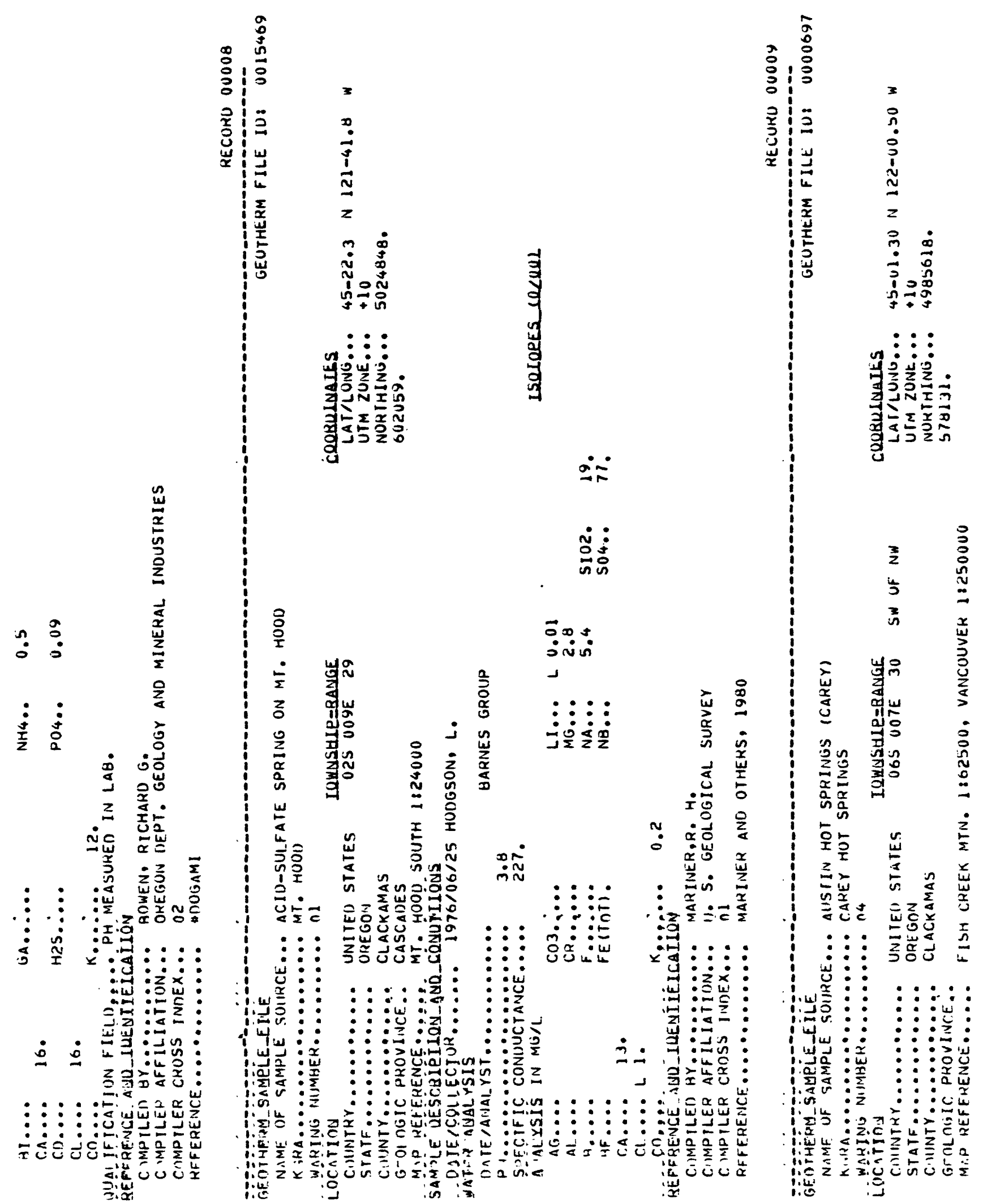




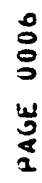
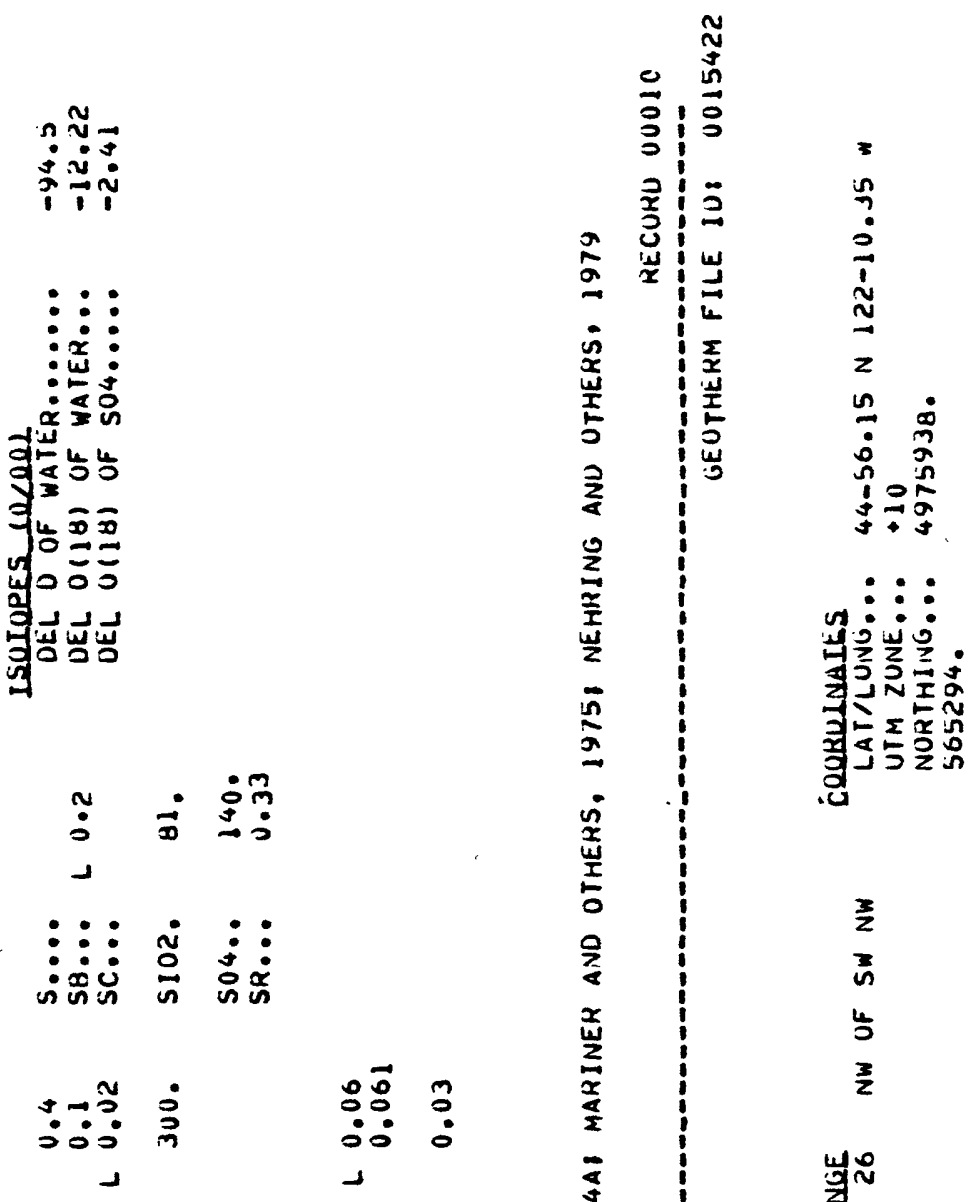

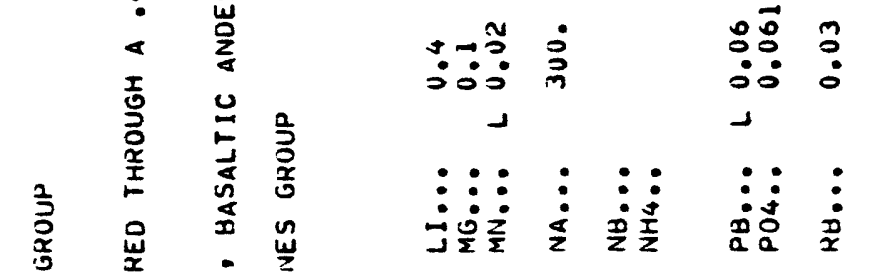

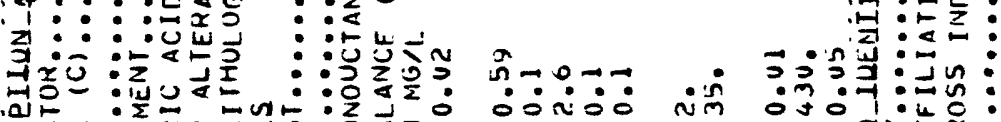

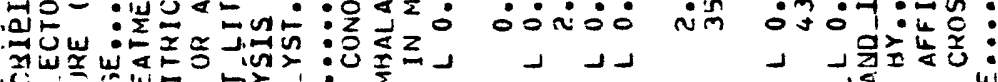

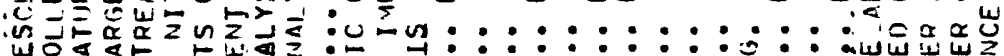

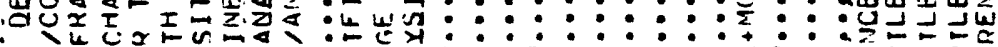

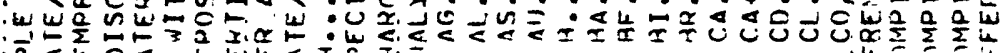

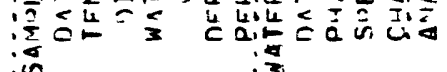


0
0
0
$y$
0
$a$
$a$

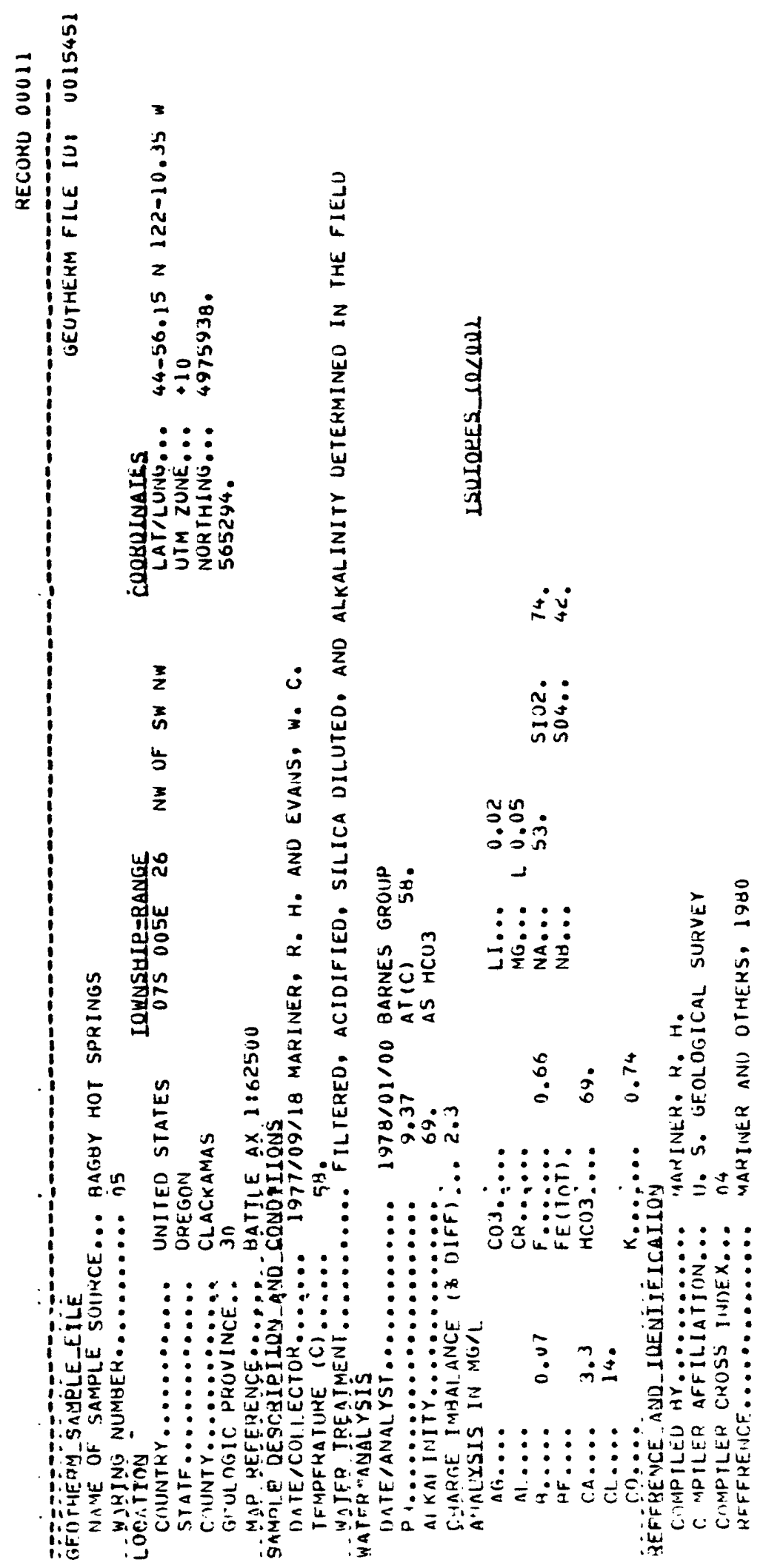




$$
\frac{200}{2}
$$
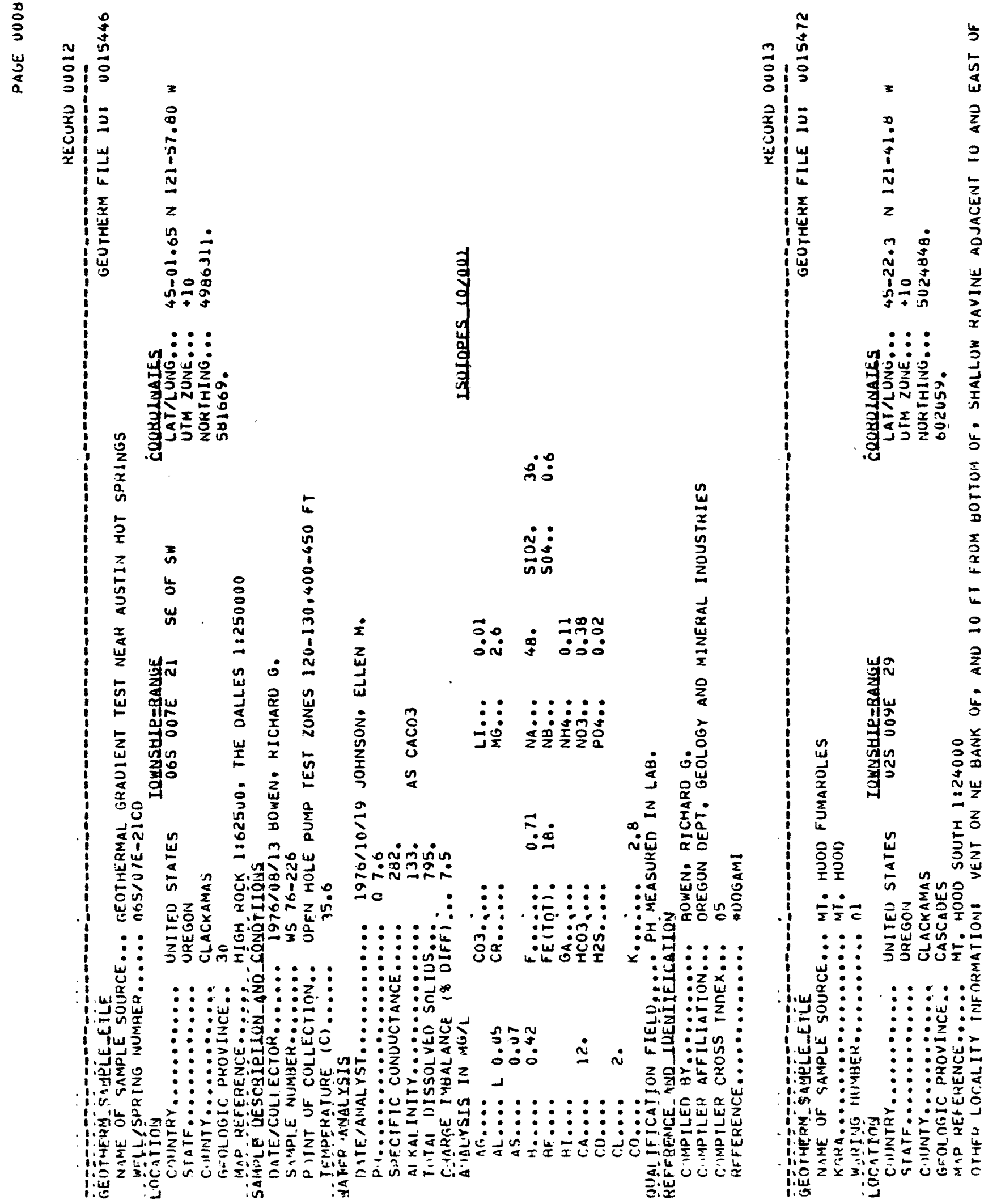

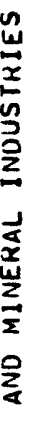

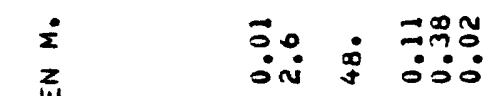

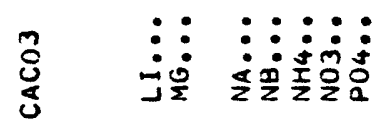

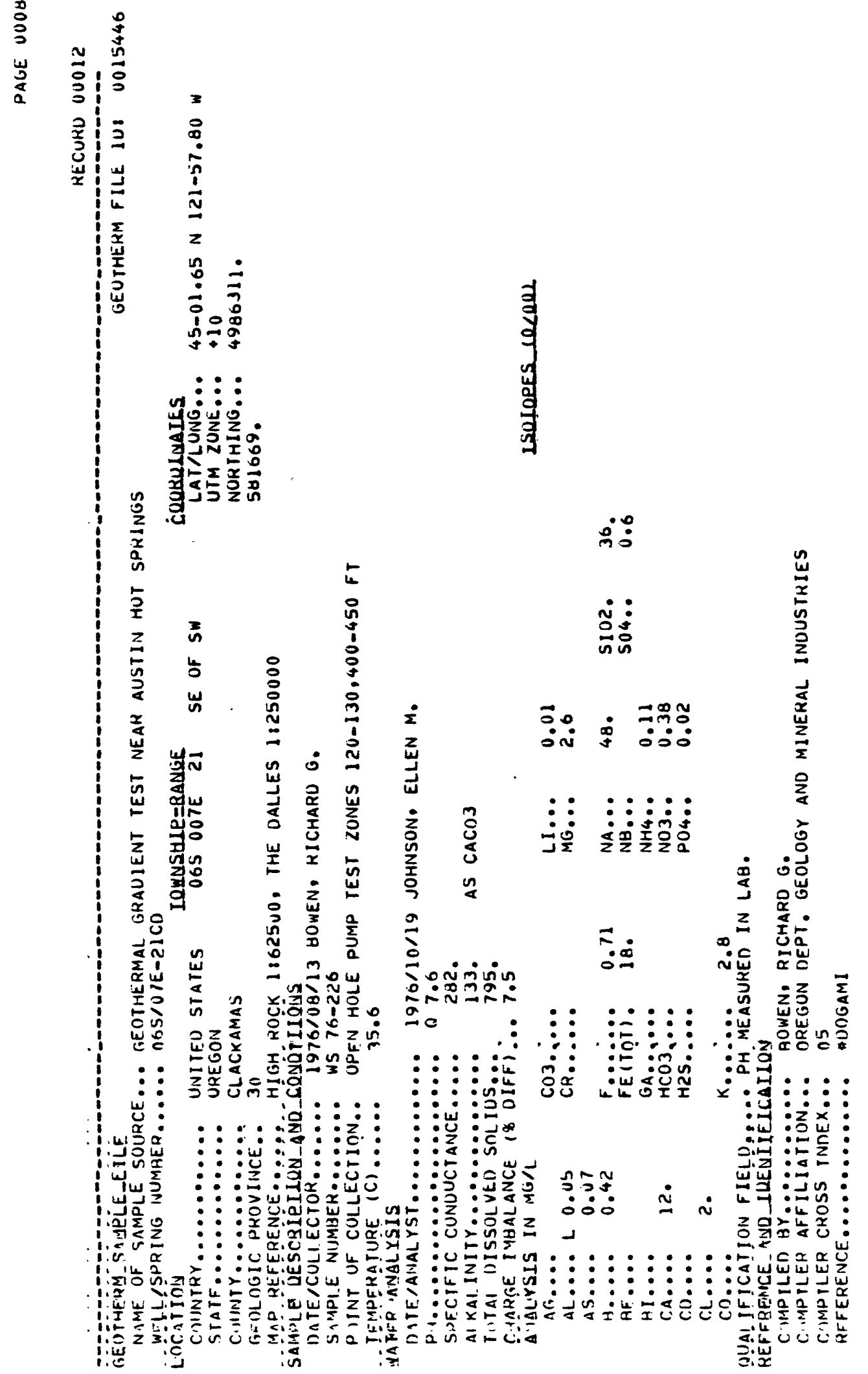

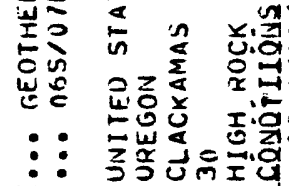
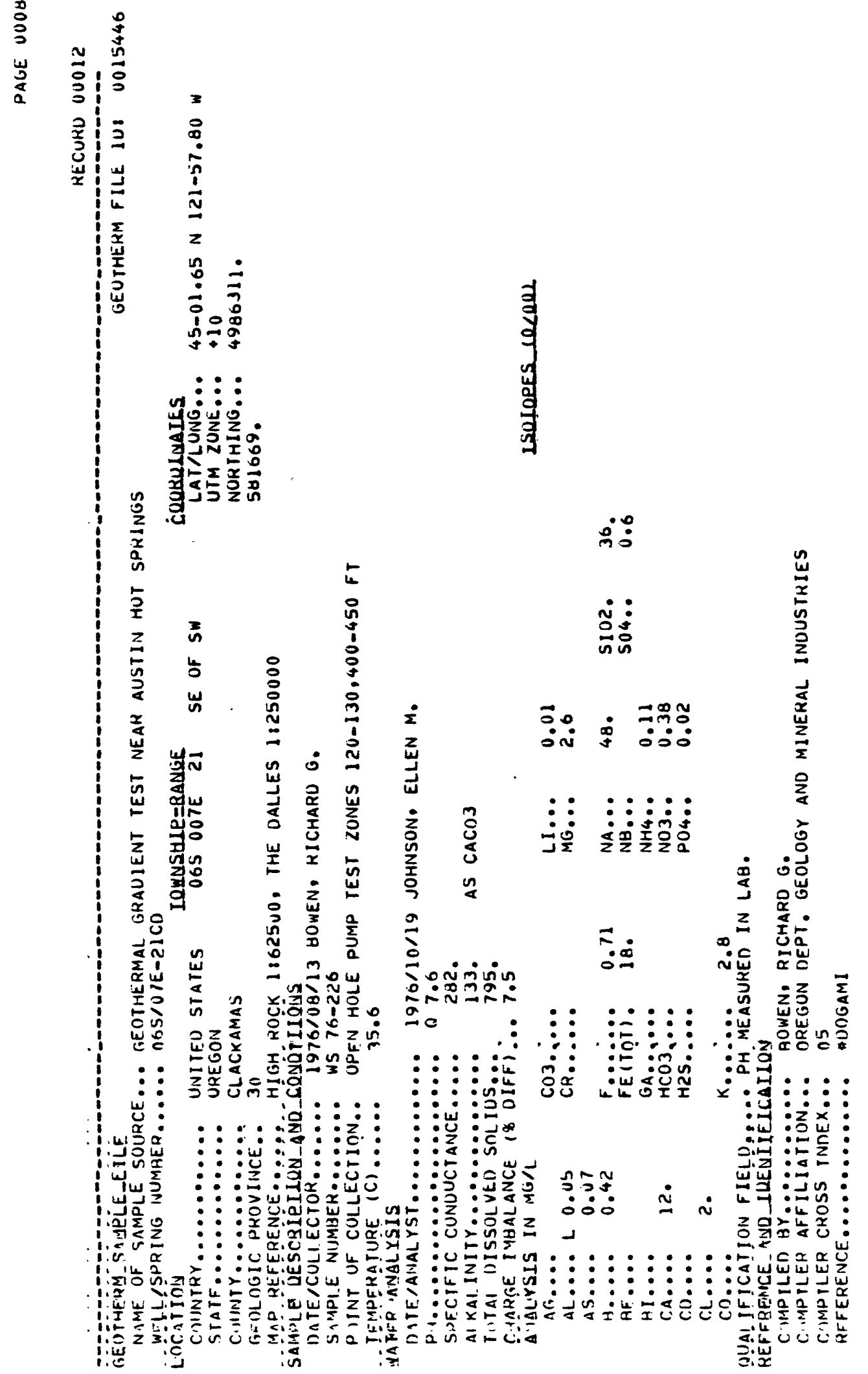

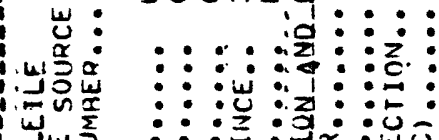

:

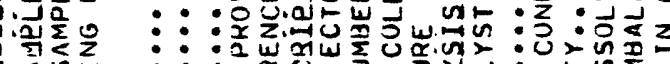

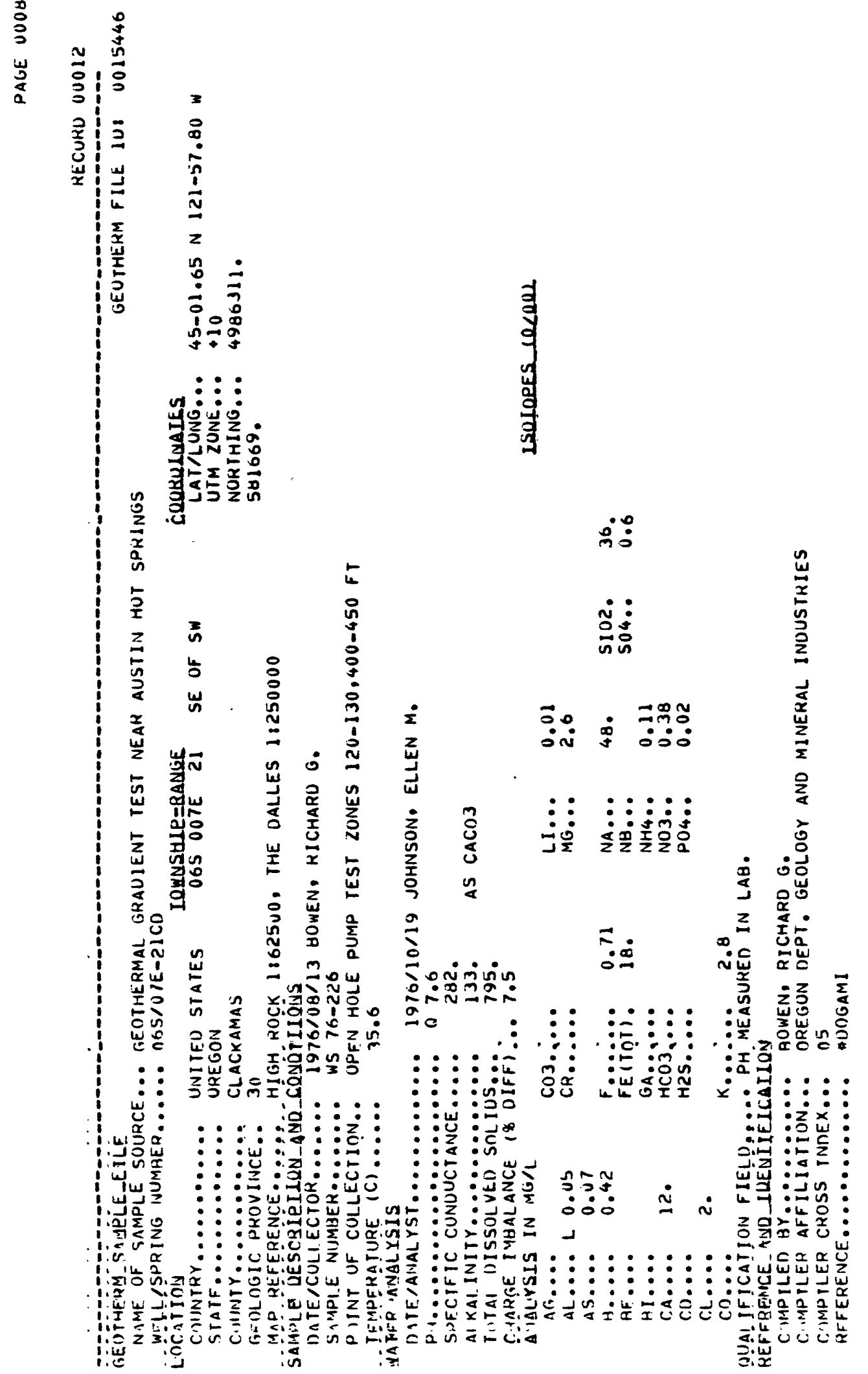

:

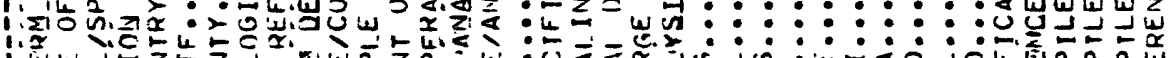

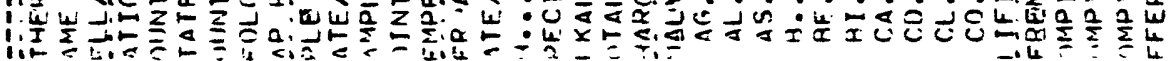

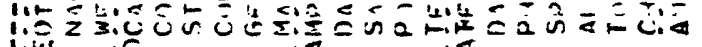
造记心 :

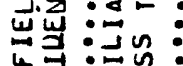
以ᄀ:

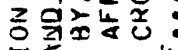

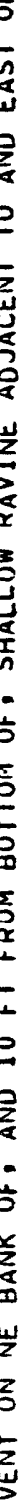

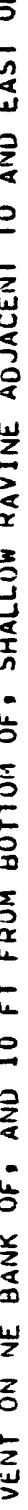

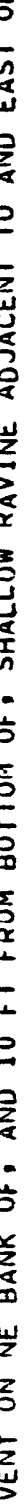

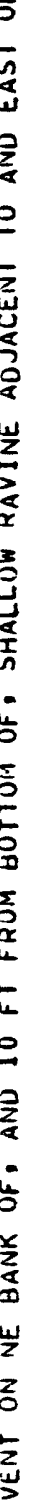

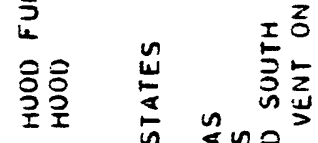

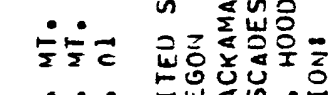

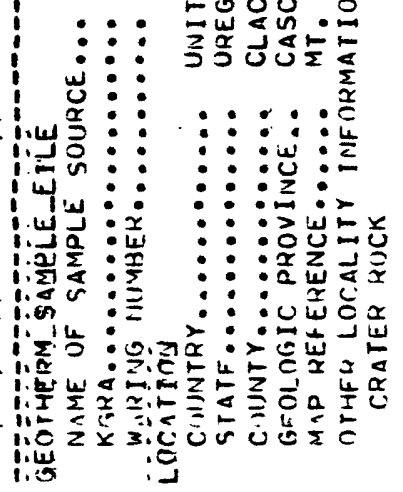


$\frac{1}{3}$

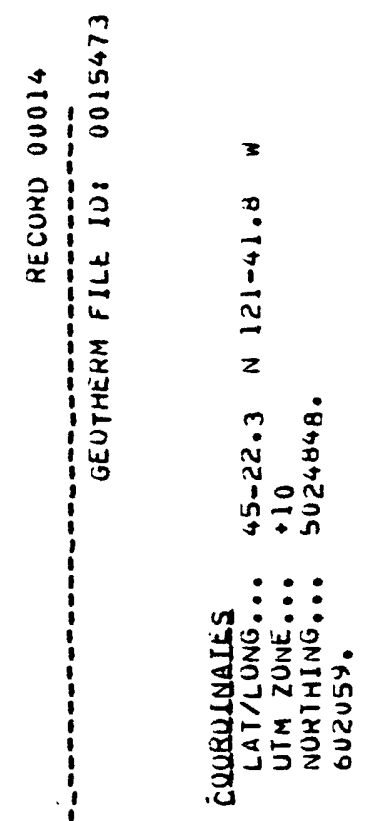

告

羿
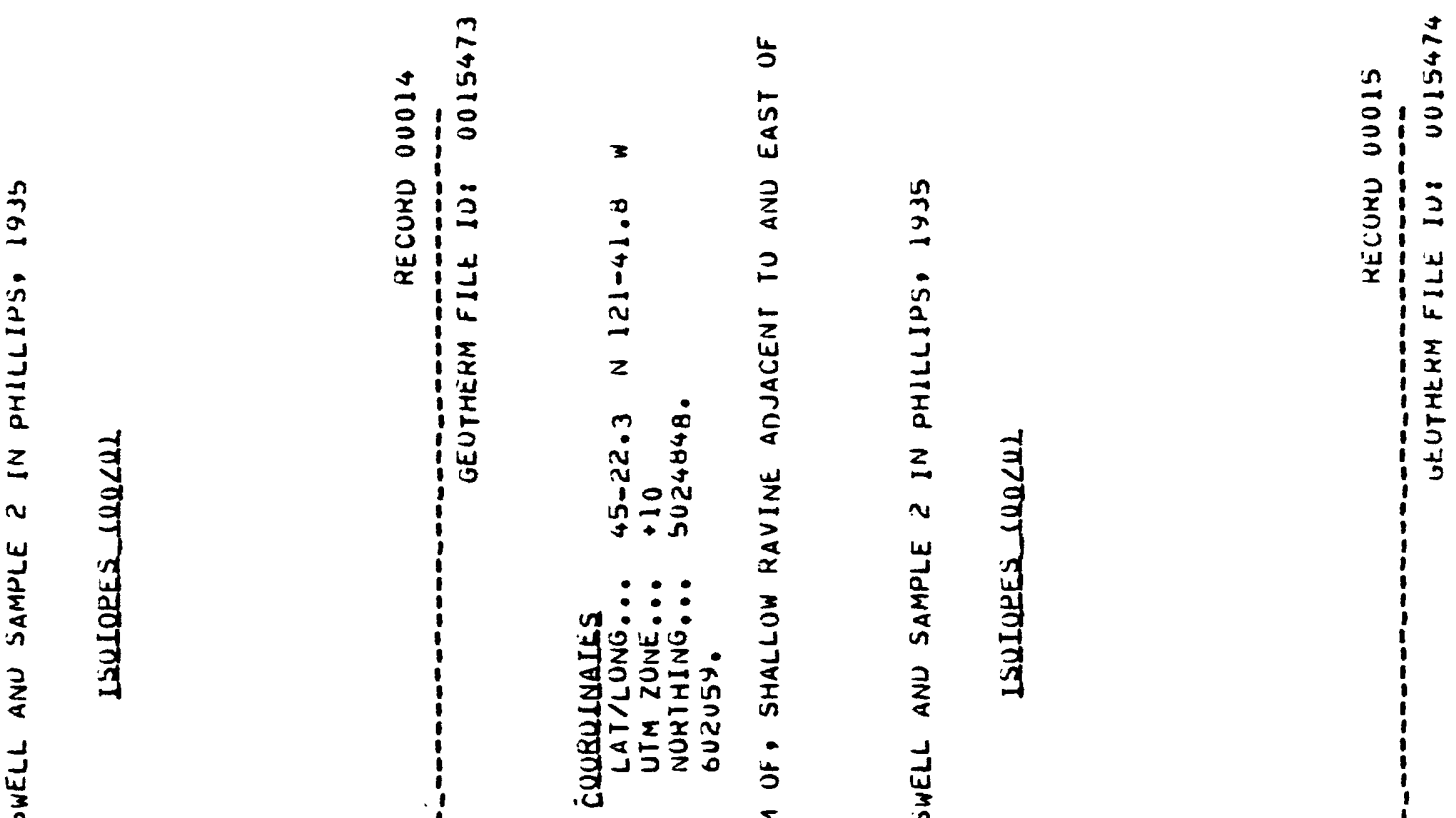

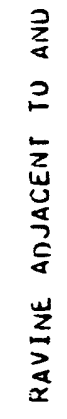

$\stackrel{n}{n}$

范

范宫

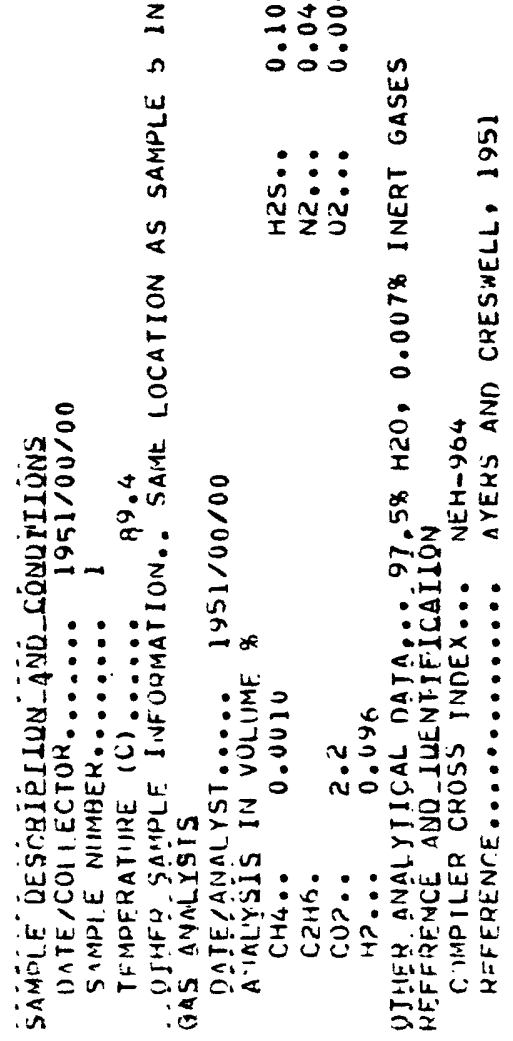

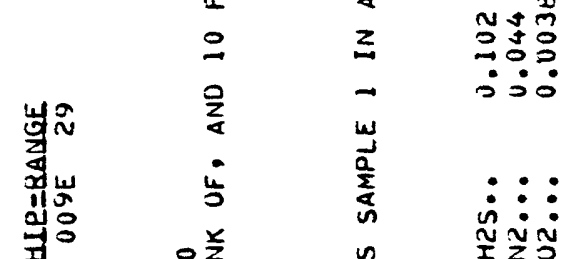

ن

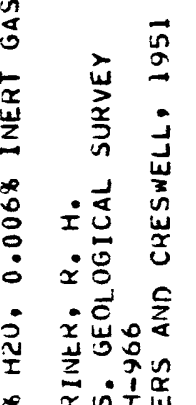

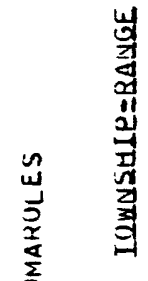

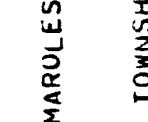

$\sum_{4}^{5}$

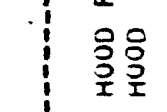

$\left\{\begin{array}{l}\text { 婄 } \\ : 0\end{array}\right.$

$\left\{\begin{array}{r}\vdots \\ \vdots \\ \vdots \\ \vdots \\ \vdots\end{array}\right.$

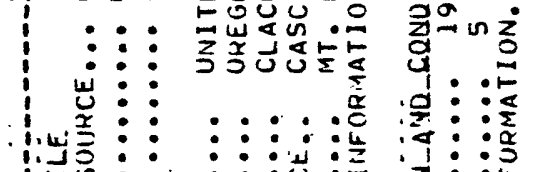

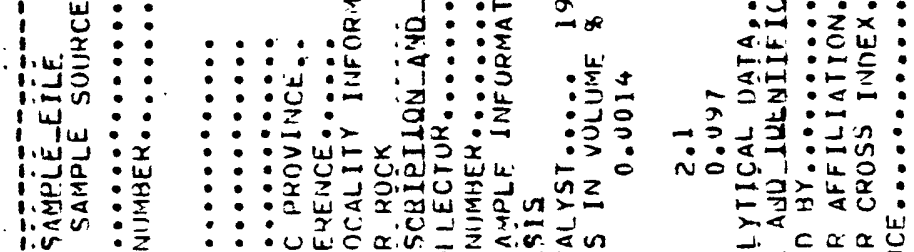
海: : : : : :

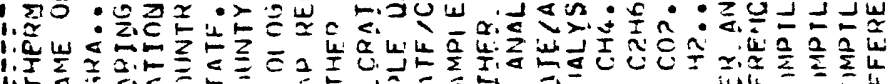

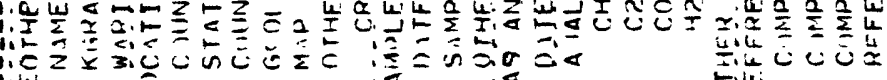
定 


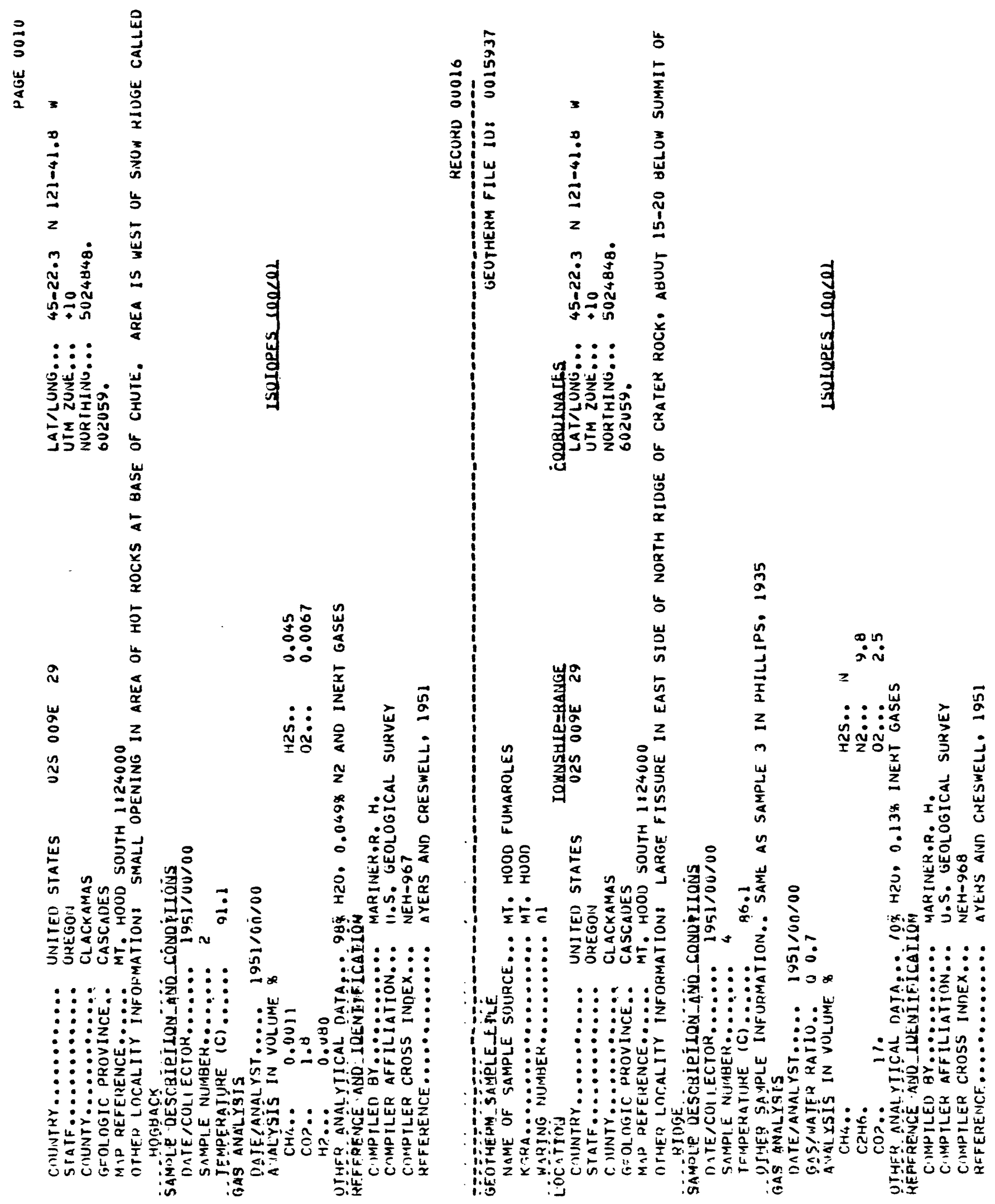


בa

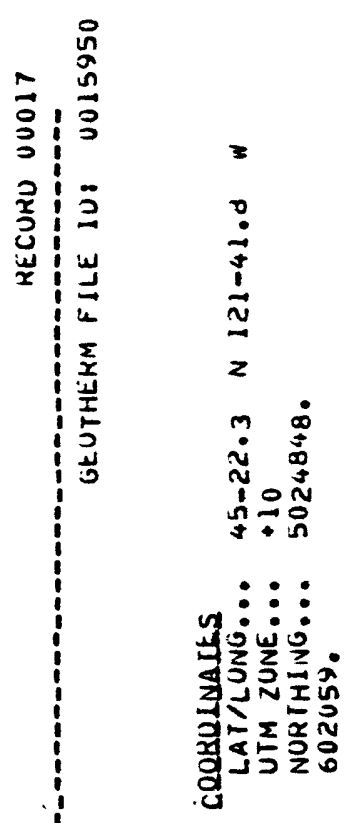

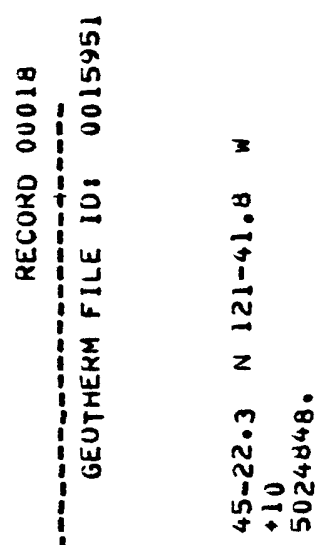

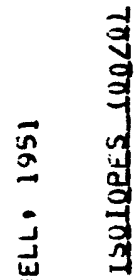

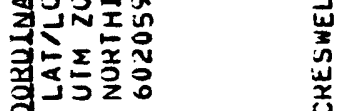

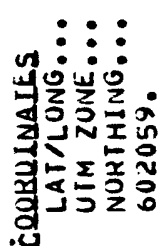

งิกิำ

००:

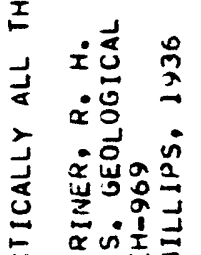
$0, \frac{1}{2}=\frac{1}{2}$ 종무

: $:$ : $::$

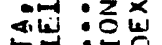
运:

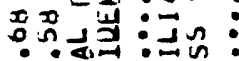
四象文出:

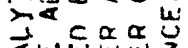

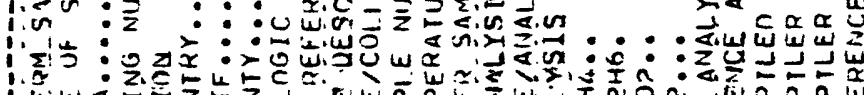

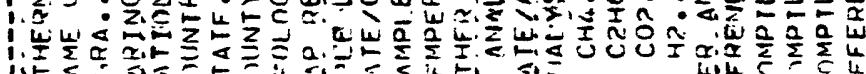

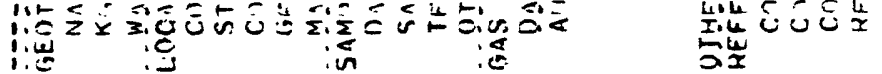




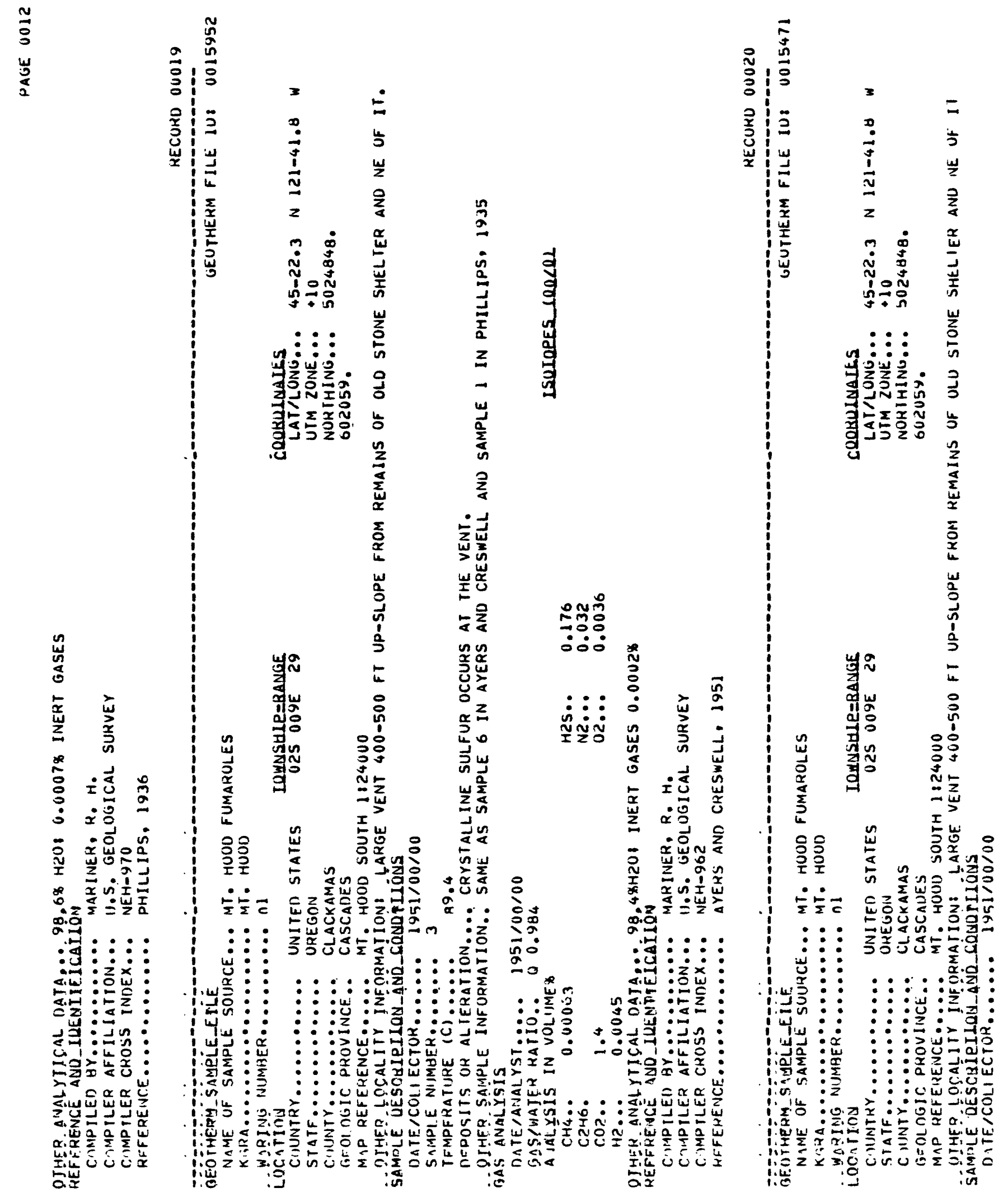


$m$
0
0
0
0
2

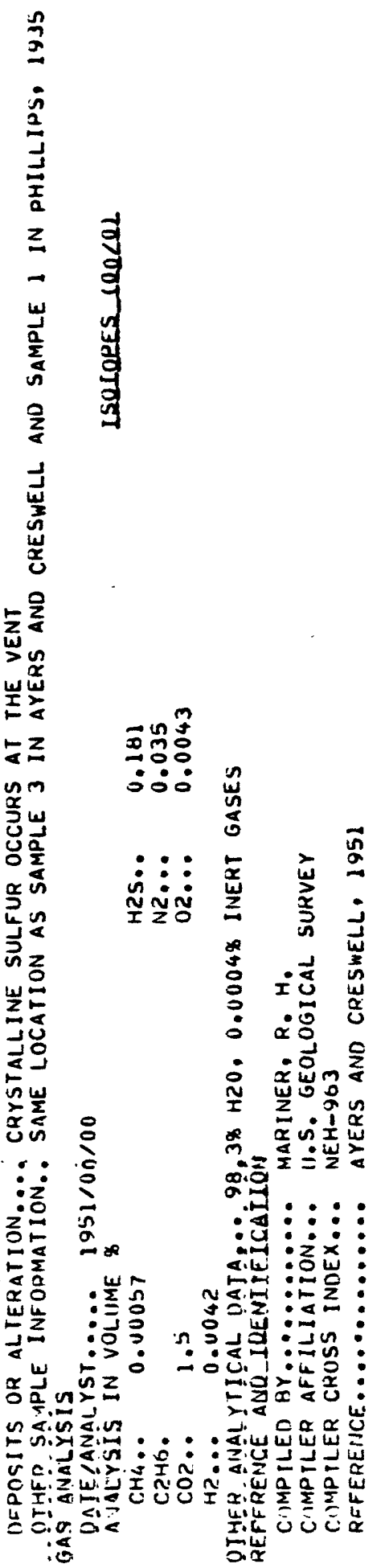

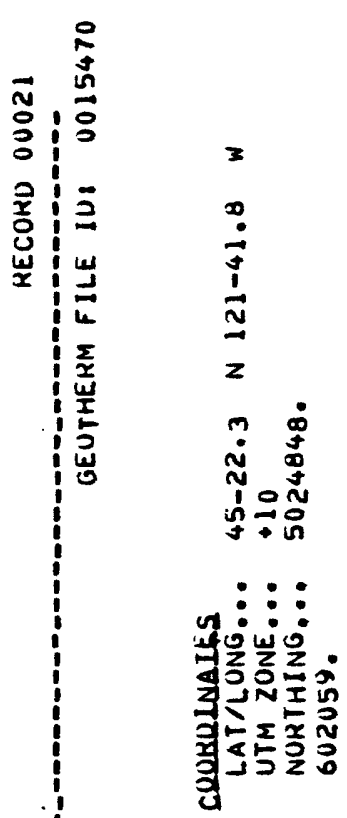

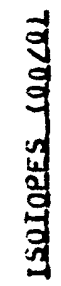

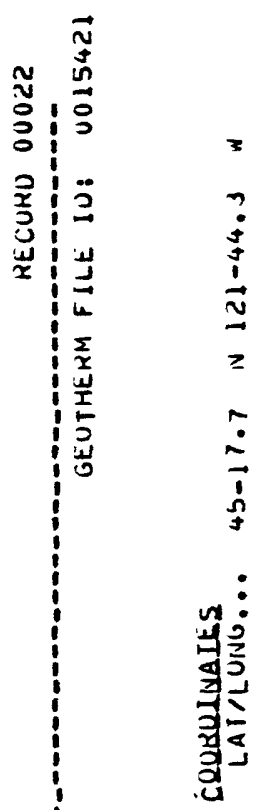

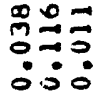

耑

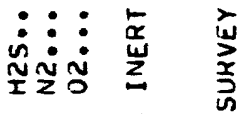
落芯

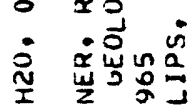

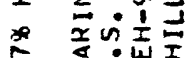

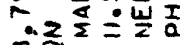
s.:

: $::::$ an:

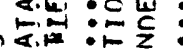
móz: : $\because$ 岂: :

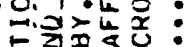

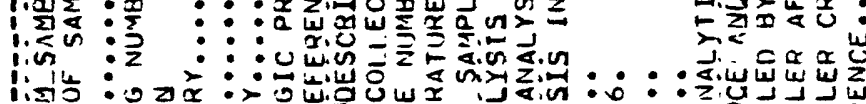

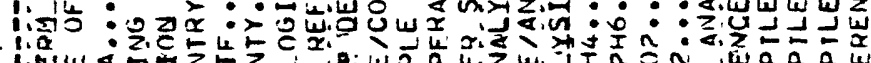

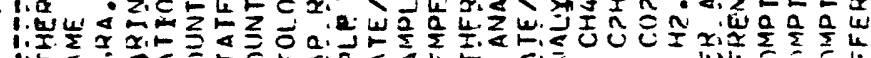

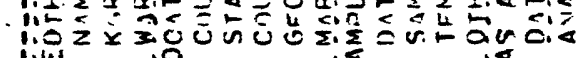
i.: 


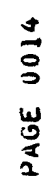

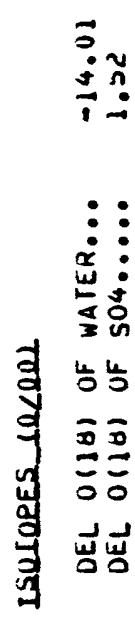

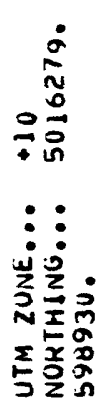

:

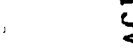

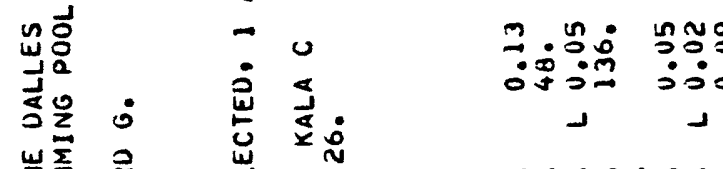

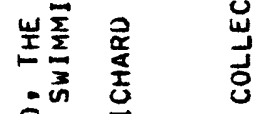

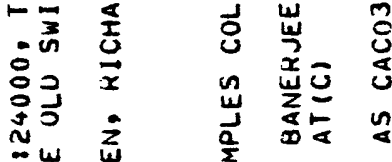

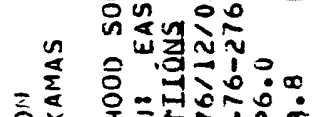

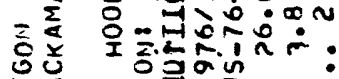

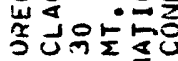

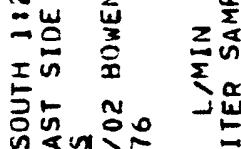

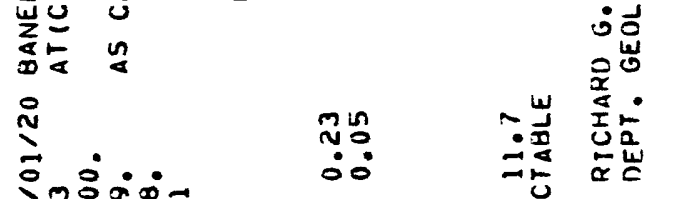

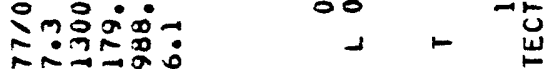

a

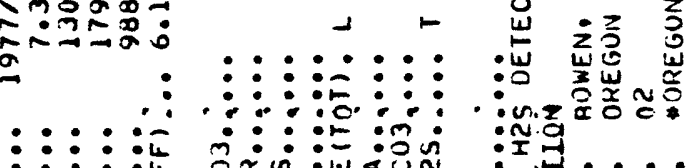

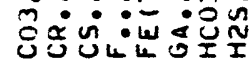

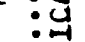

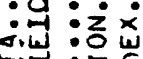

,

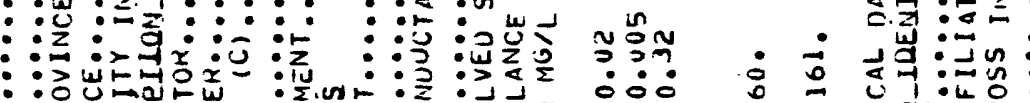

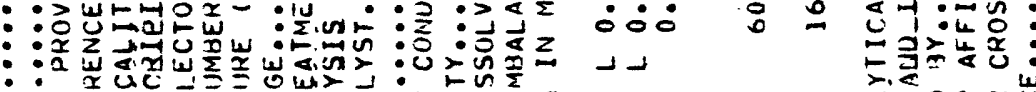

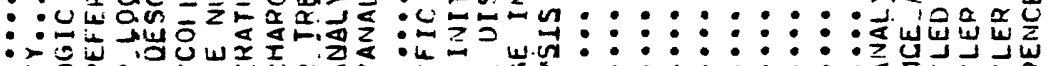

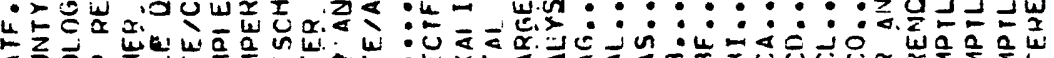

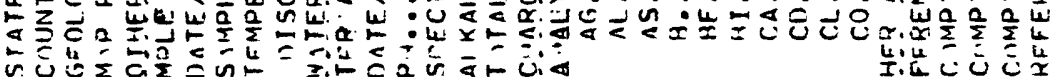

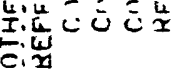

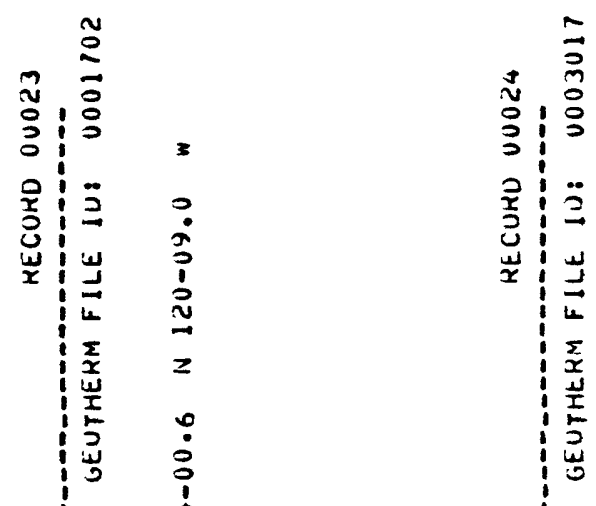

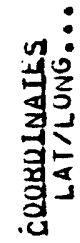

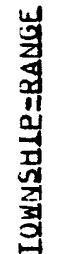

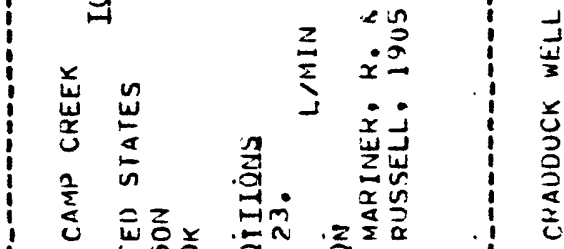

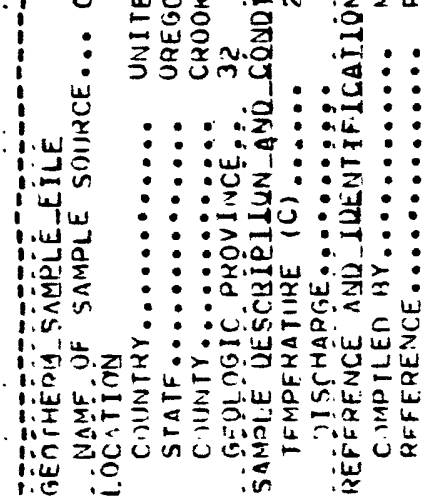



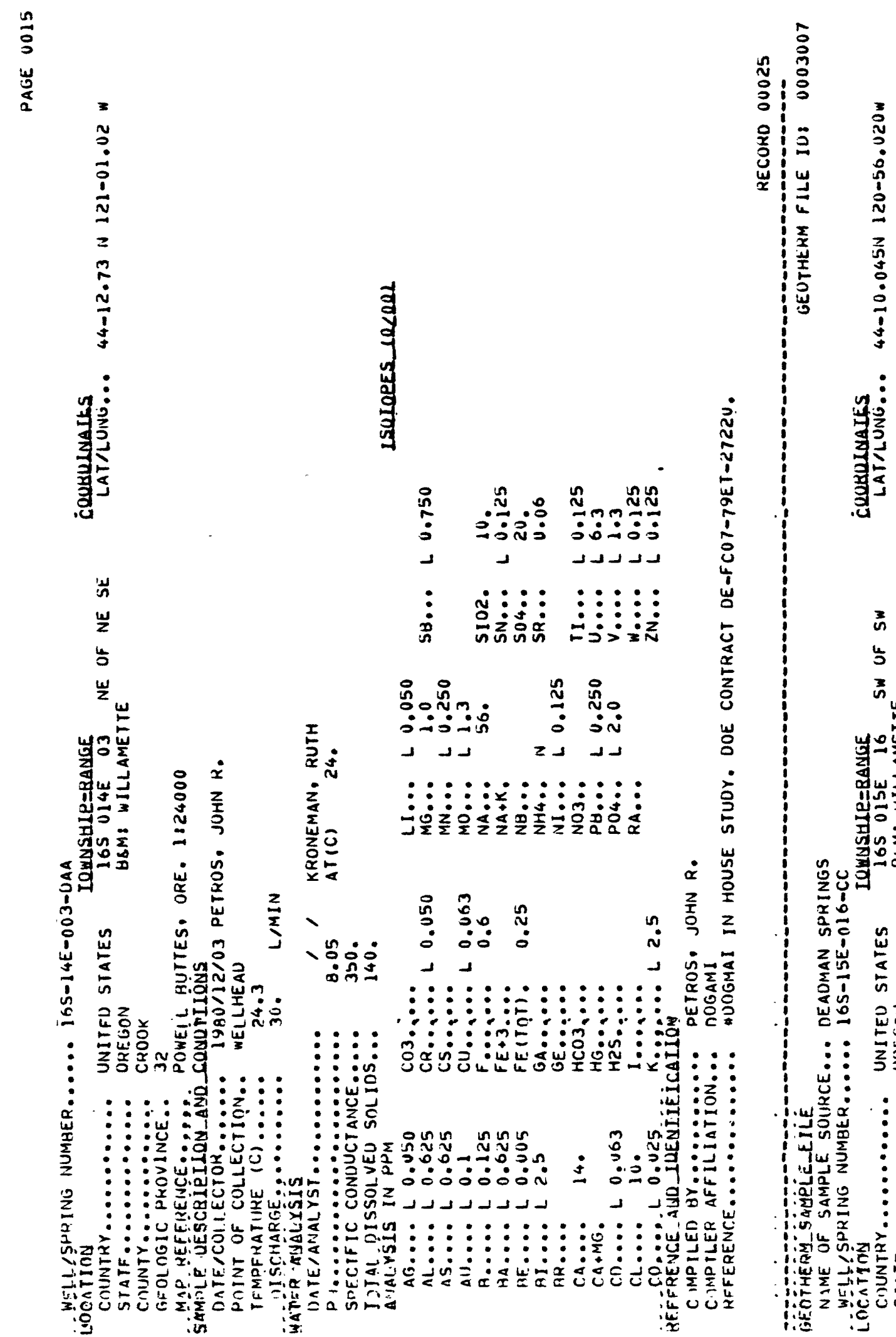


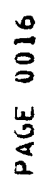

菻

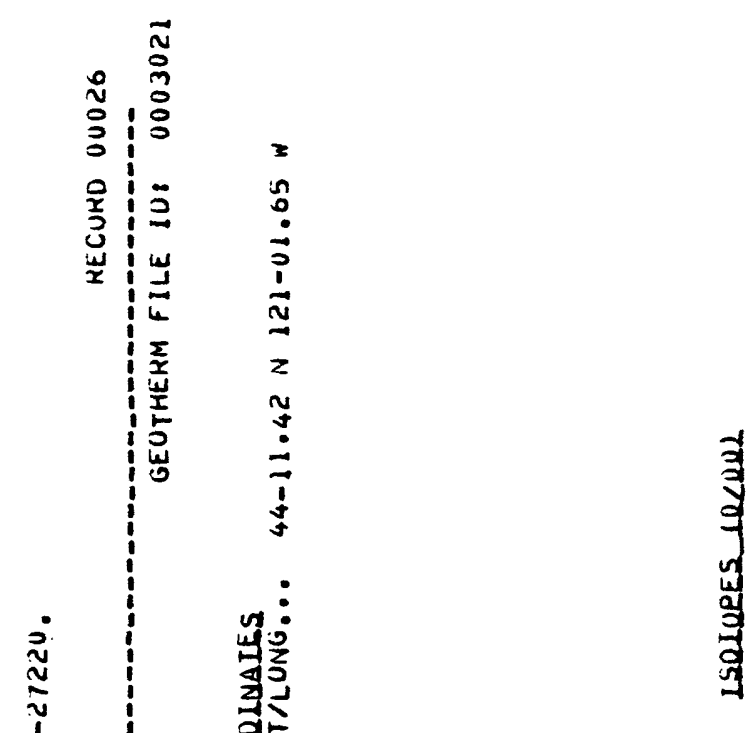

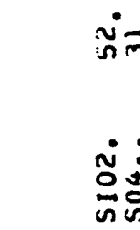

כึ:คำ

$\checkmark$

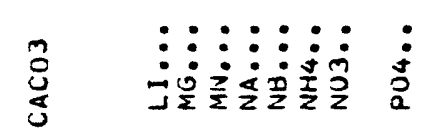

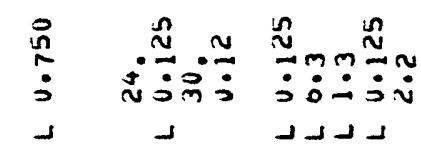

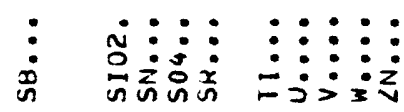

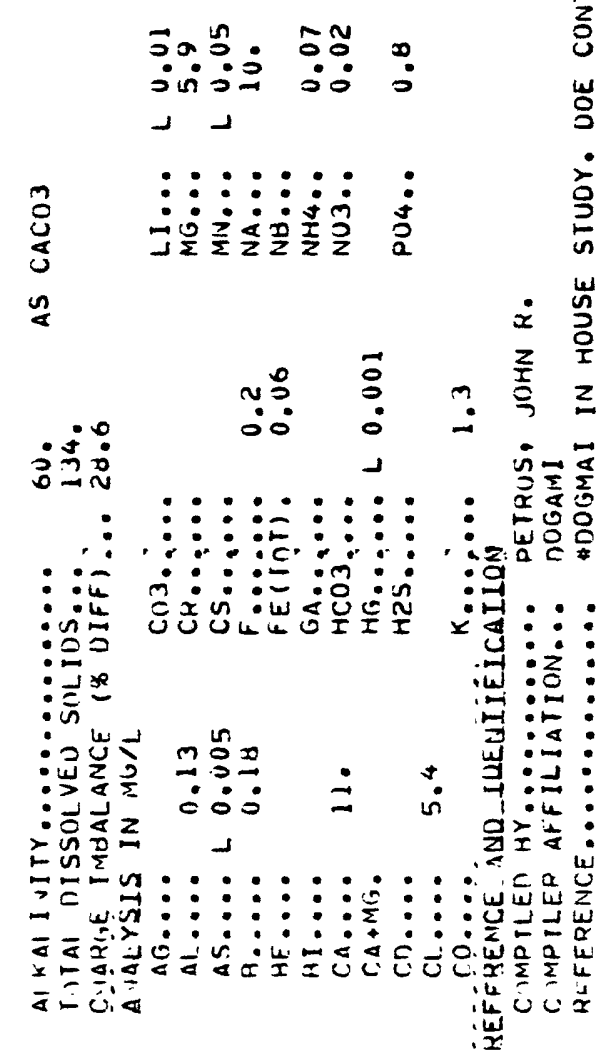

¿

屴

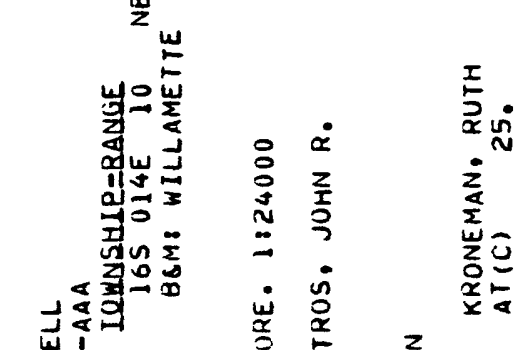

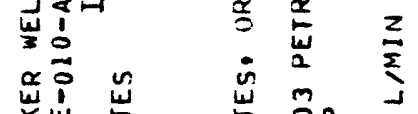

岁岁

w

: :

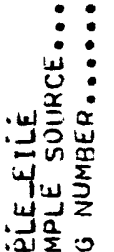

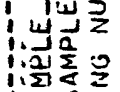

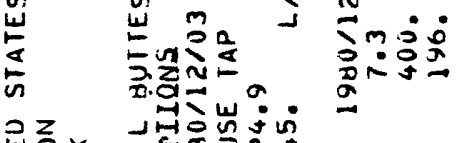

i.

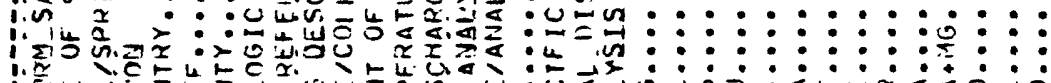

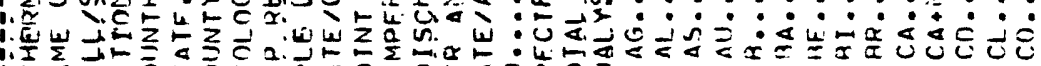

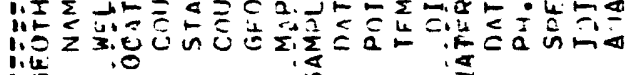



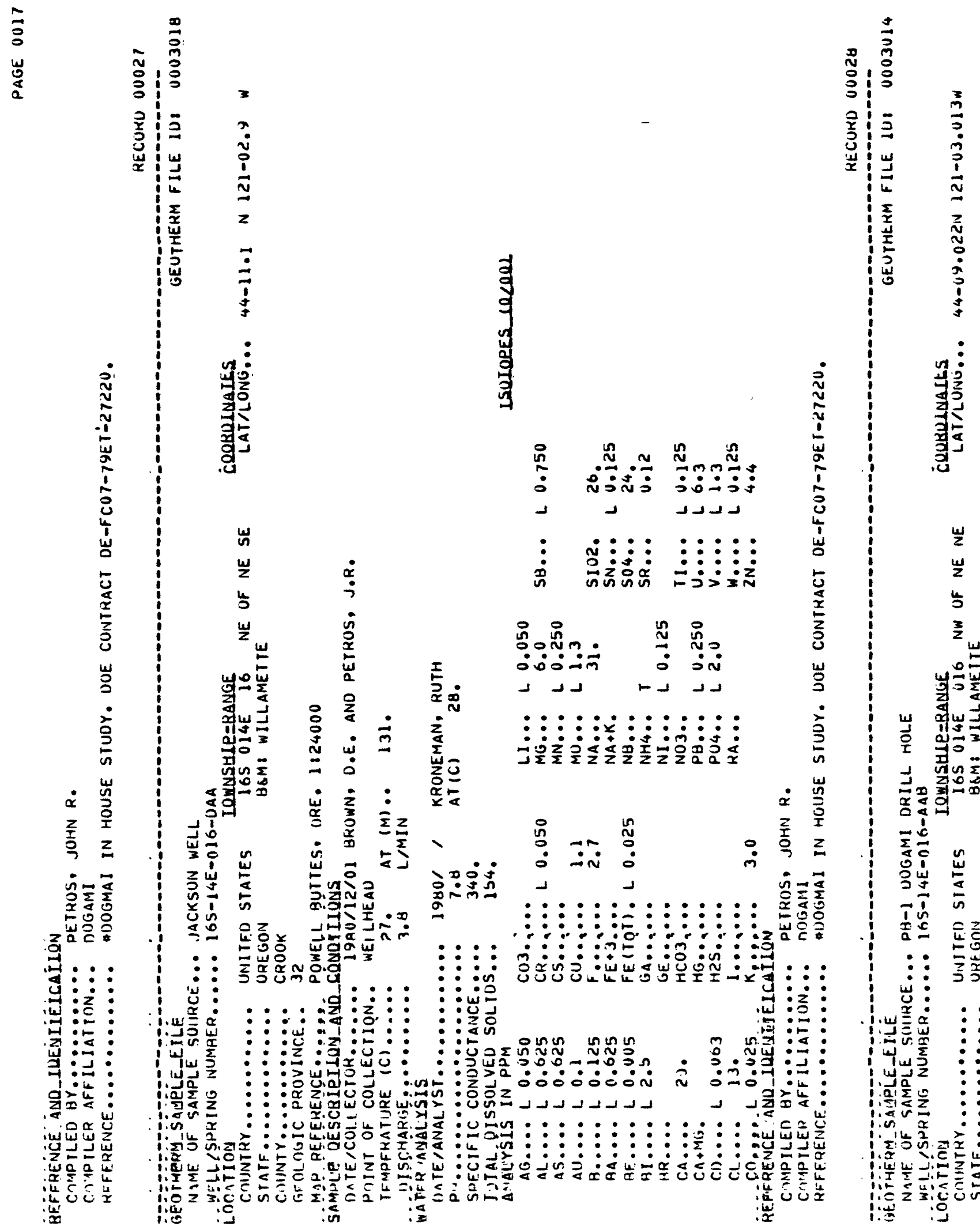
ถัก

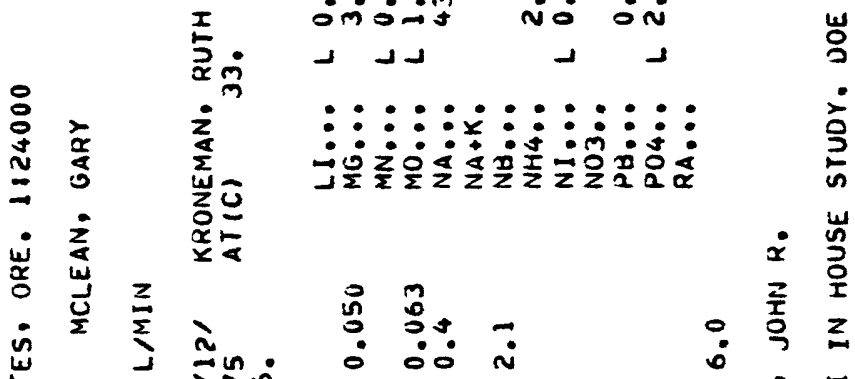

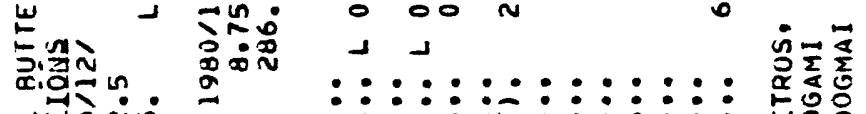

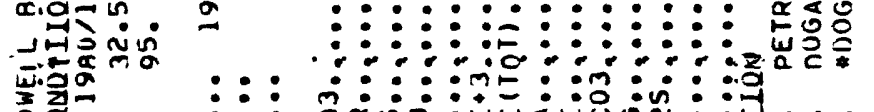
a.व्व $:::$

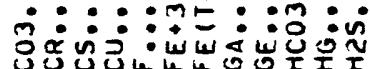

: : : : :

:웡 $: \vdots: \vdots:$ :

:

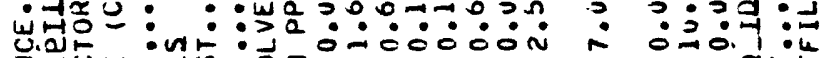

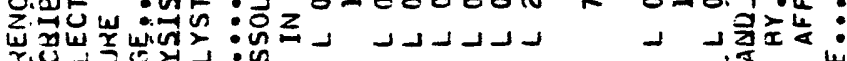

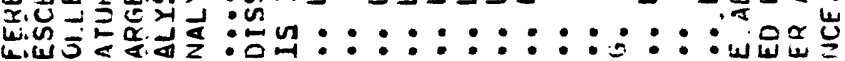

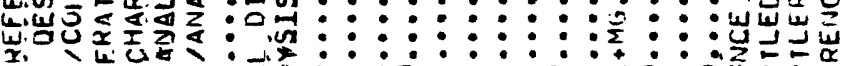
T,

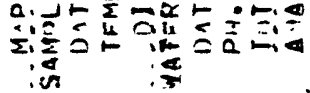
:

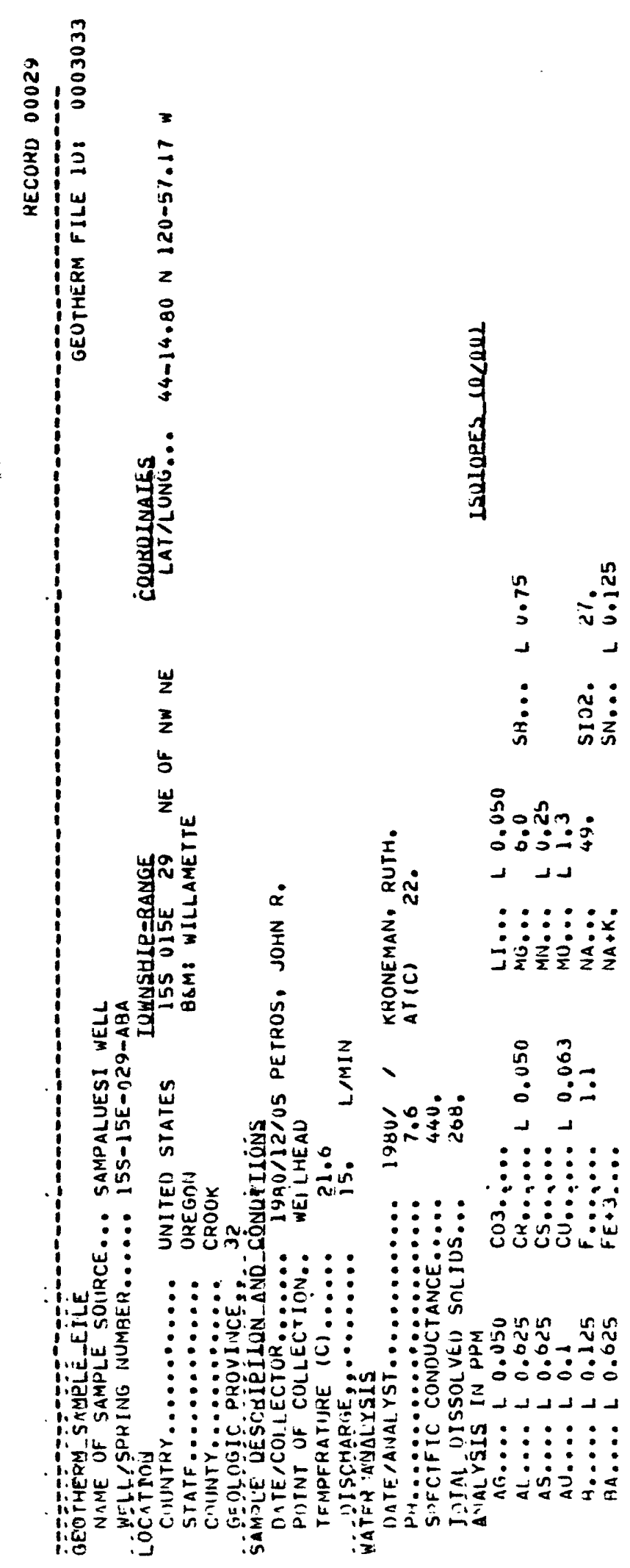


$\frac{a}{8}$
$\frac{\omega}{0}$
$a$
$a$

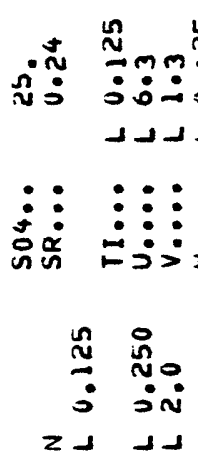

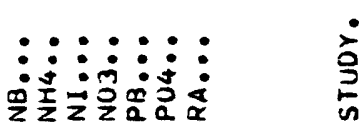

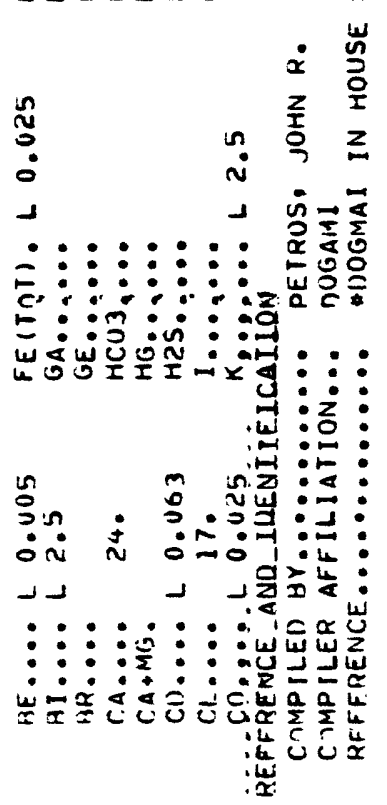

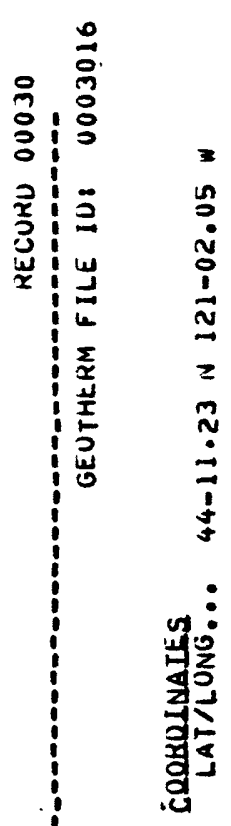

aี

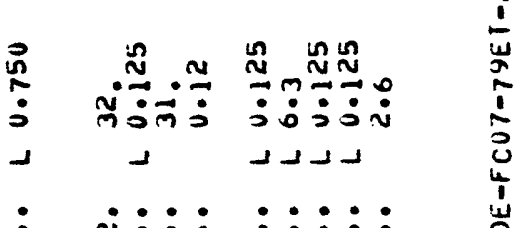

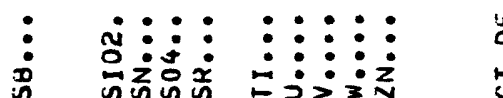

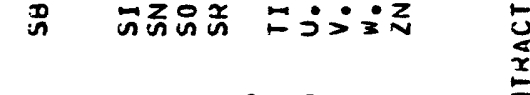

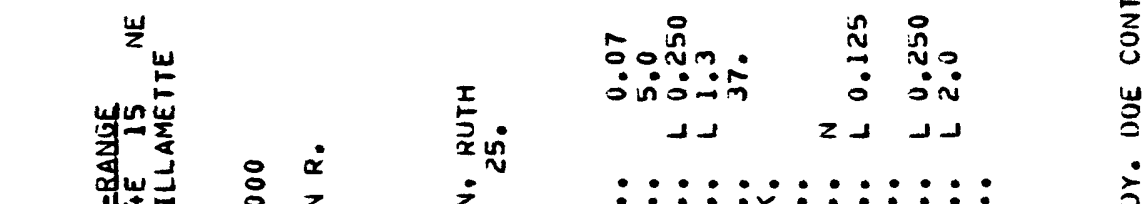

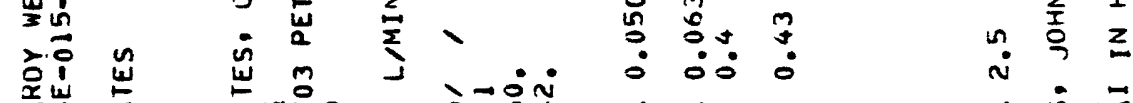

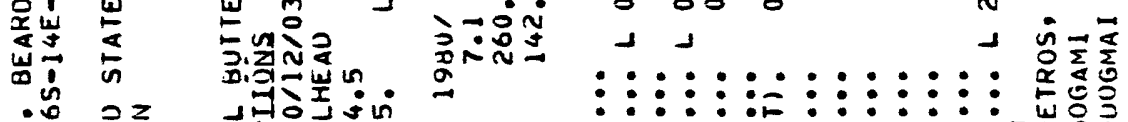

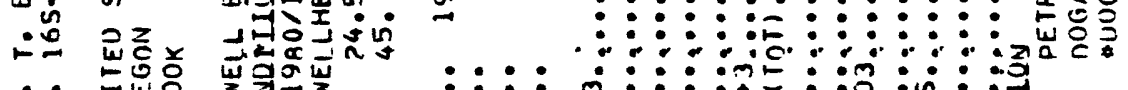

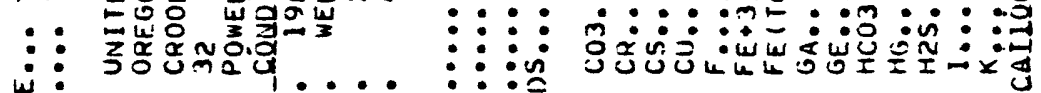

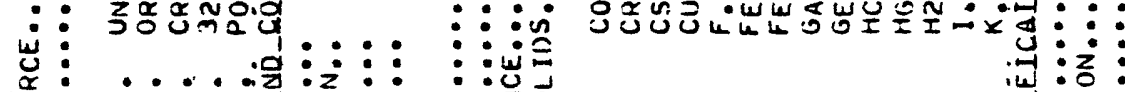
:

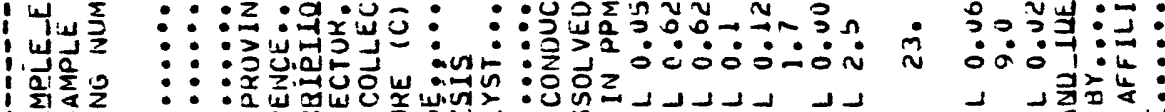

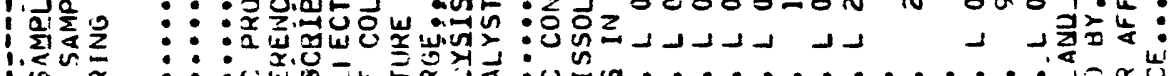

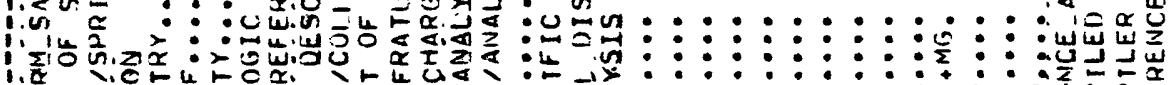

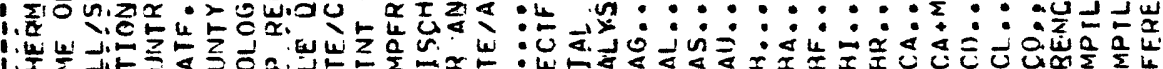

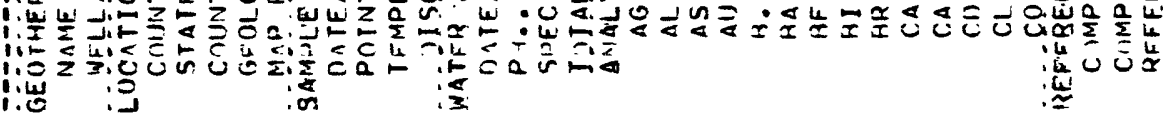



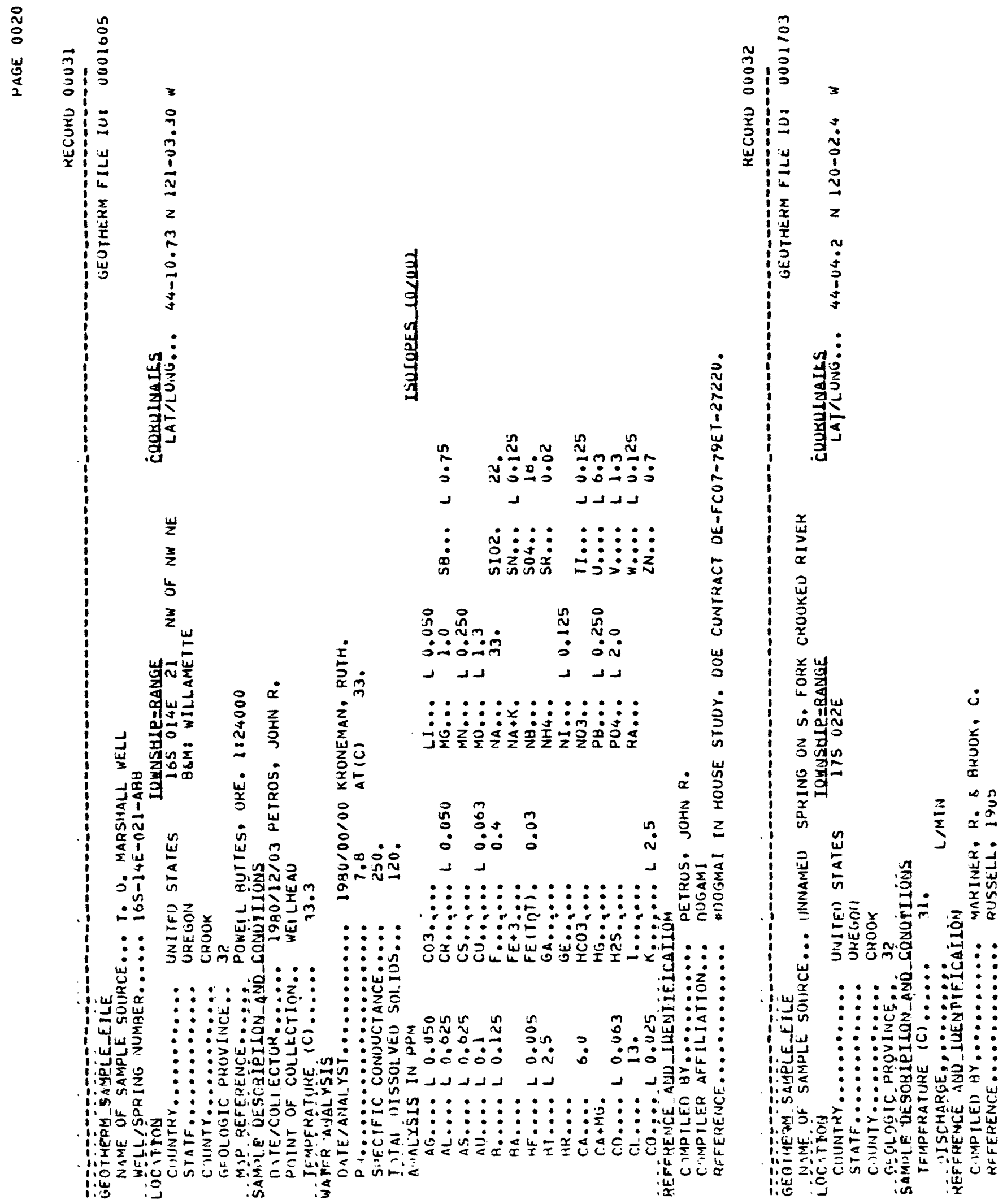


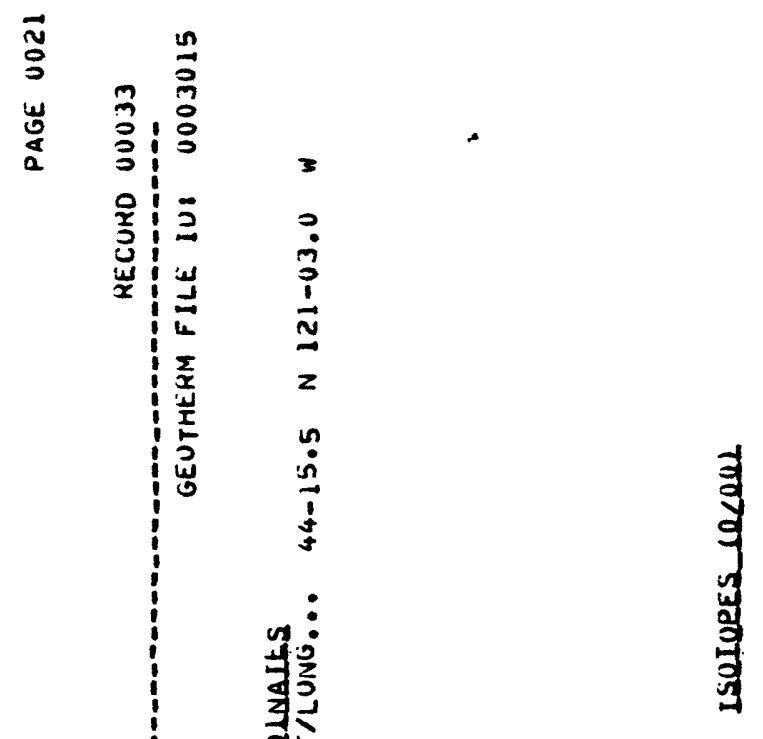

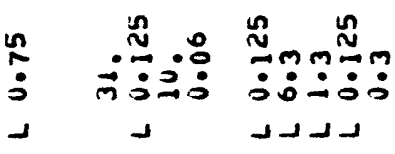

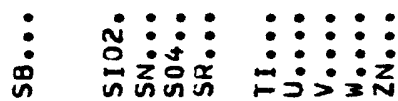

o

5

옹
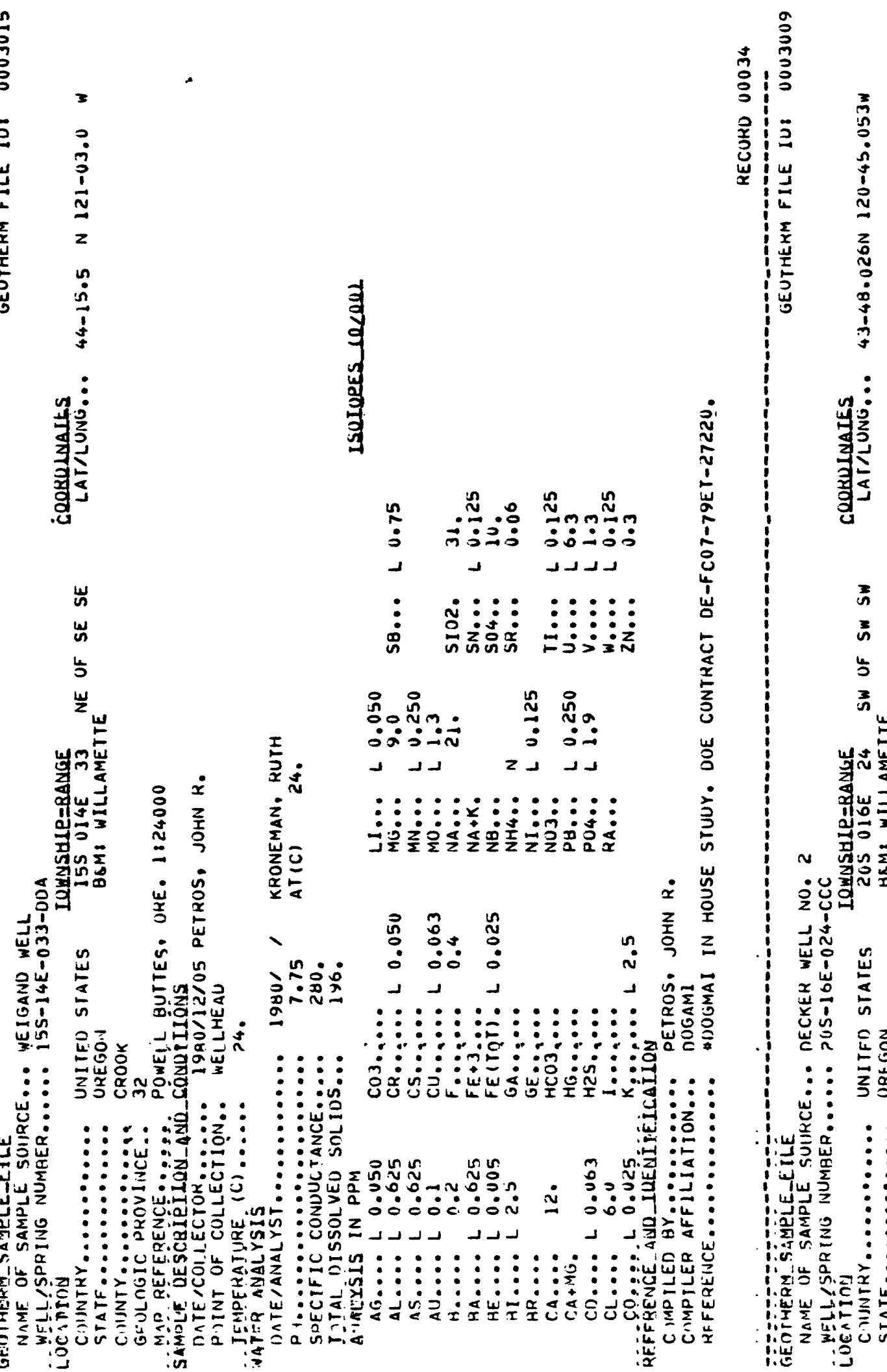

山s

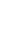




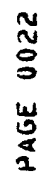

范

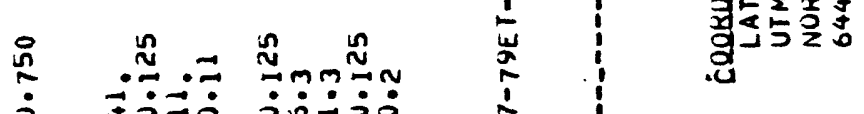

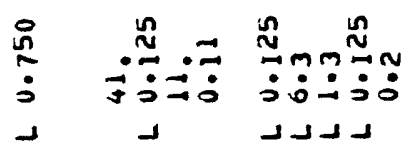

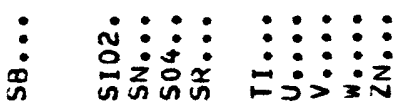

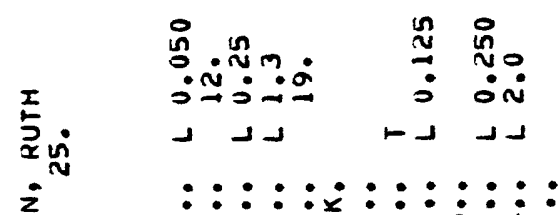

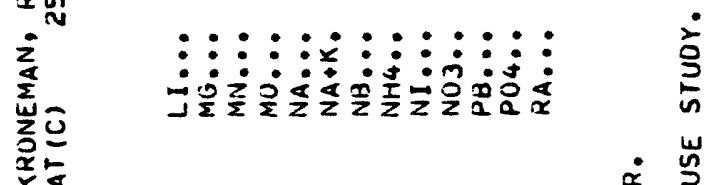

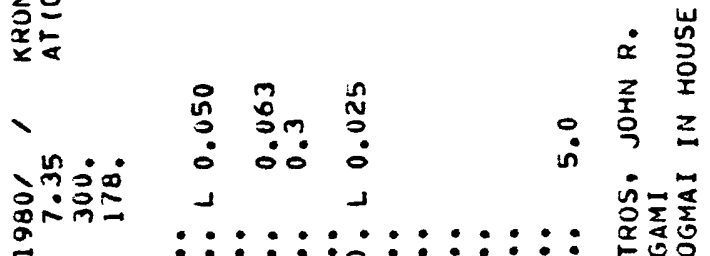
$:: \vdots: \vdots:$ : : : : : : : : : $:::$ :

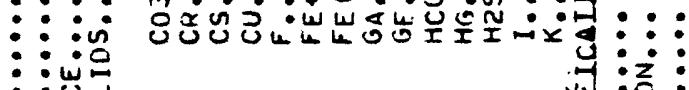
$:: 0$

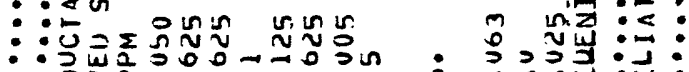
U: $:$

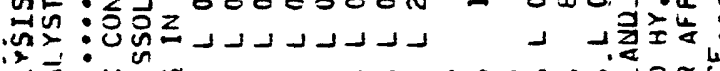

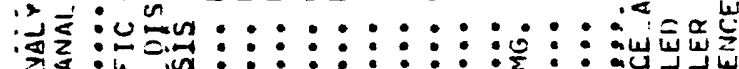

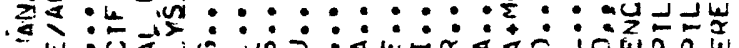

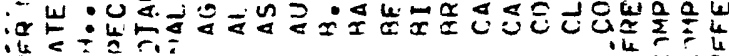

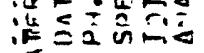
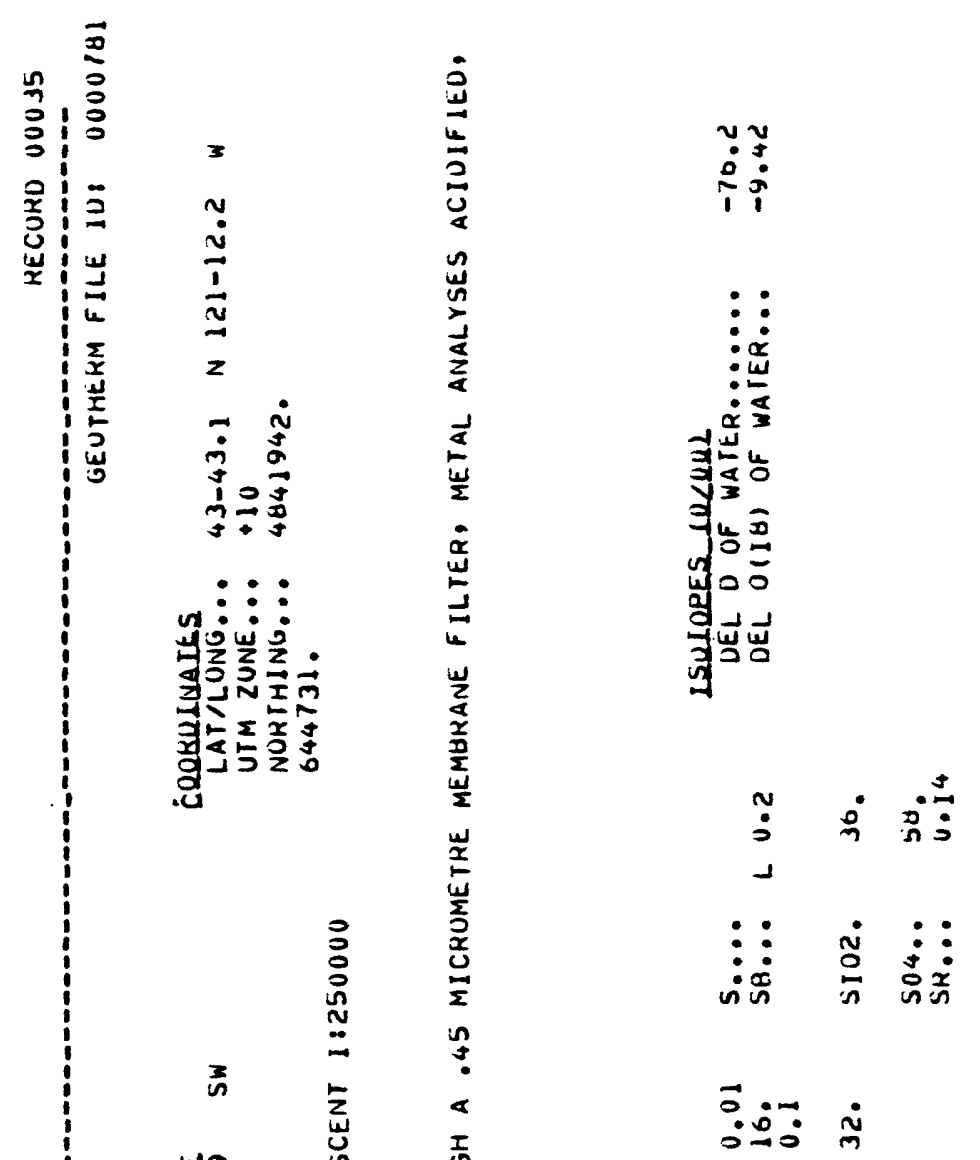

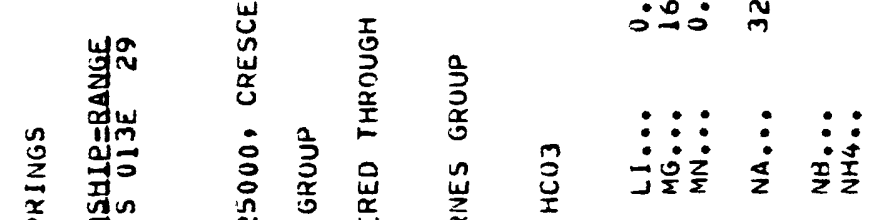

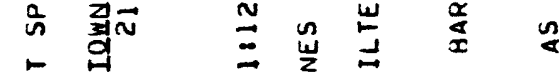

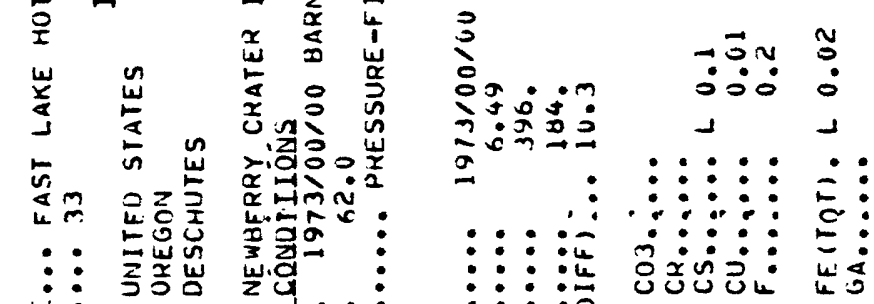
:

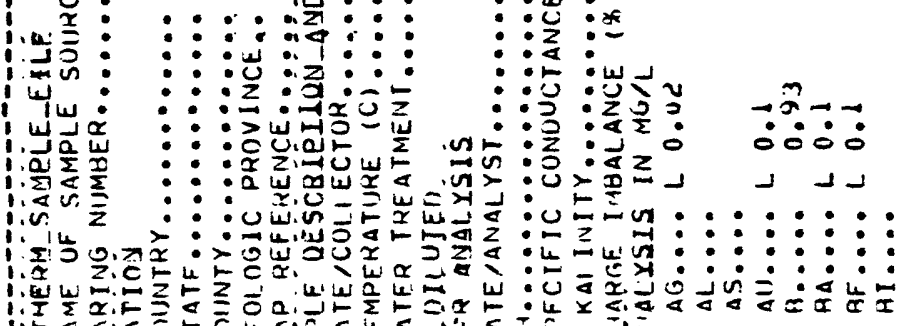

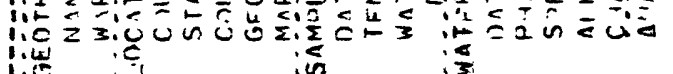




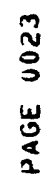

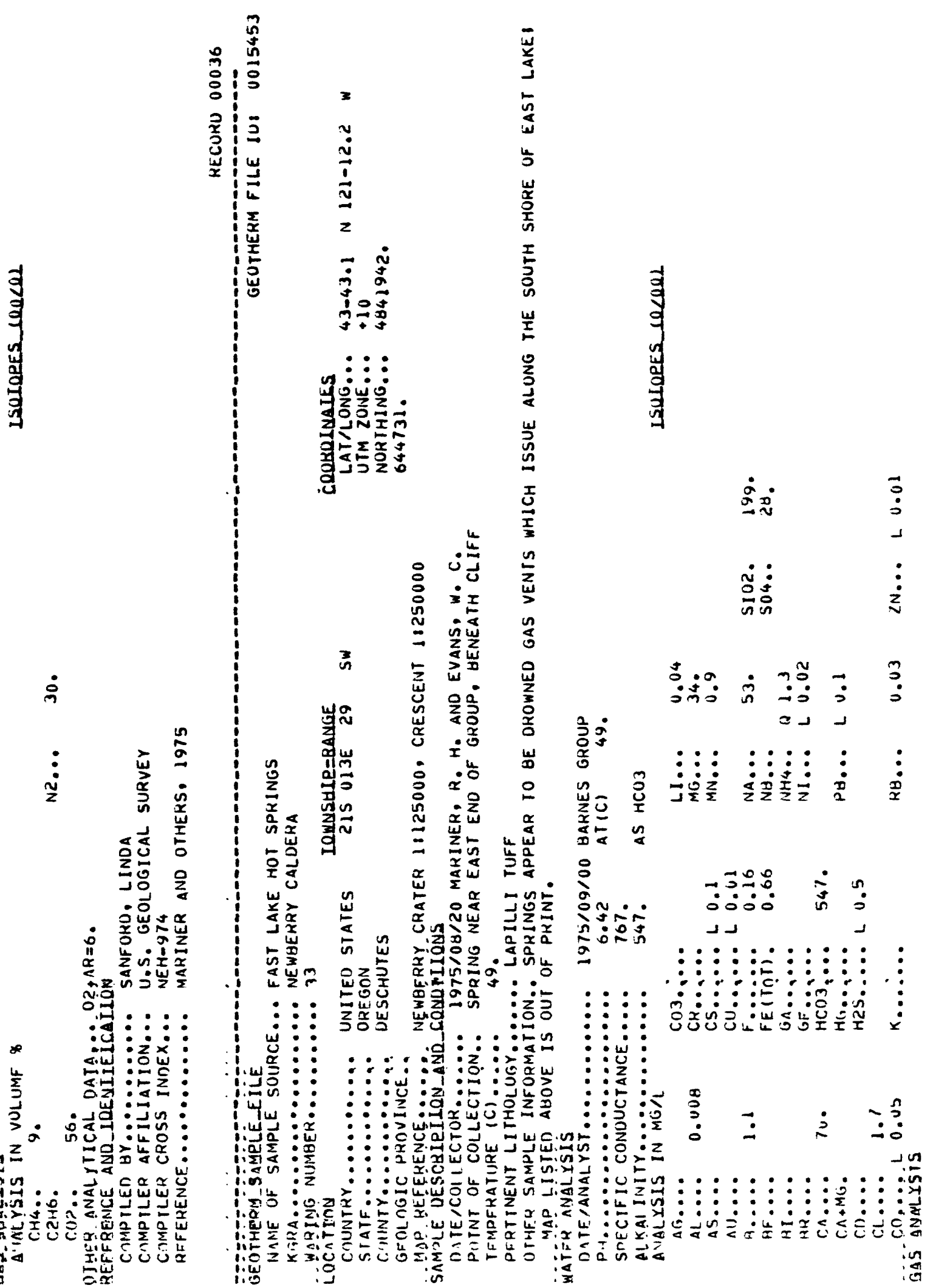


\begin{tabular}{l}
\multirow{2}{0}{} \\
0 \\
0 \\
0 \\
0 \\
0
\end{tabular}
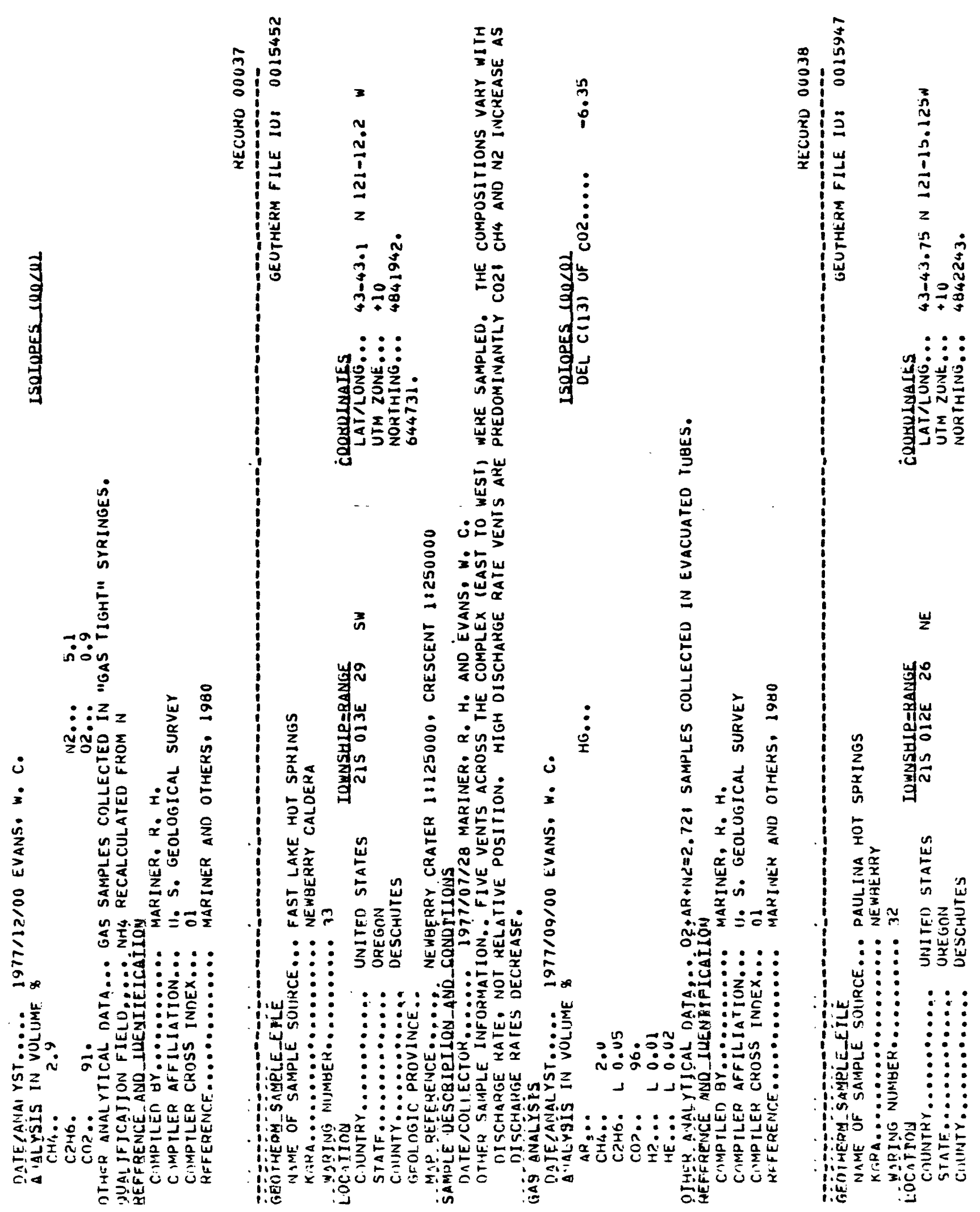
密
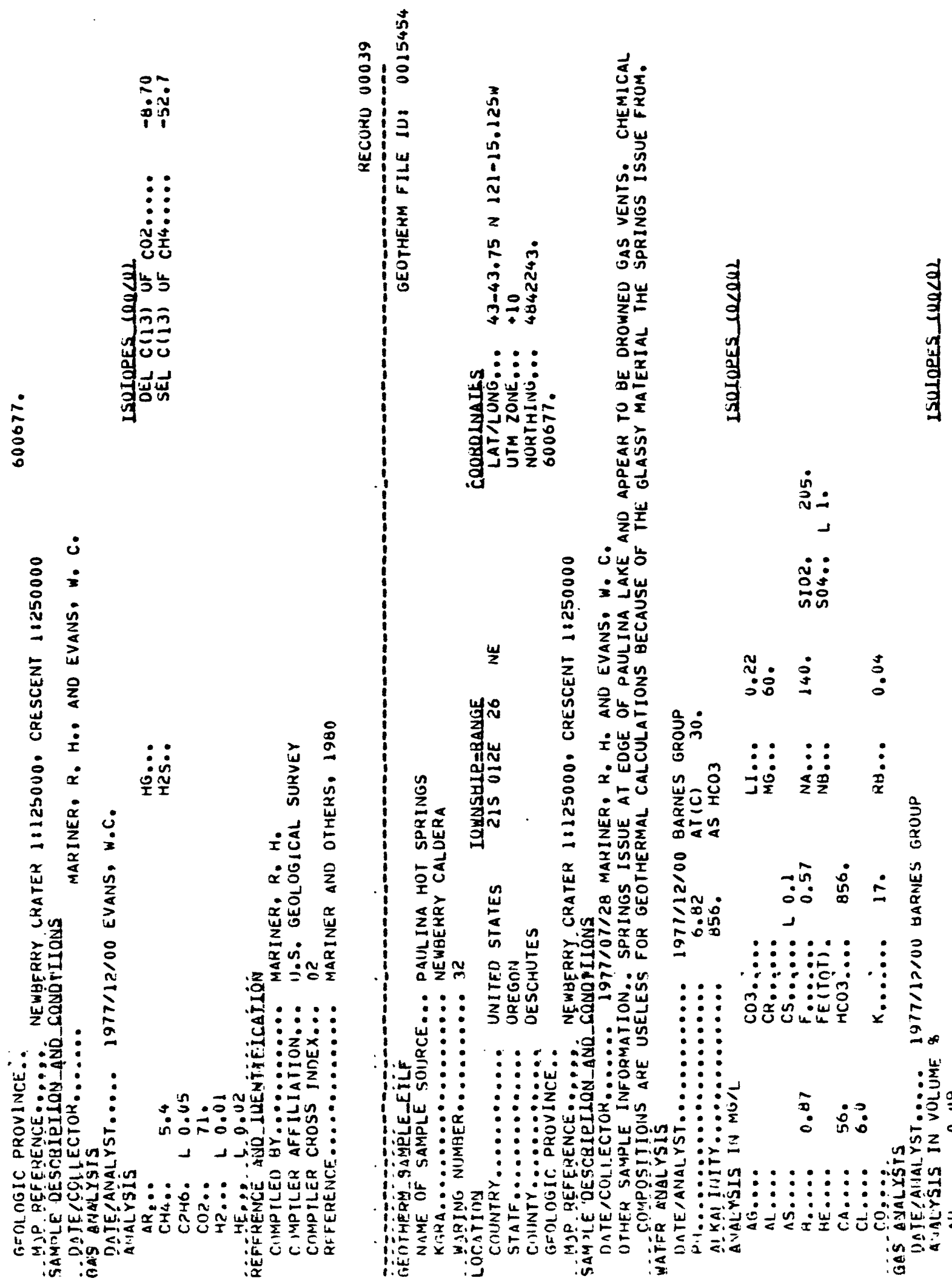

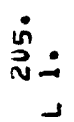

蓉离

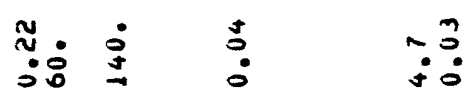

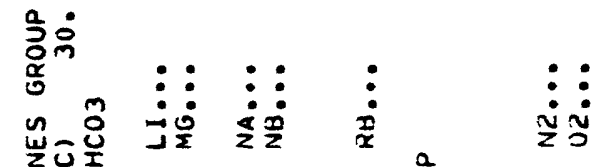

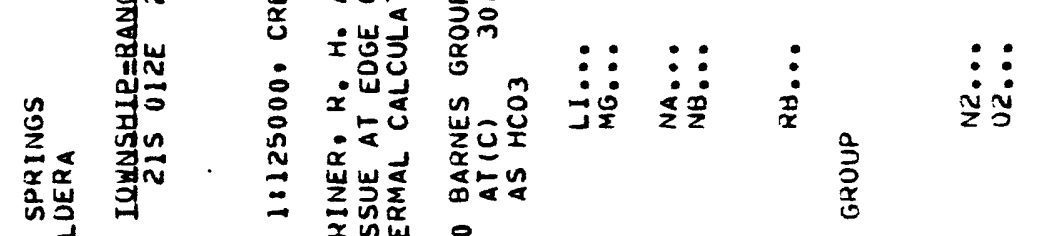

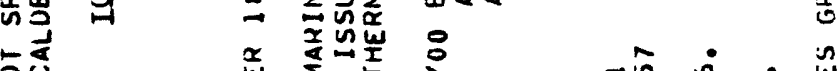

웅

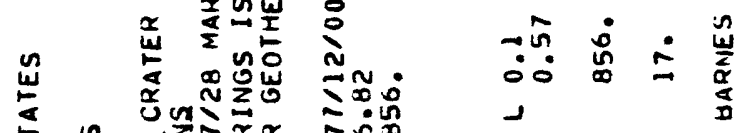

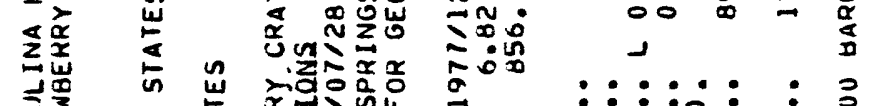

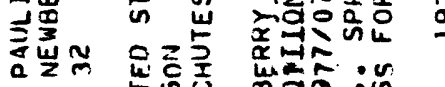

$:::$ 岂岕岕

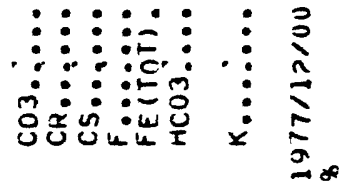

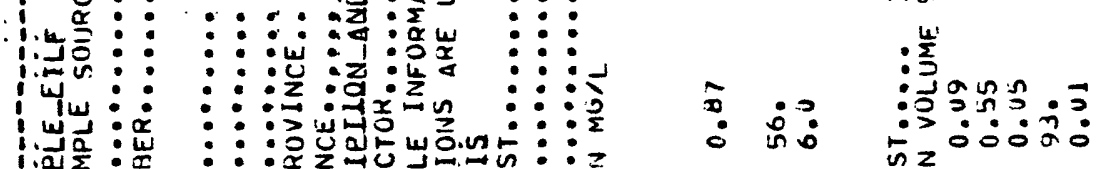

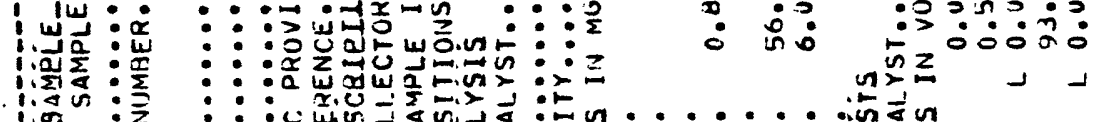
洸

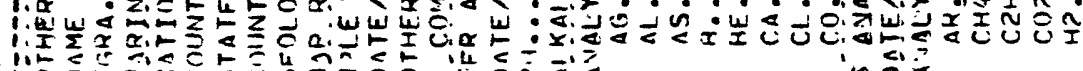

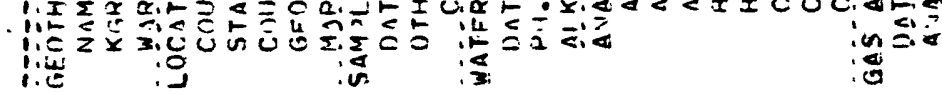




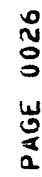

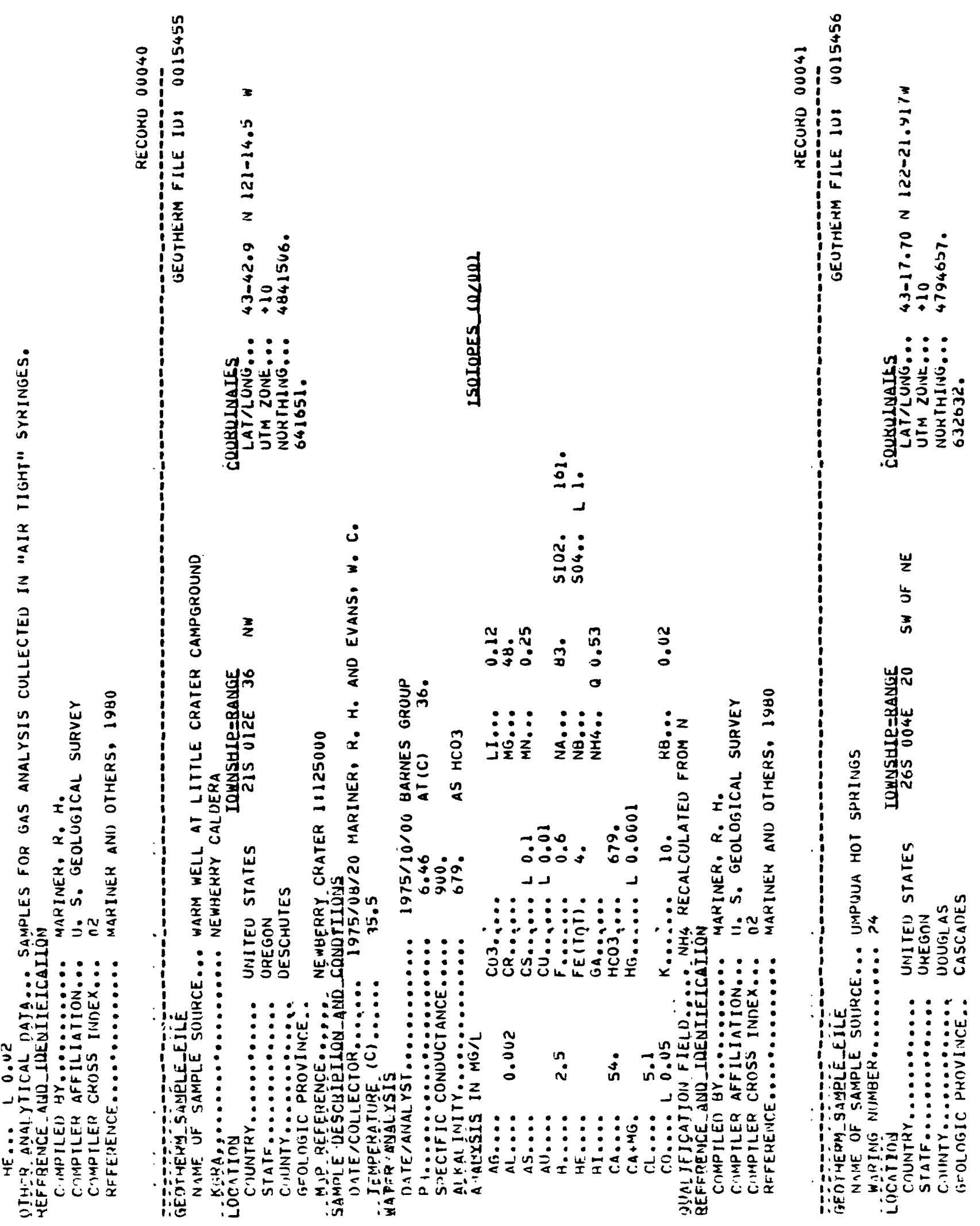




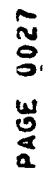
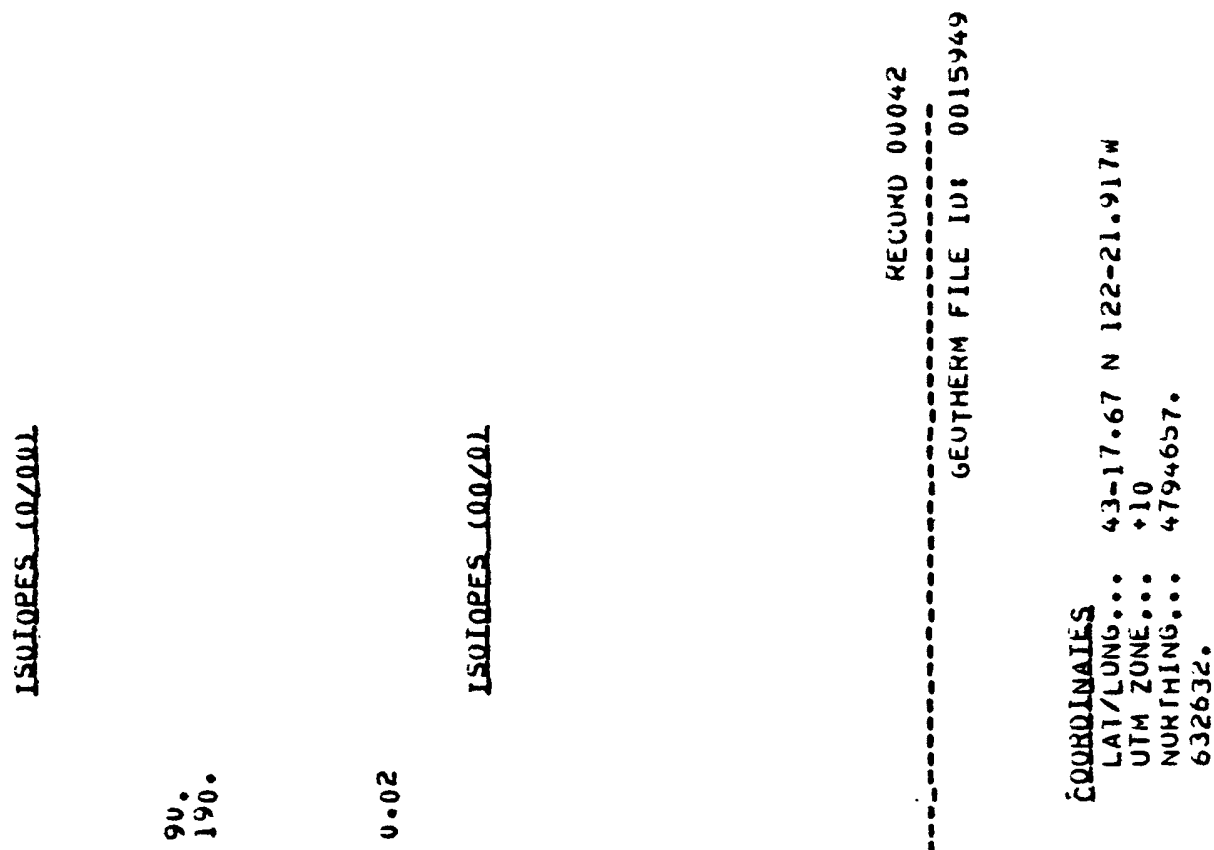

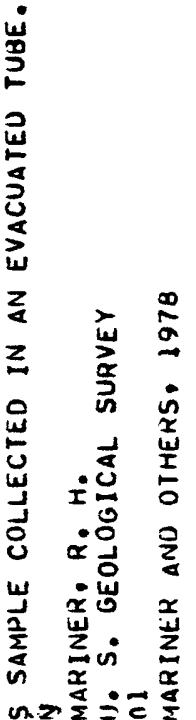

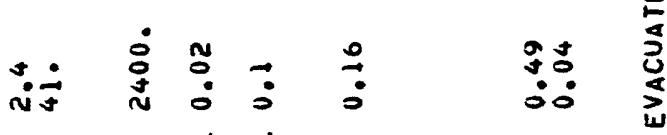

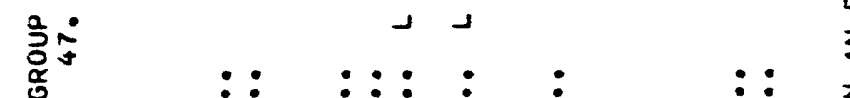

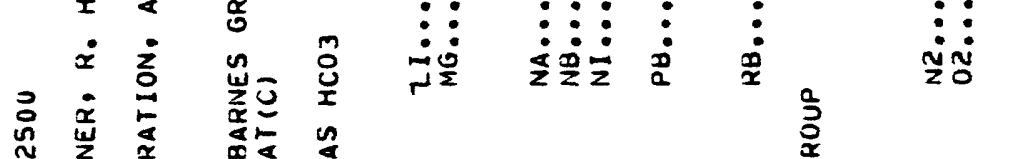

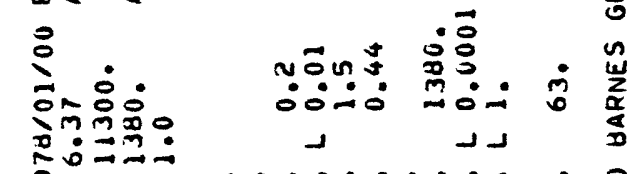

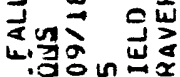

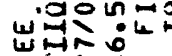

․ํํㅁำ

$\therefore$

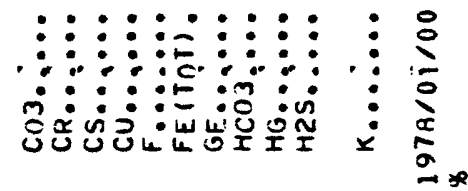

: : : :

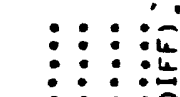

: : :

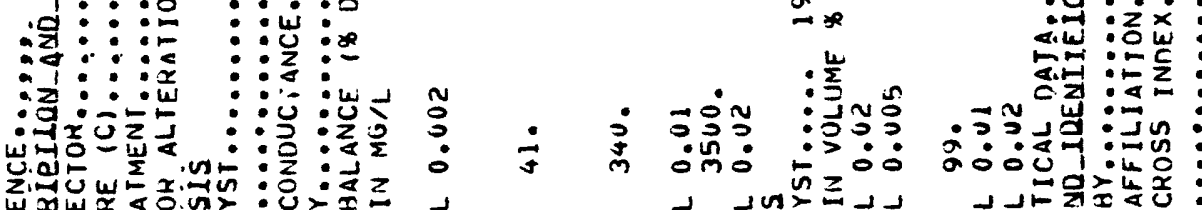

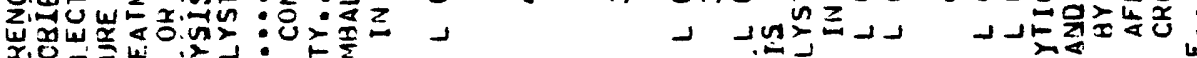

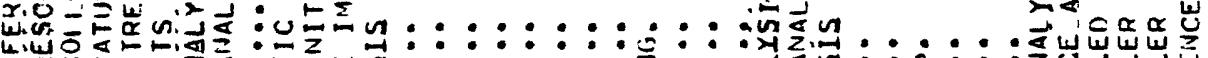

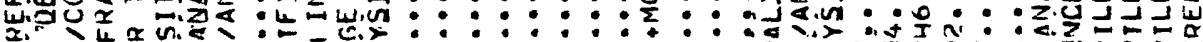

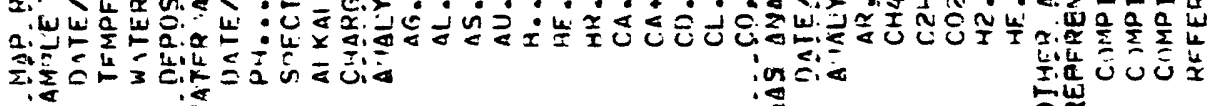
然

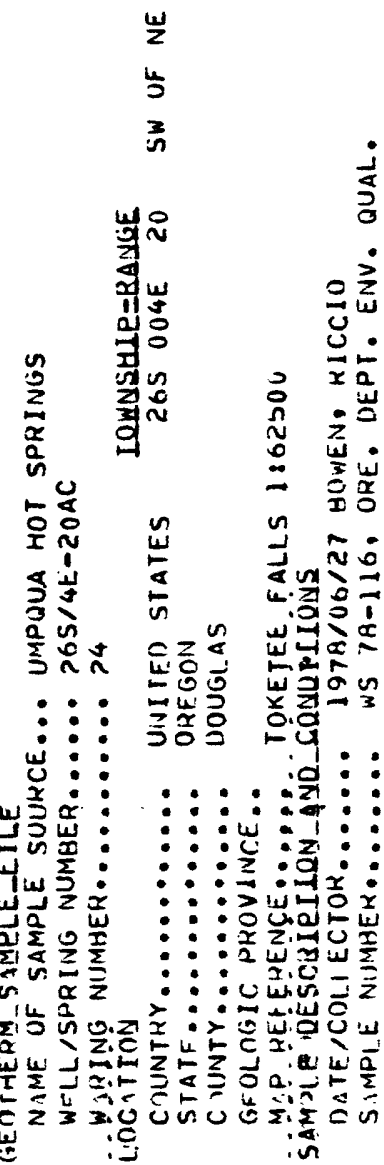




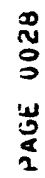

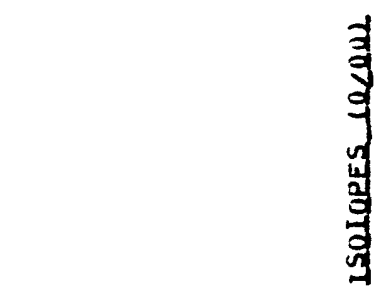
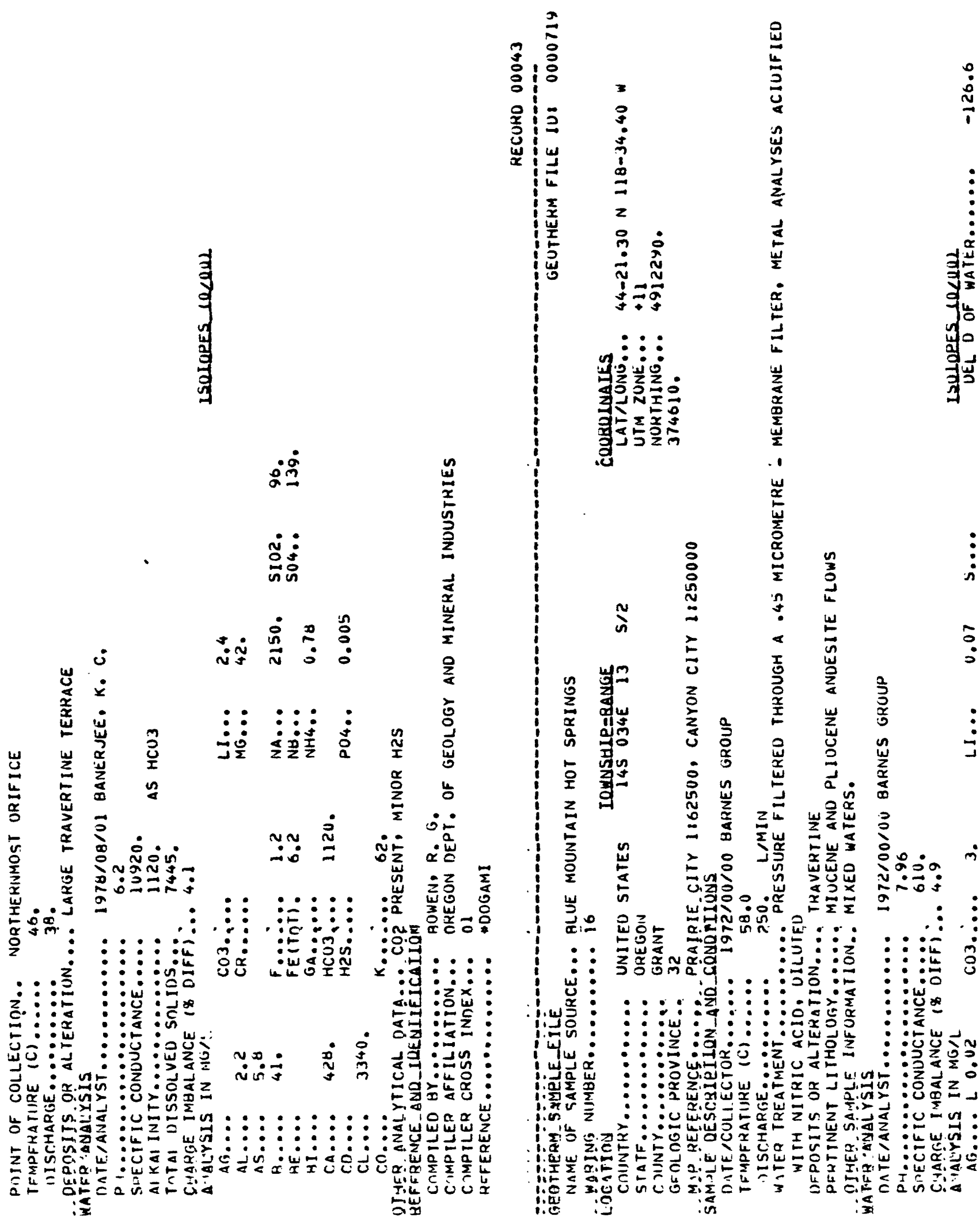

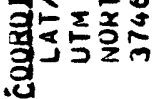

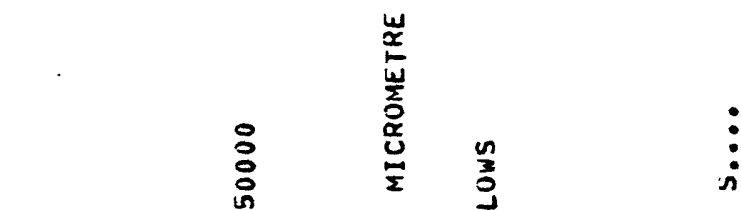

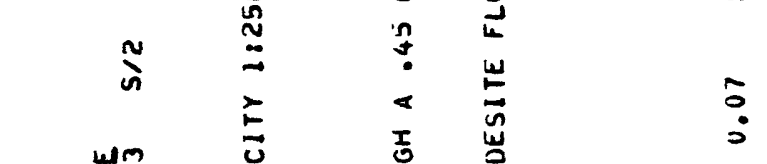

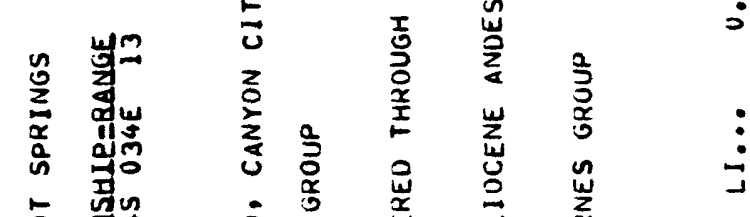

交嬊

包

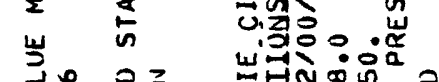

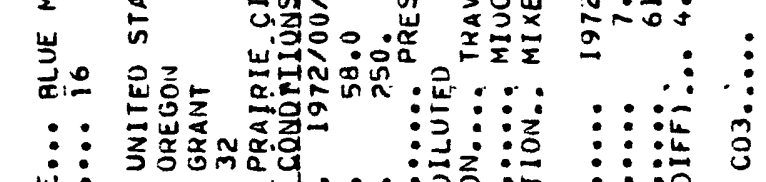

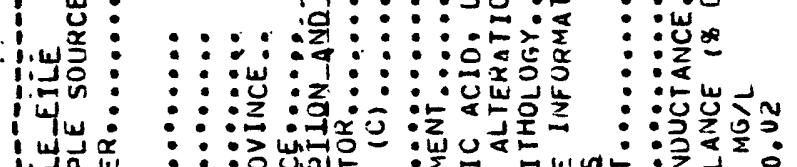

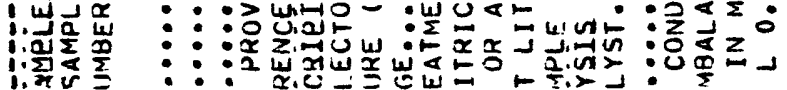

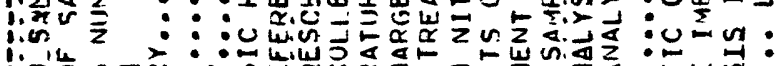

"'s

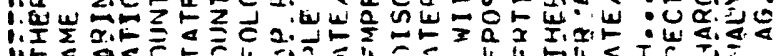

hit

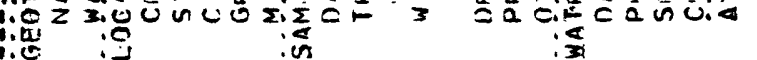




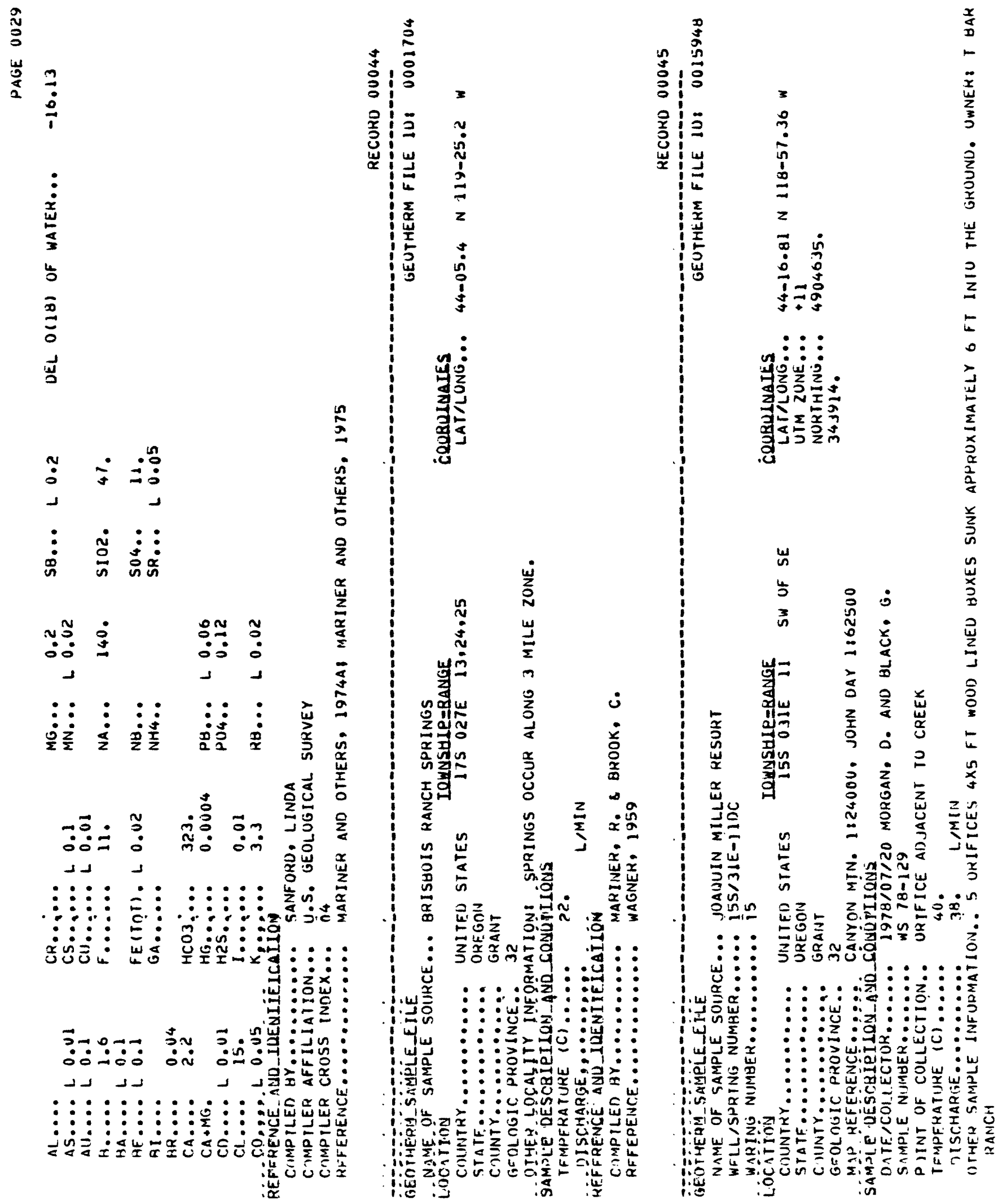


咅

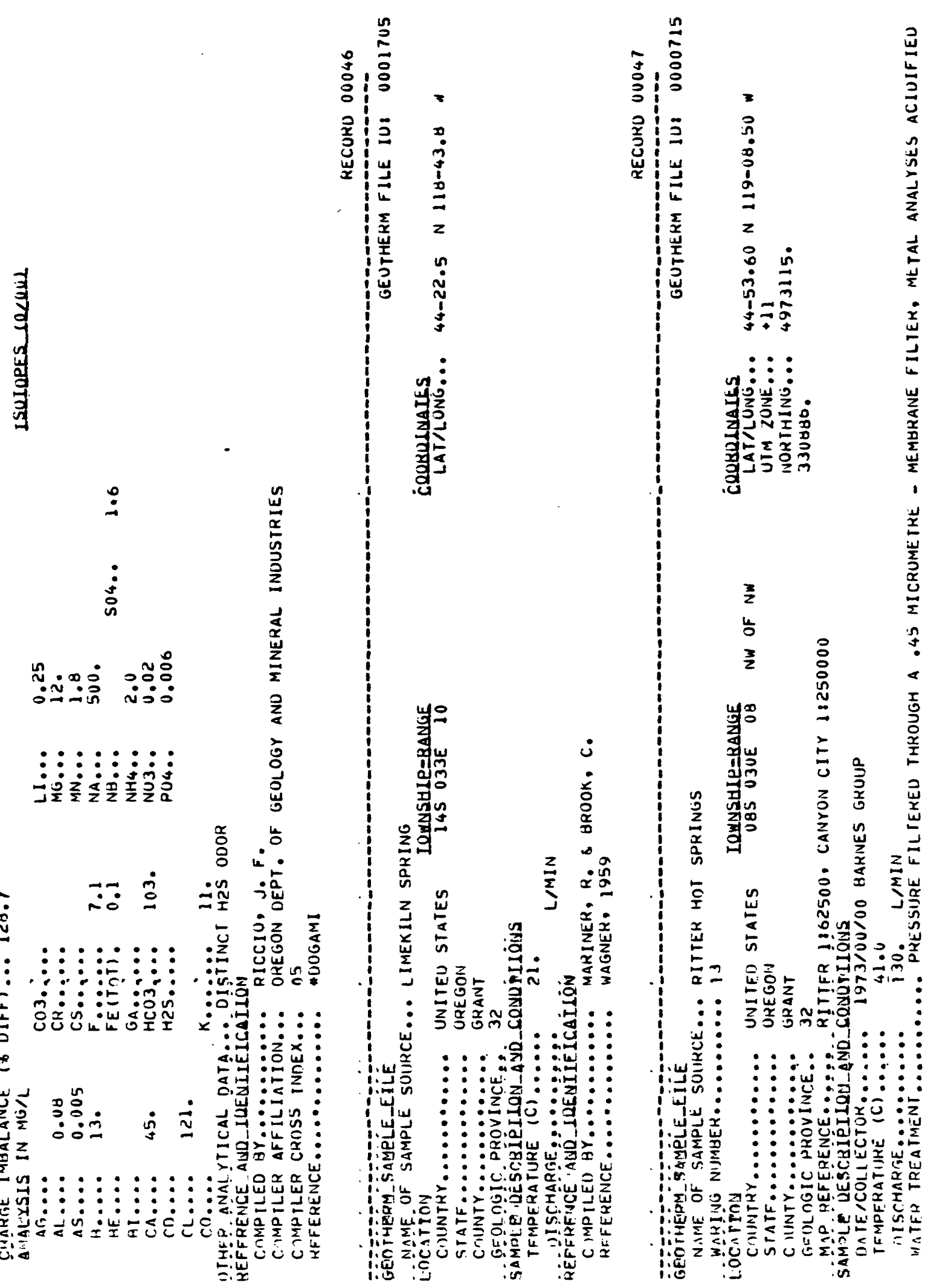


$\overrightarrow{3}$
0
0
Ju
$a$
$a$
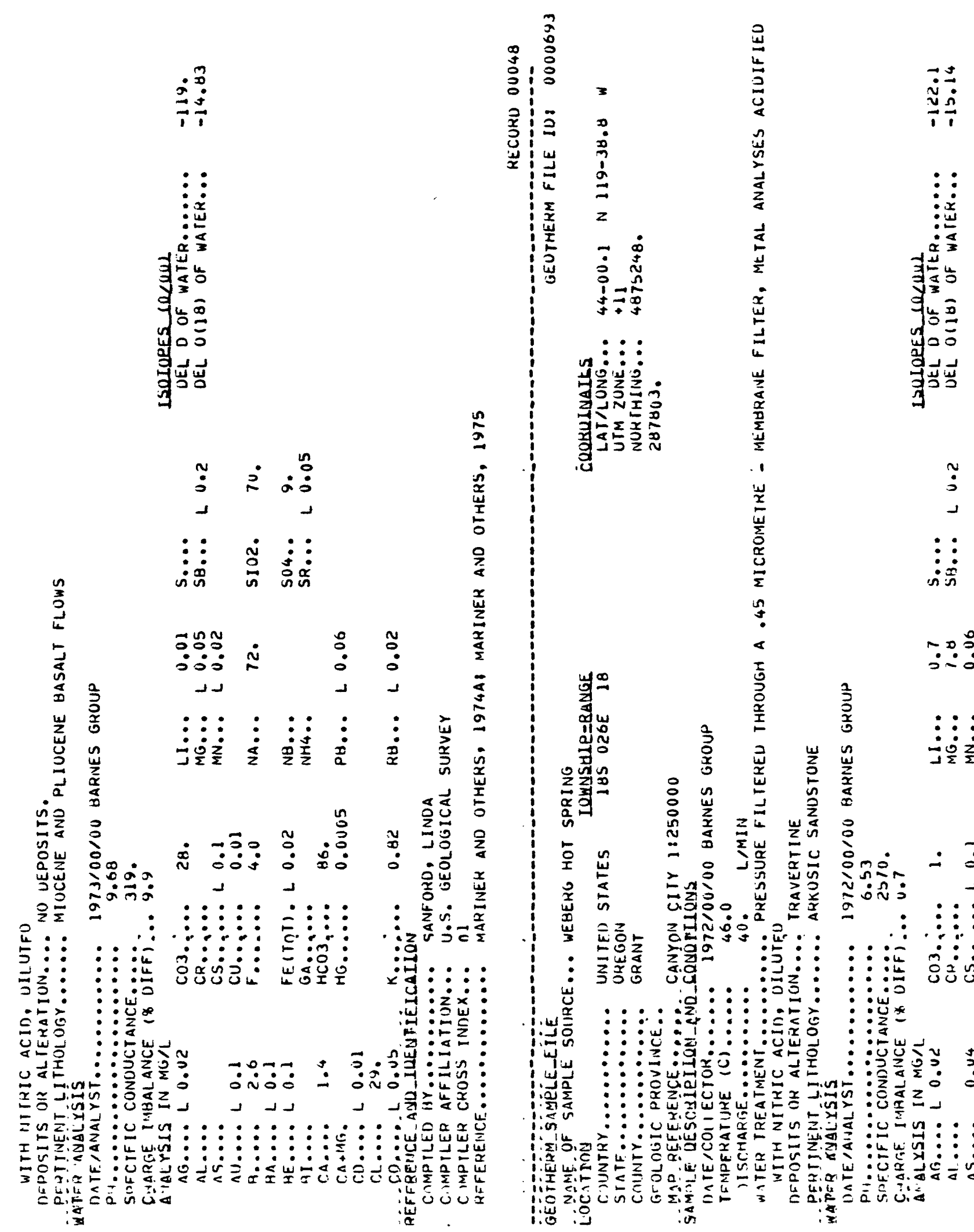

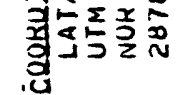

$\underset{\sim}{\stackrel{5}{*}}$

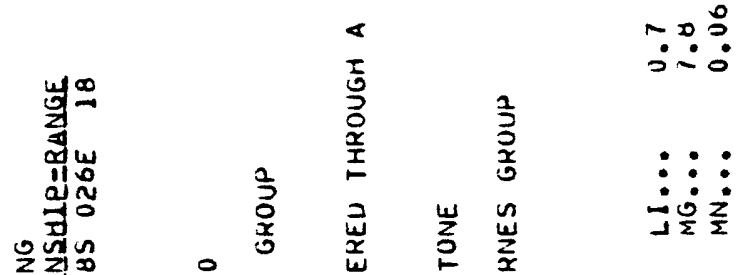

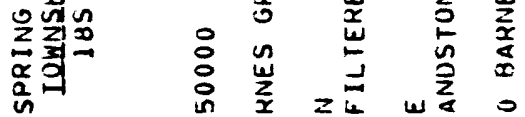

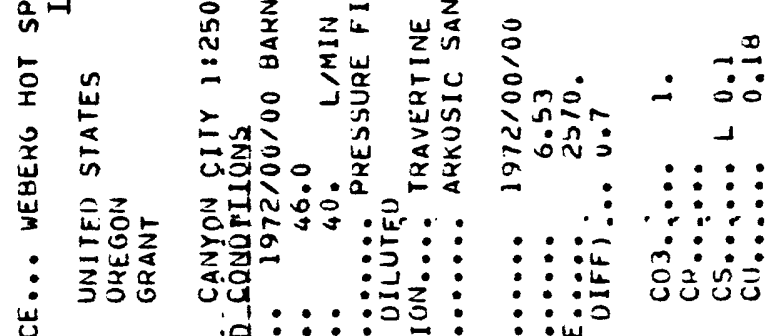

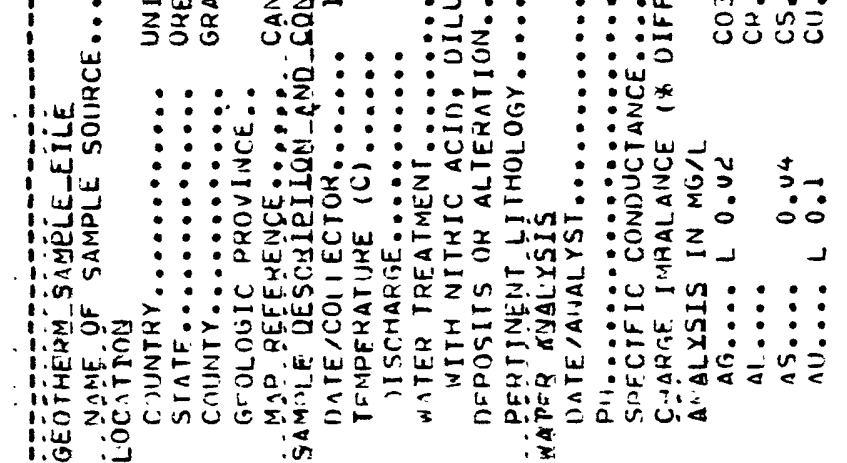




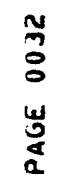

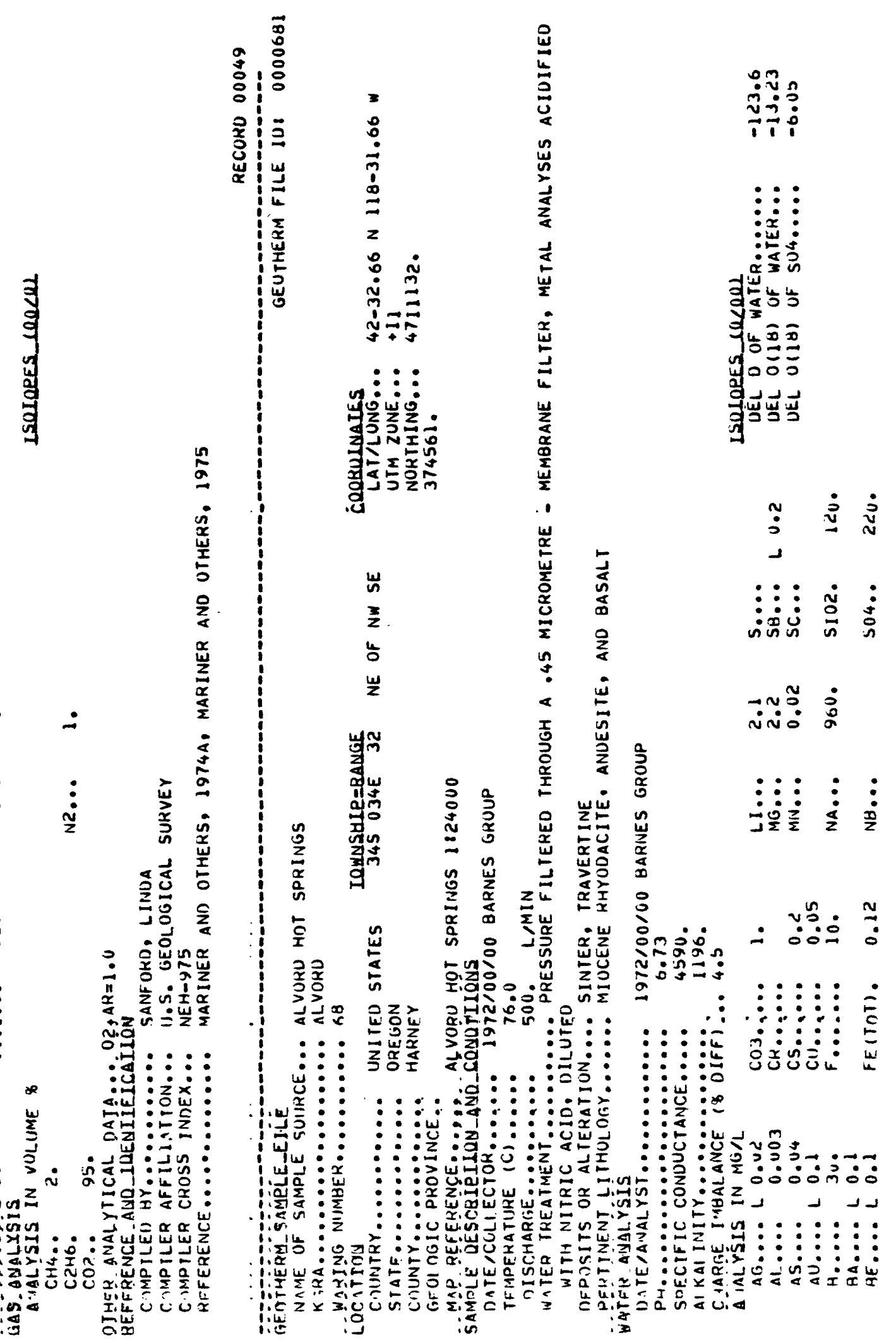


率
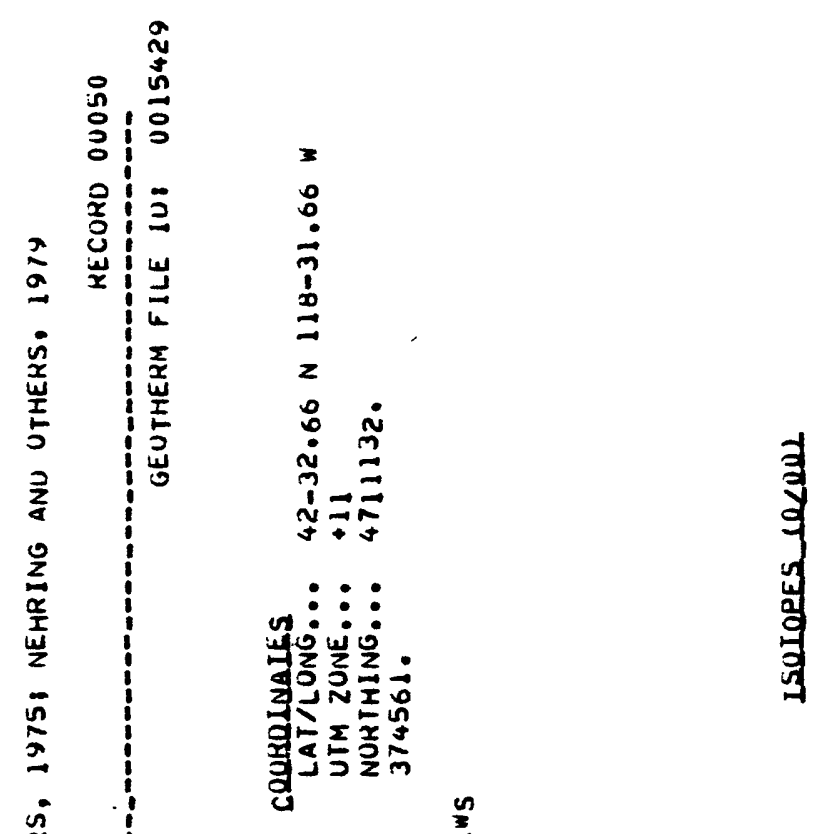

$\stackrel{\sim}{5}$

$\ddot{0} \quad \stackrel{0}{0} \dot{0} \stackrel{?}{?}$

$\vdots$

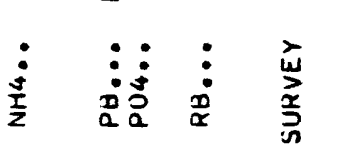

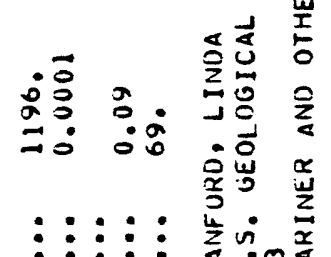

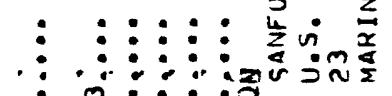

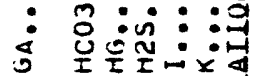

洁汇完

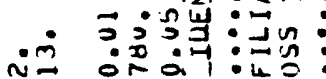

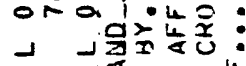

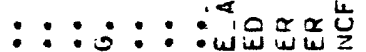

: : : : : : : :

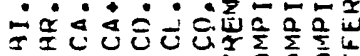

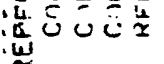

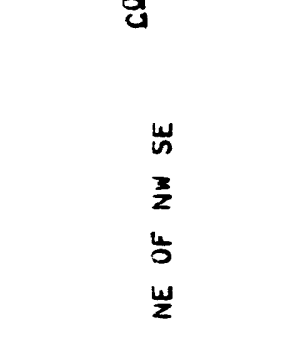

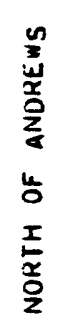

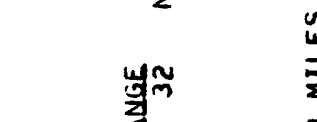

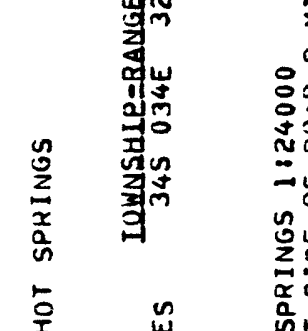

종

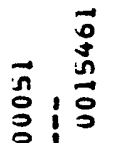

כ:

岁:

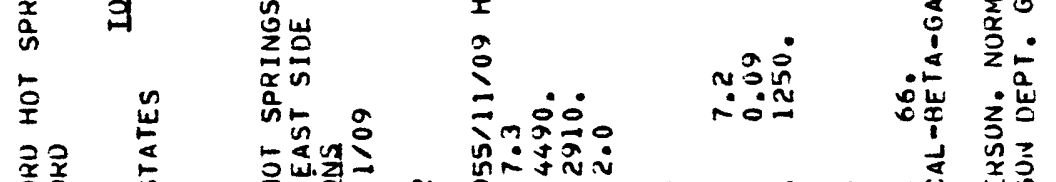

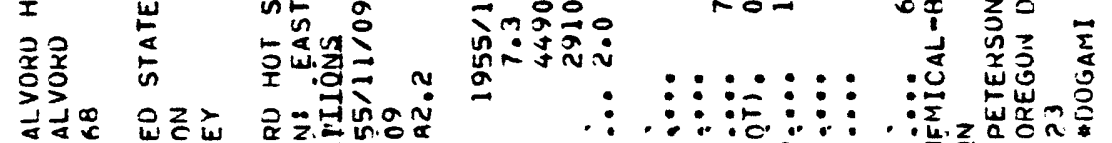

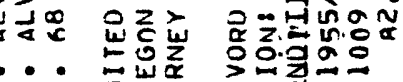

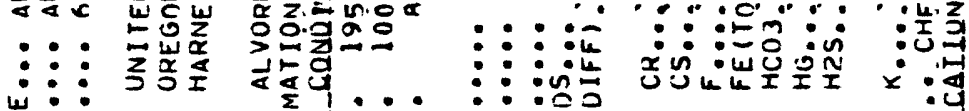

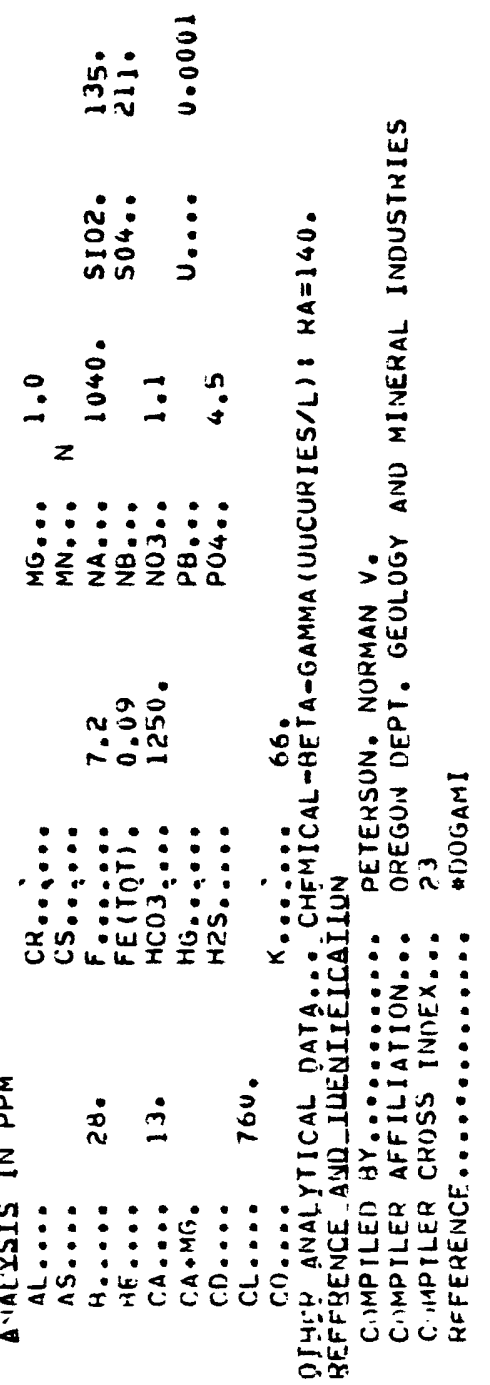


紊
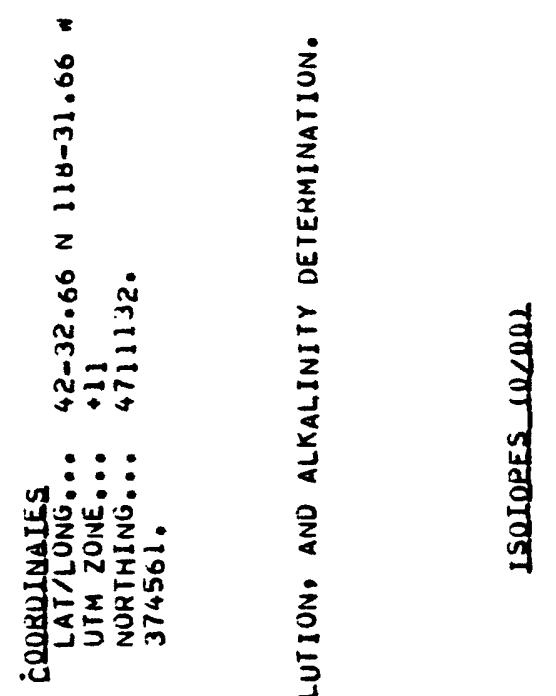

$\stackrel{\dot{D}}{\stackrel{0}{0}}$

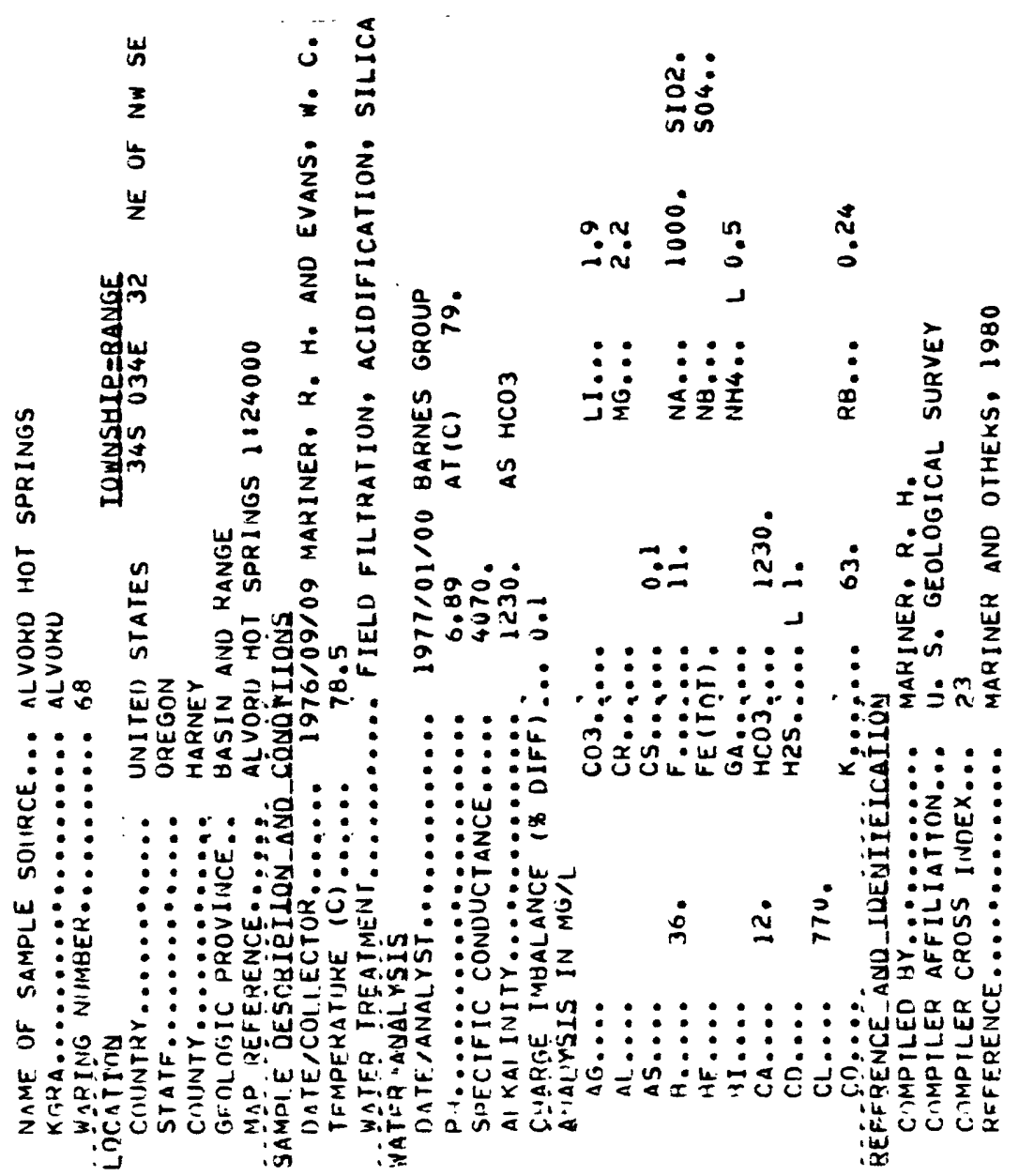

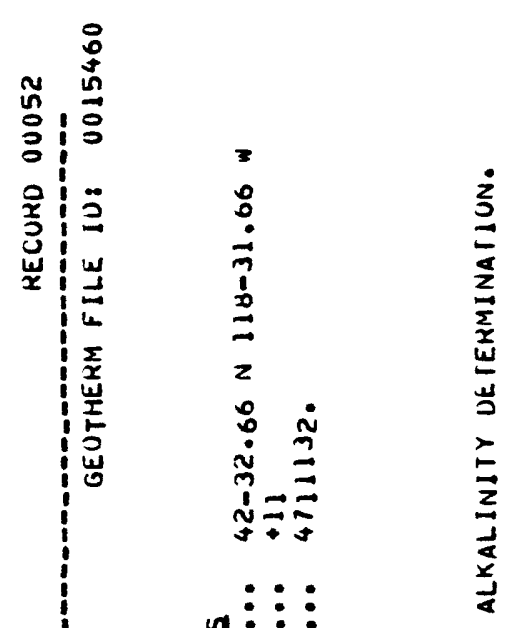
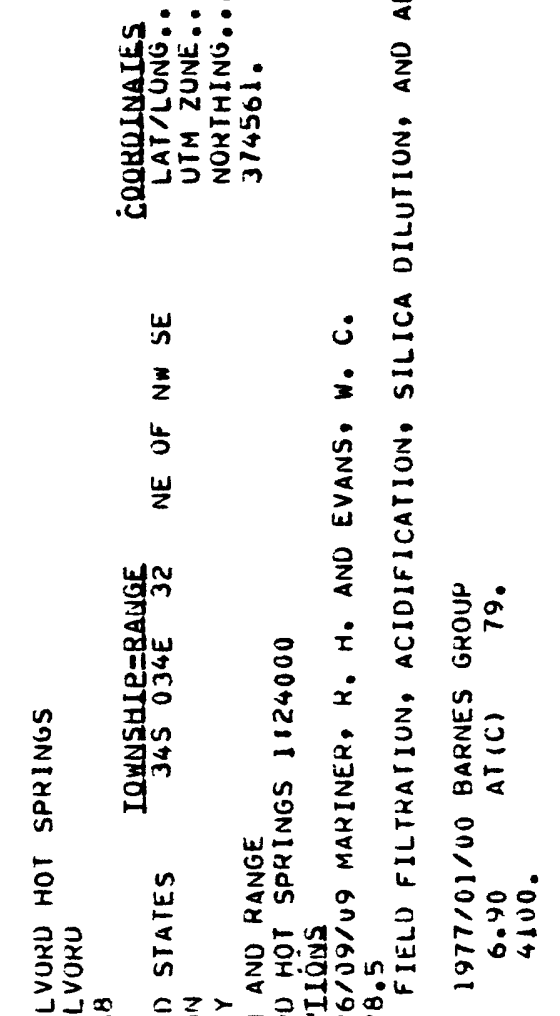

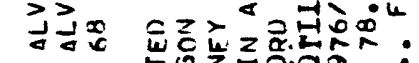

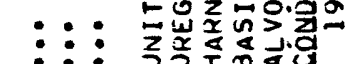

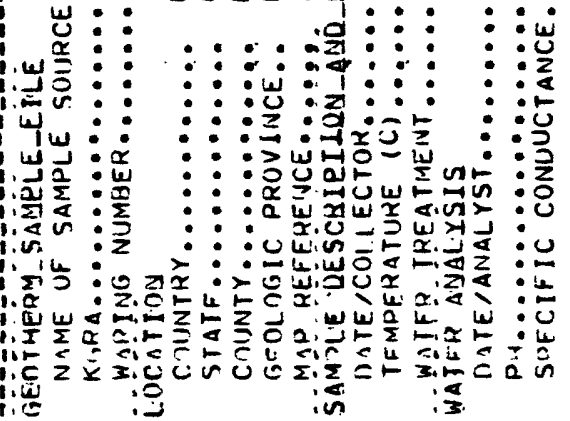


商

录

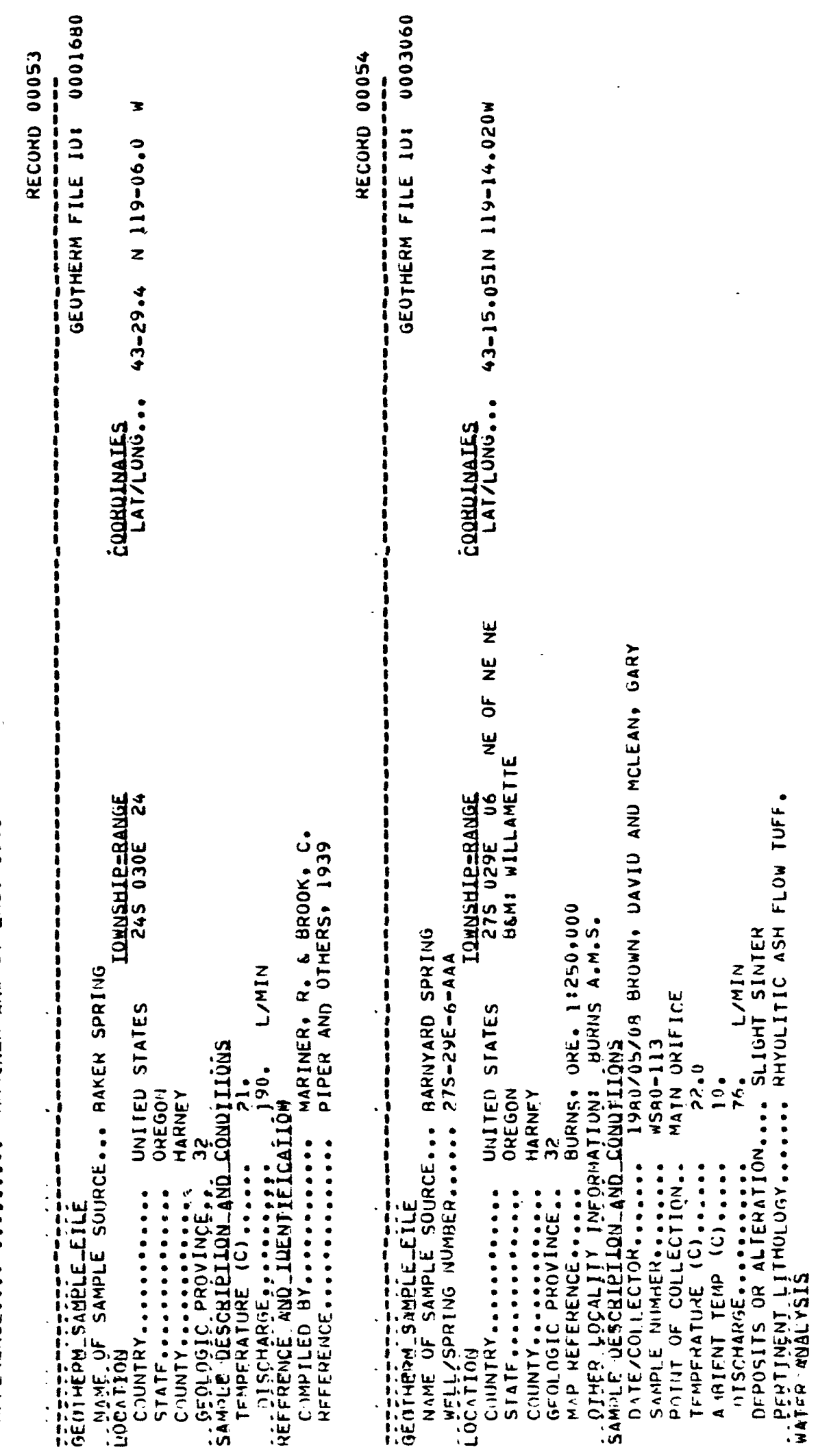


高

疍
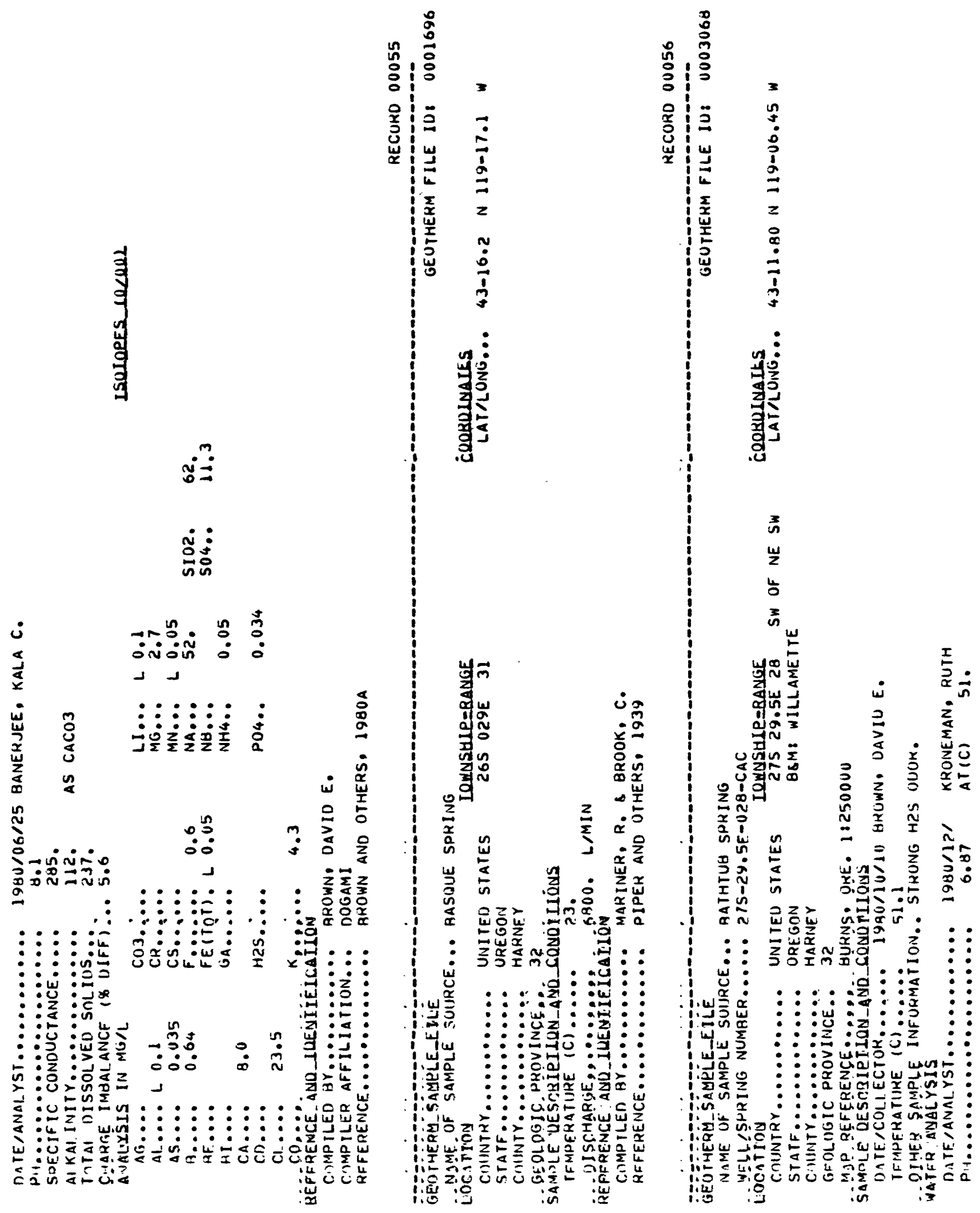


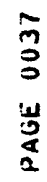

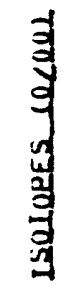

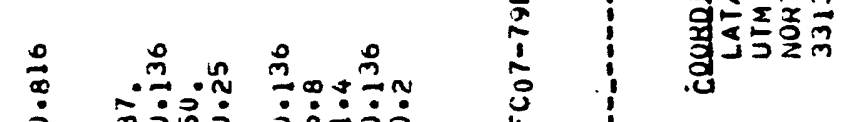

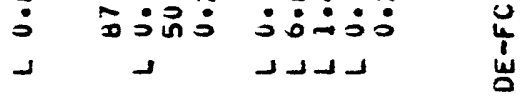

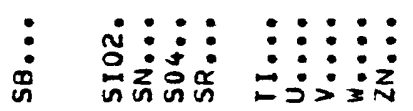

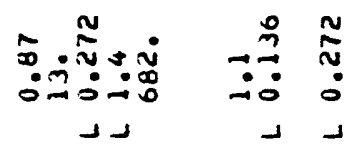

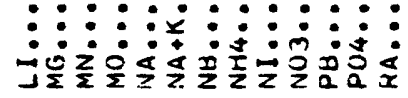

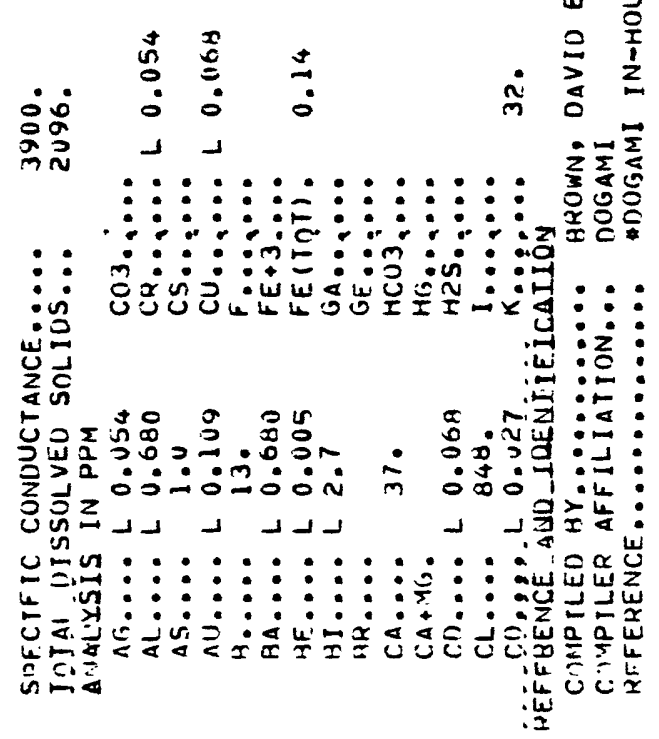

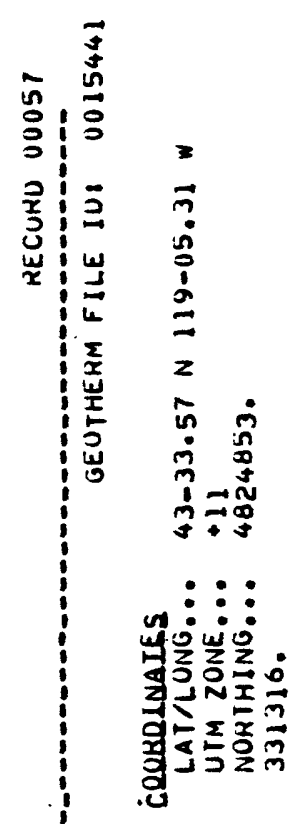

当

$\dot{0}$

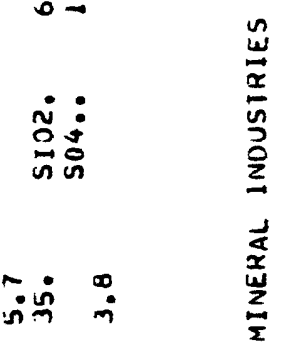

牙

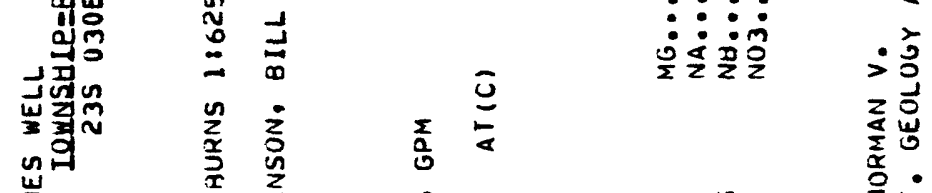

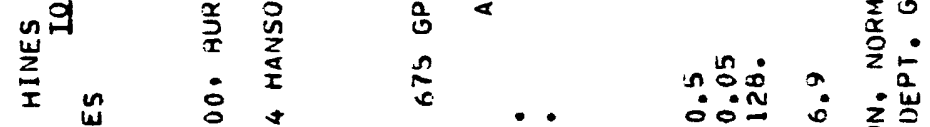

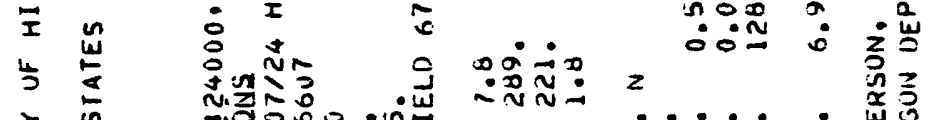

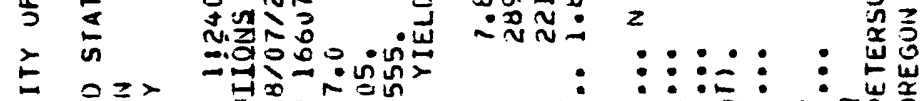

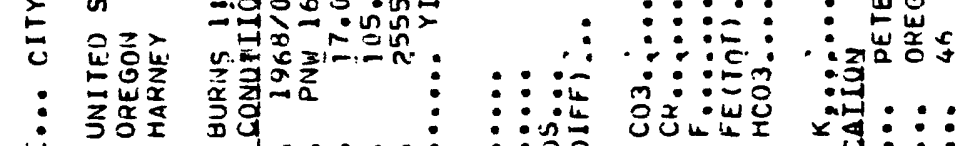

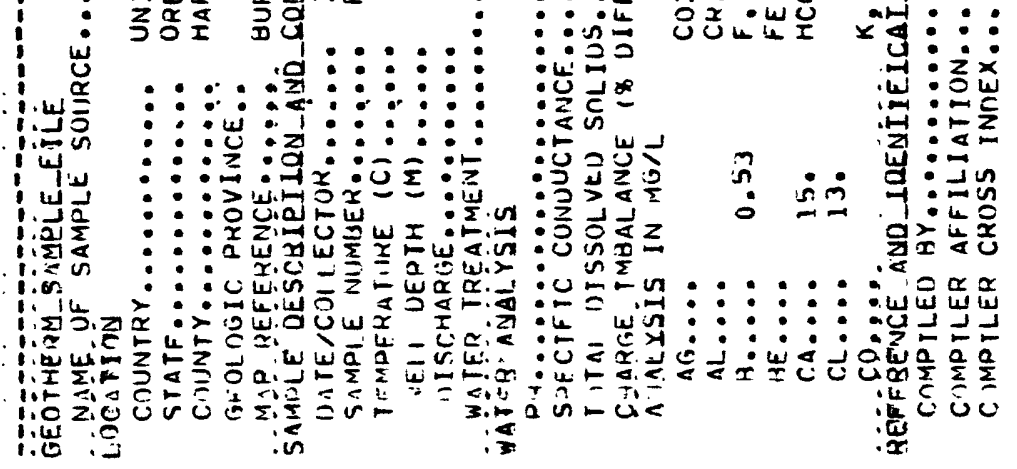




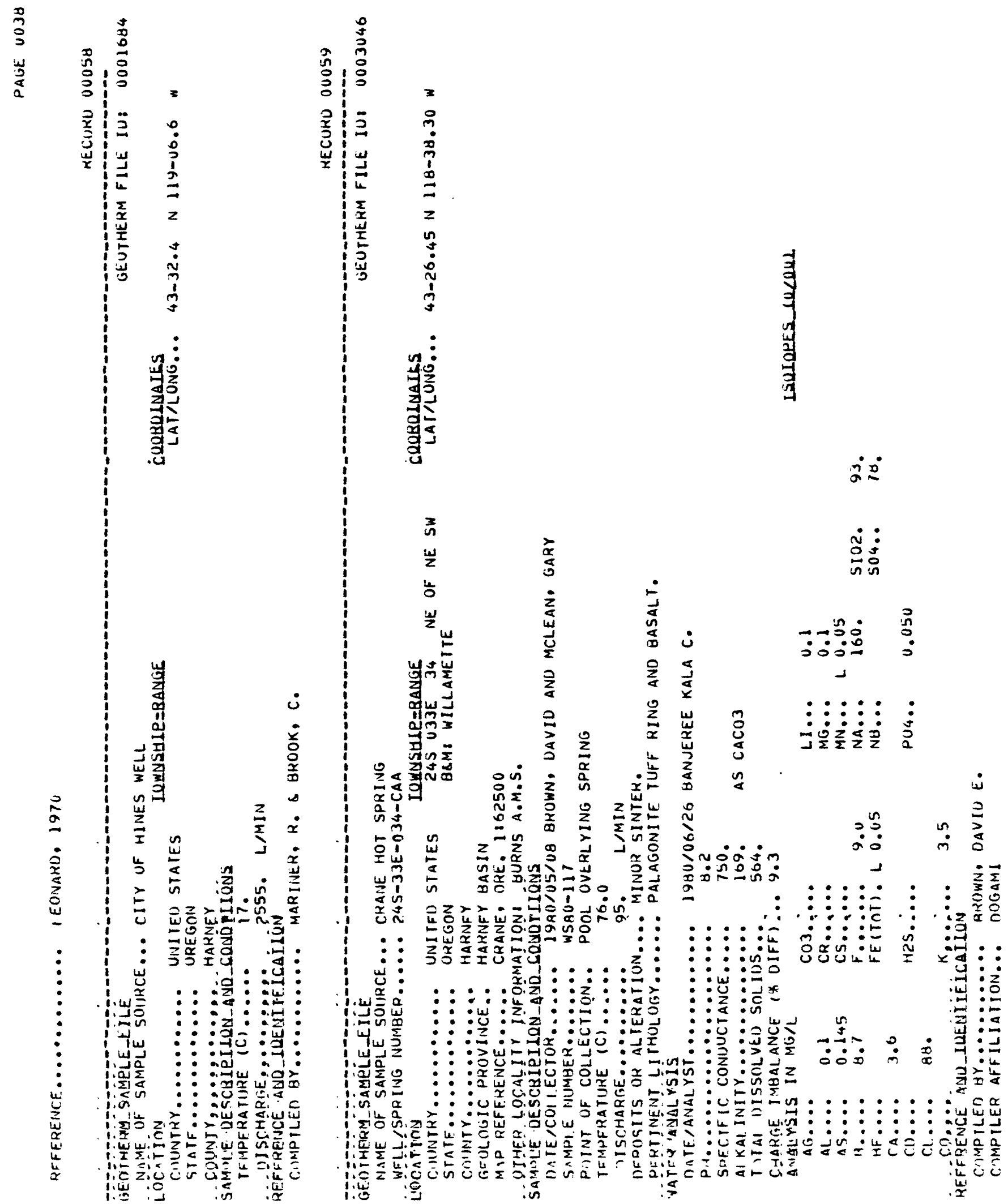



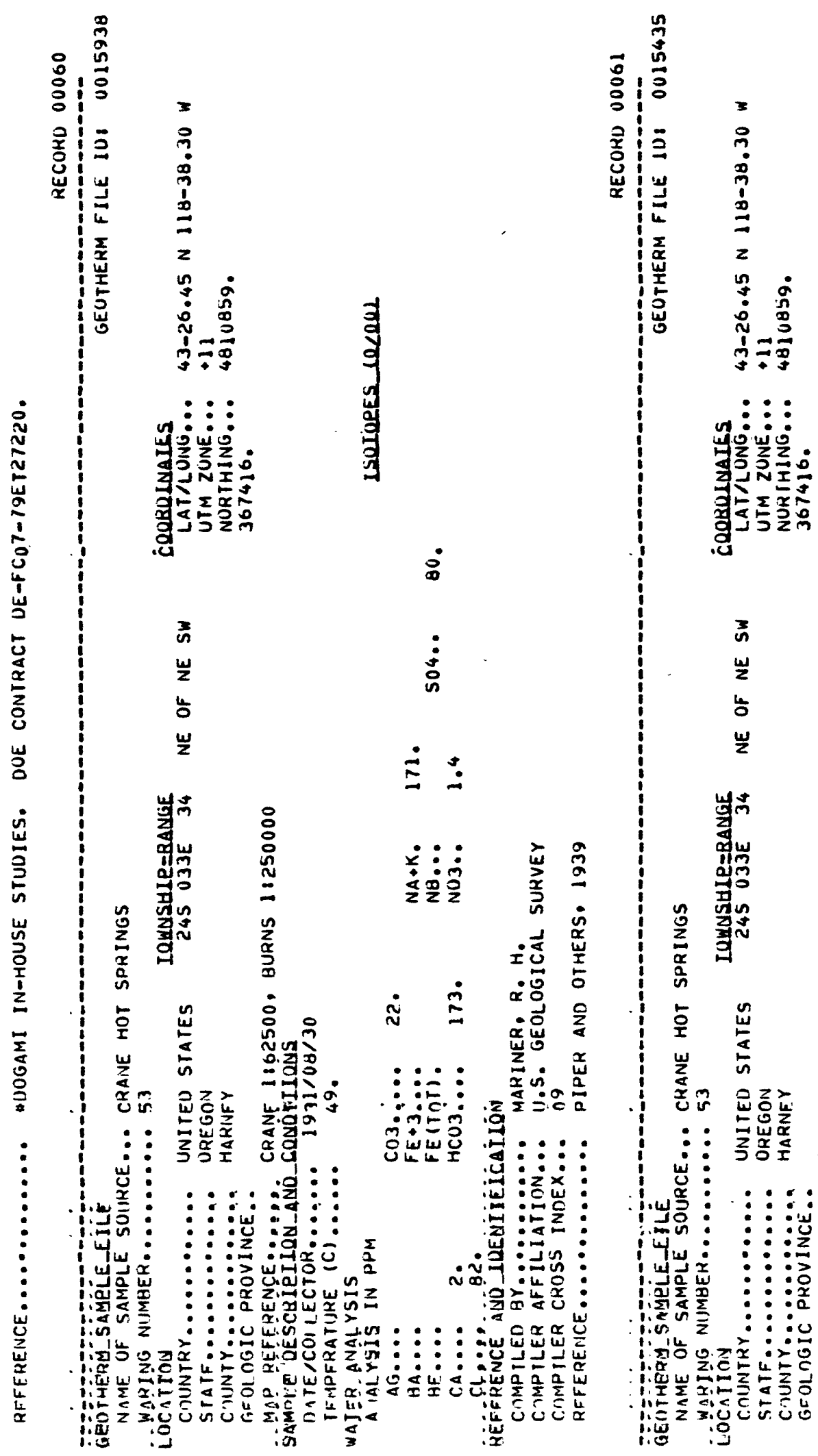

量

$\dot{\vec{x}}$

泀:

เ

宸

$\stackrel{\sim}{\mathfrak{0}}$

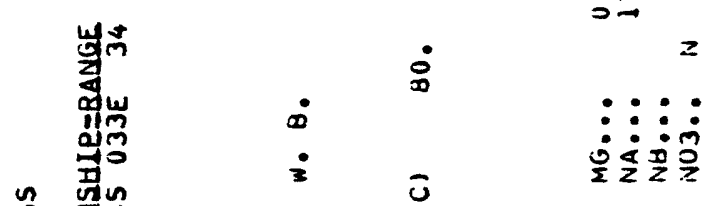

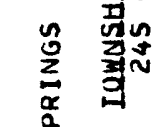

$\frac{1}{4}$

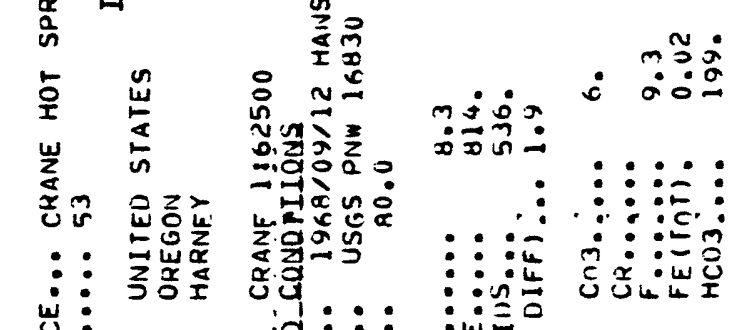

: : : : : : : : : : : : : :

出

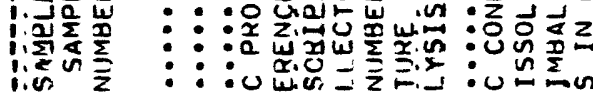

:

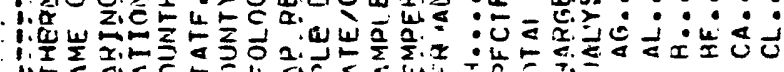

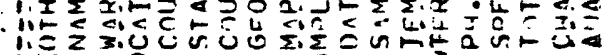

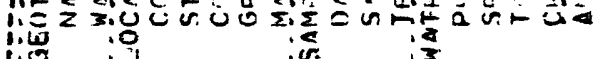




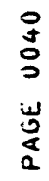

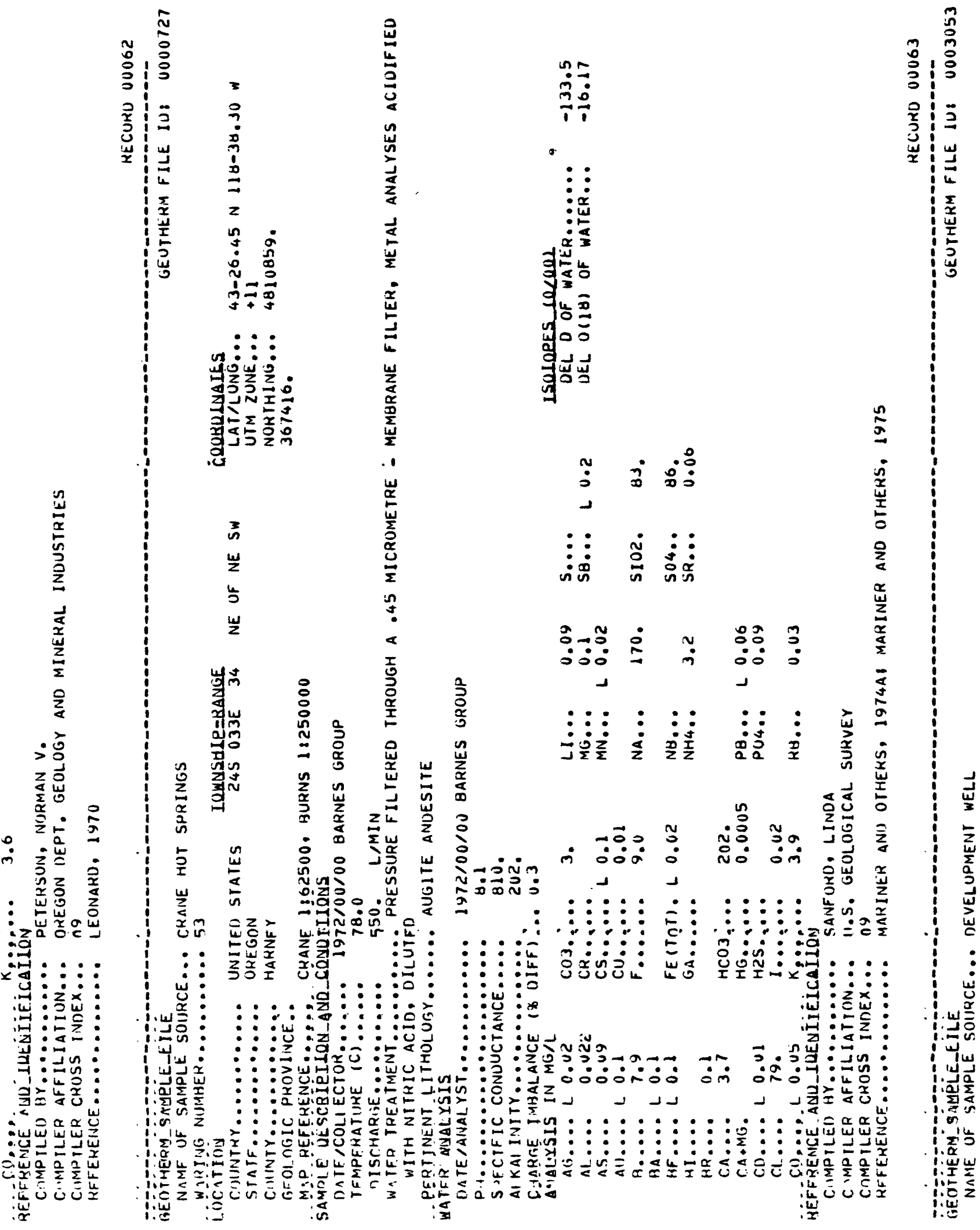



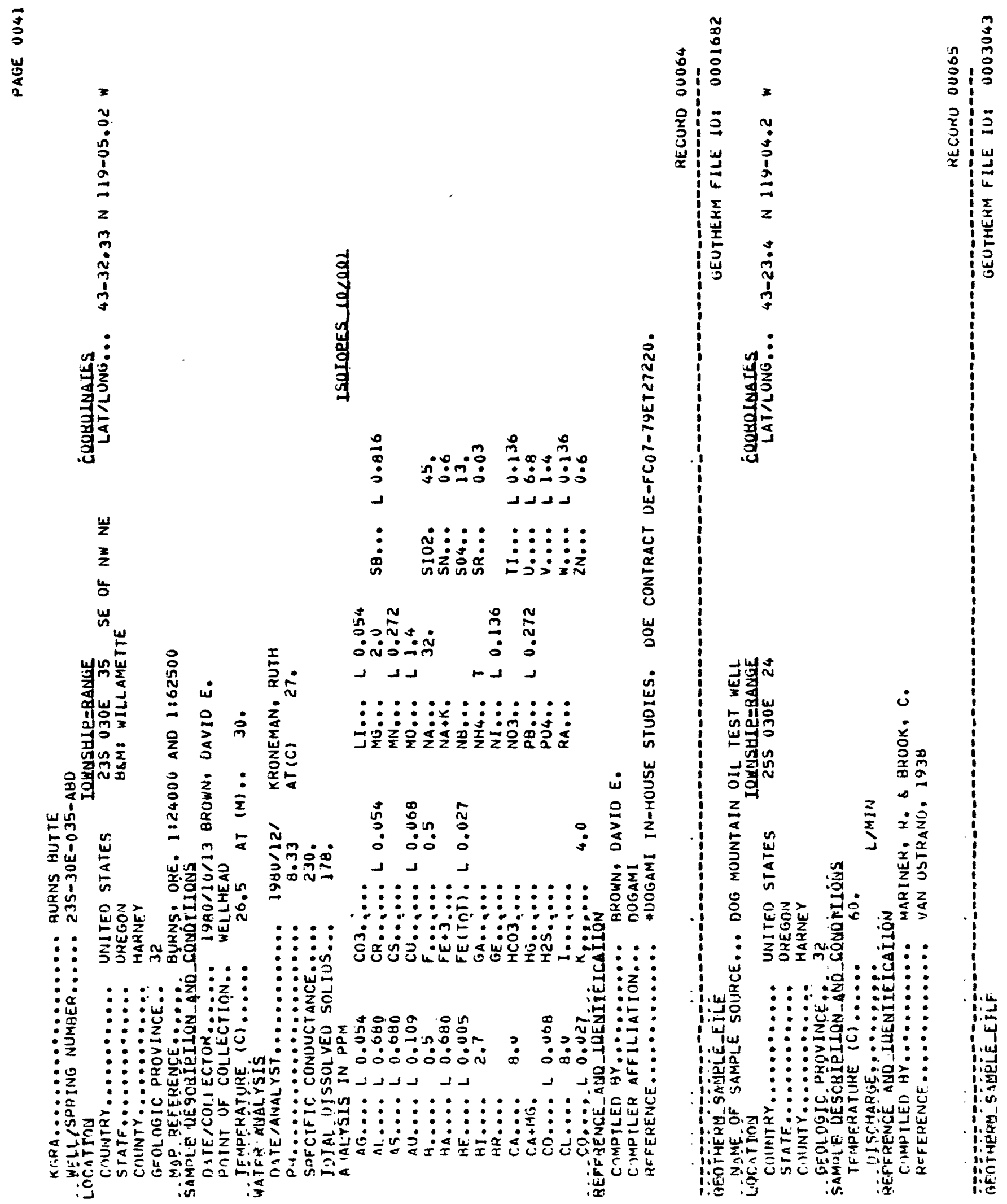
J
8
0
w
0
2
2
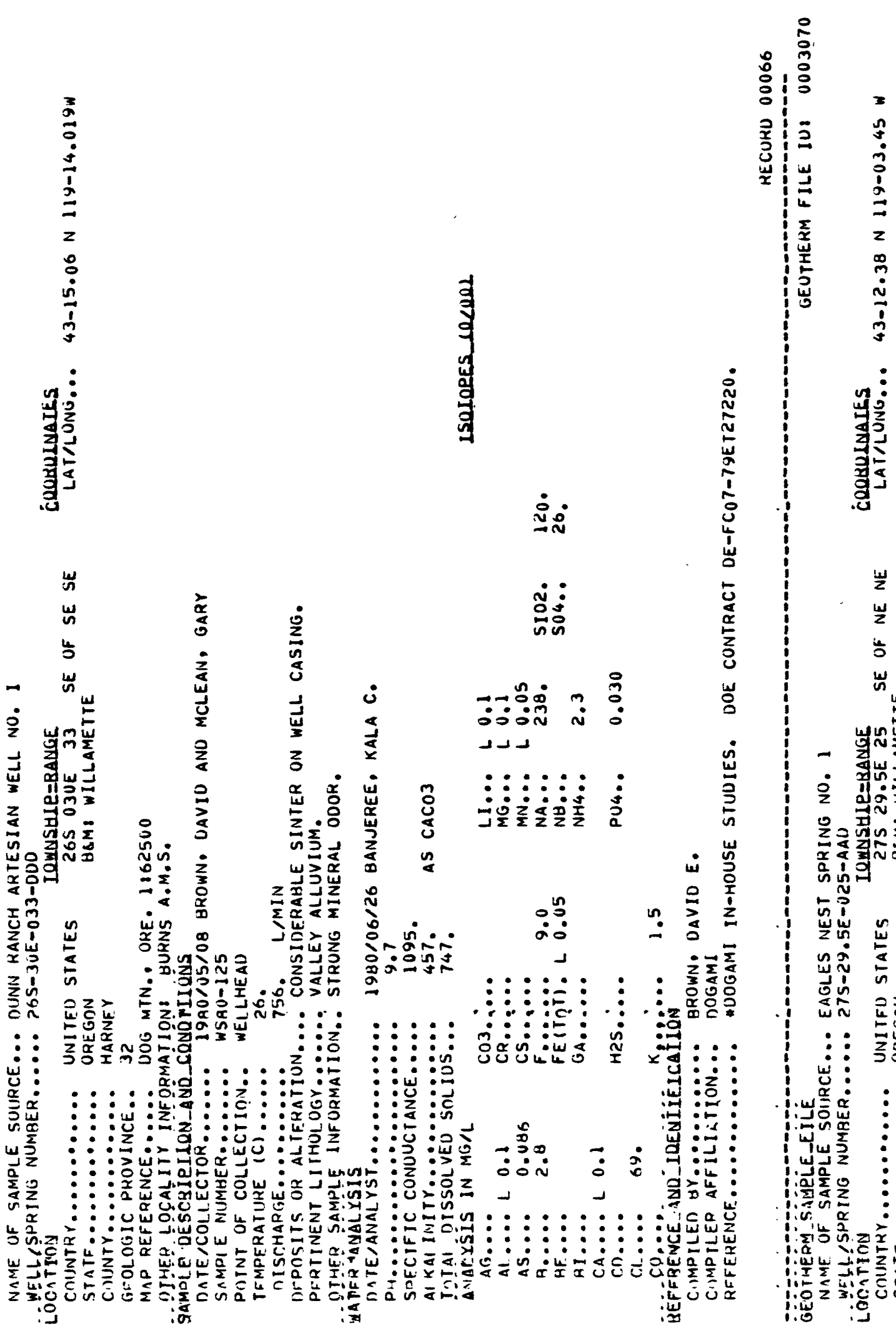<smiles>C1[Si][Si]1</smiles> 


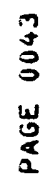

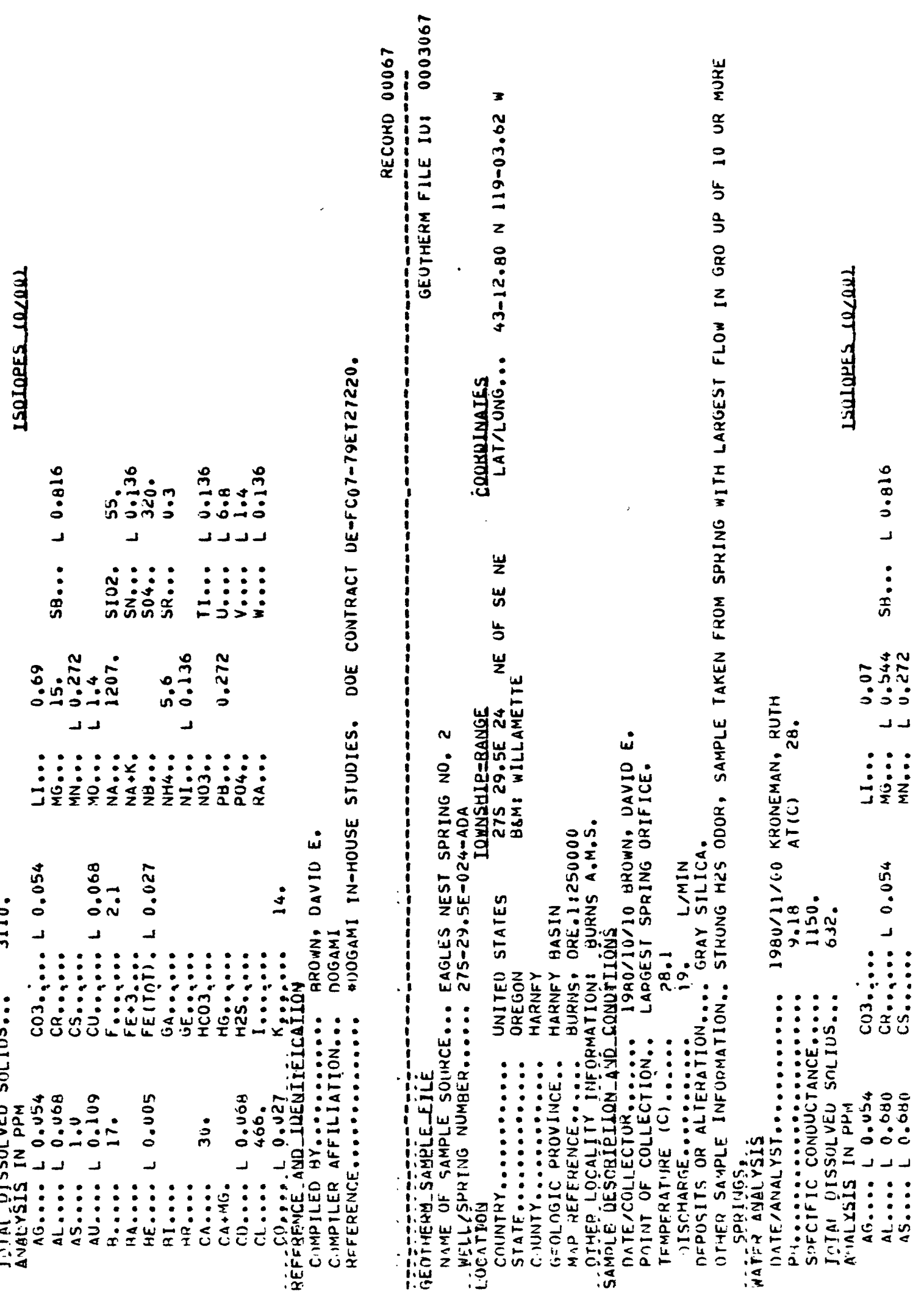




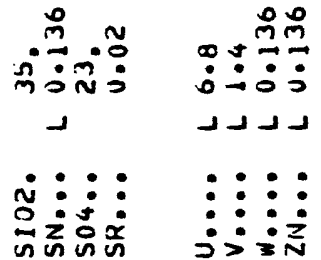

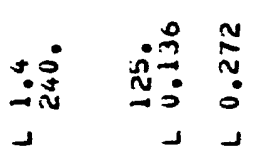

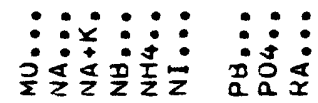

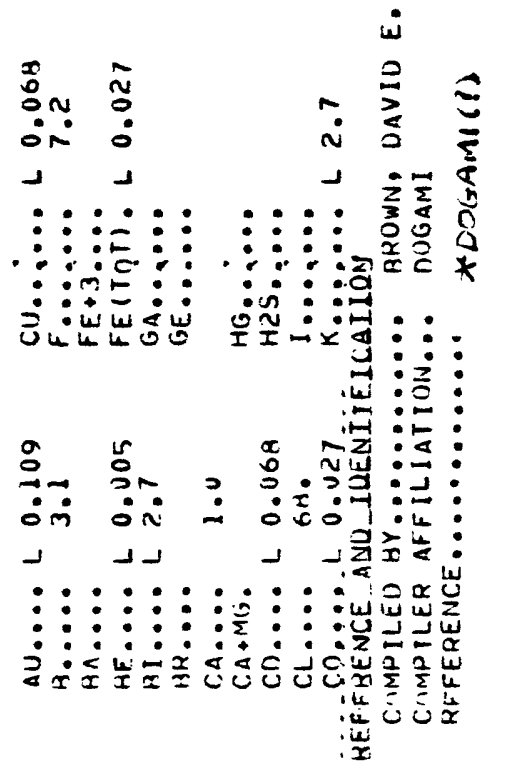

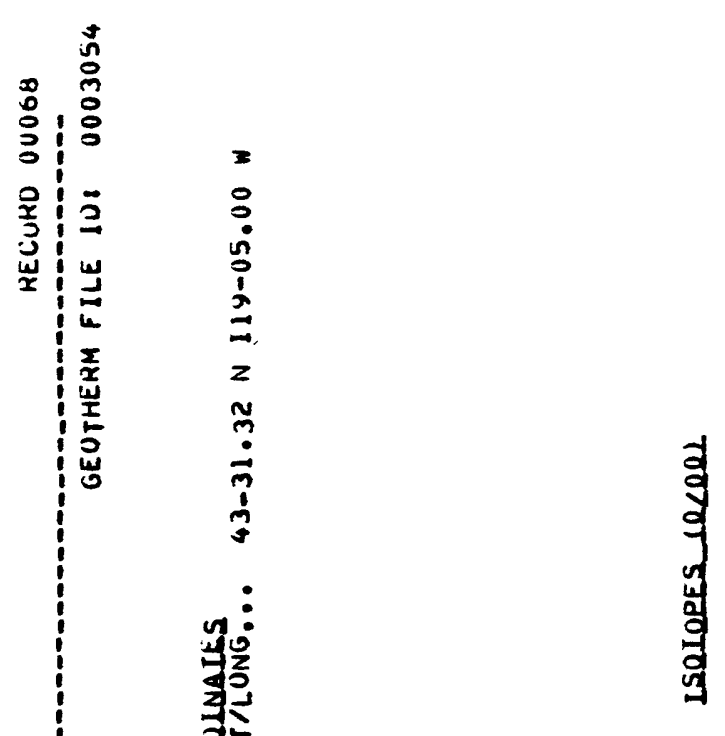

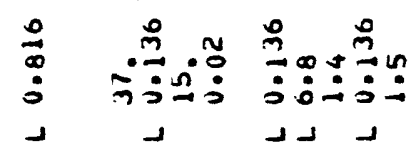

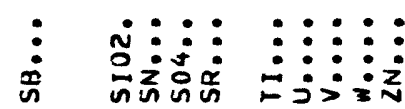

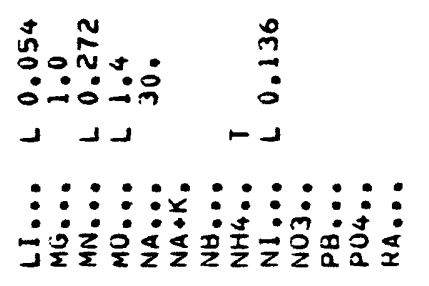

m

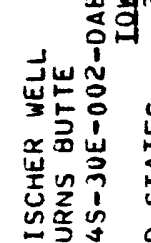

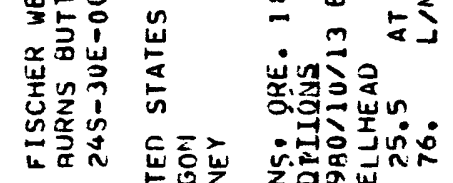

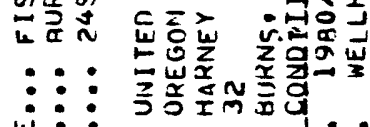

$:::: \vdots:::$ :

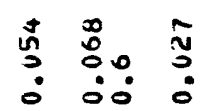

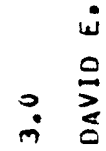

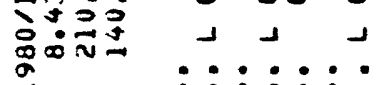

: : : : : : : :

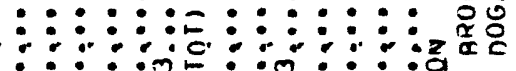

की: : : :

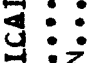

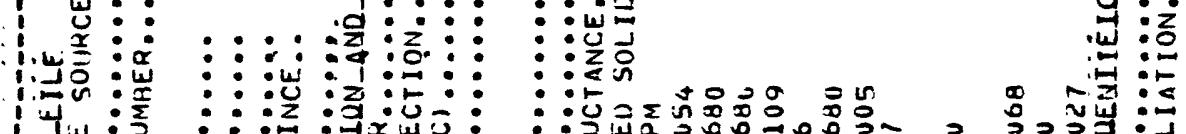

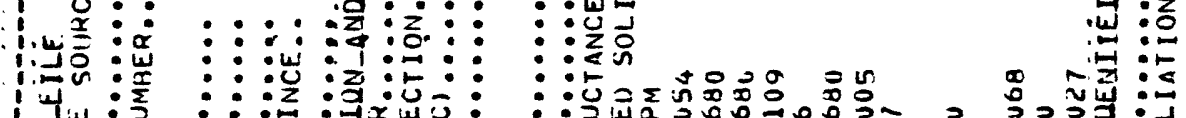

出:

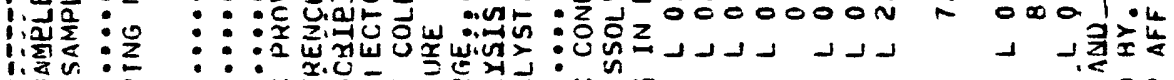

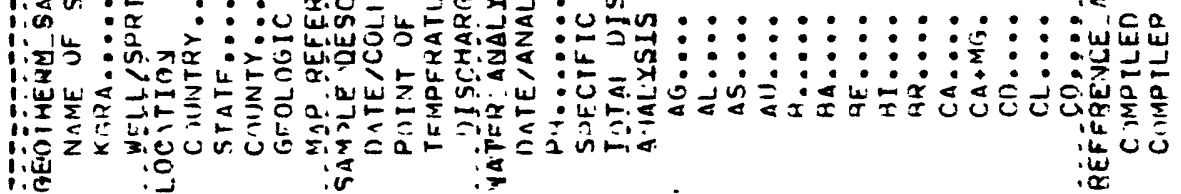




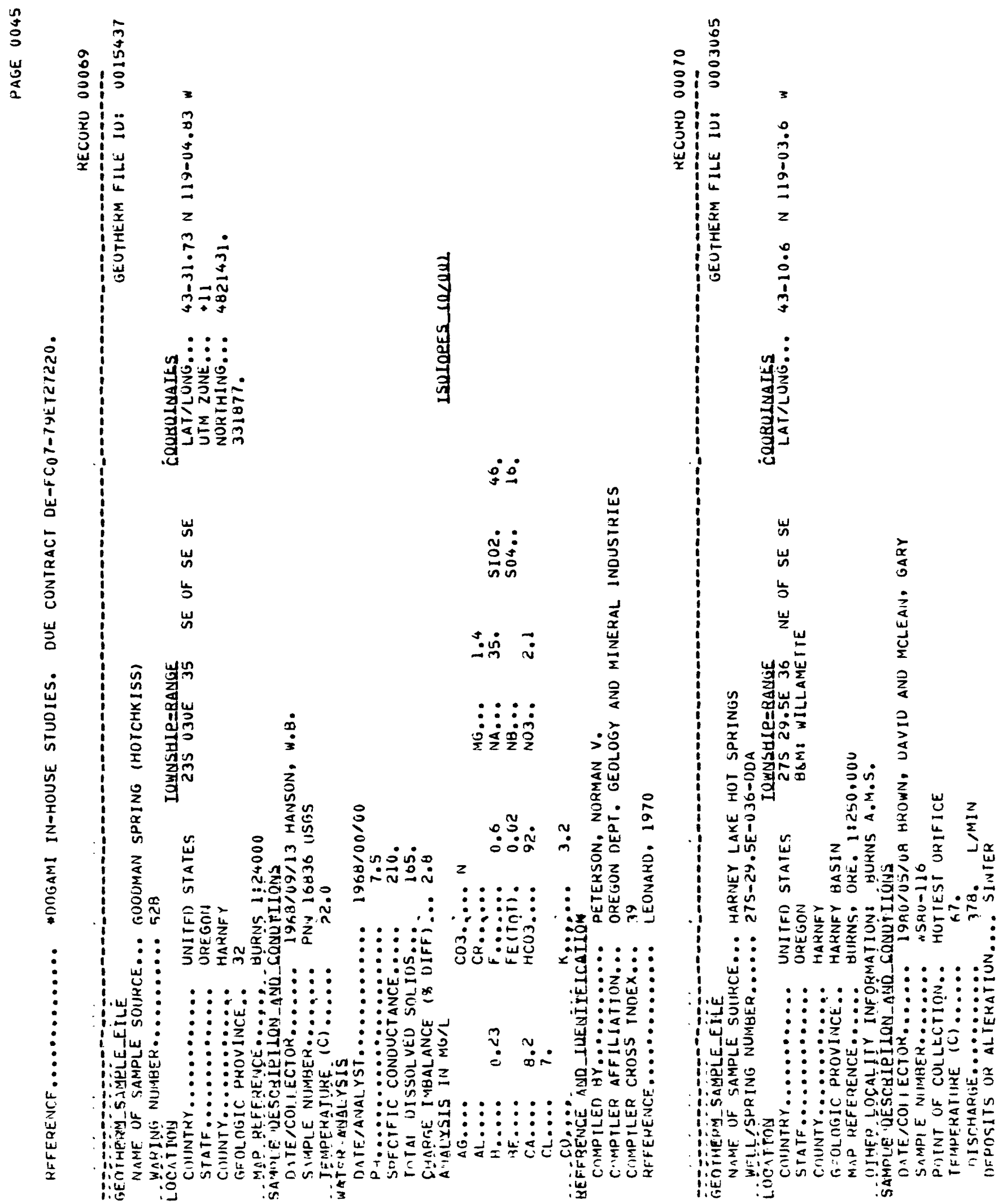




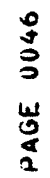
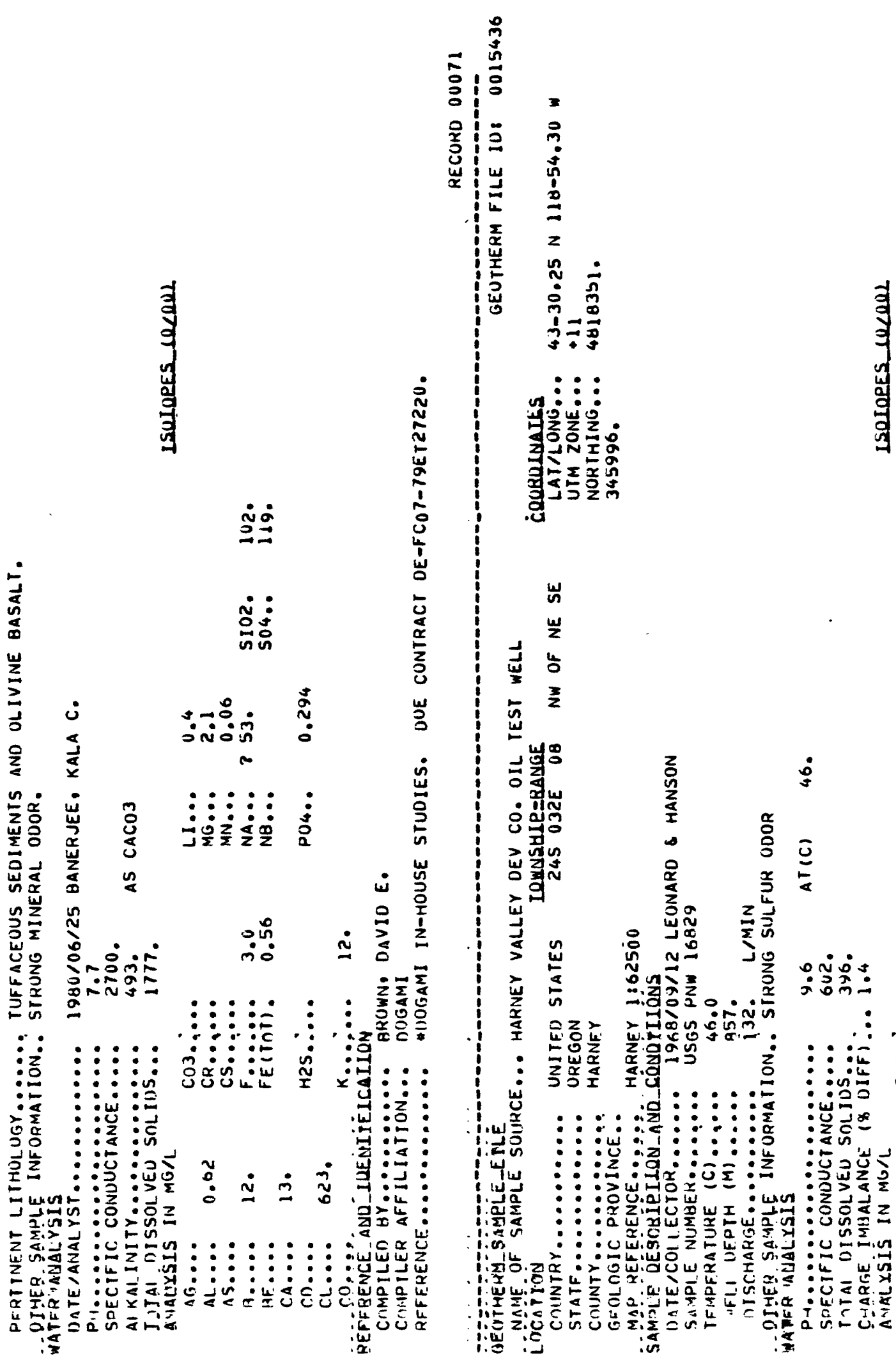

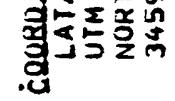
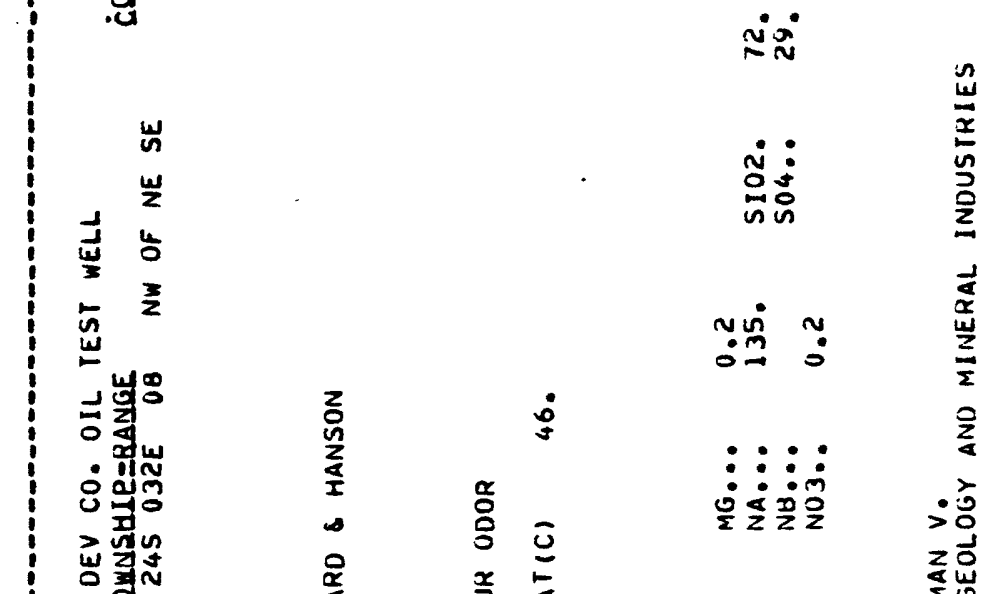

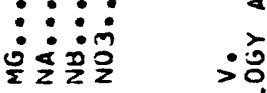

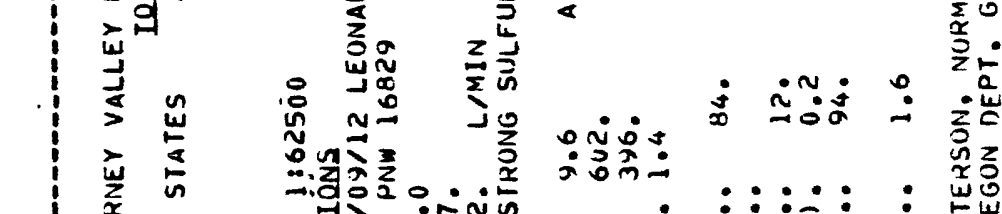

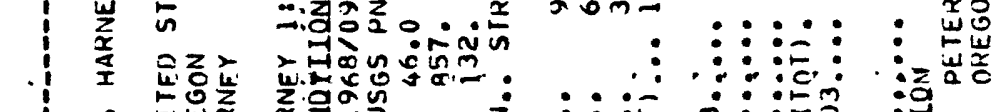

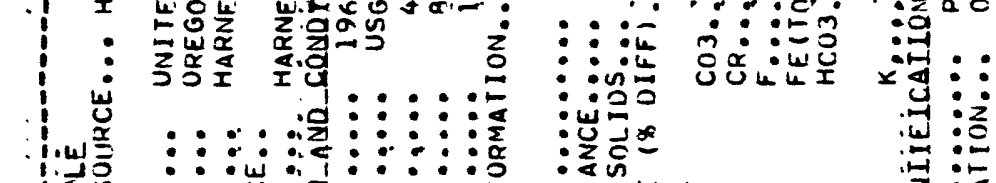

势

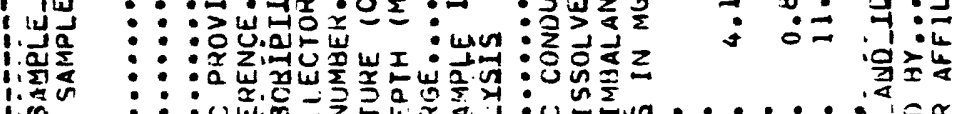

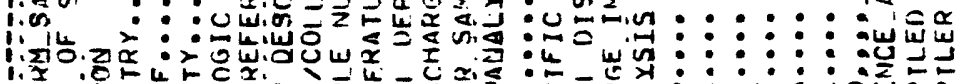

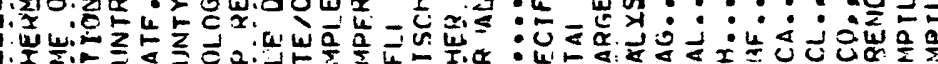

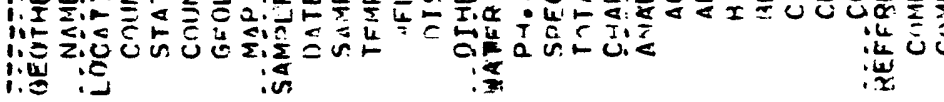




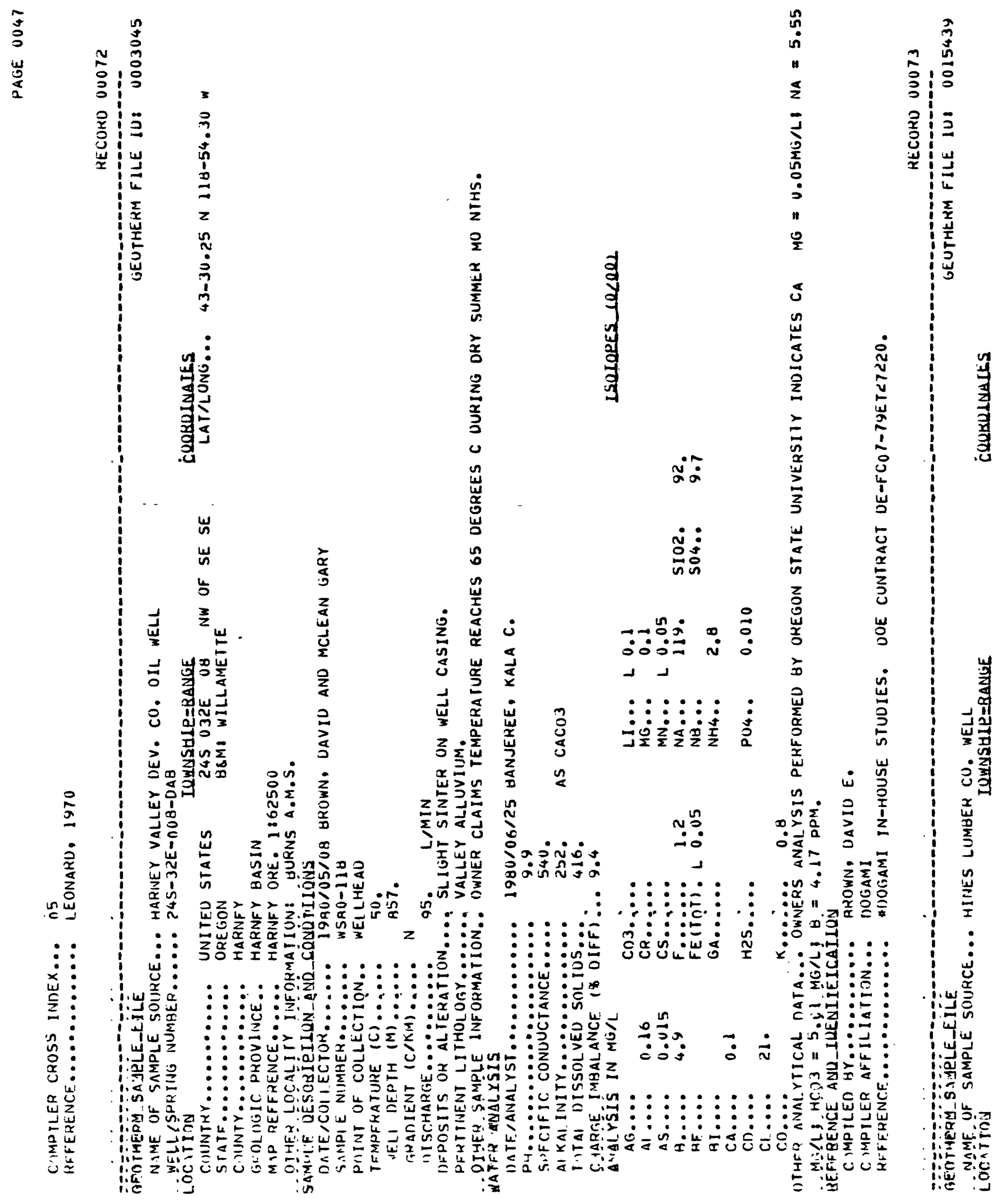



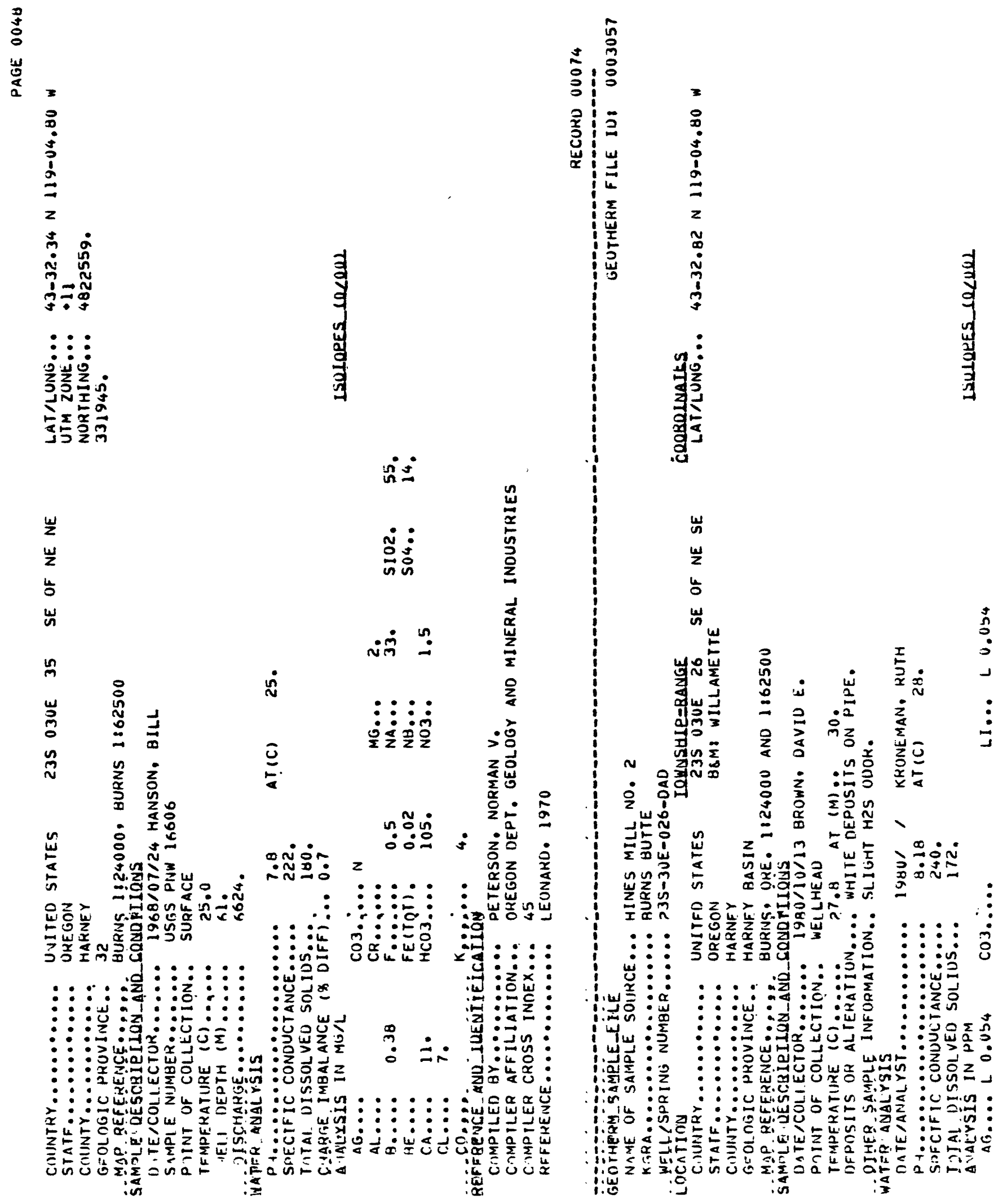
苟

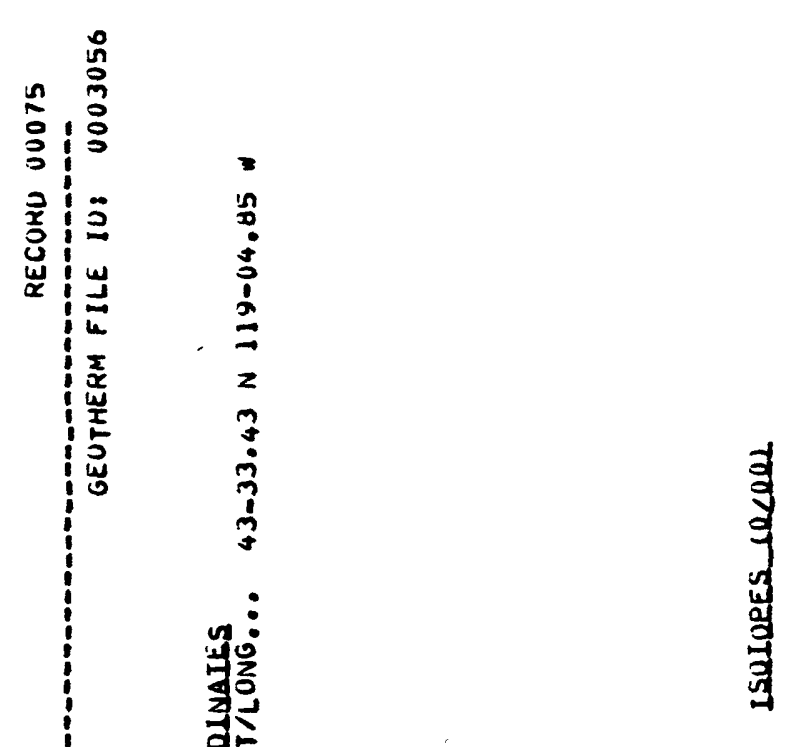

\lrcorner ـ

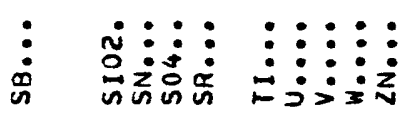

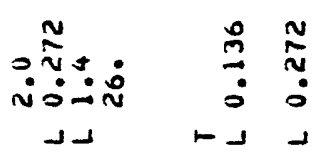

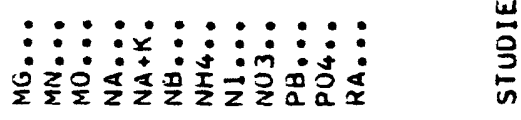
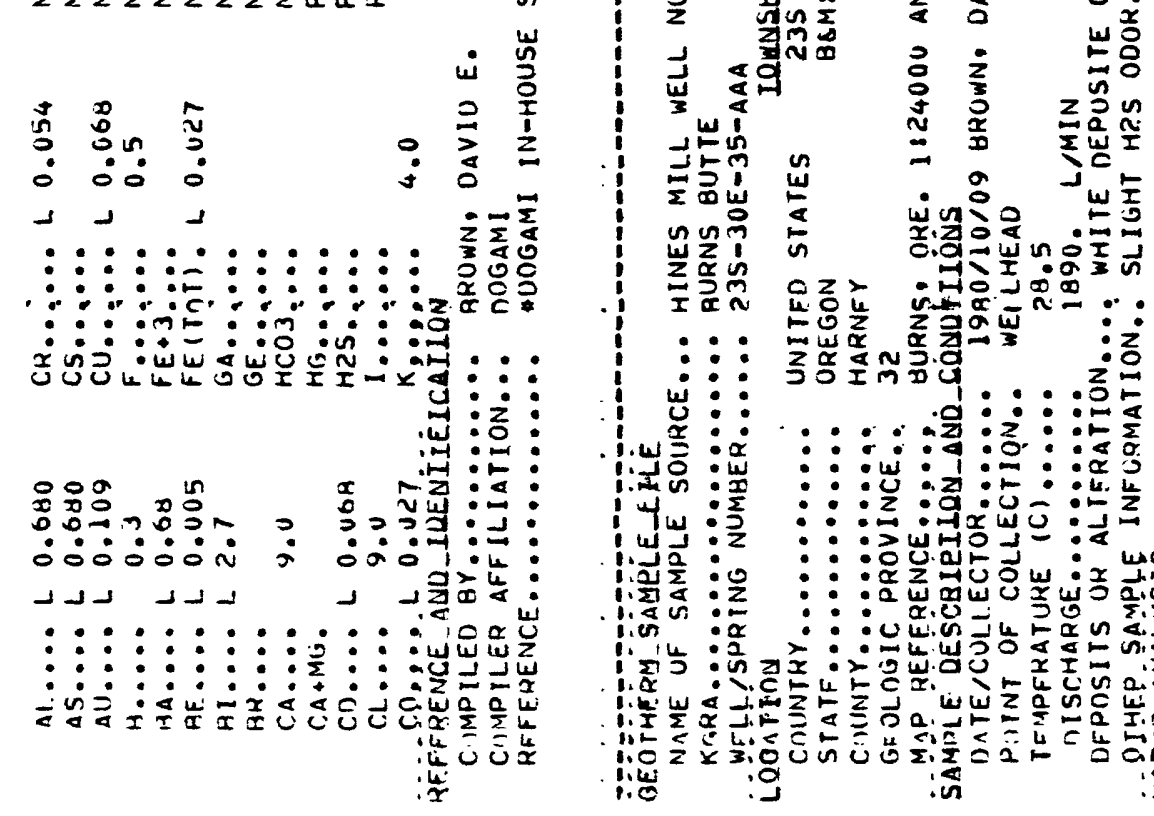

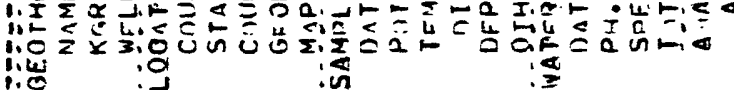

范

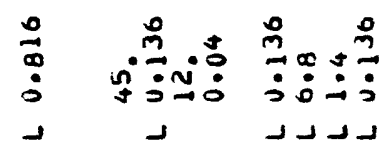

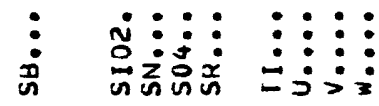

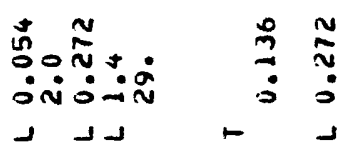

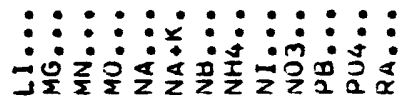

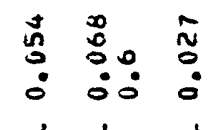

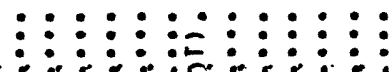

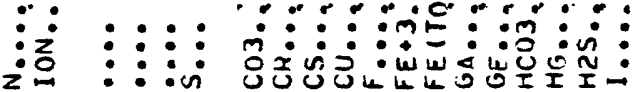

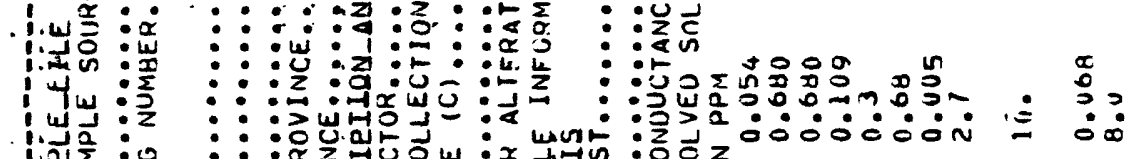

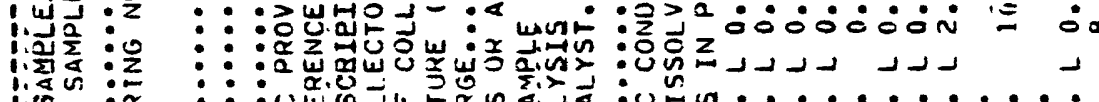

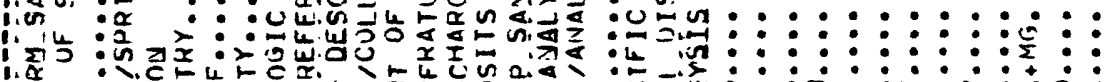

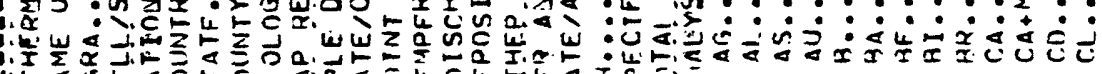


in
8
uั
:
a

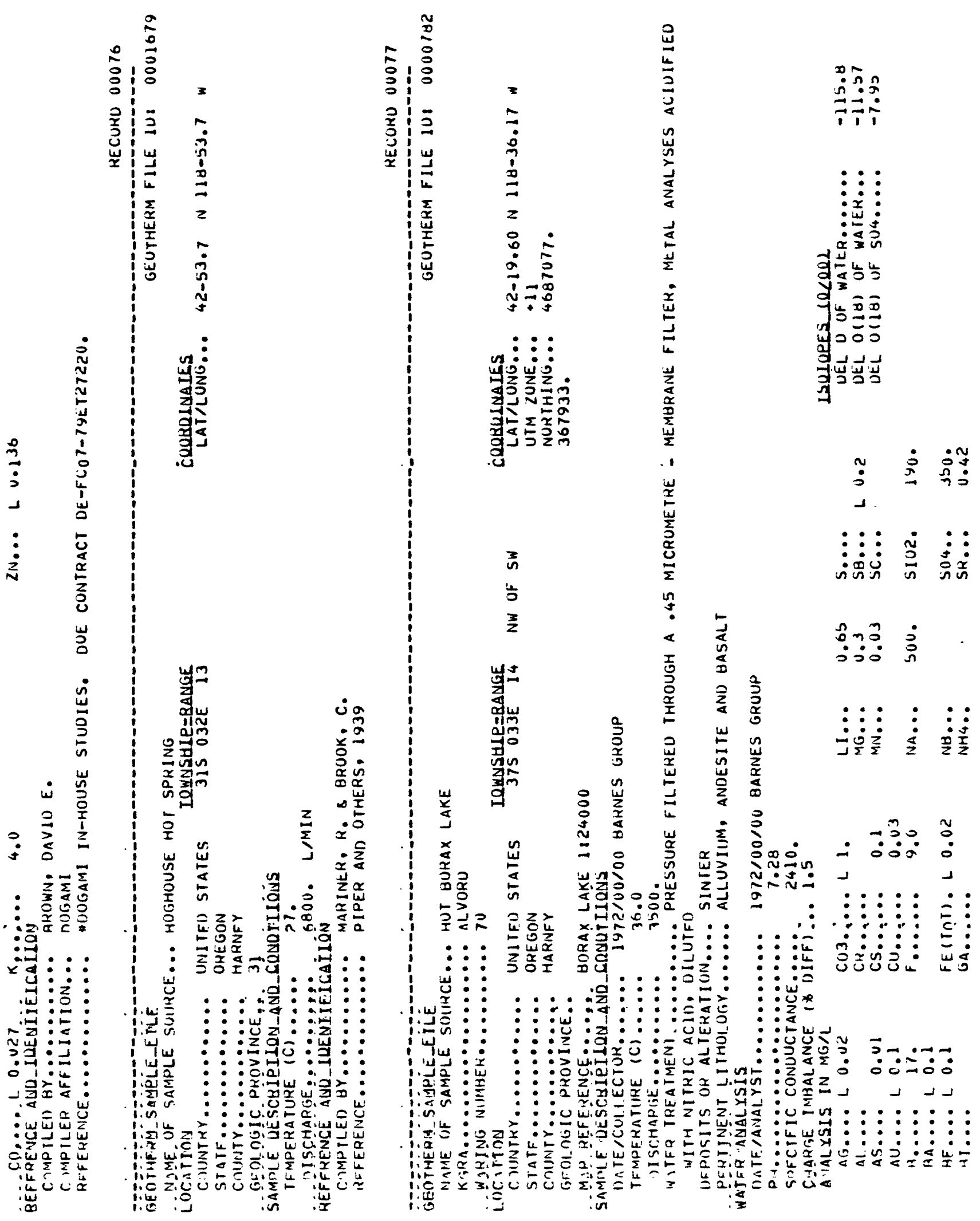


$\vec{n}$
0
$u$
0
$a$

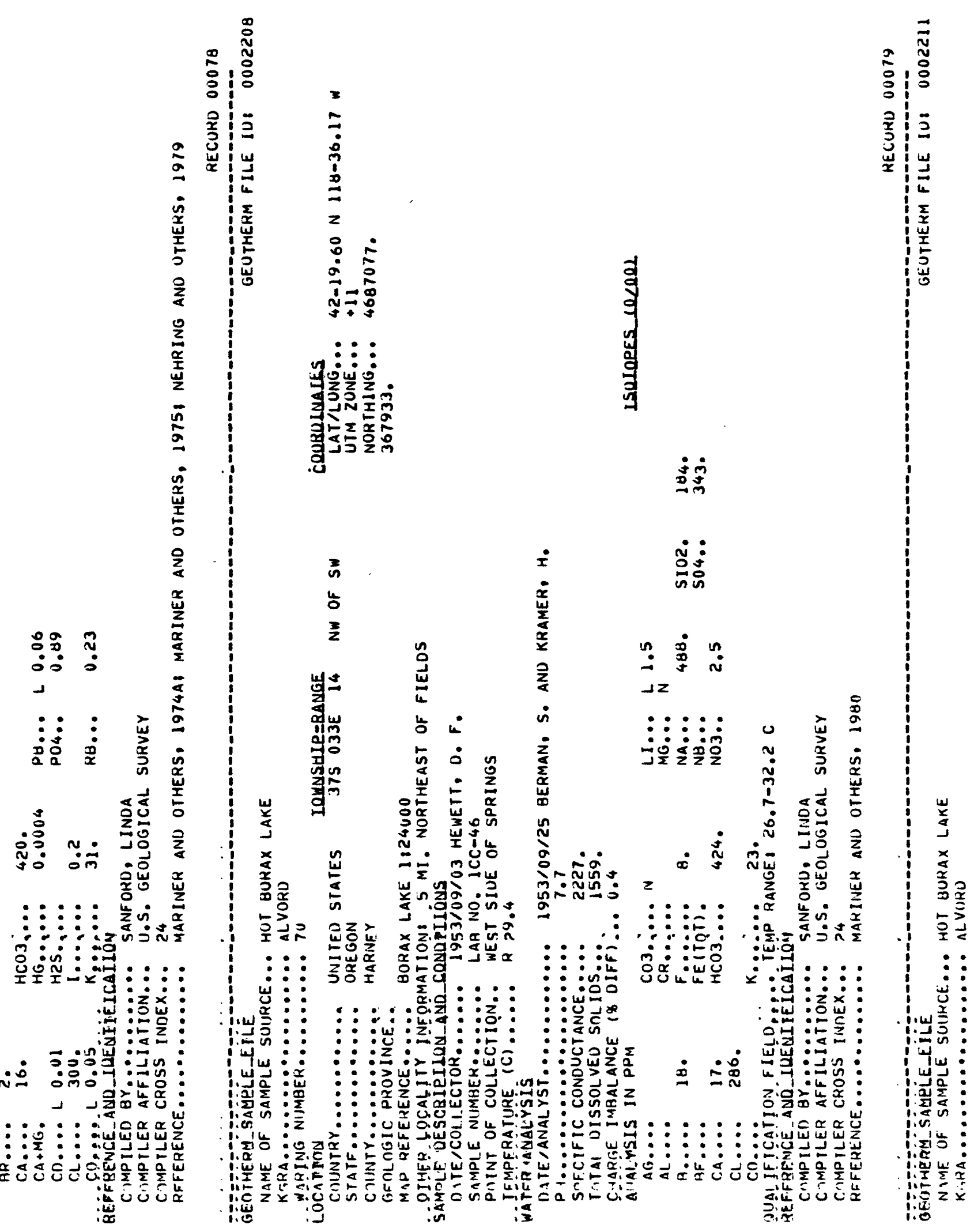


:
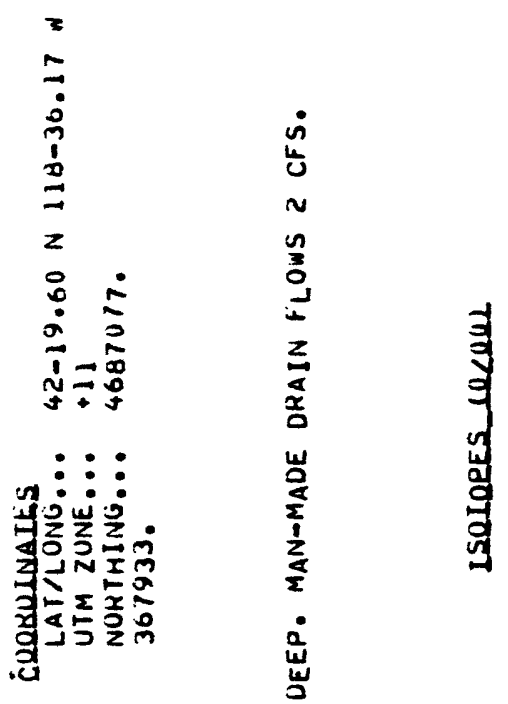

$\dot{m} \dot{0}$

苛宫

is

z

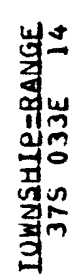

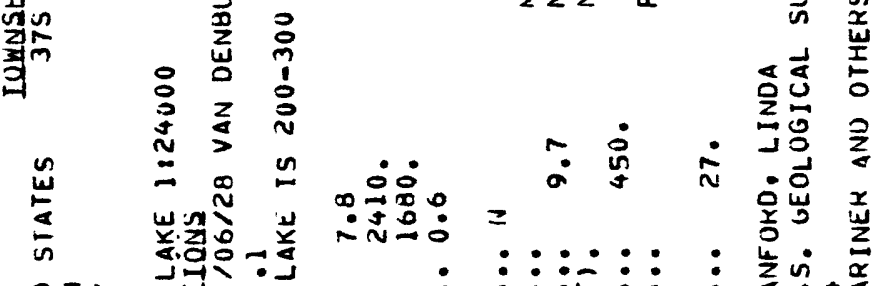

三 든

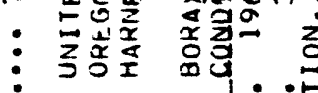

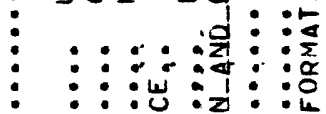

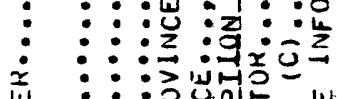

羊: : : : :

焉: : : : :

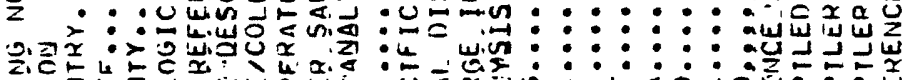

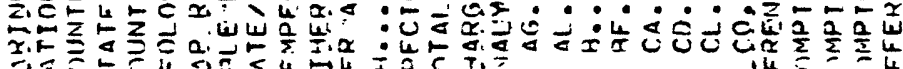

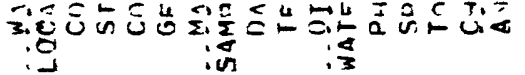

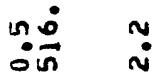

$::: \vdots$

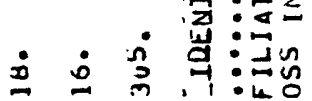

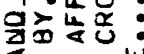

Uல

故
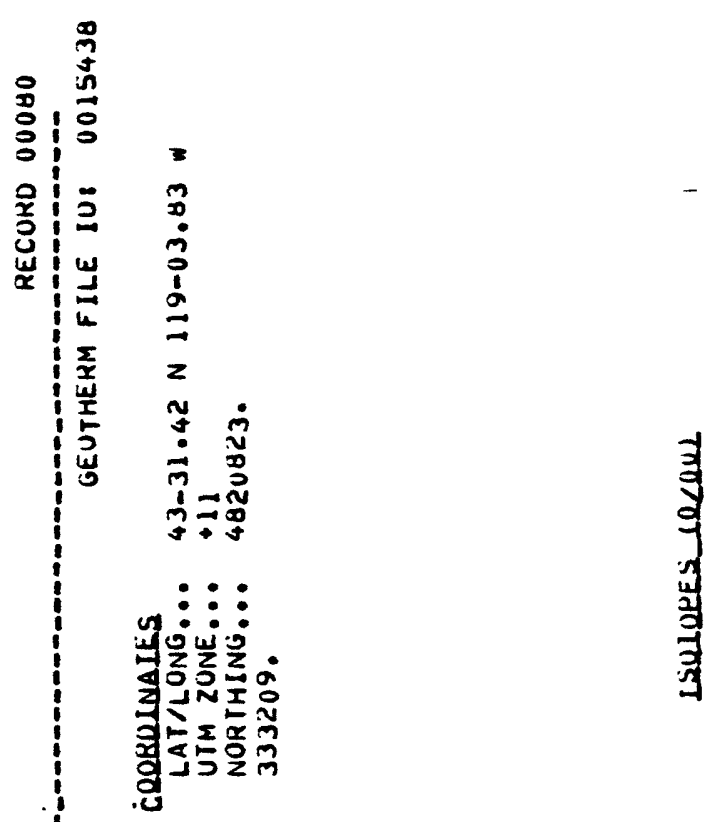

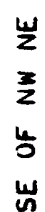

总

$\stackrel{-}{\circ}$

$\dot{\text { i }}$

$\frac{1}{4}$

: 
$\frac{\pi}{3}$

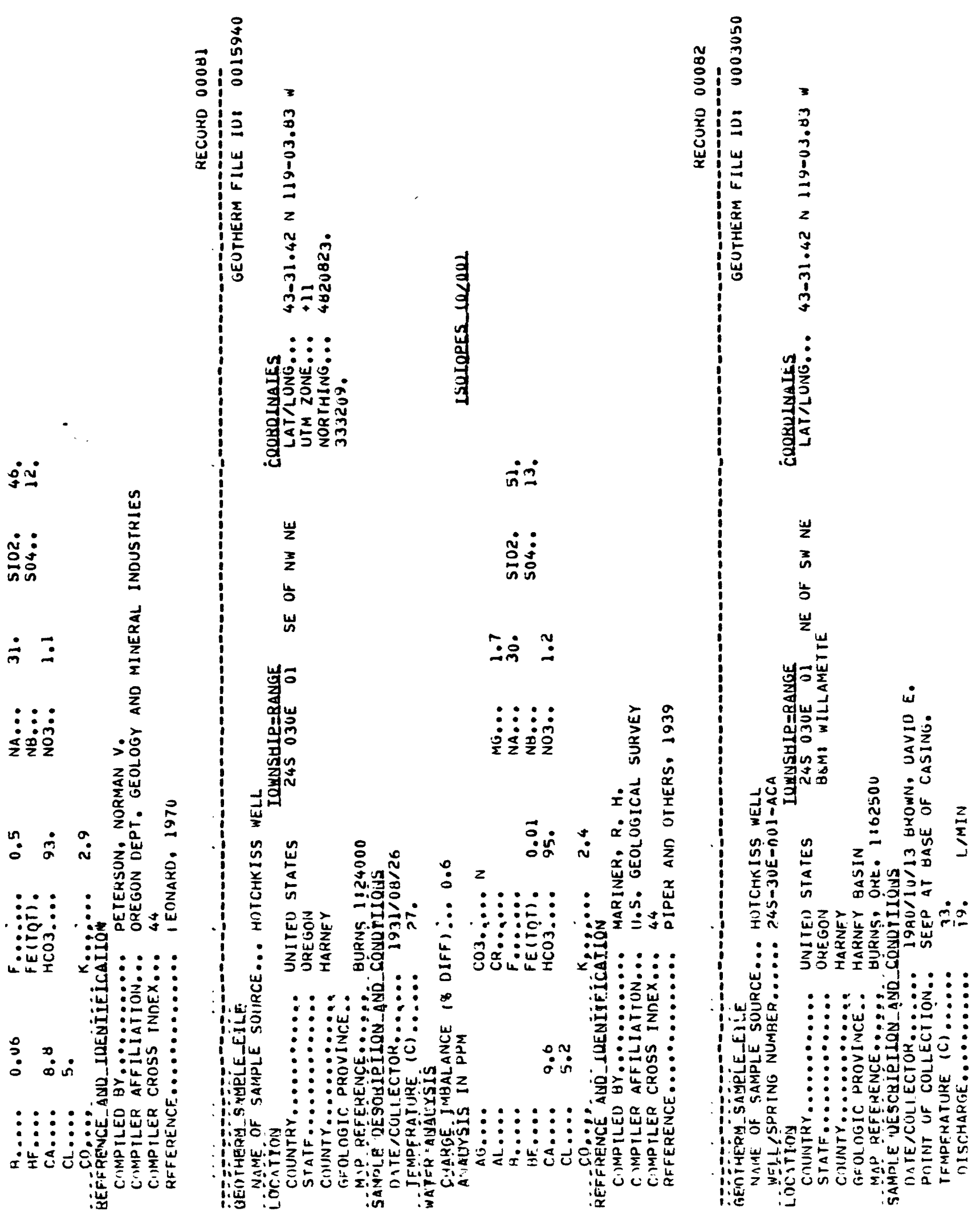


aี

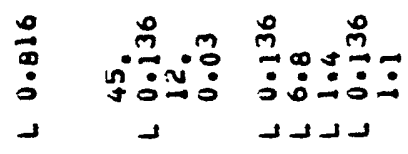

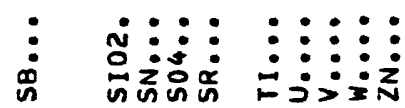

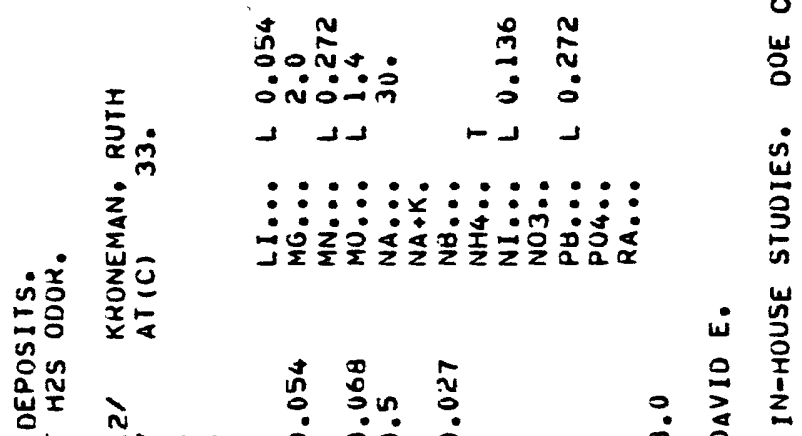

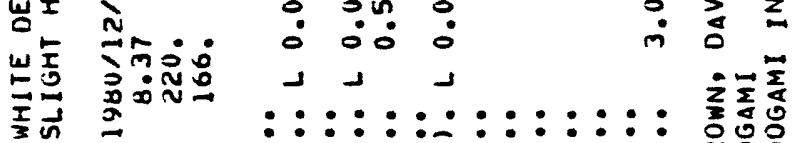
: : : : : : : : : : :

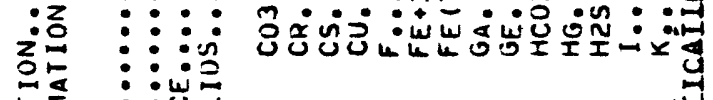

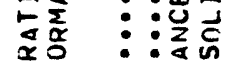

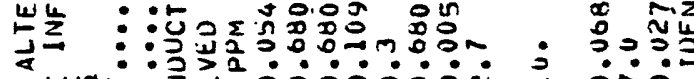

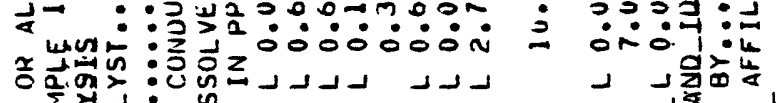

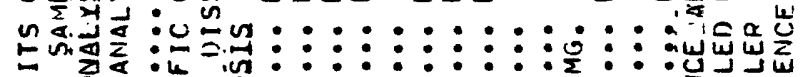

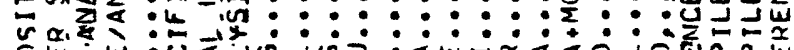

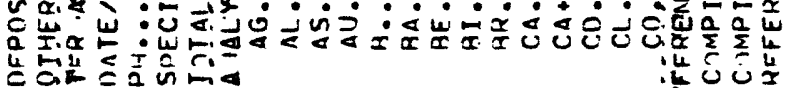

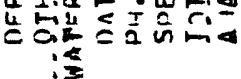

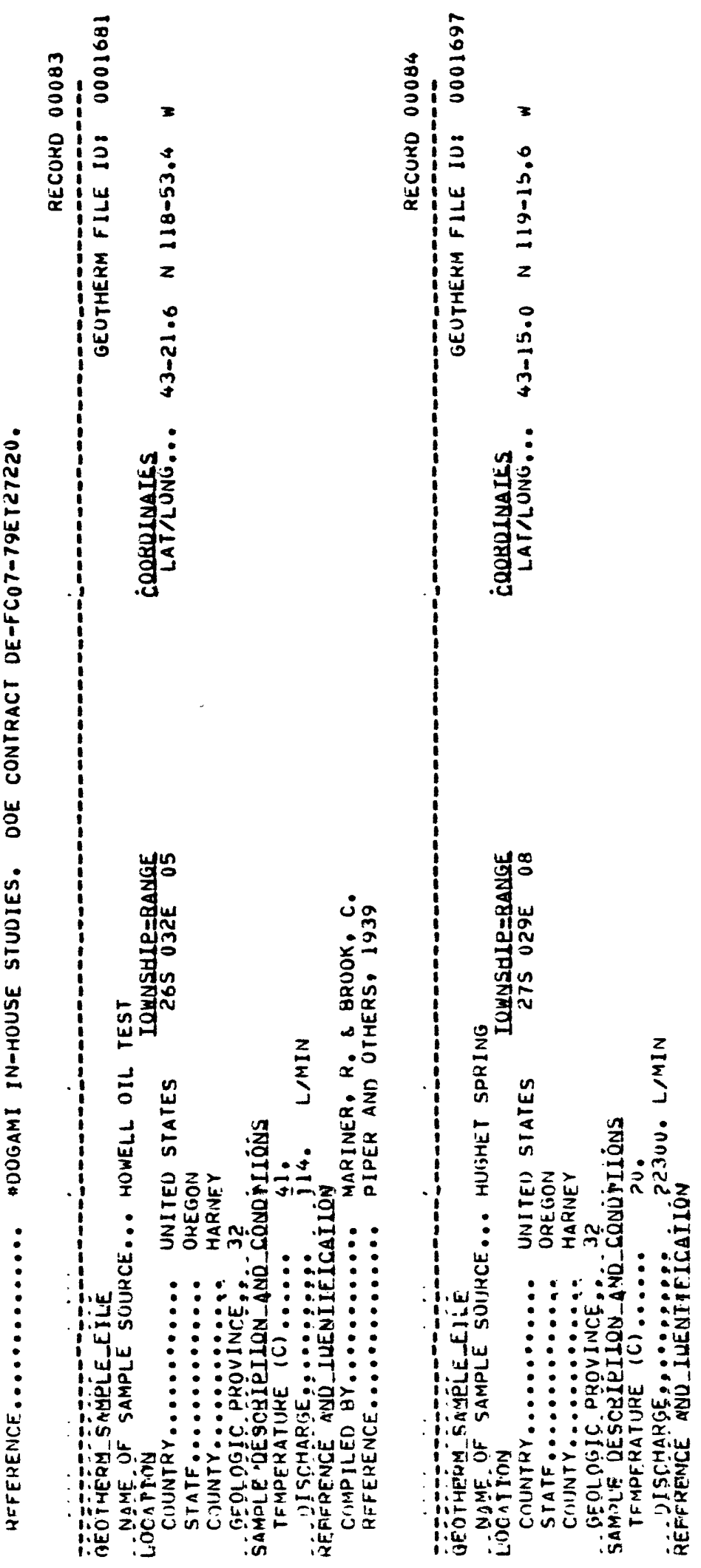


กิ
0
0
4
0
2
2

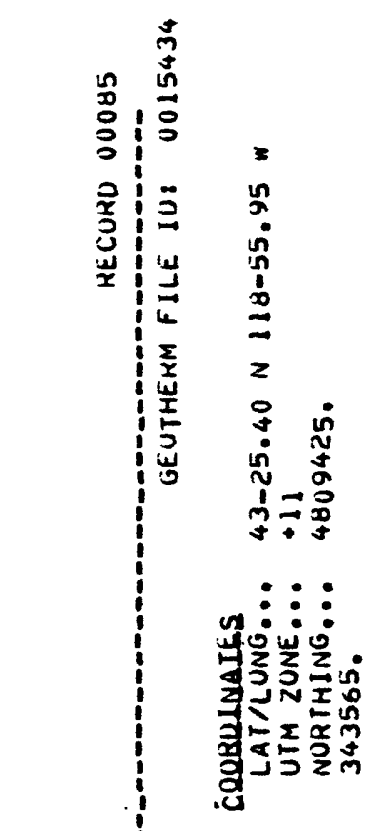

aำ

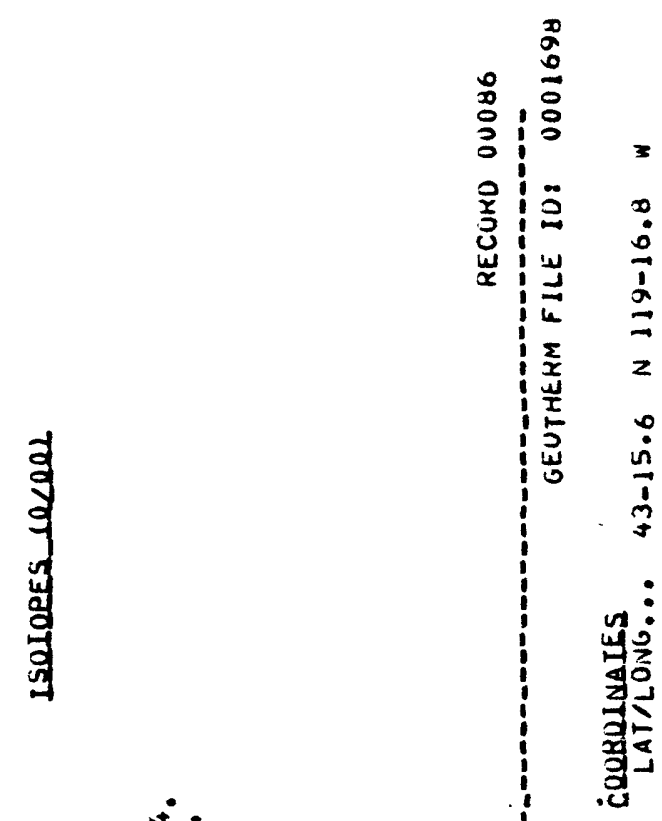

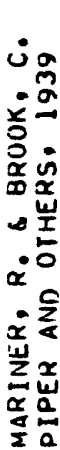

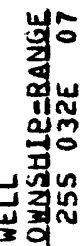

落:

点

$\dddot{5} \dot{0}:$

宪

$: \vdots: \vdots \quad$ ะ

$\frac{8}{5}$

官

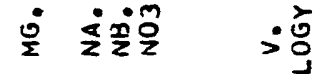

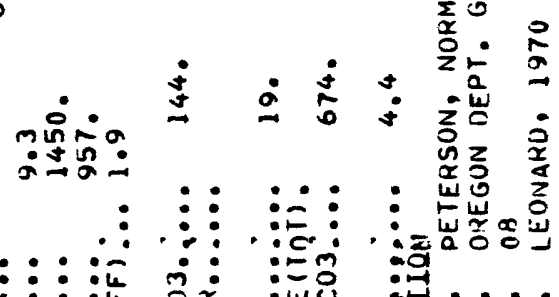

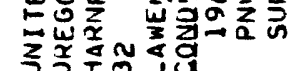

总寽 :

यु : : : :

$:::::::::::^{n}$

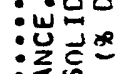

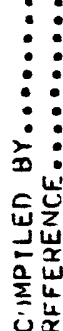

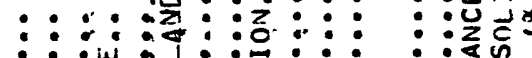

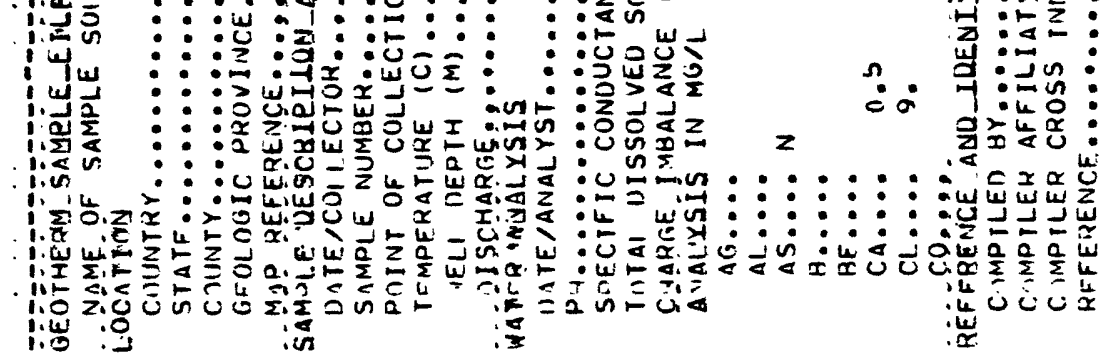

: : :

:

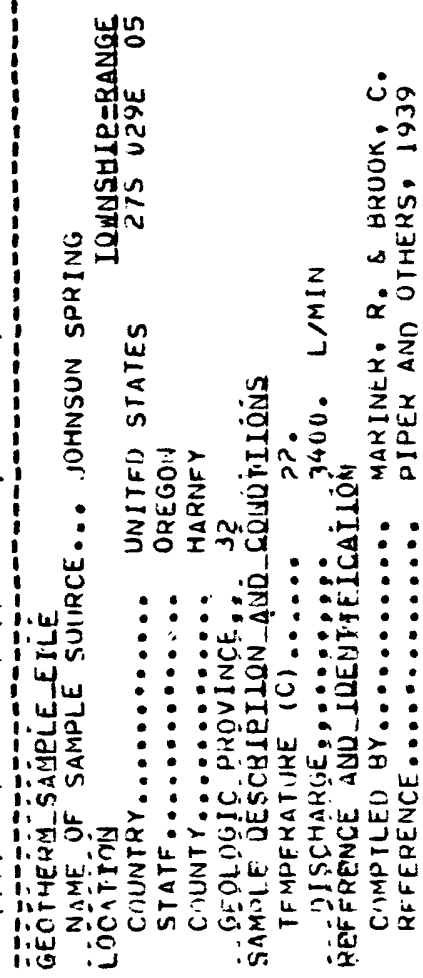




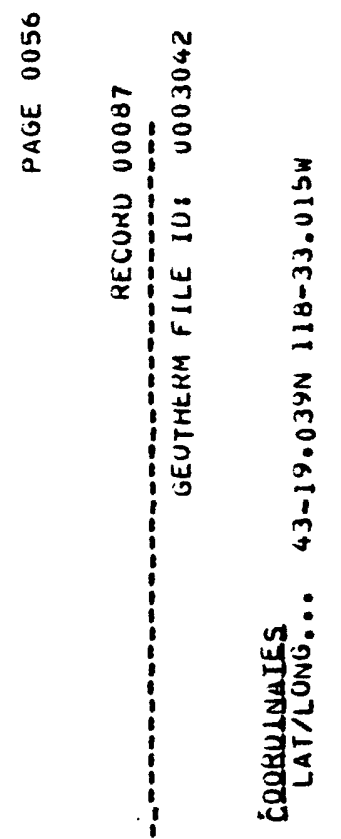

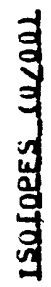

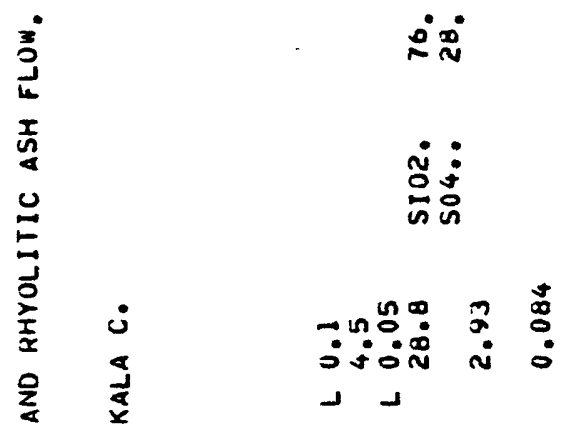

焉

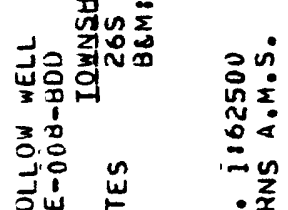

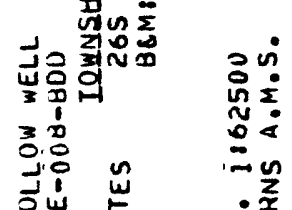

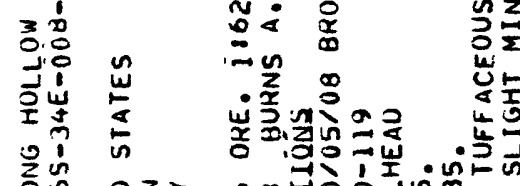

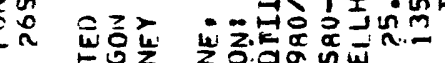

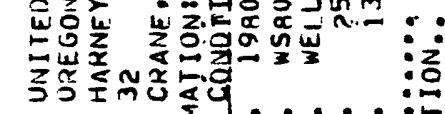

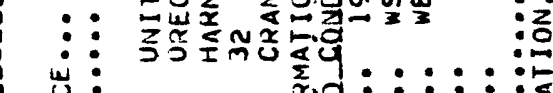

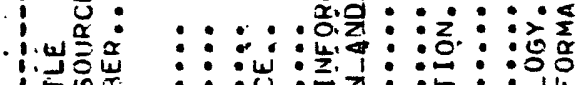

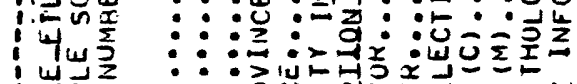

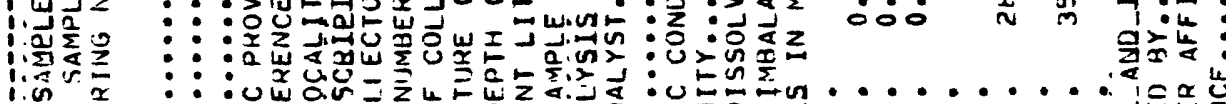

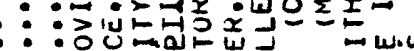

$:$ :

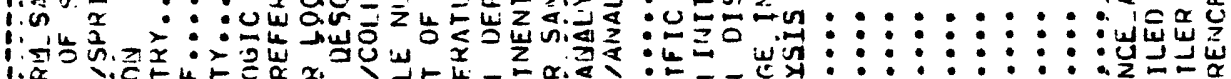

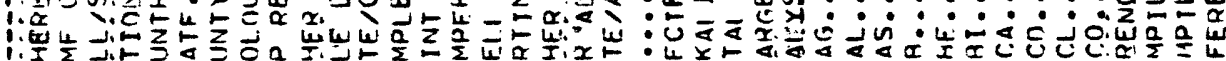

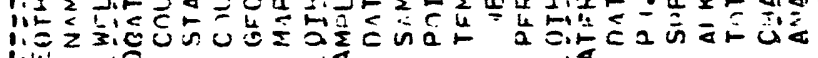

$\stackrel{0}{0}$

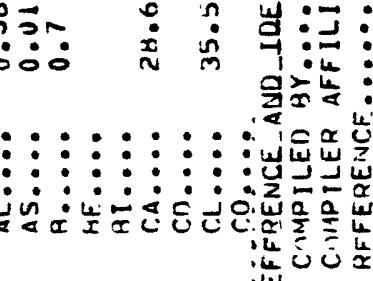

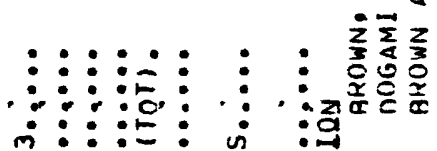

守守论出这
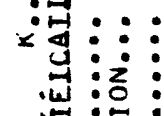


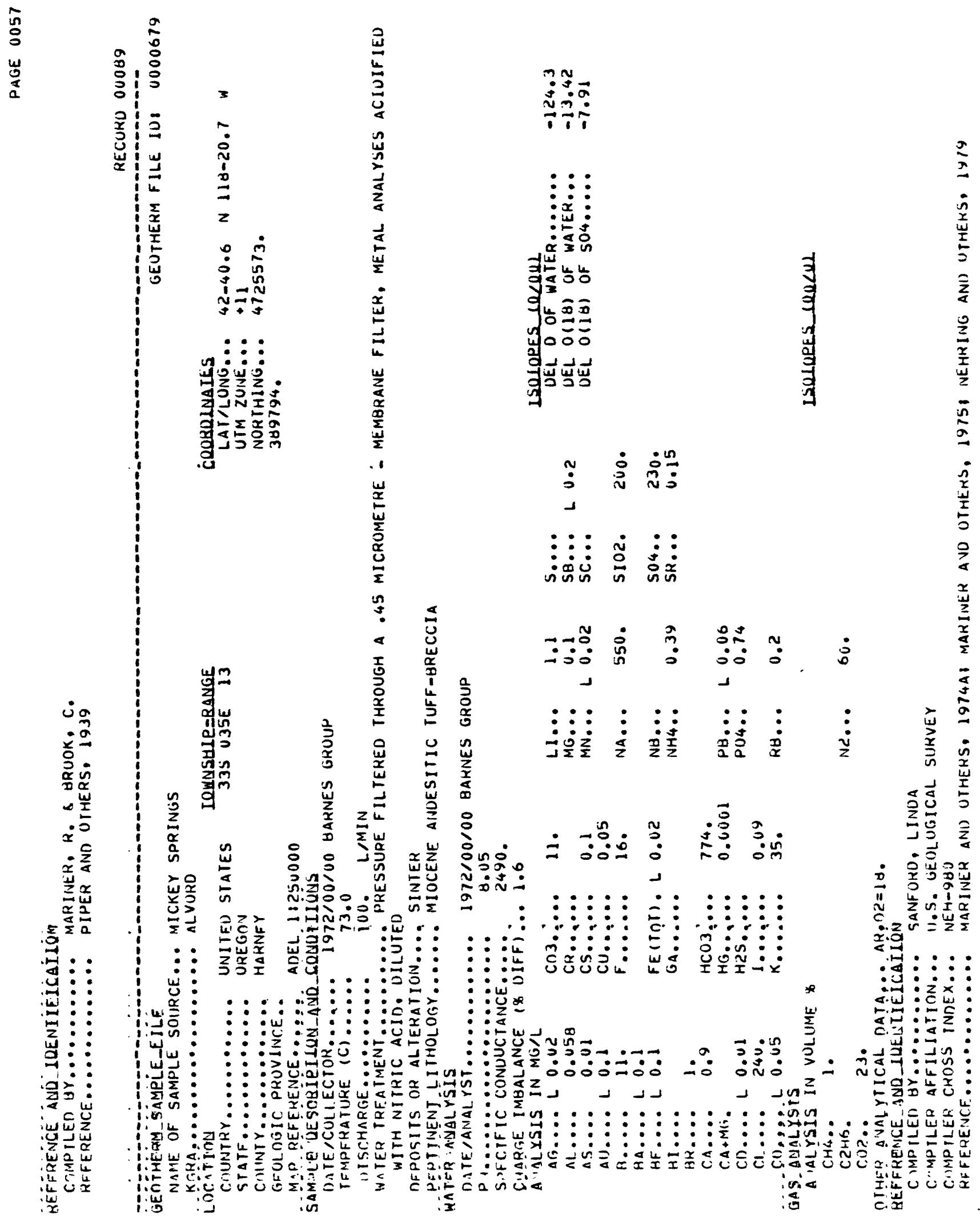




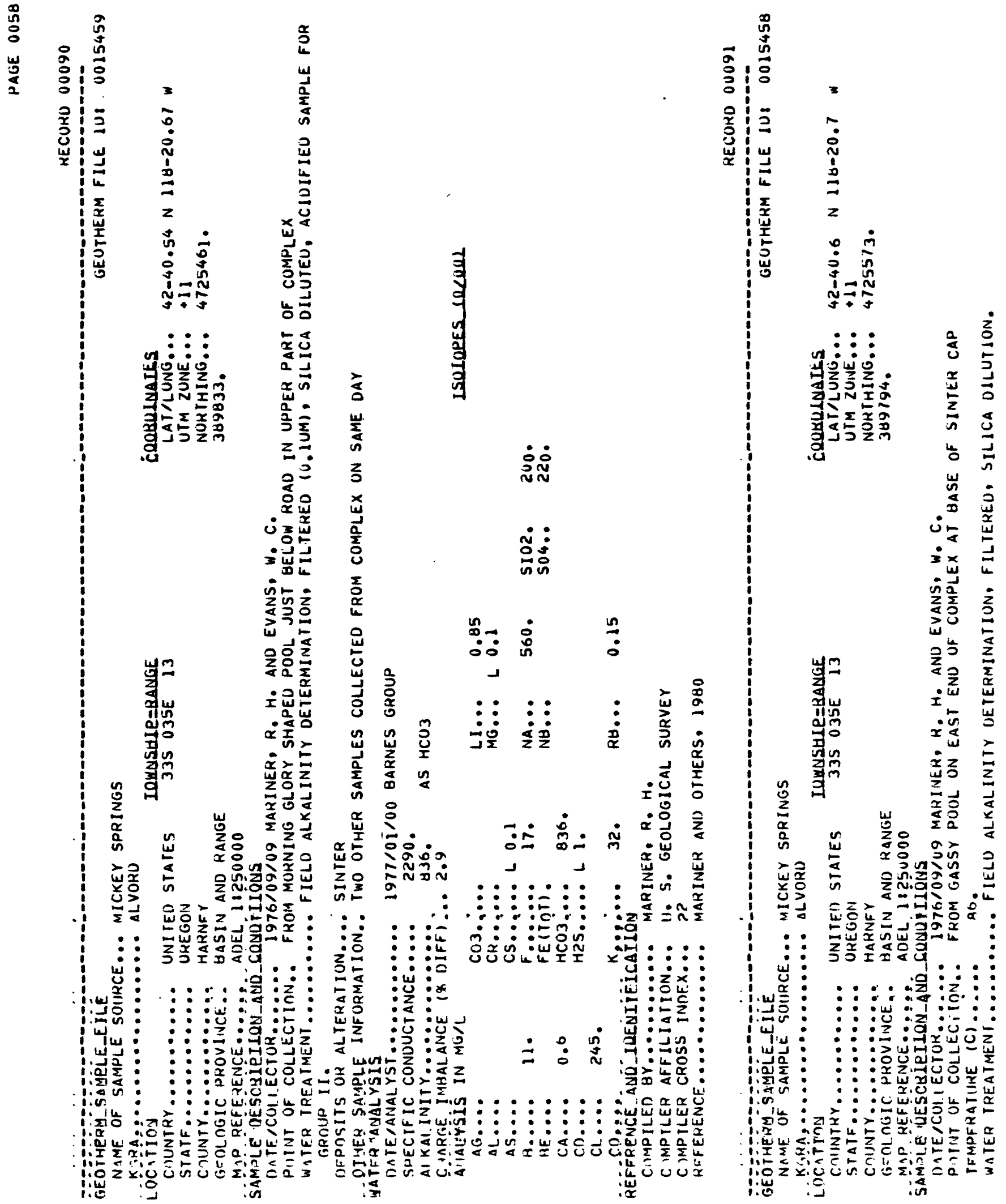


0
0
0
.
0
0
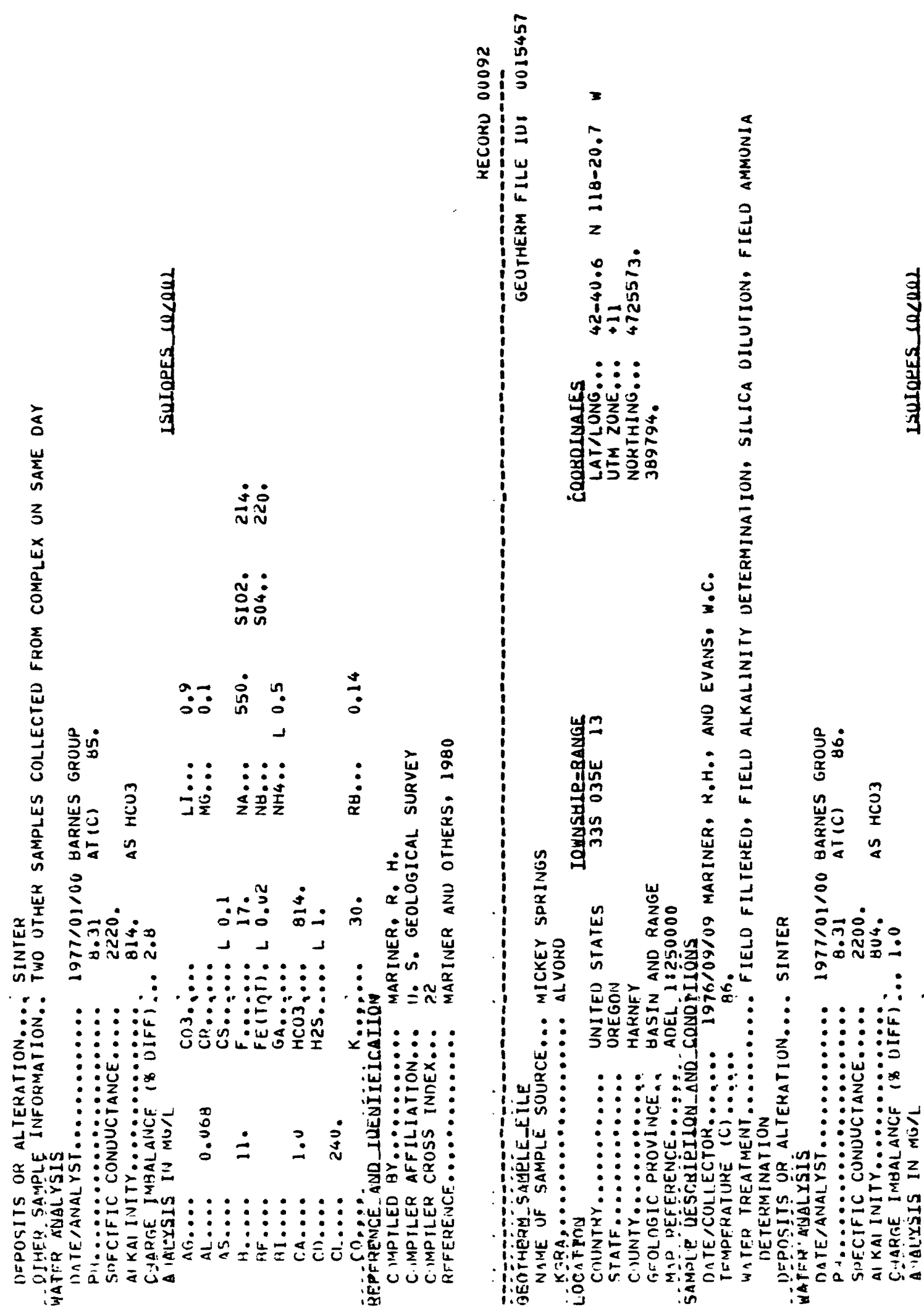

$\dot{\vec{v}} \dot{\vec{v}}$

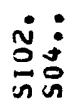

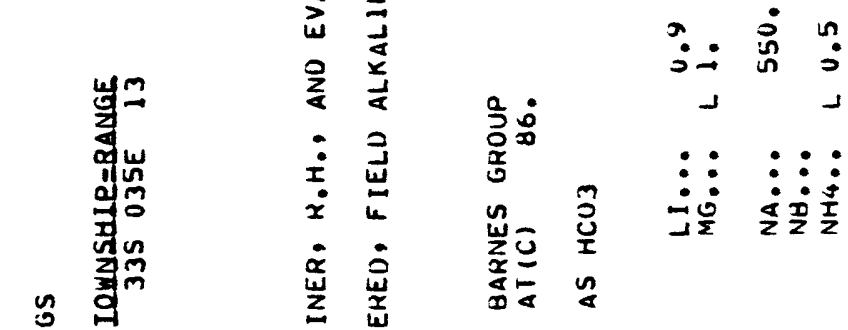

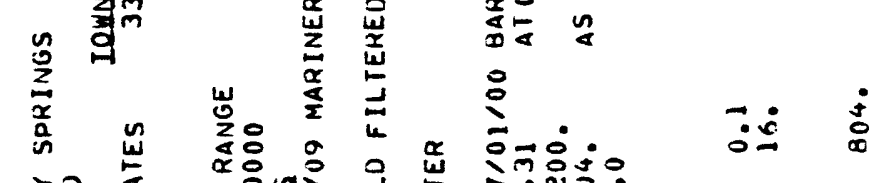

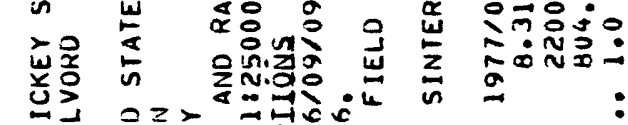

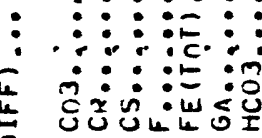

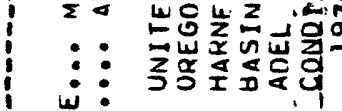

:

:

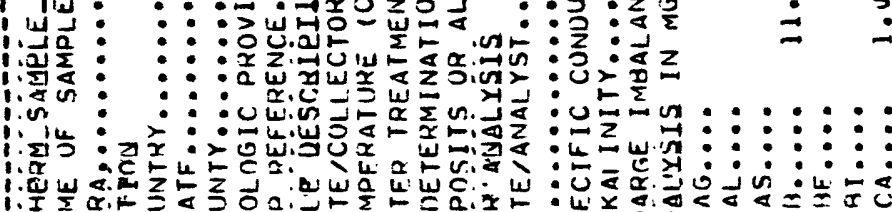

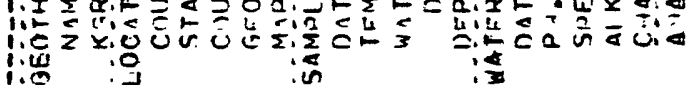




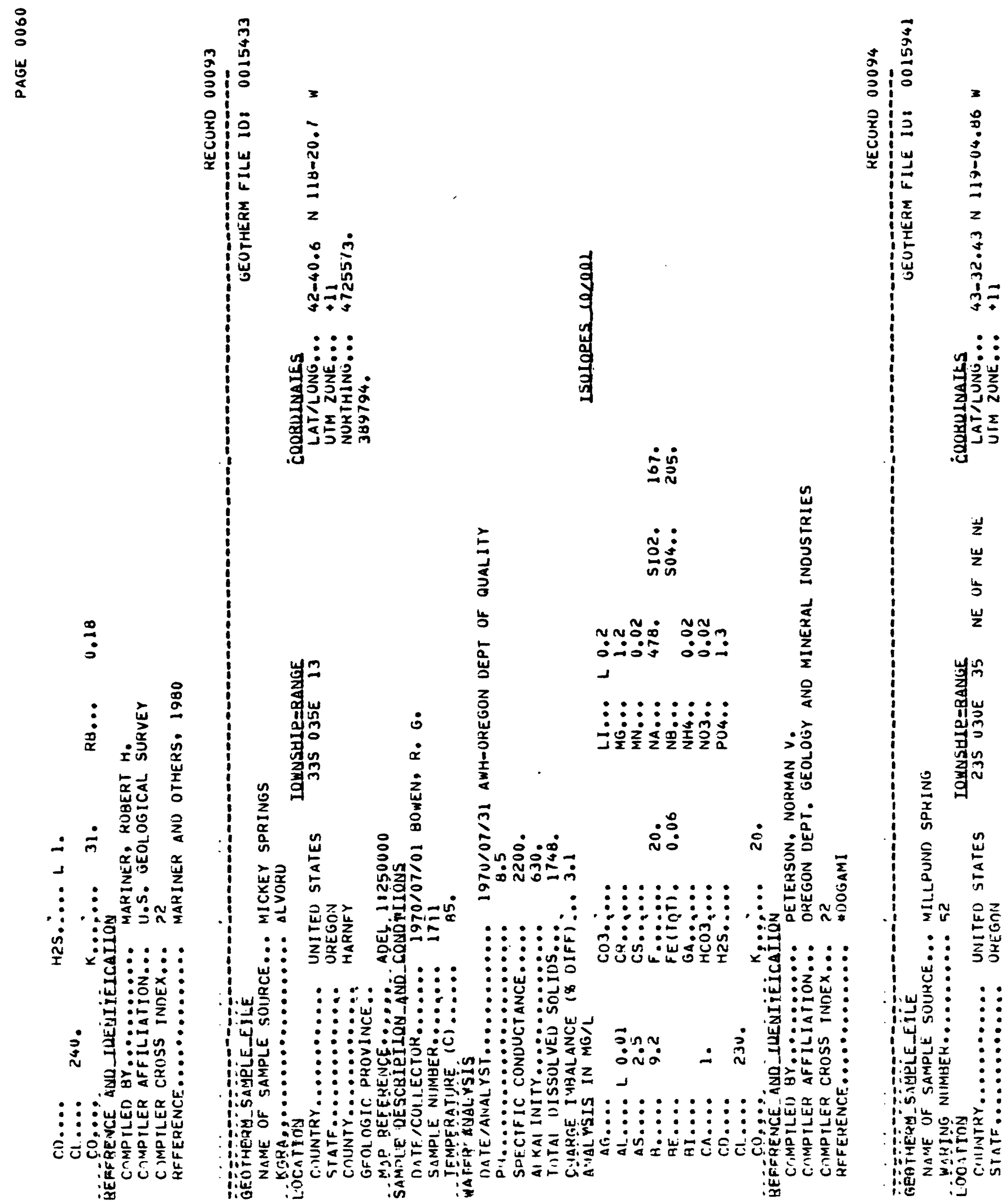


产

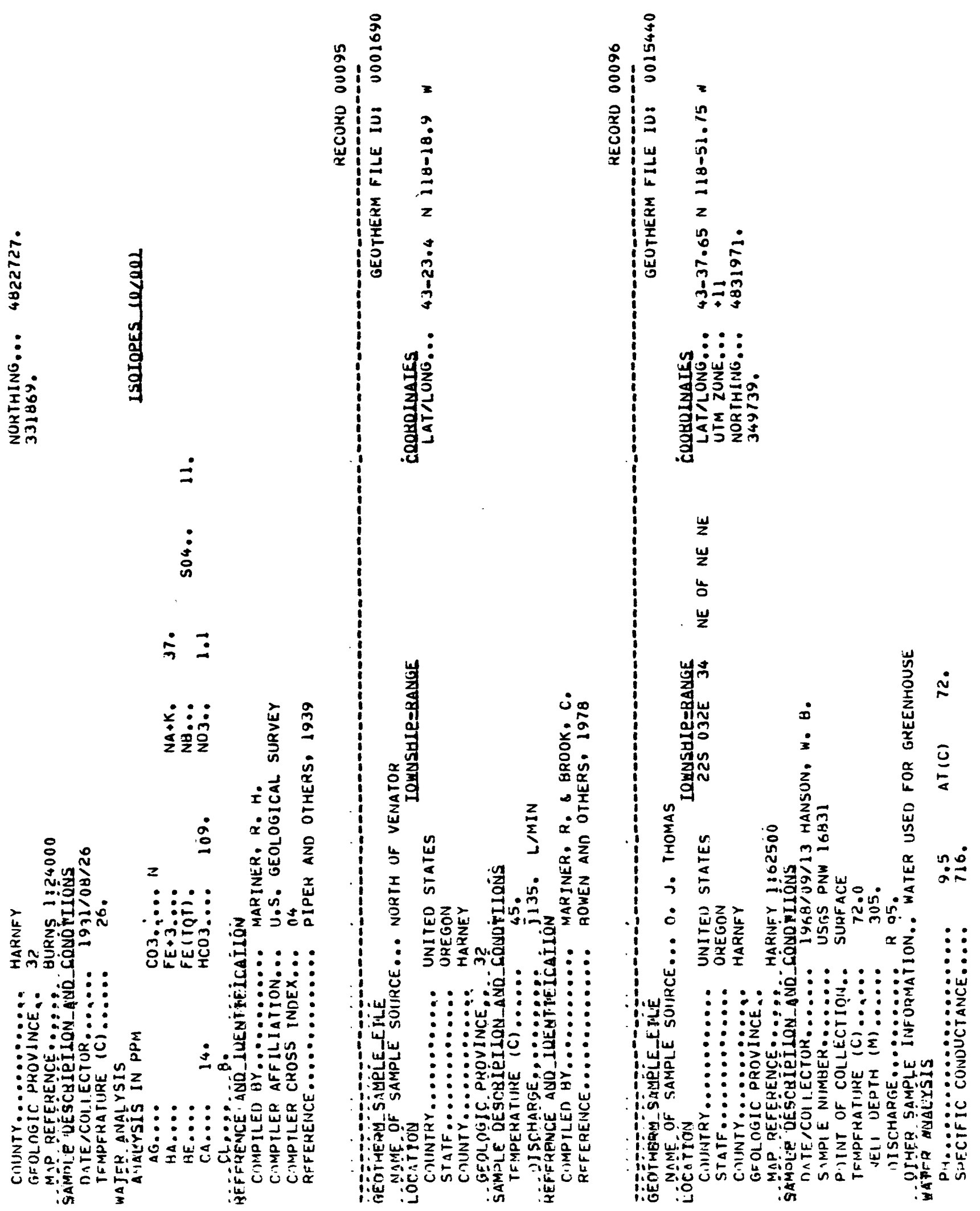




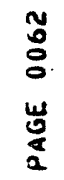
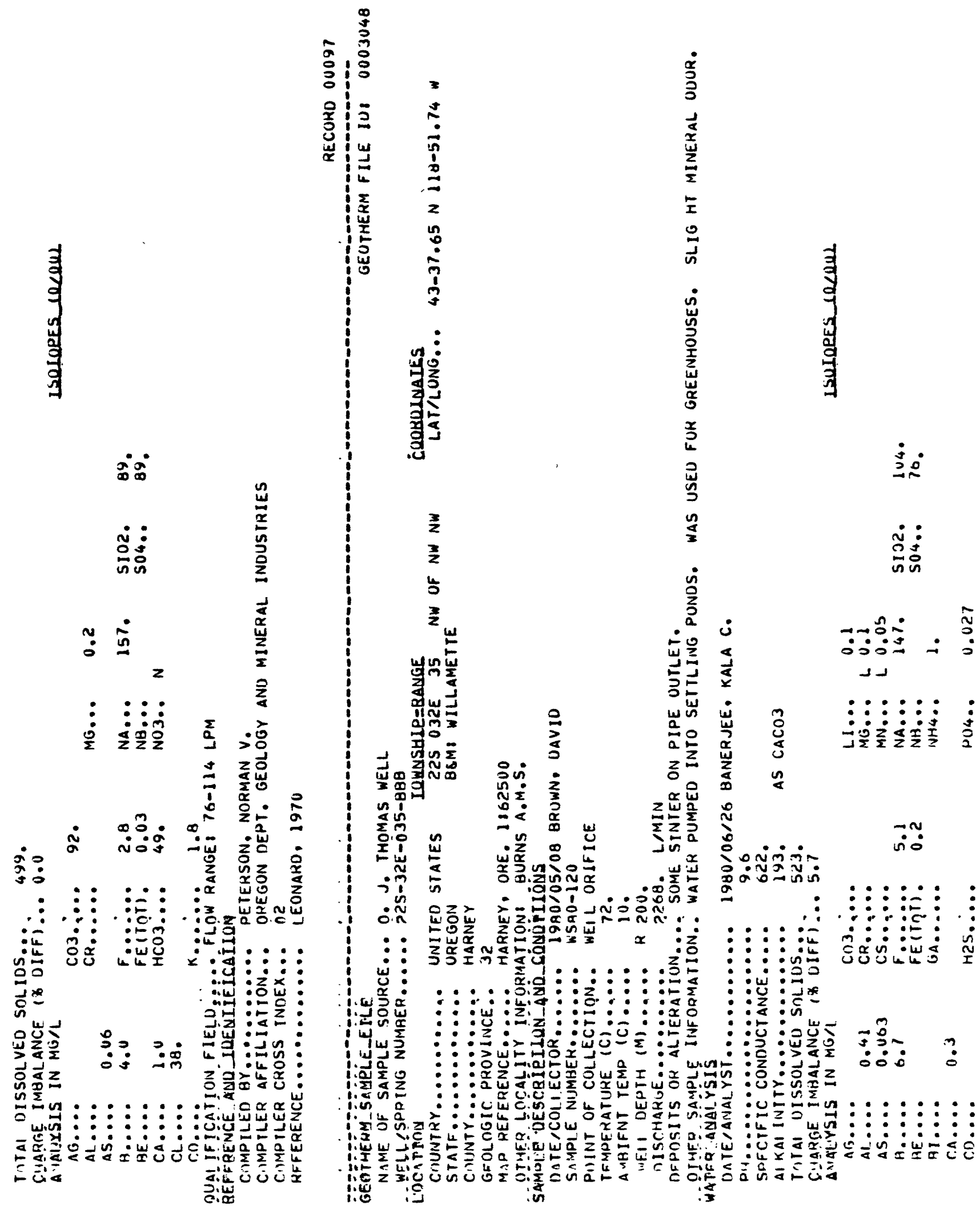

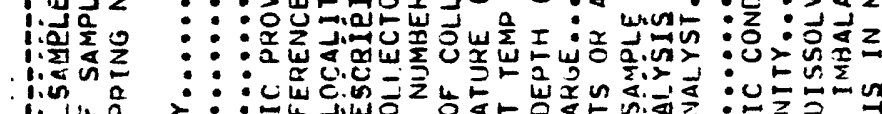

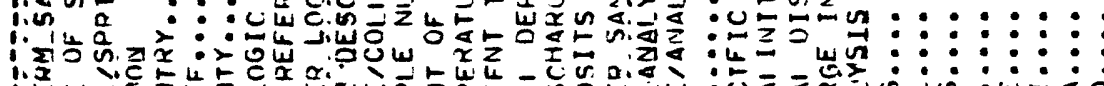

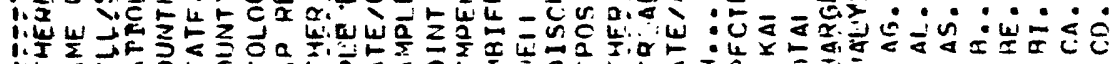

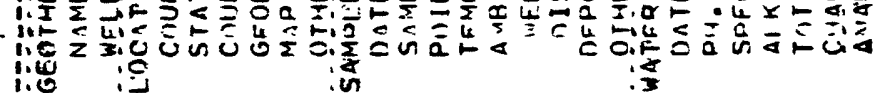


$\frac{3}{3}$

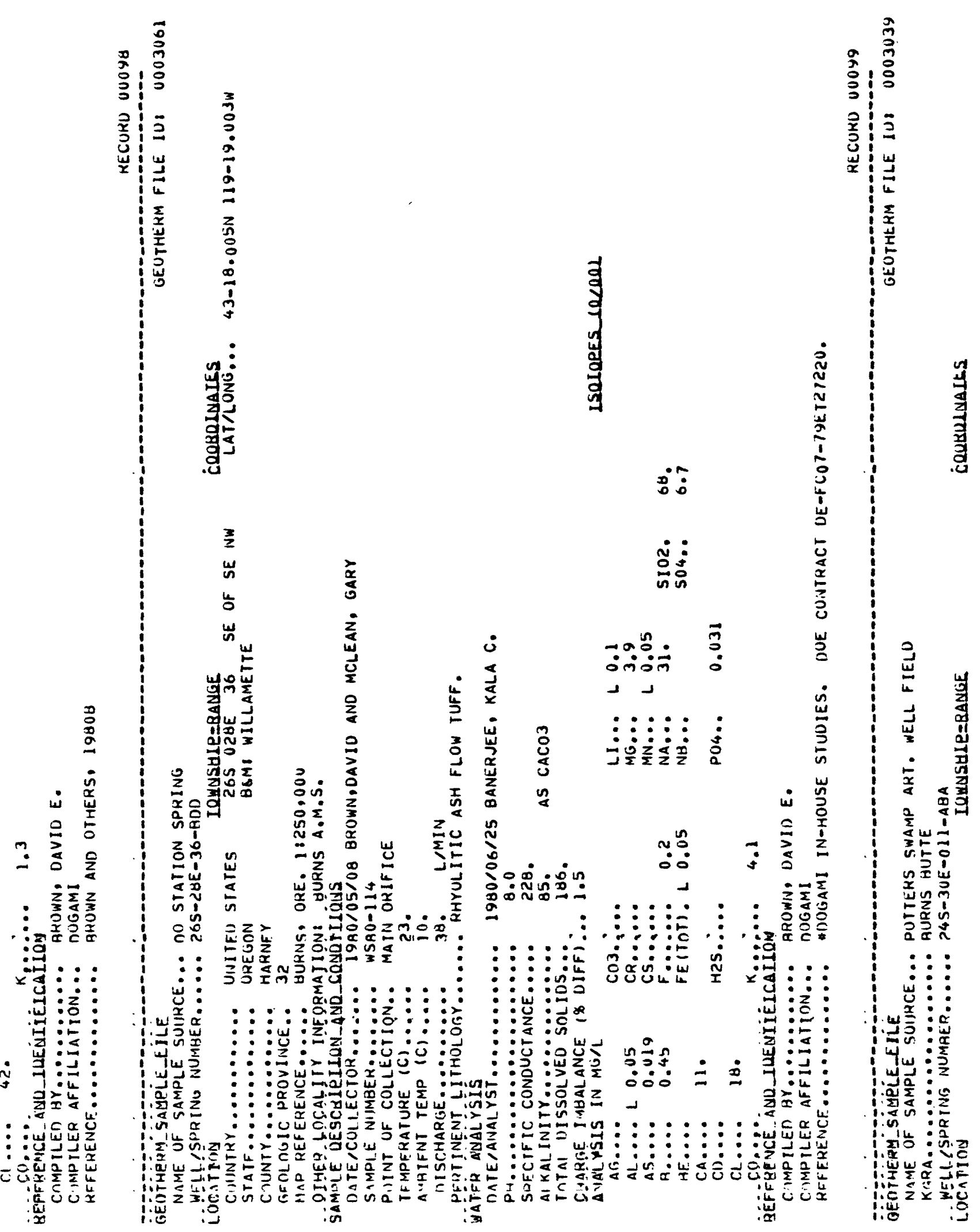



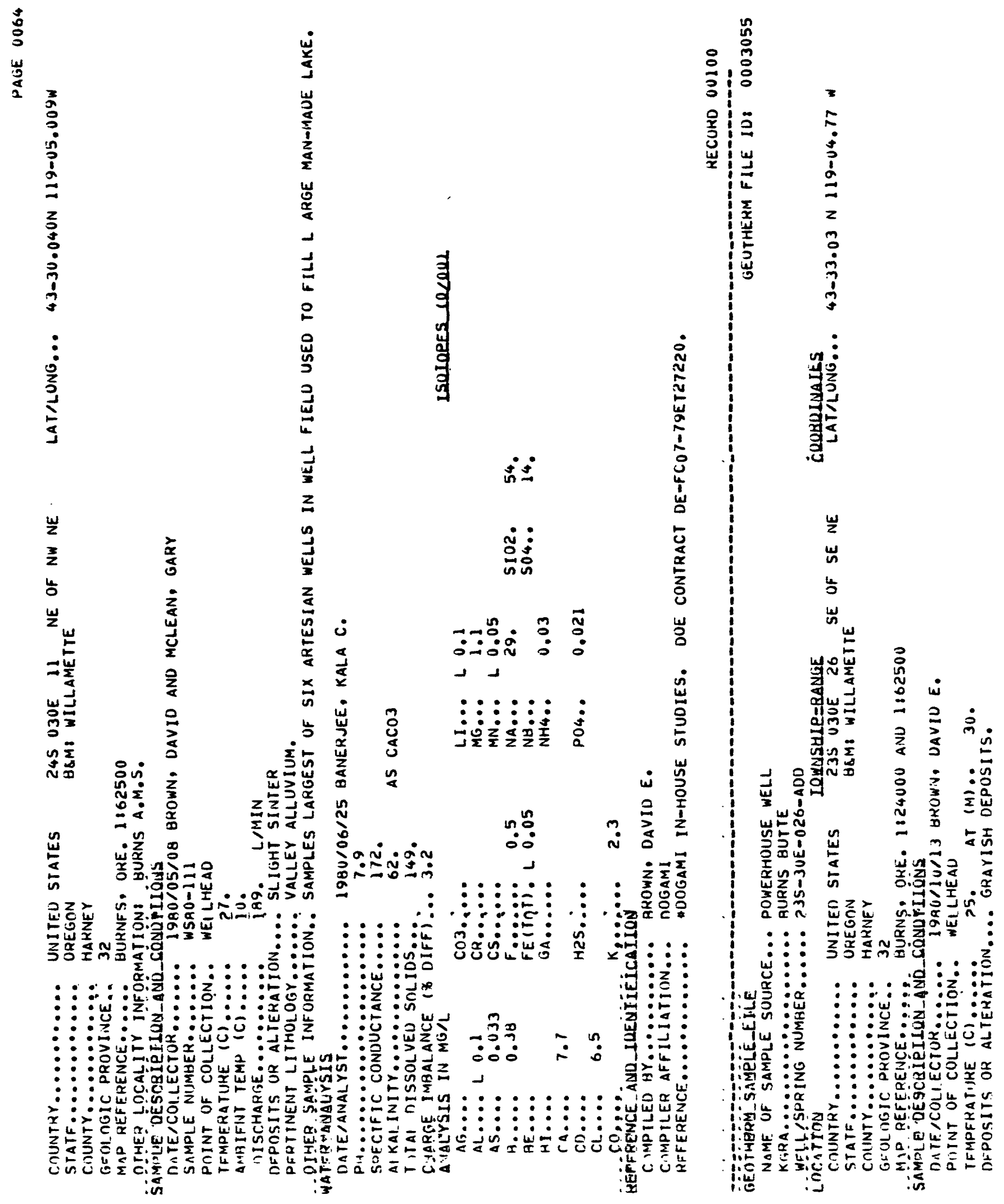

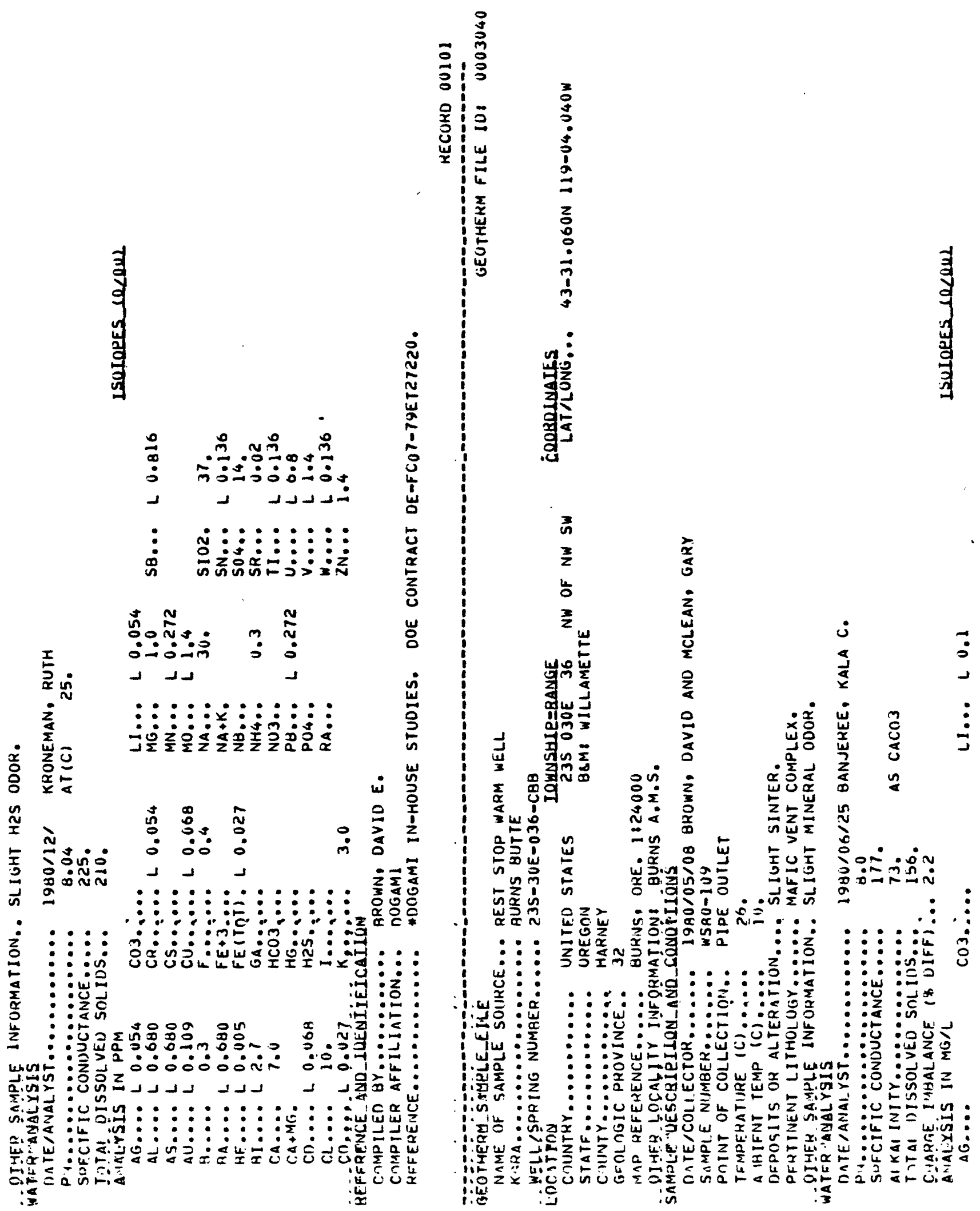
0
0
0
$w$
0
0
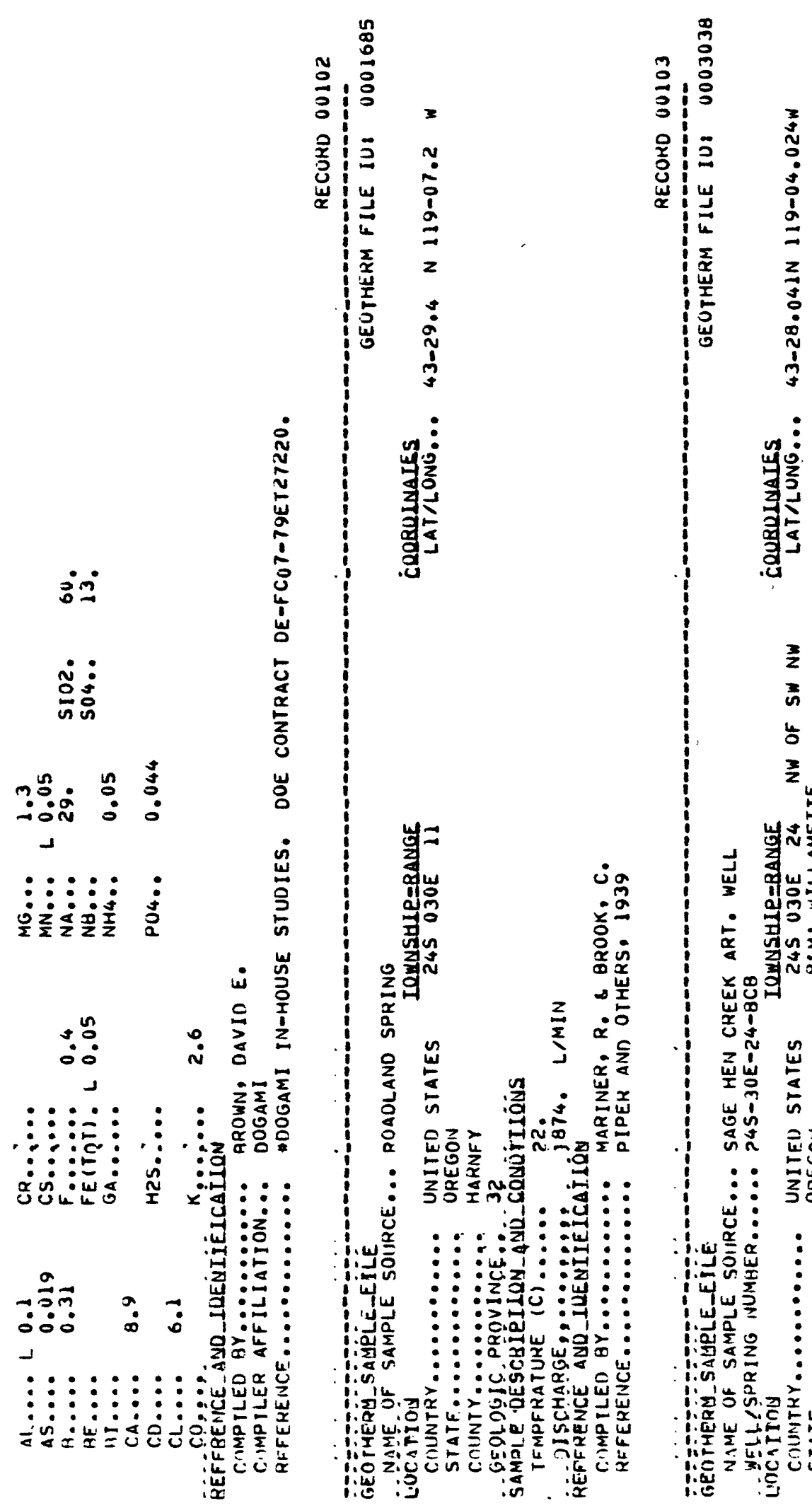


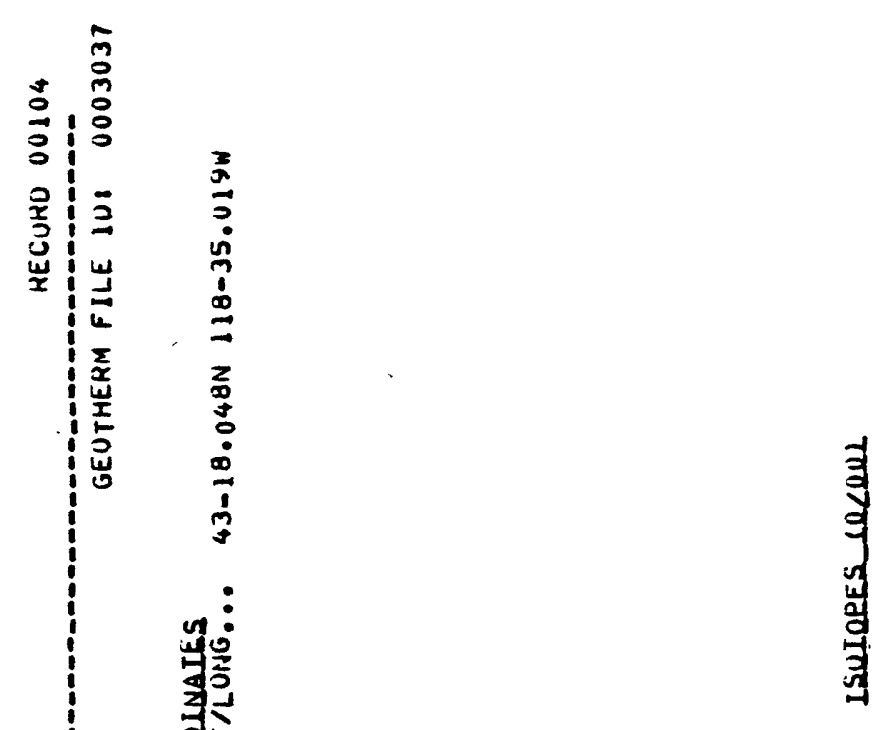

\section{$\stackrel{\circ}{5}$ \\ 它宫

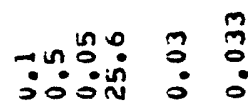 \\ \lrcorner}

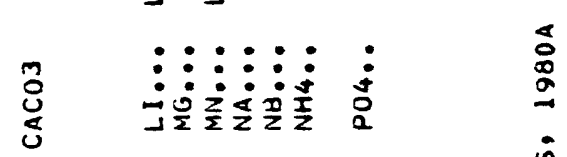
?

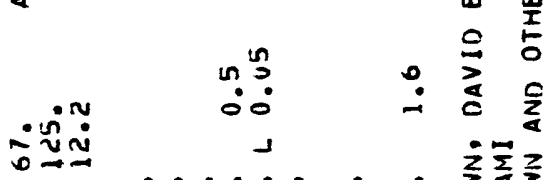

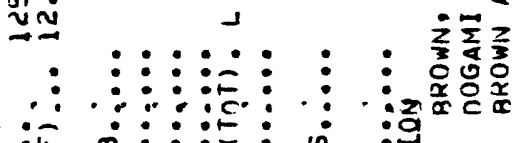
:治

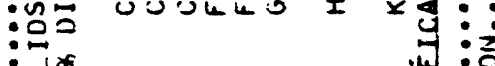

:

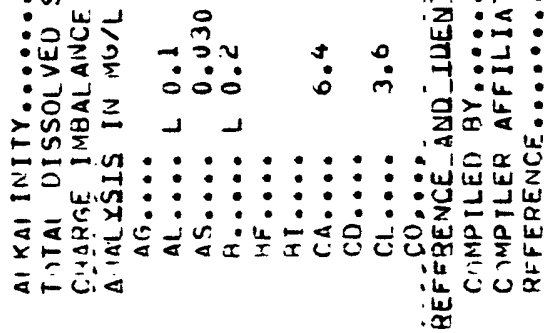

$\operatorname{lin}_{0}^{\circ}$

苍莒

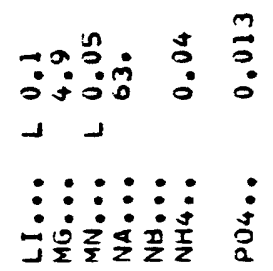

$\because \stackrel{n}{0}$ 
总

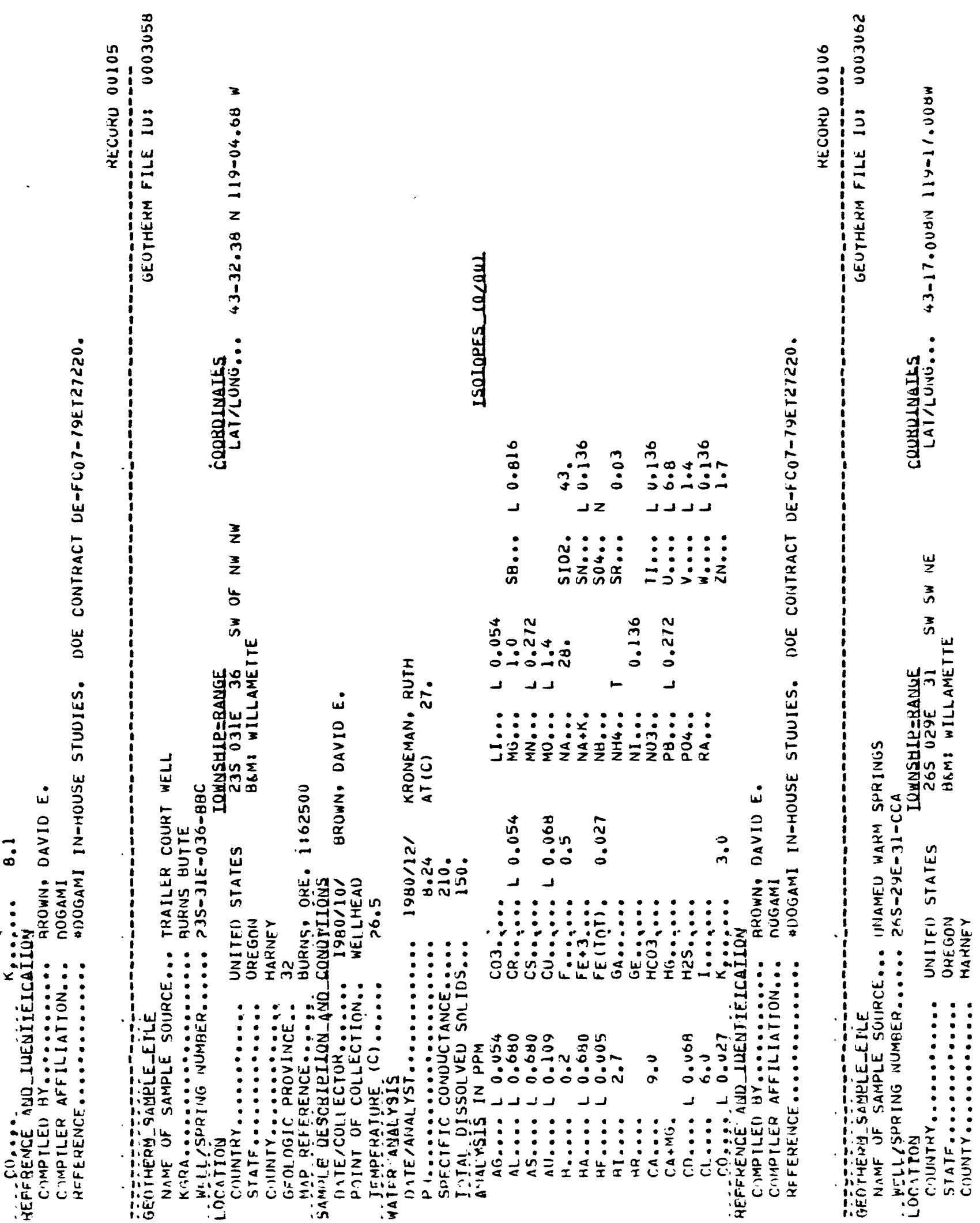




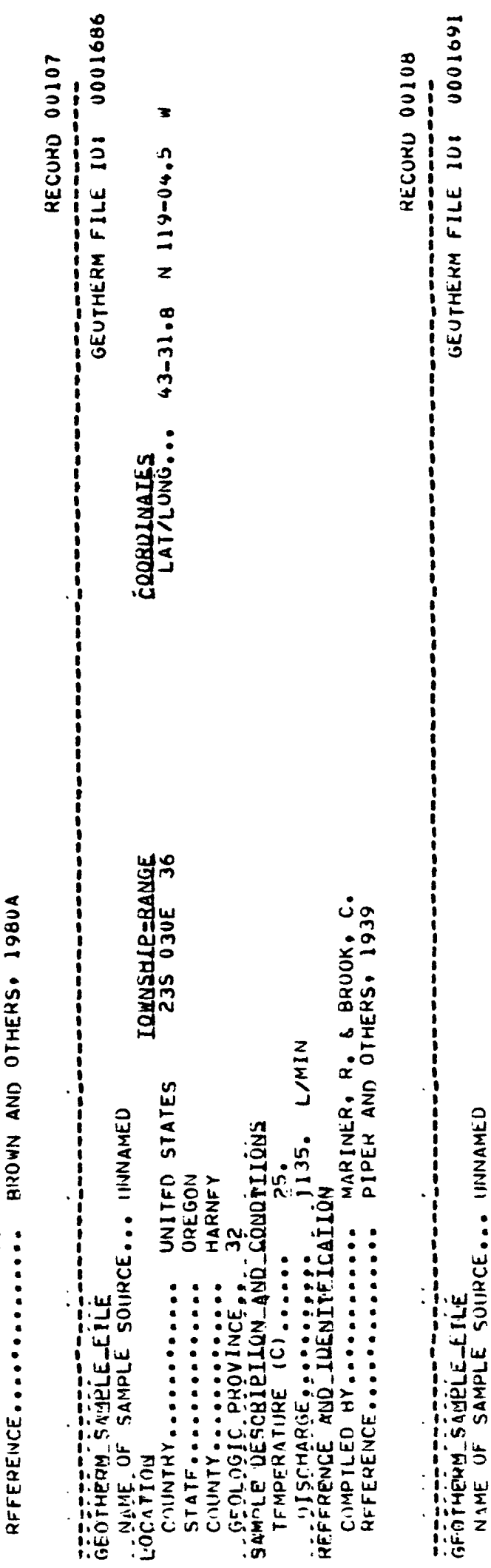




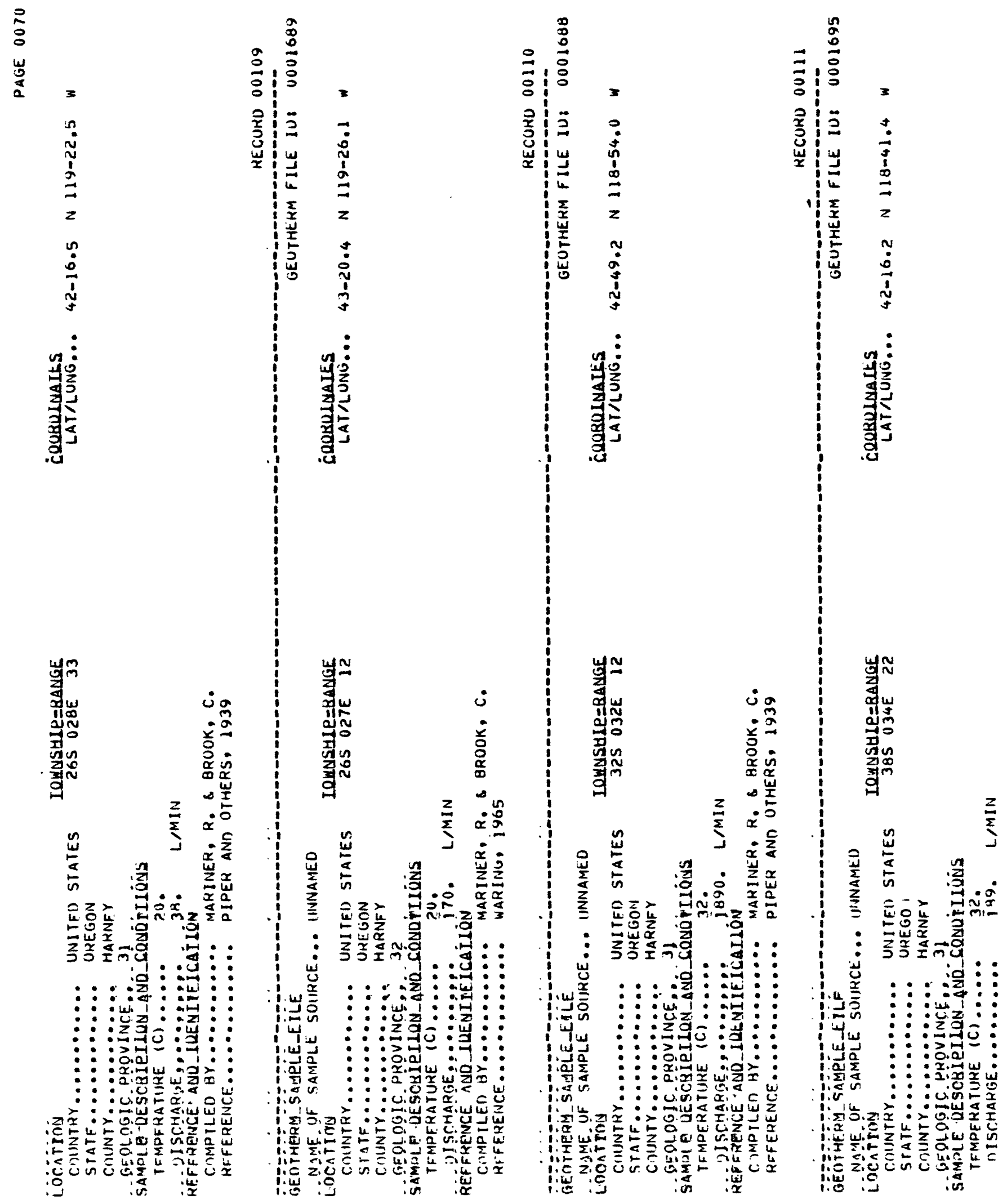




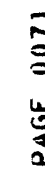

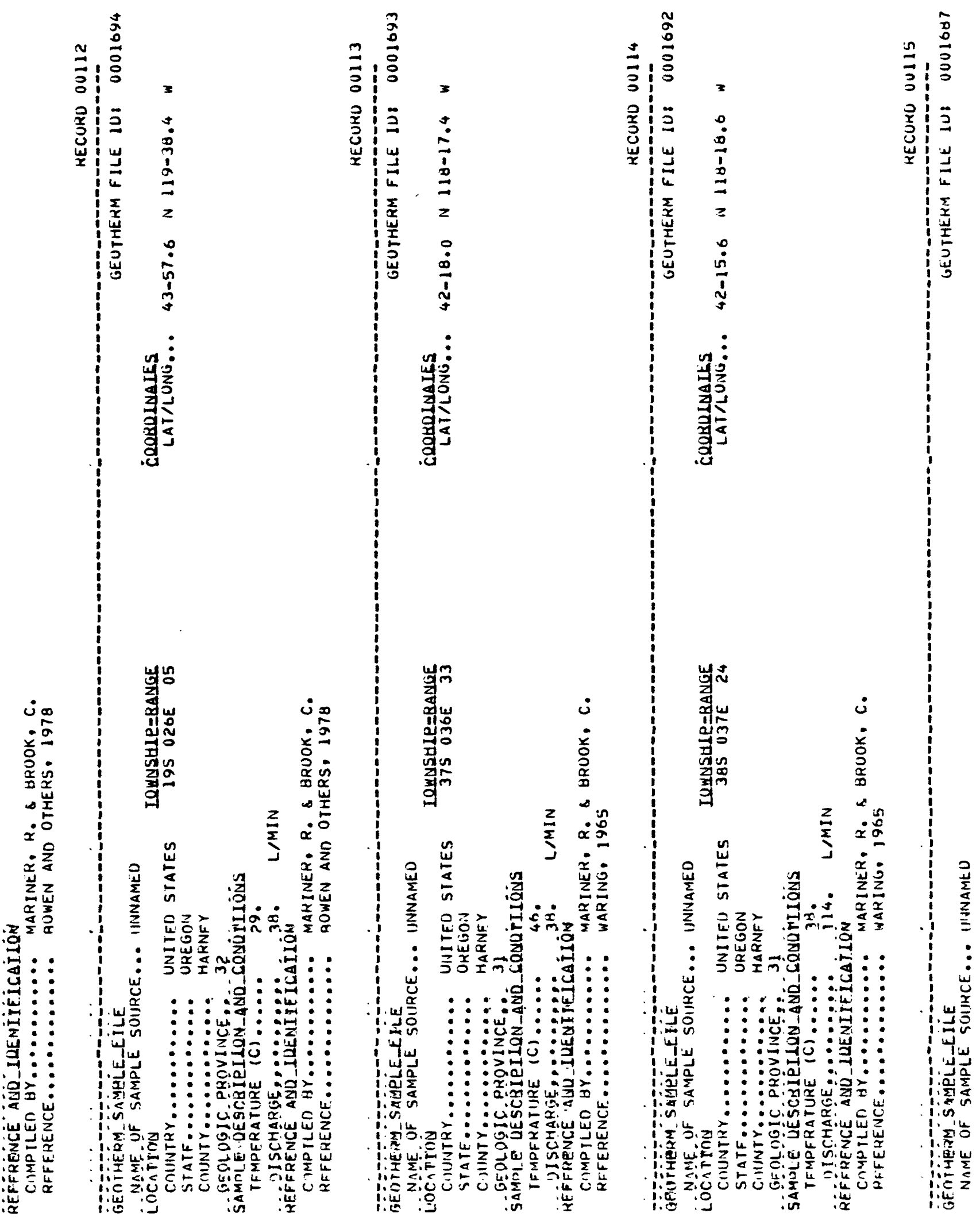




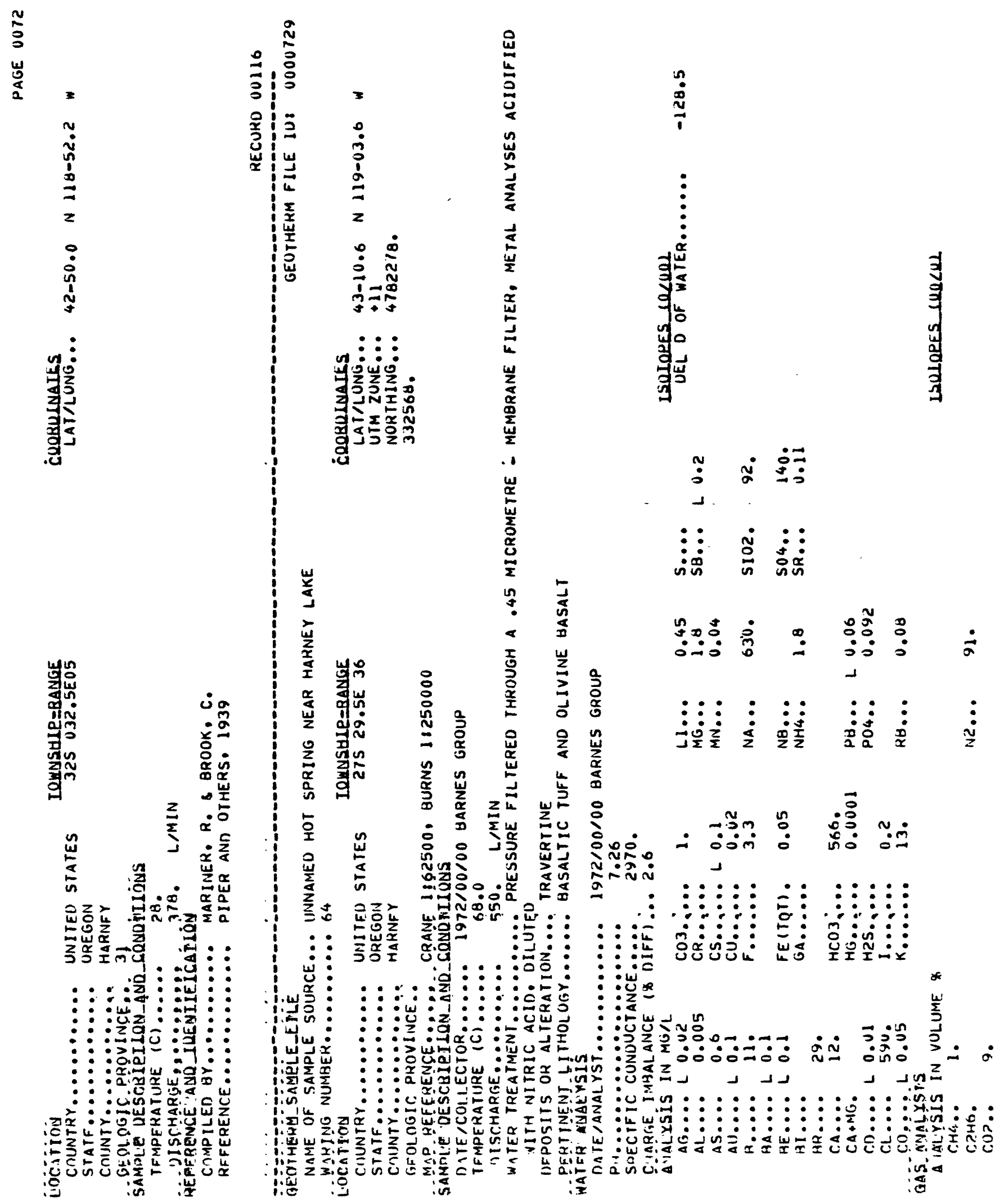




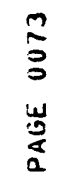
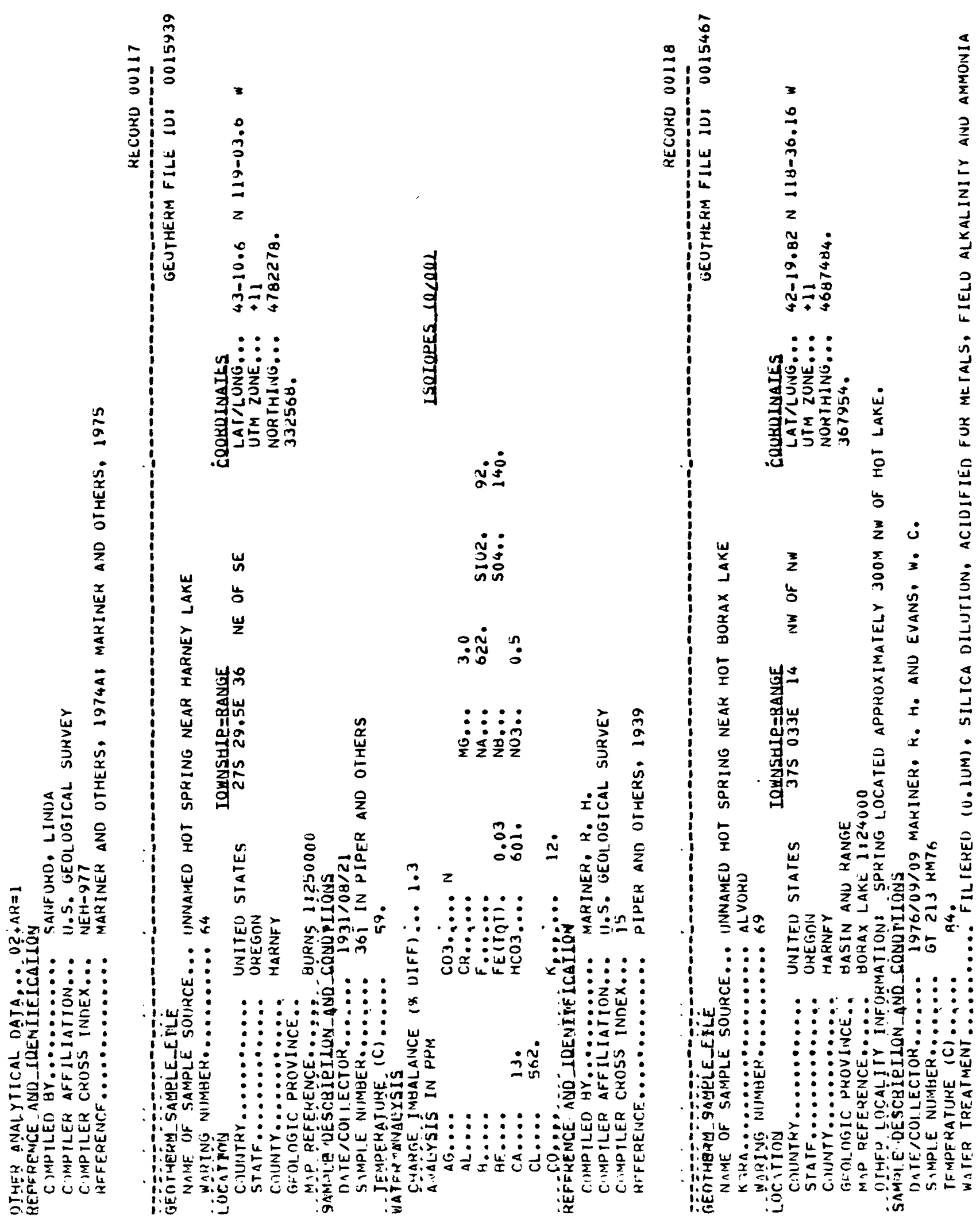

$\frac{5}{2}$
$\frac{5}{2}$
$\frac{2}{2}$
$\frac{3}{4}$
$\frac{3}{2}$
$\frac{1}{2}$
$\frac{1}{4}$
$\frac{5}{4}$

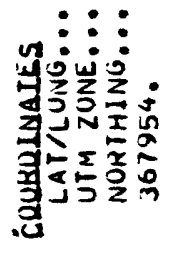

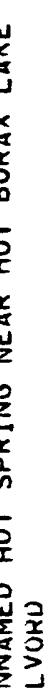

告 $\frac{3}{2}$

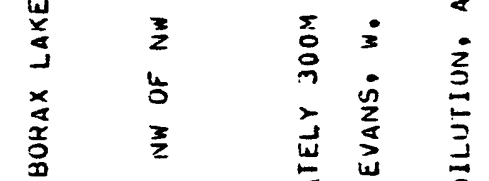

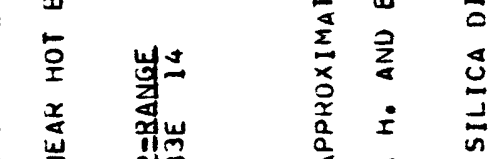

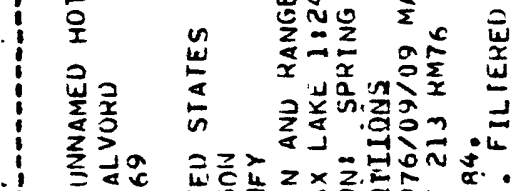

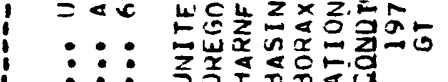

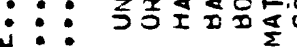

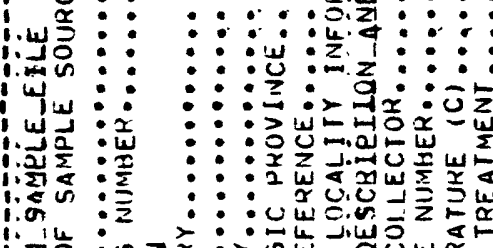

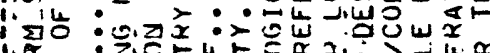

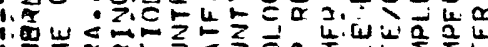

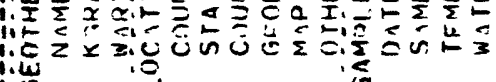


\pm
8
0
0
0
$a$

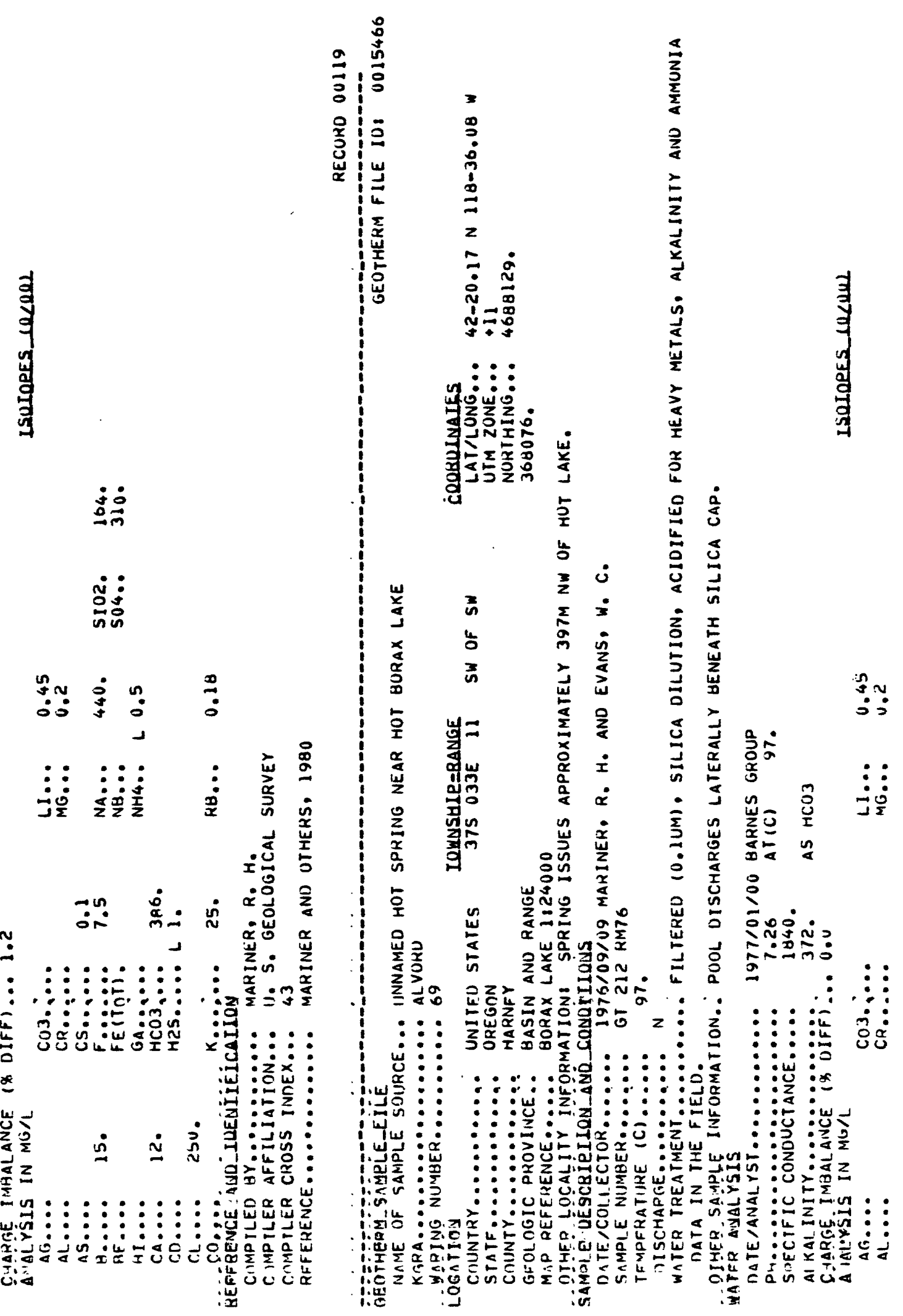




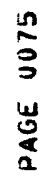

官邑

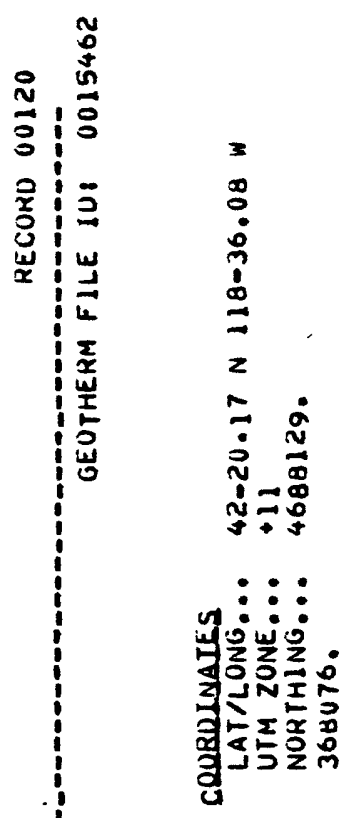

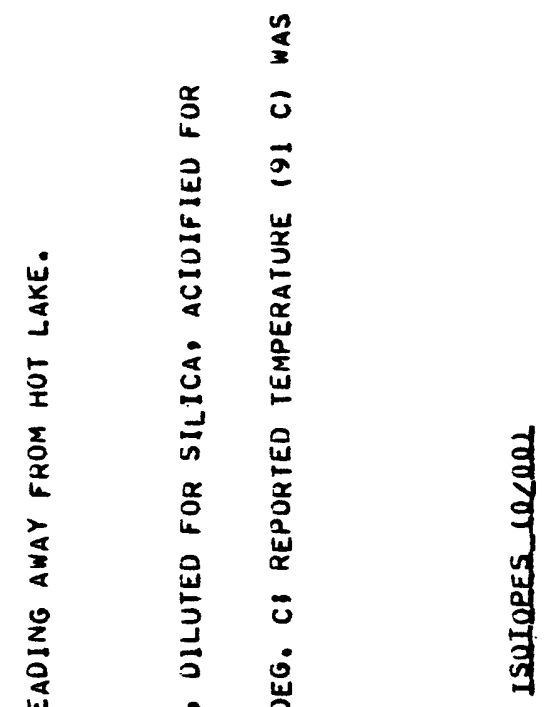

苛宫

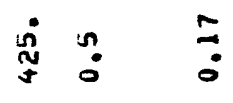

: :

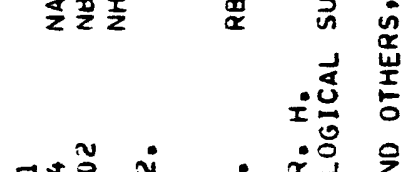

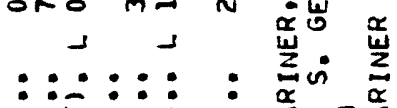

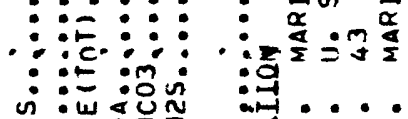

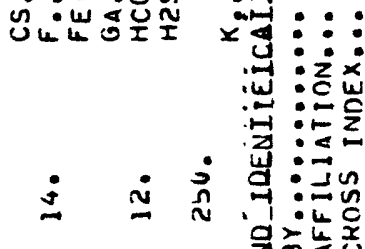

灵市视

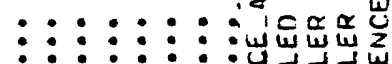

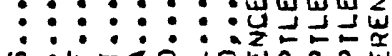

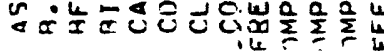

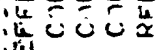

$\stackrel{\dot{D}}{\mathrm{D}}$

突递

กீ:

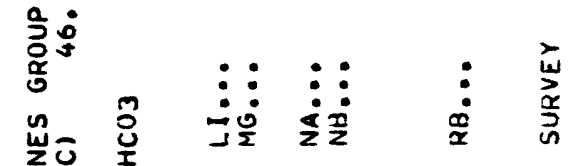

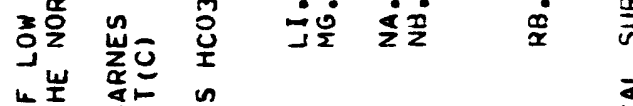

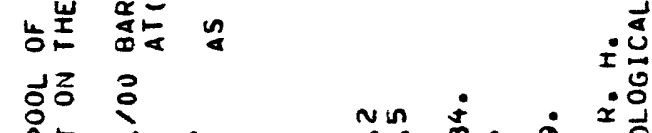

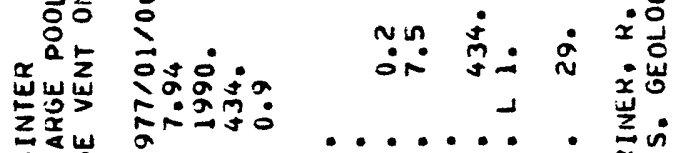

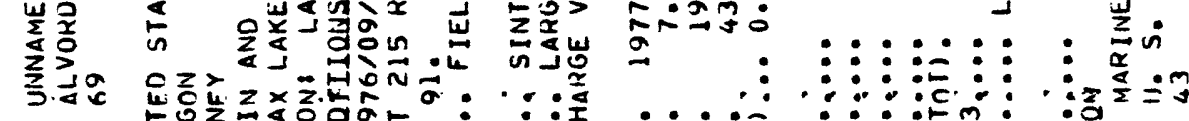
$::$ :

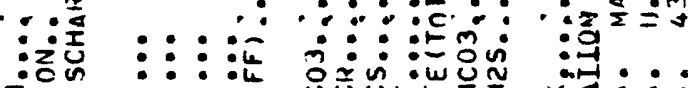

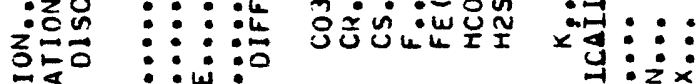
F文尔: : : :

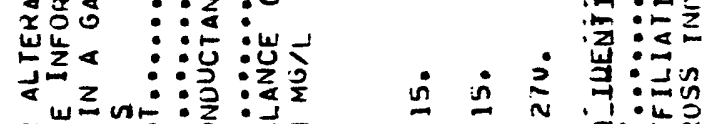

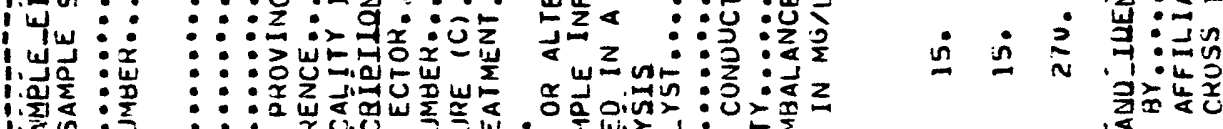
:

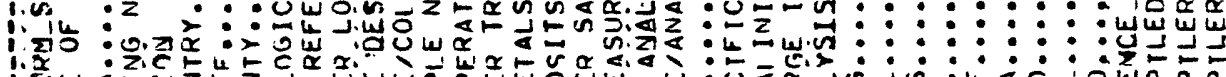

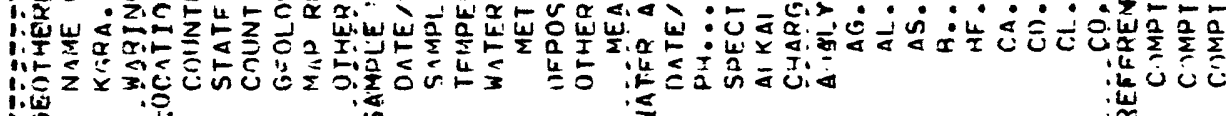




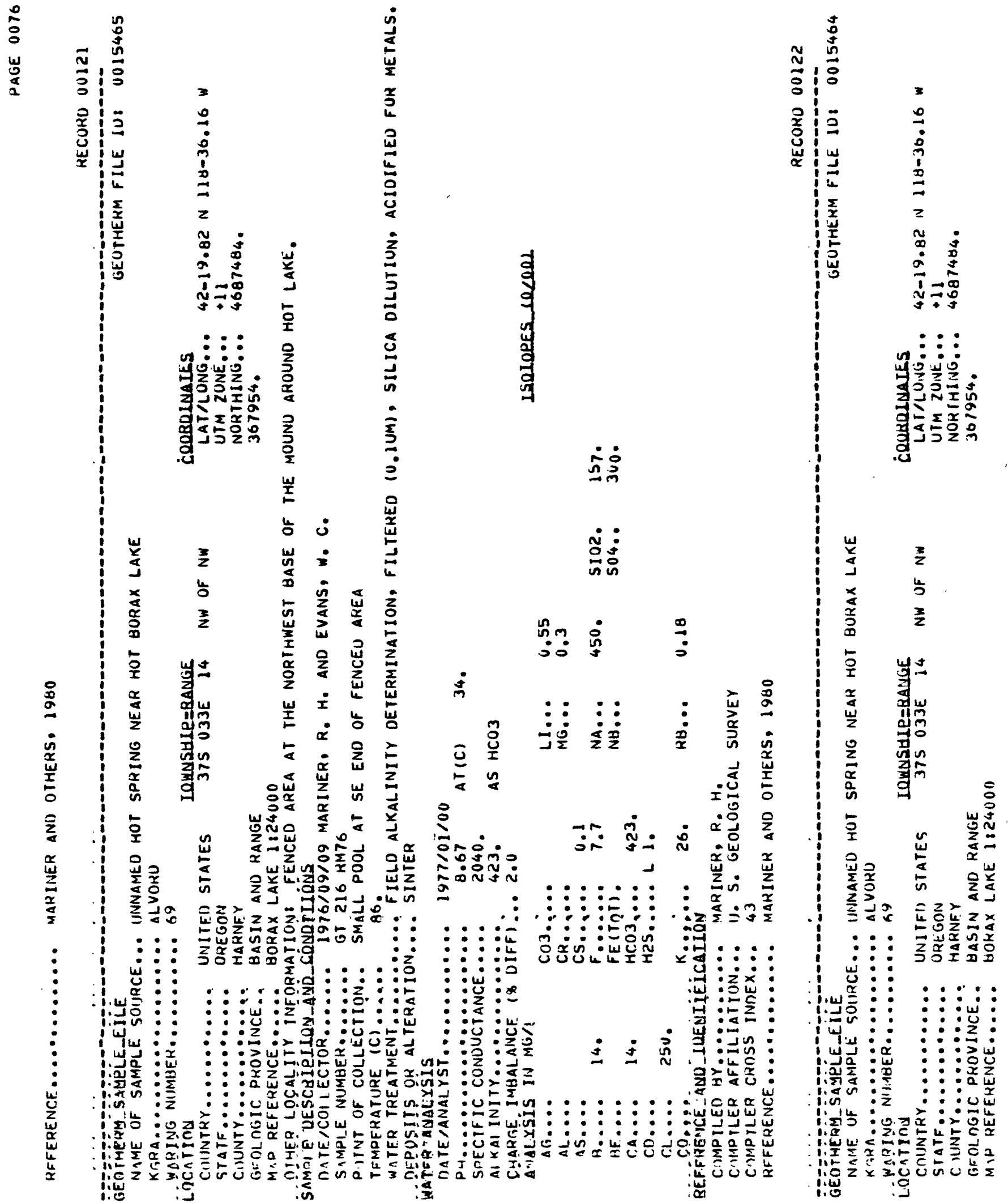



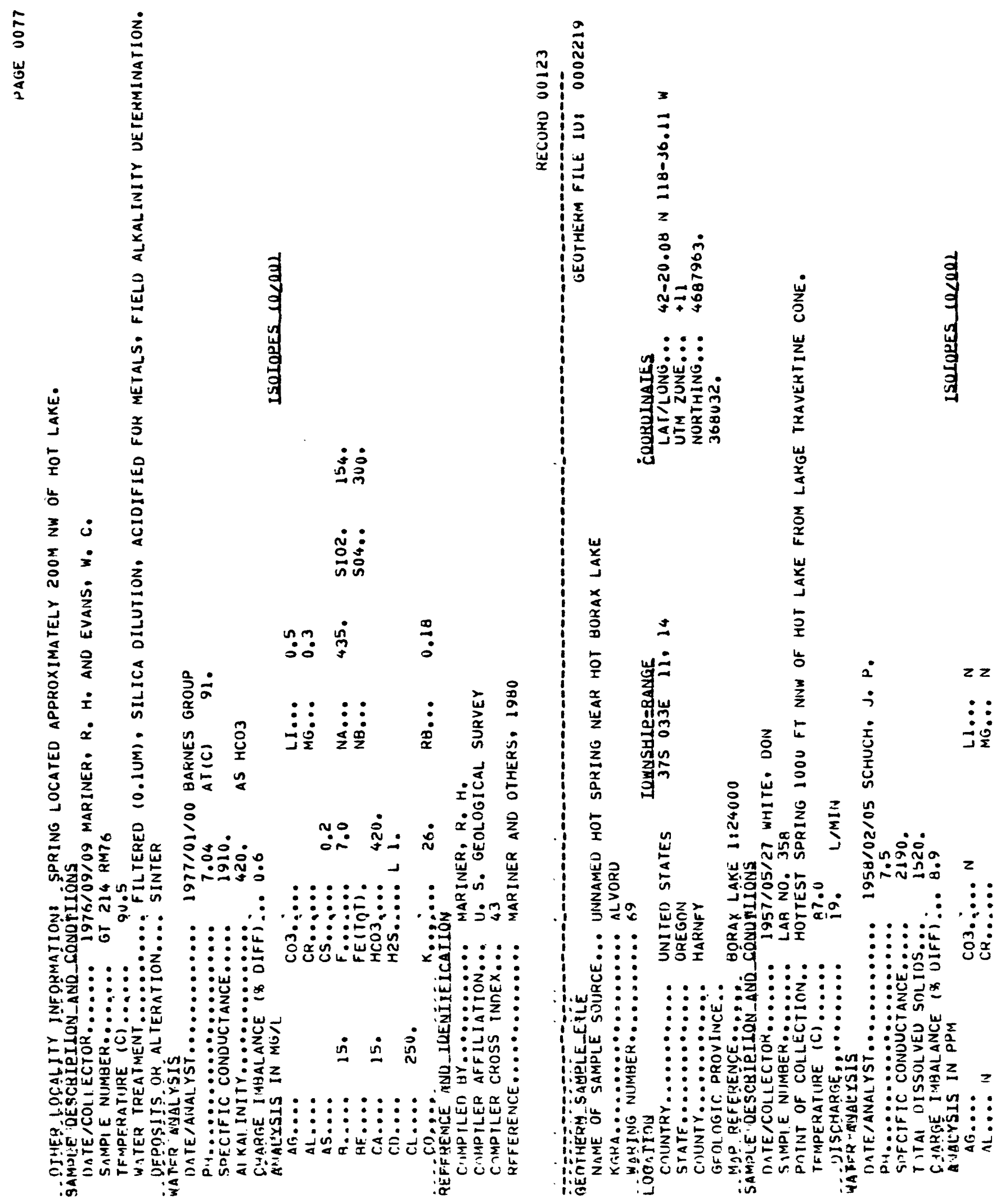


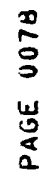

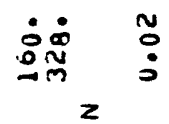

客:

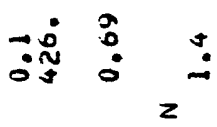

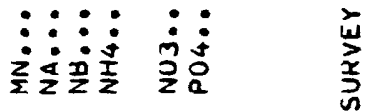

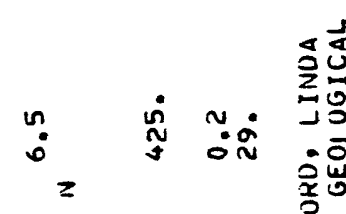

$: \vdots: \vdots: \vdots: \sum_{2}^{0} \dot{0}$

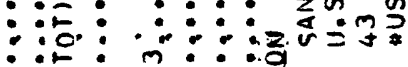

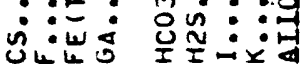

凷 : : :

ว 预文条:

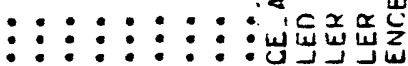
$:::::::::$ : $:$

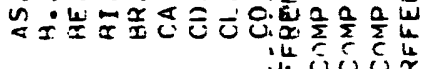
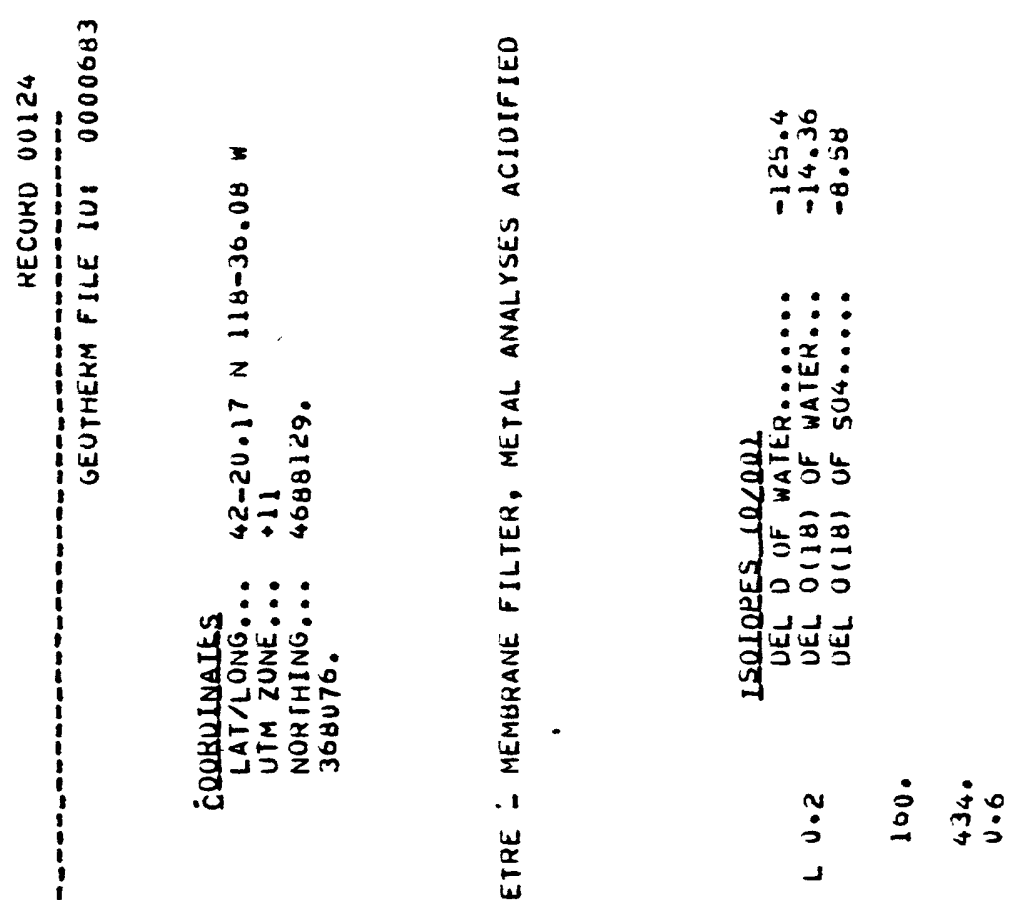

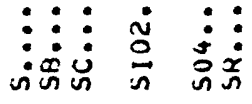

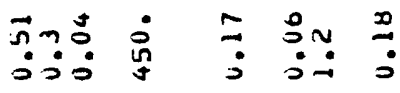

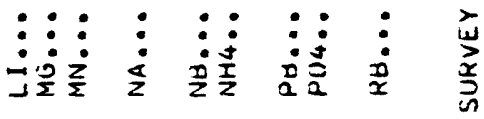

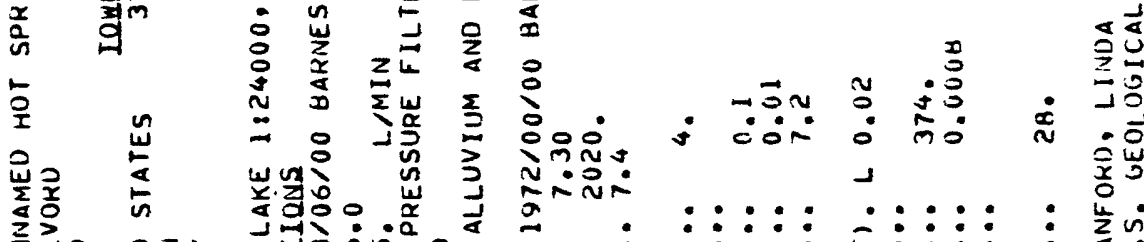

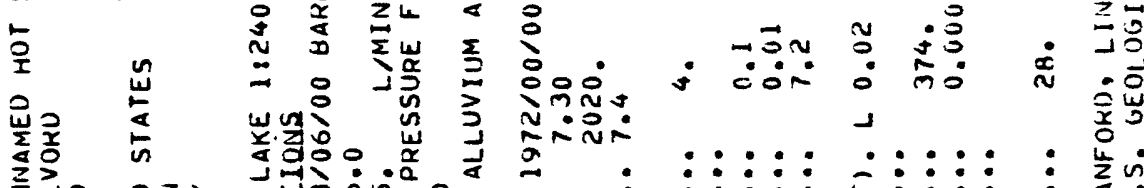

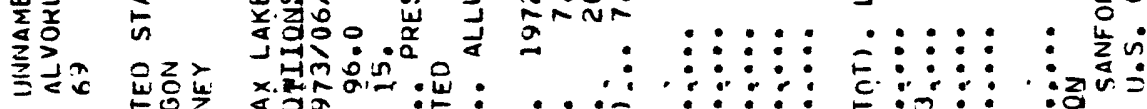

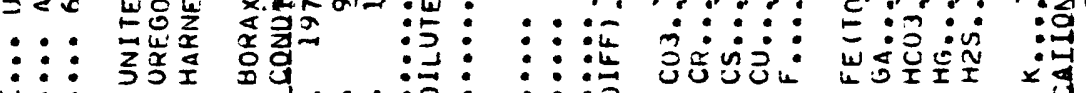

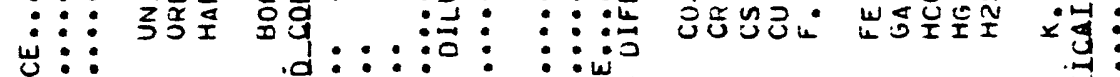

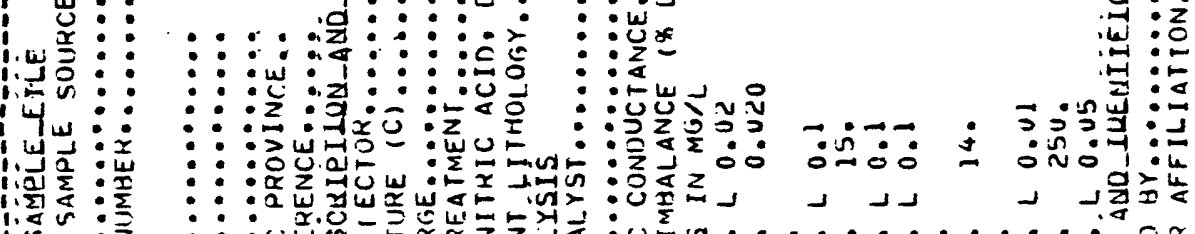

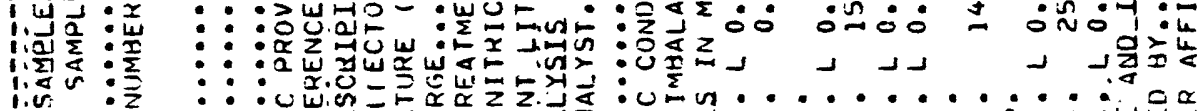

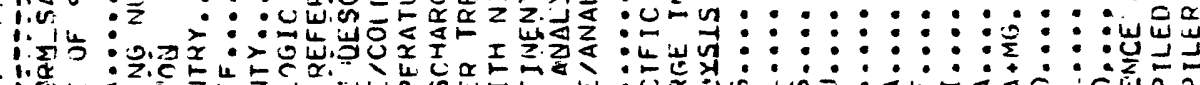
1.

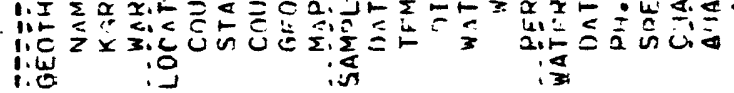




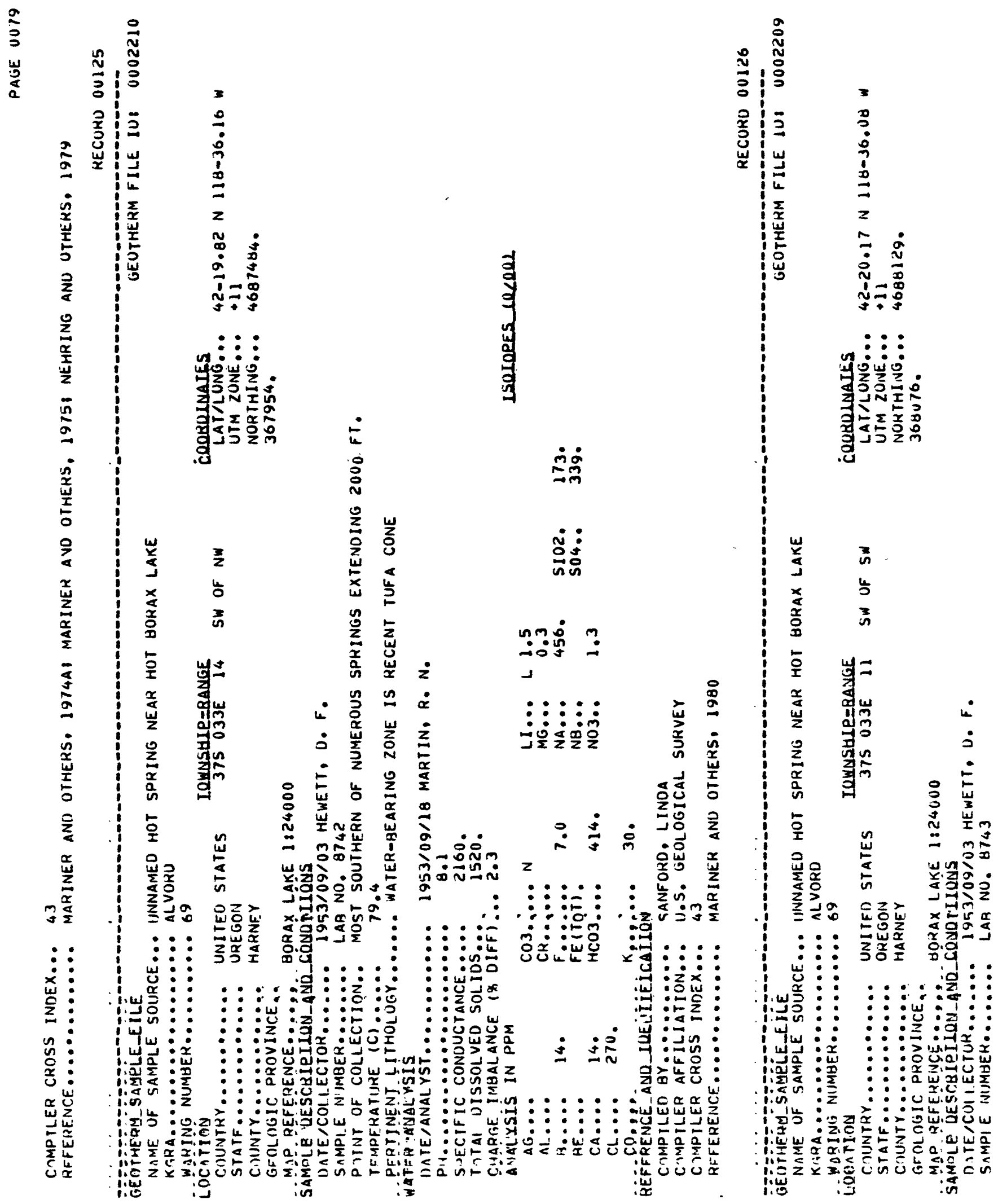




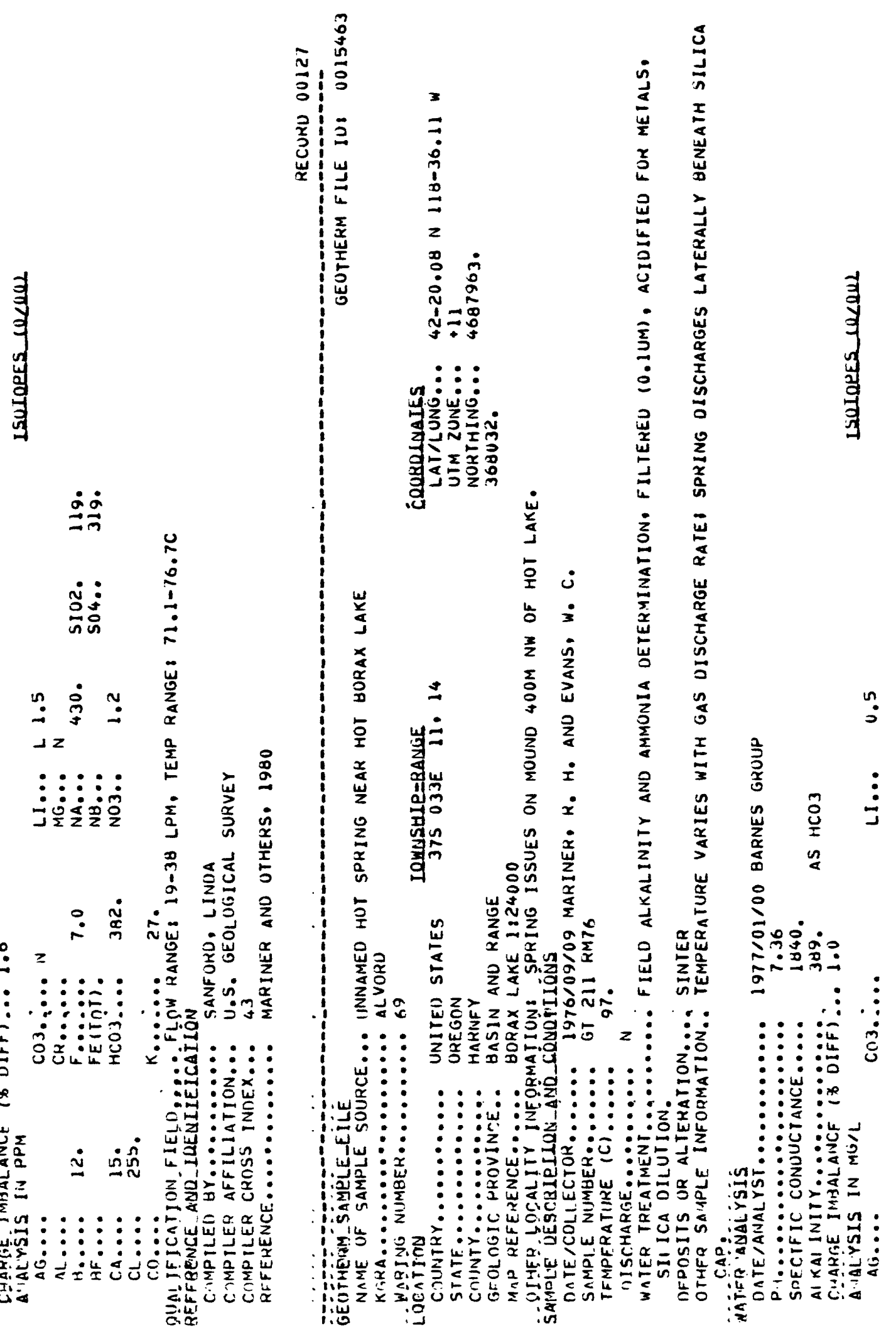




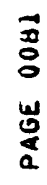
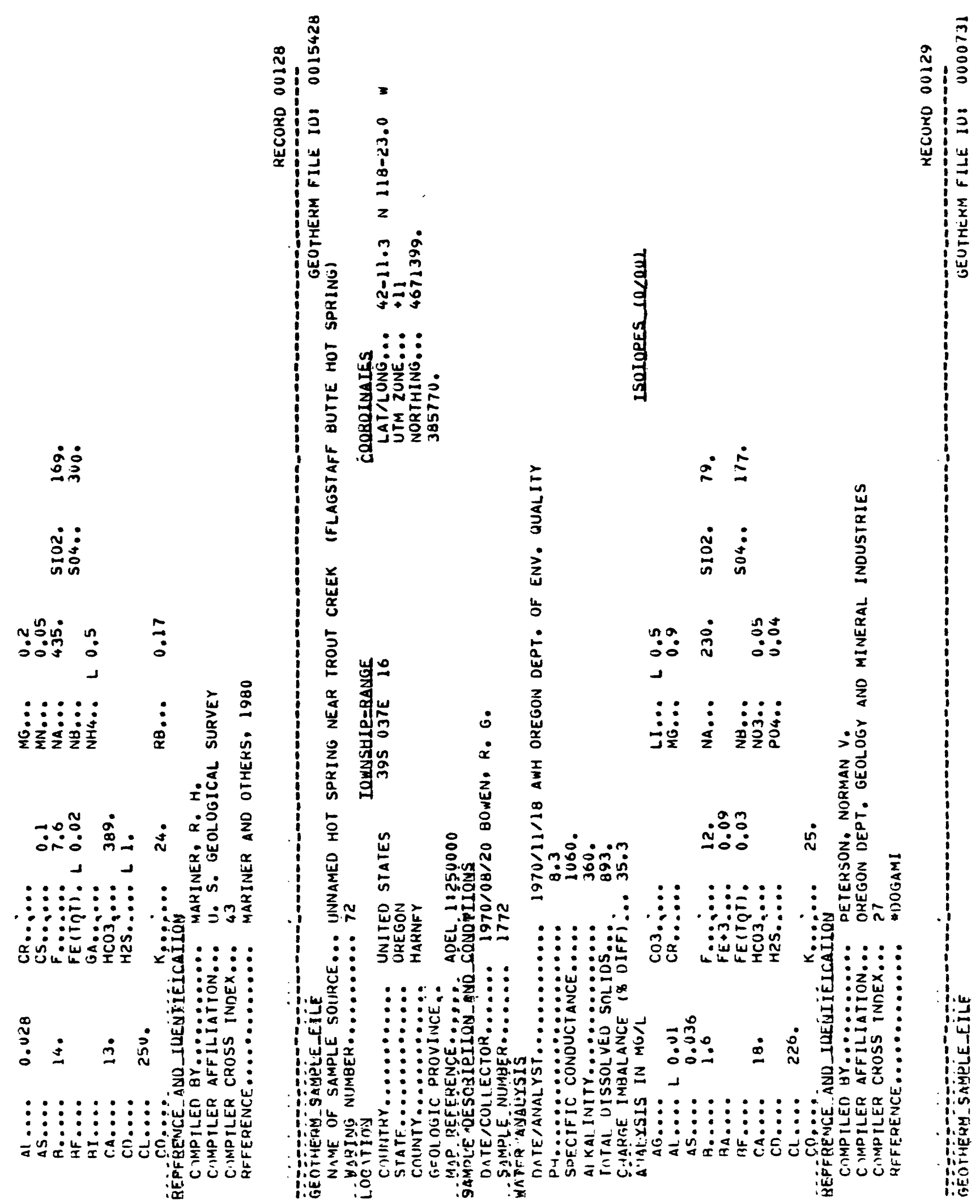


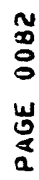
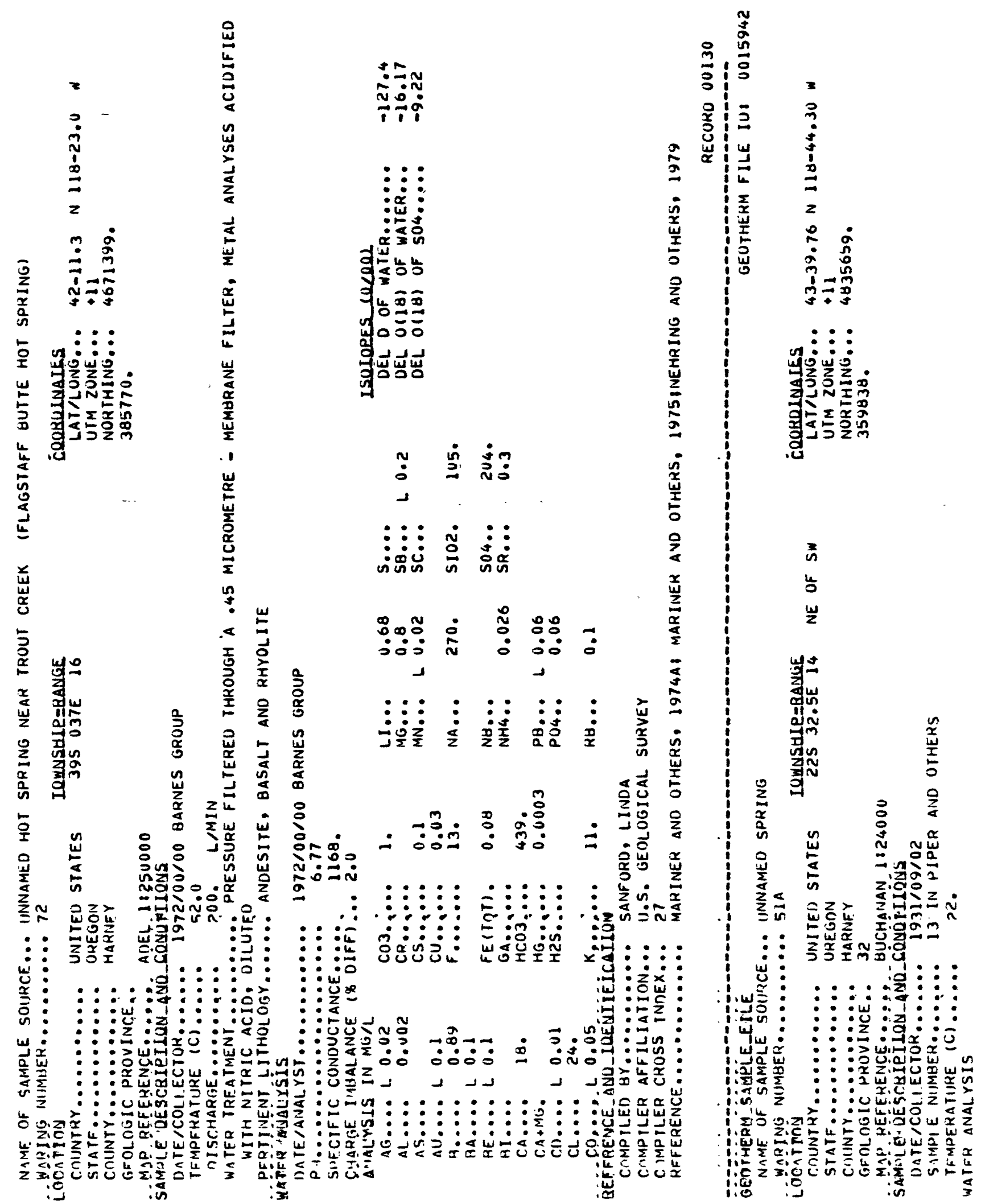
$\frac{1}{2}$

書

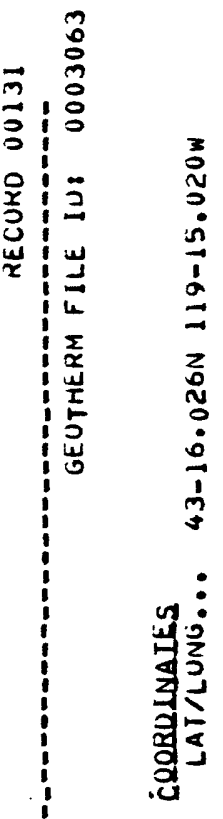

荈

¿ִ

落递

$\stackrel{\check{0}}{\simeq}$

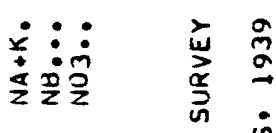

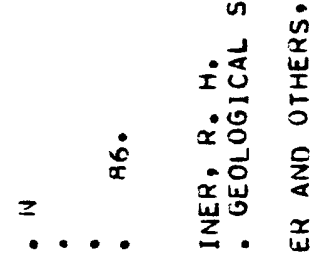

$: \vdots: \vdots \frac{x}{x} \dot{a}$

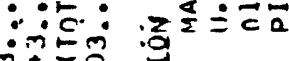

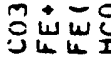

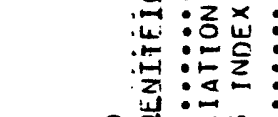

는

品

羽文过

3

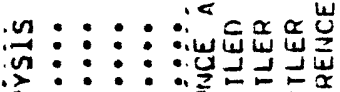

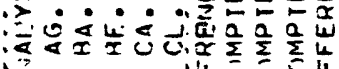

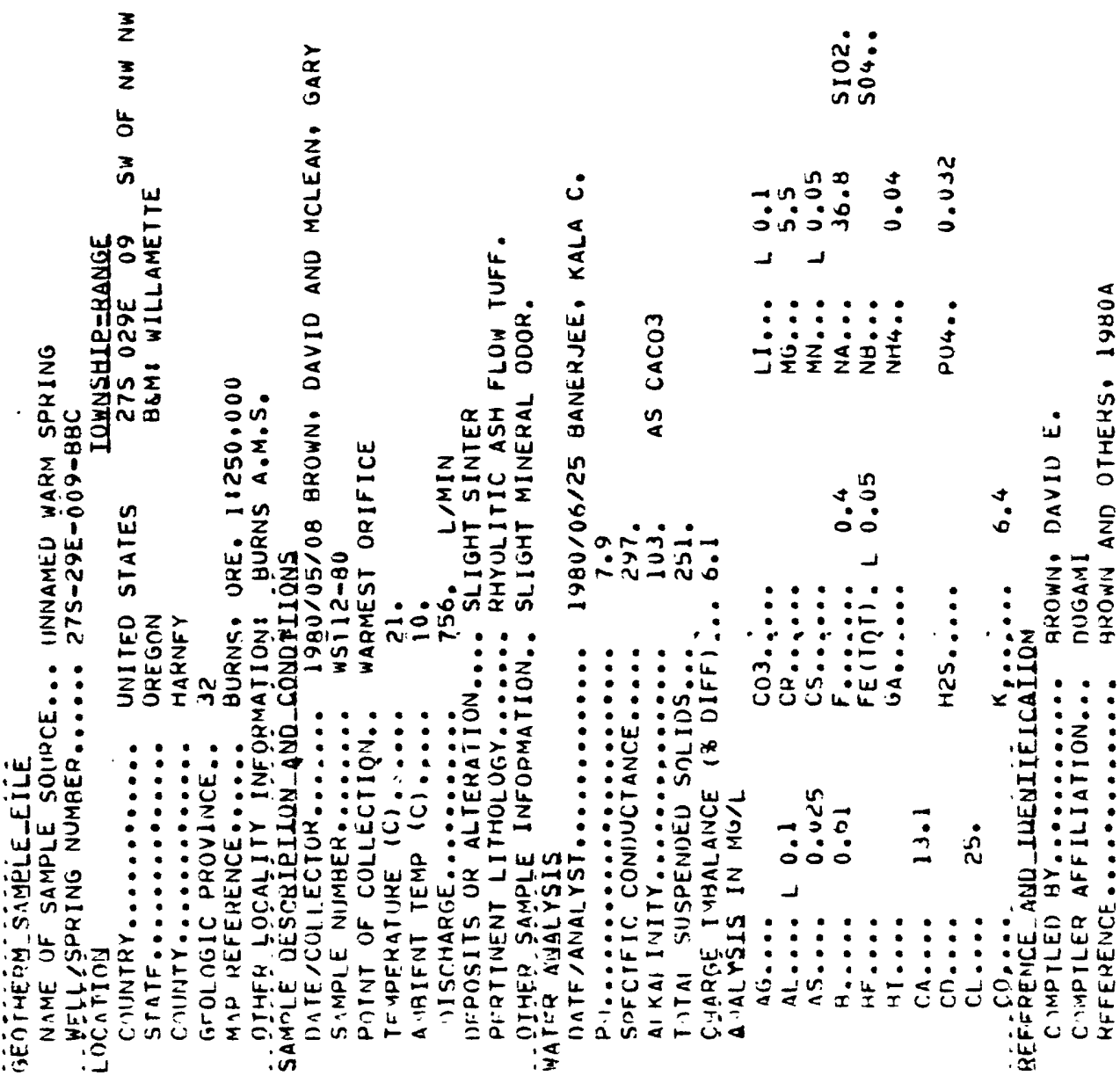




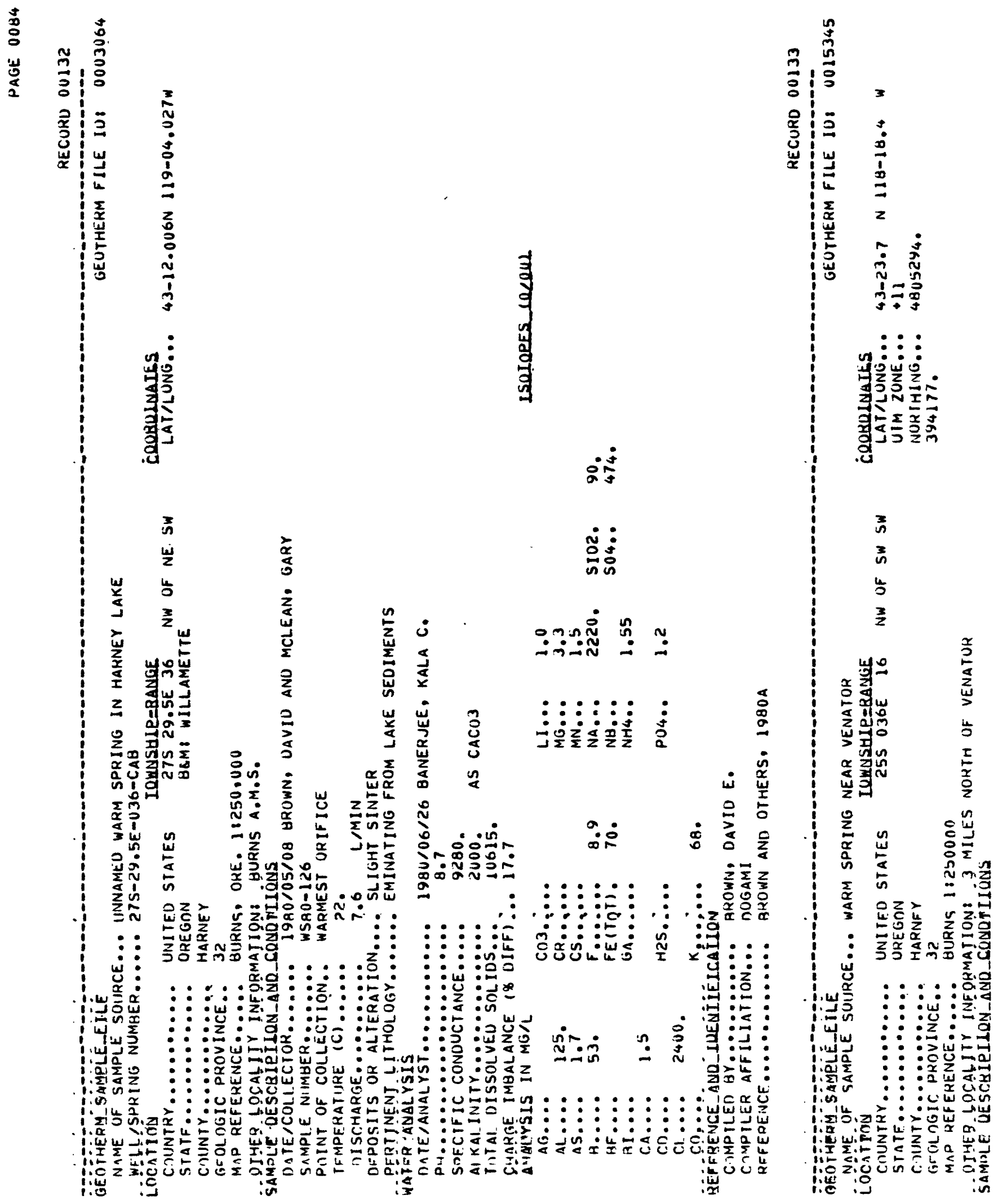




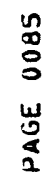

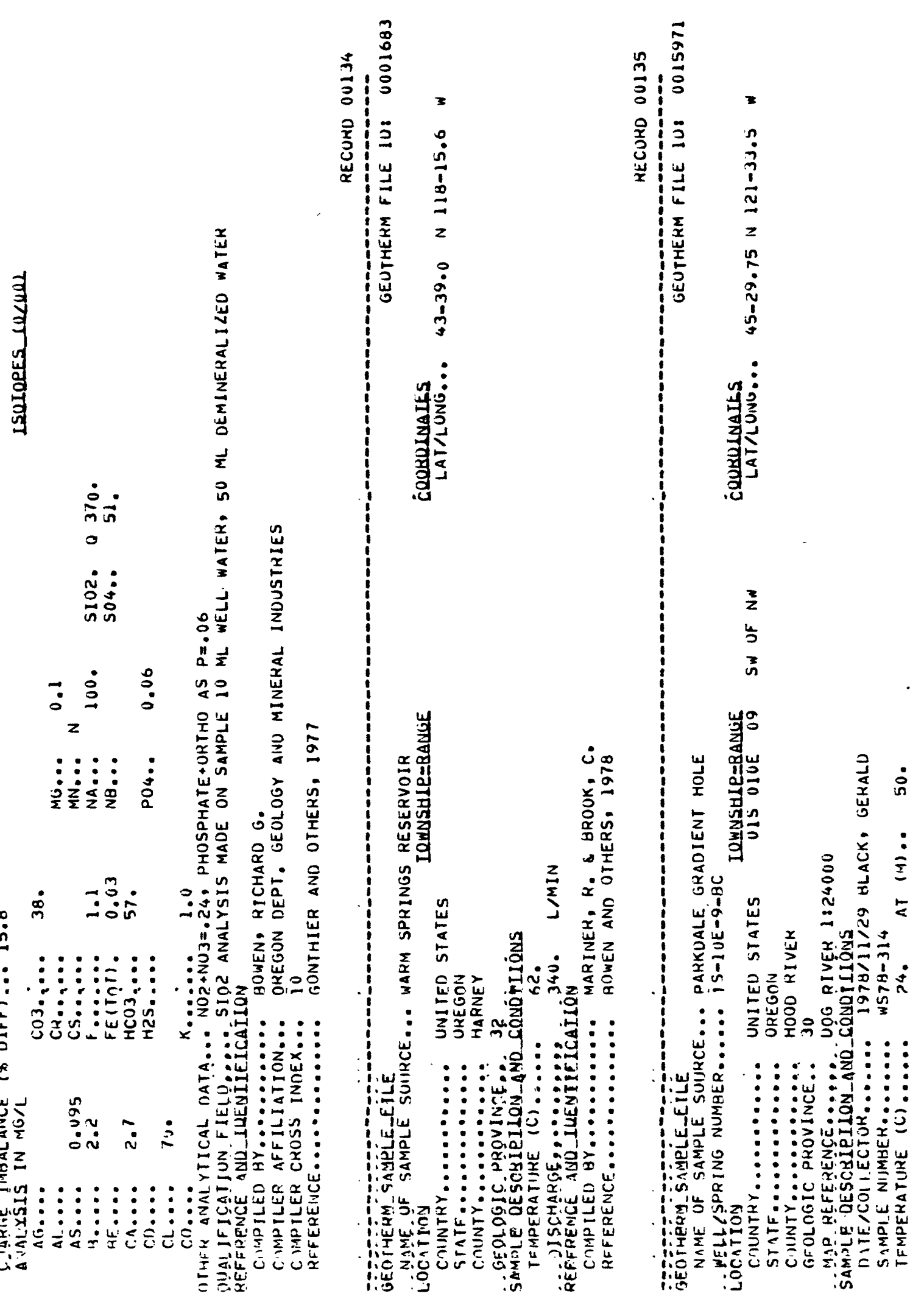




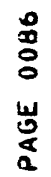

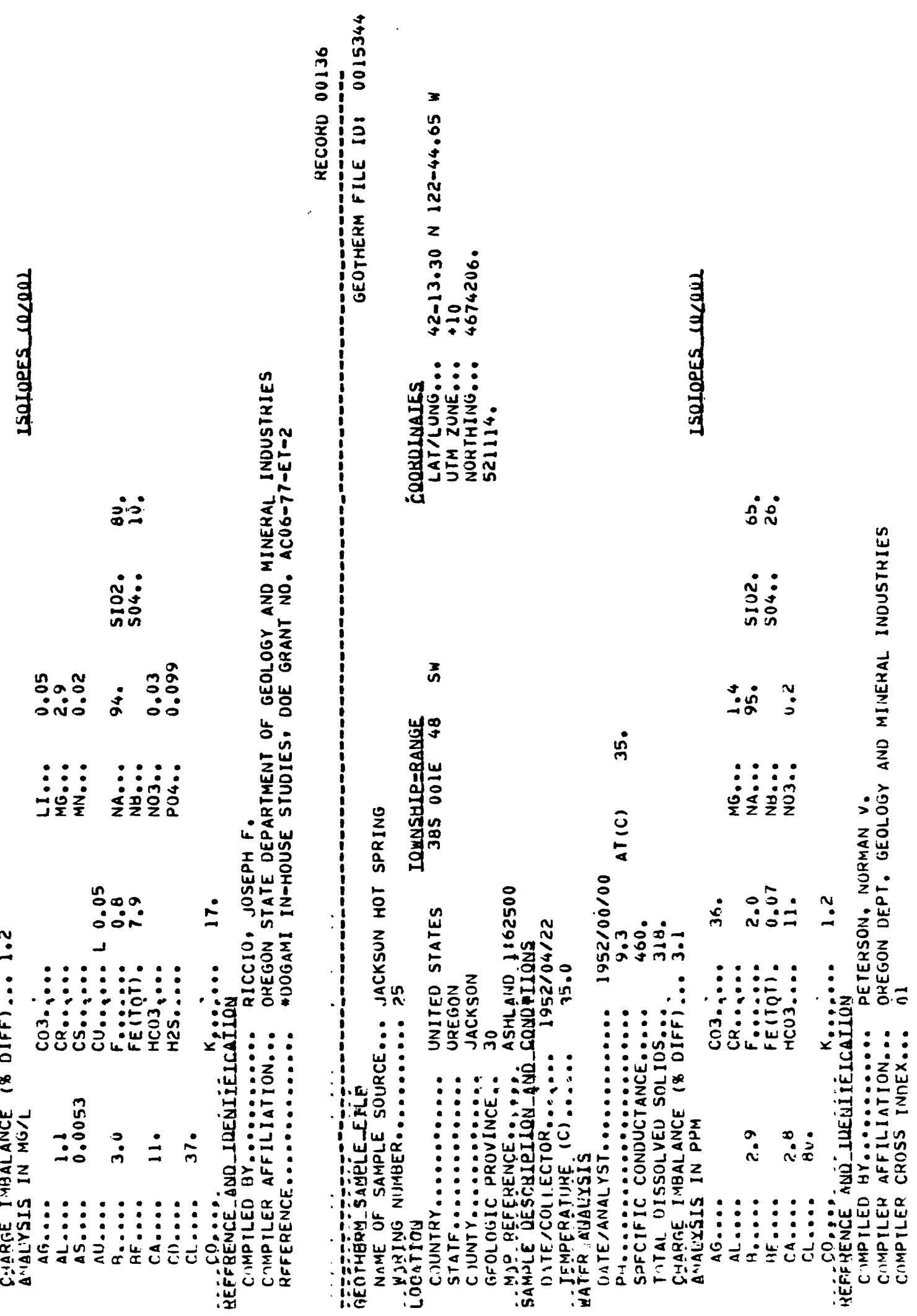




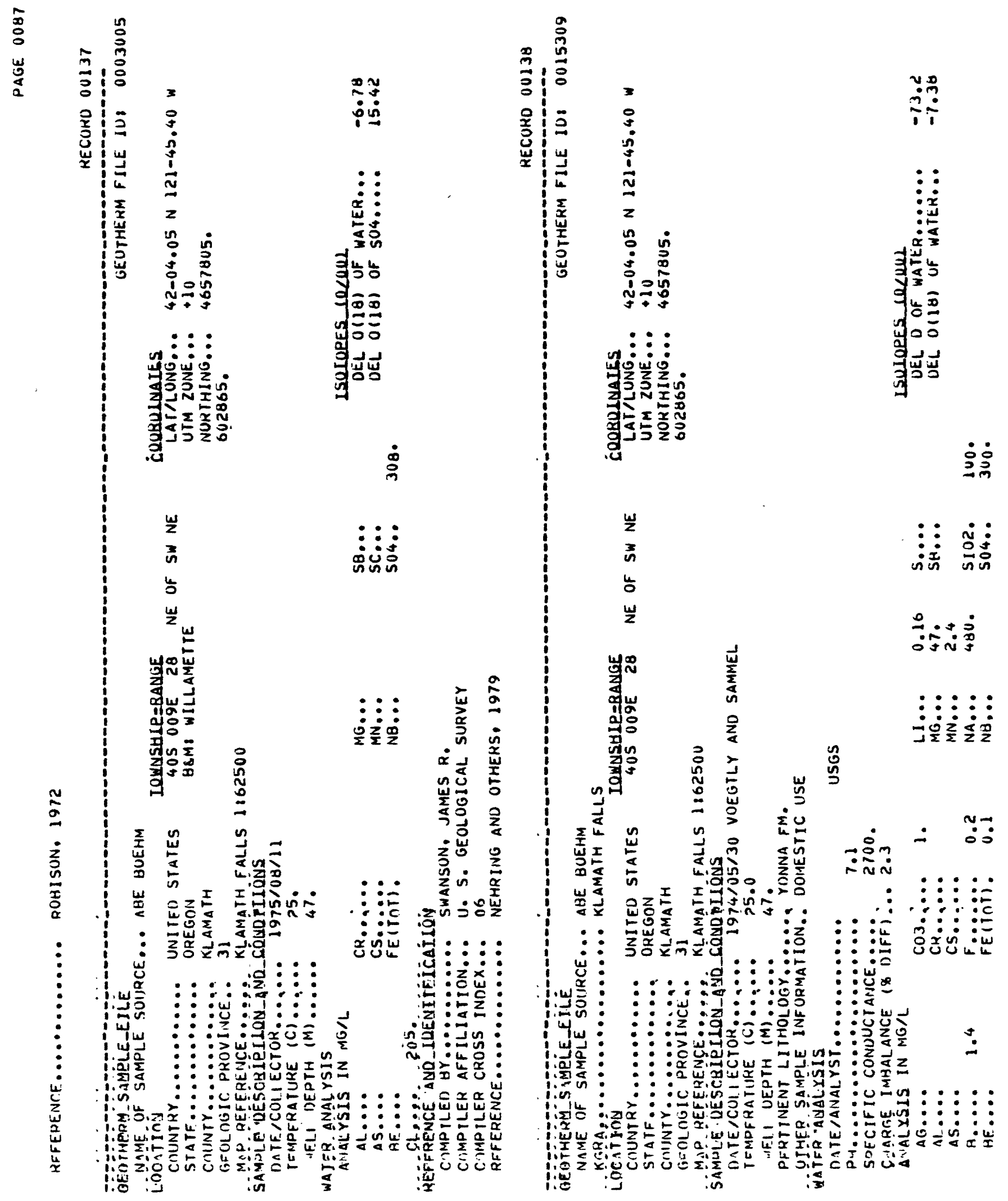


\begin{tabular}{l}
$\infty$ \\
\hdashline \\
0 \\
0
\end{tabular}
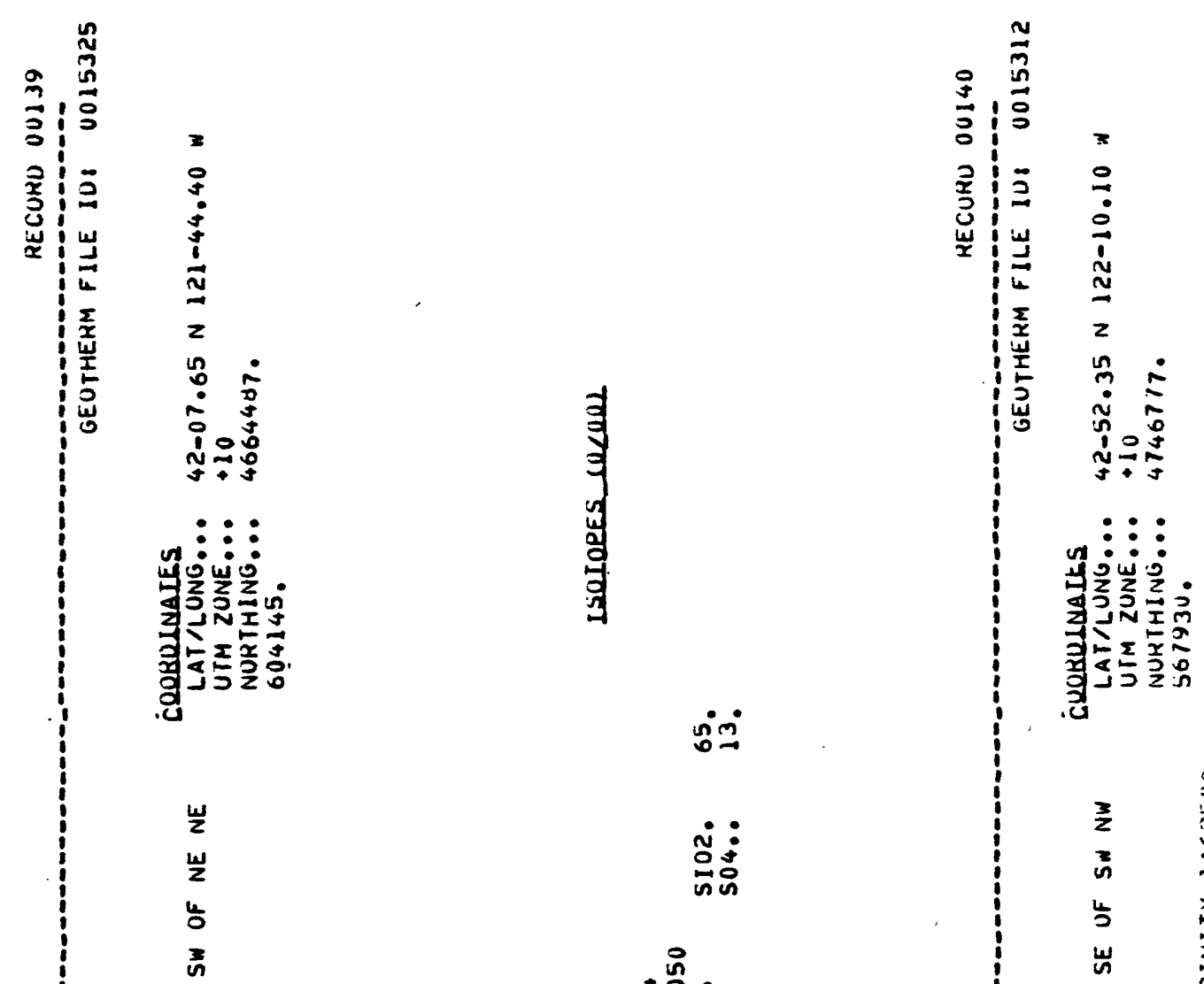

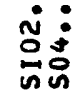

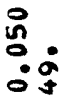

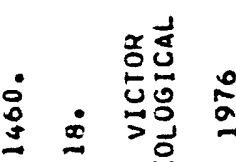

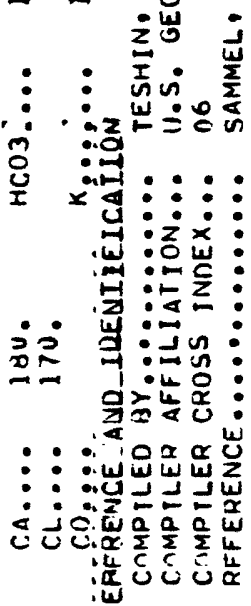

岁年

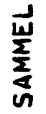

$\vdots: \vdots:$

乌ั

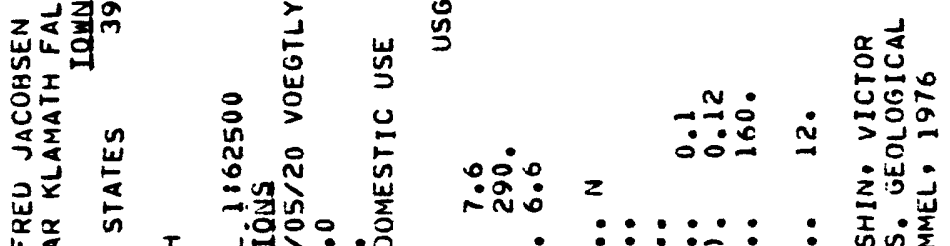

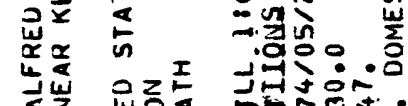
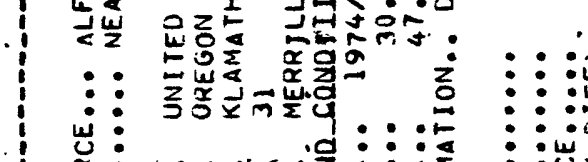

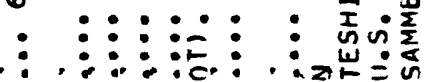

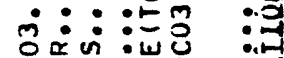

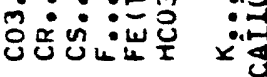
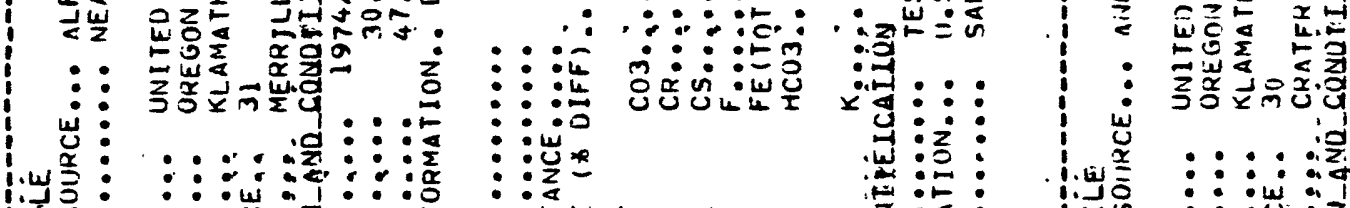

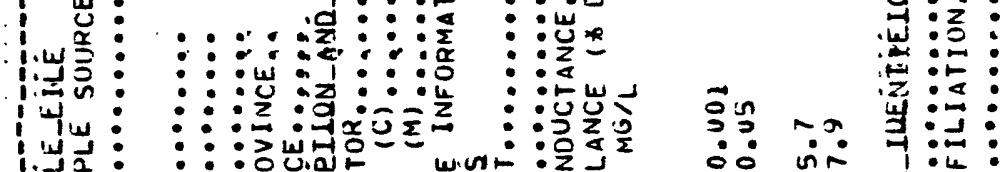

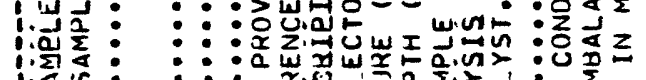
$\therefore$ in

尼文安

:

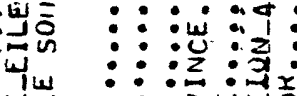

: $: \vdots:$ :

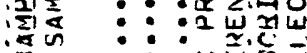
:

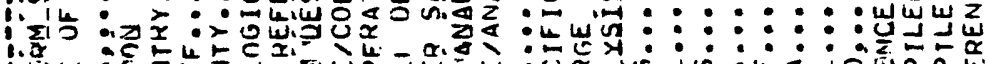

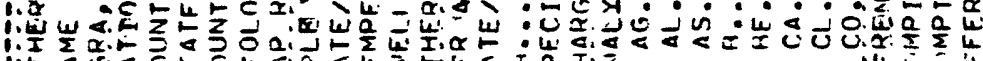

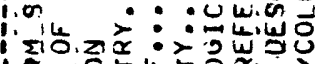

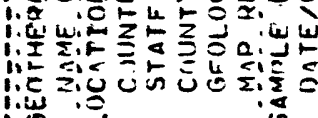




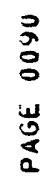

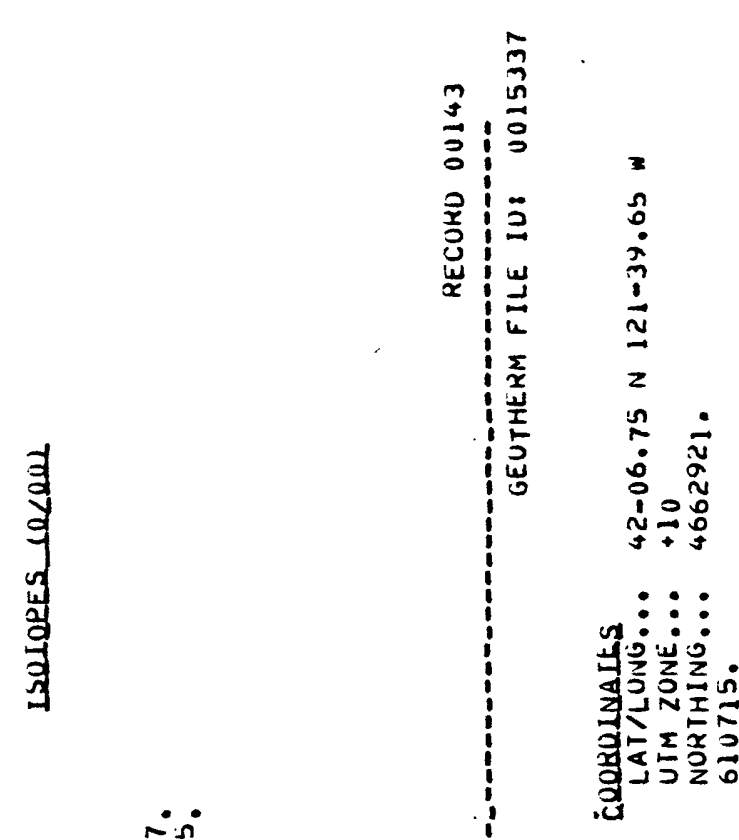

菻

$\stackrel{\substack{3 \\ \stackrel{2}{0}}}{0}$

in

突:

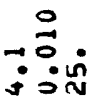

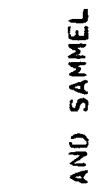

:

㟧

(3)

竞

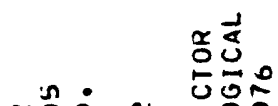
总

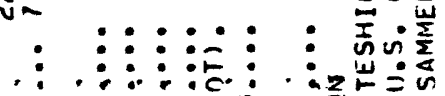

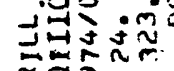

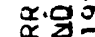

的楸矛

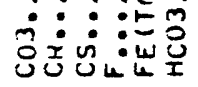

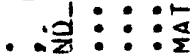

: : $:: \frac{2}{2}::$

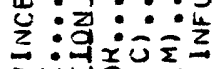

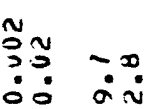

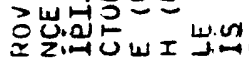

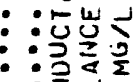

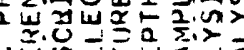

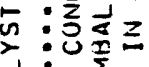

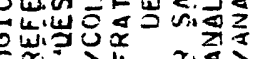

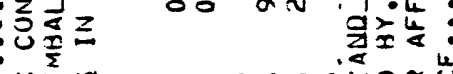

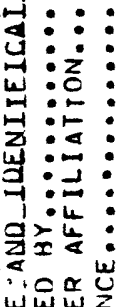

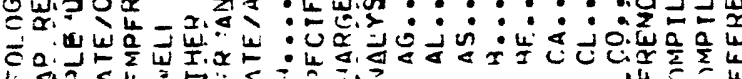

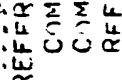

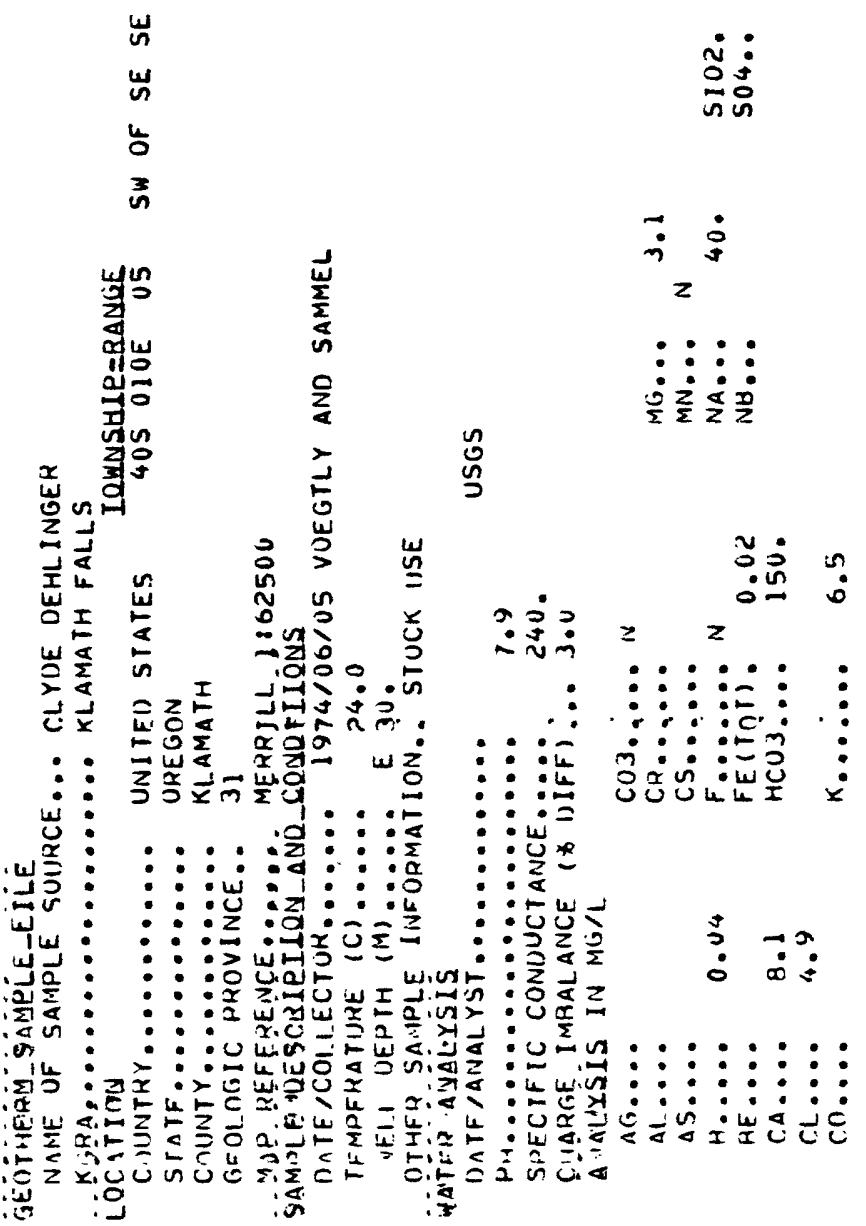




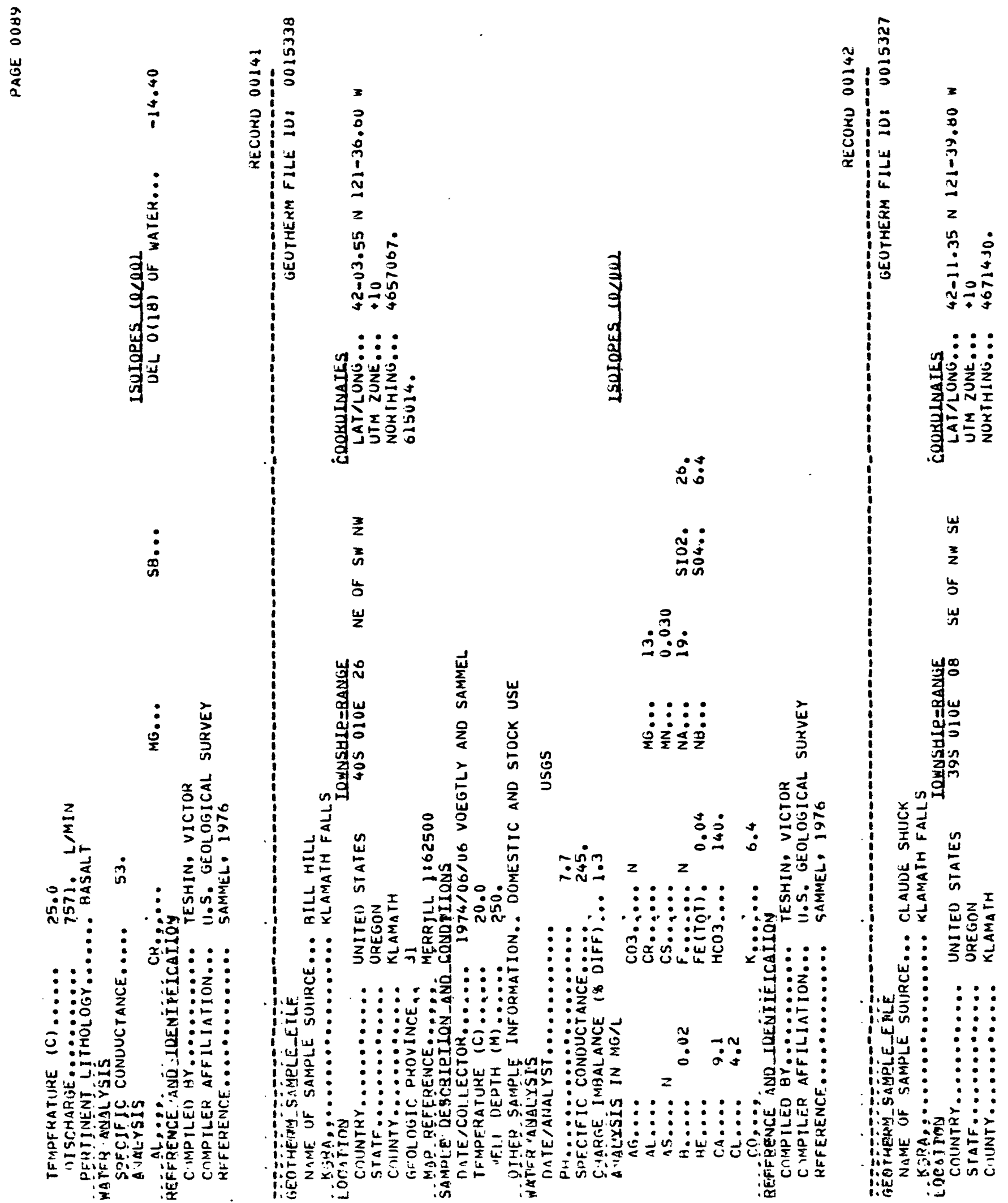


$\vec{a}$
0
0
ù
$a$
$a$

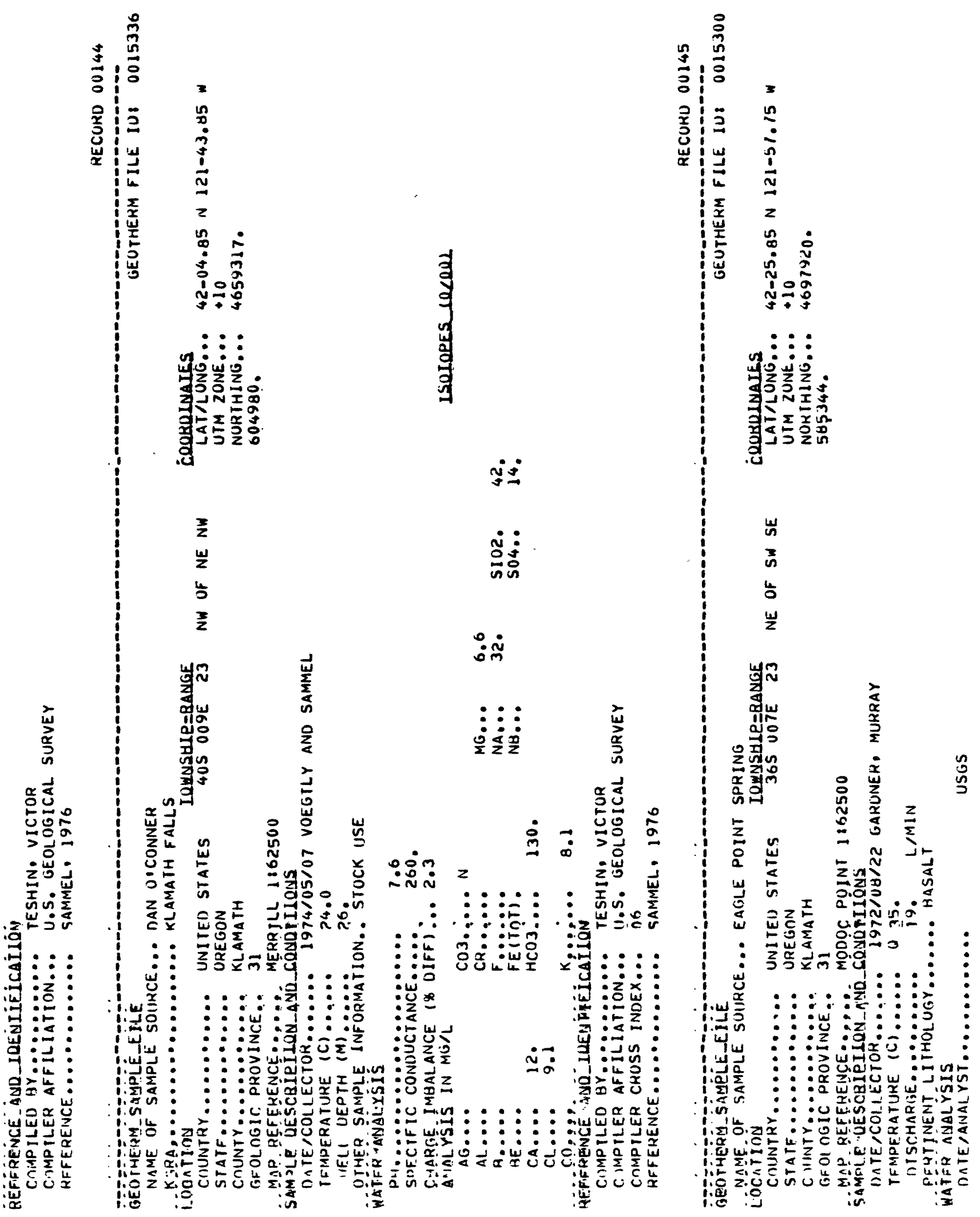




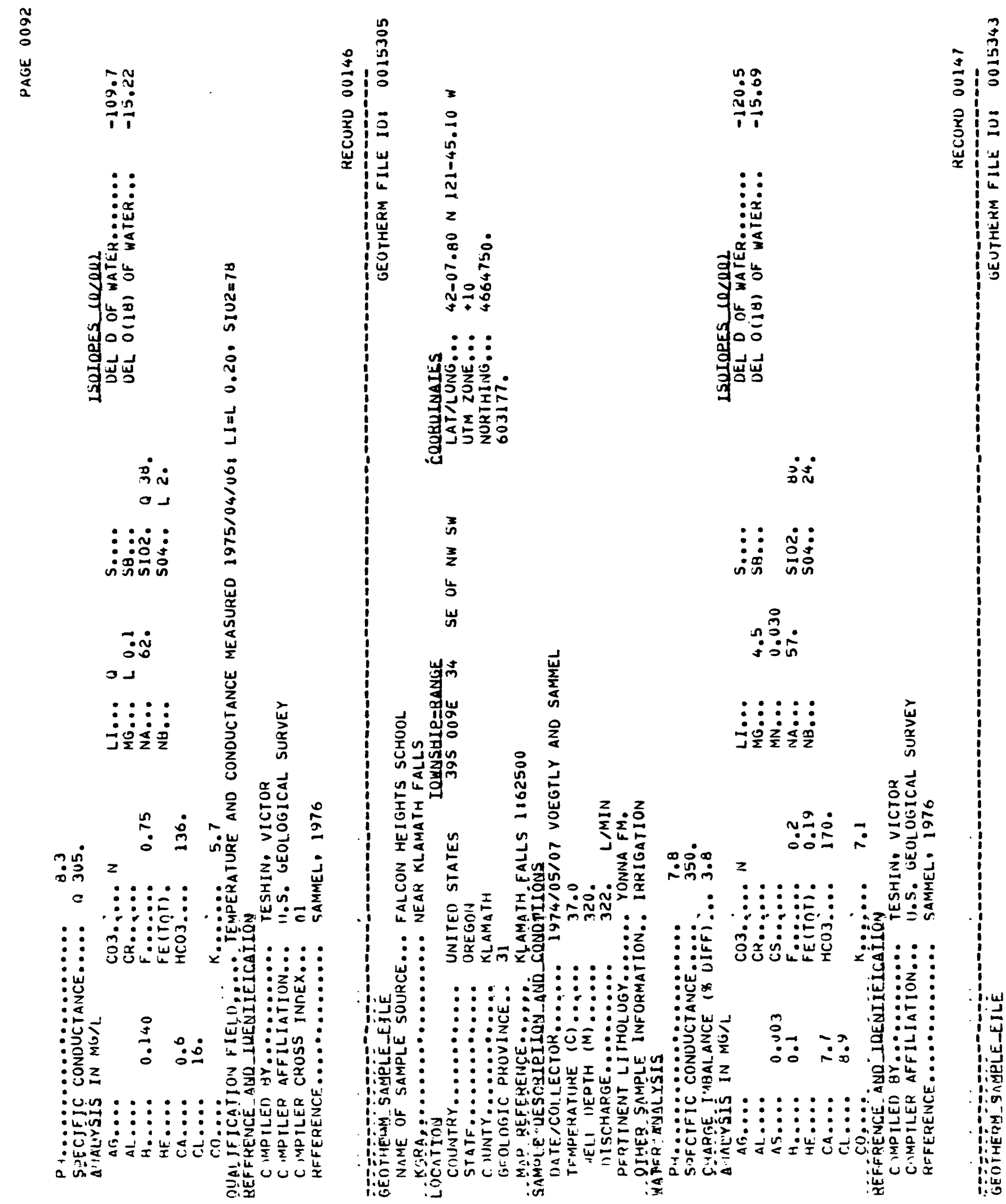




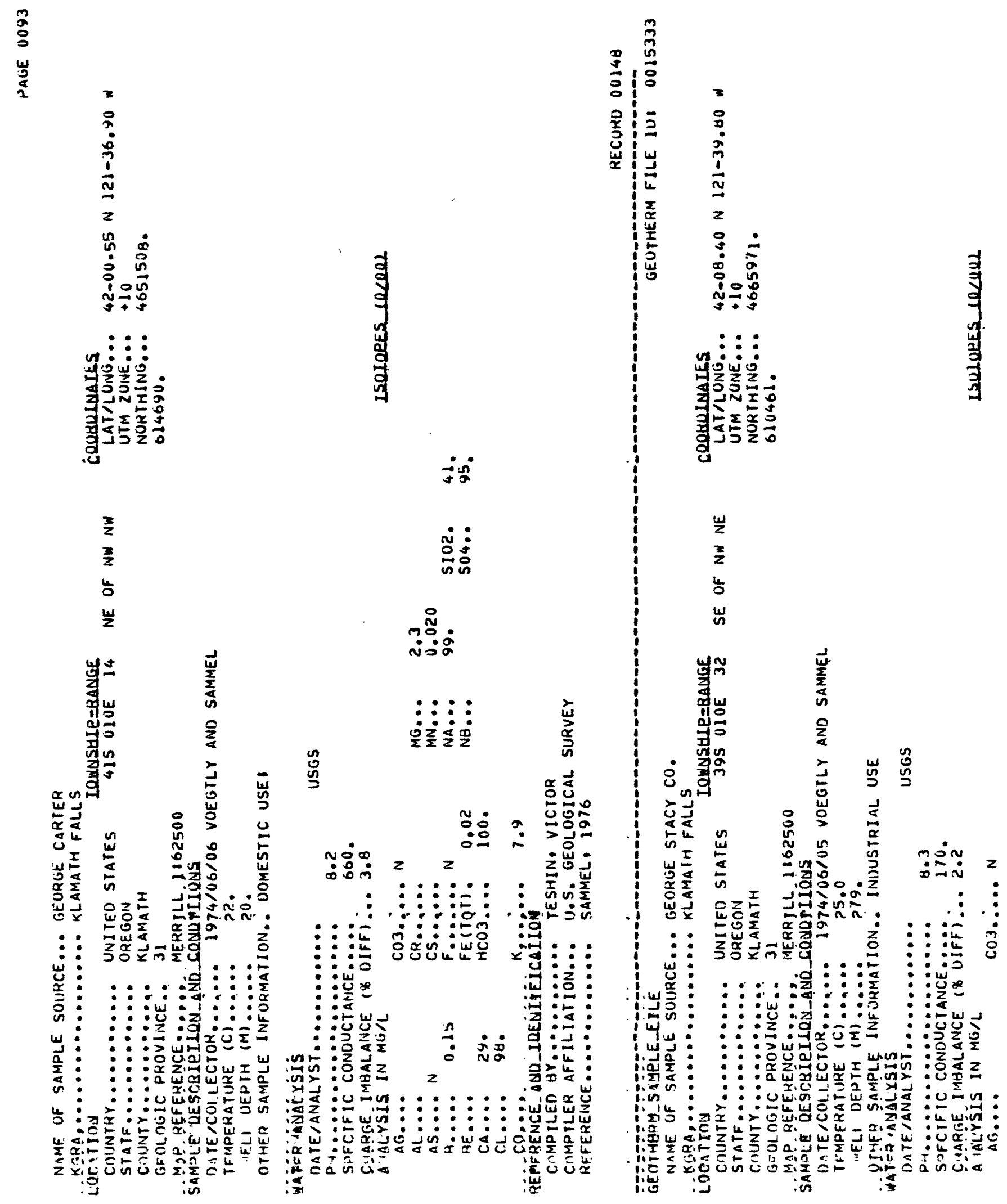


\begin{tabular}{l}
\multirow{2}{8}{} \\
8 \\
y \\
$a$ \\
$a$
\end{tabular}
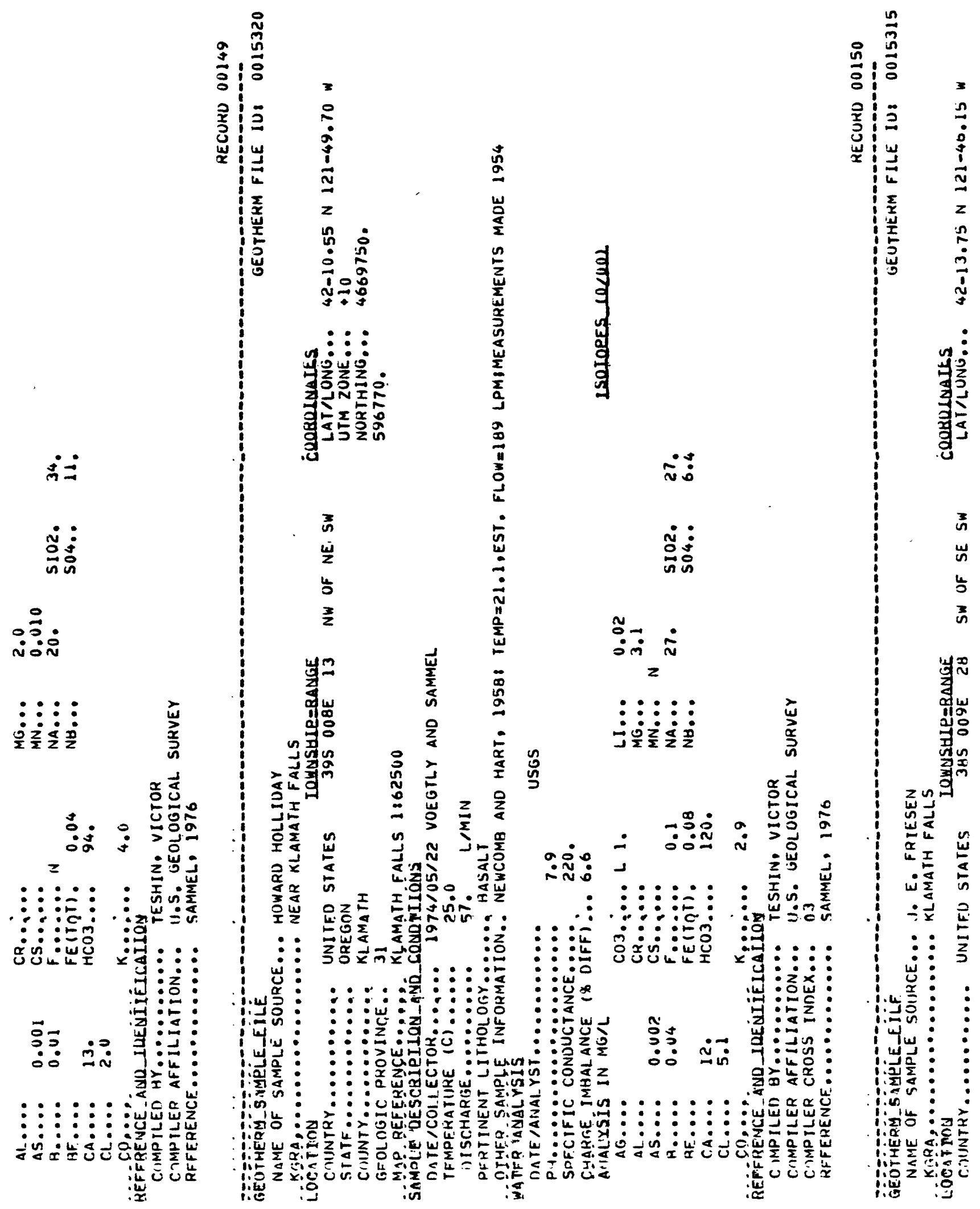

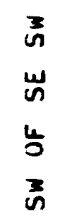
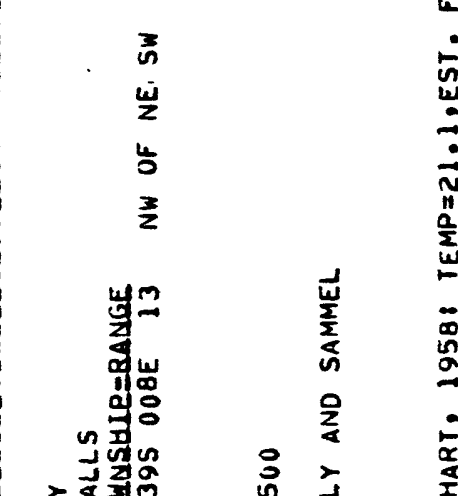
$n$
2
0
0
$y$
0
0

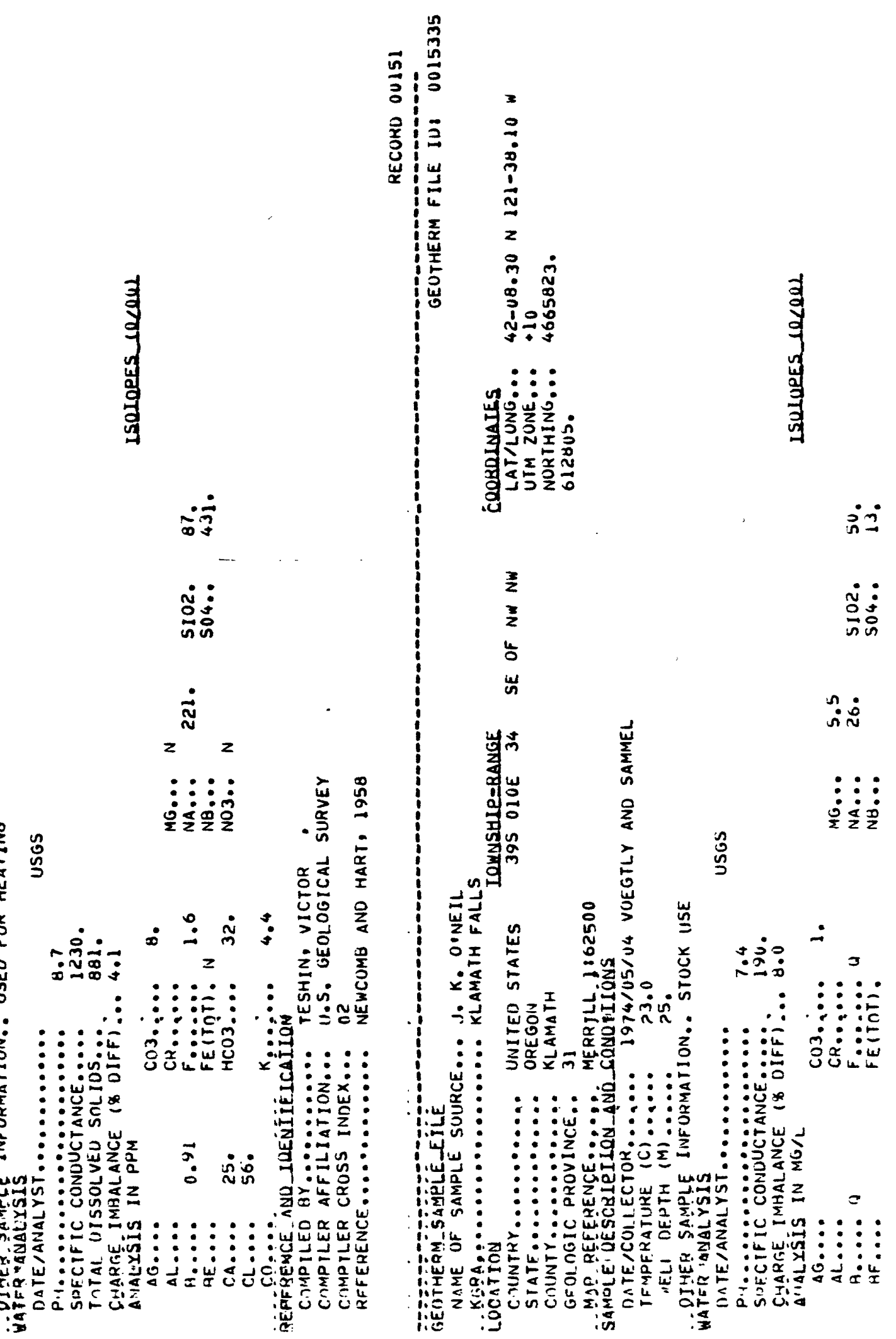

aี
בี
जो
aี
3
जี

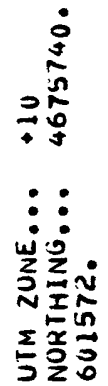


$\frac{8}{3}$
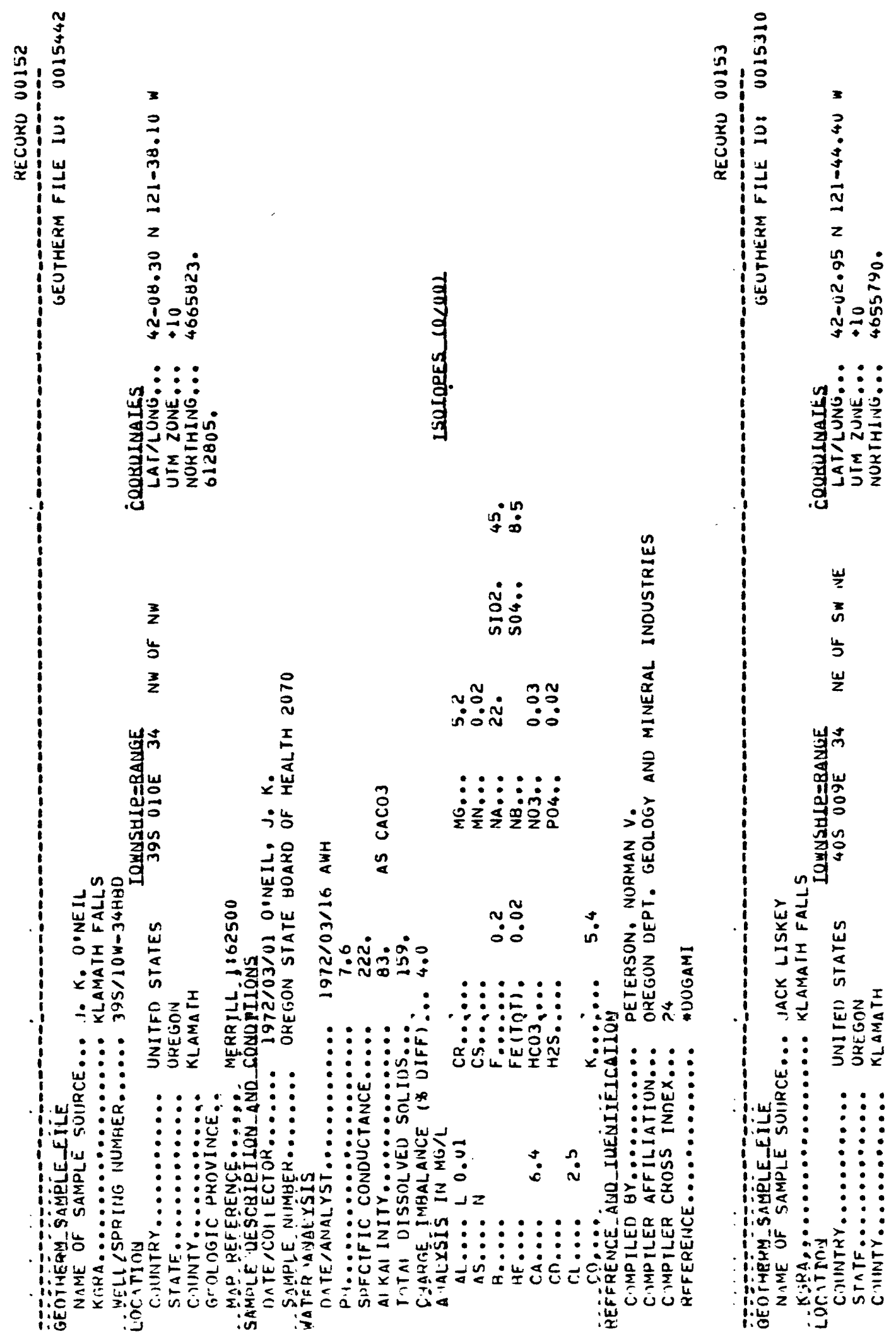

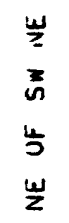

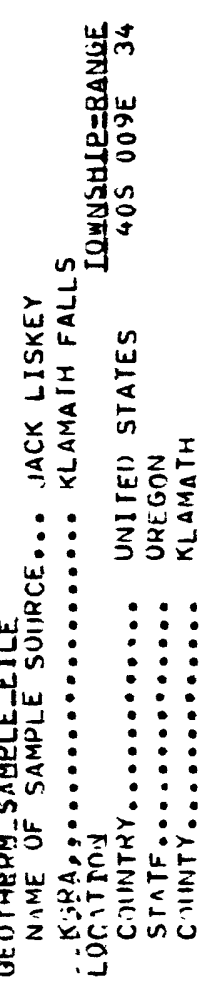


旁

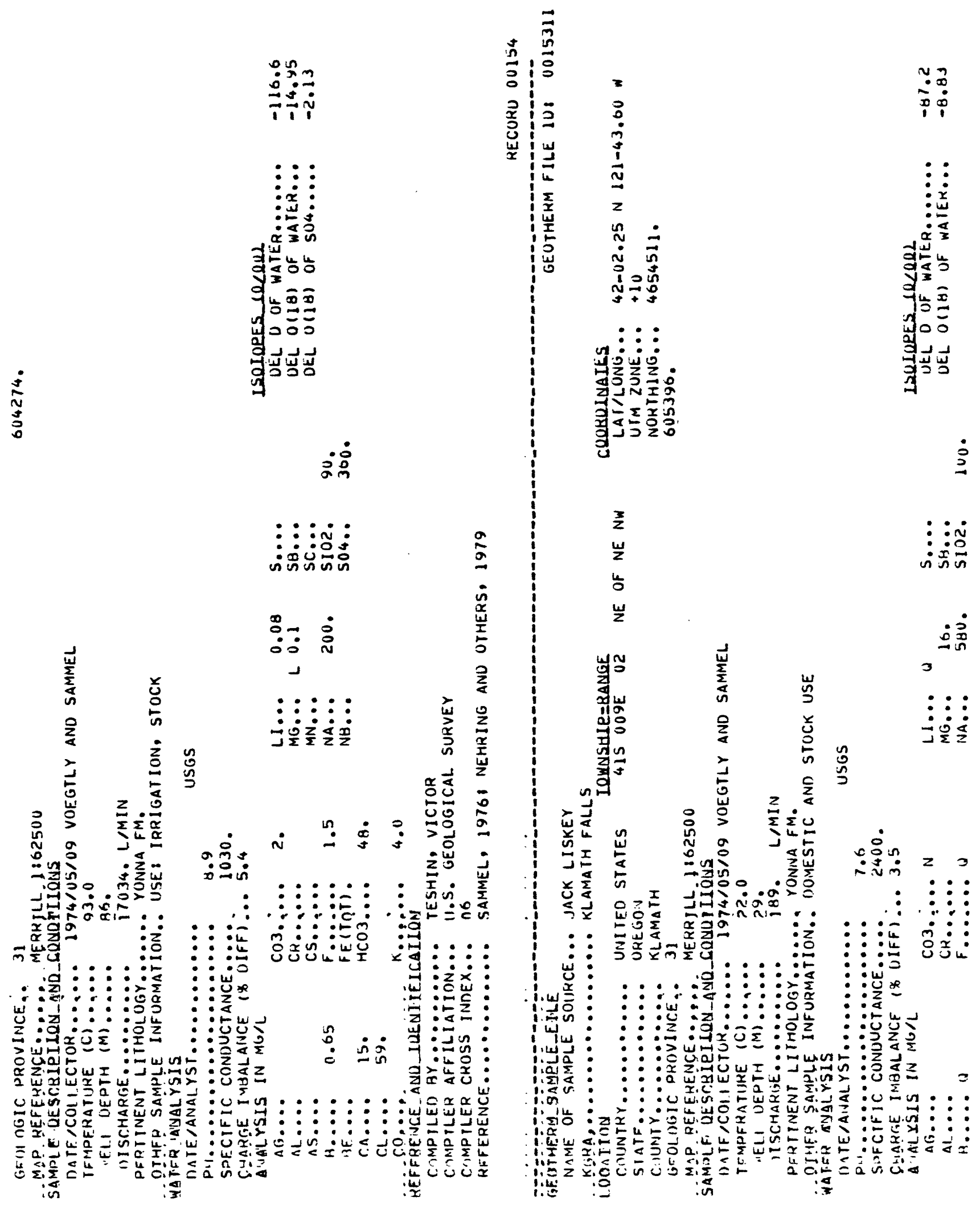




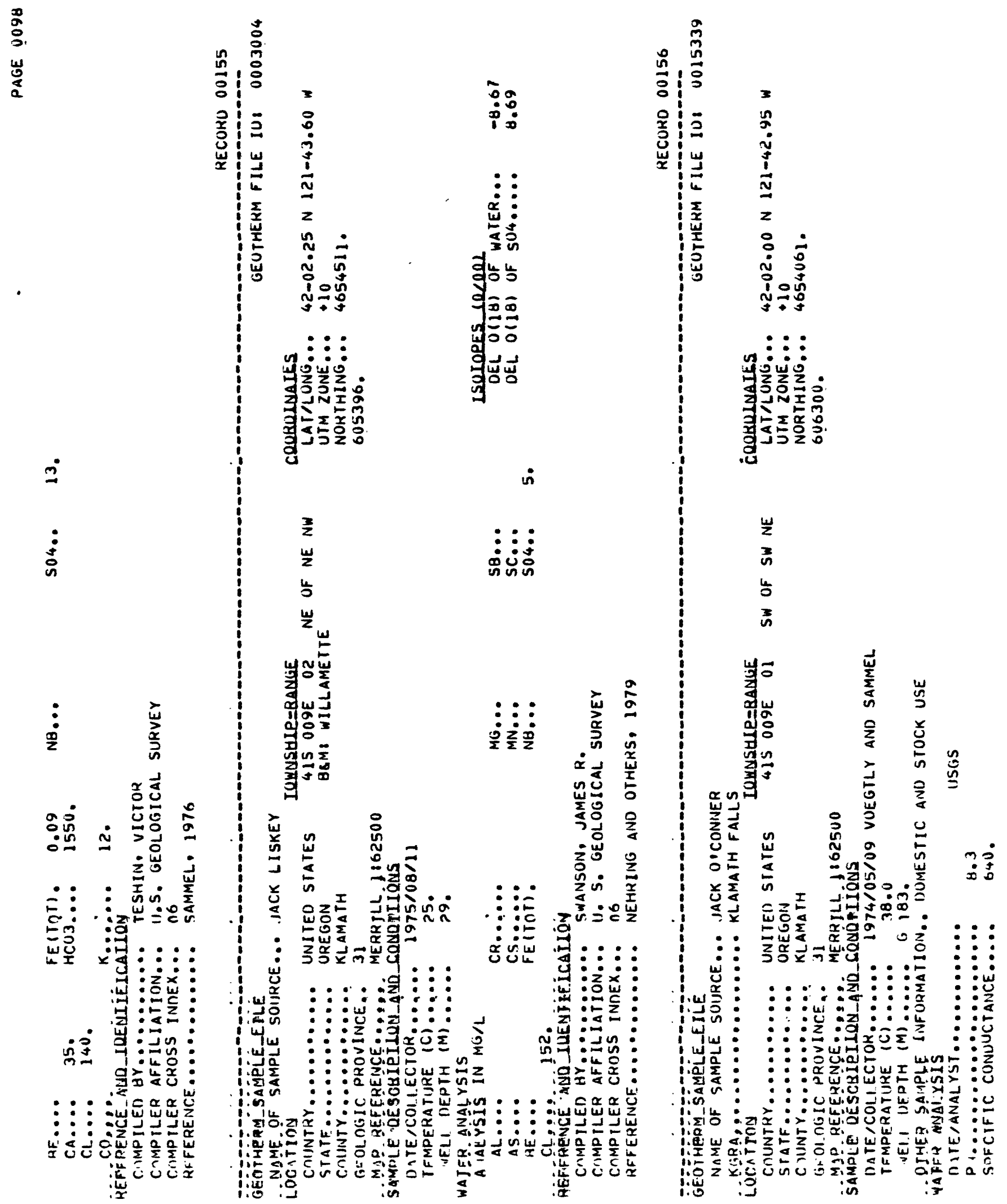


2
$\dot{a}$
0
0
$a$
$a$

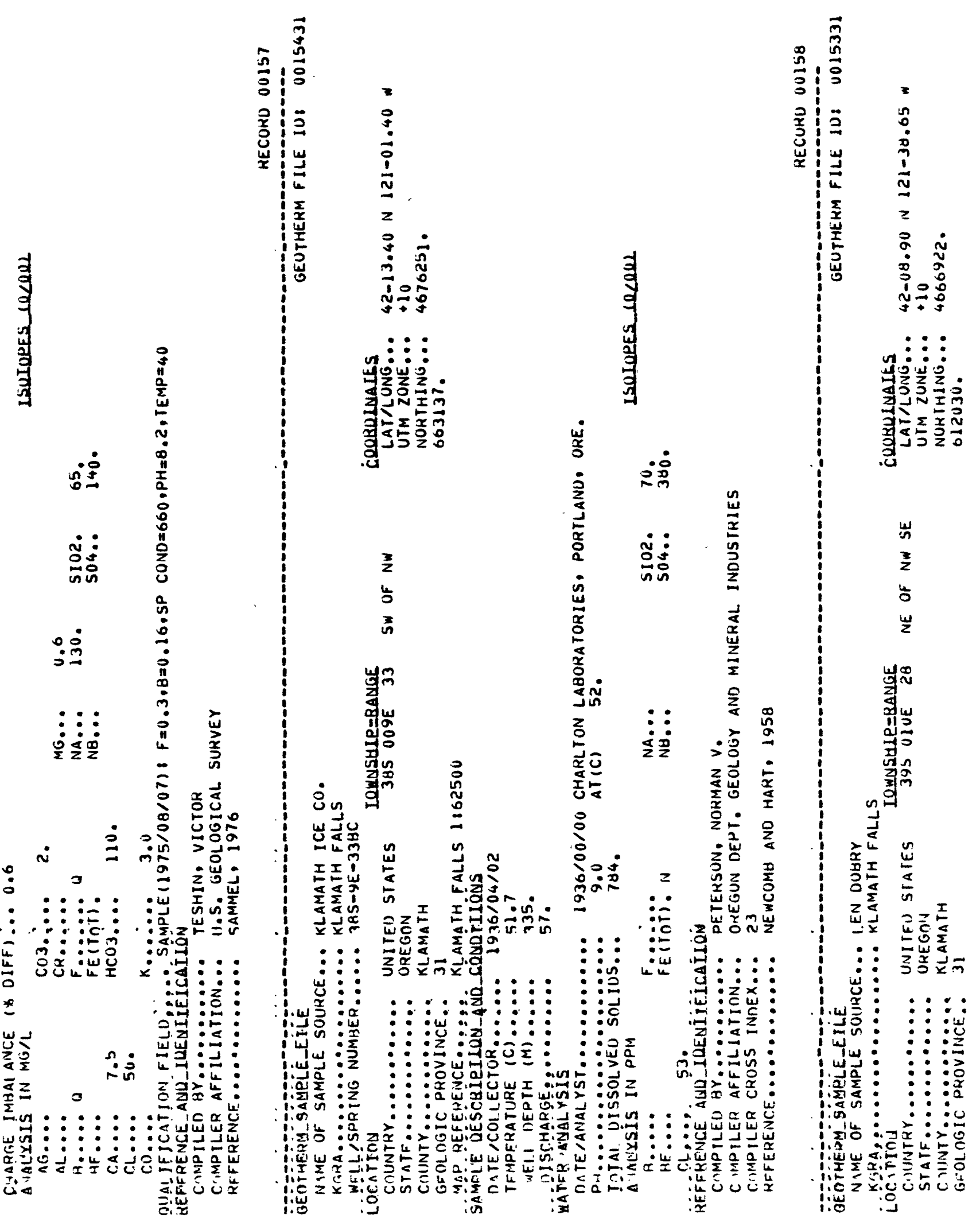


0
$\vdots$
0
0
$\vdots$
2

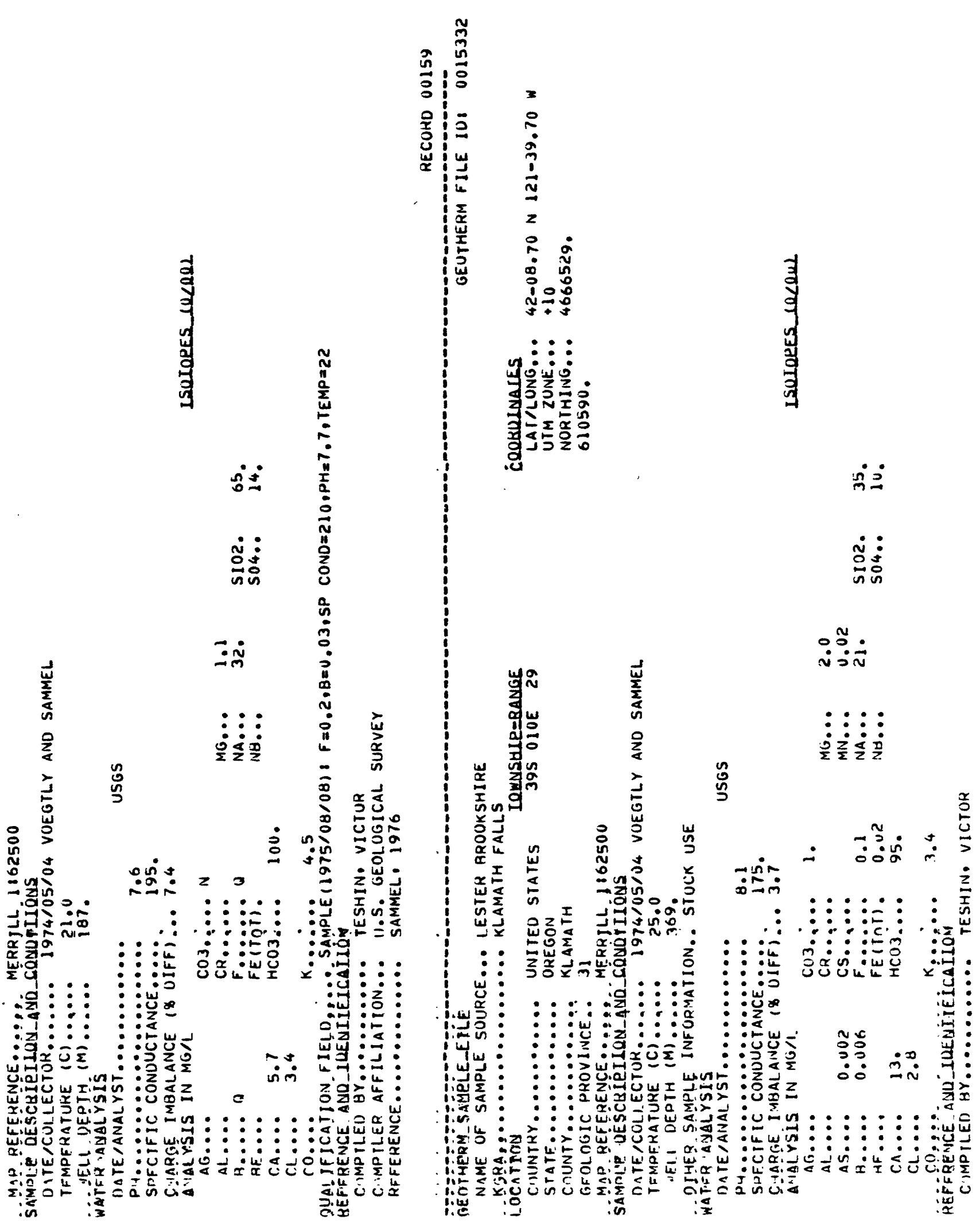


$\overrightarrow{0}$
0
0
0
0
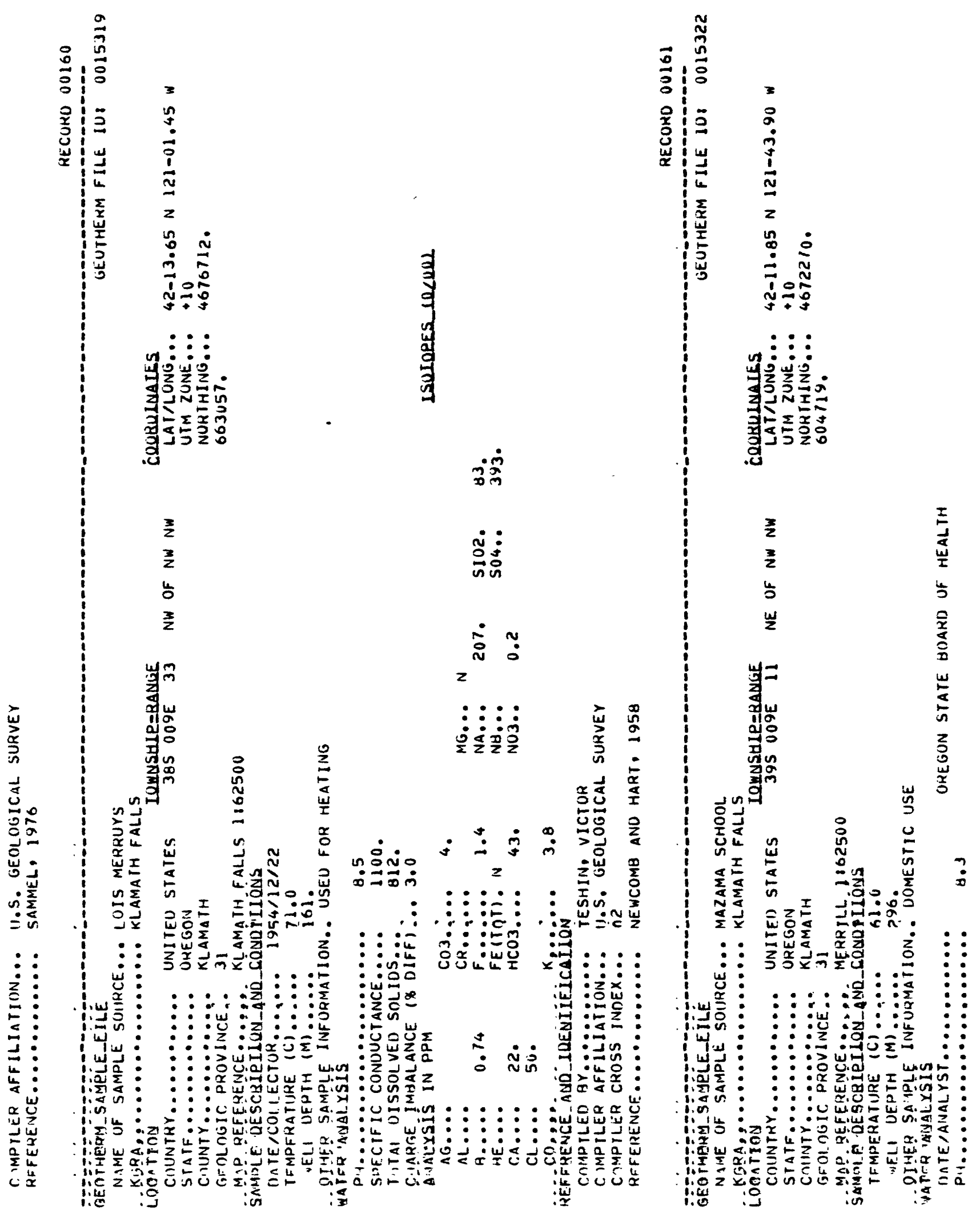


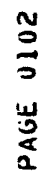
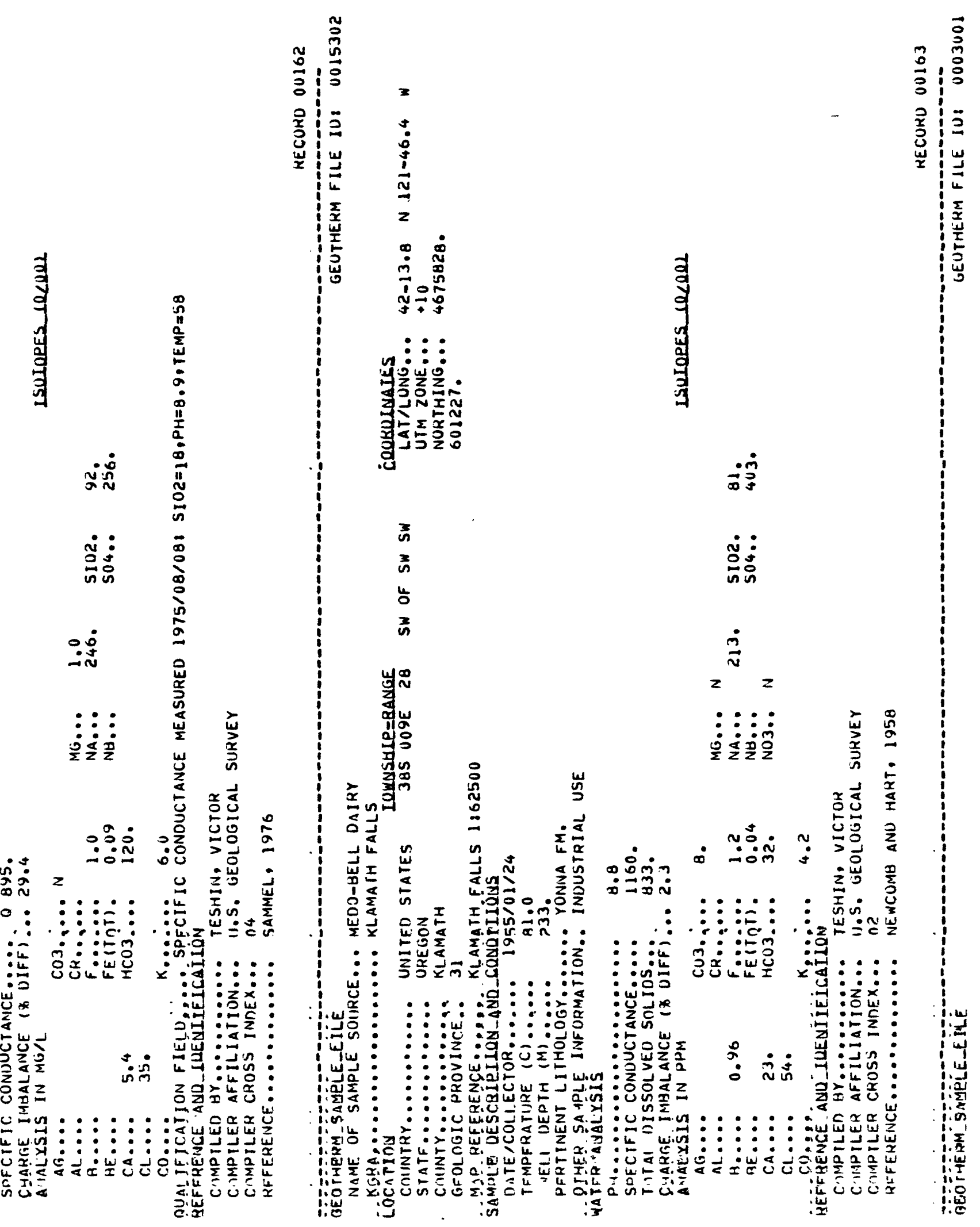
0
0
0
0
0
0
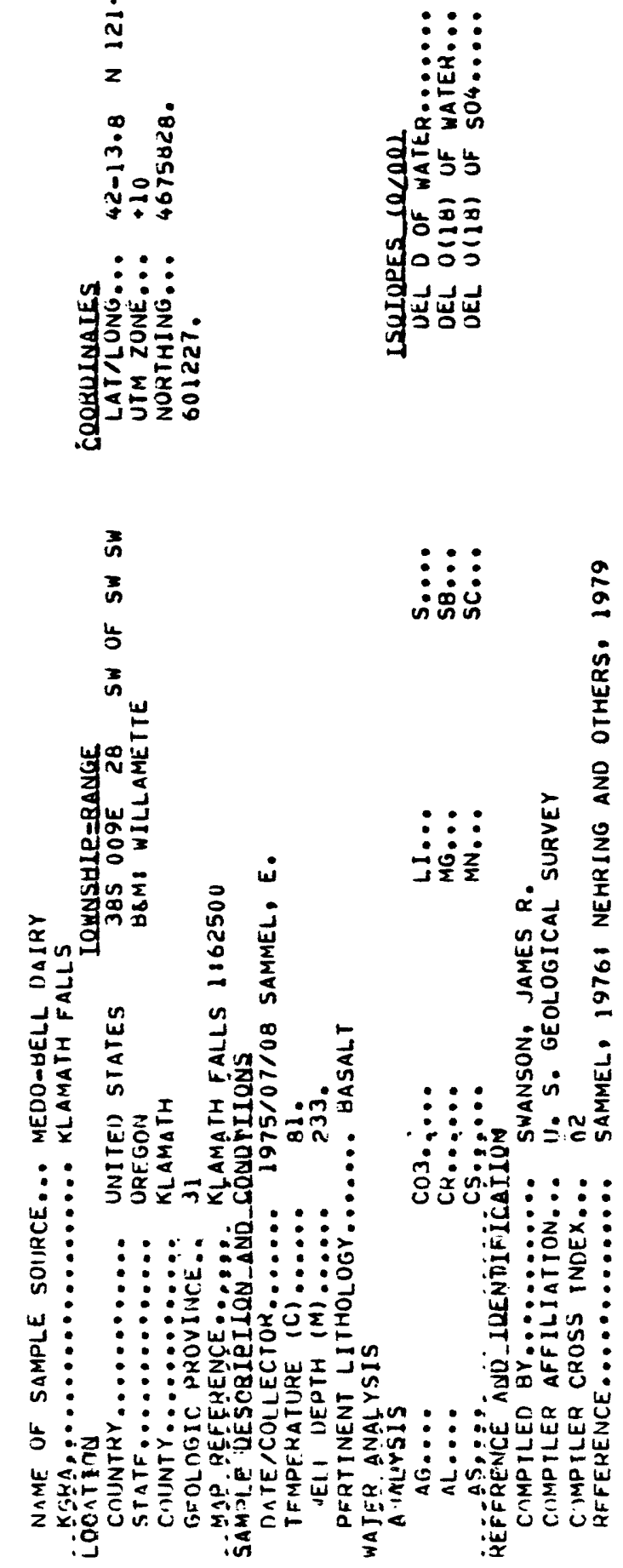

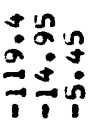

离

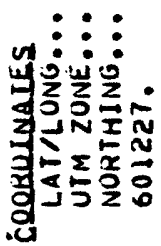

岂岕这

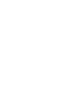

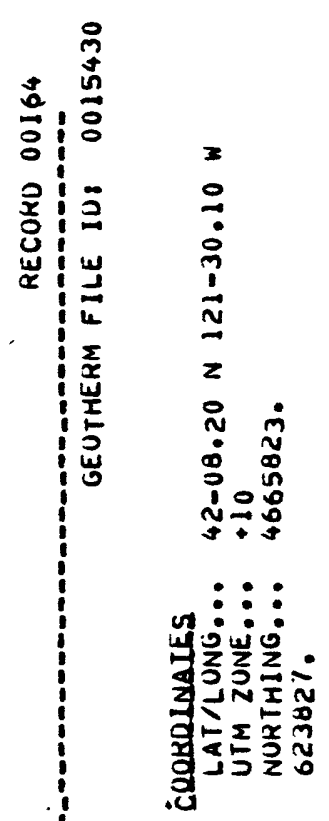
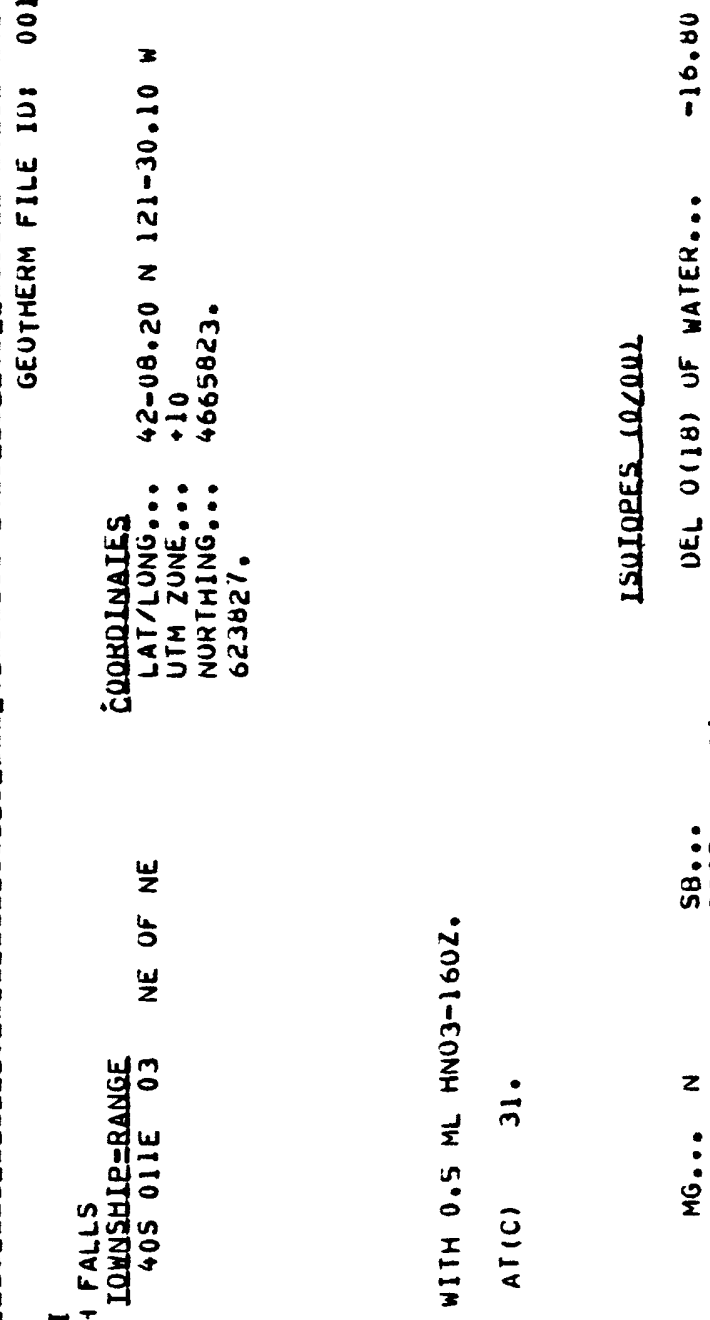

$\stackrel{\dot{0} \infty}{\doteq}$

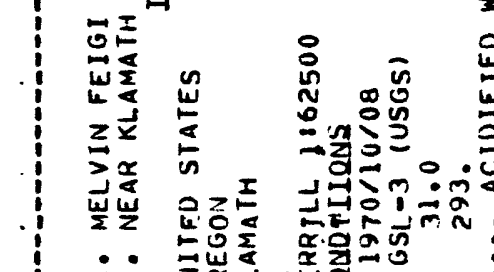
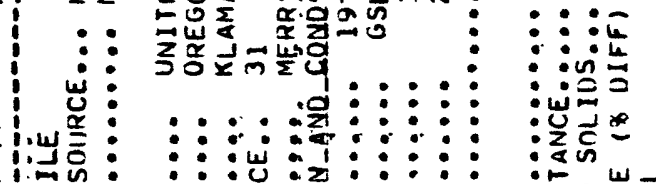

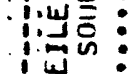

لـ

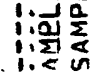

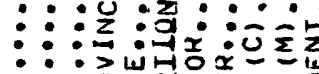

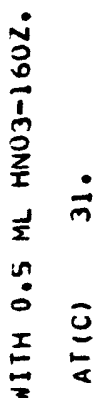

㩆范

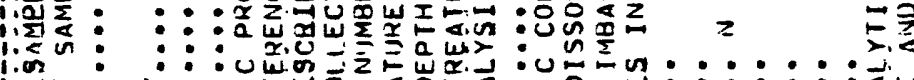

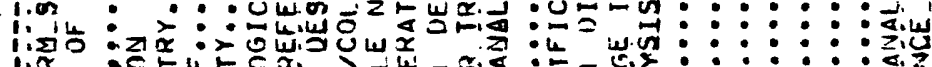

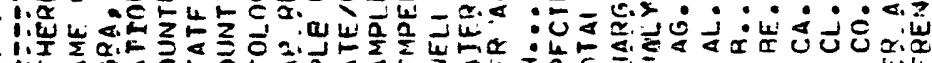
i.t

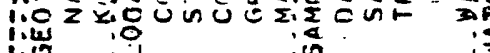




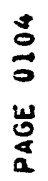

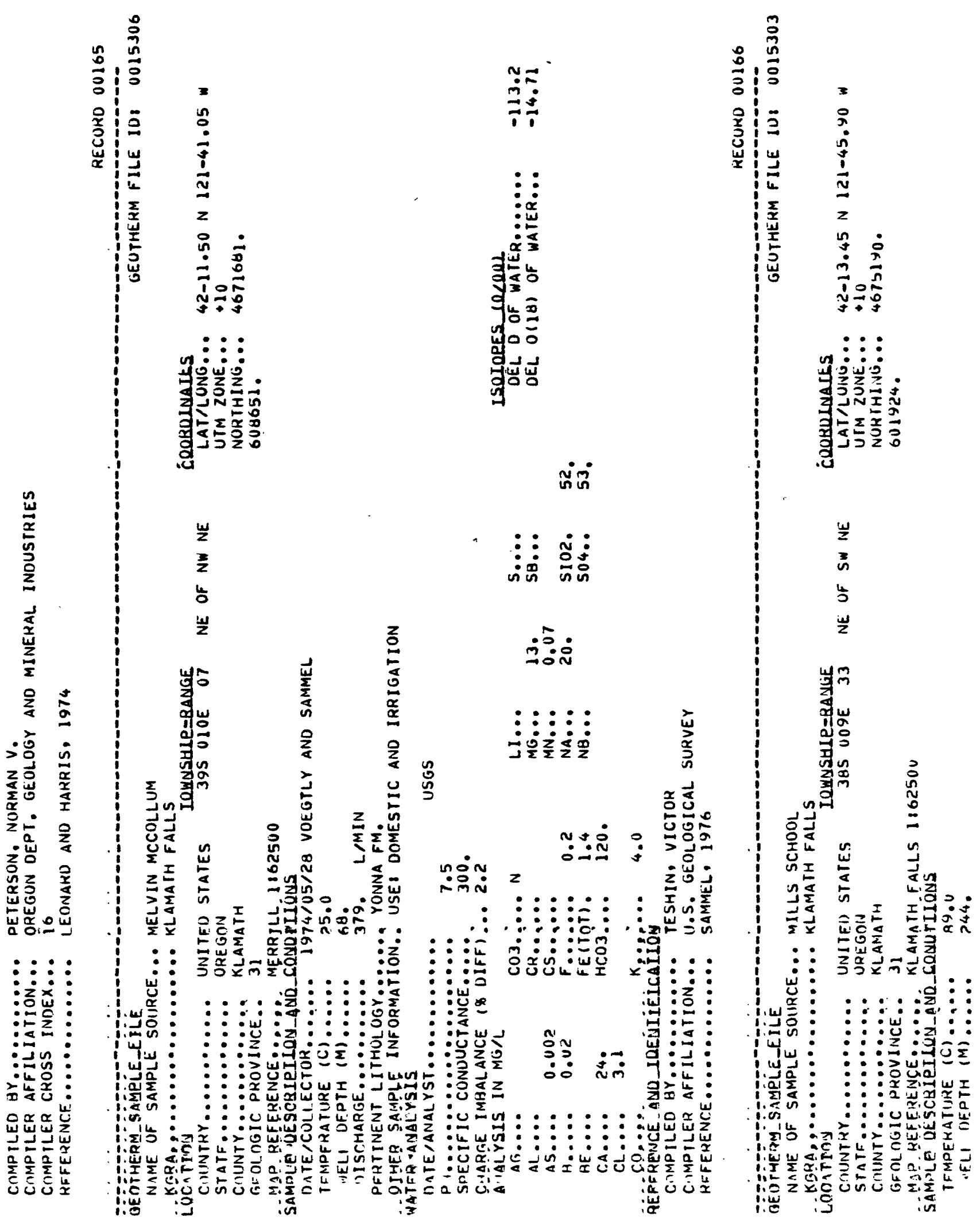




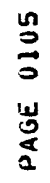
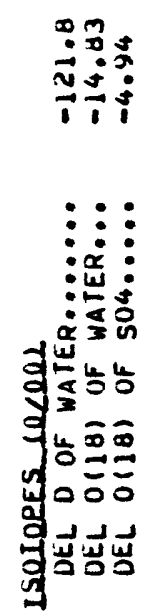

就

: $: \vdots:$ :

莃

崖

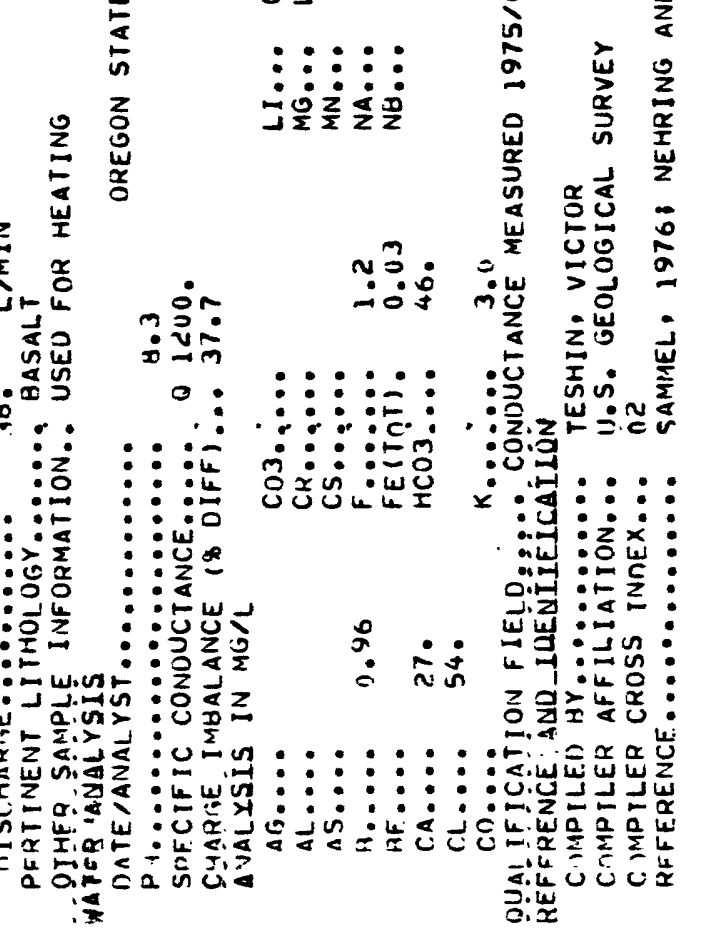

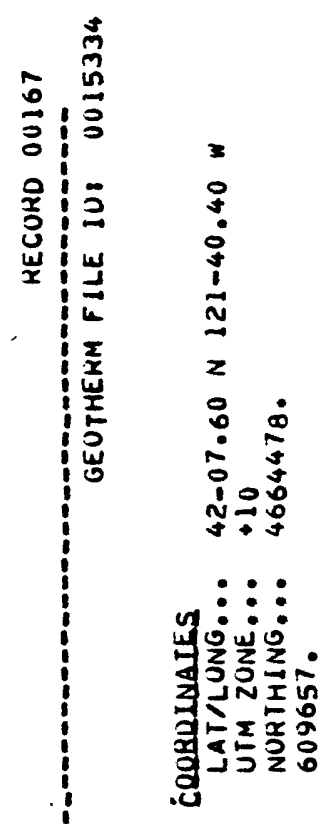

范

我

ज
क
c
un

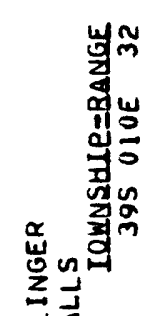

这

$\dot{\sim} \dot{\sim}$

$z$

$:: \vdots:$

ڤั

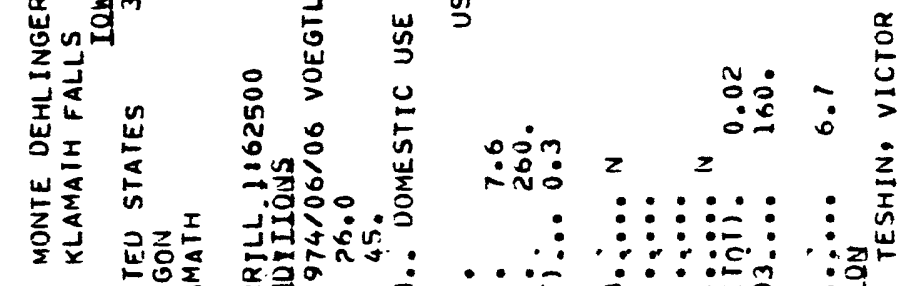

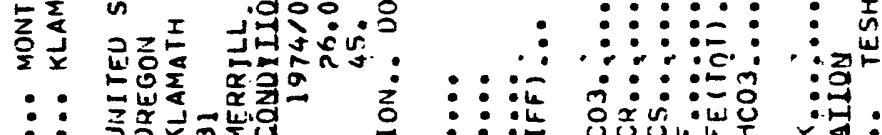

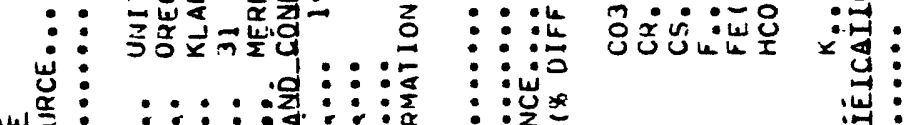

:

:

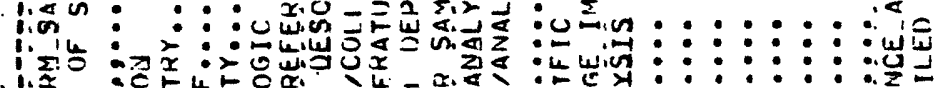

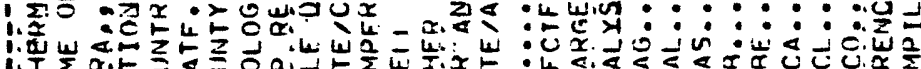
i.

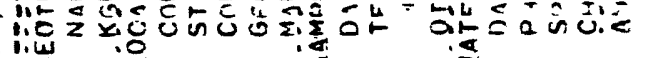

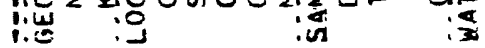




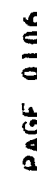
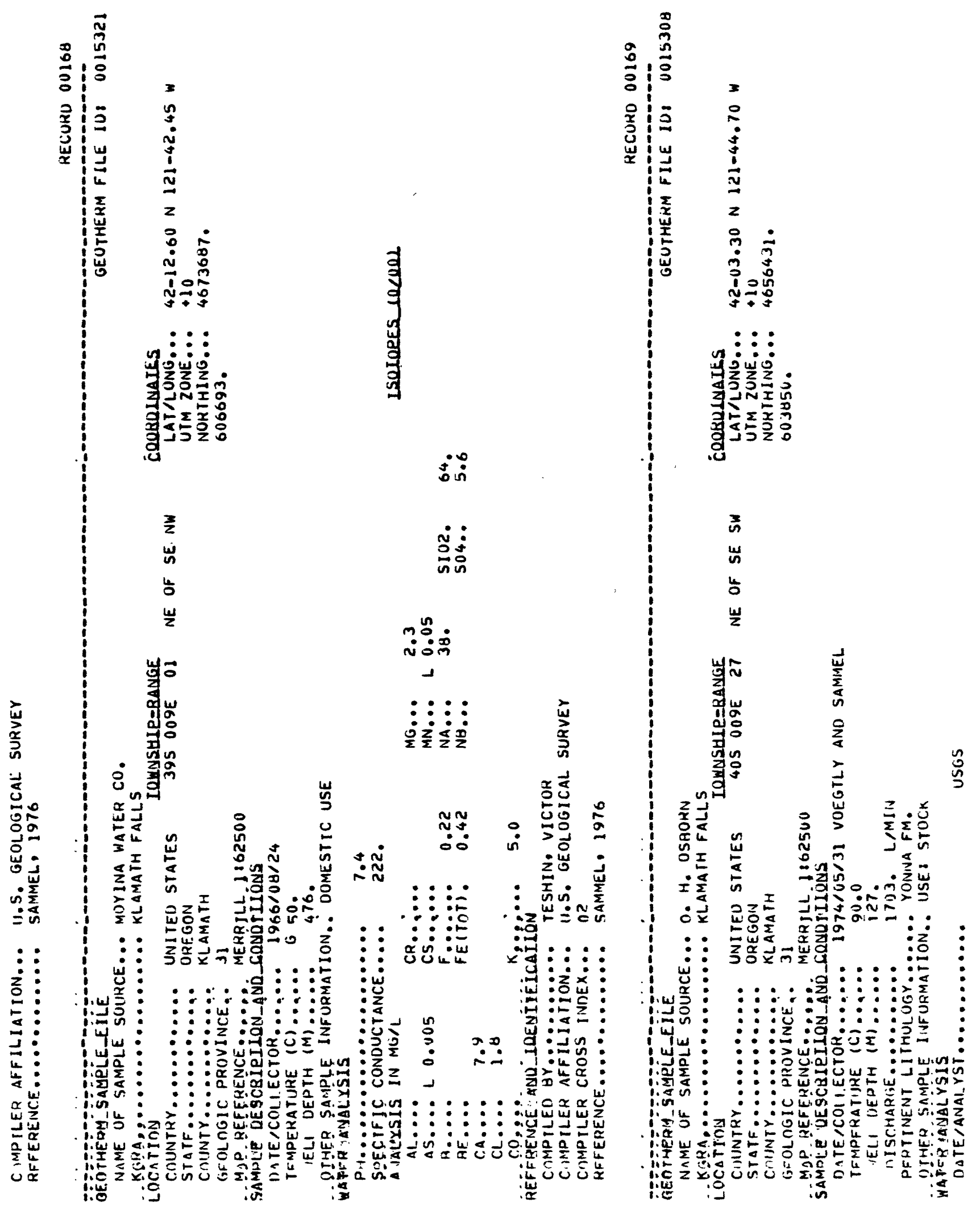
$\hat{o}$
$\vdots$
$\frac{\omega}{0}$
$a$
$a$
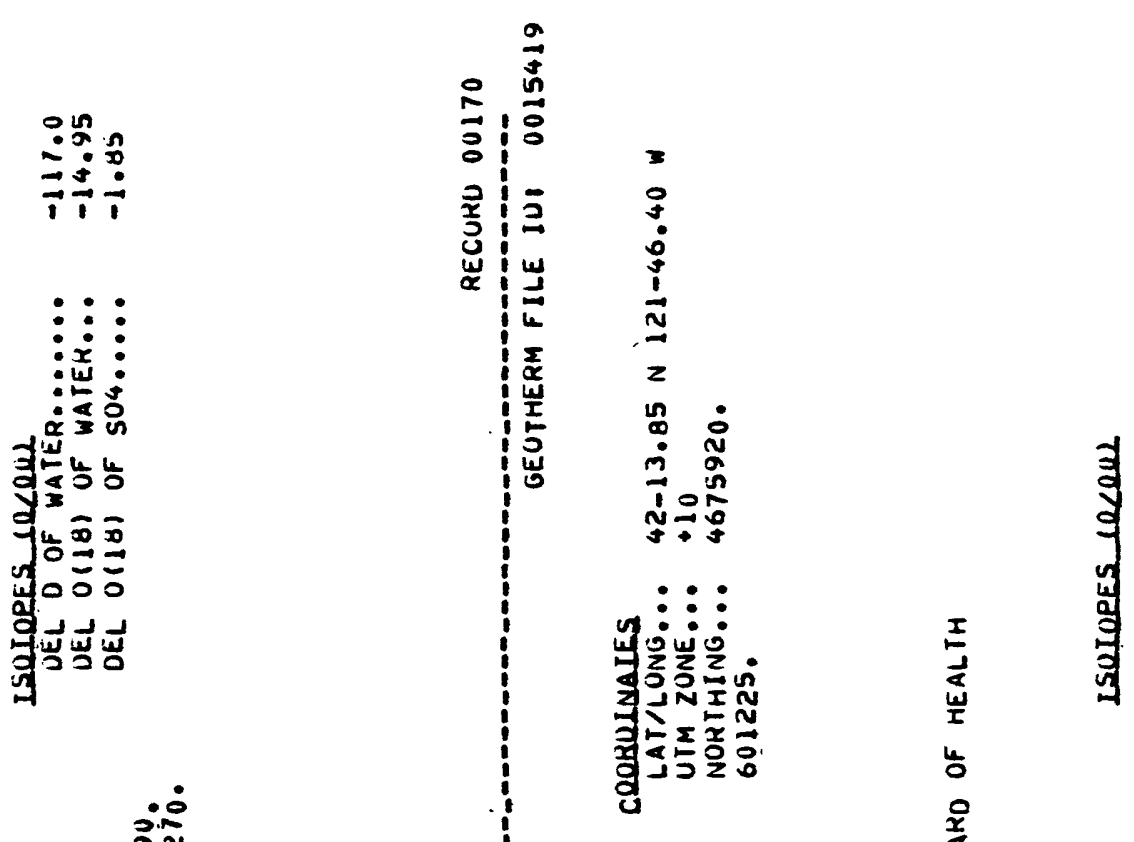

$\dot{a}: \dot{R}$

: : : : :

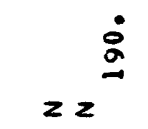

$\begin{array}{lll}: & \vdots \\ \vdots & :\end{array}$
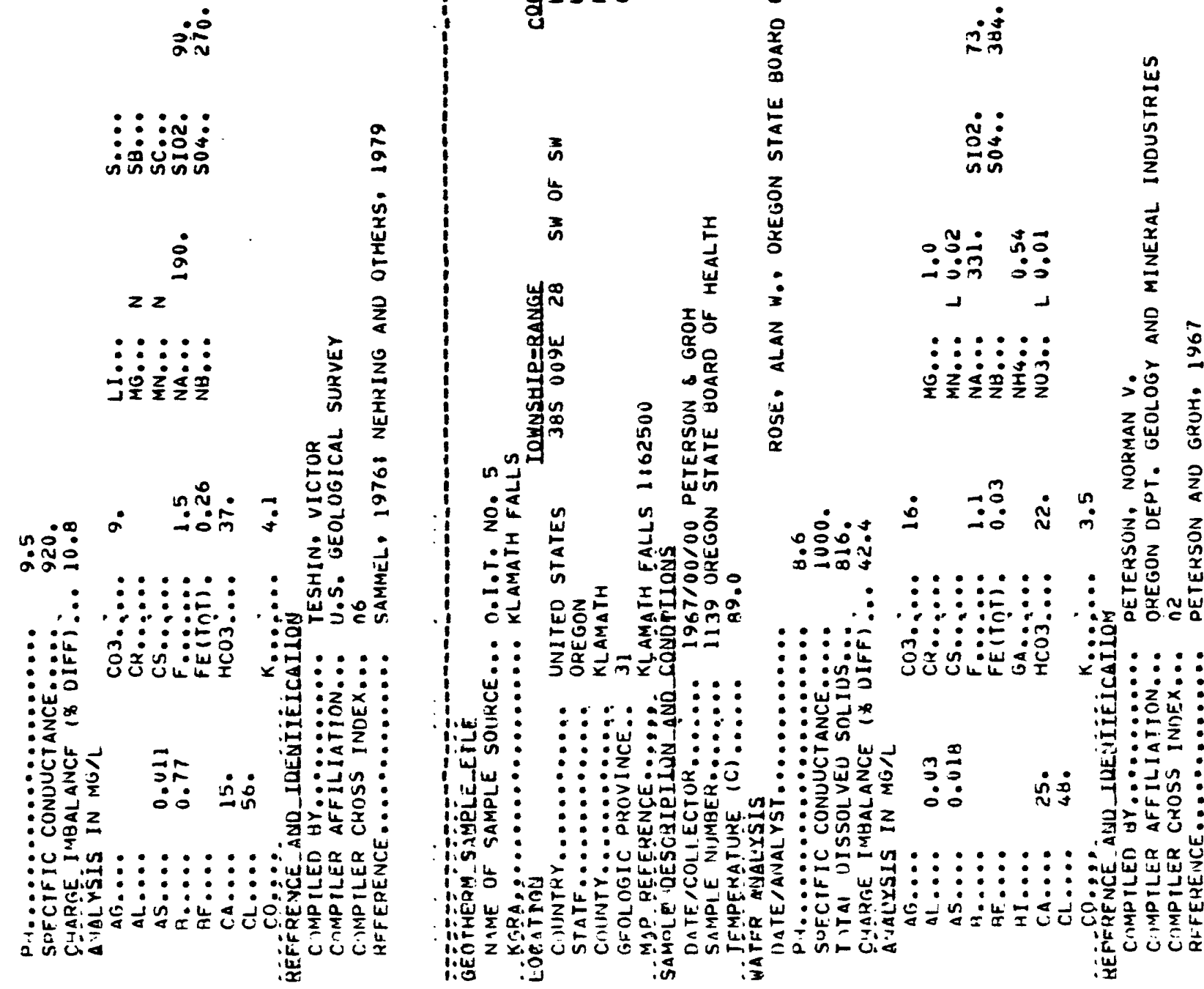

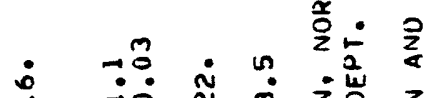

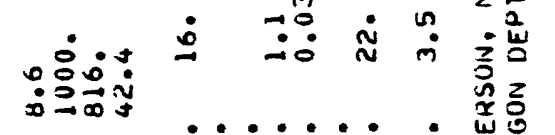

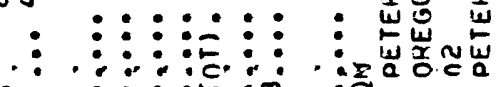

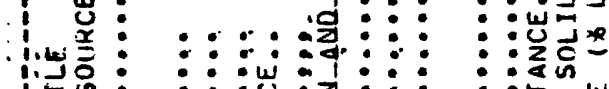

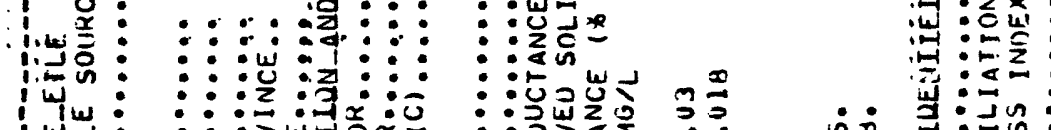
!

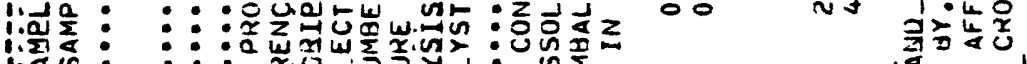
: 珠

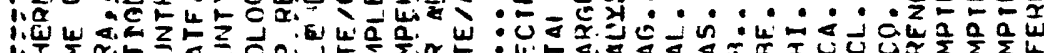

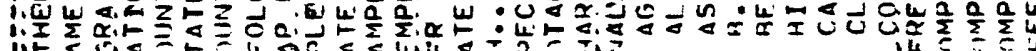

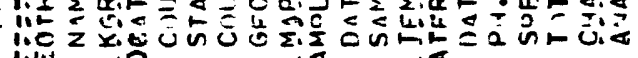

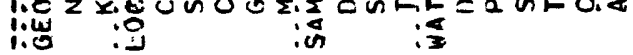



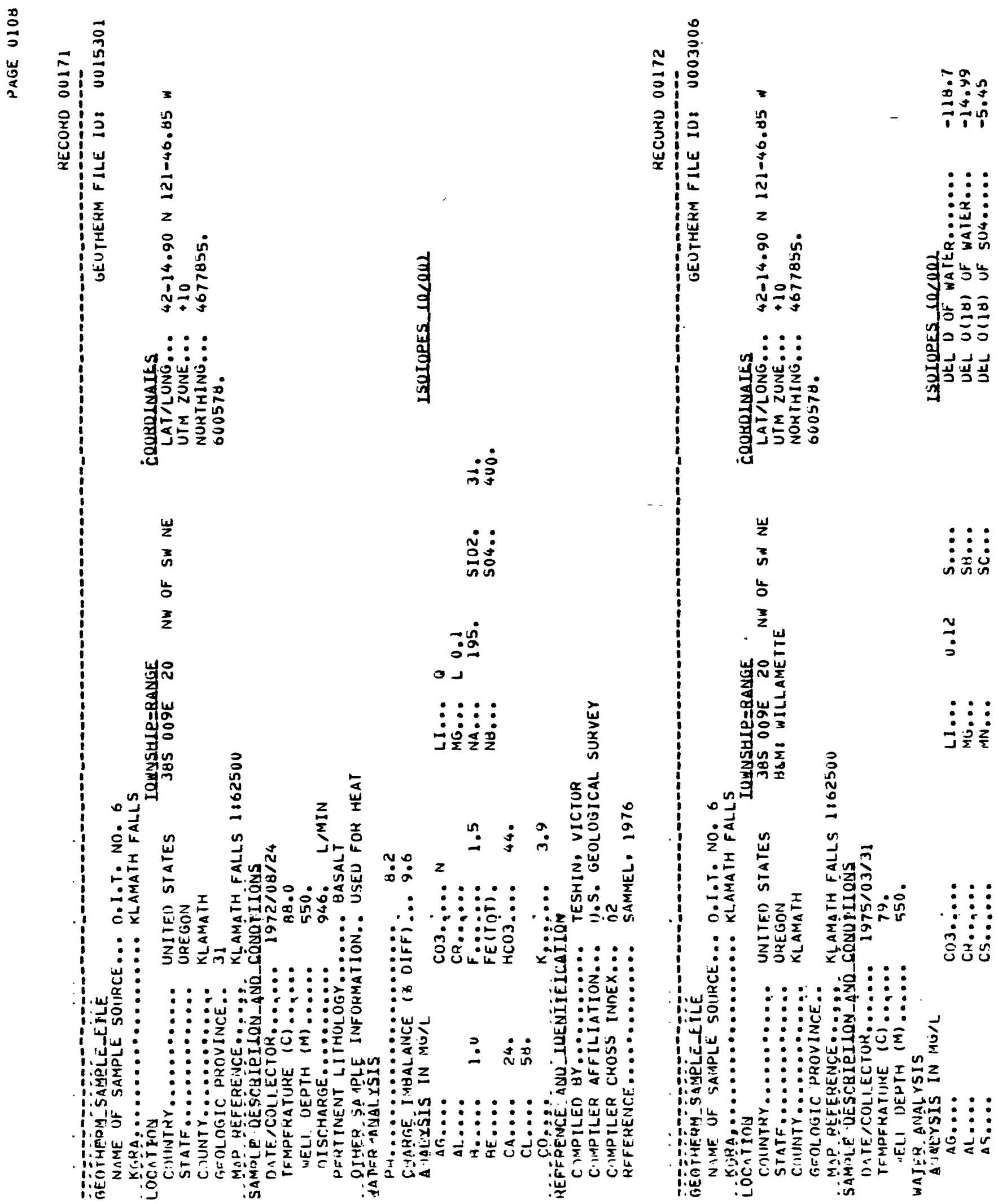


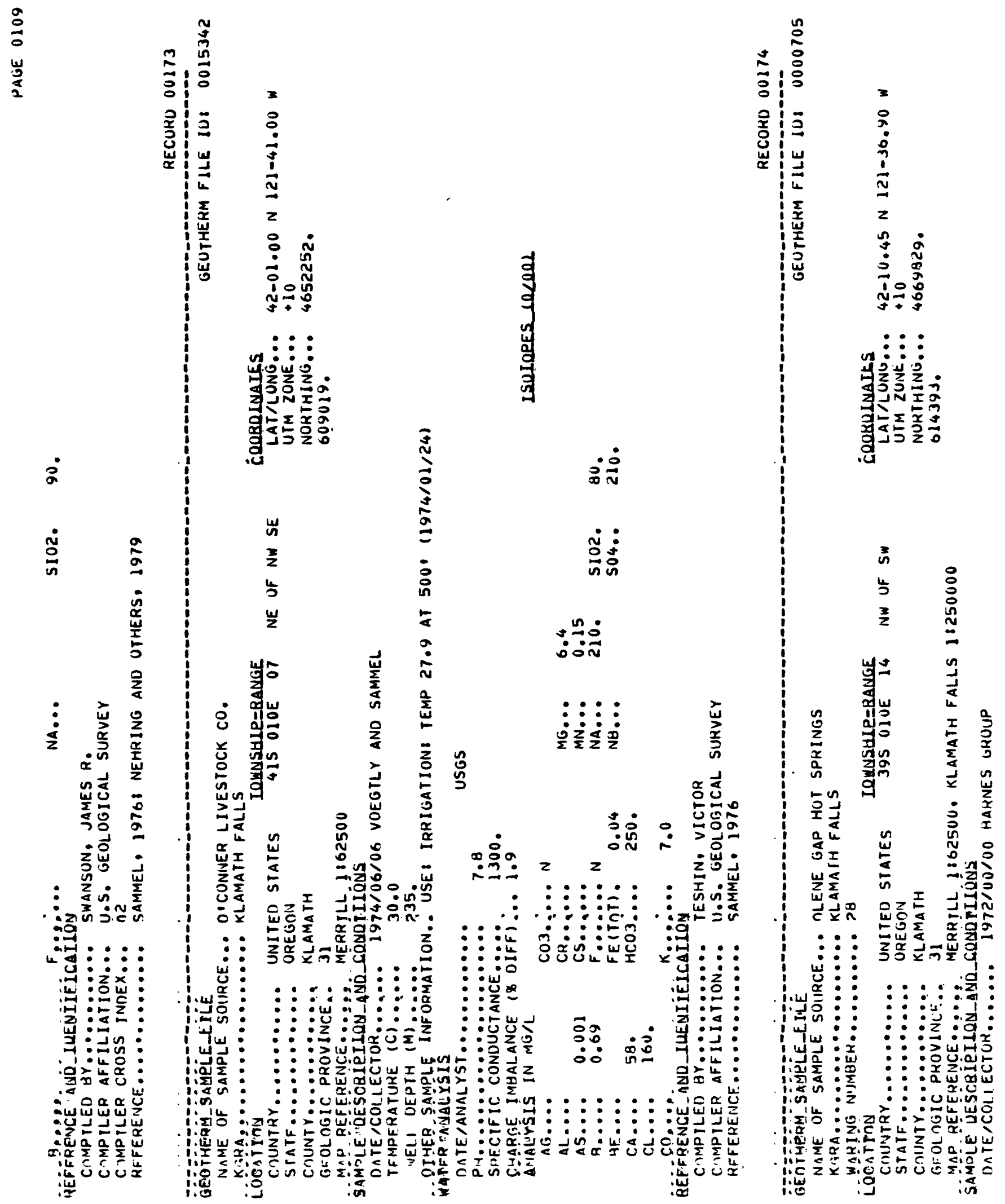




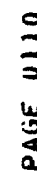

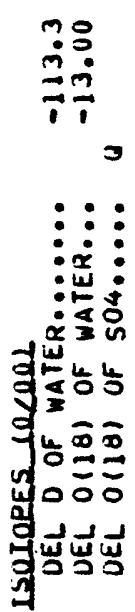

ㅇํㅇ
+

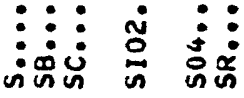

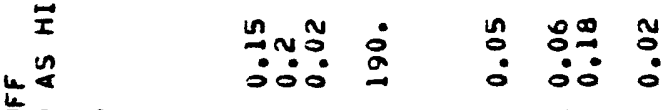

志

$\because: \frac{\text { 范 }}{2}$

숭 :

: :

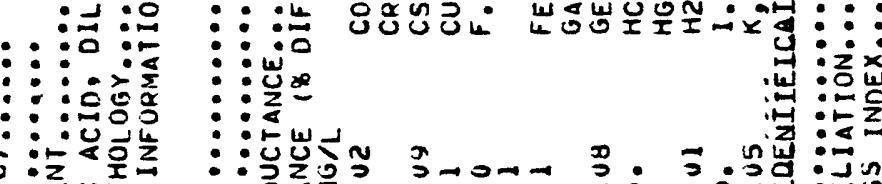

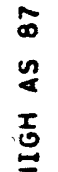

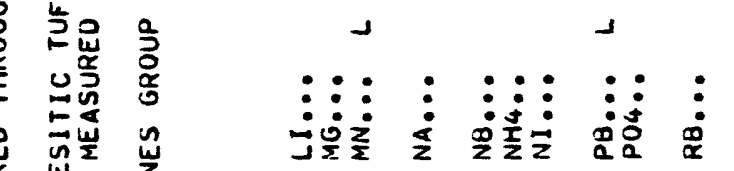

经出

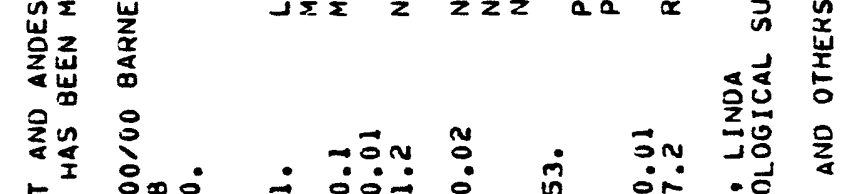

施 $-\dot{0} \div$ : 0 in on

经产

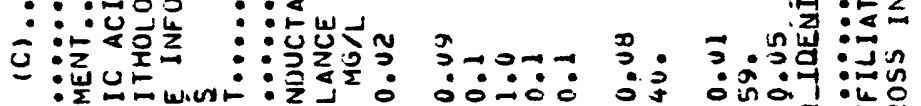

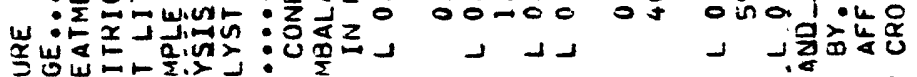

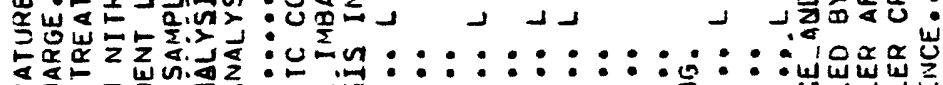

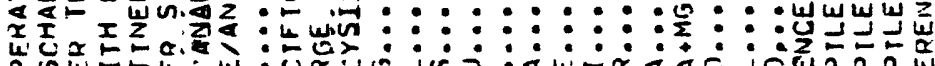

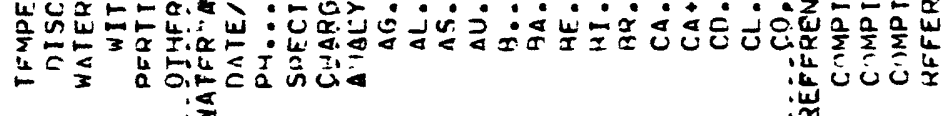

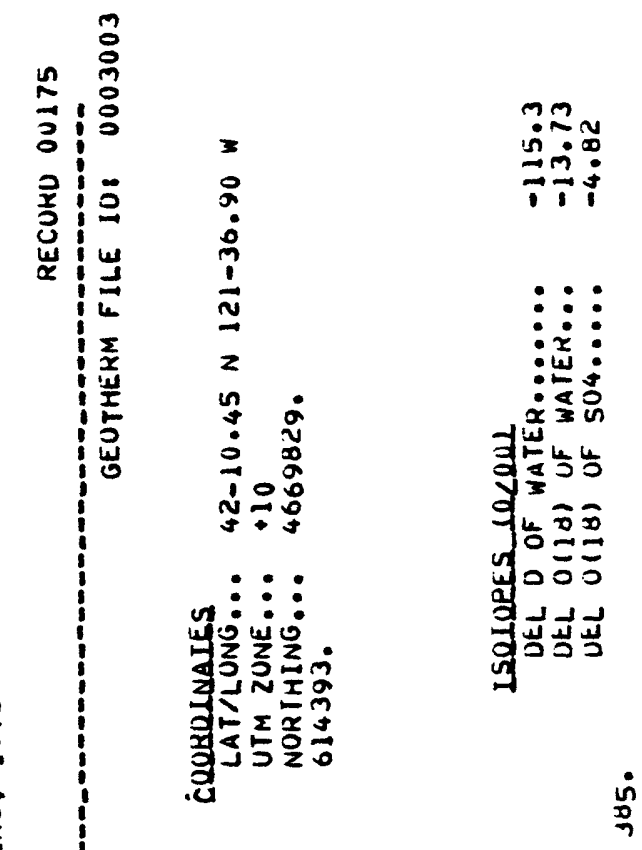

$\vdots: \vdots:$

ธ

z

氙点

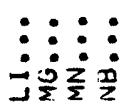

峁

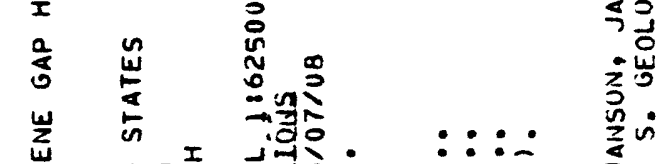

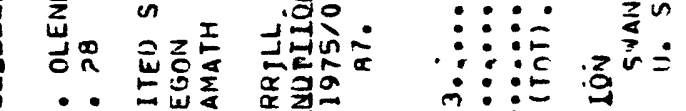
: : :

|

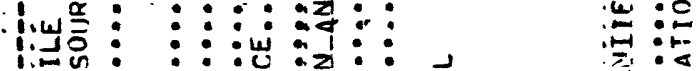

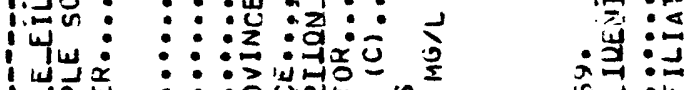

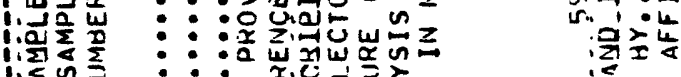

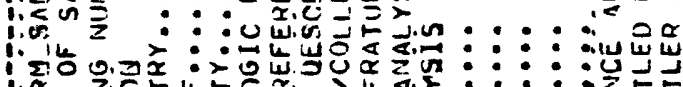

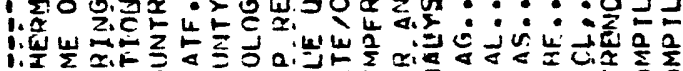

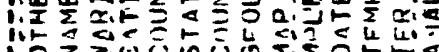

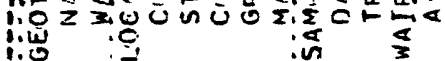


$\overrightarrow{3}$
$\overrightarrow{3}$
$\overrightarrow{0}$
$a$
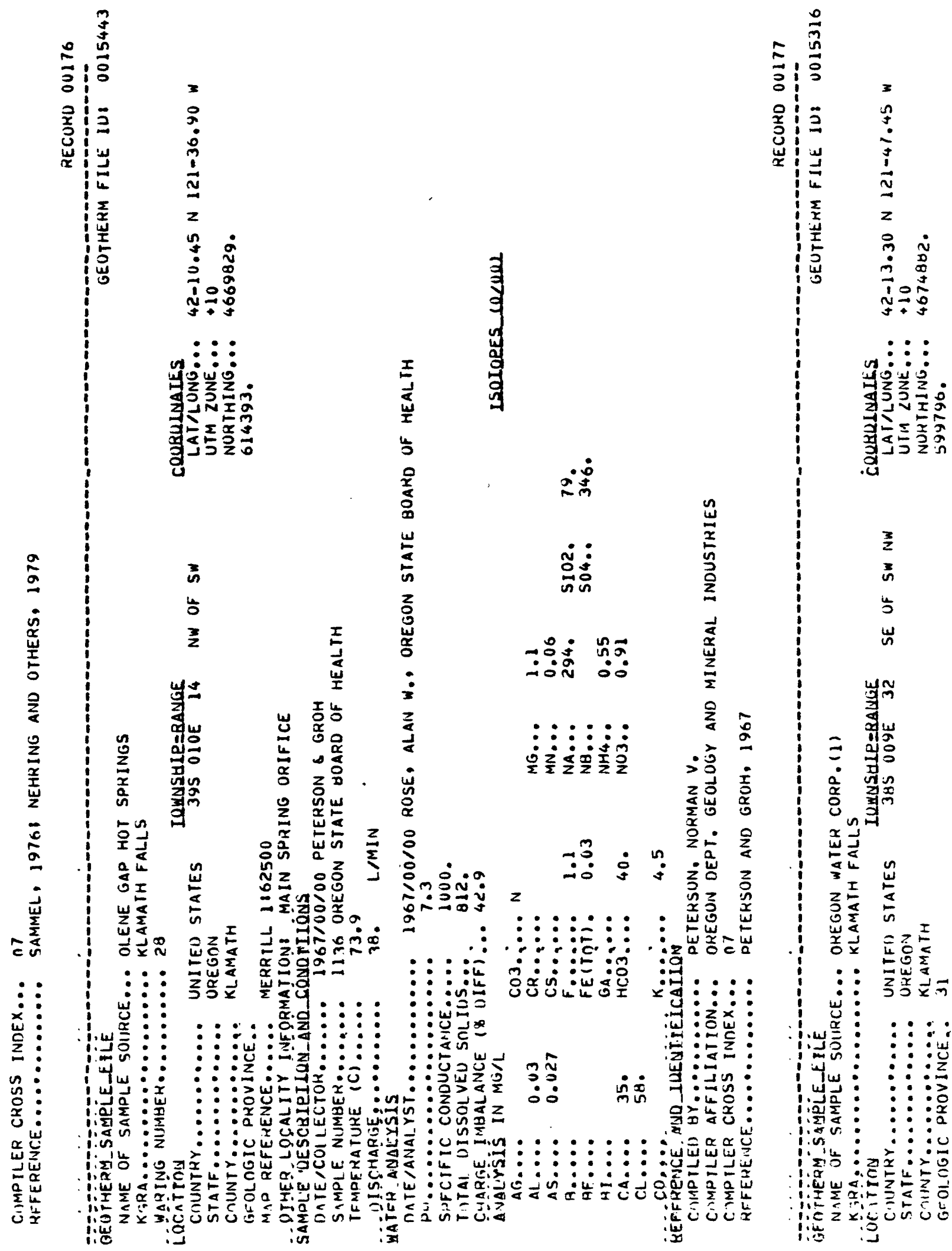

$\frac{3}{2}$
s
s
un

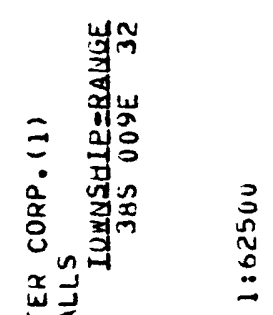

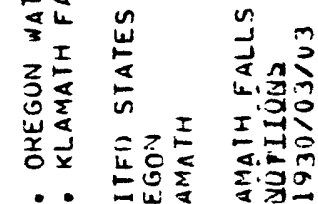

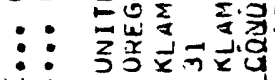

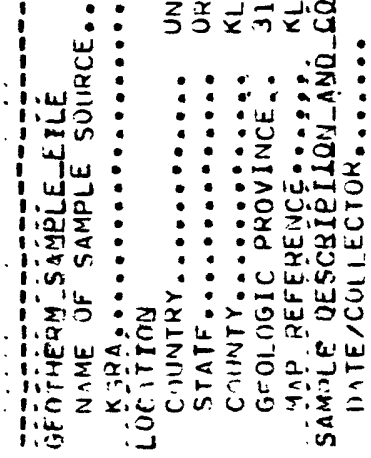


$\cong$
3
0
0
$a$
$a$

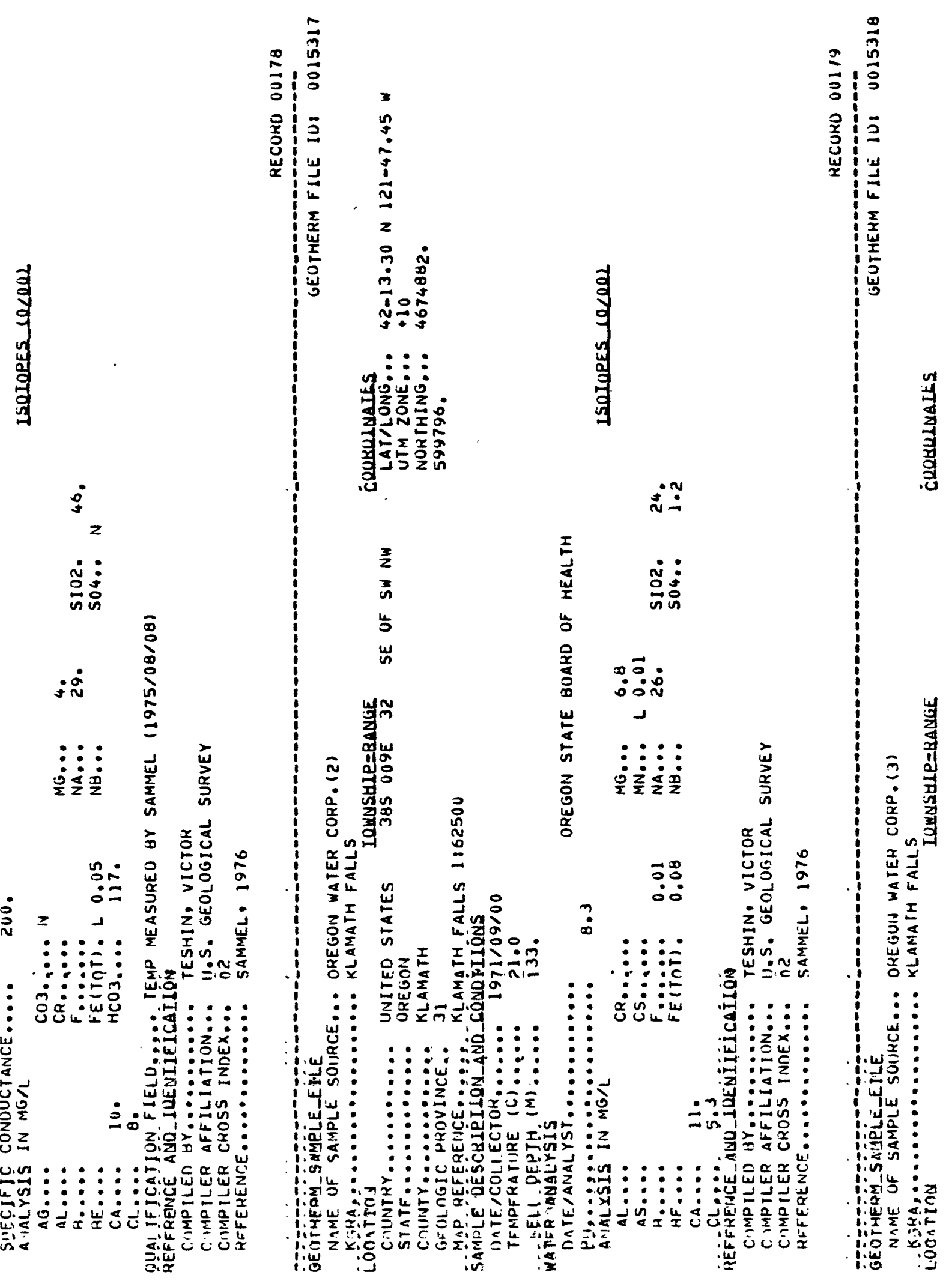




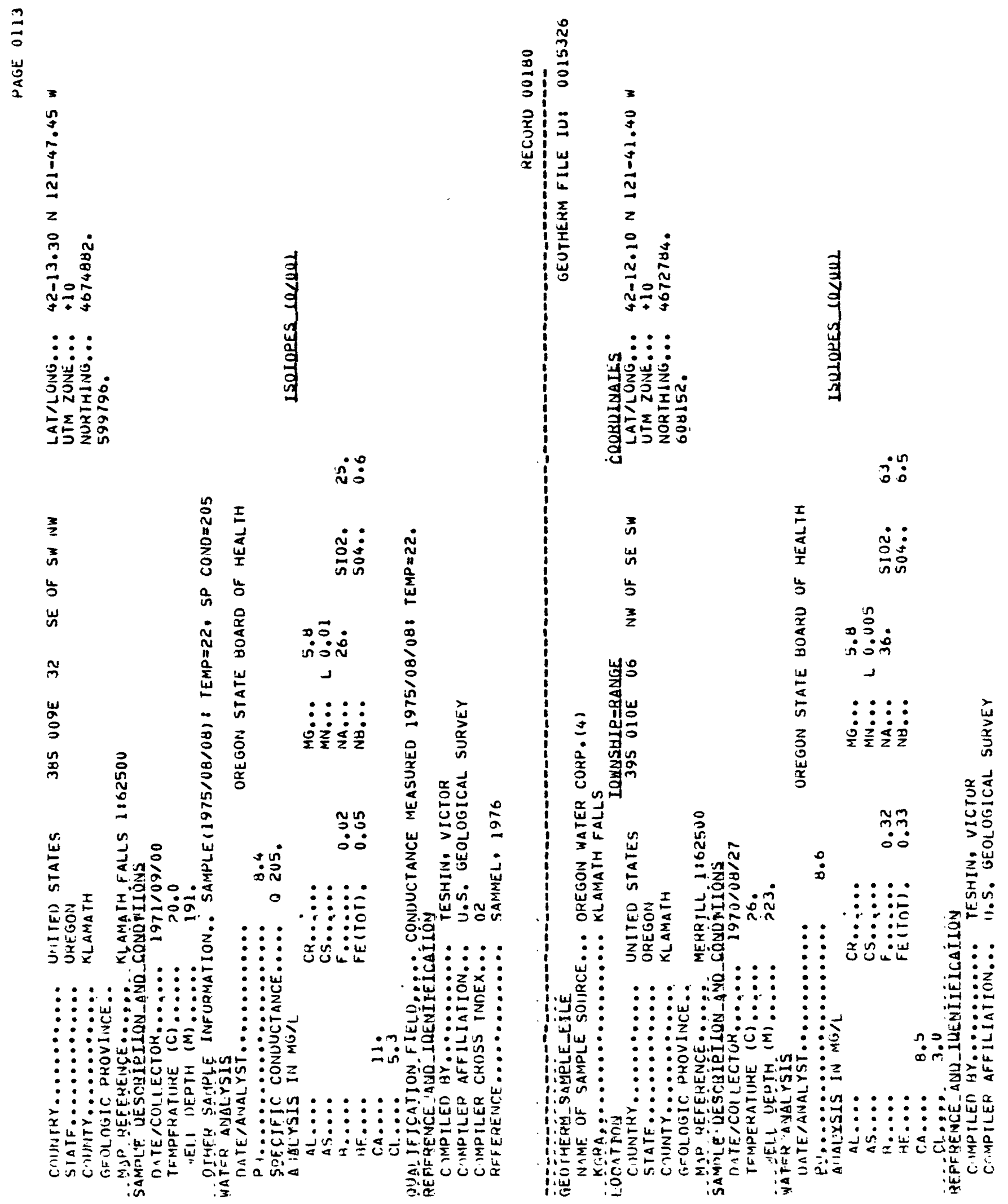



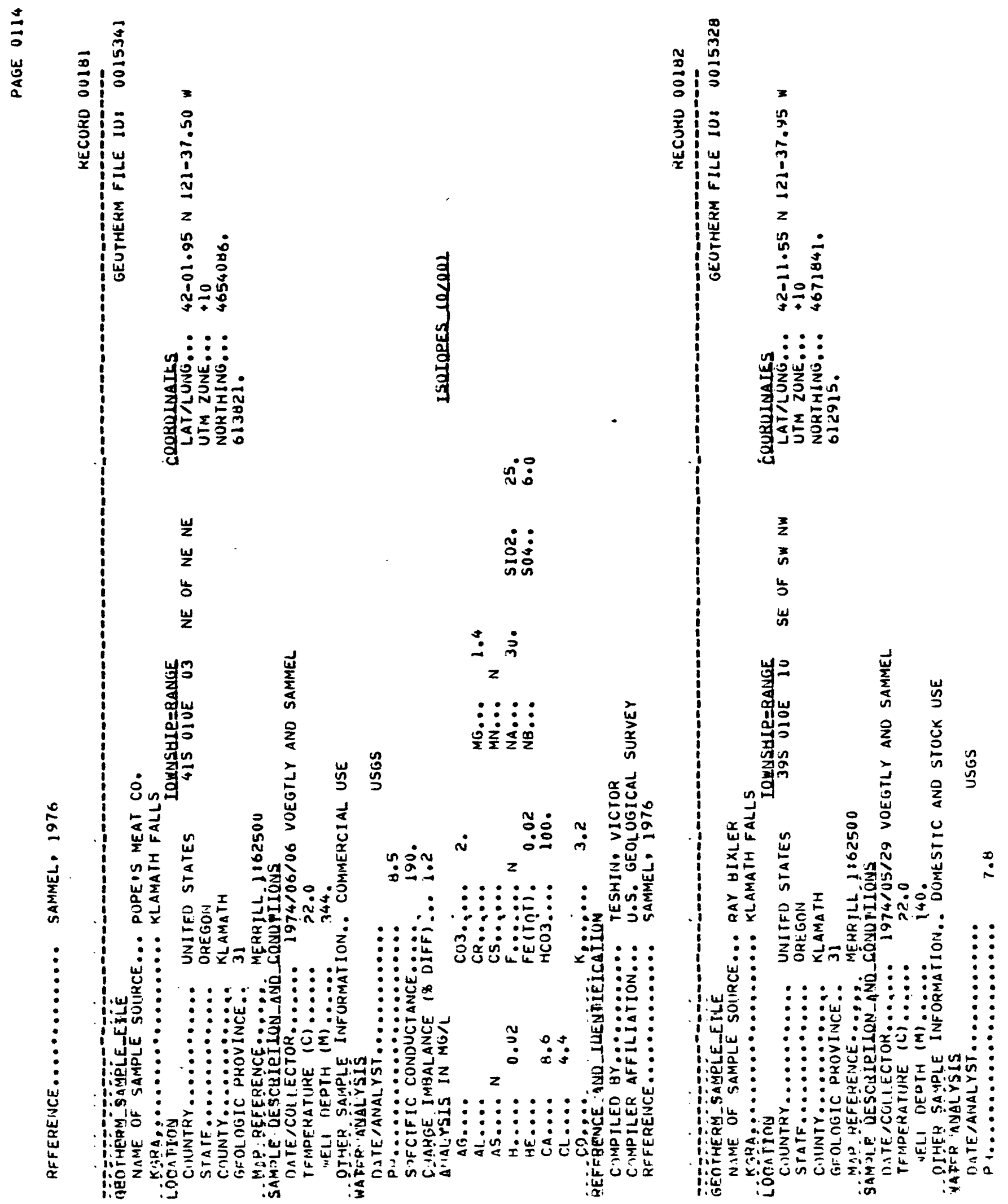


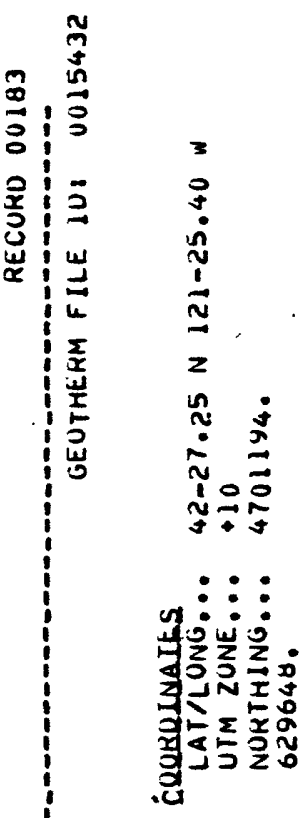




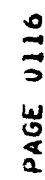

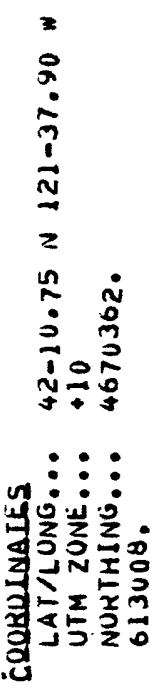

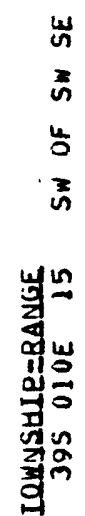

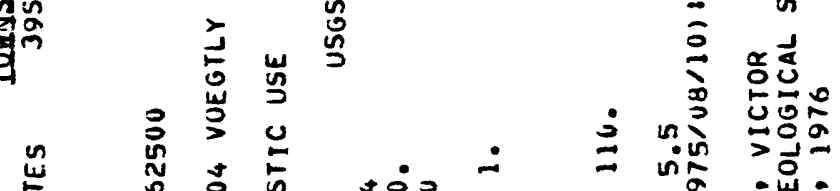

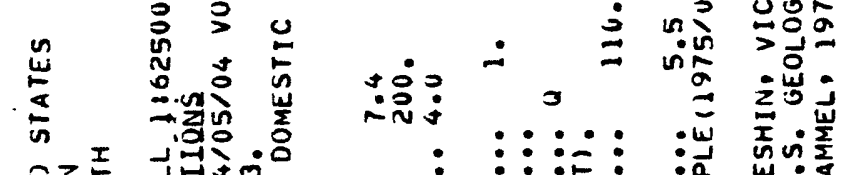

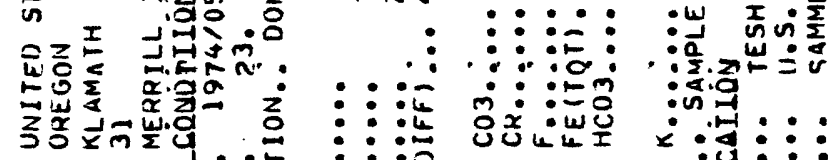
무: : :

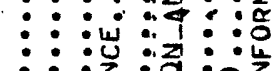

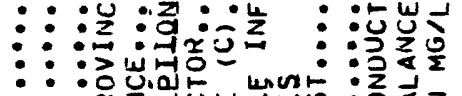
$::$ : :

줄 :

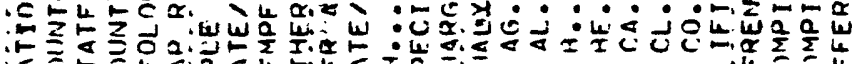

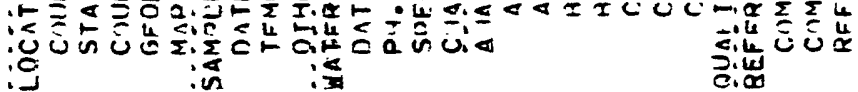

a

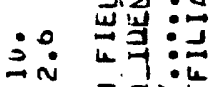

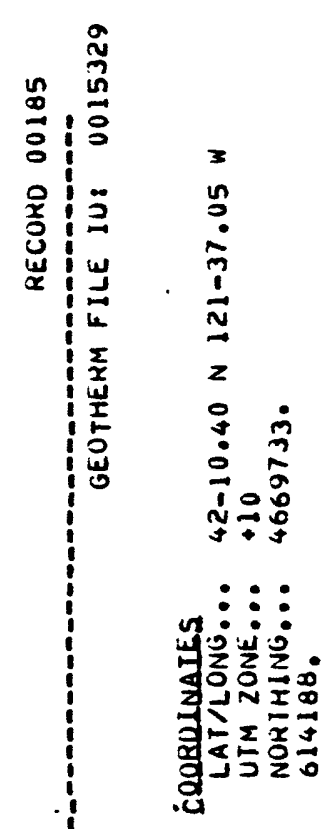

荈

$\dot{0} 0 \dot{0}$

苛寉

$\because \dot{\sim}$

$:: \vdots:$

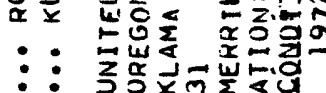

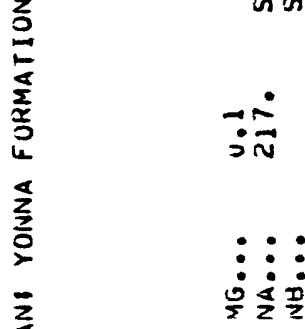

: : : :

nั่ง $\because \dot{0}$

บ这: $z$

o: : : : :

$\therefore \quad:::$

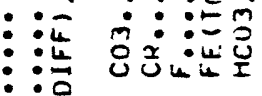

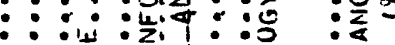

: : $:$ : $:: 0$ :

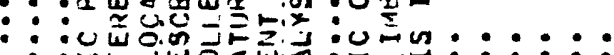

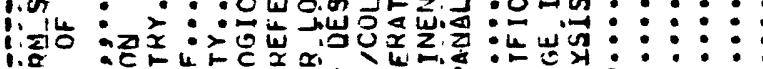

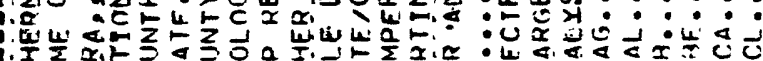

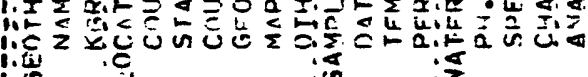


3
3
0
0
$a$
$a$

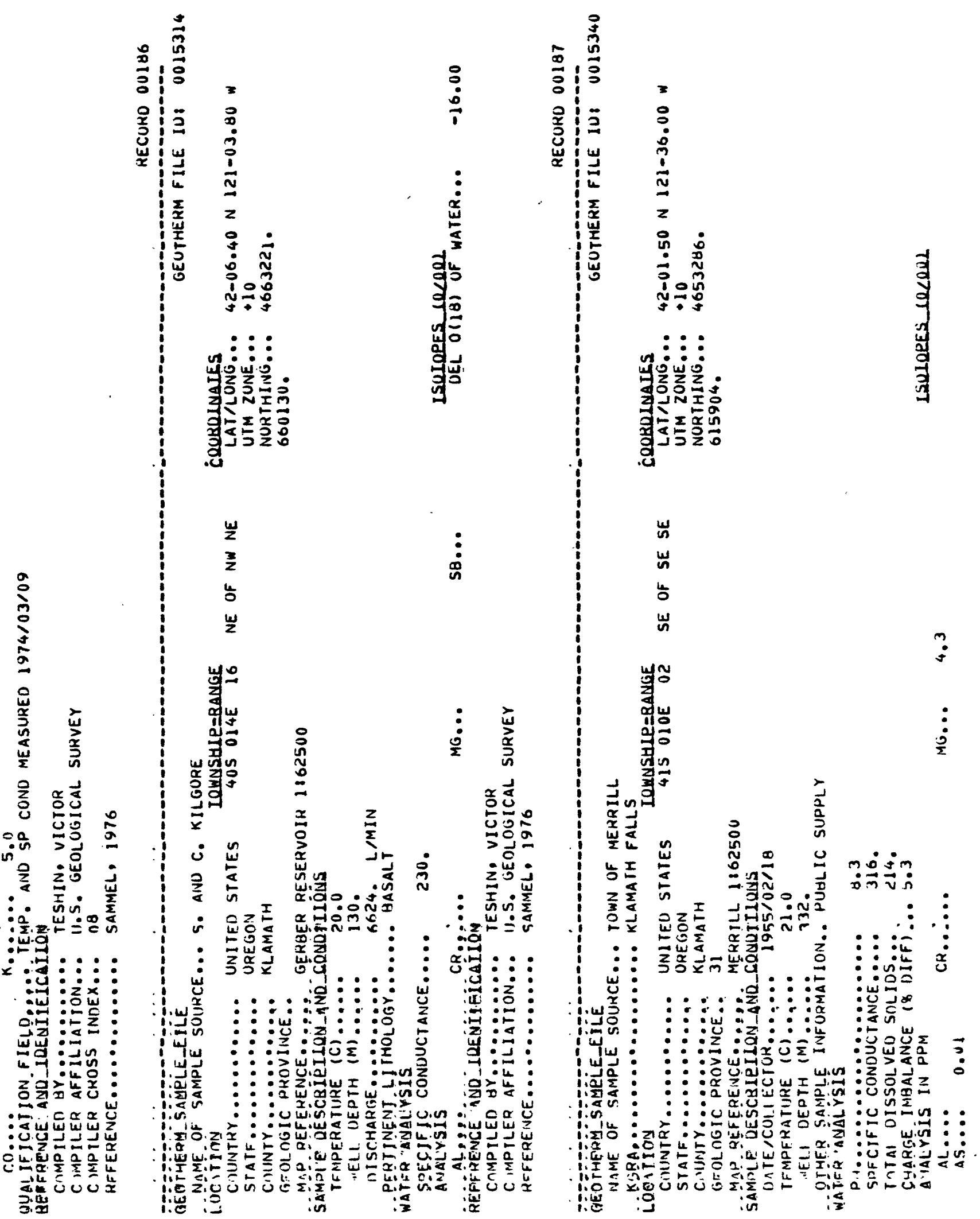


高

$\ddot{m} \vec{n}$

苛宫

ง

$::: \quad$ 岁品

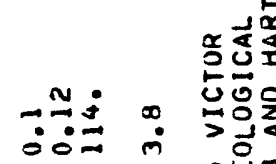

...

:

证皆

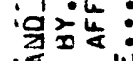

$::::$ :

:: : : :

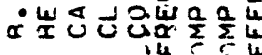

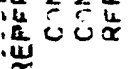
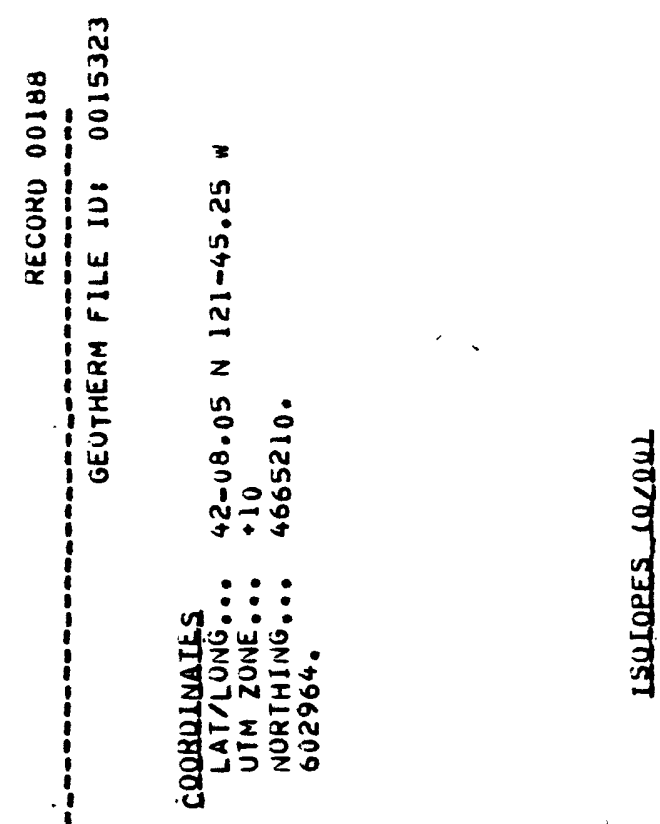

콕

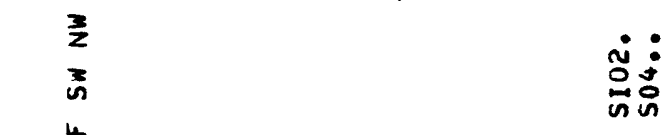

与

is

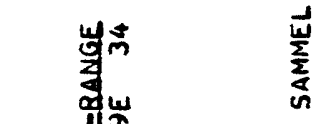

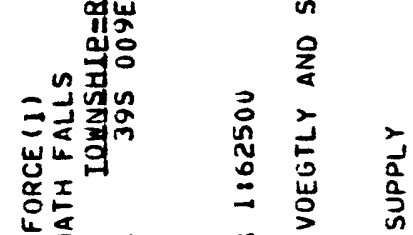

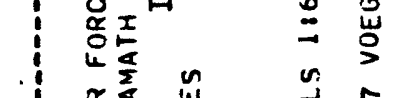

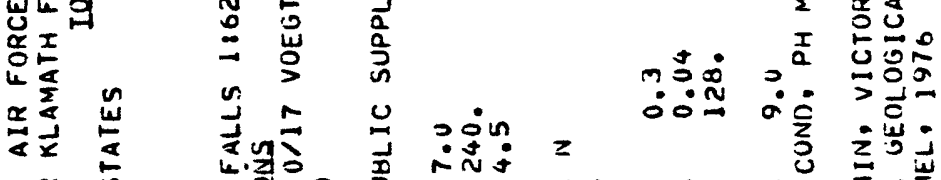

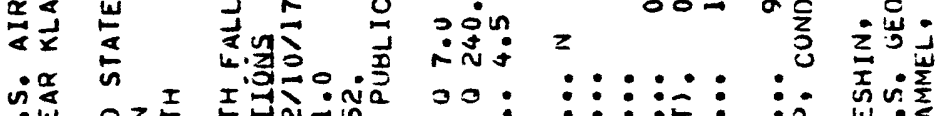

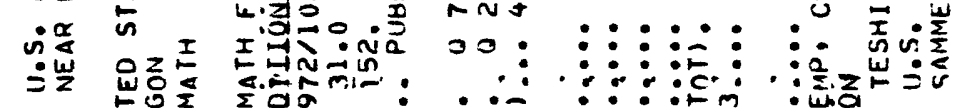

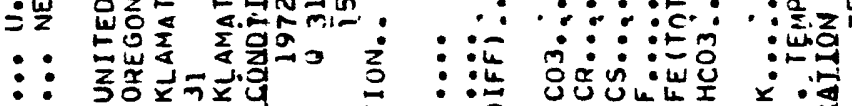

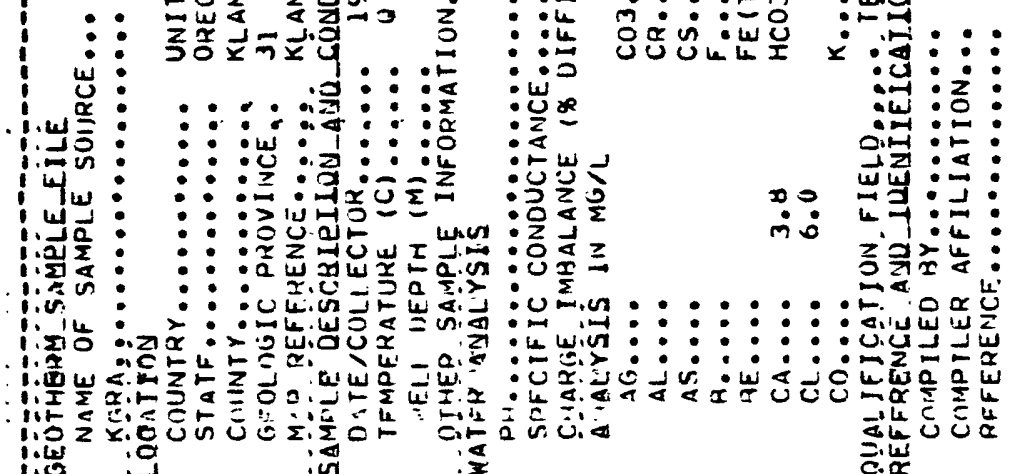

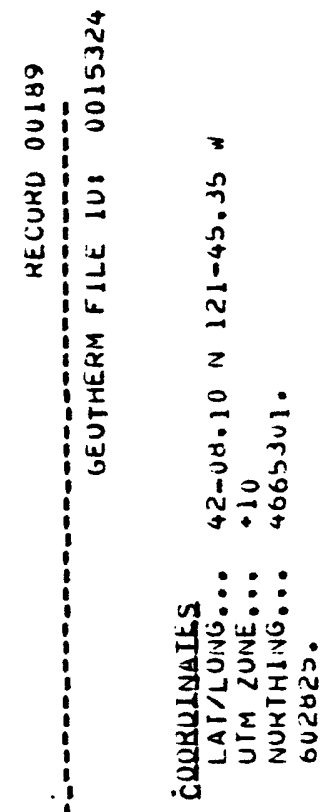

$$
\begin{aligned}
& 3 \\
& 3 \\
& \text { in } \\
& 5 \\
& \text { s }
\end{aligned}
$$

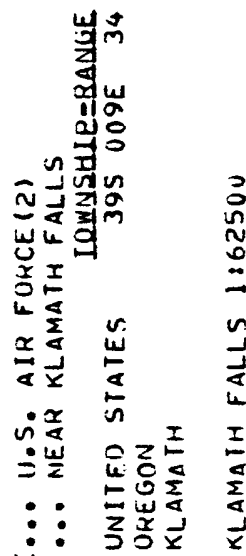

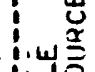

:

: بل:

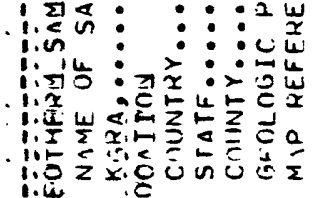


$\stackrel{a}{\frac{2}{2}}$

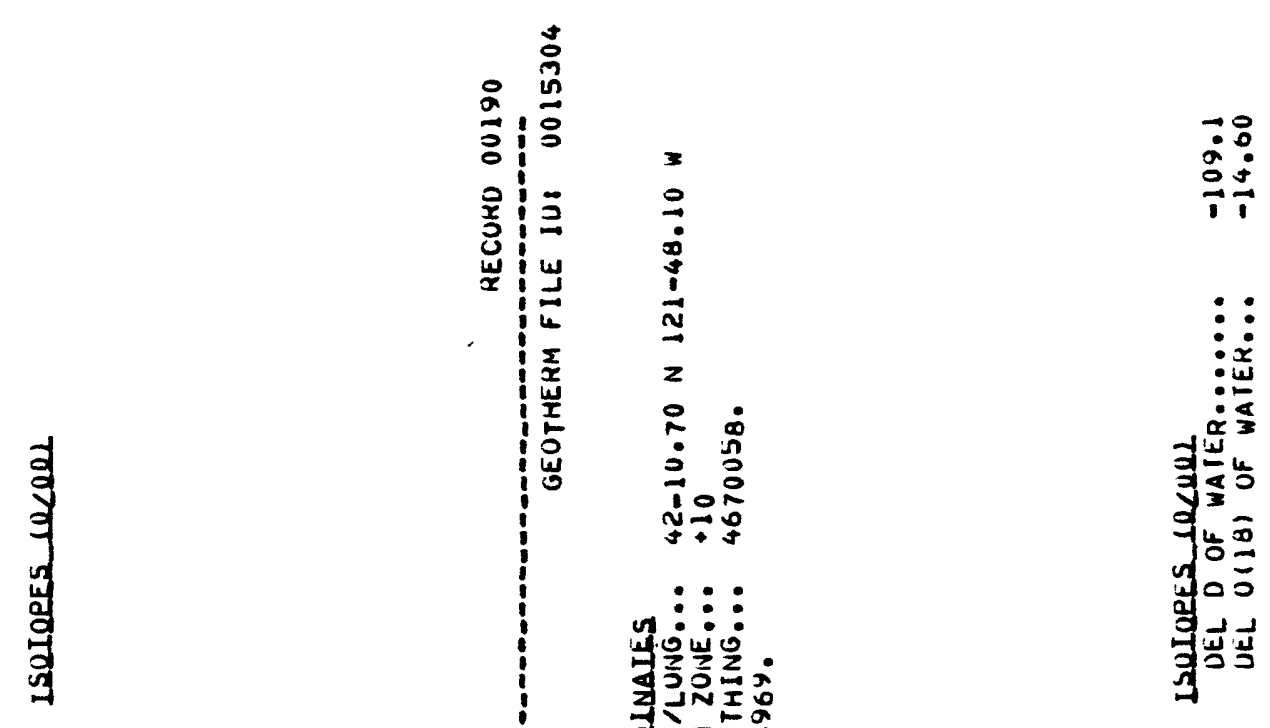

$\frac{\vec{w}}{\sum_{2}^{2}}$

जี่:

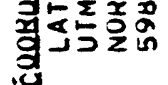

:

$\stackrel{0}{0}$

交

तั0.

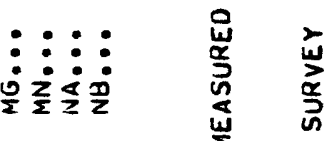

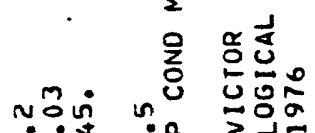

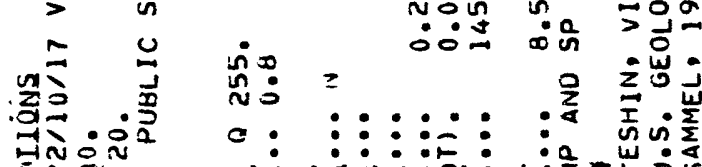

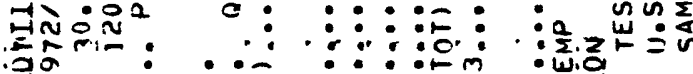

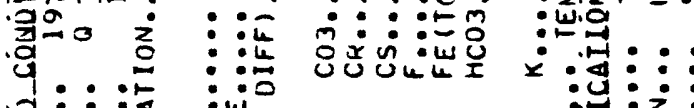

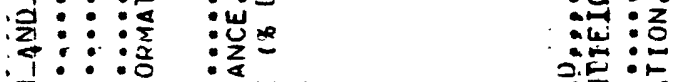

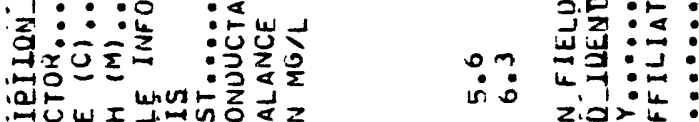

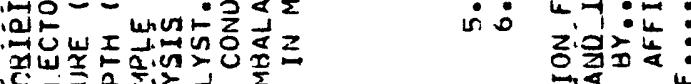

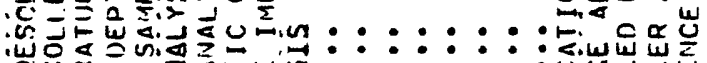

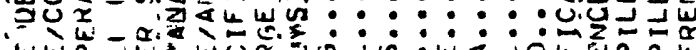

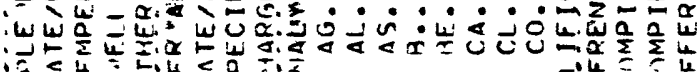

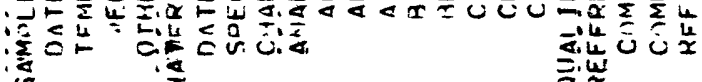

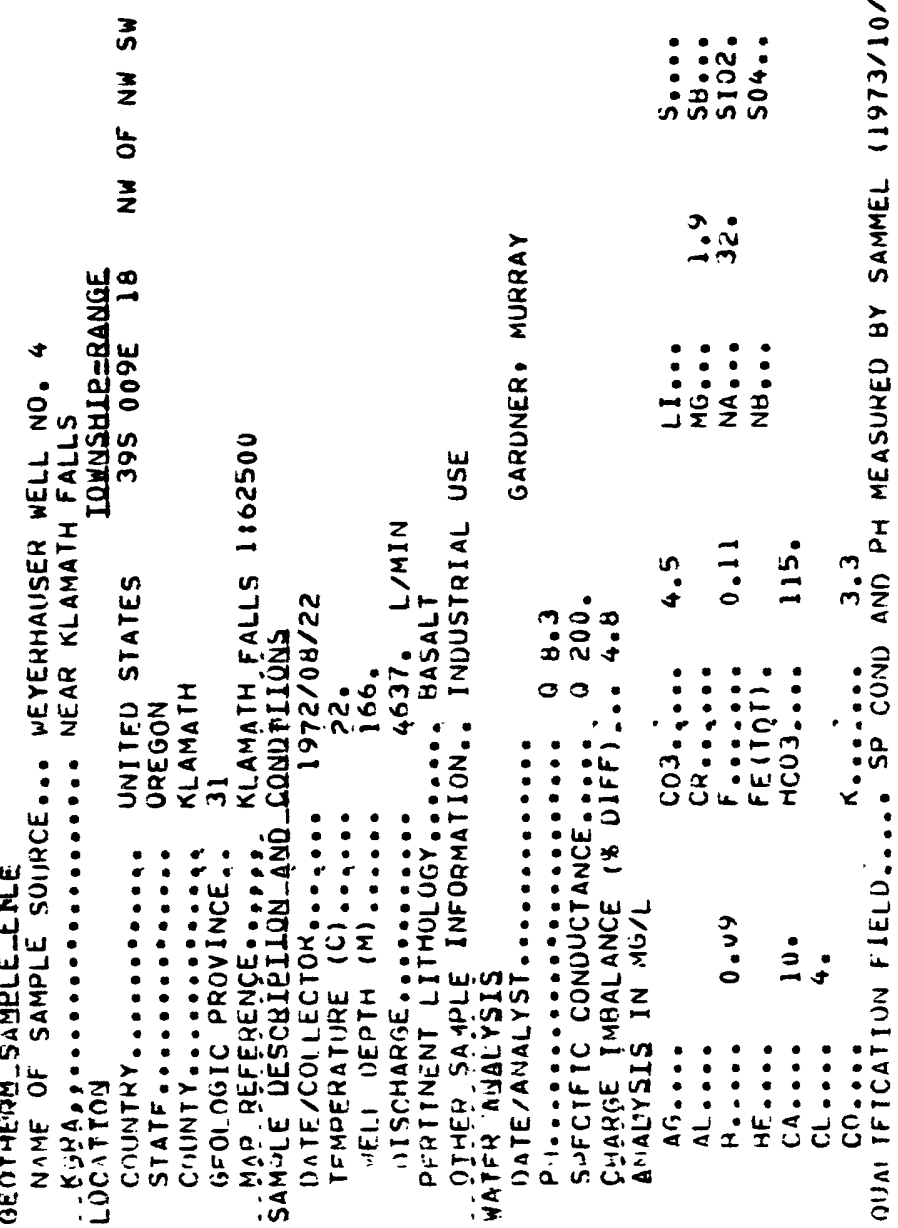




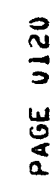

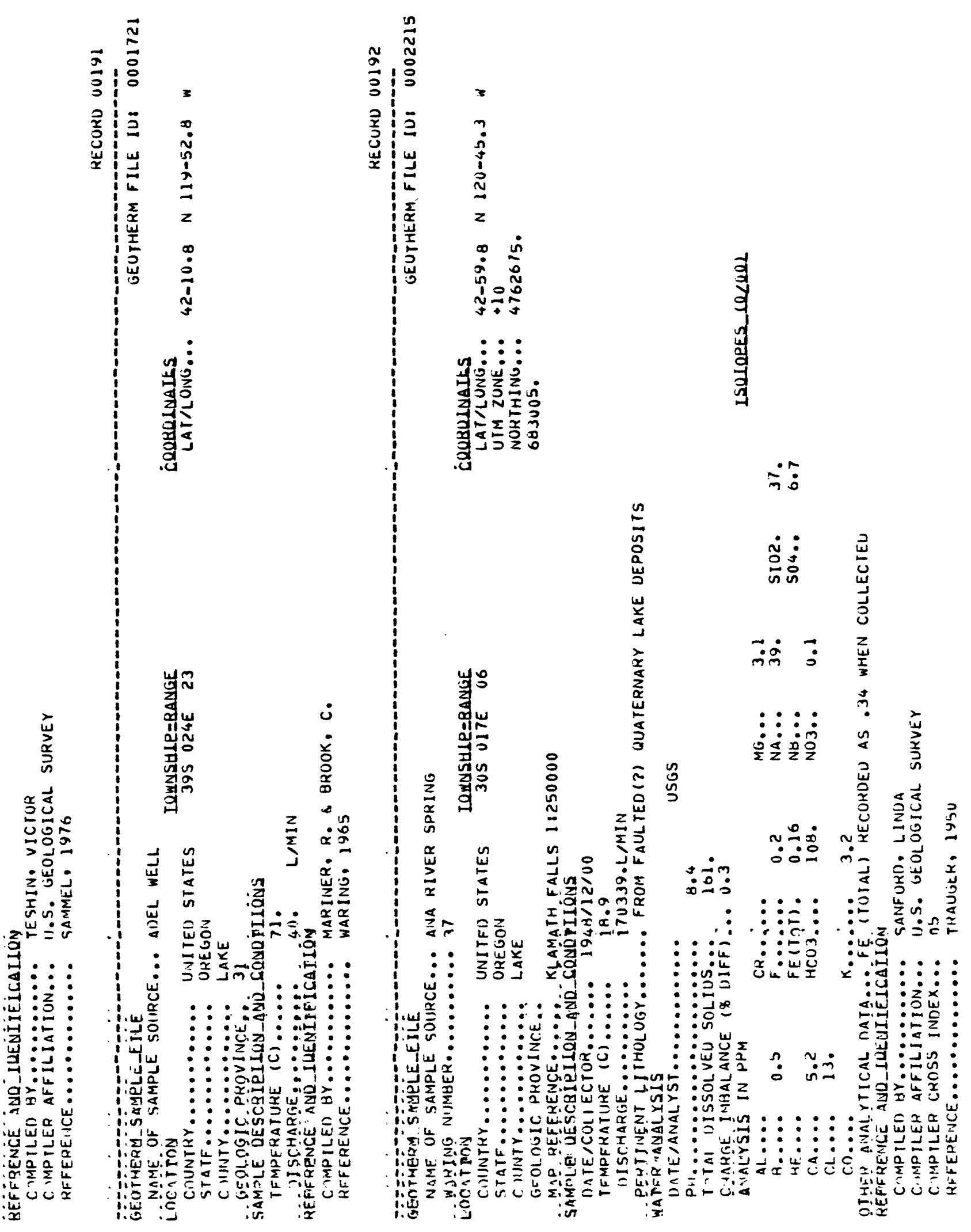



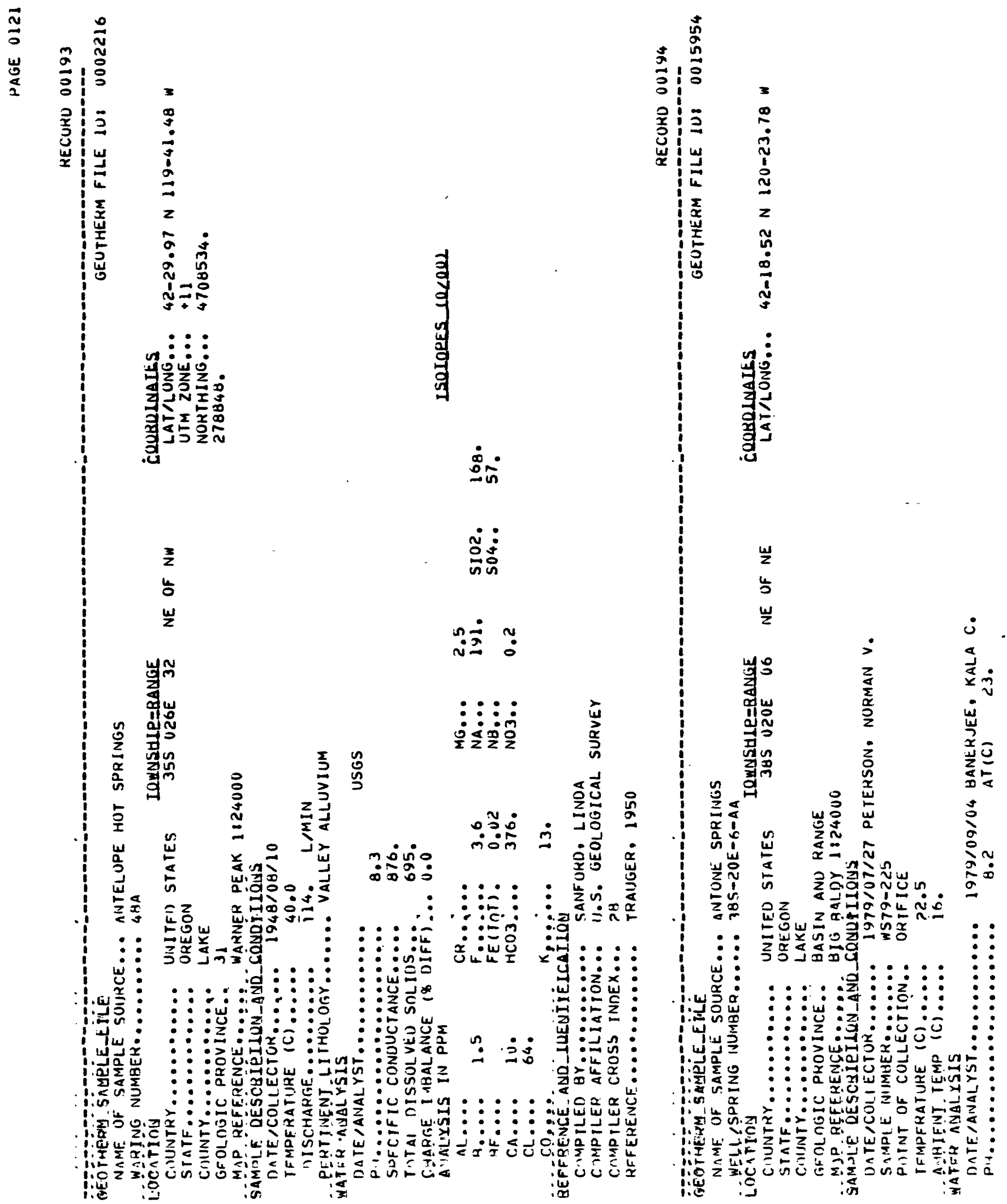


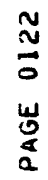

寻

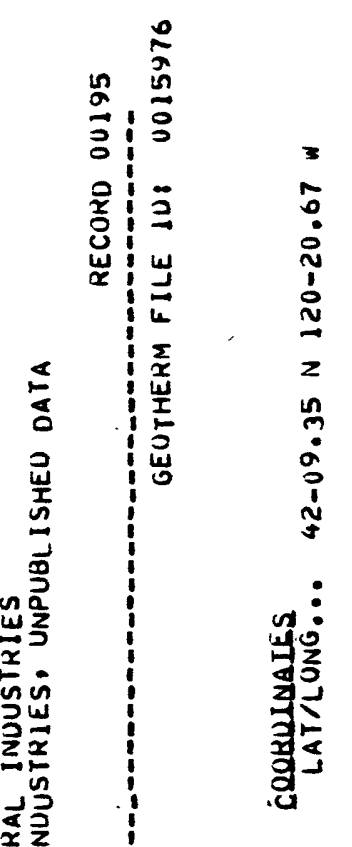

节

$\dot{i} \dot{u}$

实宫

ü
岁
$\frac{3}{2}$

is:

คิกน

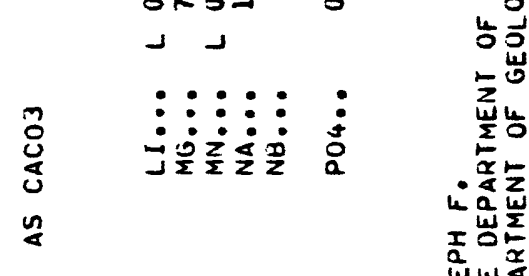

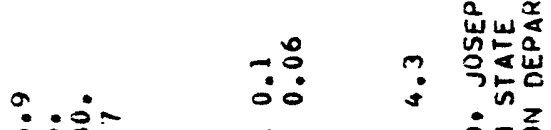

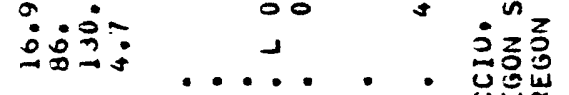

$\therefore: \vdots: \vdots: \vdots \vdots \vdots \vdots$ :

: : : :

岁:

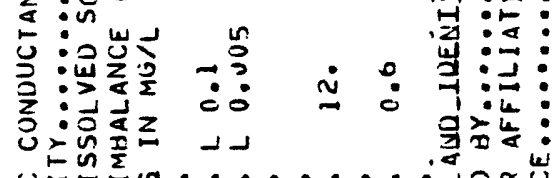

즌대 : : : : : : : : :

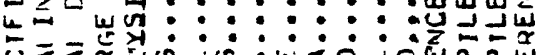

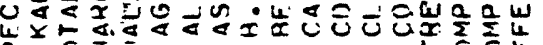

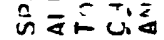

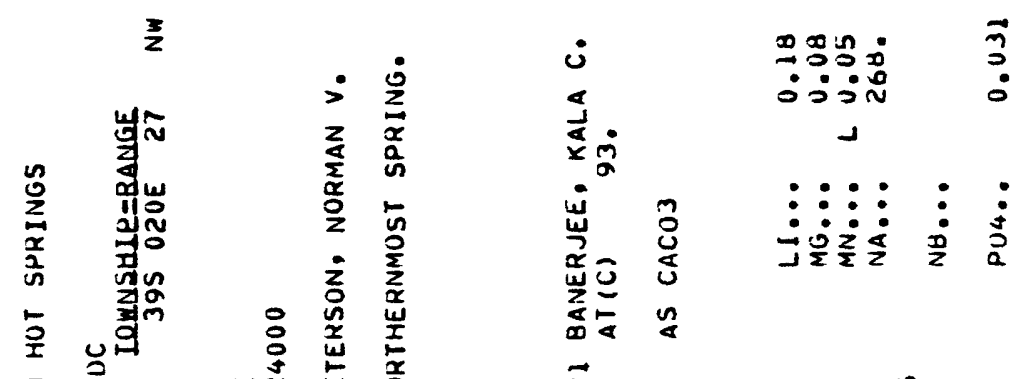

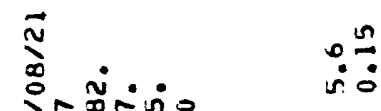

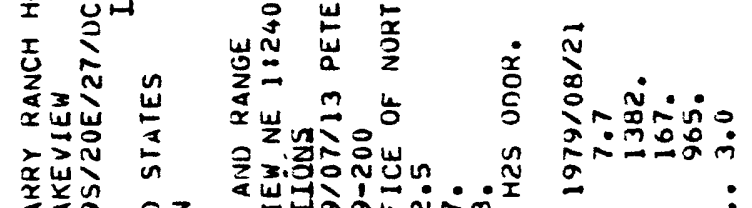

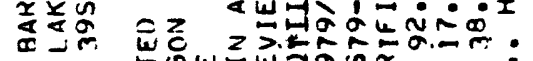

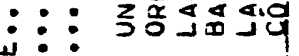

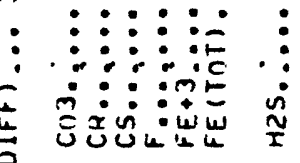

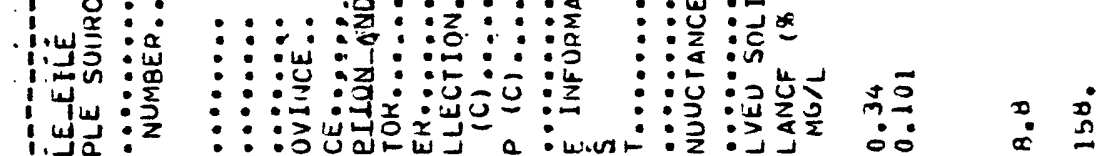

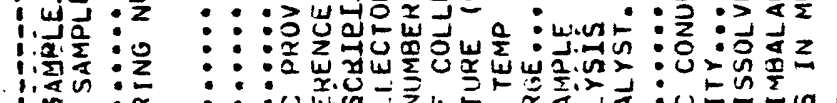

liv : : : : : :

in : :

l.

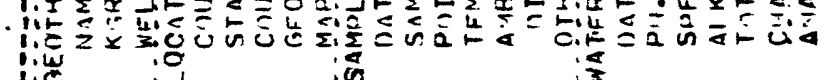




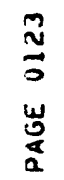

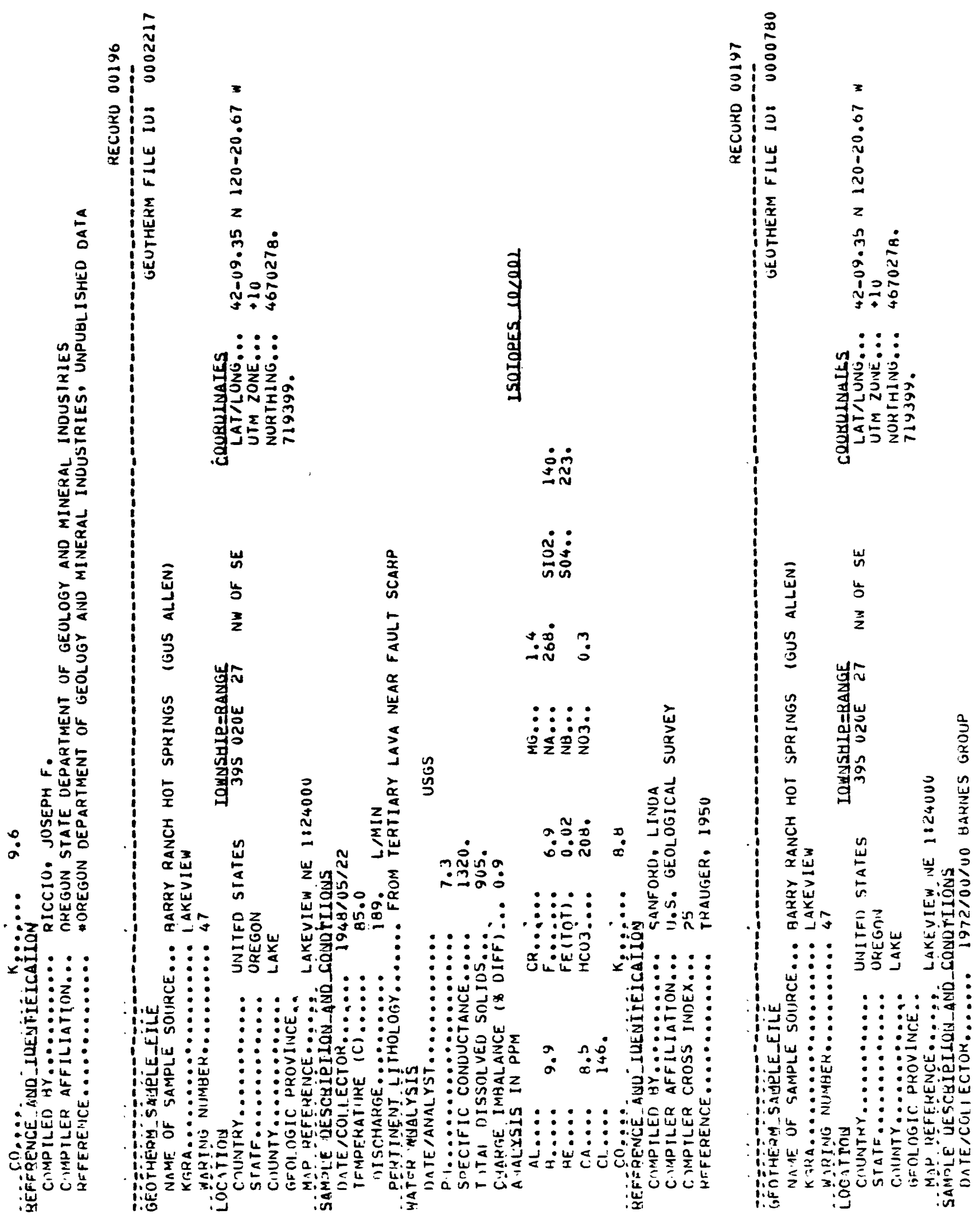



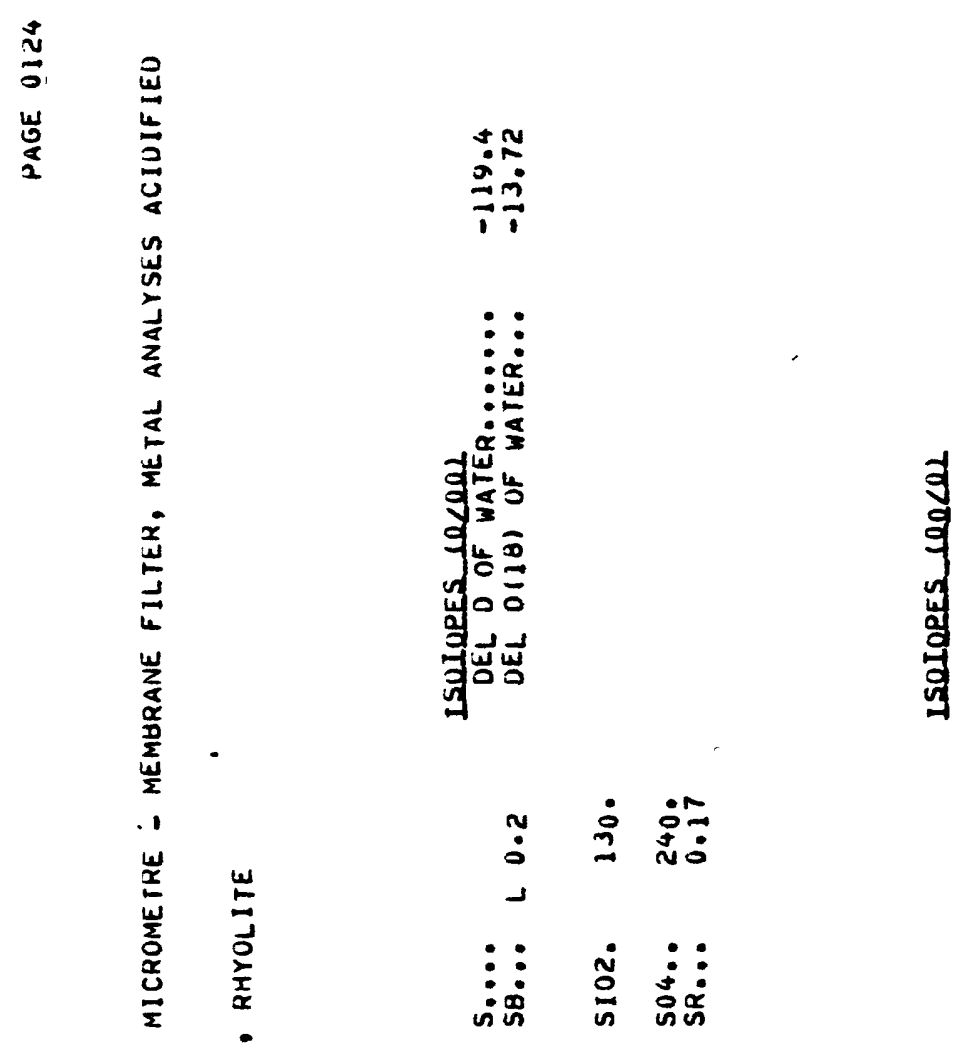

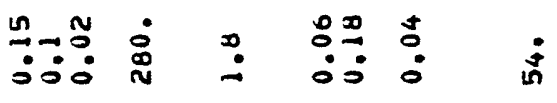

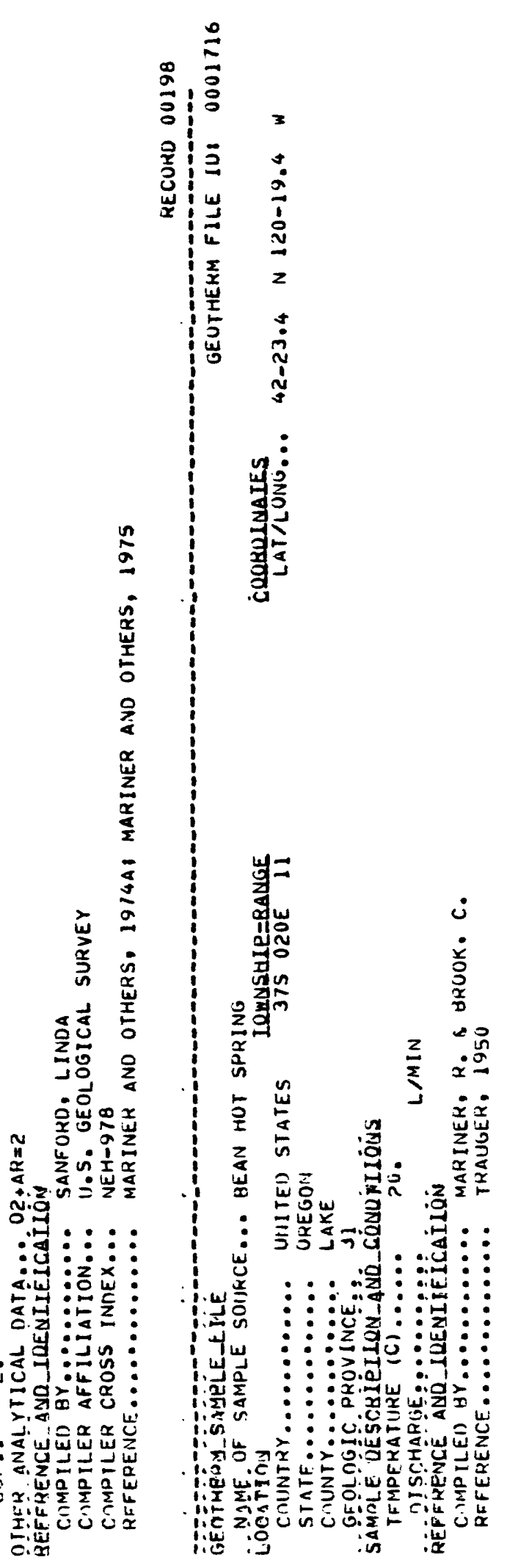

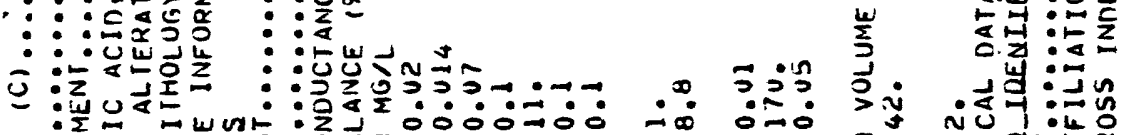

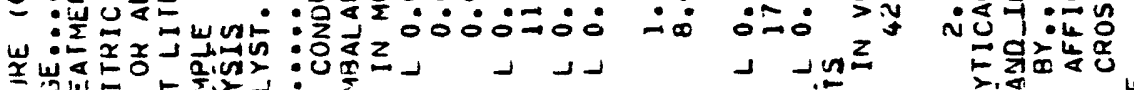

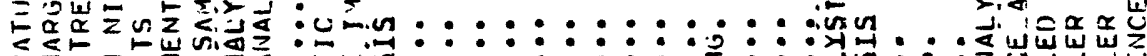

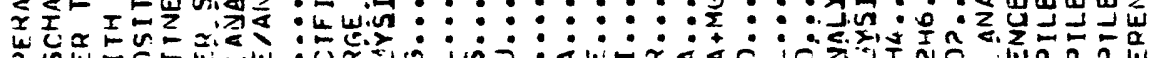
ํㅗㄴ

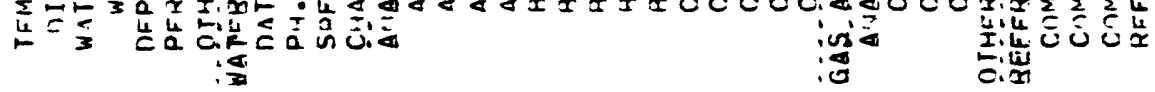

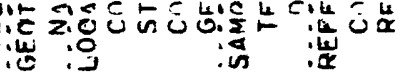



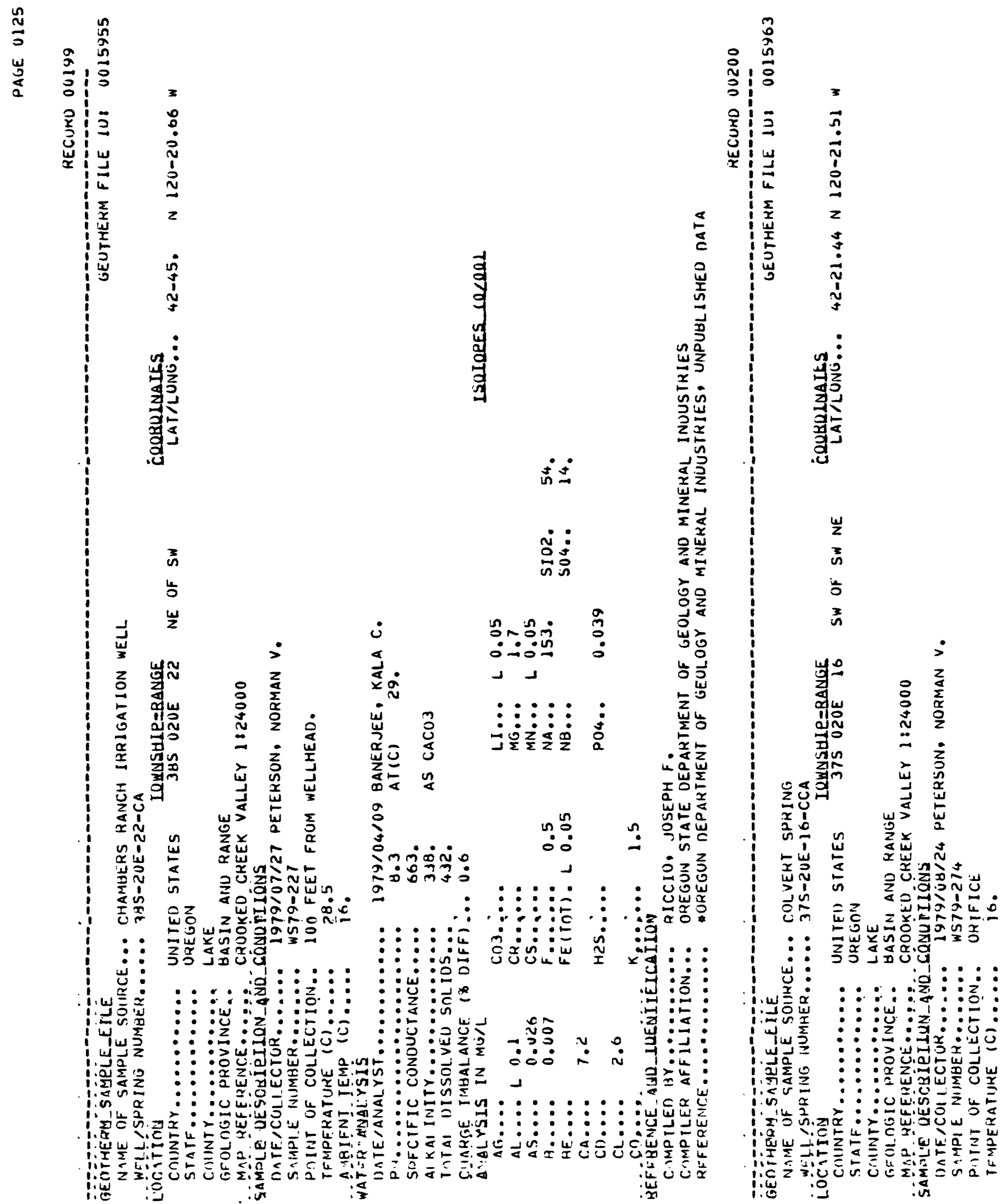
บ
0
$u$
0
0
2

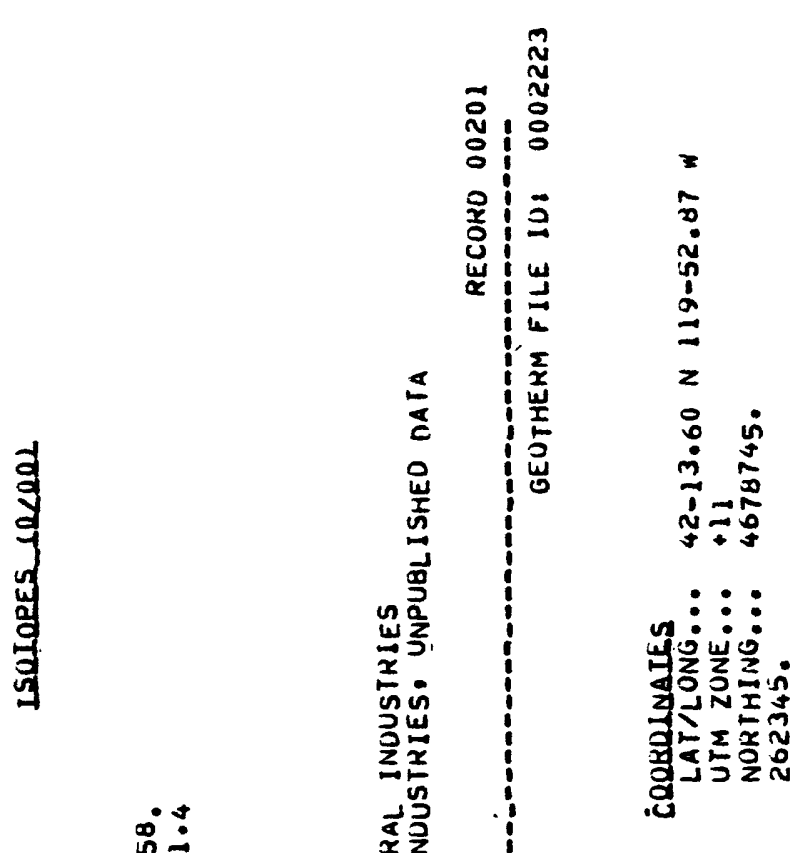

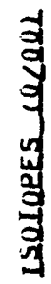
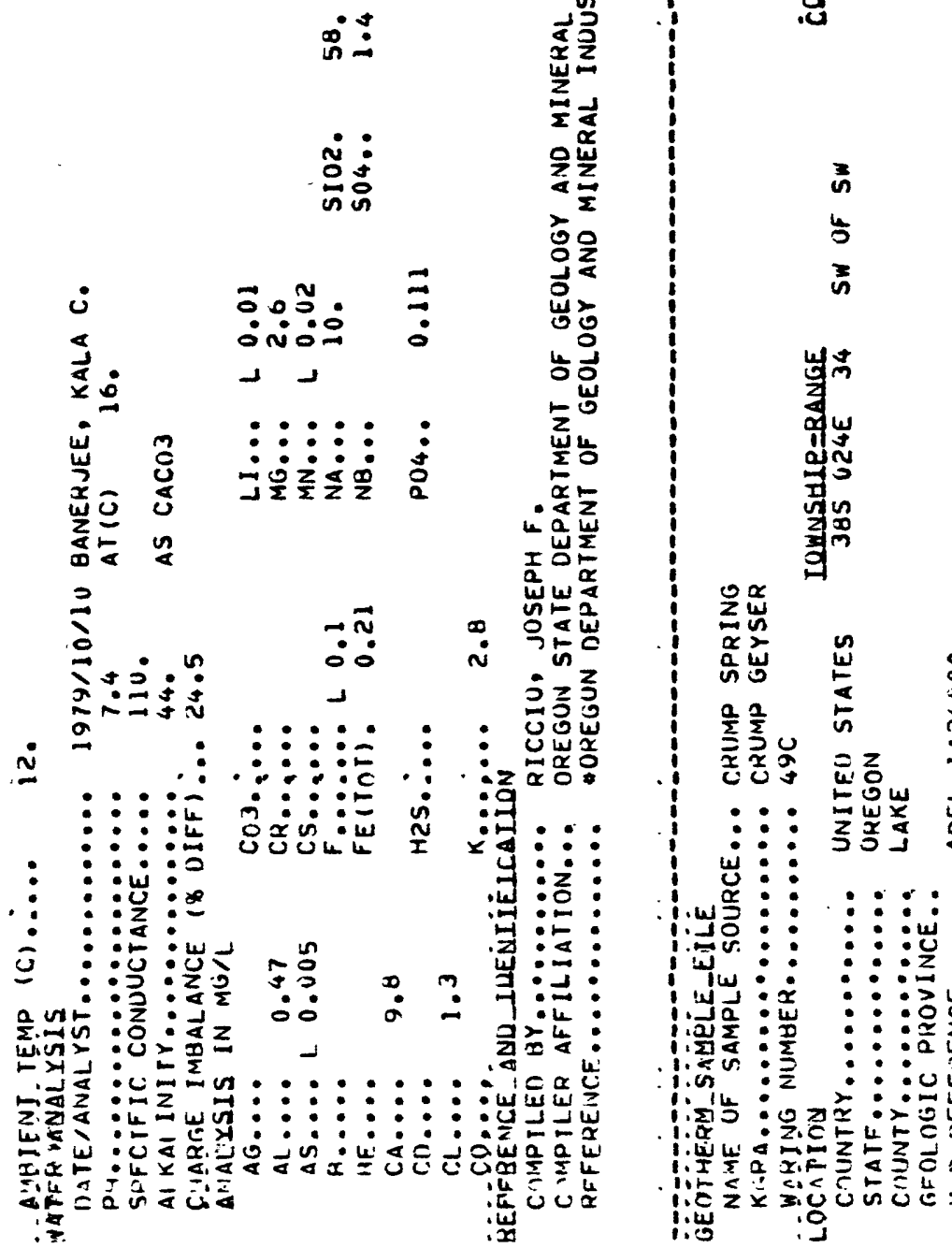

กั:

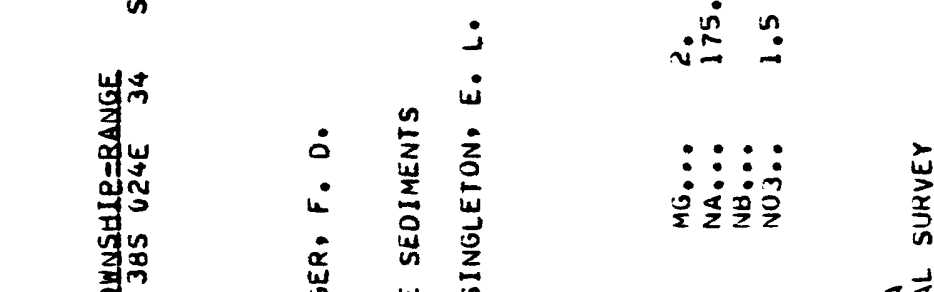

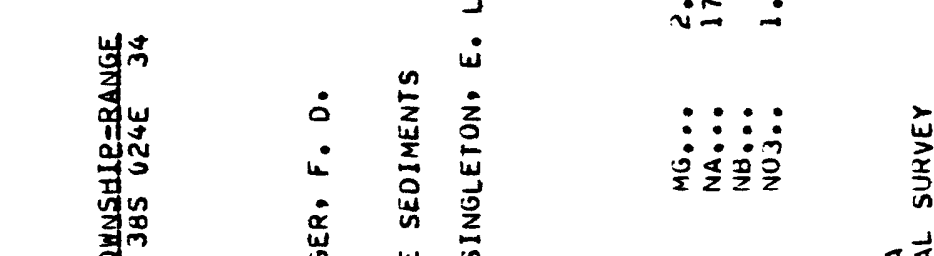

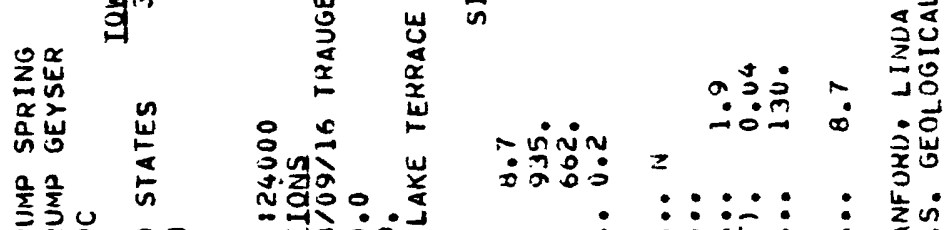

ज

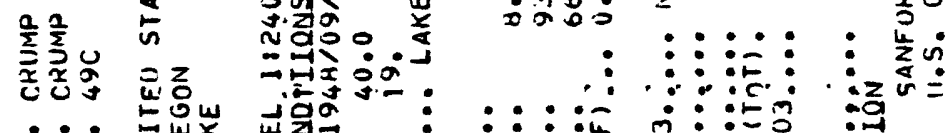

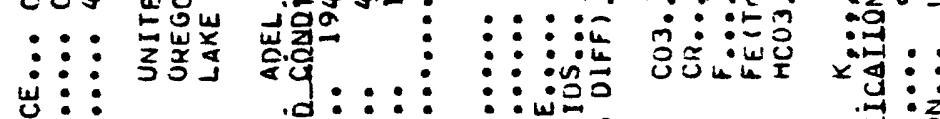

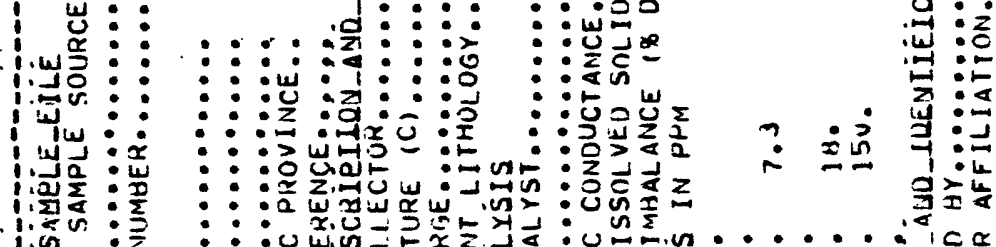

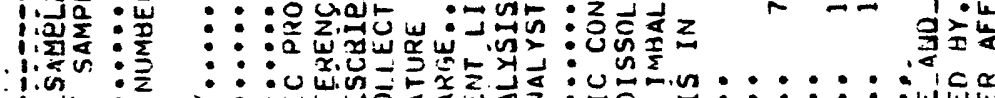

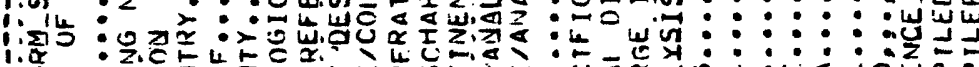

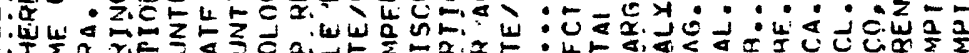

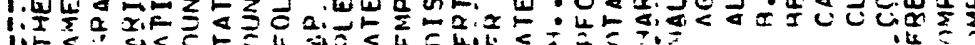

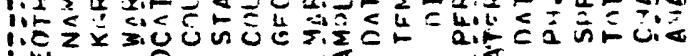




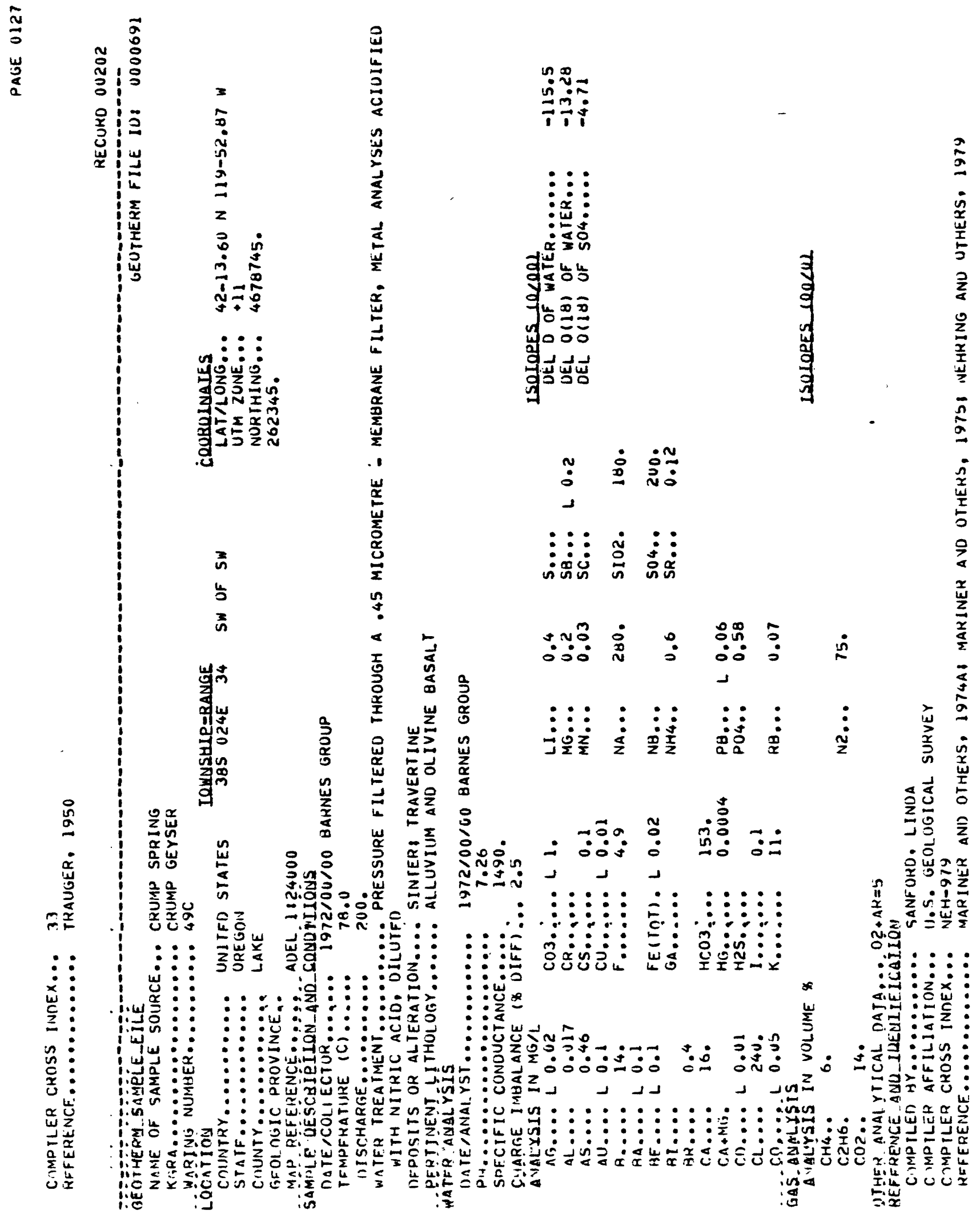




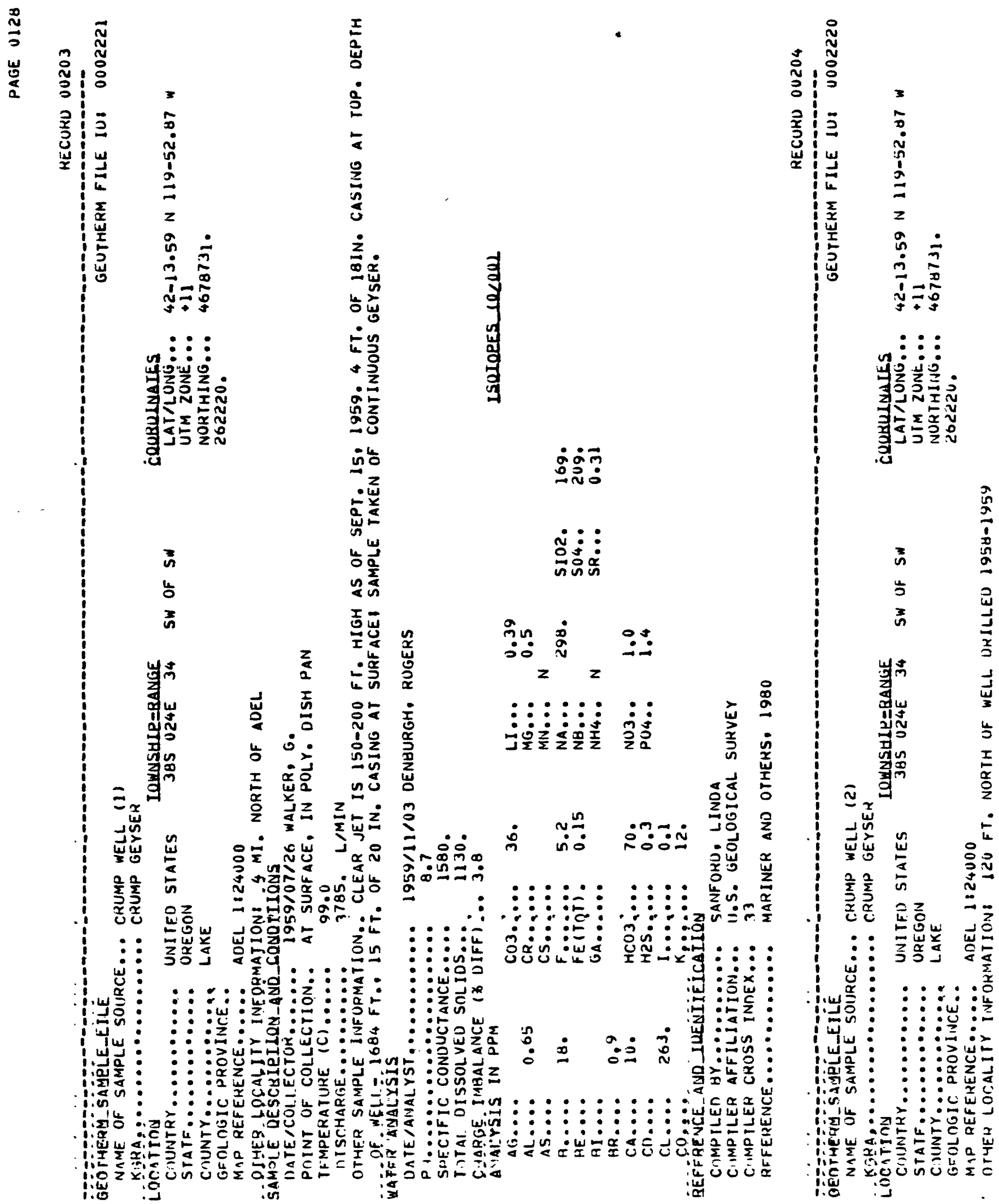


$a$
0
0
$u$
$a$
$a$
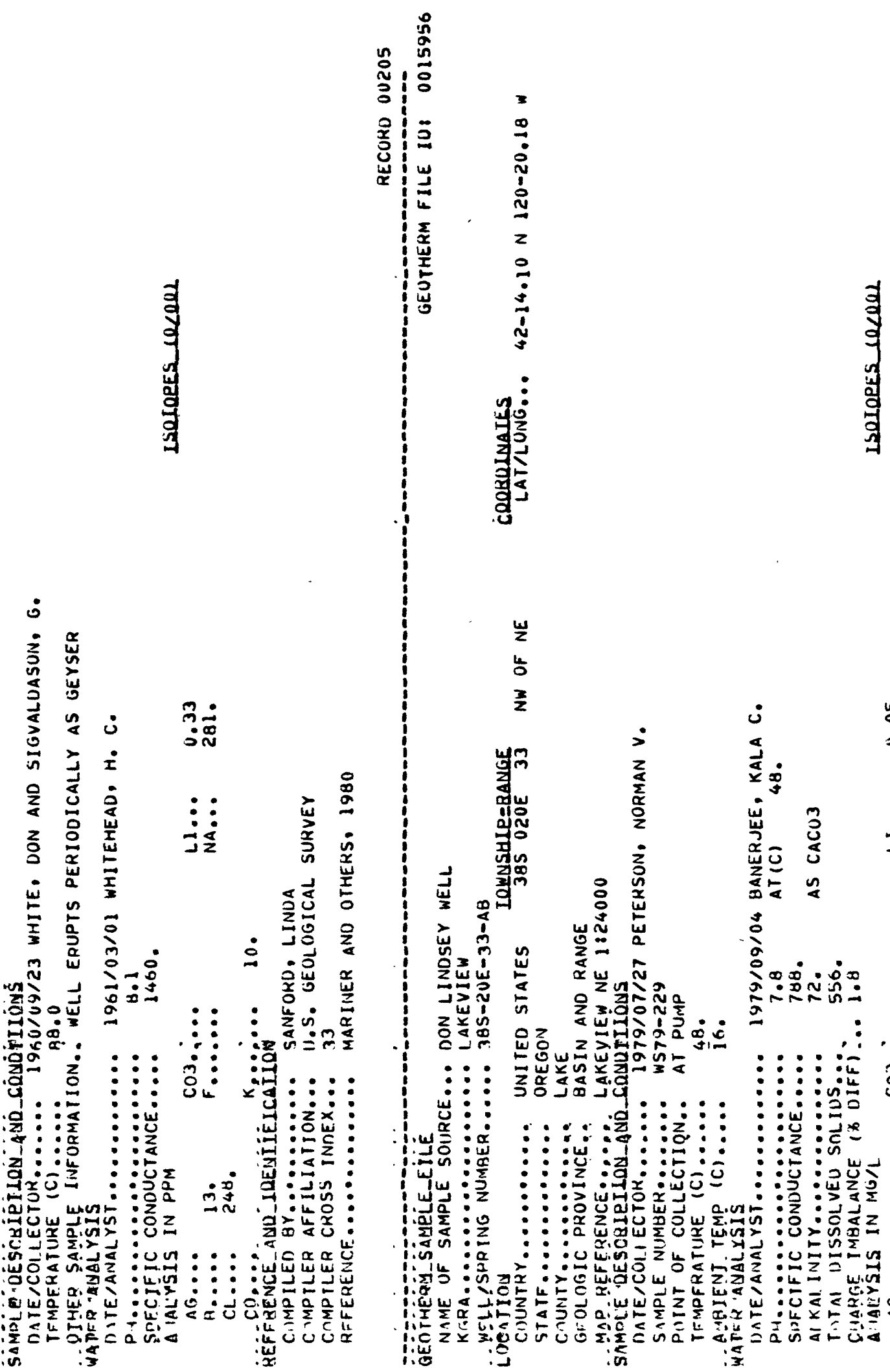

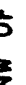

$\dot{0}: \frac{i}{\sim}$

节:

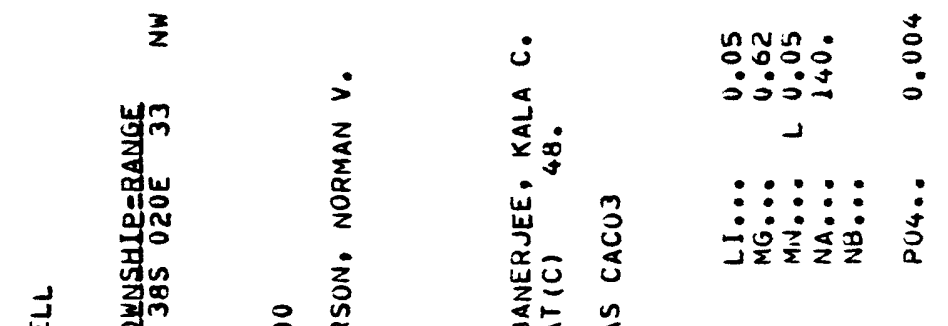

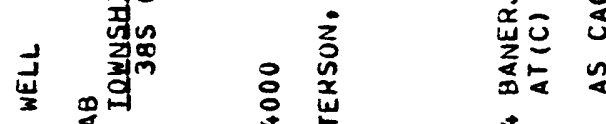

No

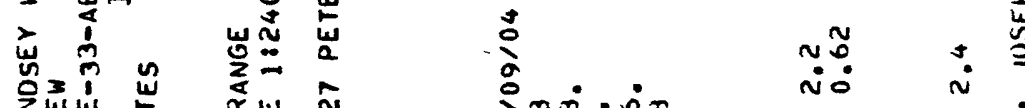

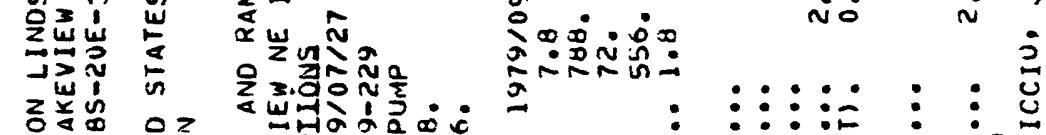

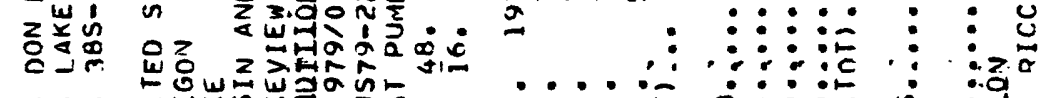

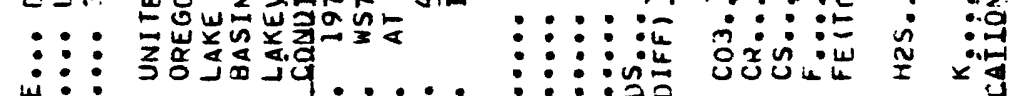

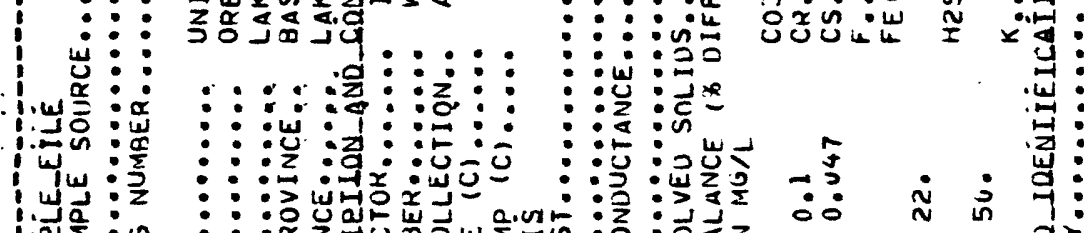

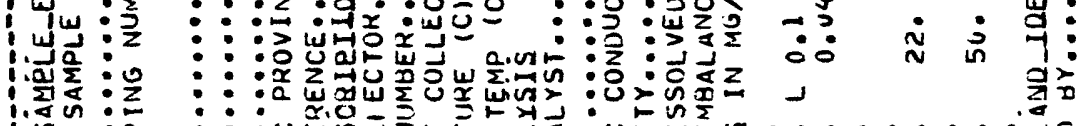
泾:

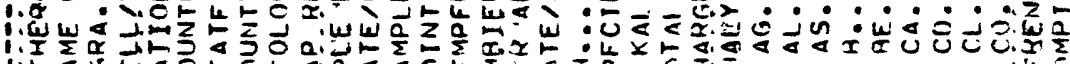

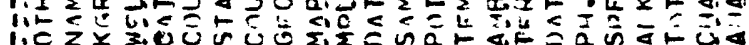
li. 

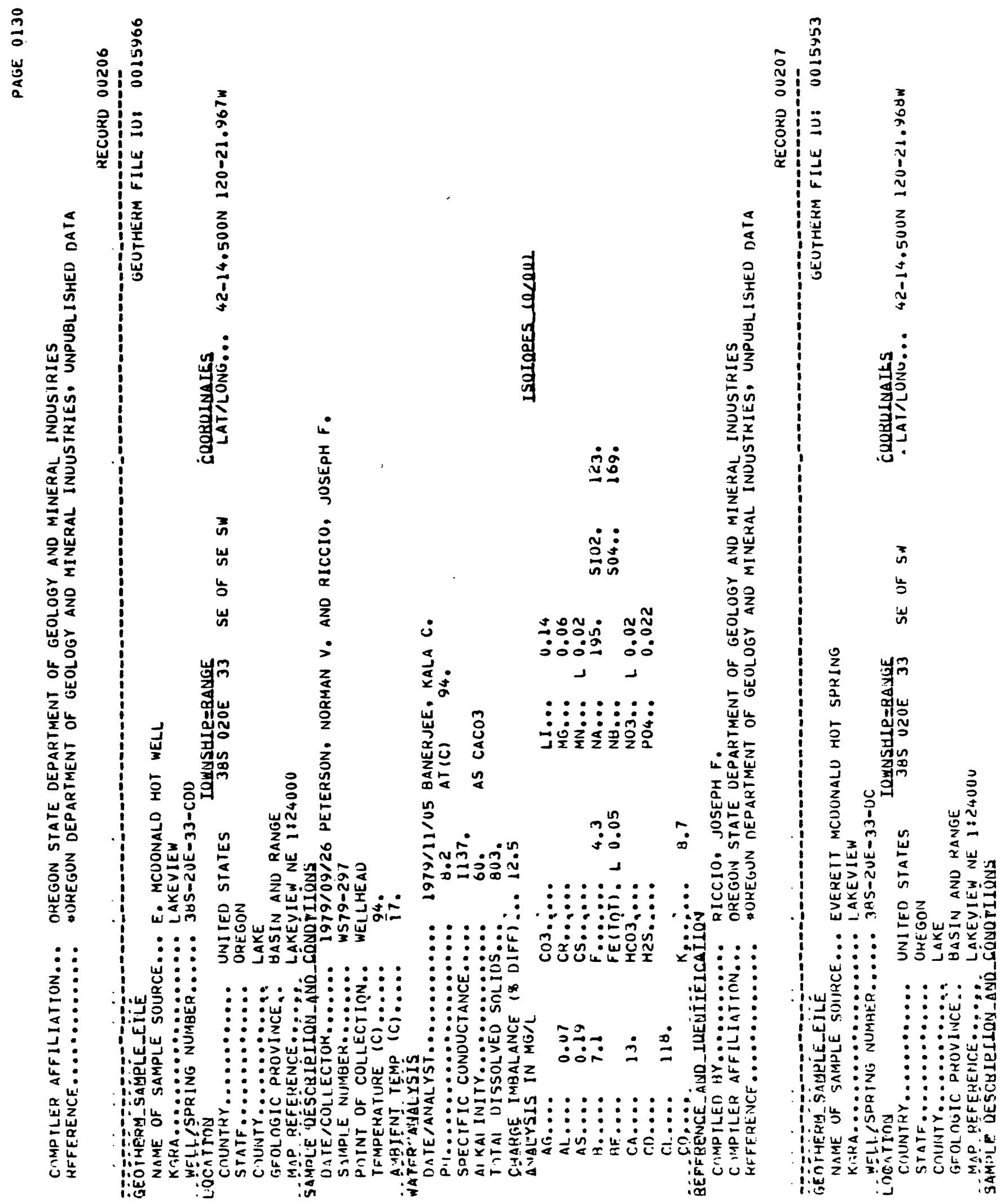


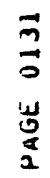
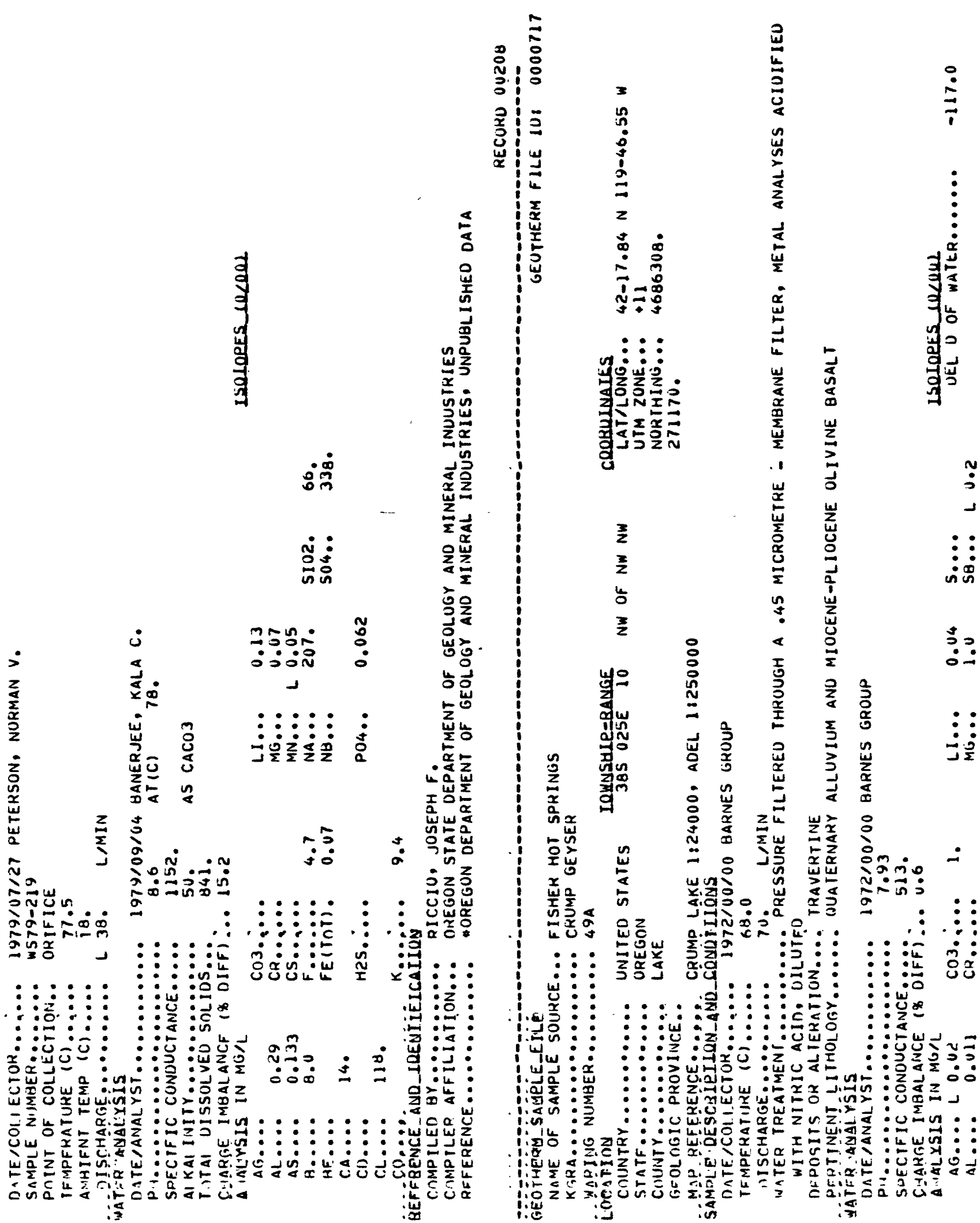


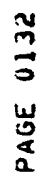

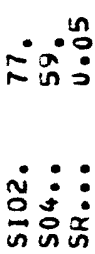

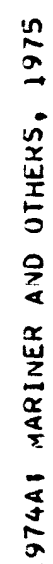

$\stackrel{\sim}{\stackrel{\sim}{\sim}} \stackrel{0}{\stackrel{0}{0}} \stackrel{0}{0}$
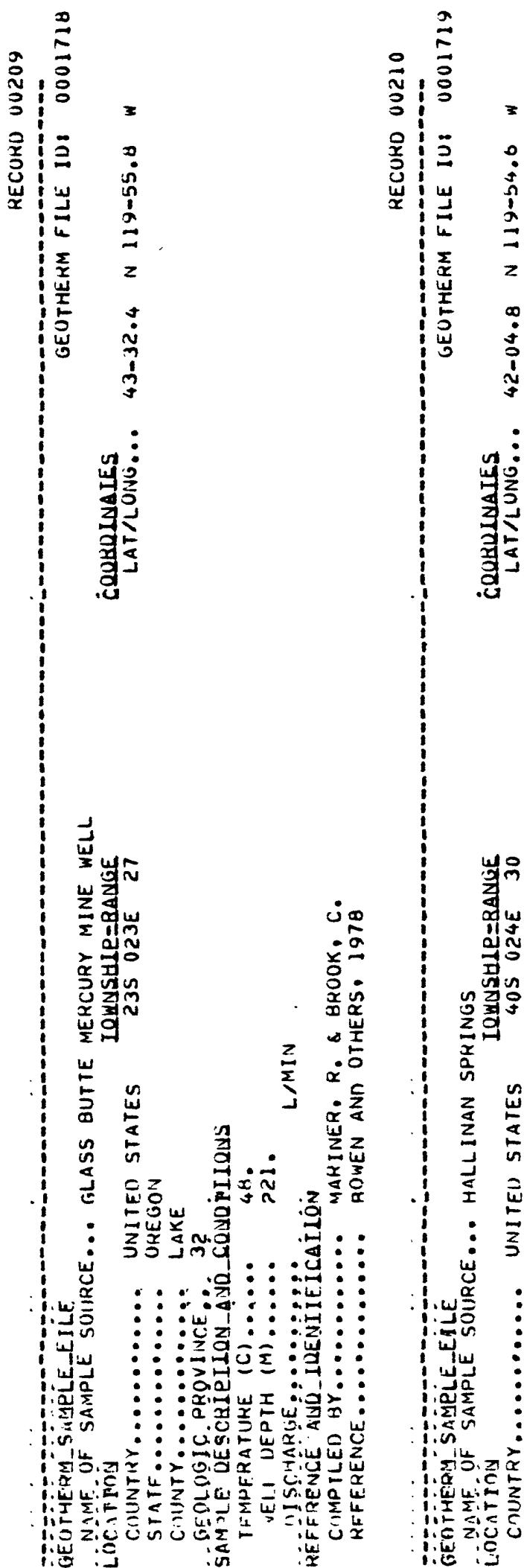

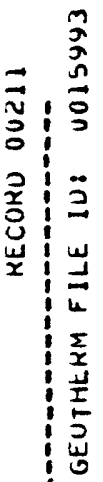

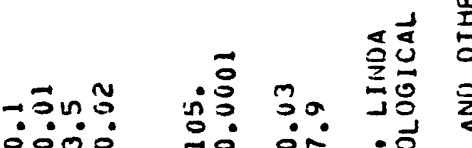

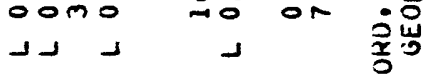

$::: \vdots: \vdots:: \vdots: \sum_{\frac{1}{4}}^{4} \frac{x}{4}$

$\because:: c$ :

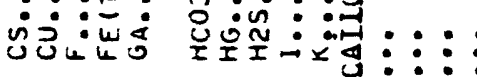

击:

汽之

무워

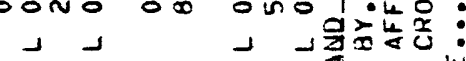

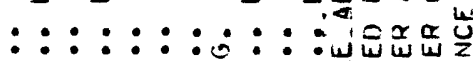

$:::::::: 2:::$ : : $:$

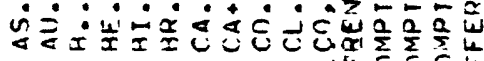

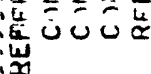

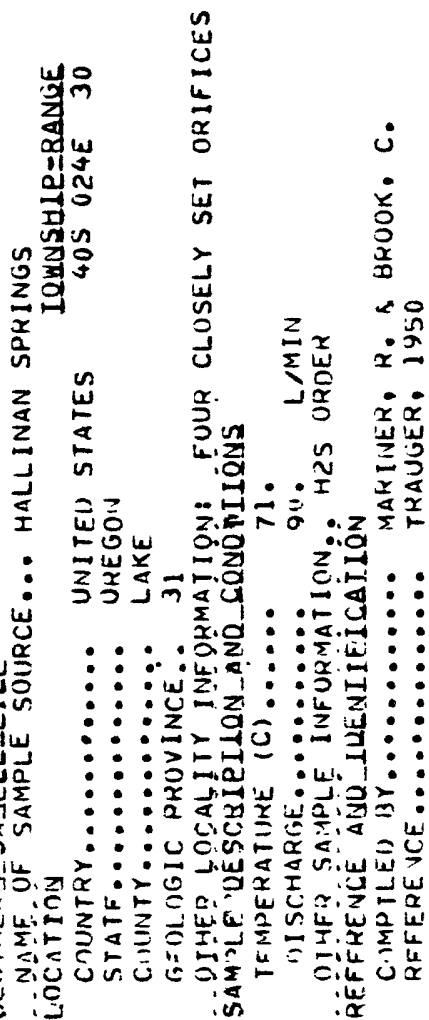

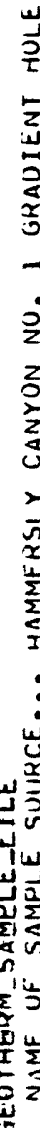


$m$
3
0
0
0
0
0
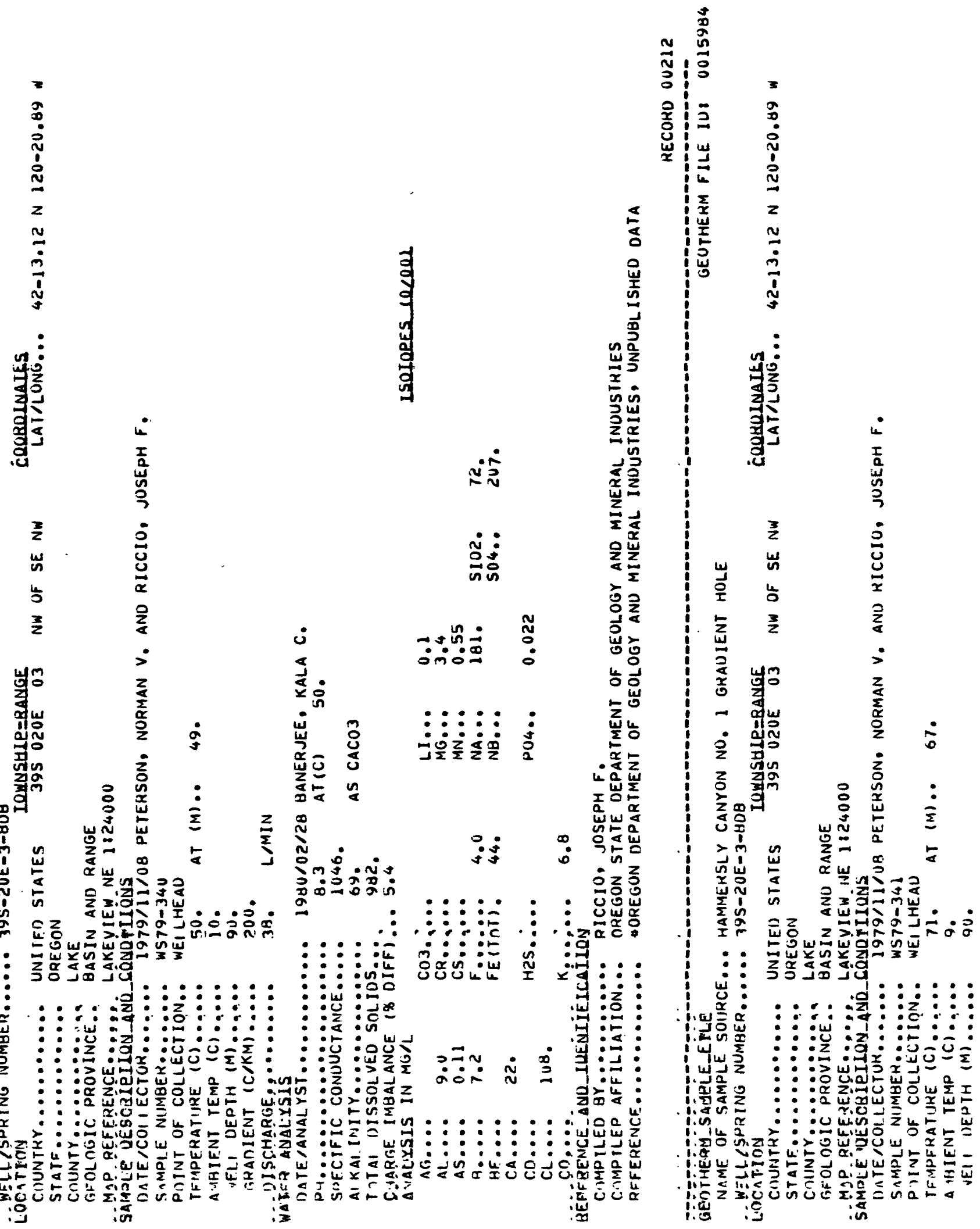
5
$\frac{5}{3}$
5
$\frac{5}{2}$
2

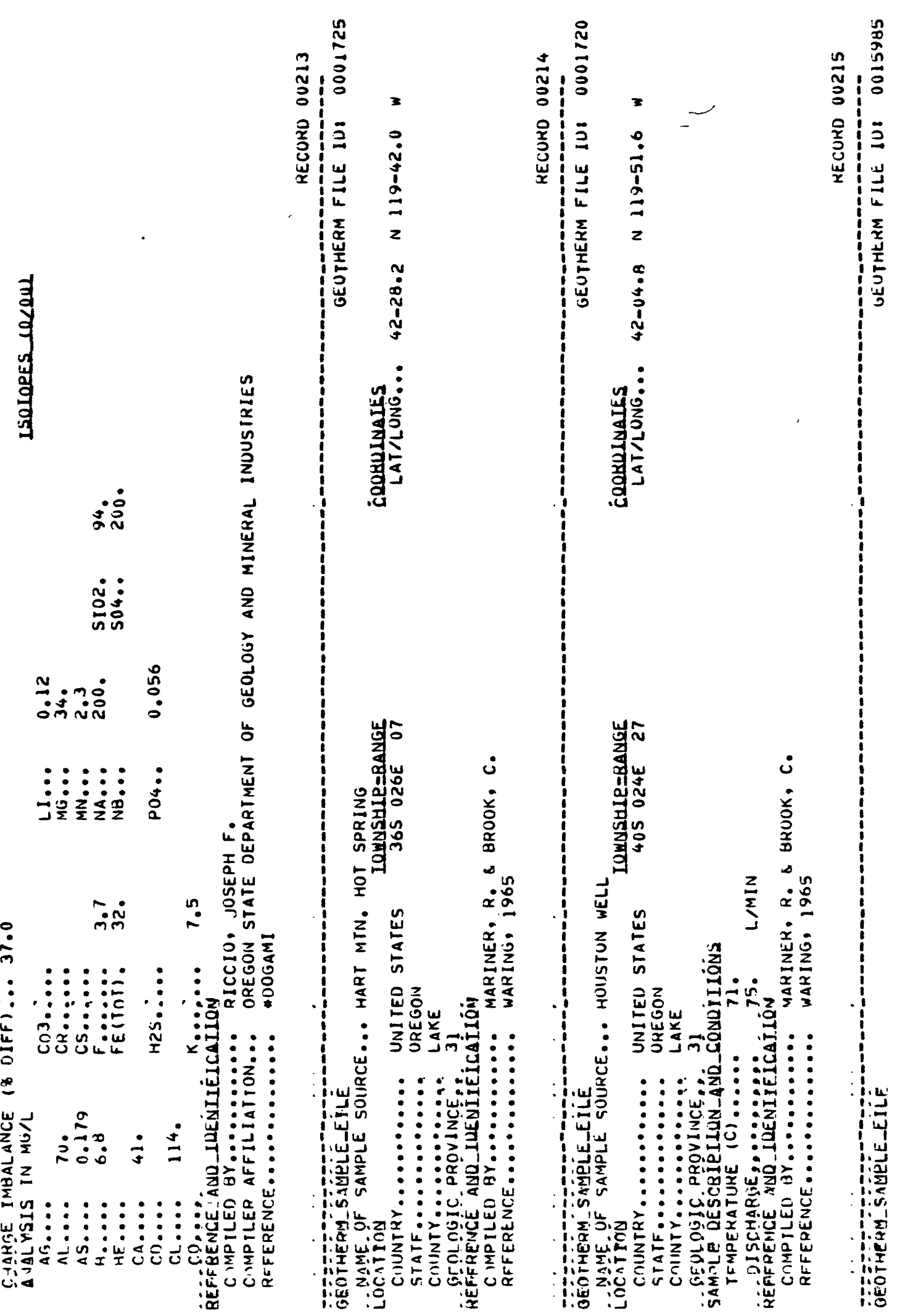




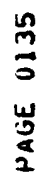
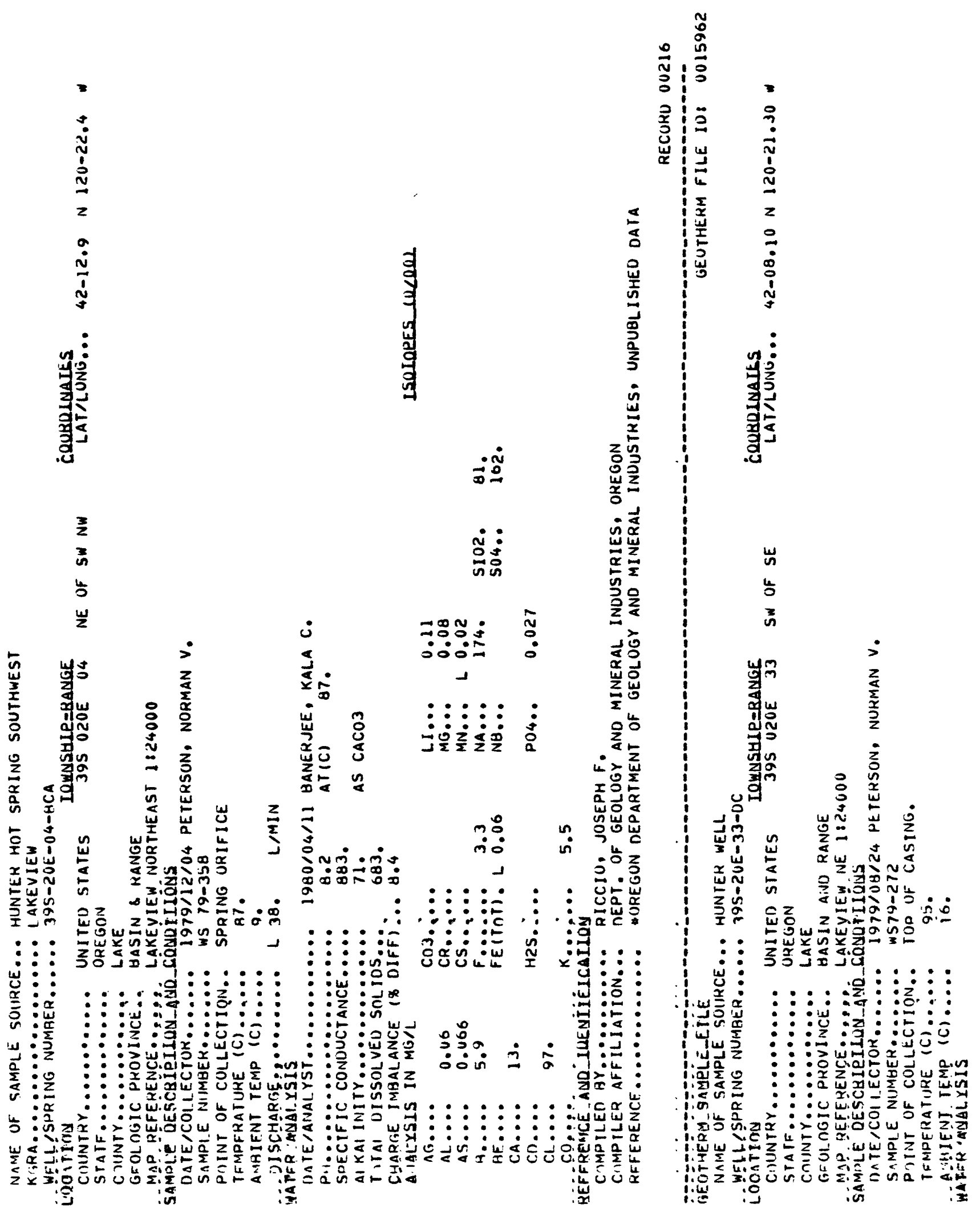


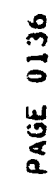

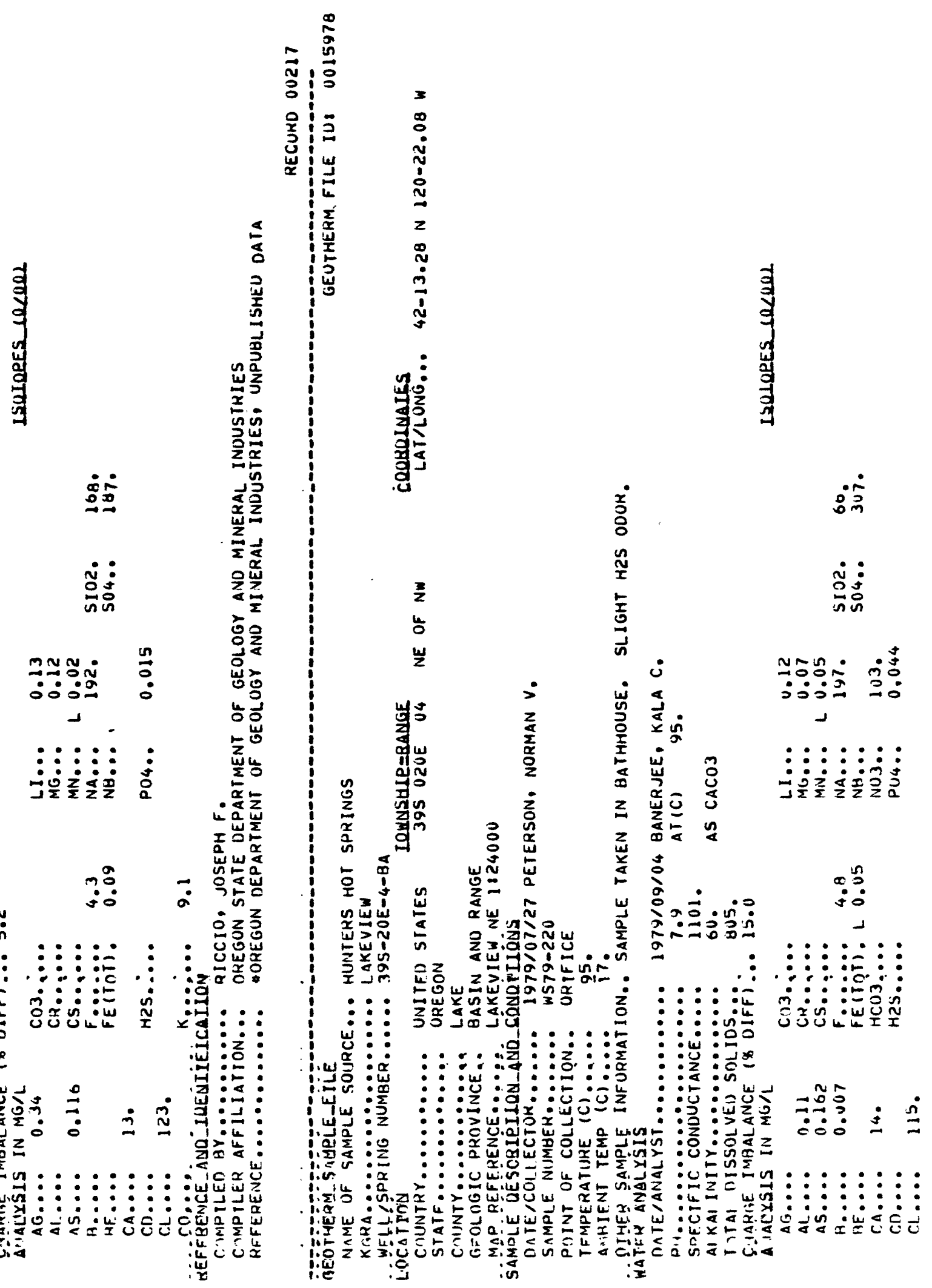




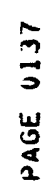

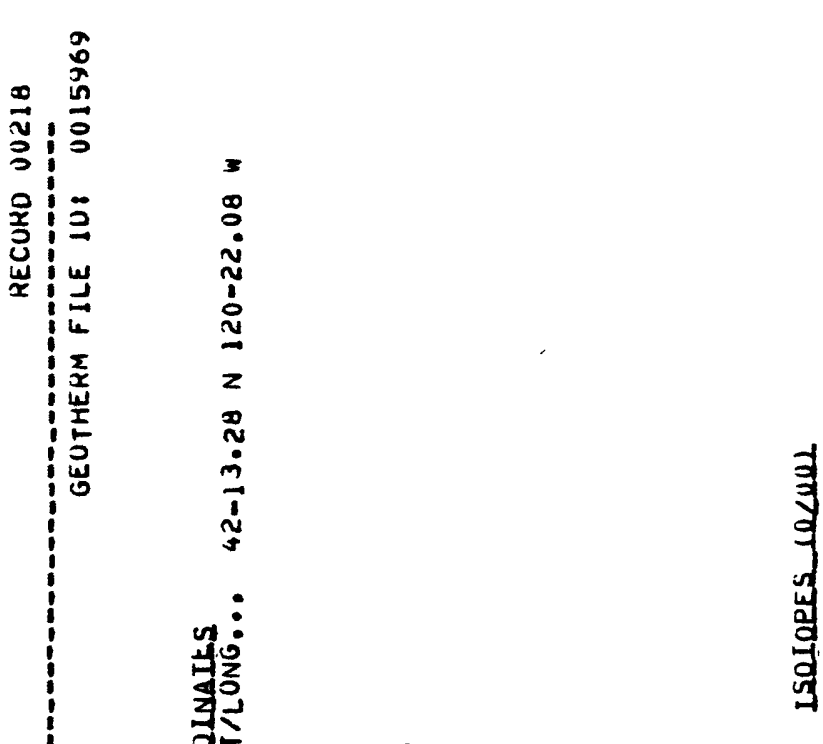

矛

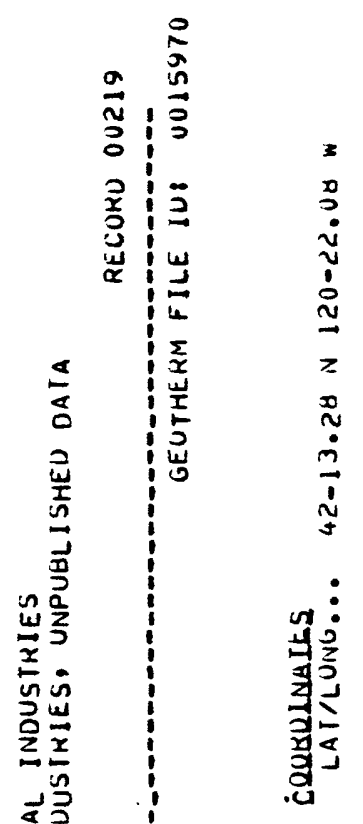

$\frac{\pi}{2}$
$\frac{u}{5}$
$\frac{w}{2}$

落:

$\underset{\frac{1}{2}}{\frac{1}{2}}$

:

i $\quad$ mm气

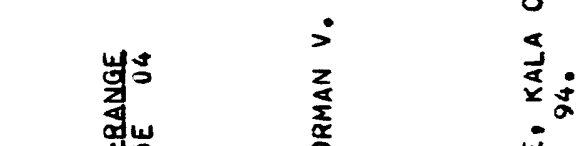

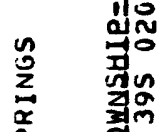

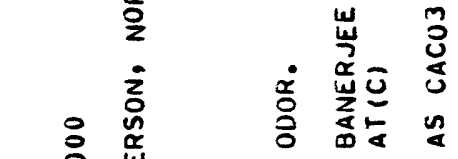

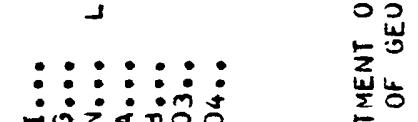

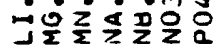

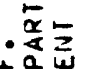
嵌离

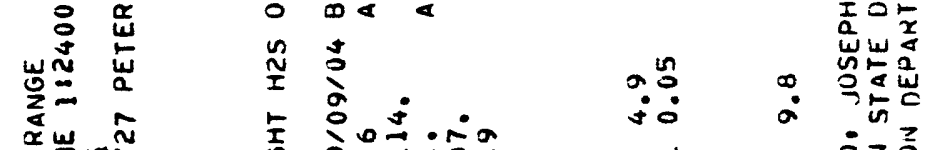
。马约 递文

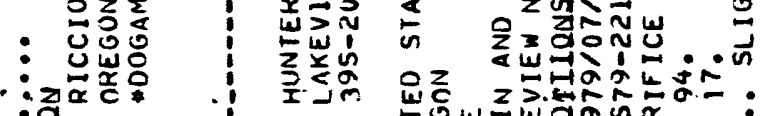

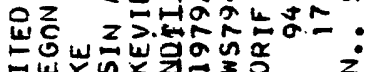

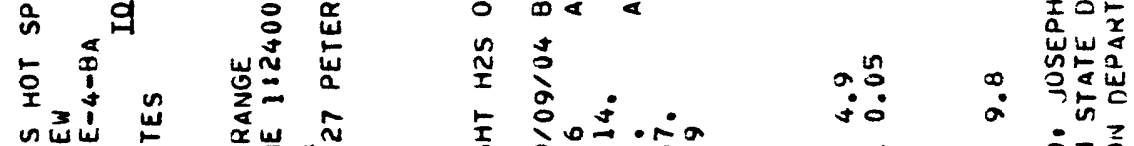

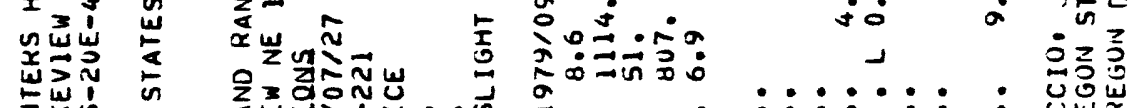
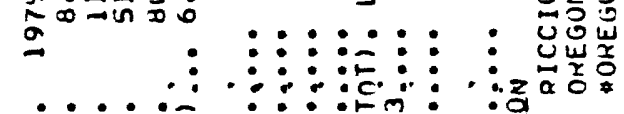

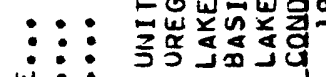
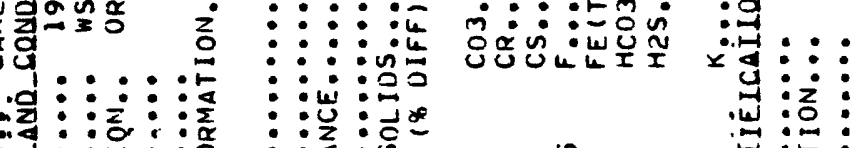

$\because \frac{\text { nn }}{2} \frac{n}{0}$

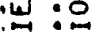

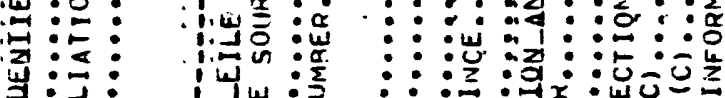

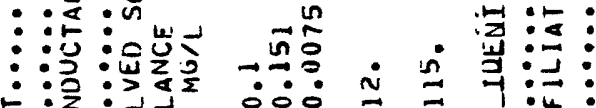

:

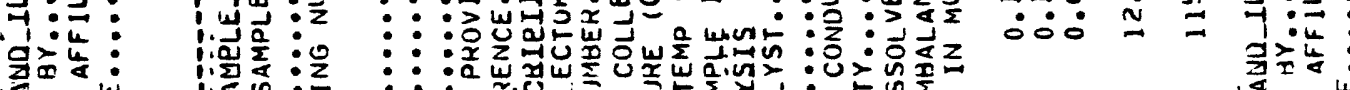

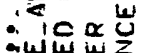

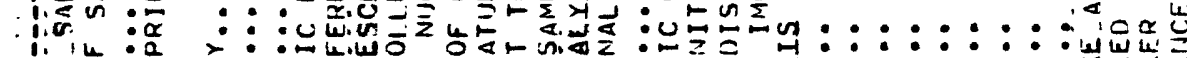
Vut :

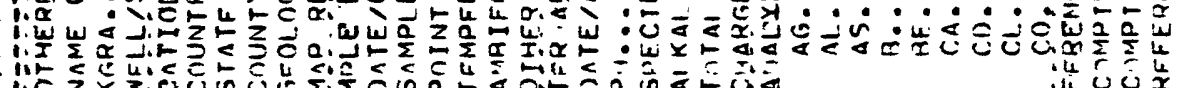




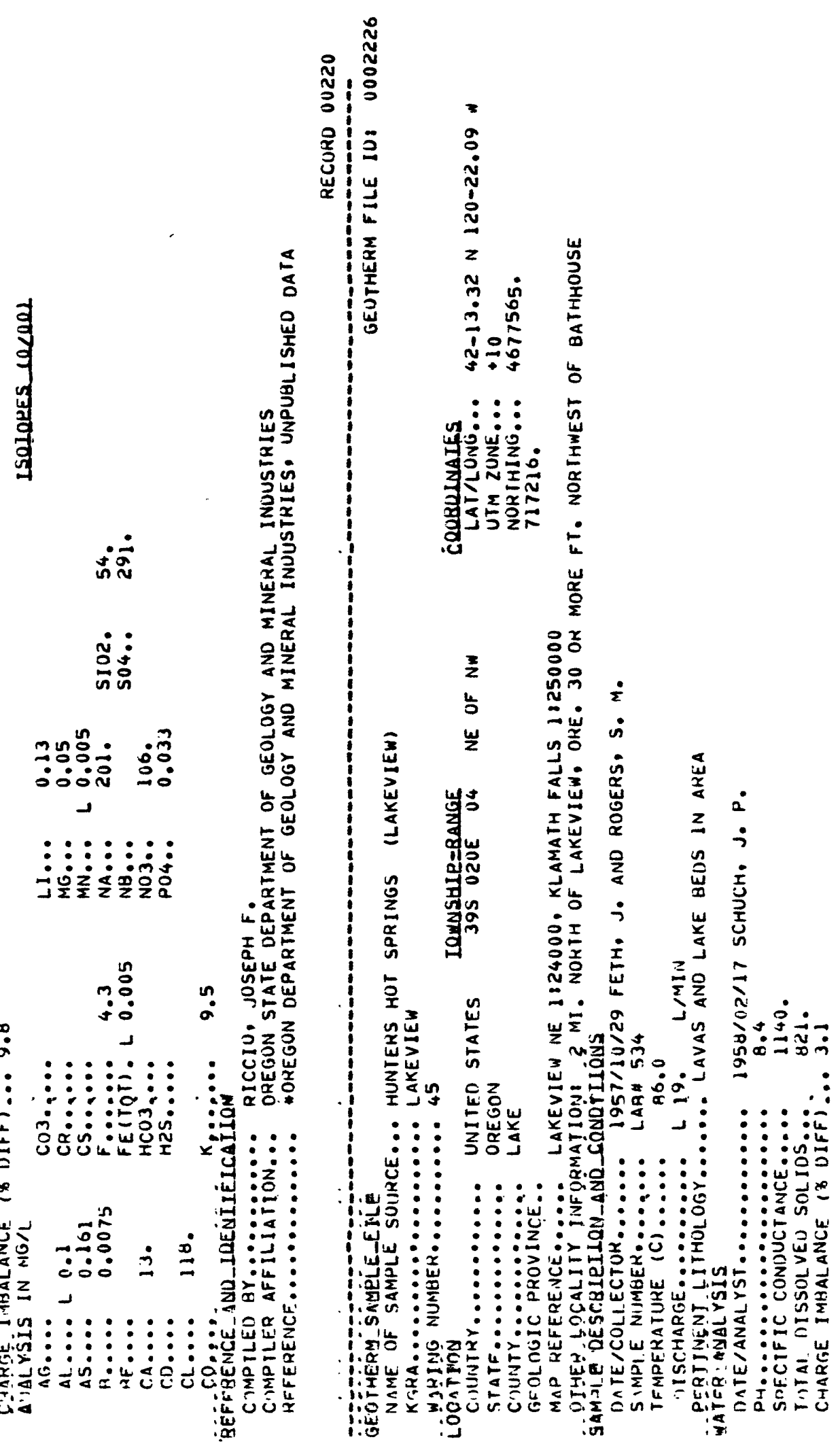


$\stackrel{2}{3}$
$\stackrel{3}{0}$
$\stackrel{0}{a}$

aี

$\dot{0} \dot{0}$

$\overrightarrow{\tilde{N}}: \stackrel{\tilde{O}}{0}$

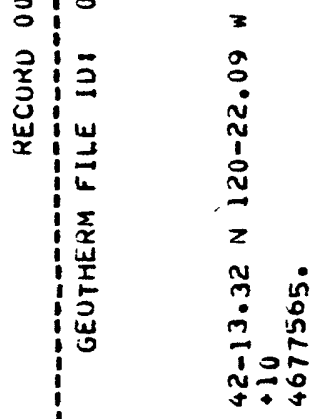

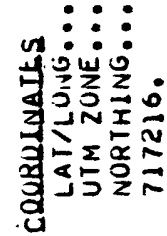

3ี่

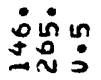

紊导 $\quad$ :

$\stackrel{+}{i} \stackrel{\dot{0}}{\stackrel{0}{*}} \stackrel{m}{0}$

$:: \vdots:: \vdots:$

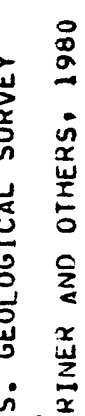

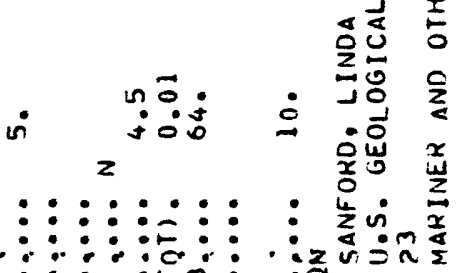

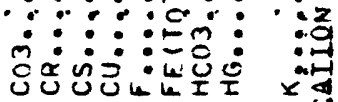

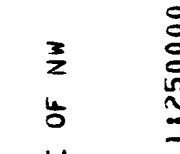

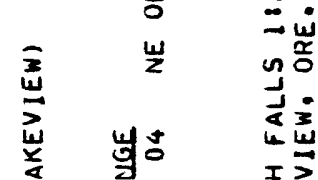

:

王离

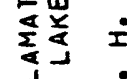

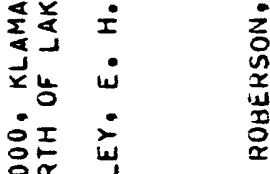

家吉

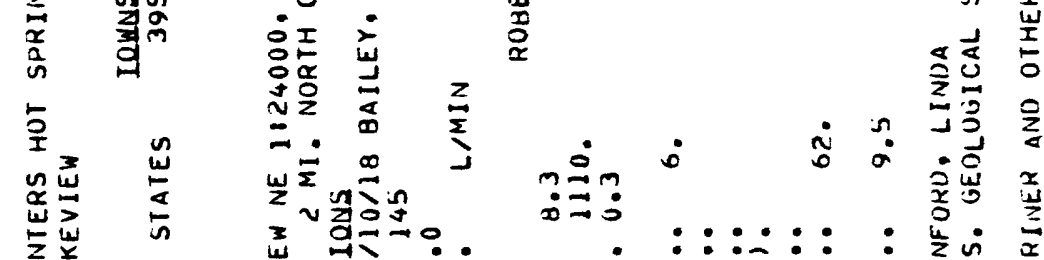

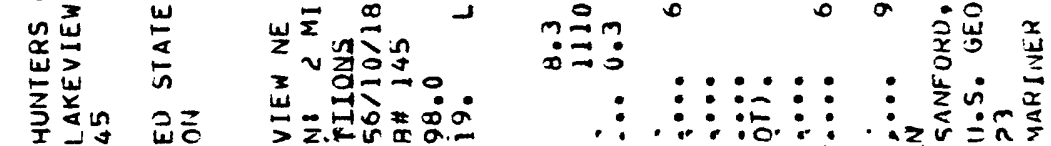

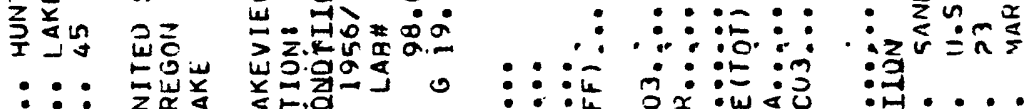

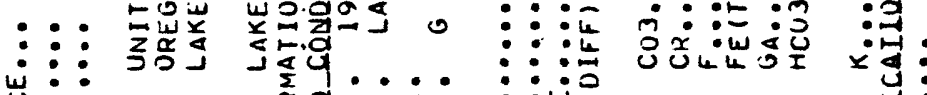

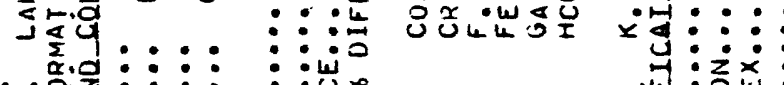

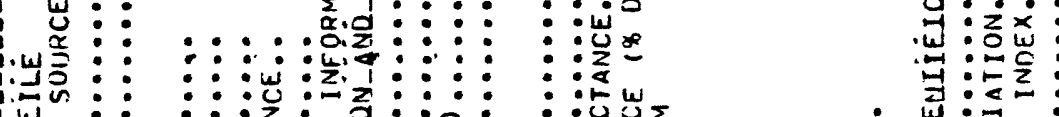

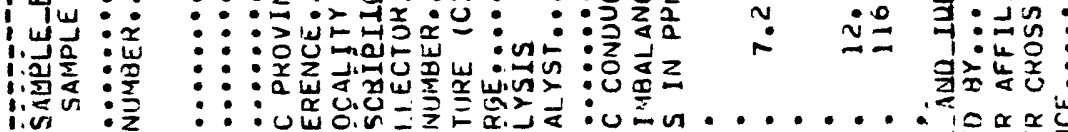
ivin : : : : :

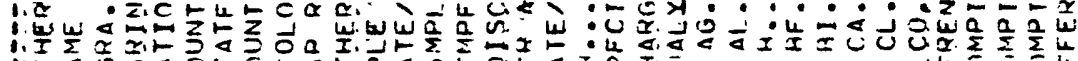
i.

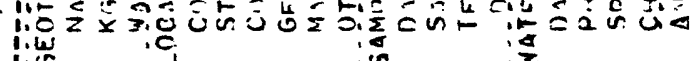




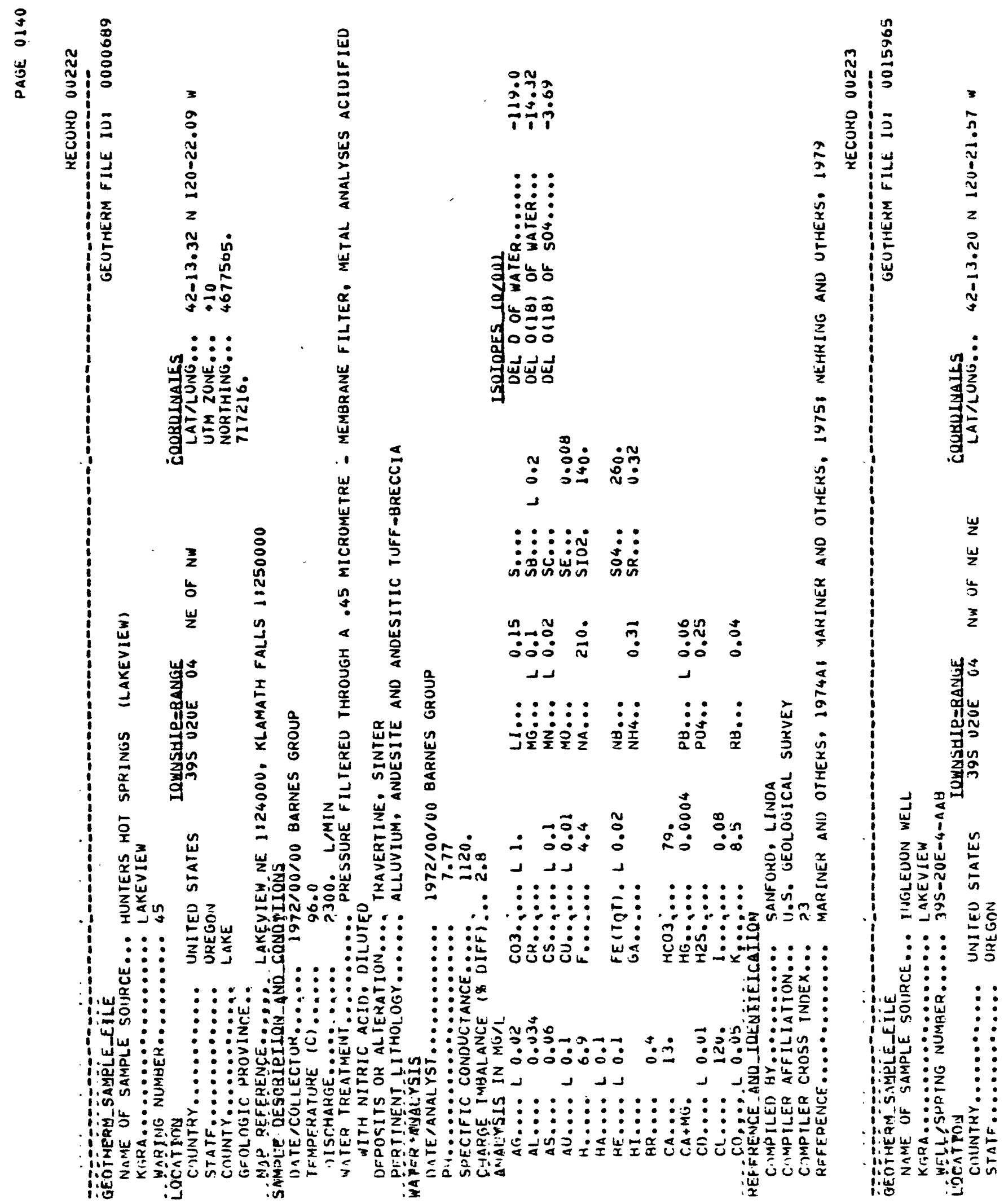




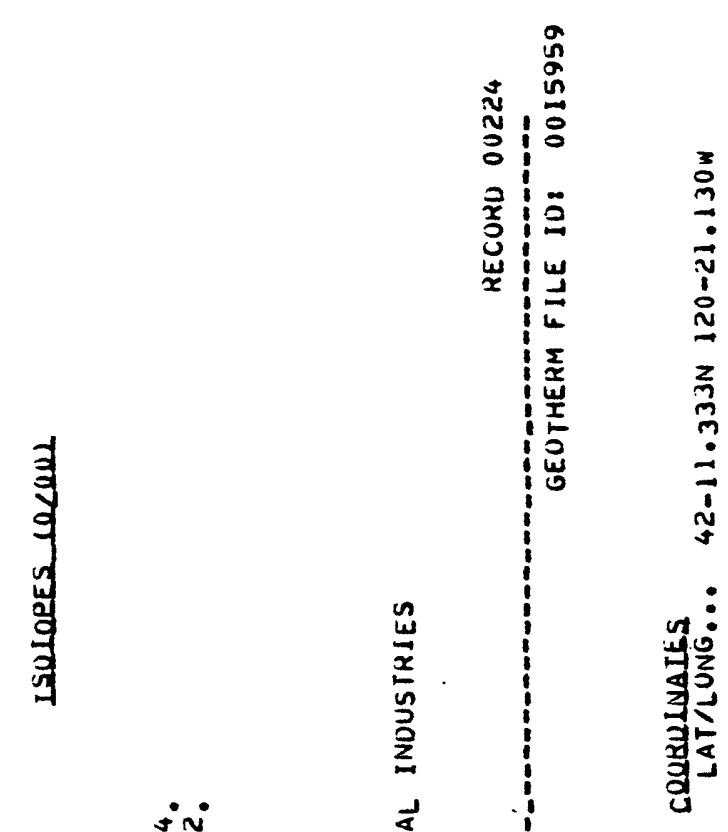

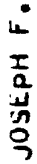

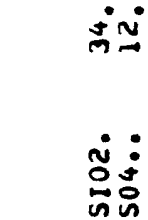

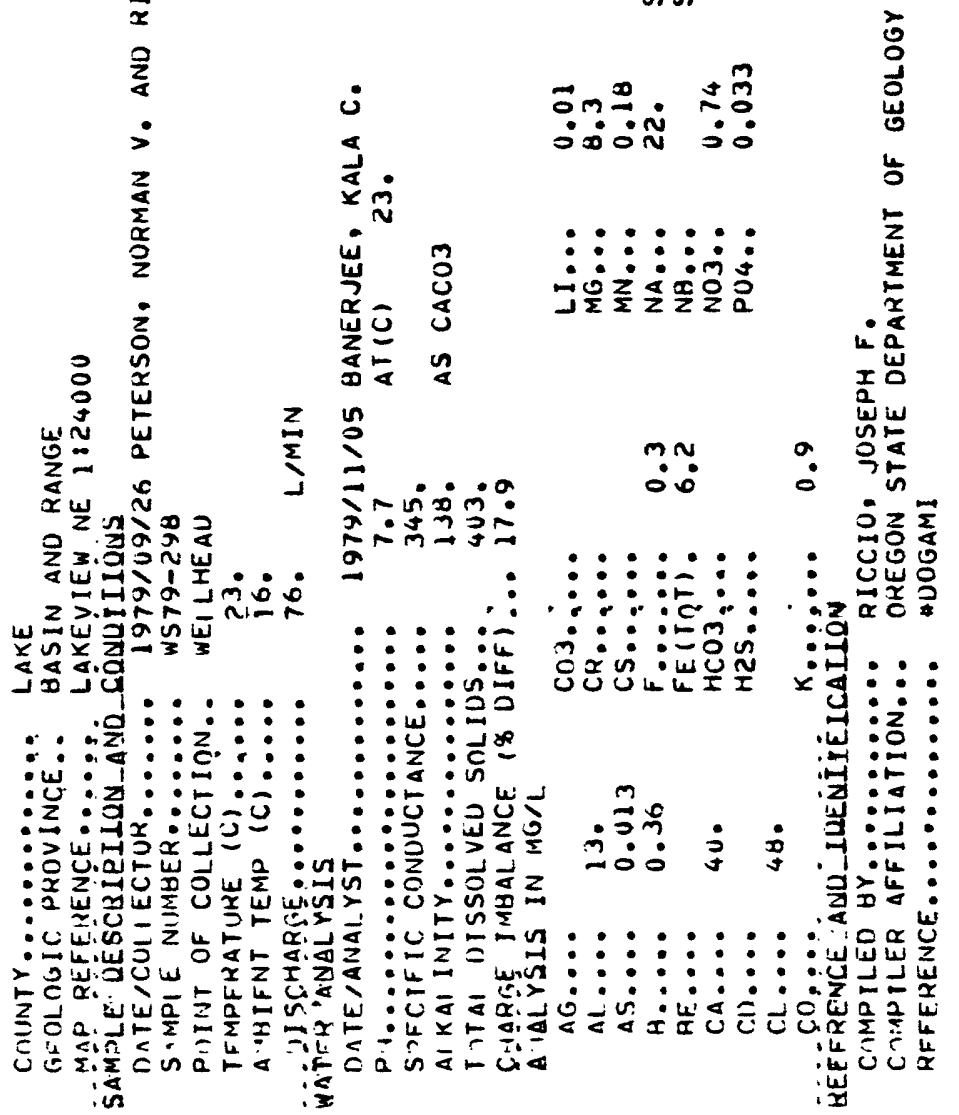


誉

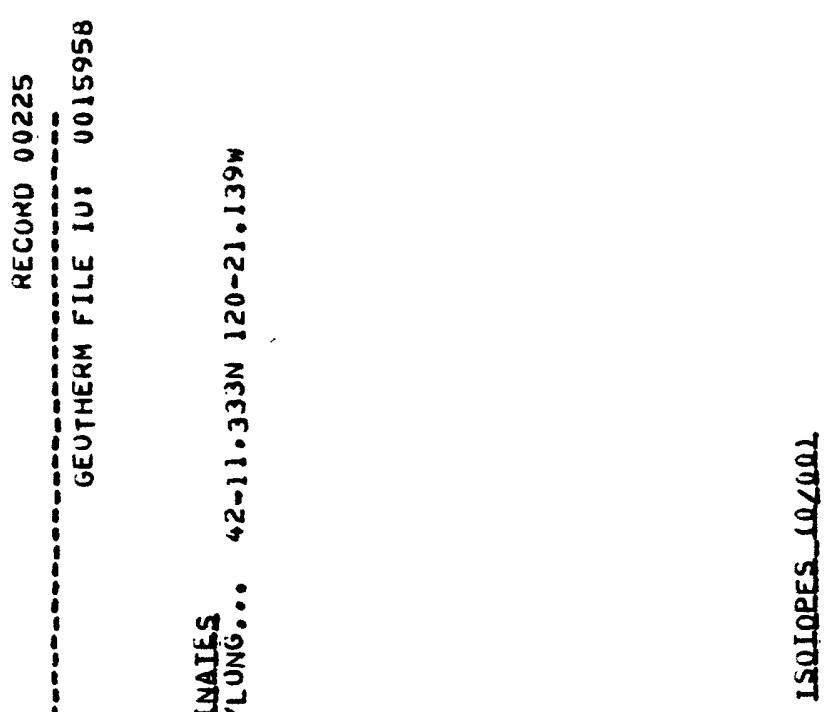

$\frac{n}{\underline{x}}$

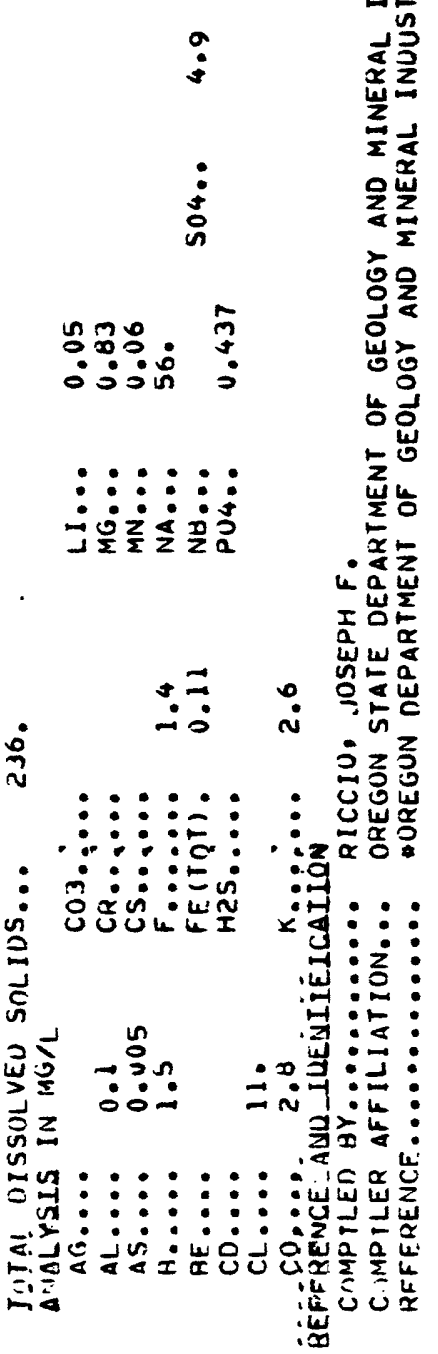

㭊

告

$\underline{2}$

$\stackrel{2}{2}$

苛范

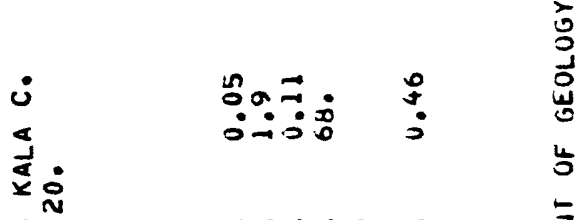

+

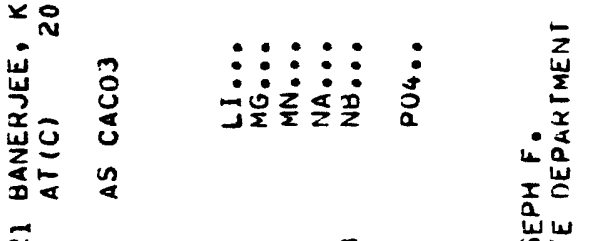

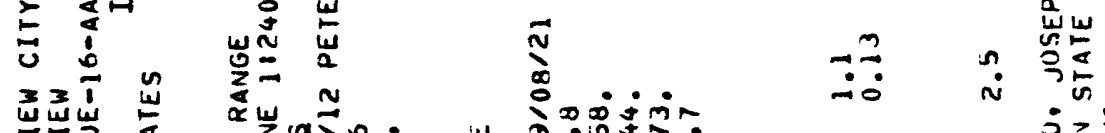

至焉岂

$\dot{0} \bar{z}$

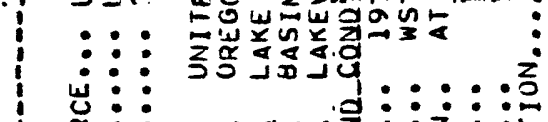

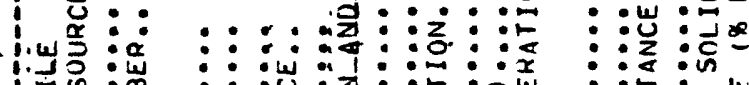

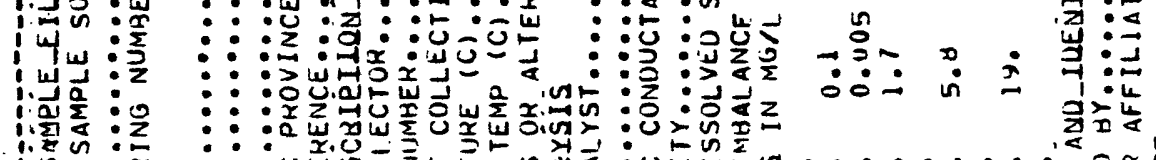

lin : : : : : :

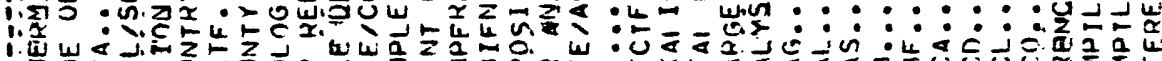

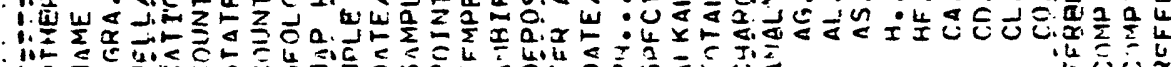

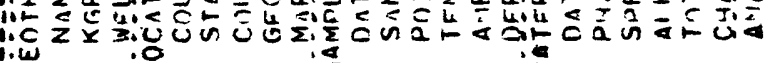




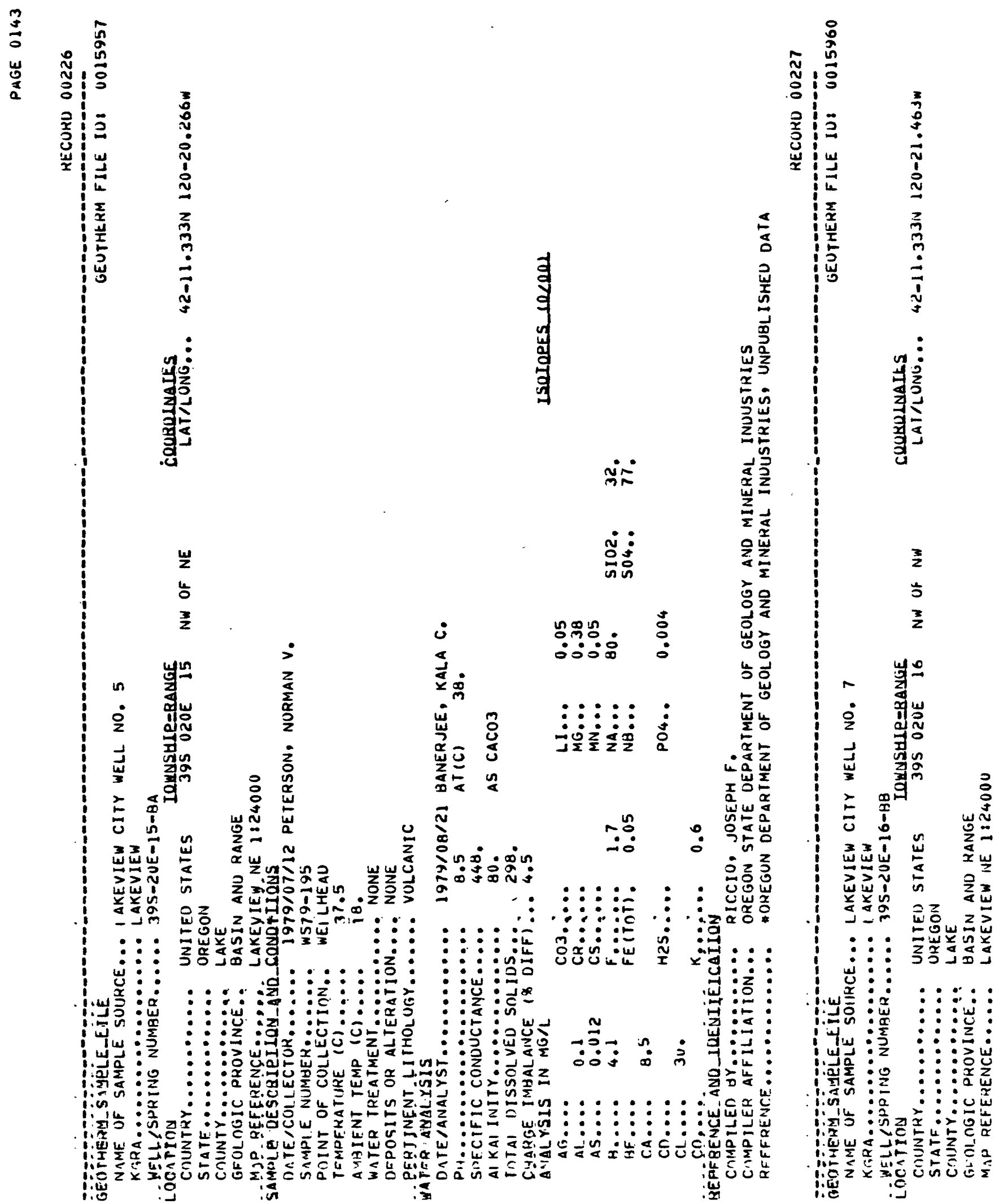



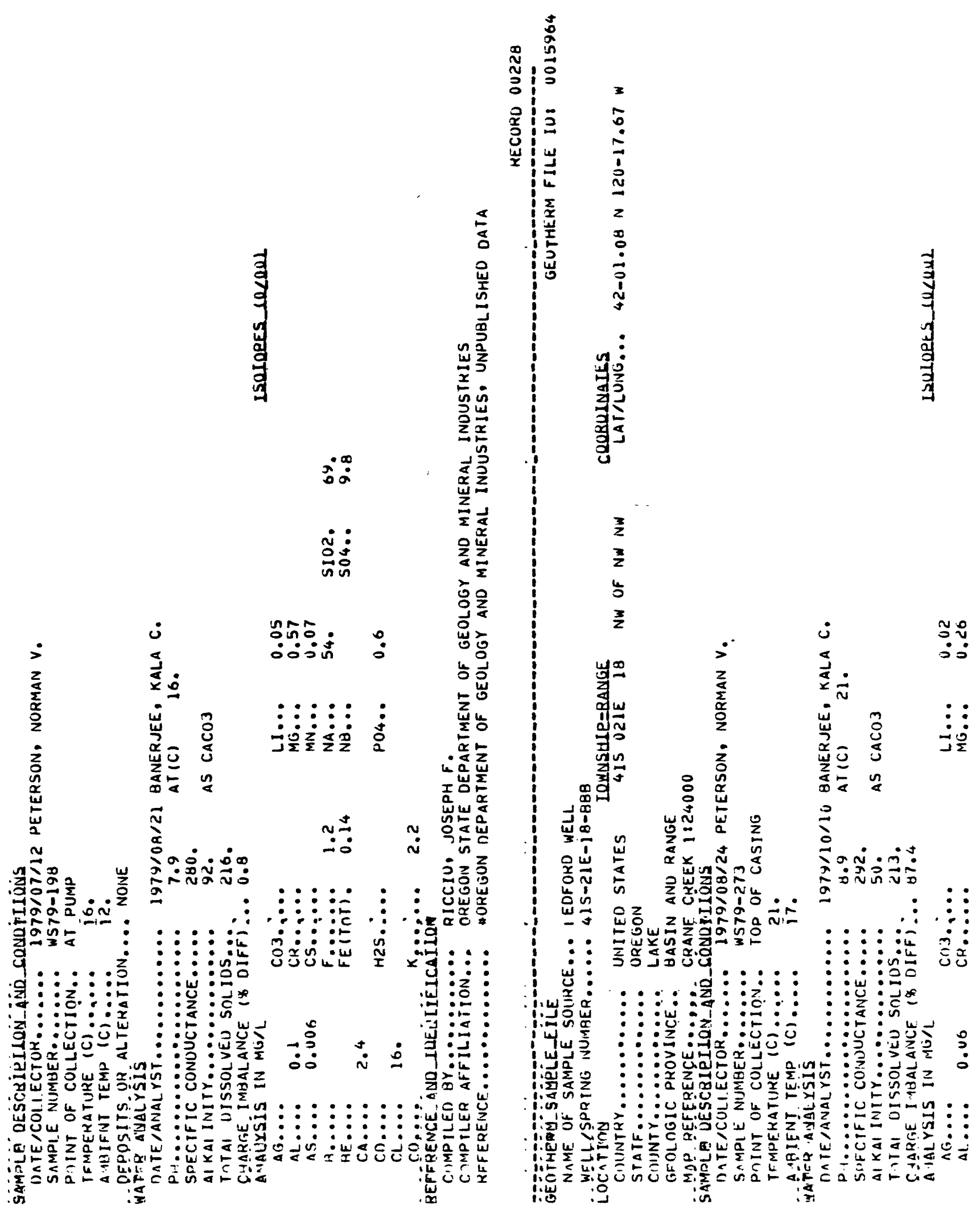
$\underset{\substack{0 \\ 0}}{0}$

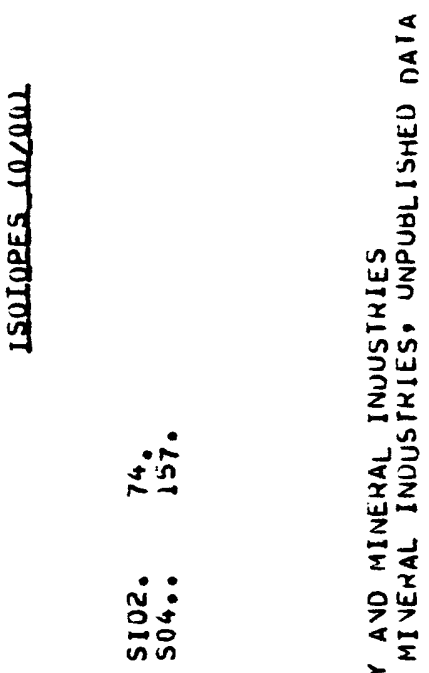

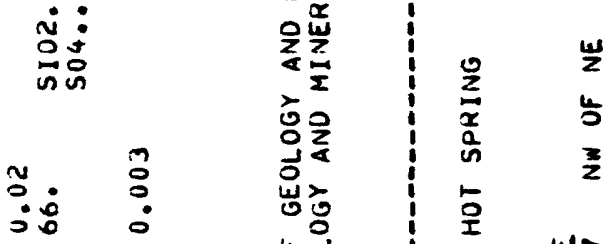

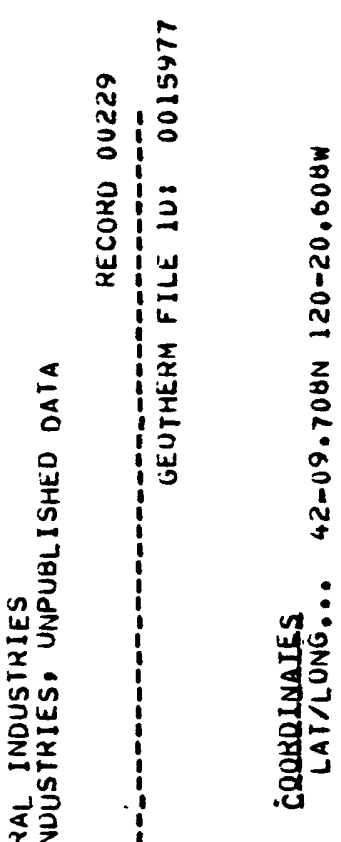

$\frac{a}{5}$

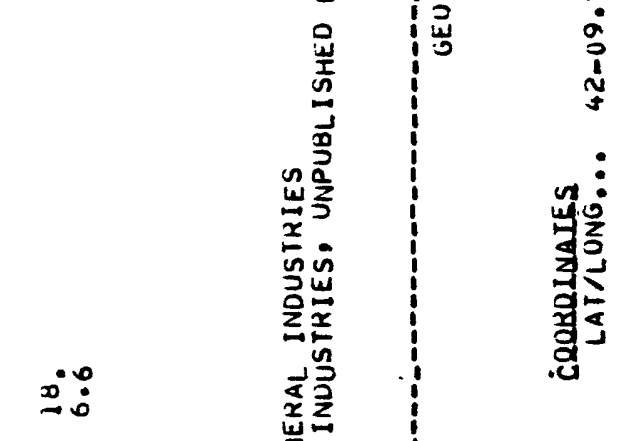

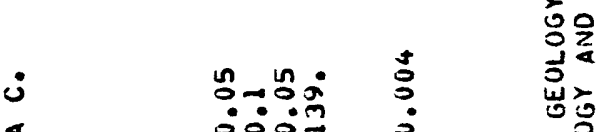

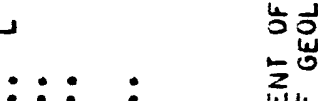

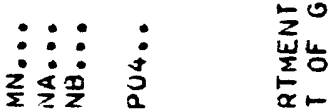

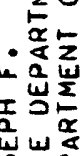

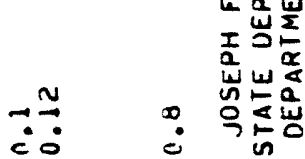

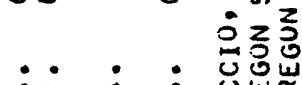

$\vdots: \vdots: \vdots:$ :

:

占

牙宅育:

$:::::::$ :

$::::::$ : :

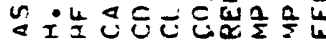

治论岂

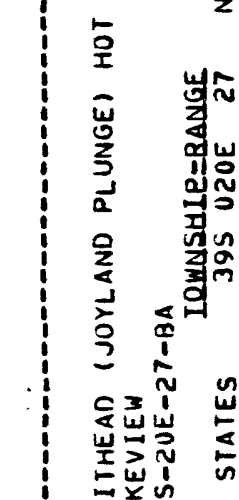




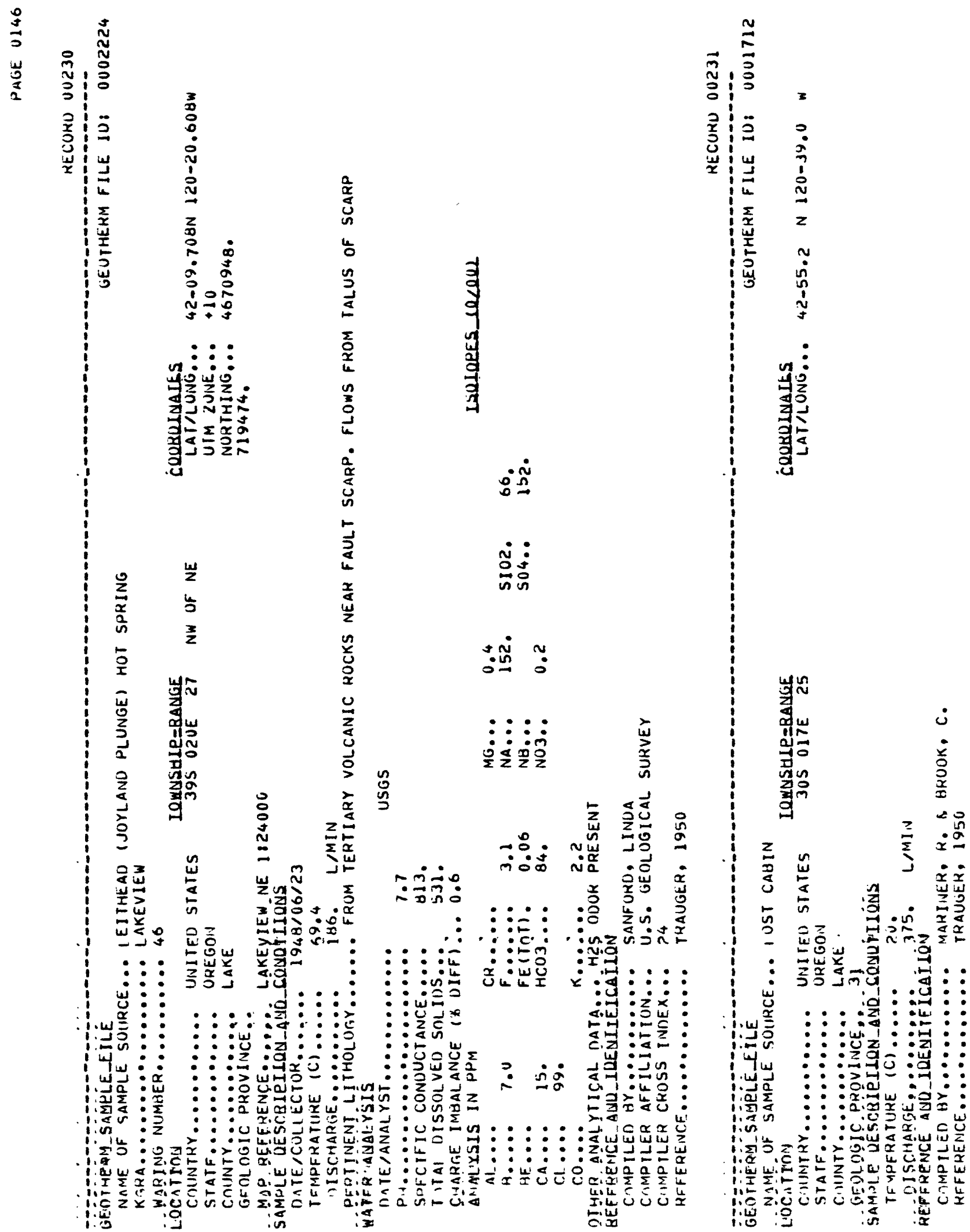




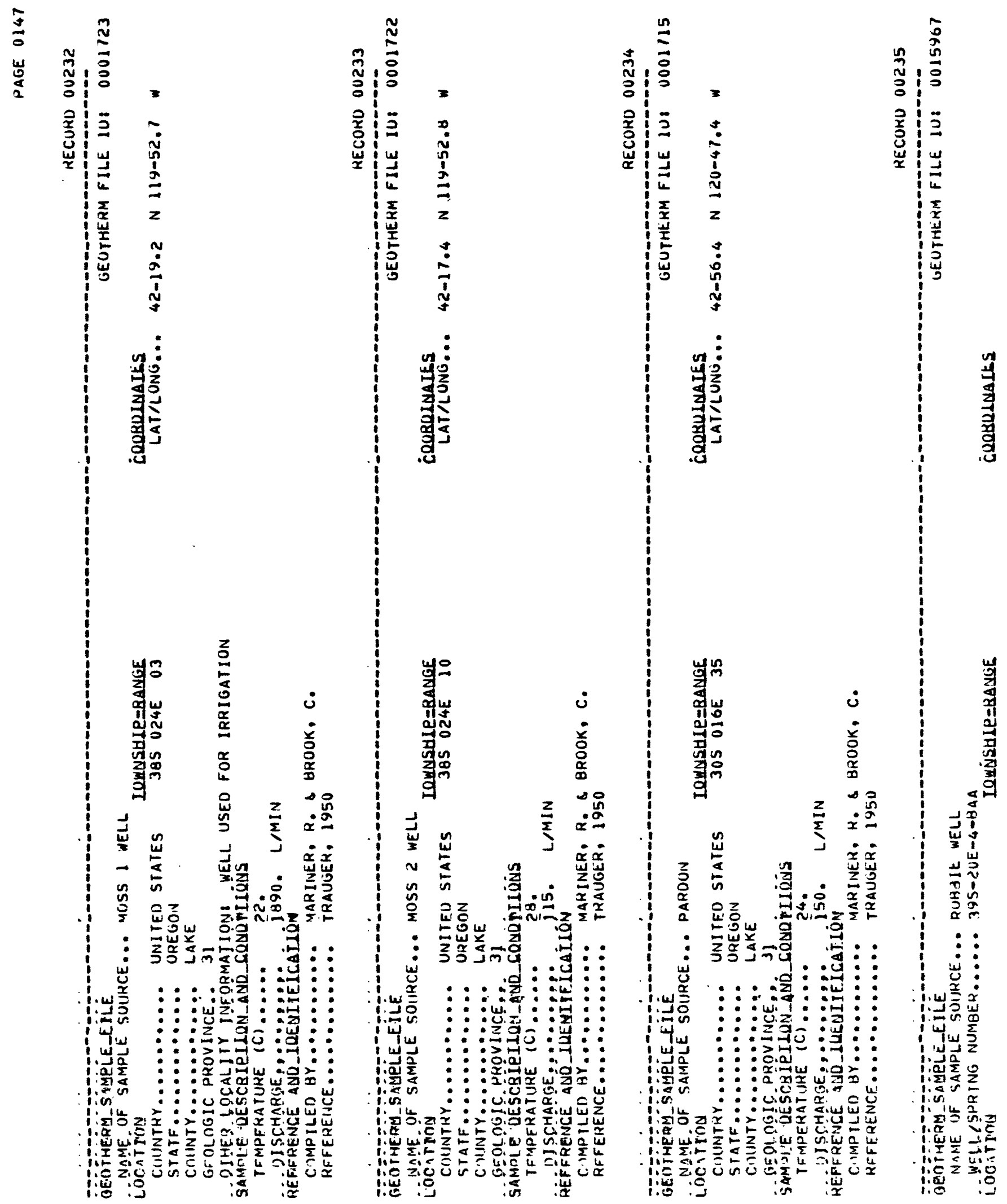



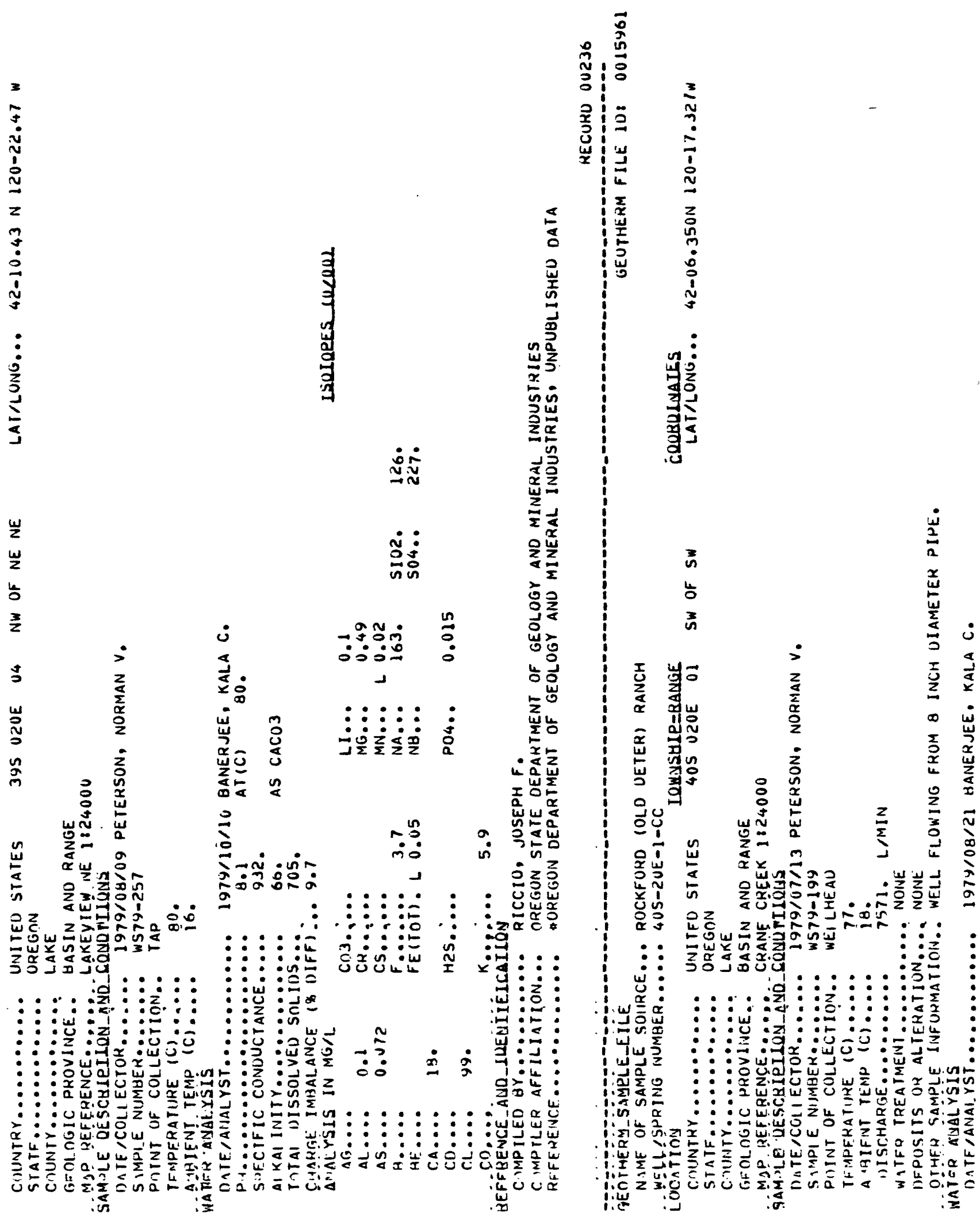


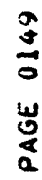

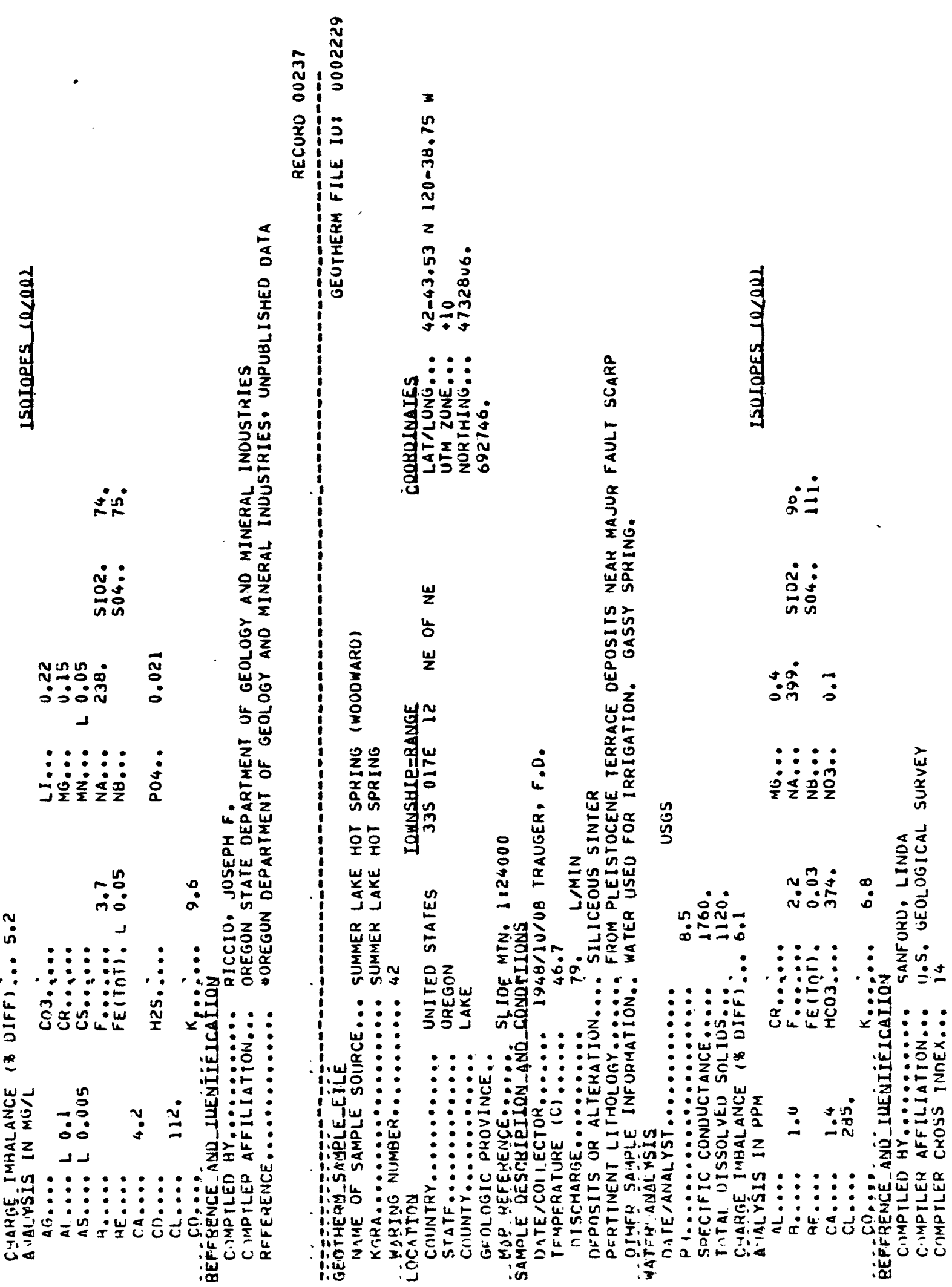




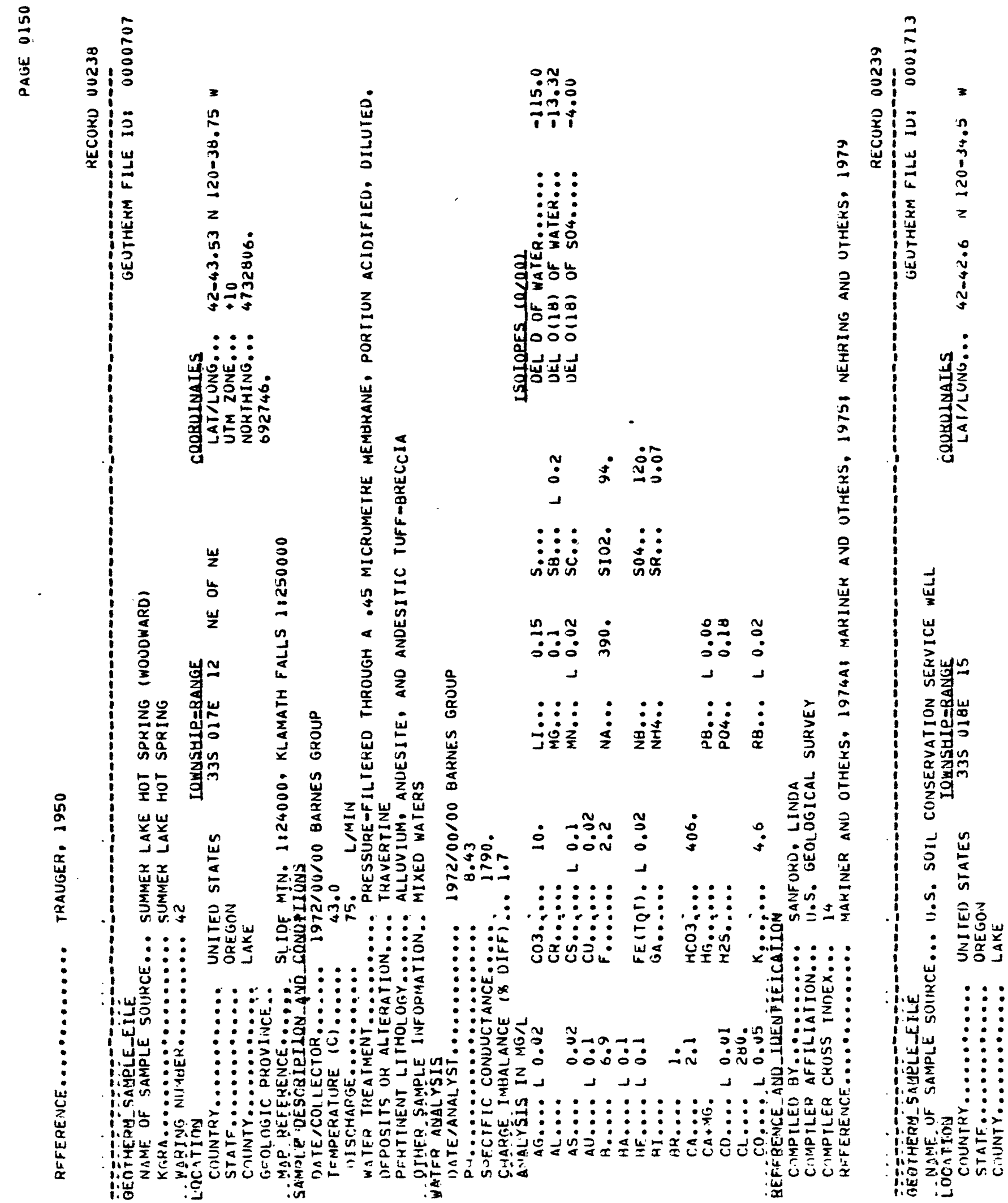


$\frac{5}{3}$
แั
$\frac{0}{2}$

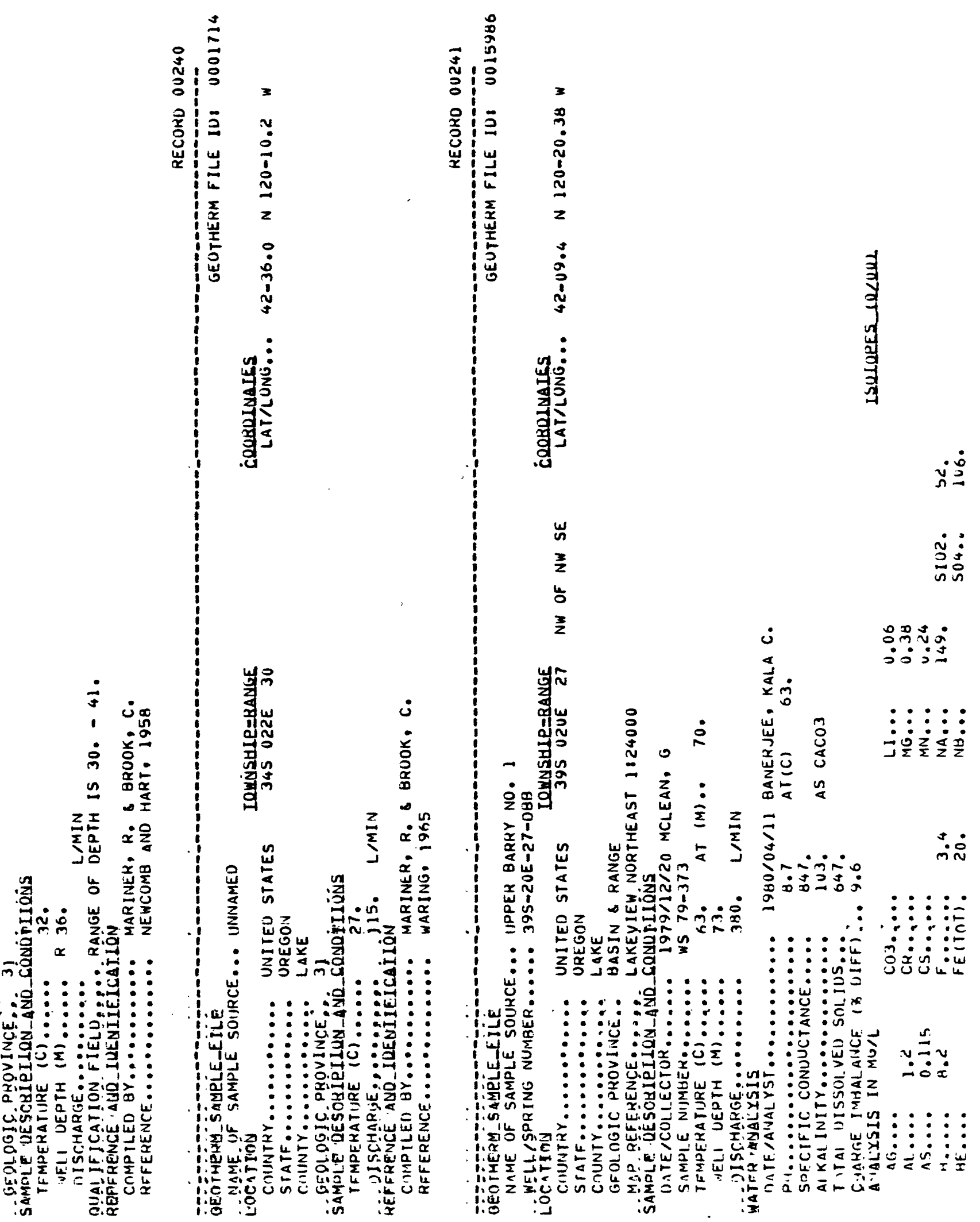




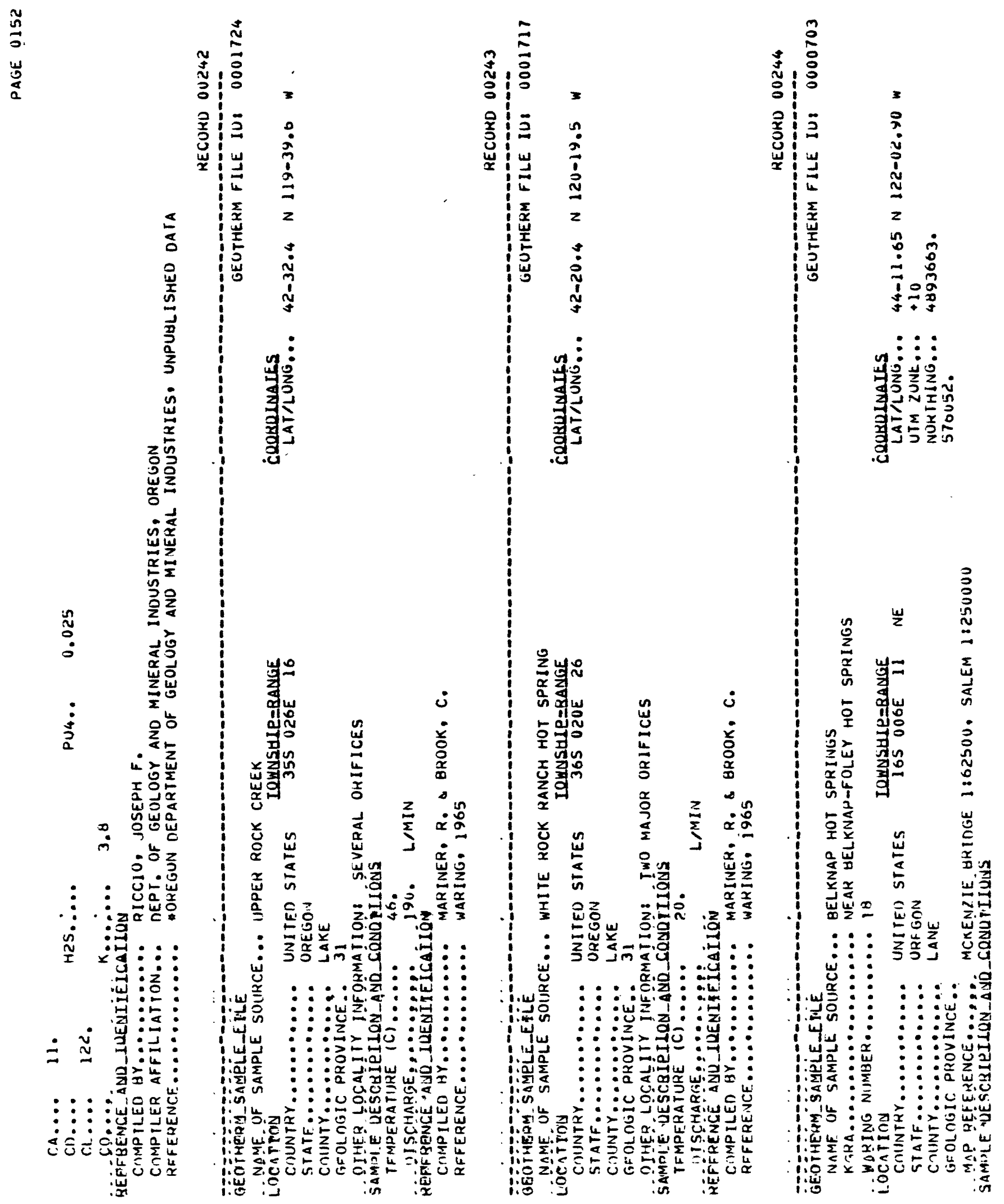




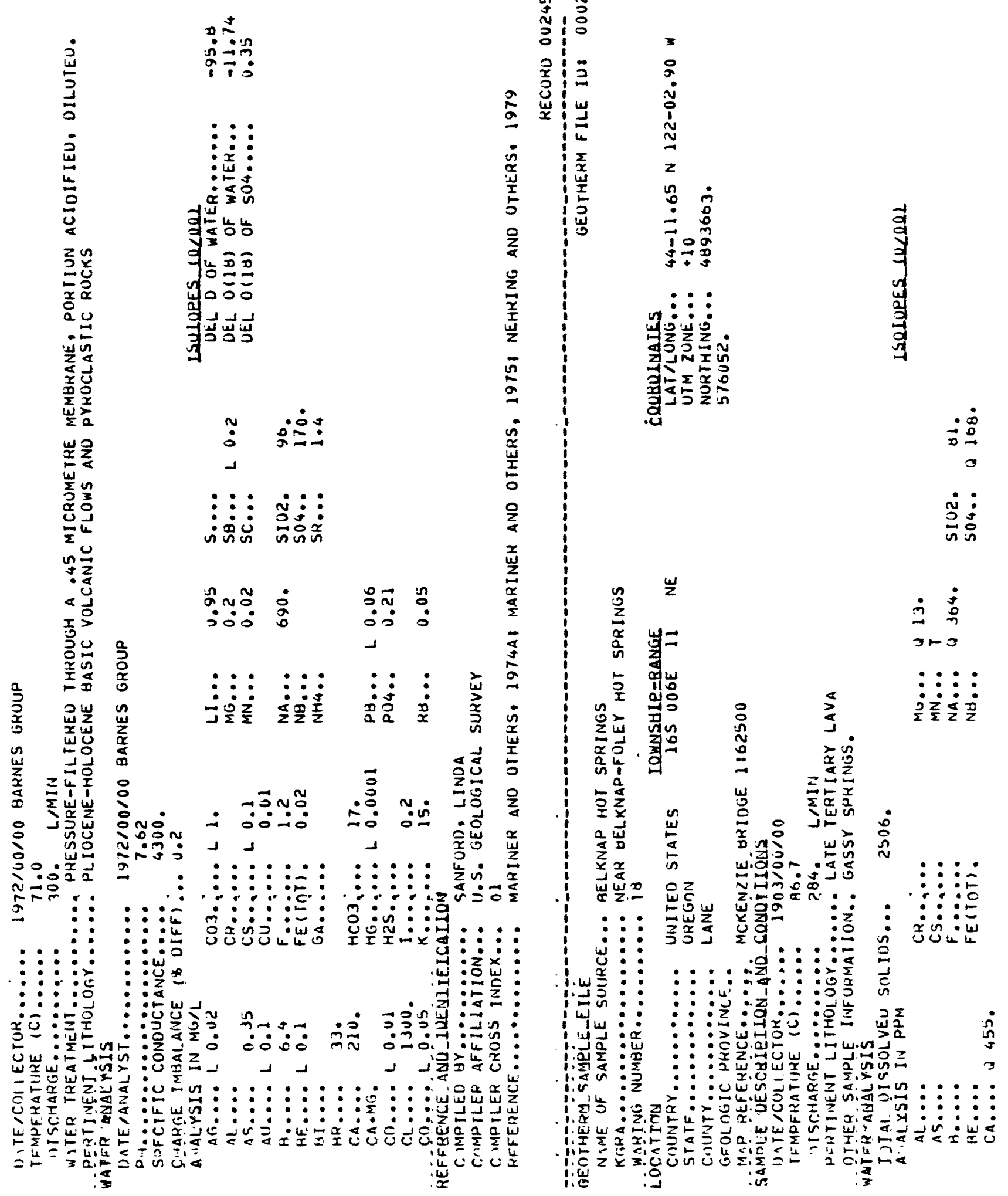




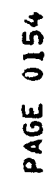

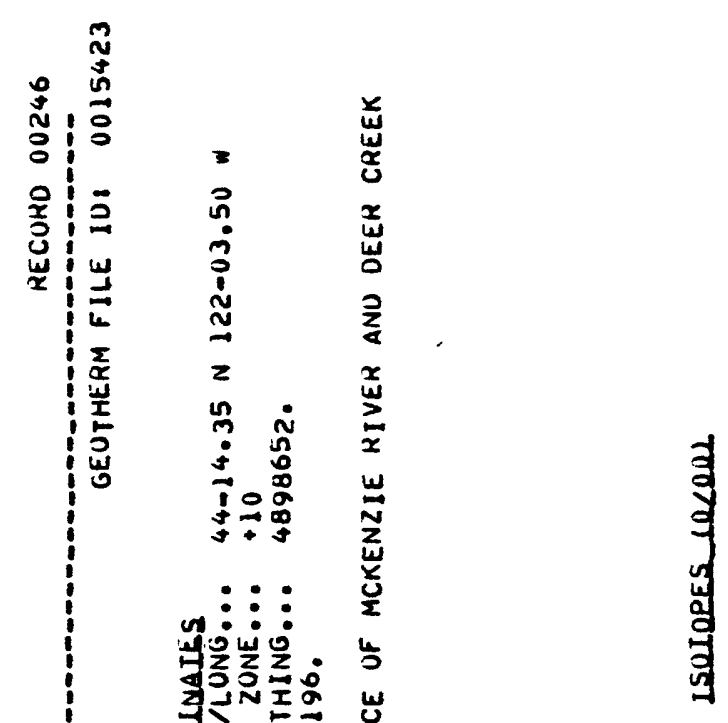

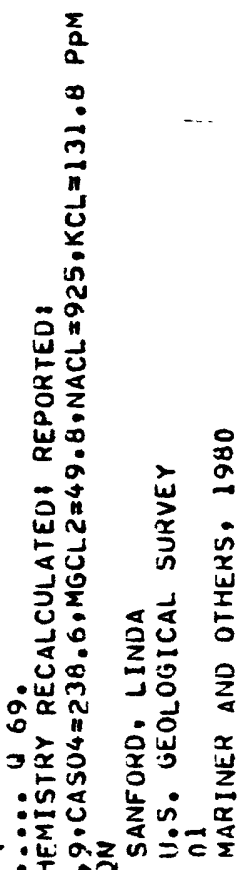

:

:견

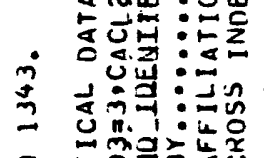

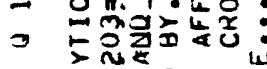

: : :

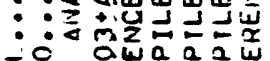

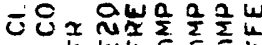

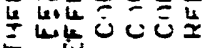

当
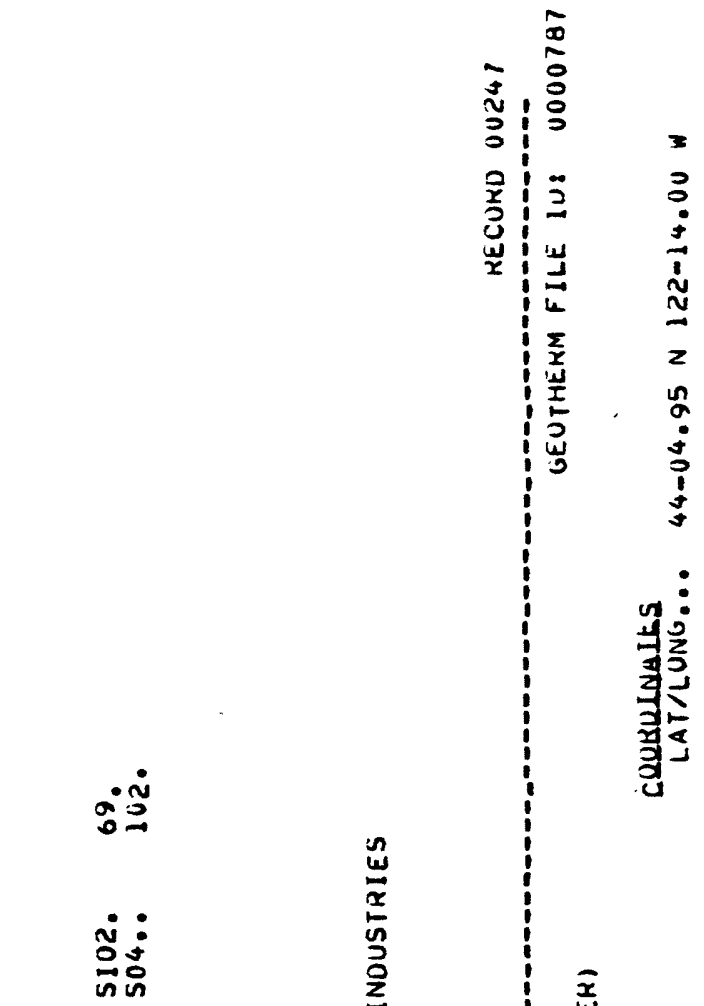

岂

ᄂ

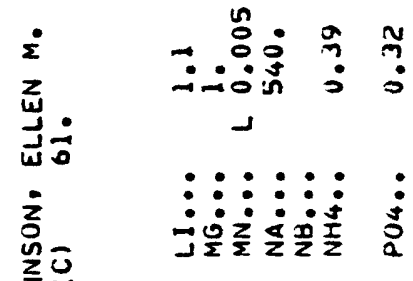

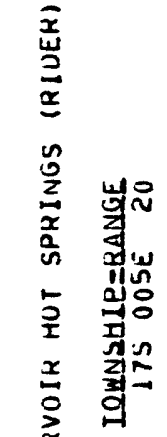

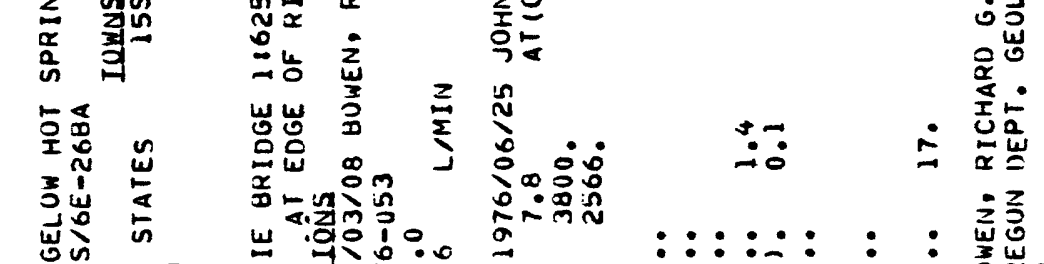

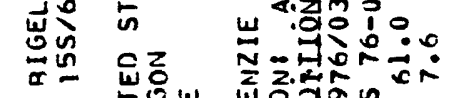

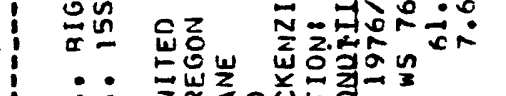

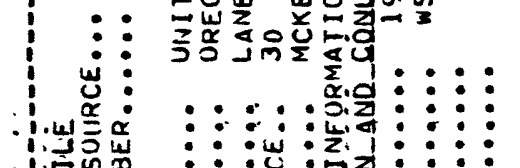

$:: \vdots:$ :

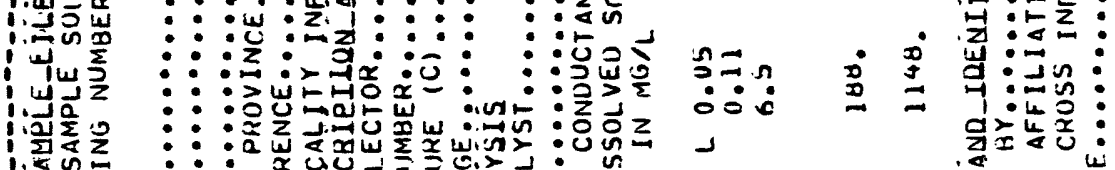


$\underset{\frac{n}{2}}{\frac{n}{2}}$
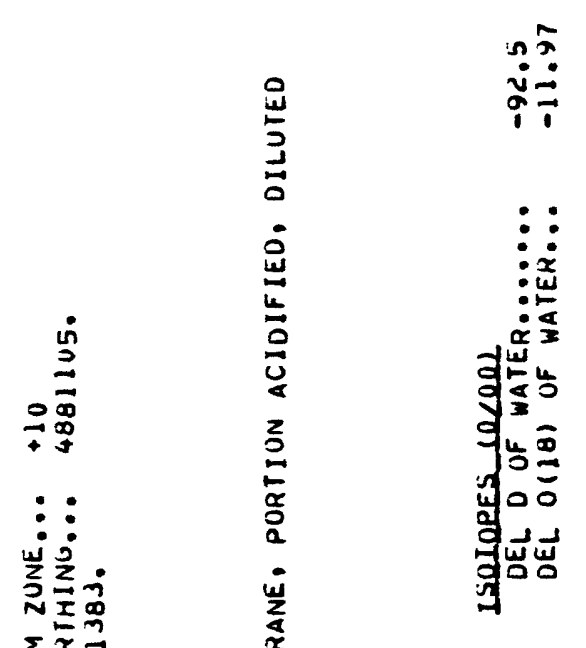

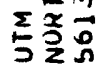

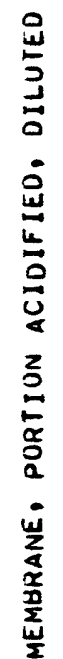

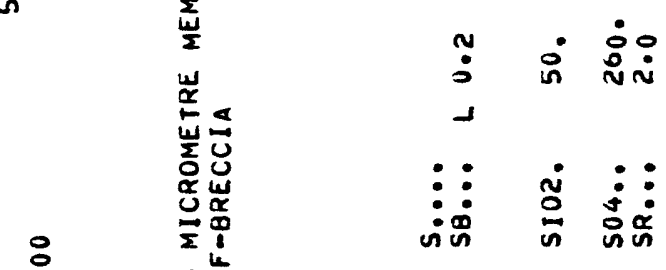

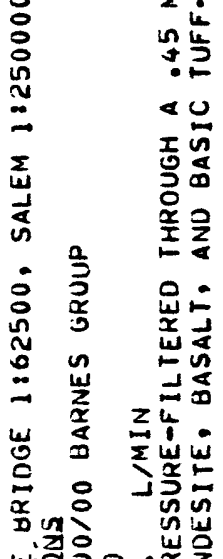

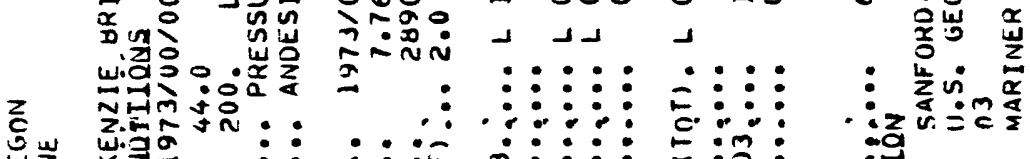

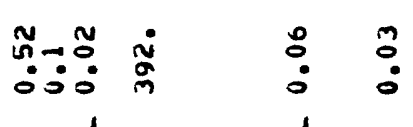

ڤ2)

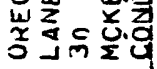

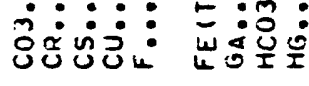

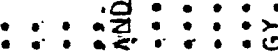

$: \vdots \vdots::: \frac{\pi}{0}$

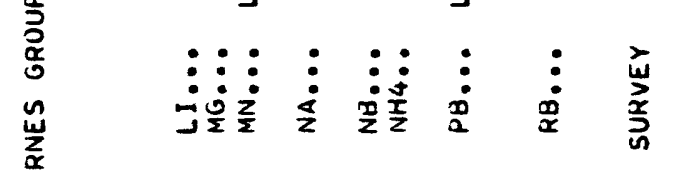

$::$ : $: 2:::: 0$

:

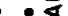

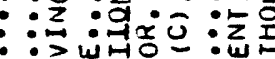

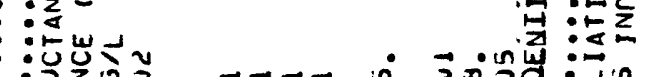
: 岁过 :

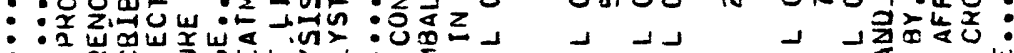

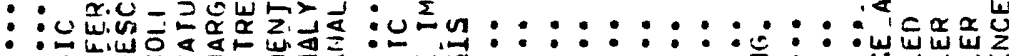

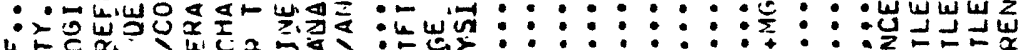

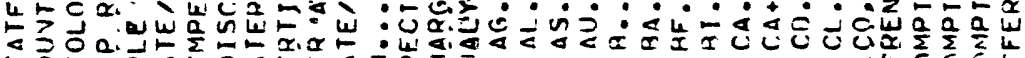

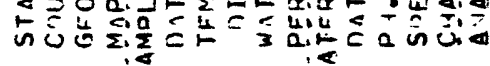


$\stackrel{3}{0}$
$\frac{0}{0}$
$\frac{0}{a}$
$a$

马ี

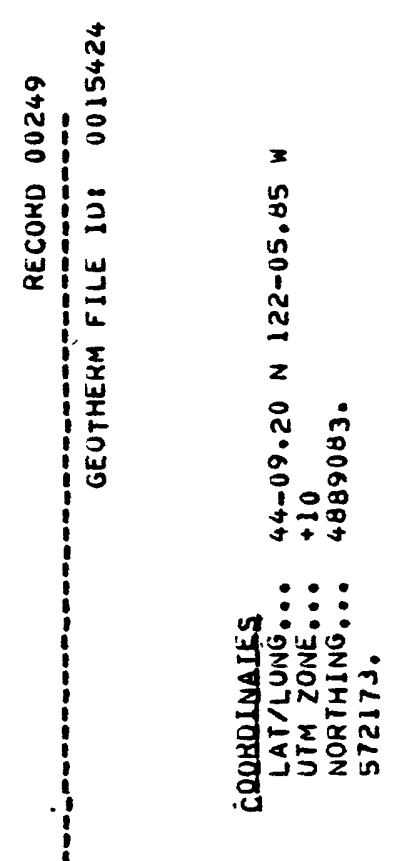

寻

\section{$\div$}

落:

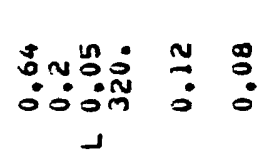

$::::::$ :

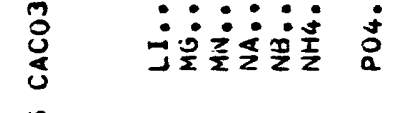

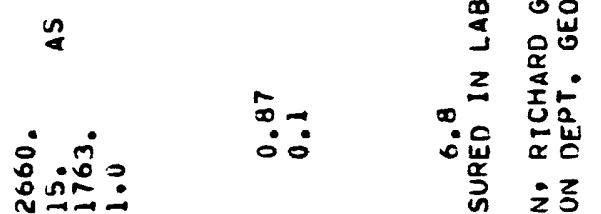

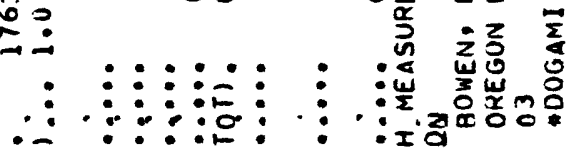

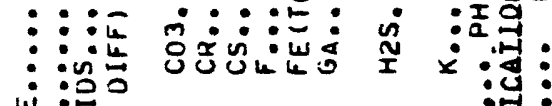

岁: :

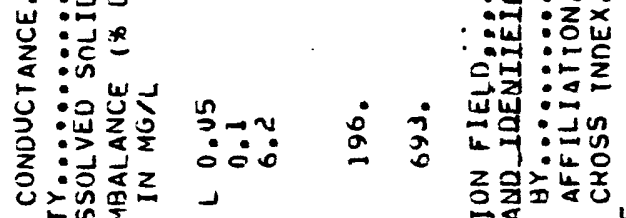

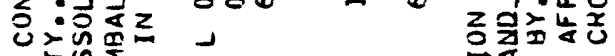

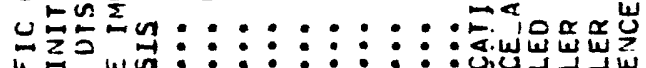

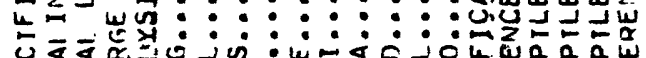

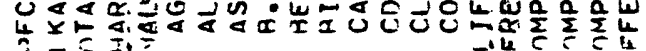

的手政

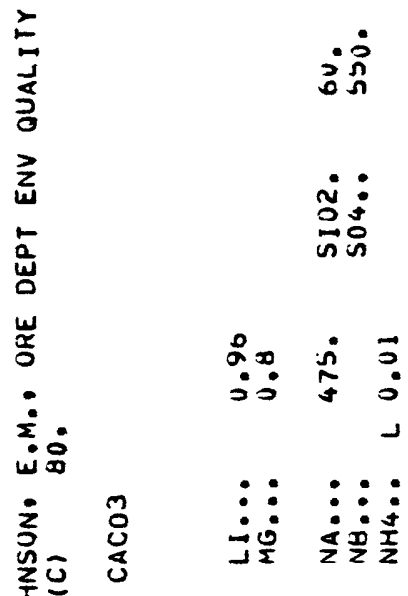

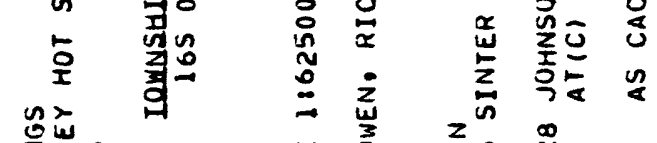

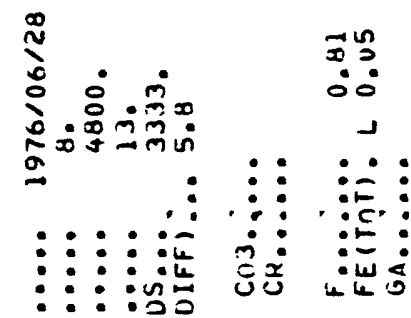

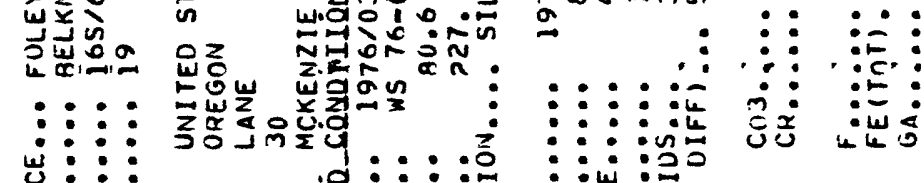

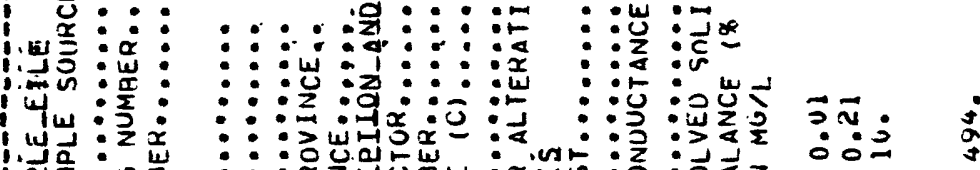

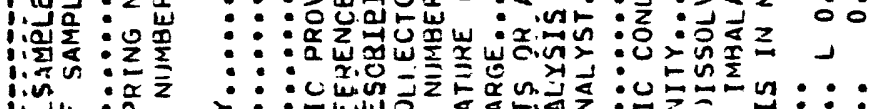

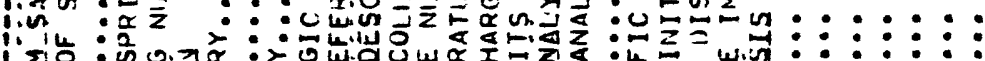

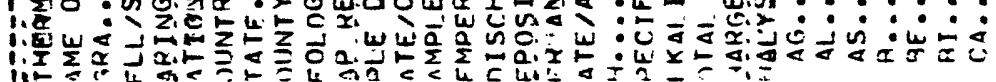

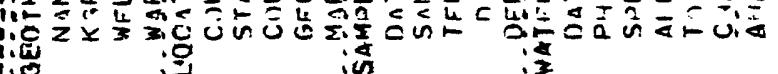




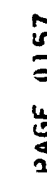

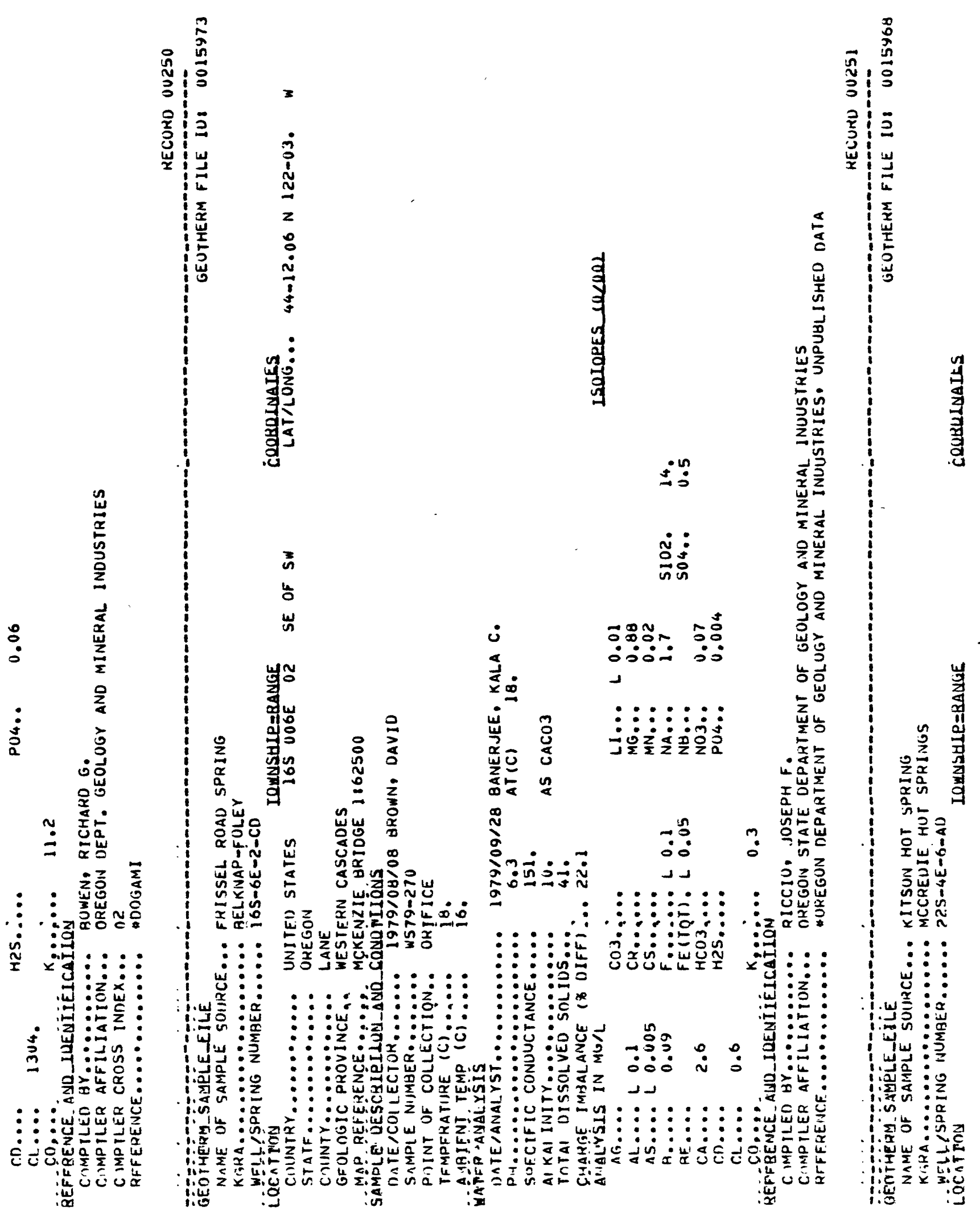



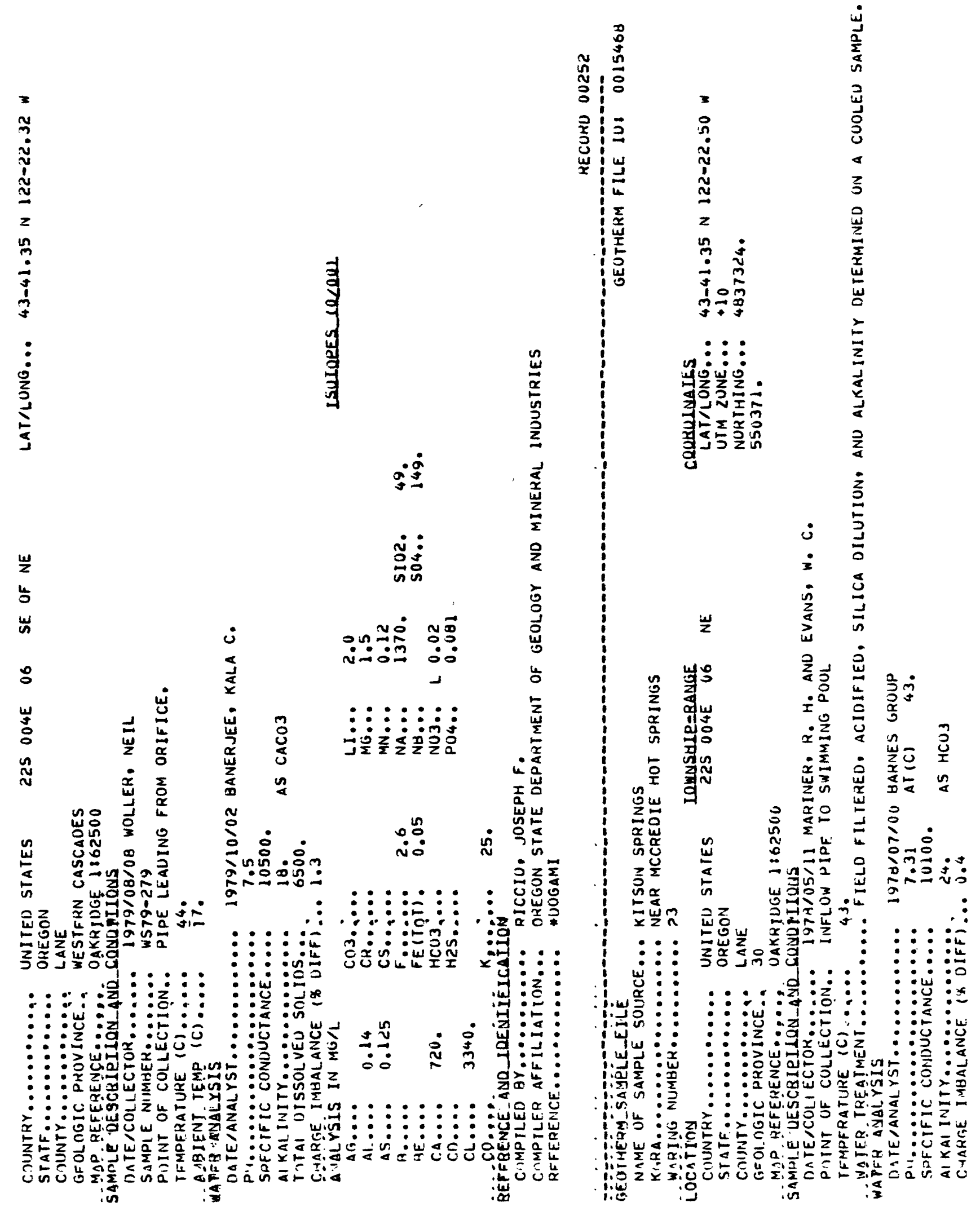

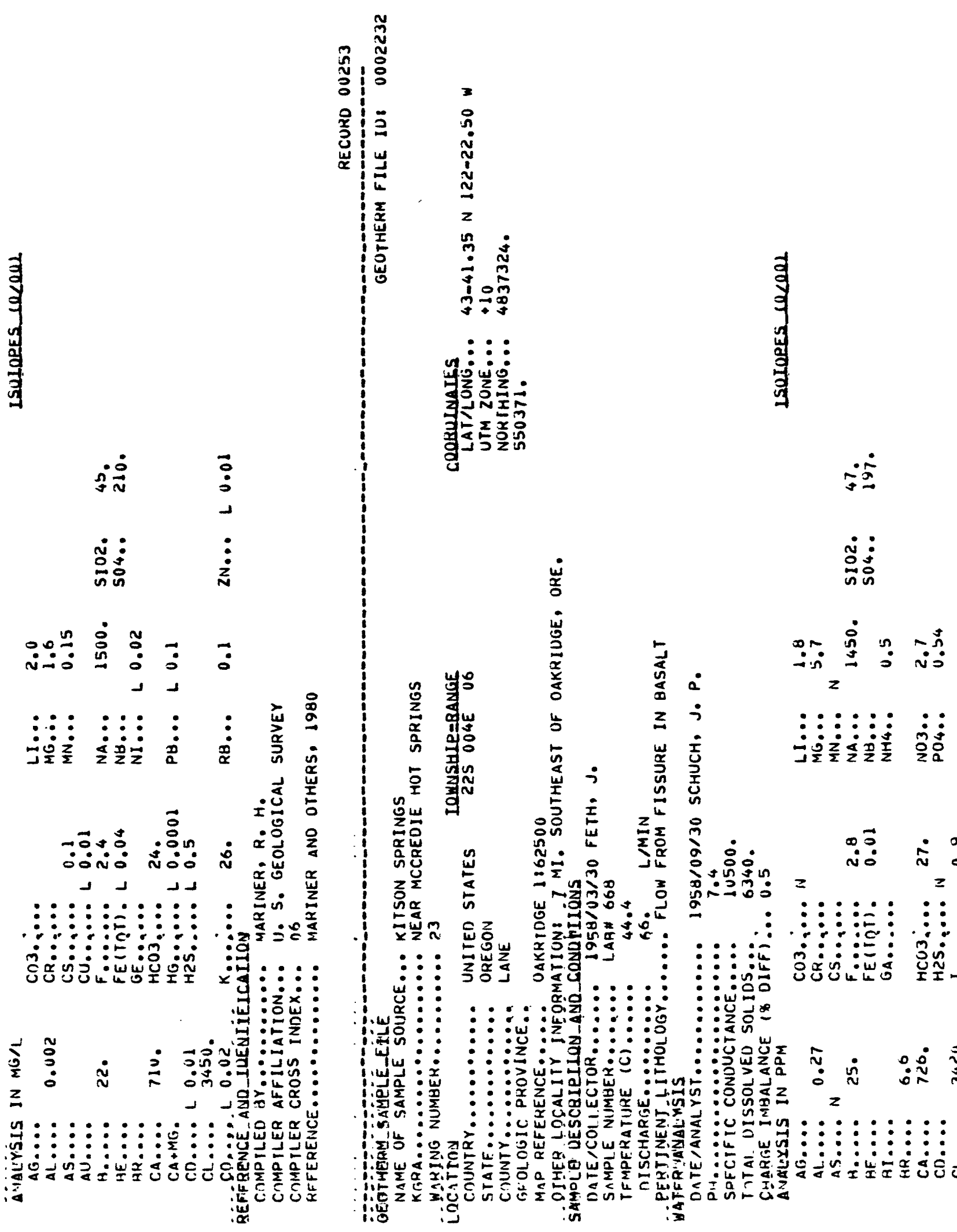

象空

送岕

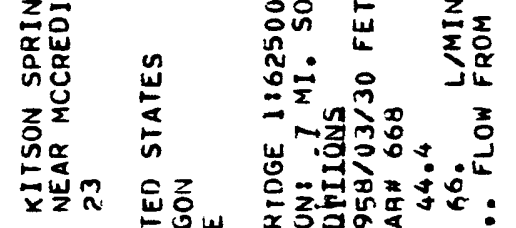

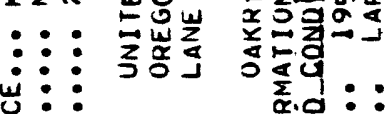

$$
\begin{aligned}
& \text { 我 } \\
& \text { 范宫 }
\end{aligned}
$$

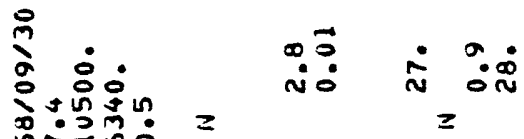

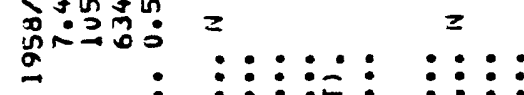

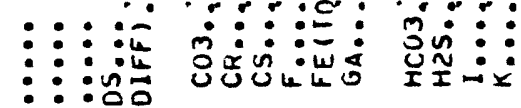
仙然: : : : : : : :

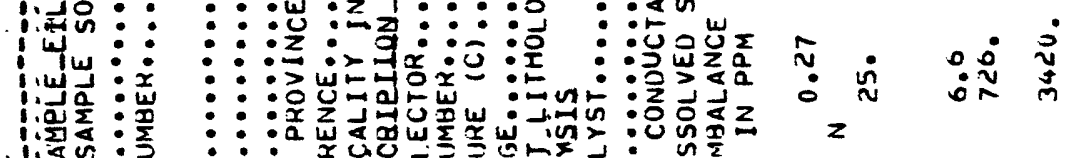

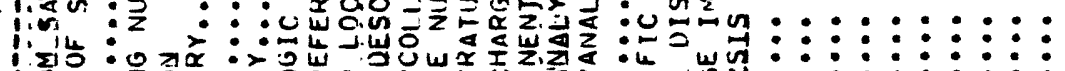

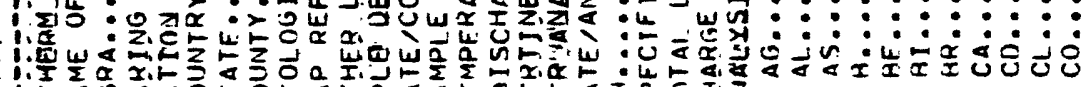

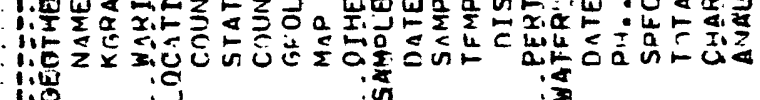




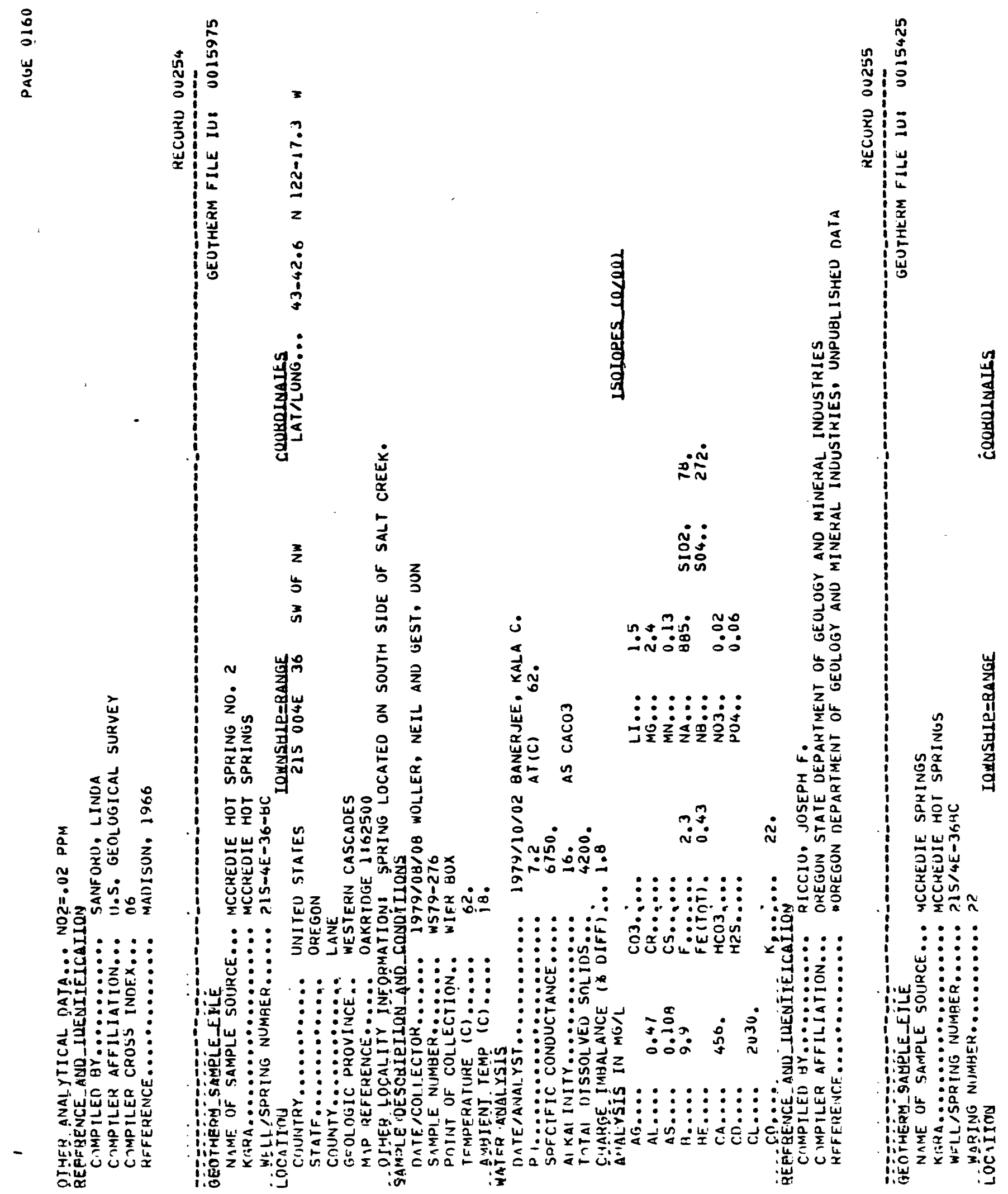




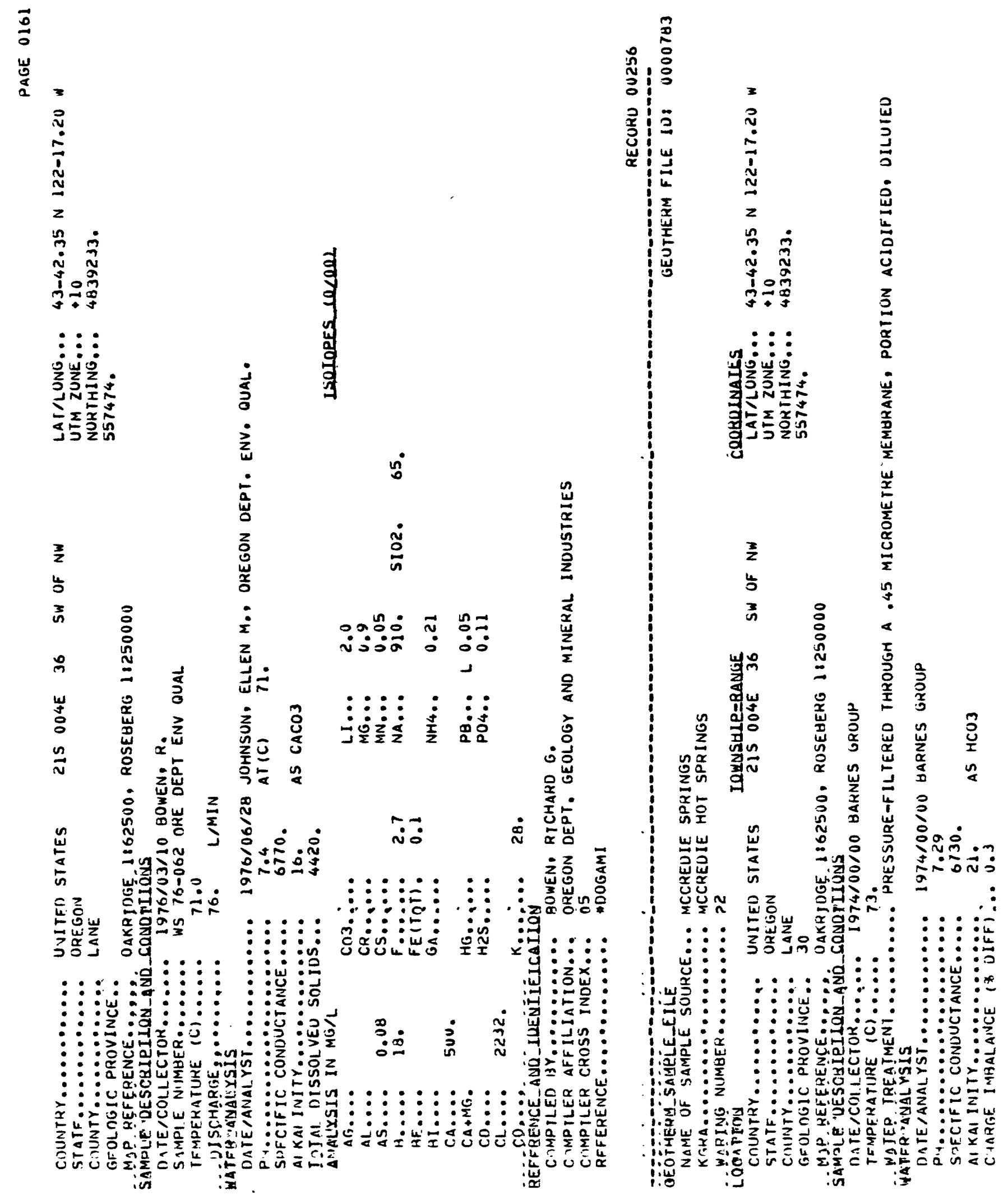



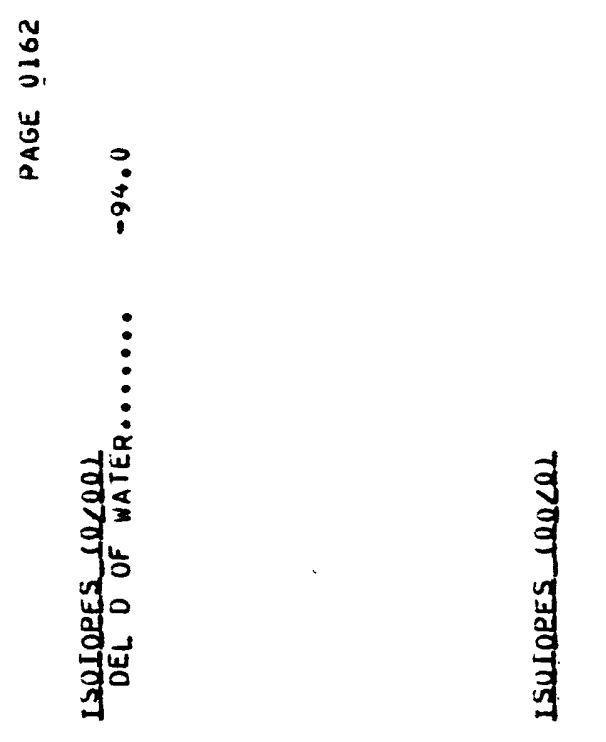

$$
\begin{aligned}
& \text { วับ } \\
& \text { 药 }
\end{aligned}
$$

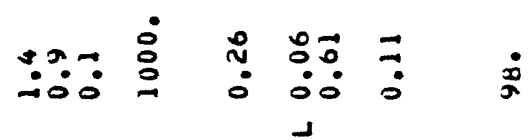

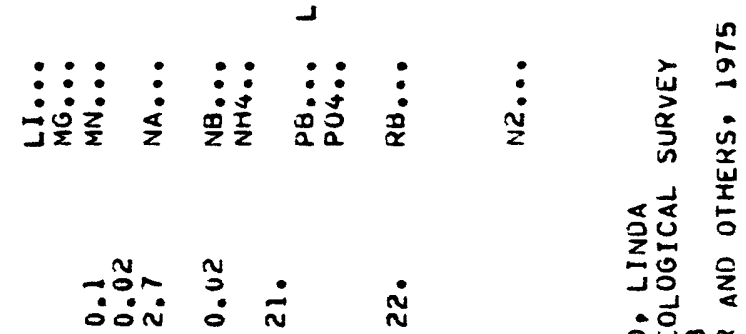
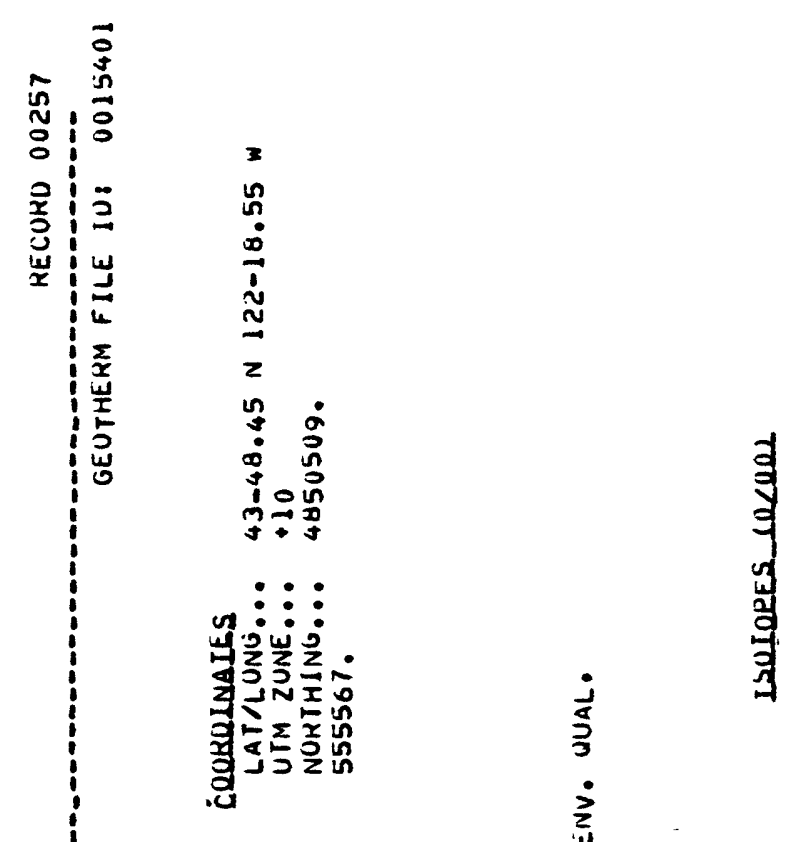

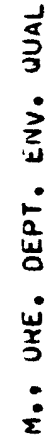

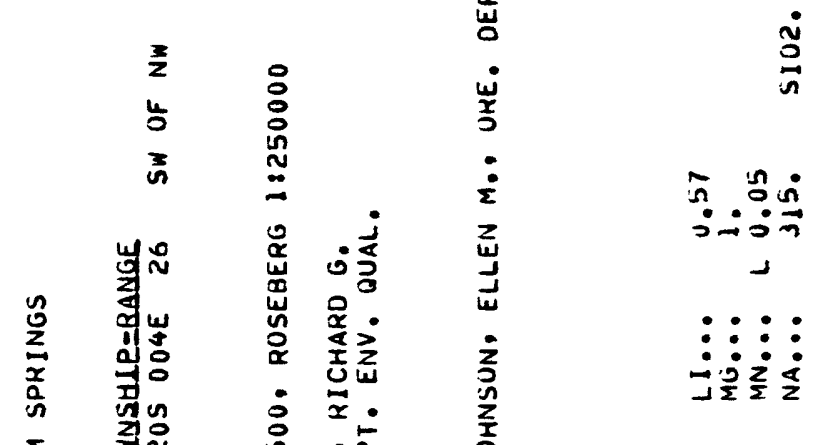
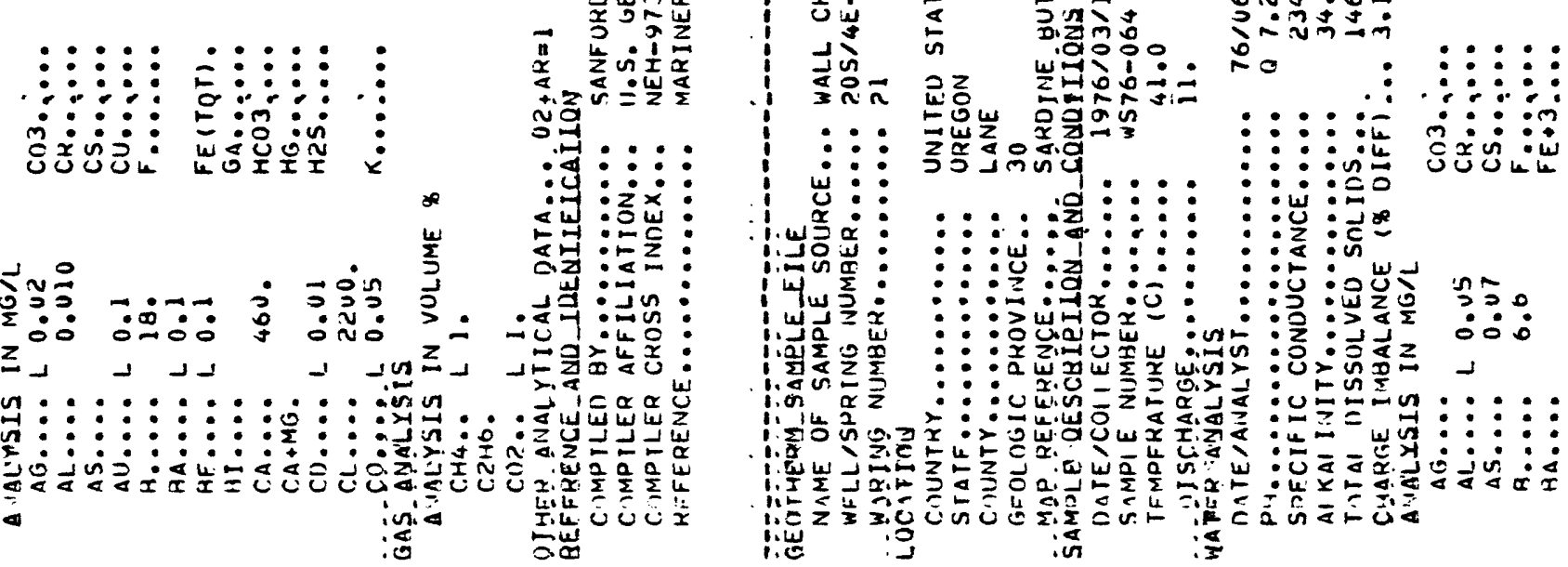
3
0
0
$\omega$
0
0
$a$

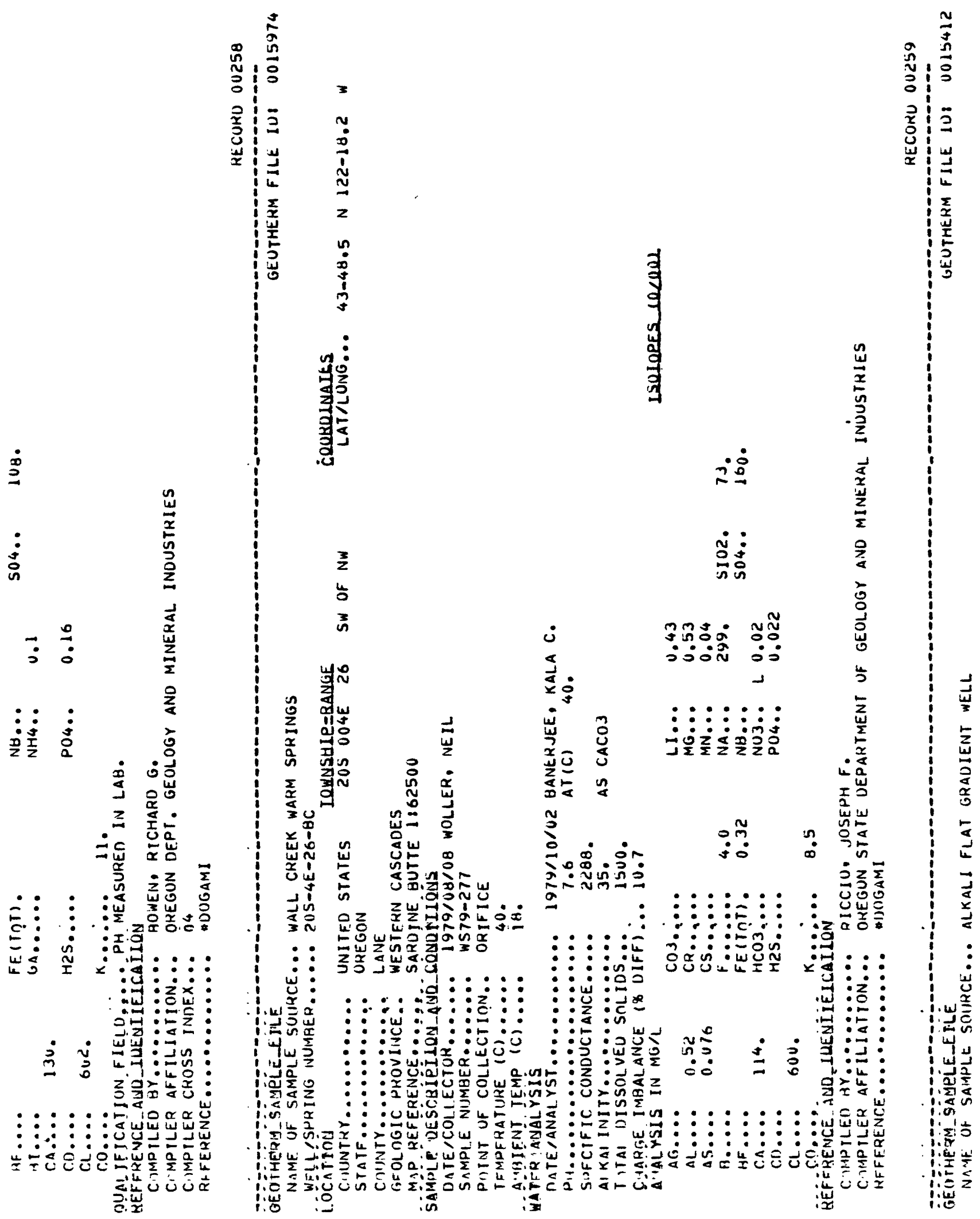


:
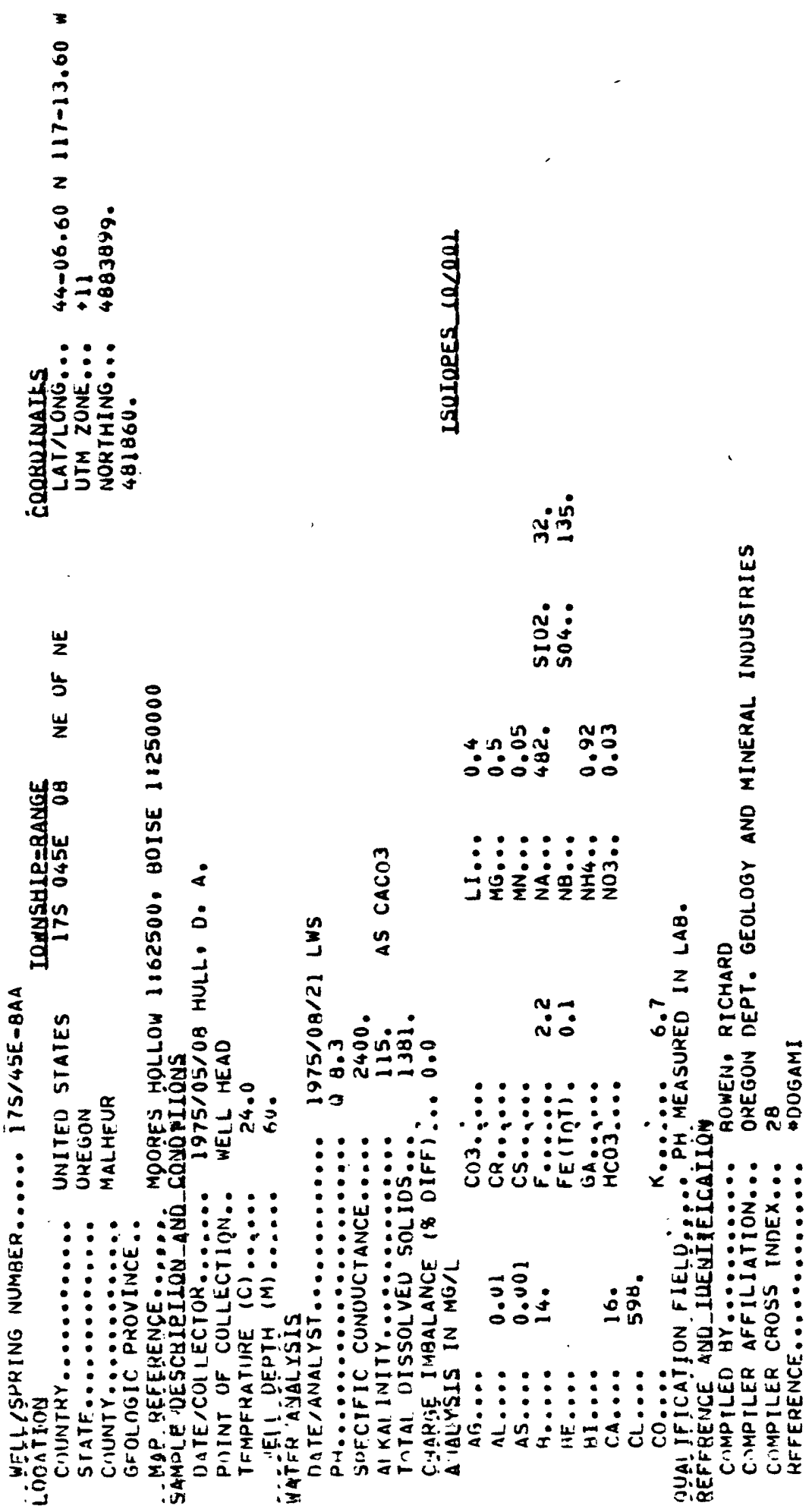
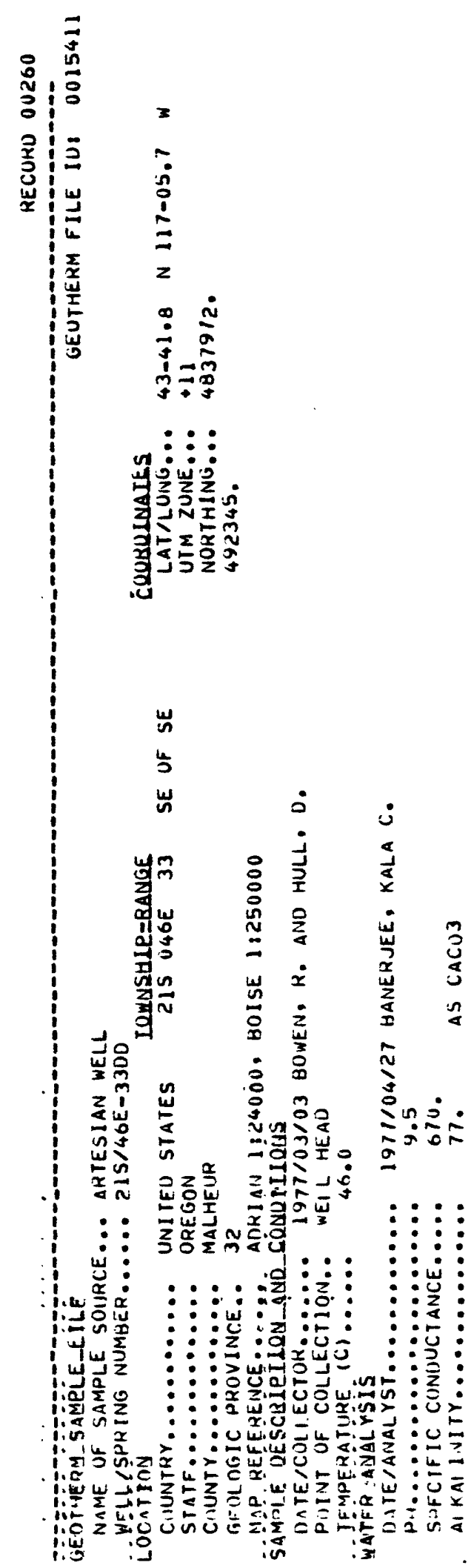


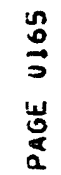

范
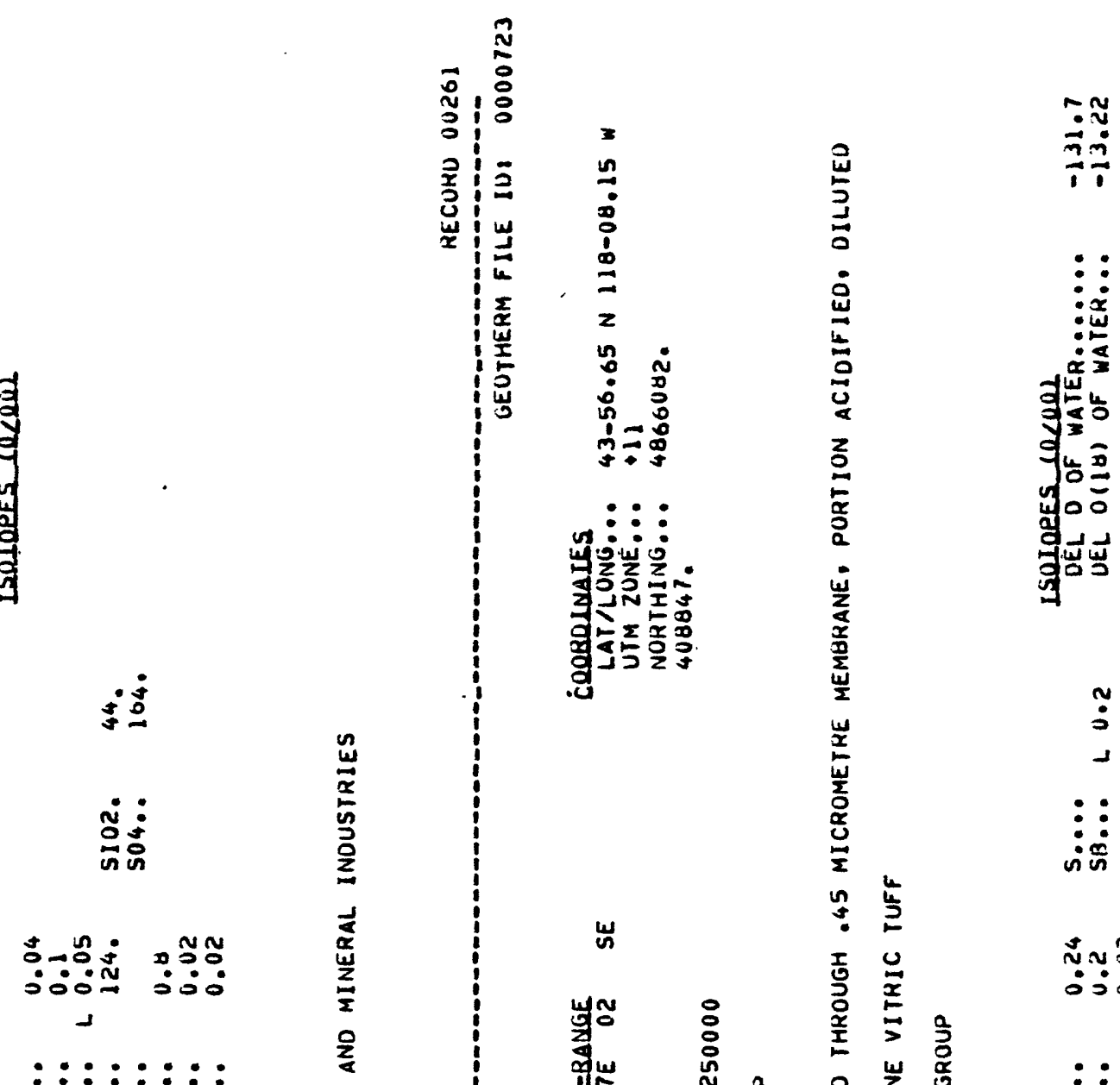

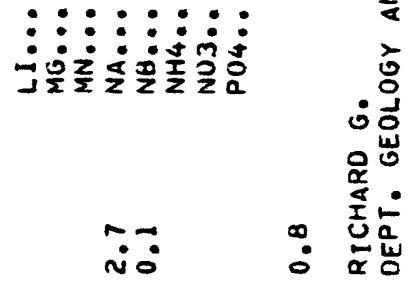
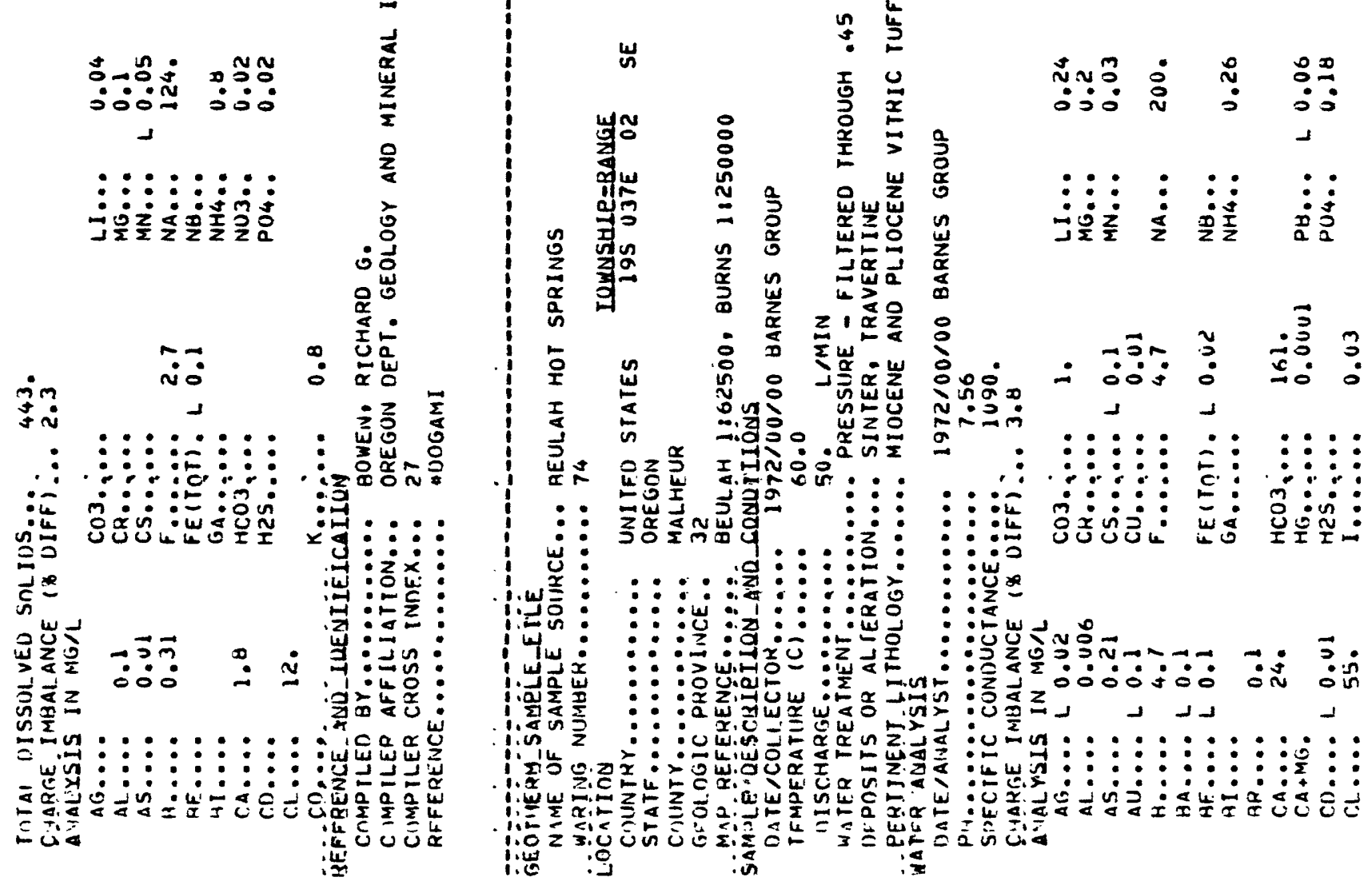
$:$
0
0
$\vdots$
0
2
2
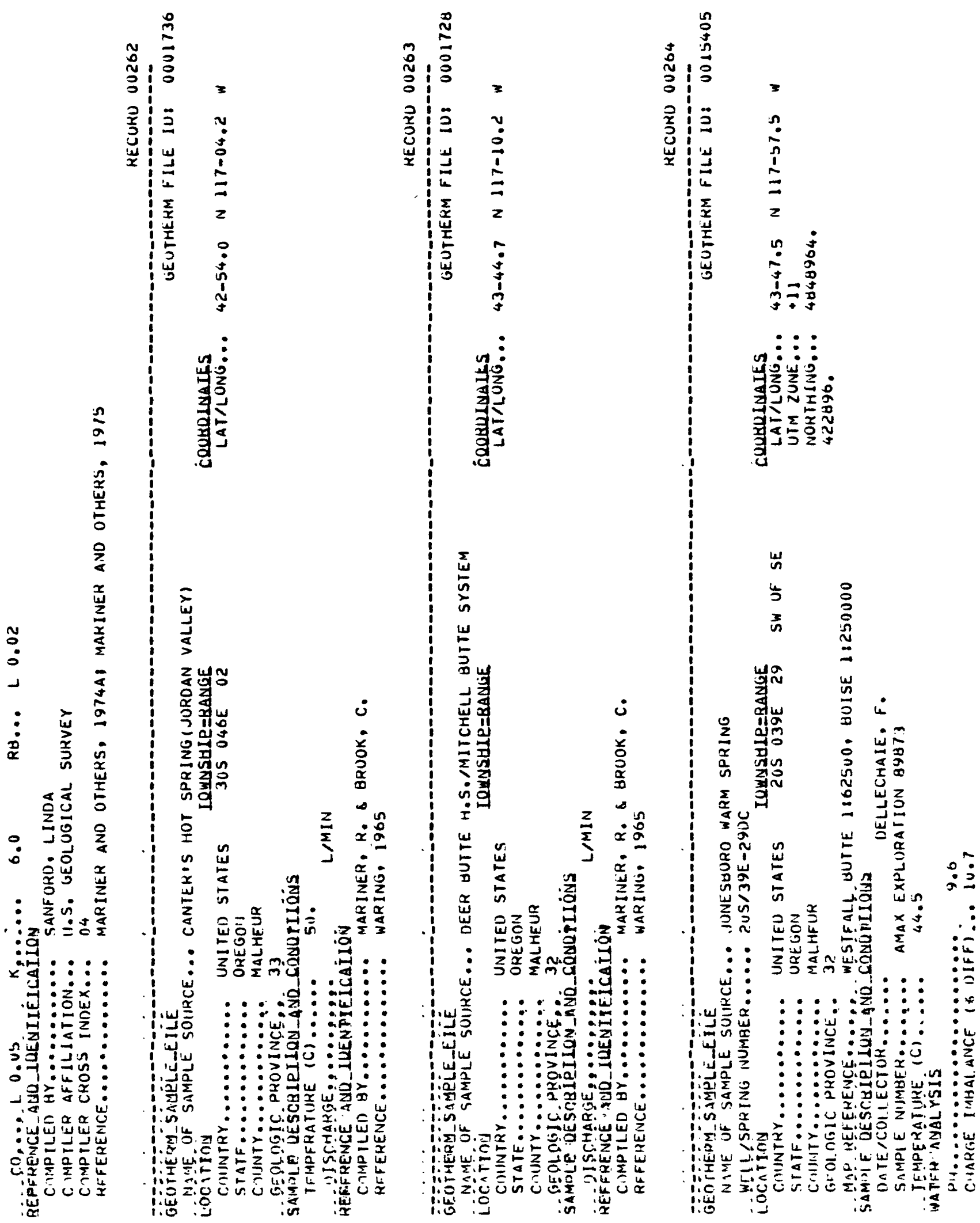
$\hat{0}$
0
0
0
0
0

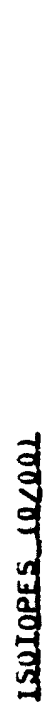

3
3
3
3
3
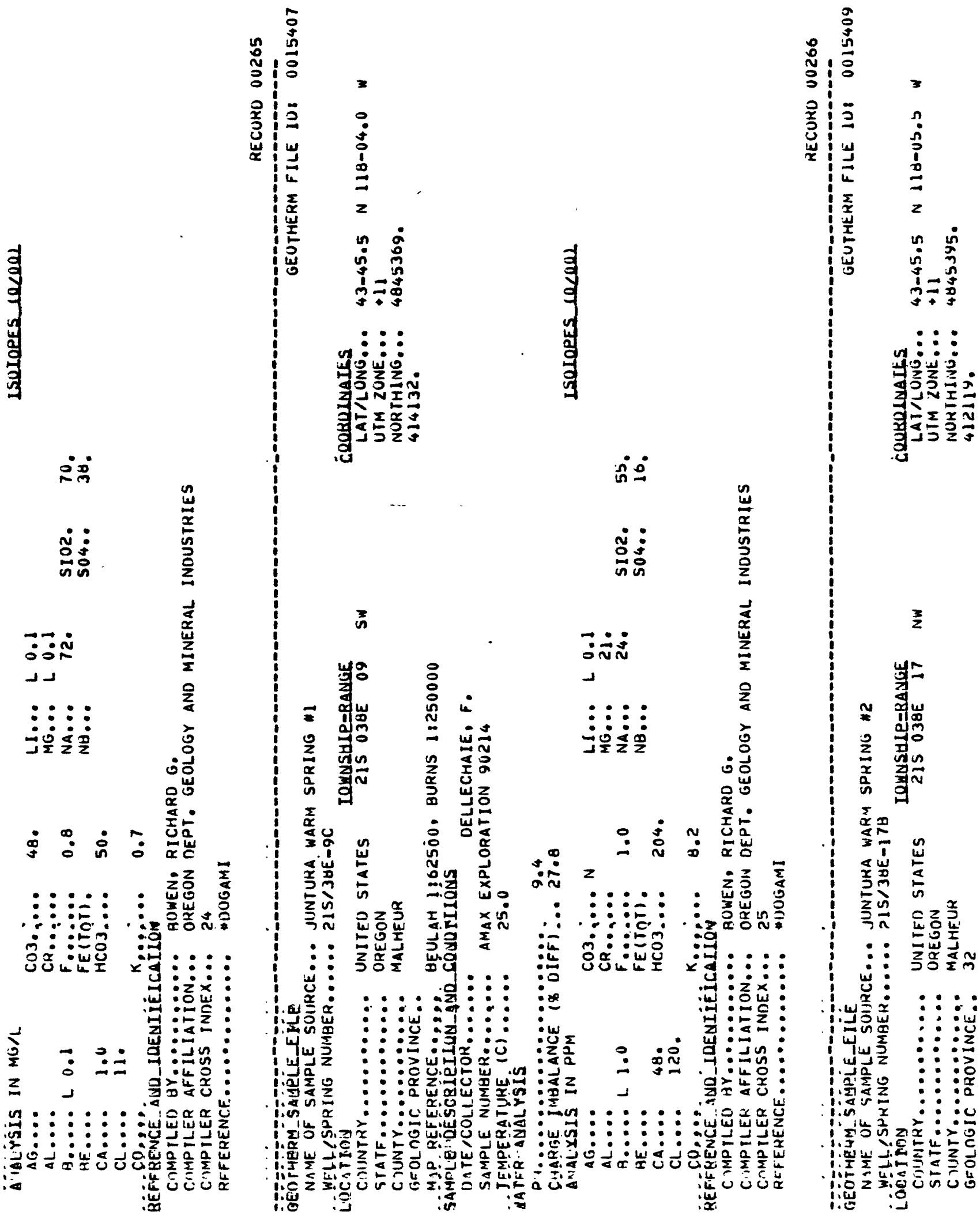
0
0
0
0
0
0
2
2

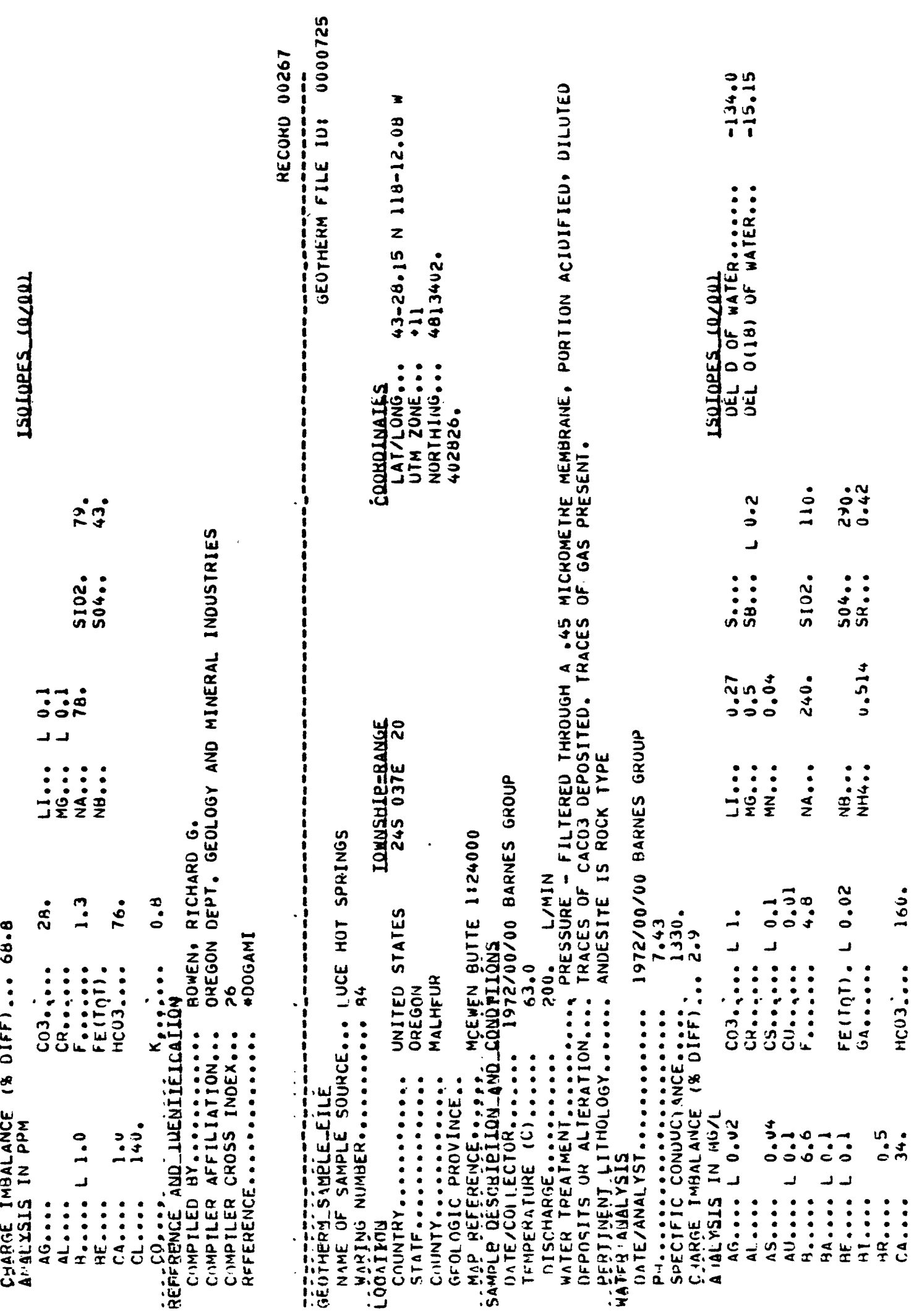


$\frac{2}{0 !}$

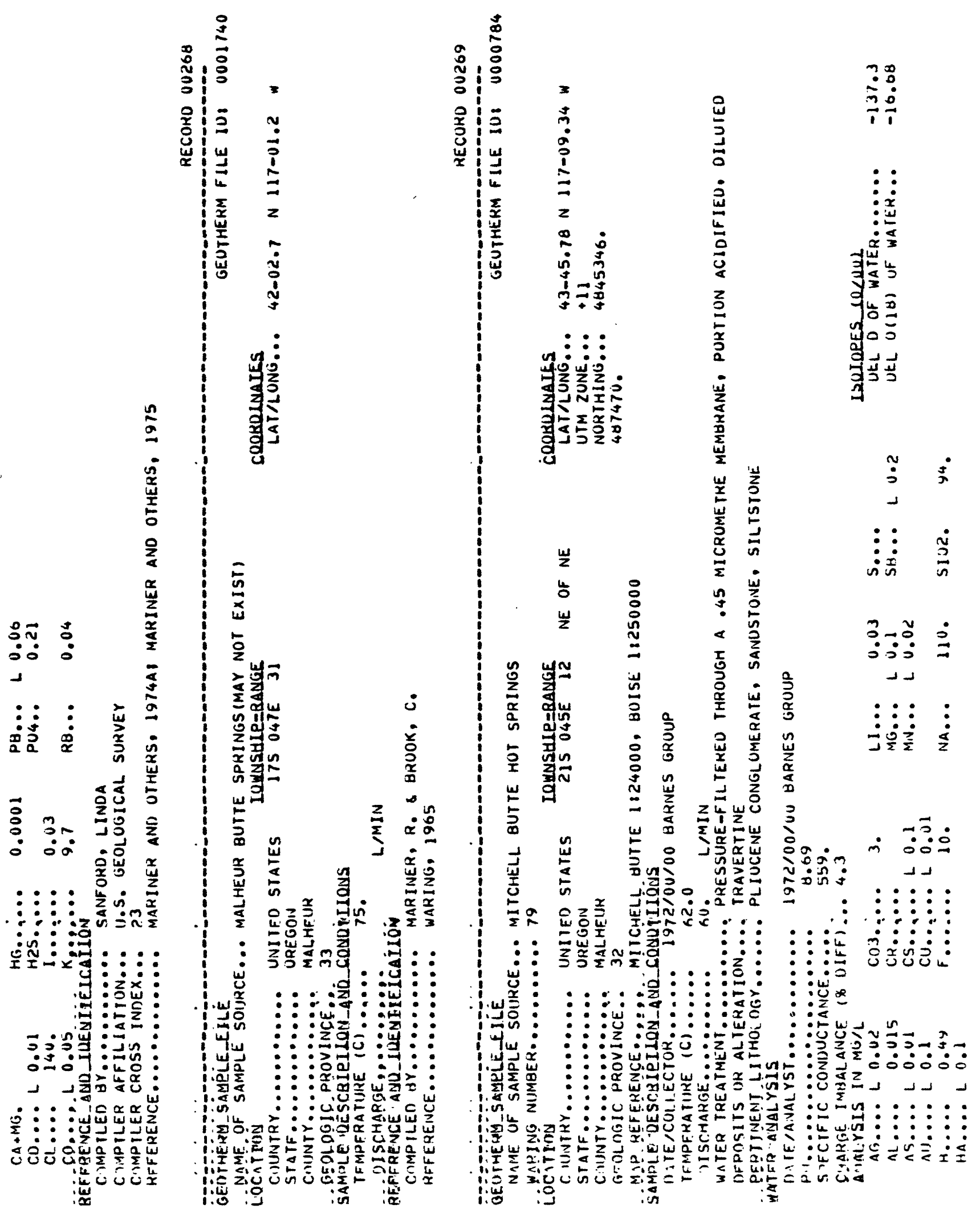


0
5
5
$\frac{5}{0}$
2
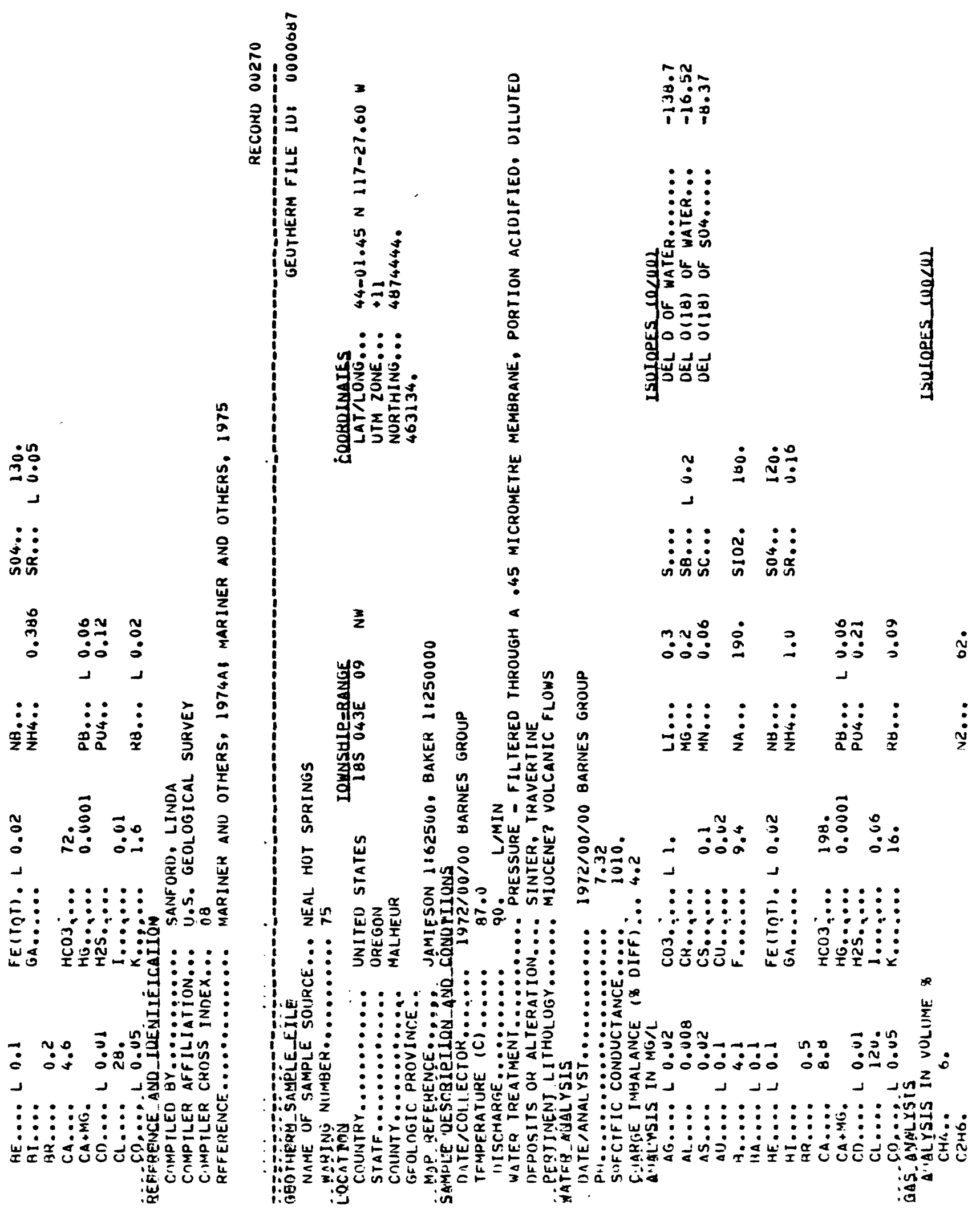
$\vec{\vdots}$
0
$\underline{3}$
$\vdots$
2

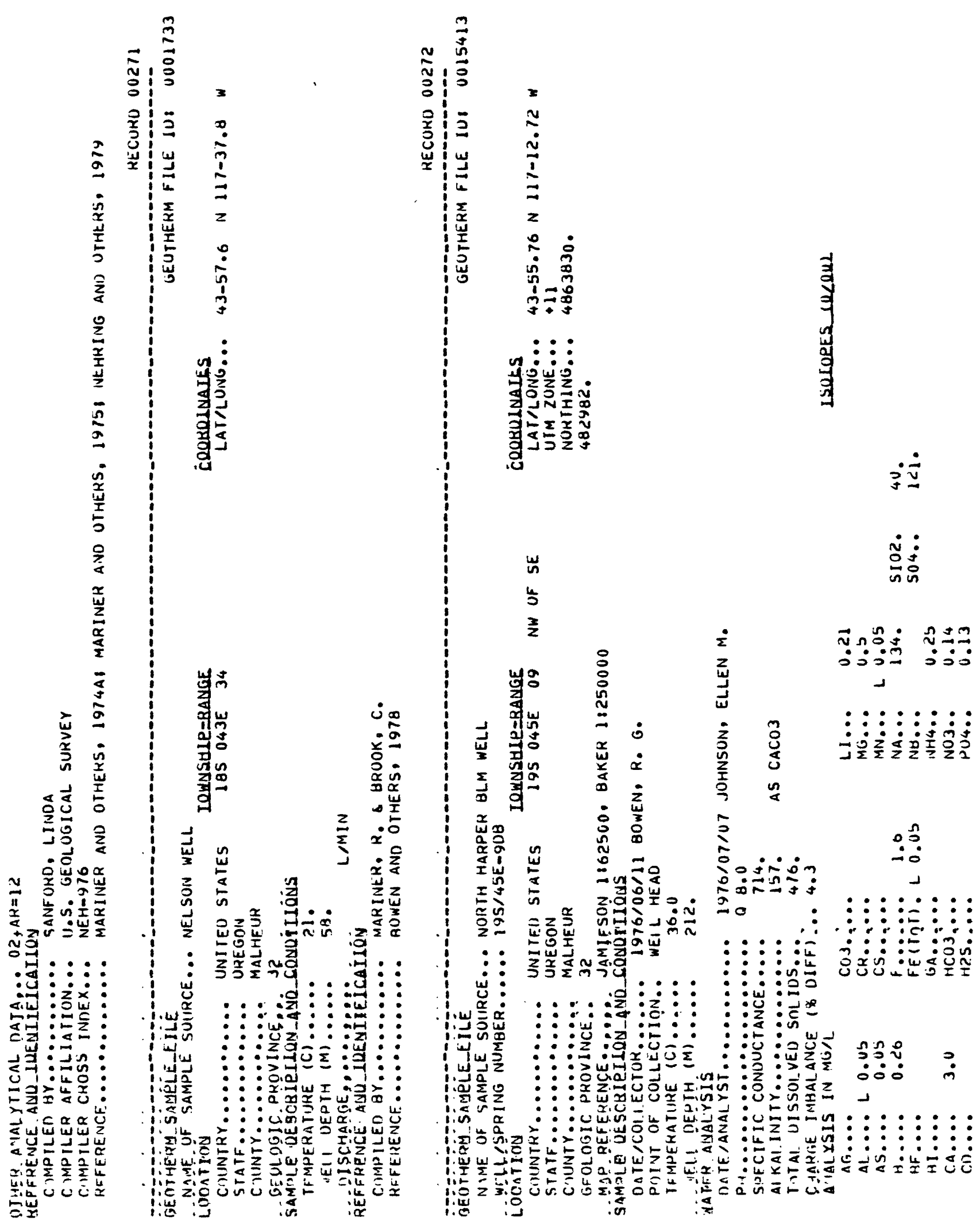




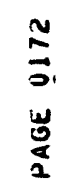
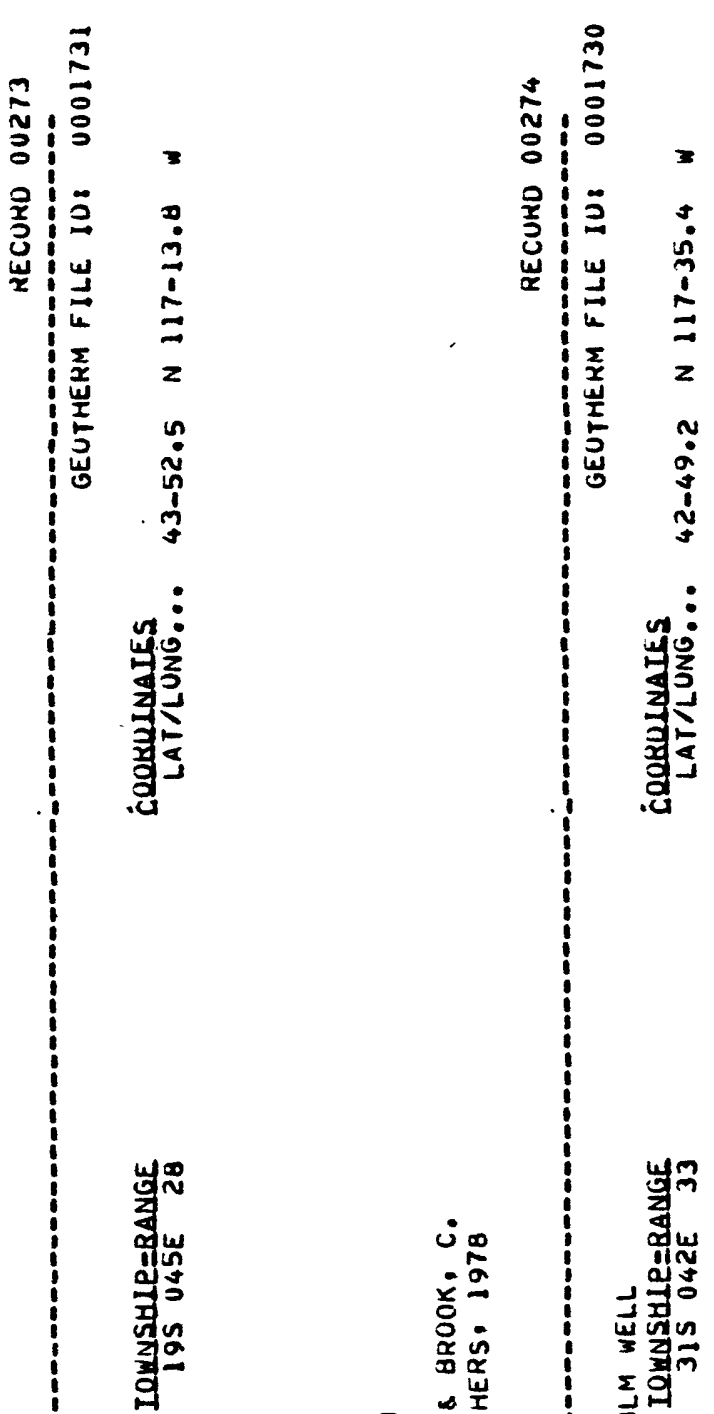

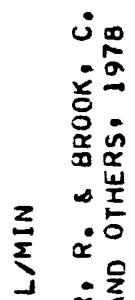

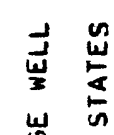

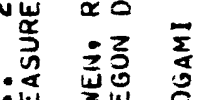

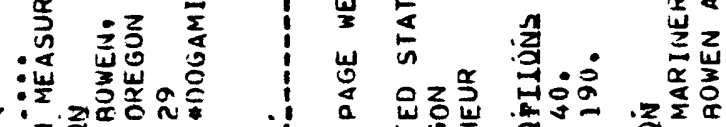

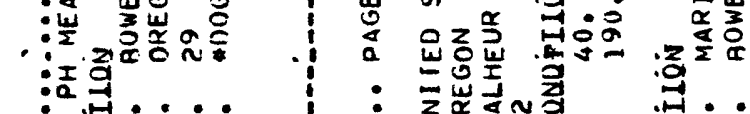

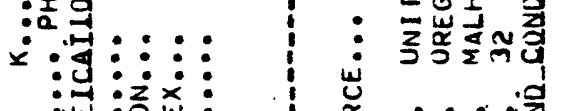

岁:

:

o븐 : $\frac{c}{2}:$

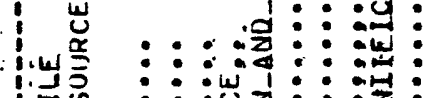

紧 $: \vdots:$ :

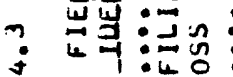

ب.

:

$:$ : : व.'

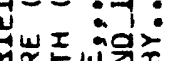

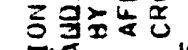

lid : : : : :

: : :

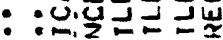

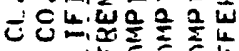

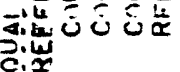

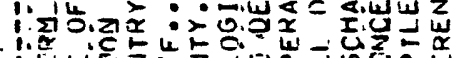

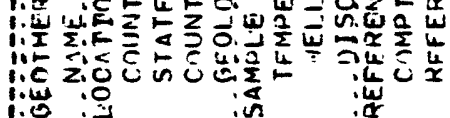
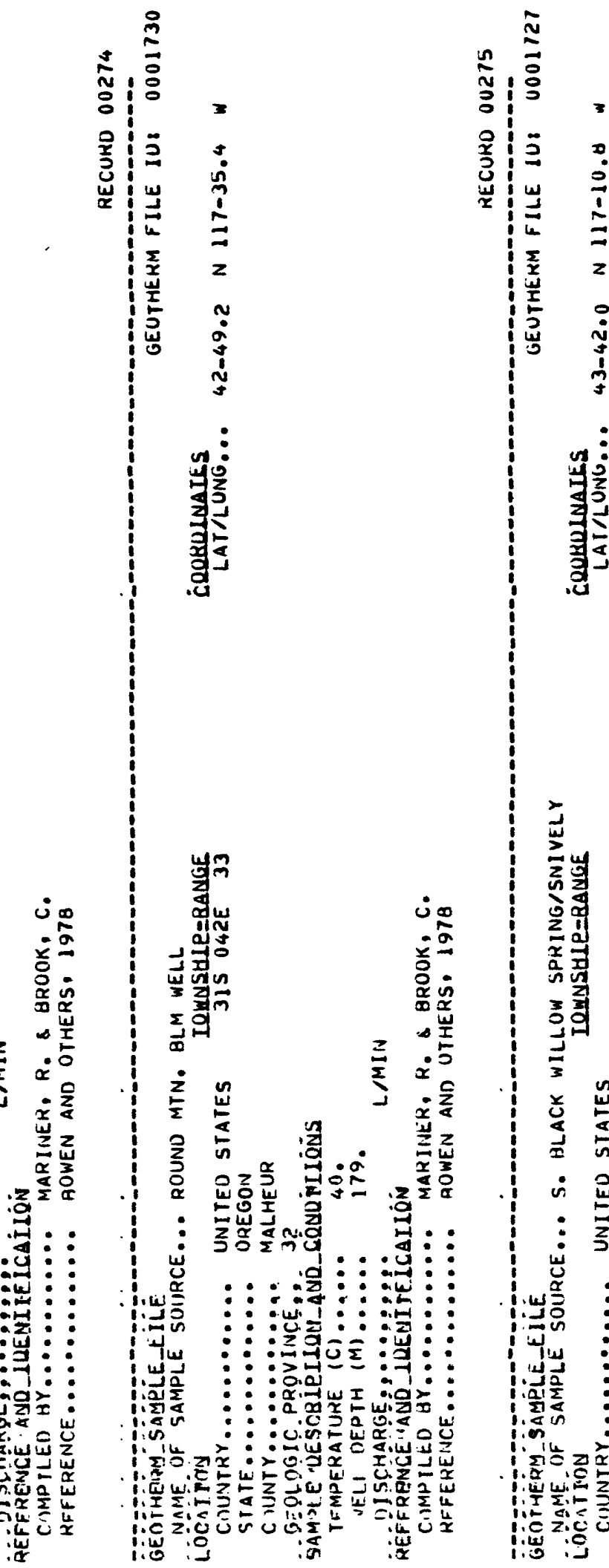

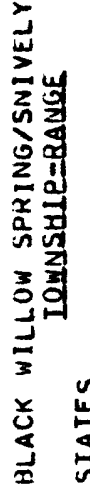

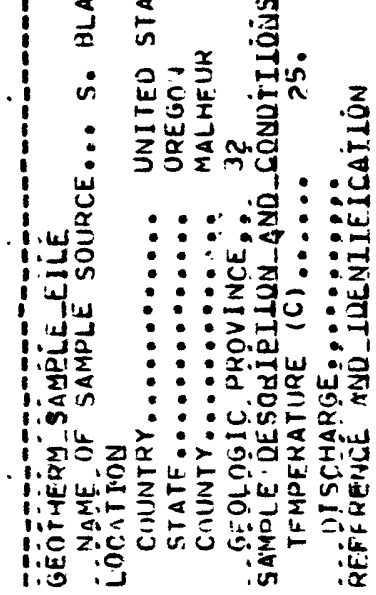



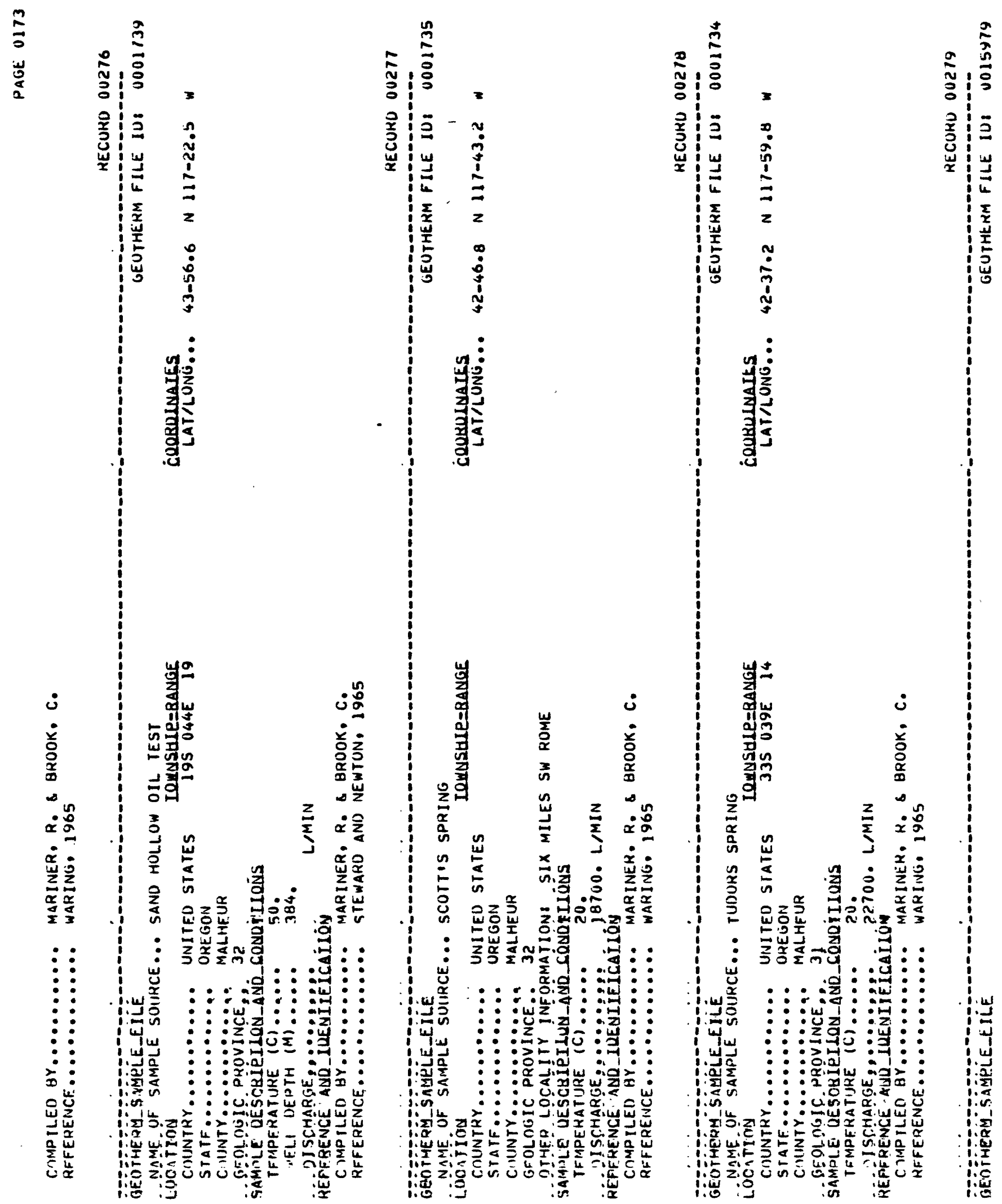
$\frac{2}{\partial !}$

量
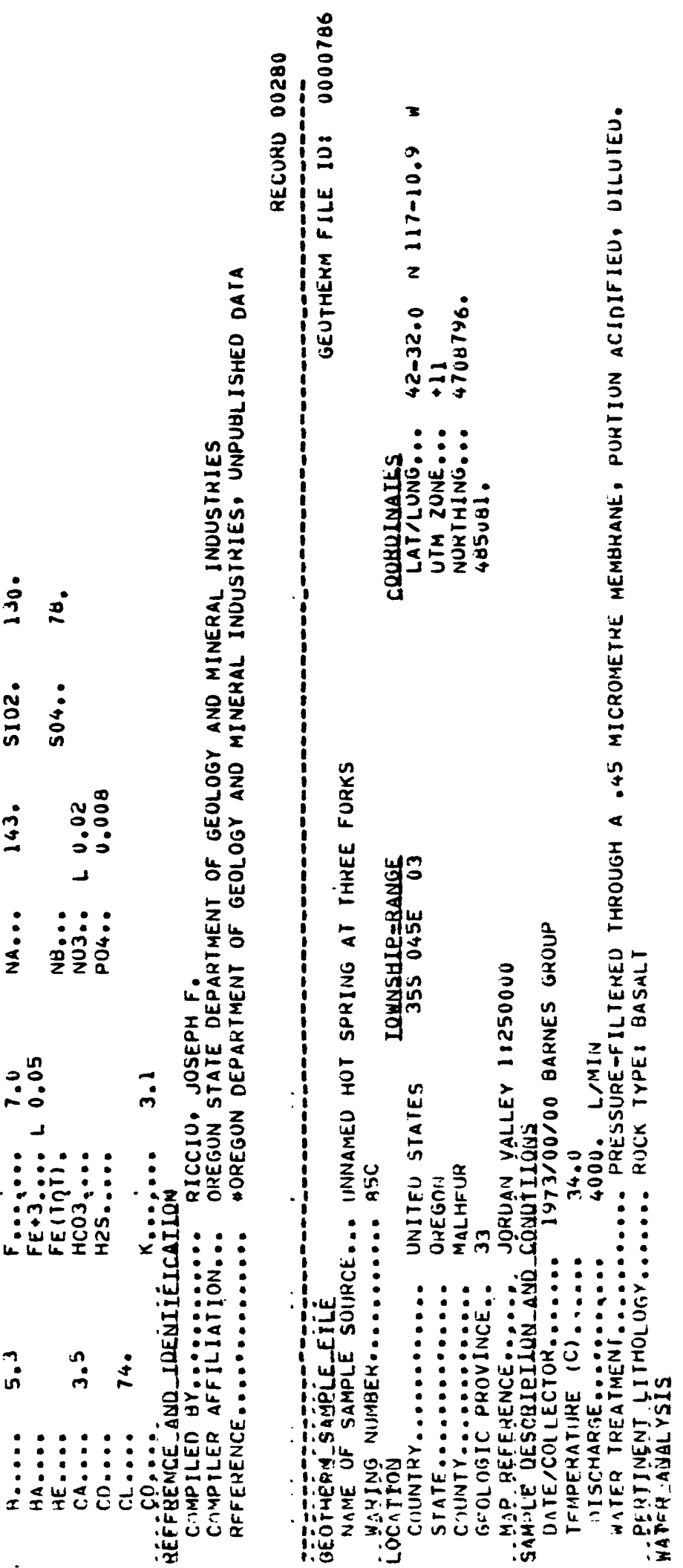
$\stackrel{1}{a}$
0
0
0
0

บุ:
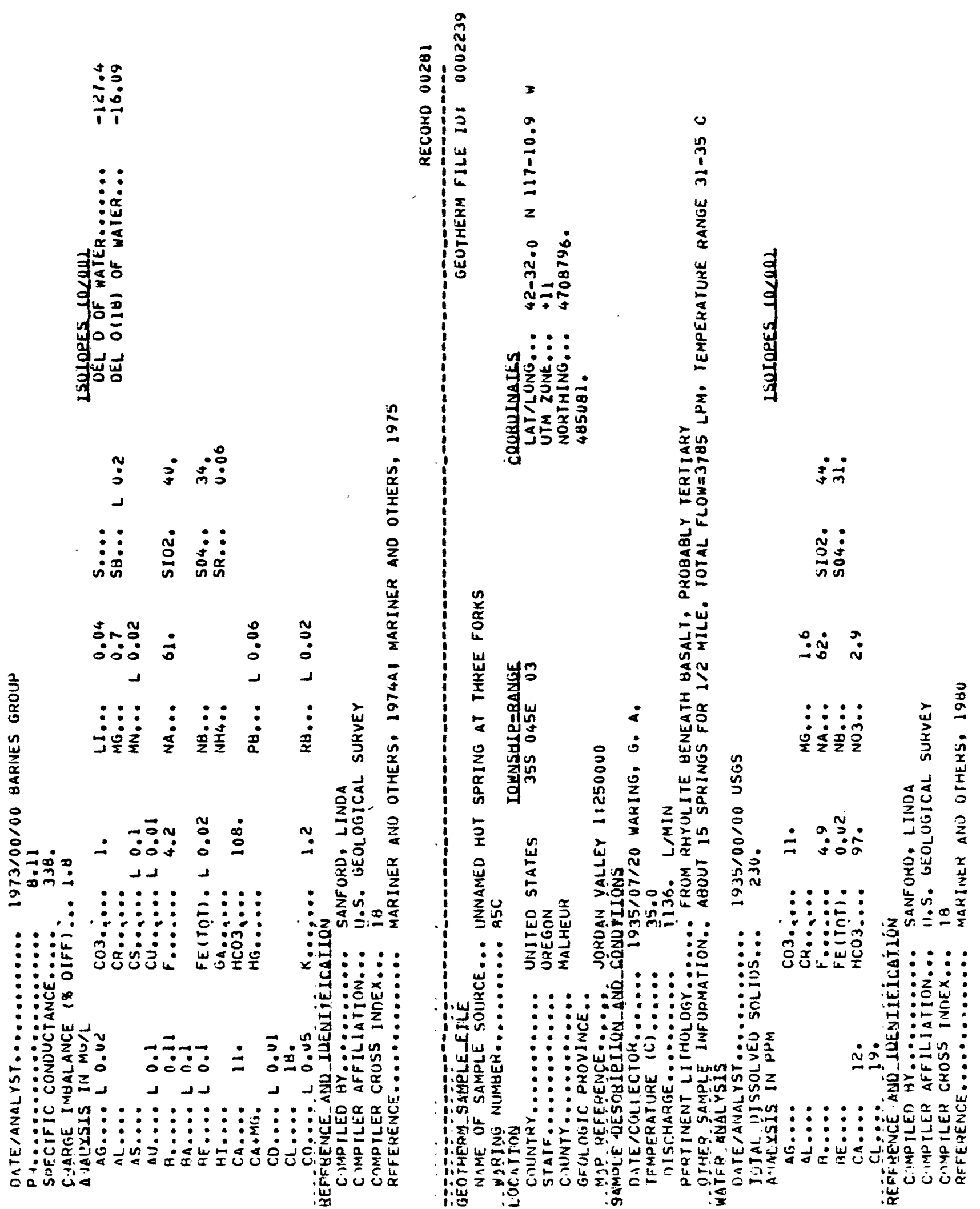
$\stackrel{0}{a}$

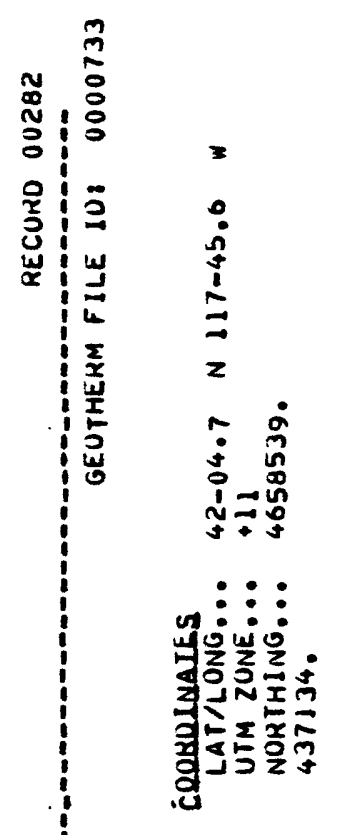

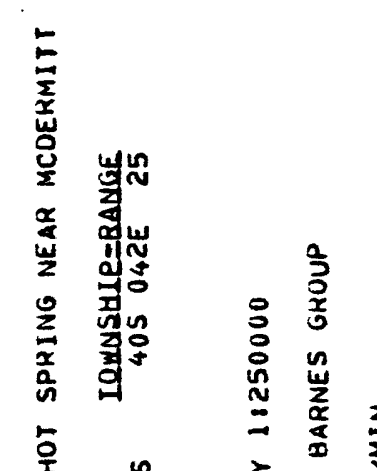

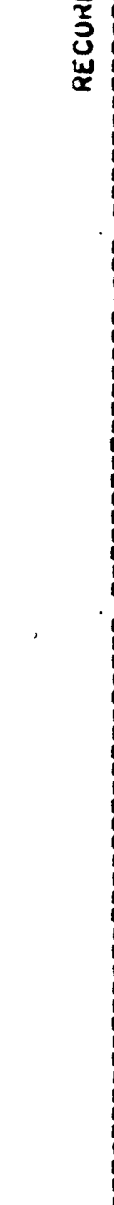

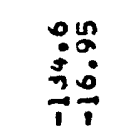

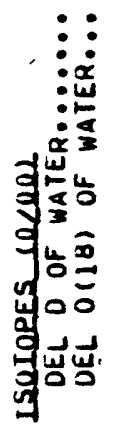

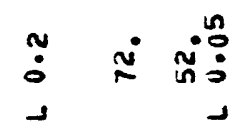

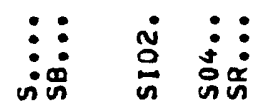

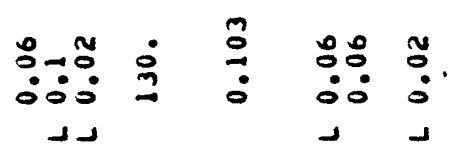

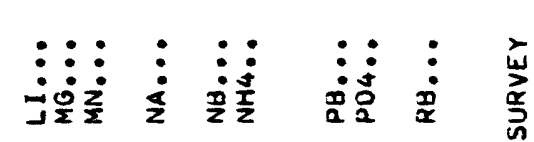
$\rightarrow$ 压

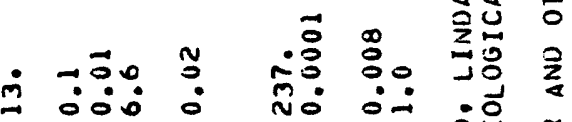

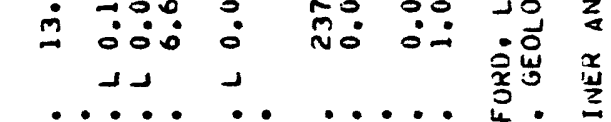

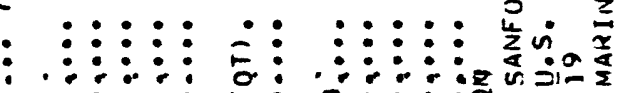
: : : :

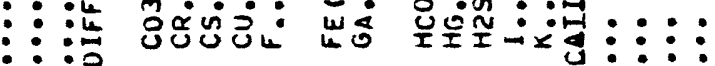
: : : : $::$

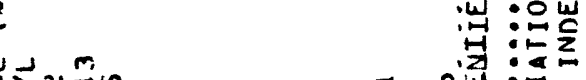
(1): :行: : : :

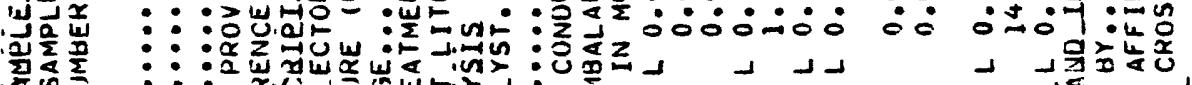

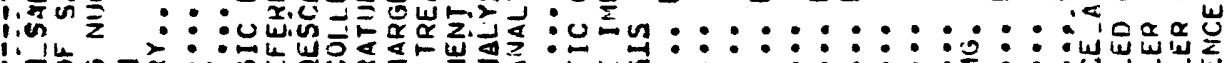

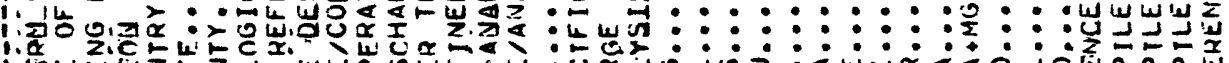

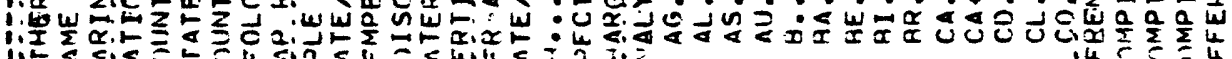

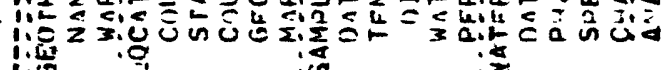

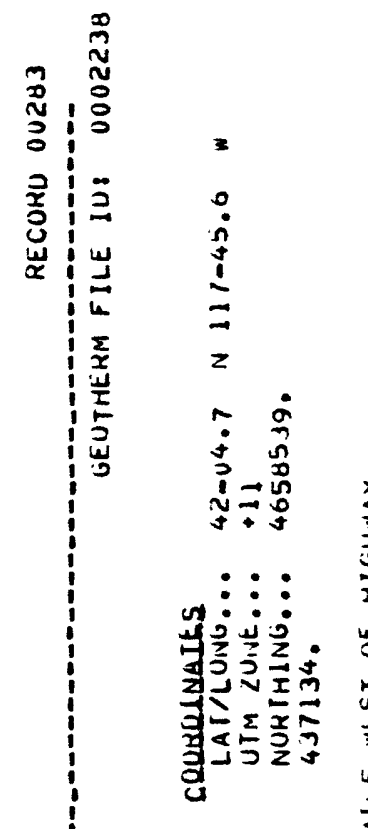

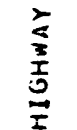
\begin{tabular}{l}
5 \\
5 \\
5 \\
3 \\
4 \\
\hline \\
$\frac{1}{2}$
\end{tabular}

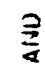$$
\stackrel{\vec{z}}{\underset{\Sigma}{\Sigma}}
$$

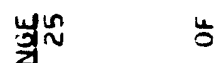

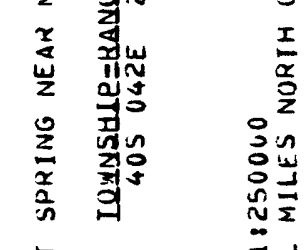

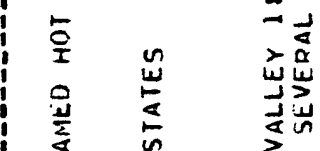

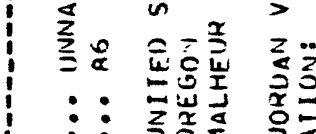

:

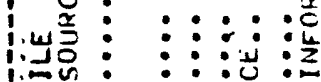

: : : : : : : : :

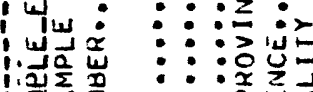

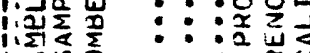

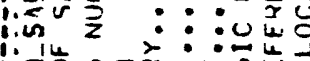
诲告

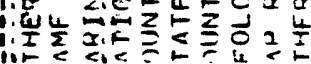

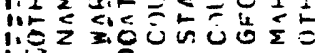


$\hat{1}$
0
4
$\frac{5}{2}$
2
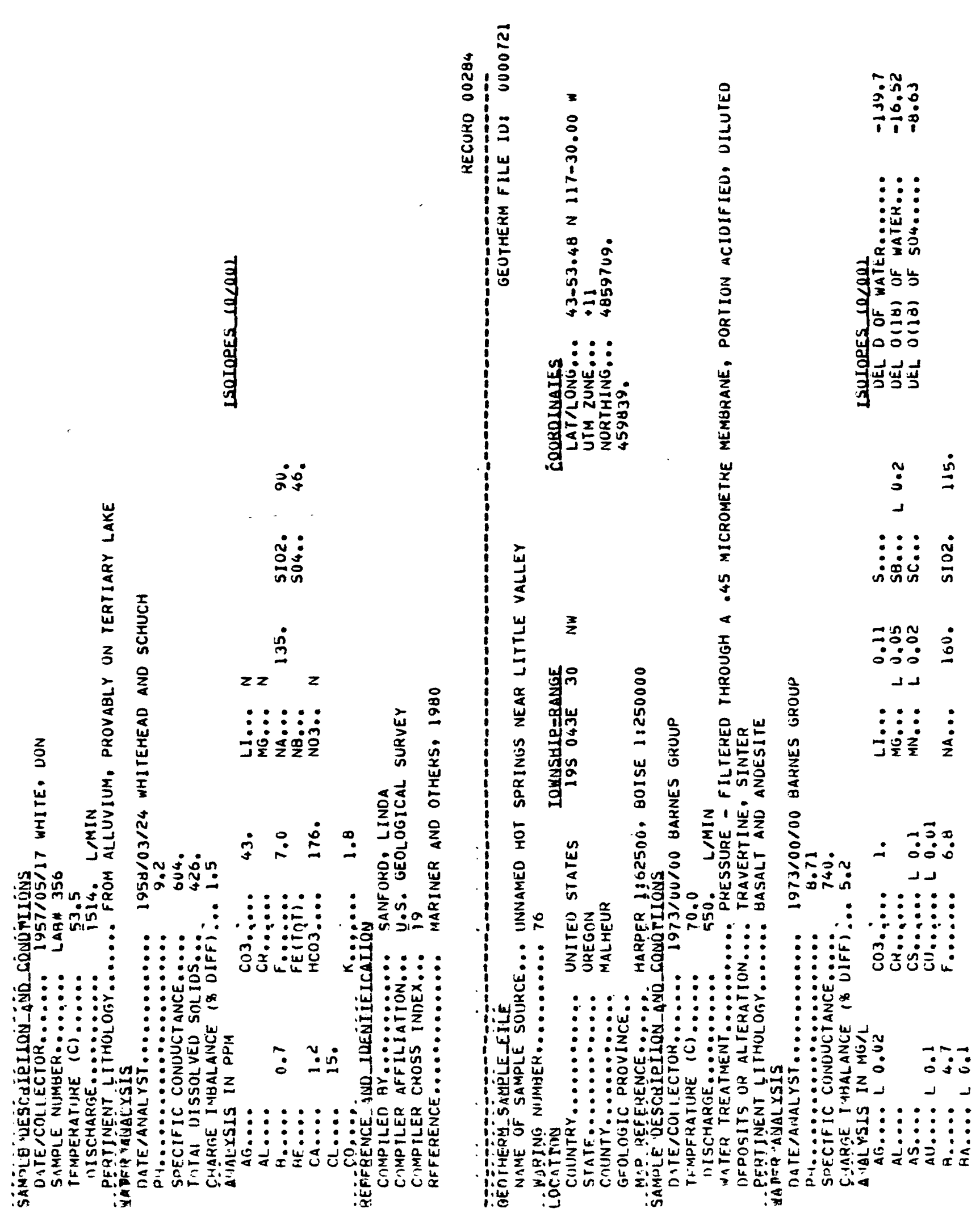
$\frac{\underline{z}}{3}$

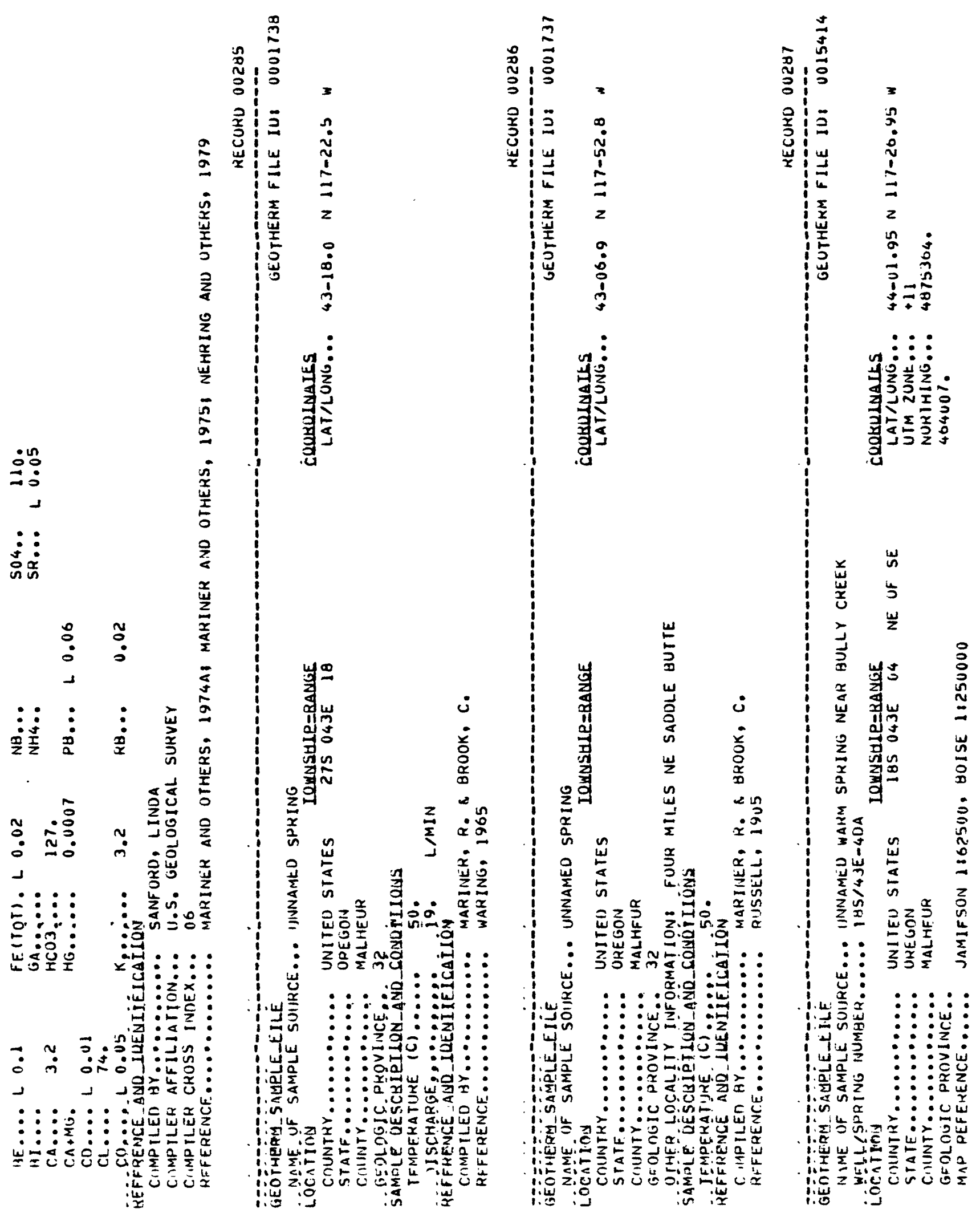




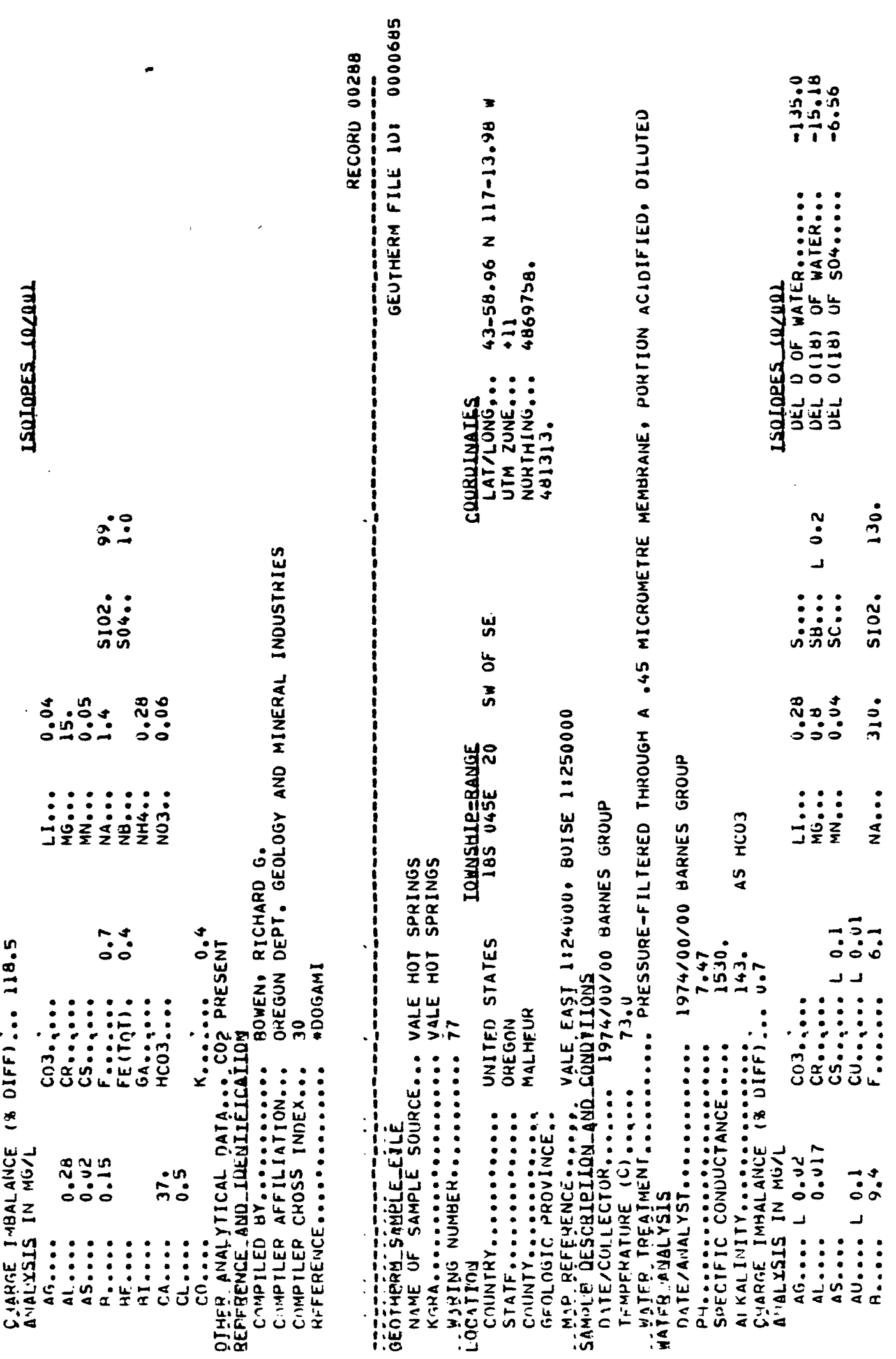


$\frac{1}{3}$
$\frac{1}{3}$
$\frac{3}{3}$
$\frac{3}{3}$

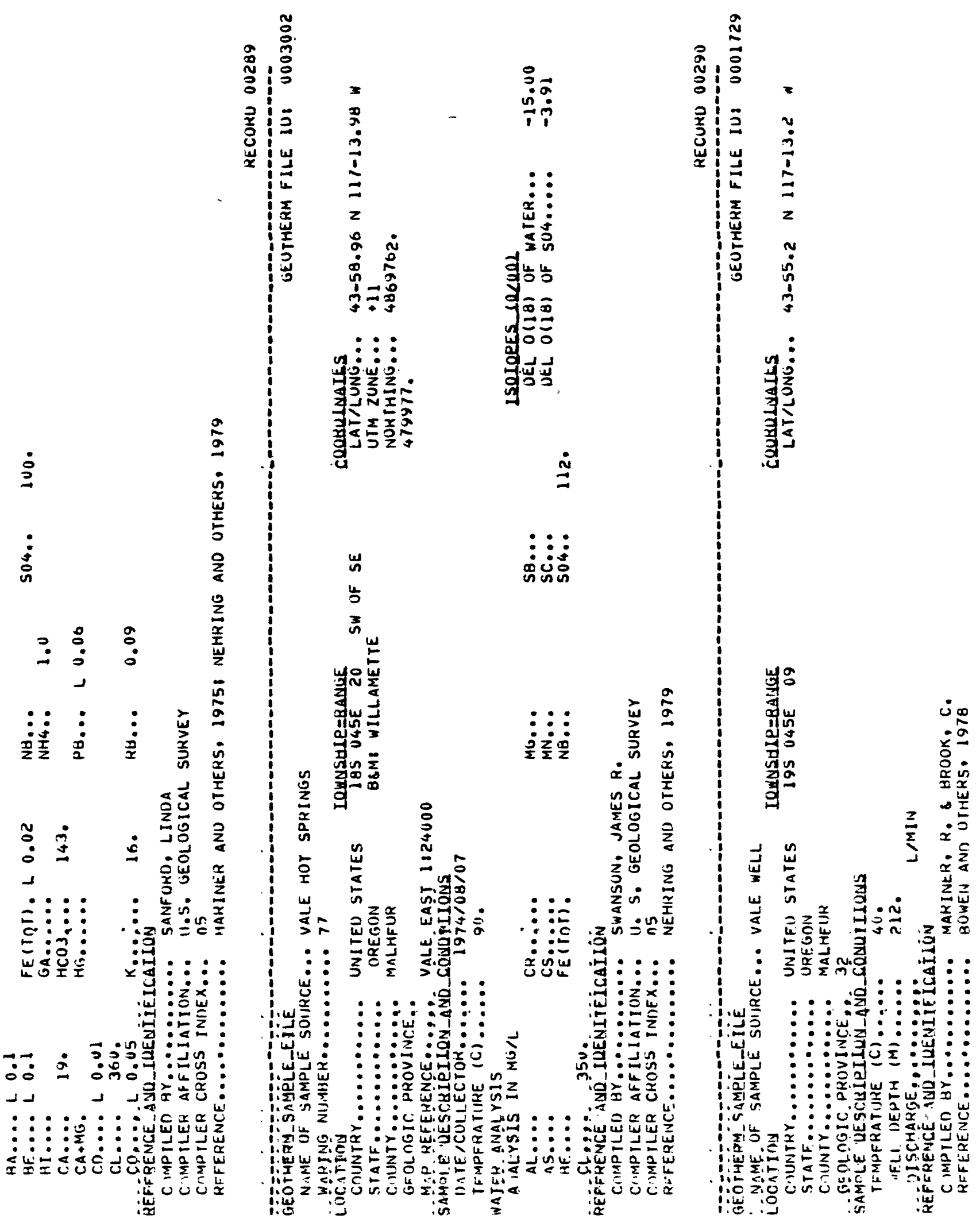


$\vec{x}$
0
$\vdots$
$\frac{3}{a}$
$a$

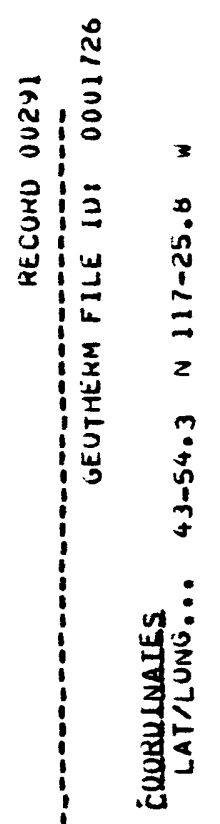

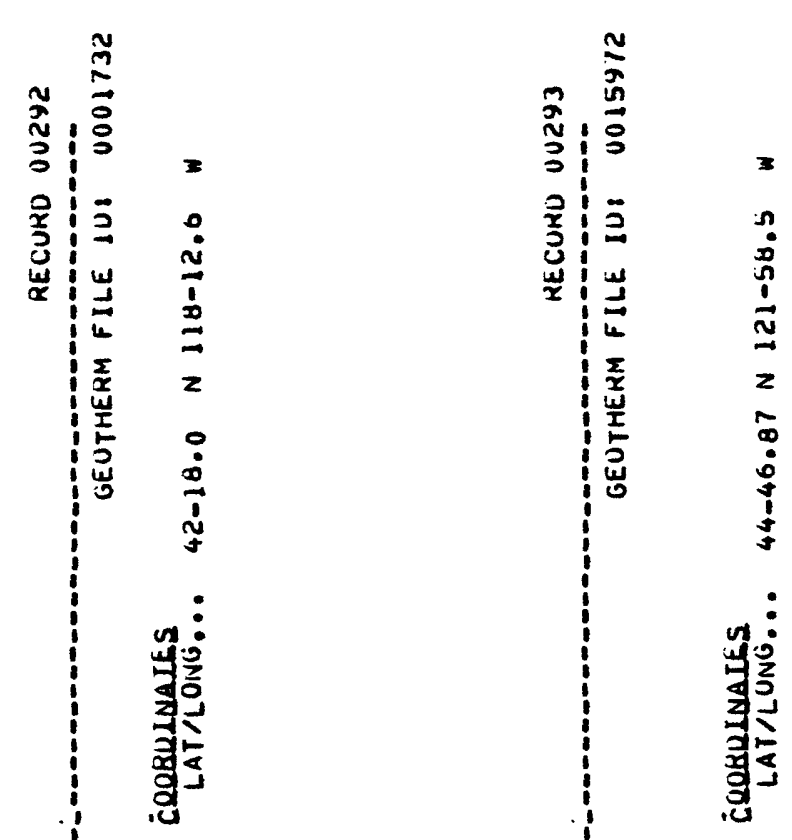

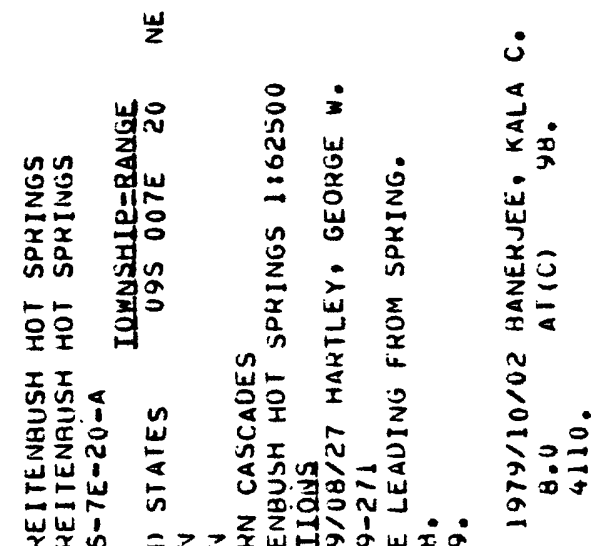

ช

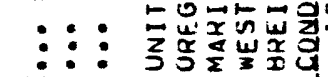
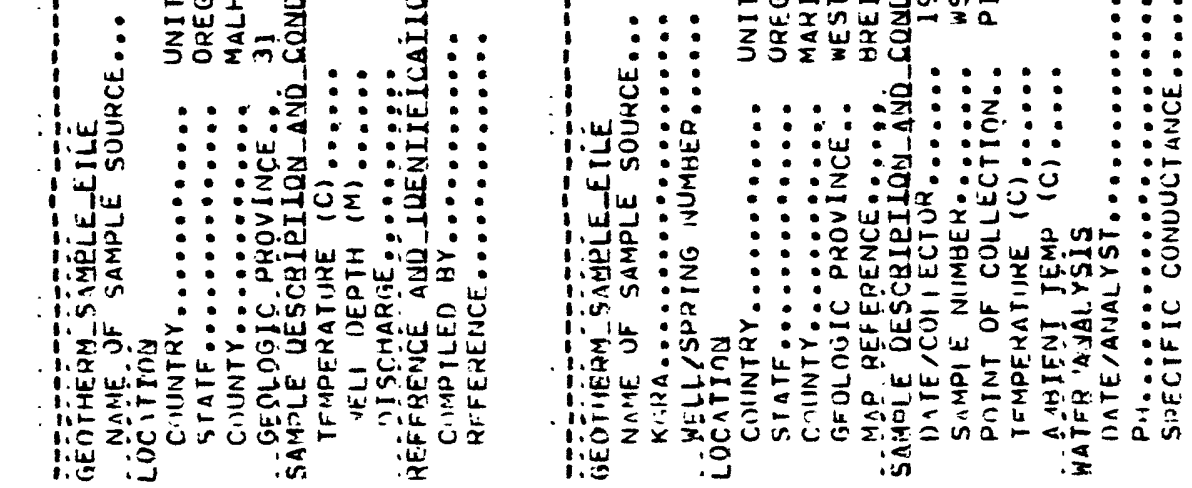
产
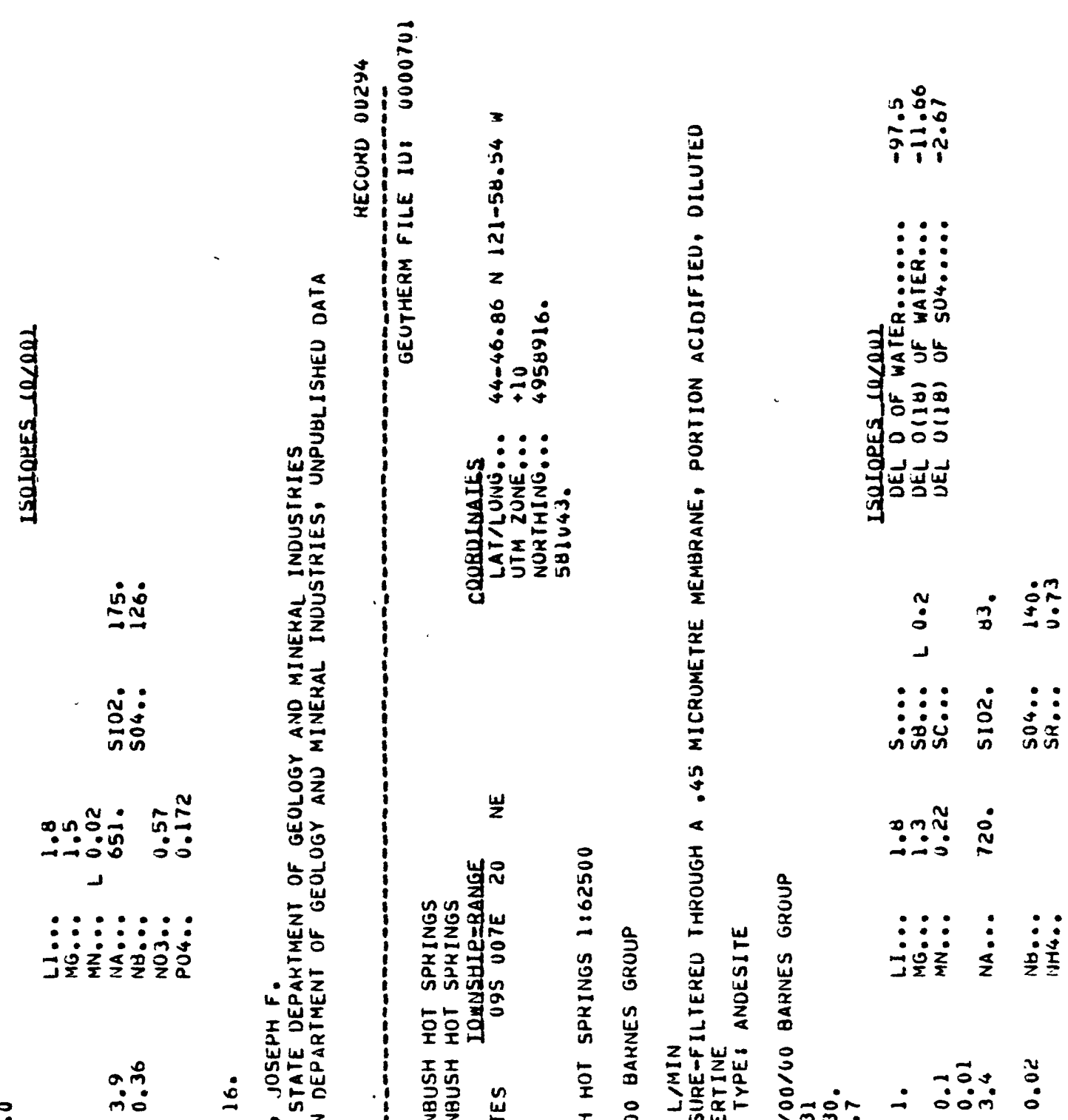

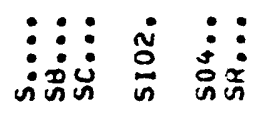

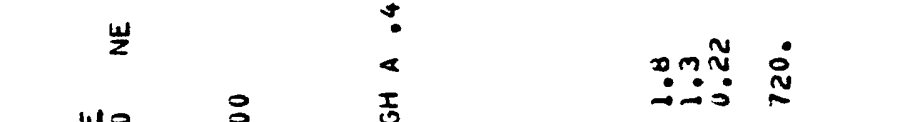

$\stackrel{0}{30} \stackrel{0}{: 0}$

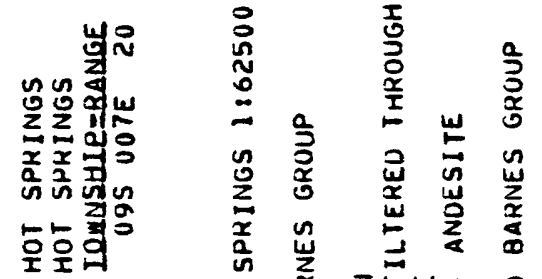

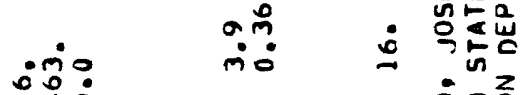

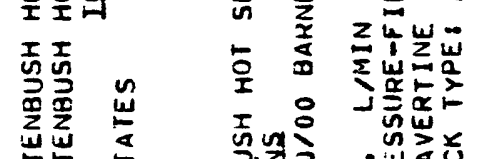
(1)

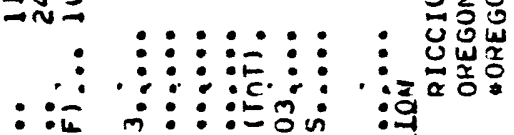

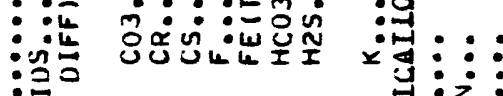

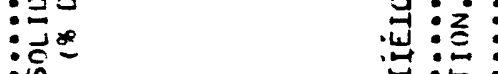

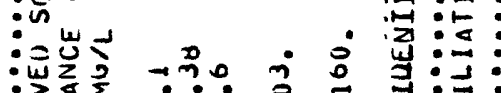

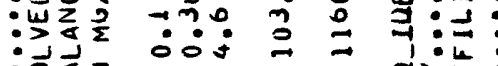

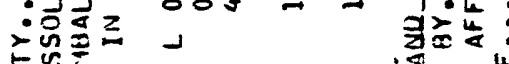

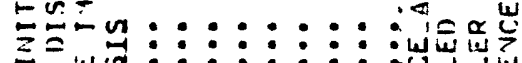

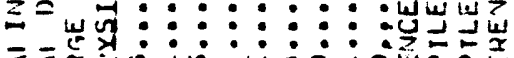

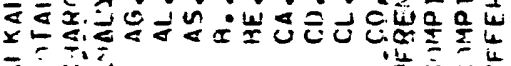
䀡就

出:

浔足:

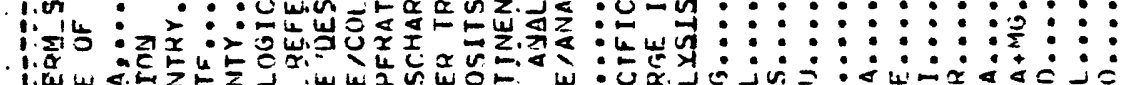
l.

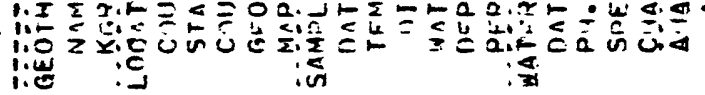




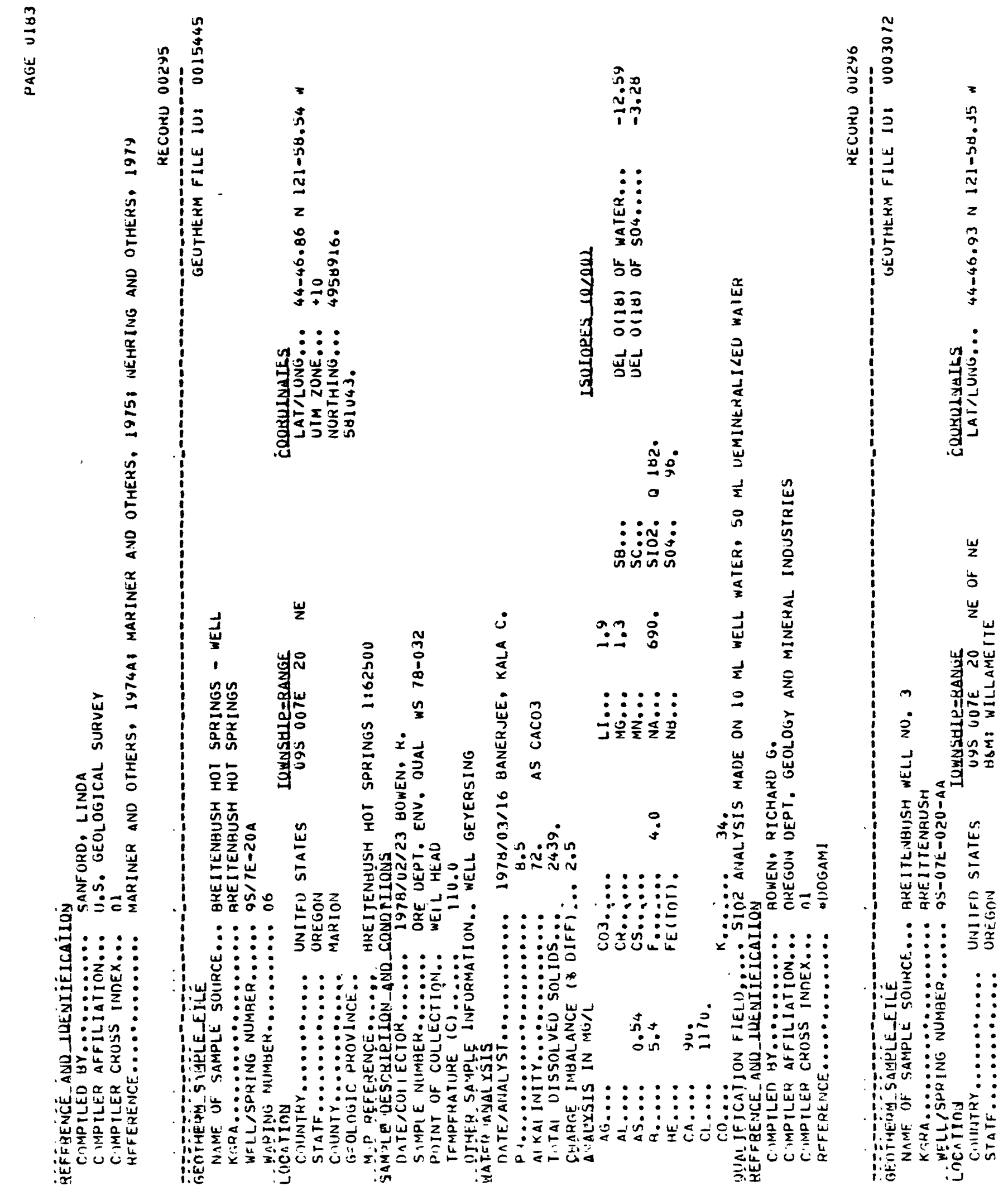



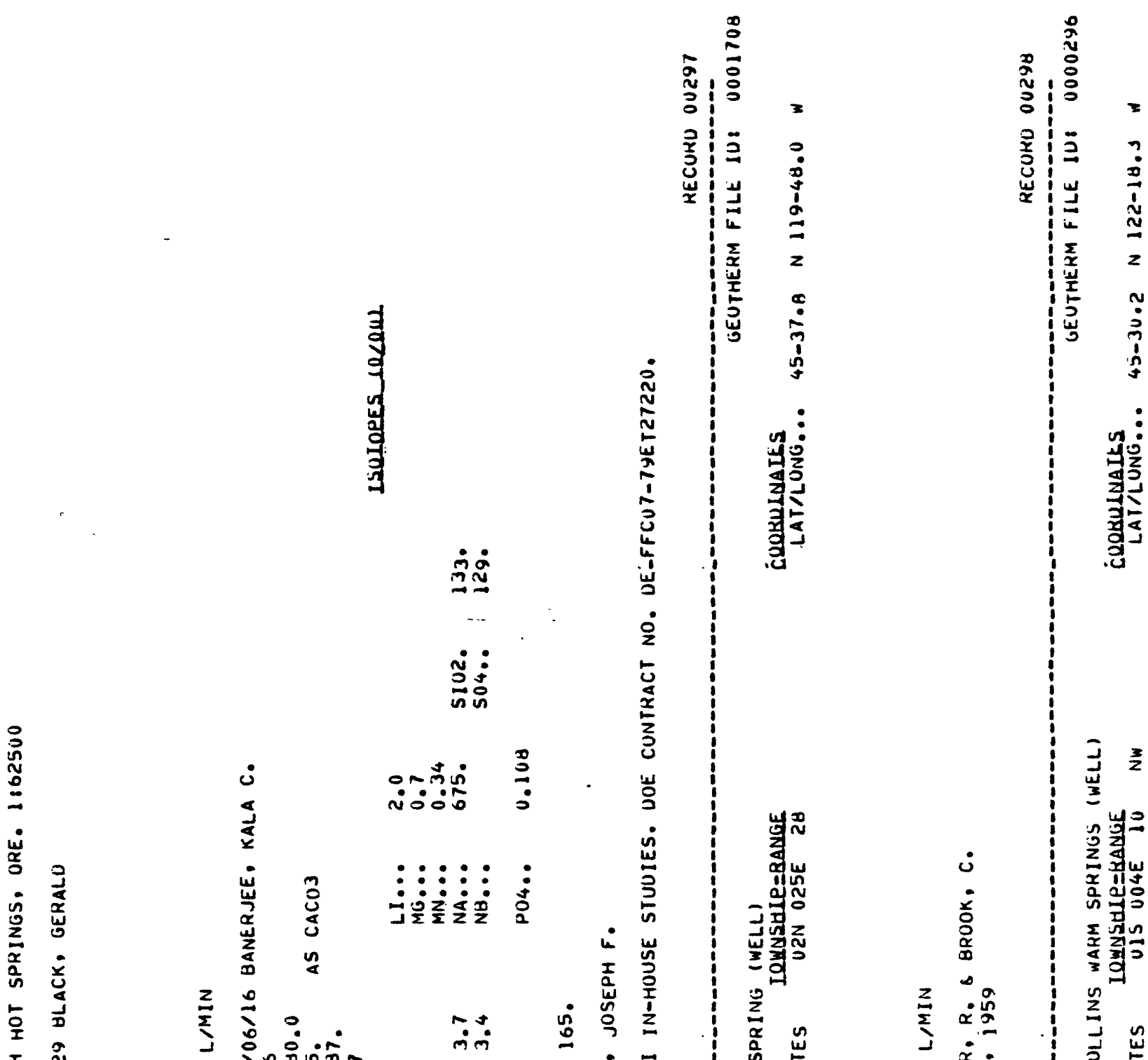

$\underset{\mathrm{J}}{\underline{3}}$

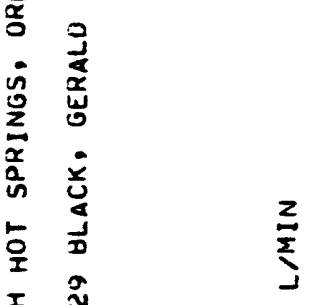

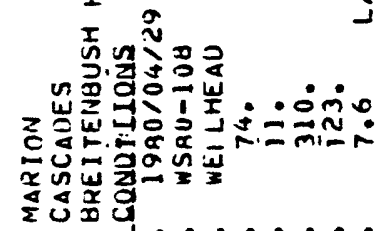

मिं

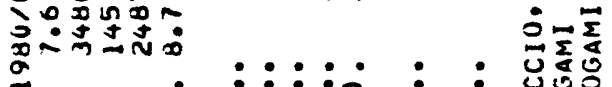

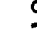

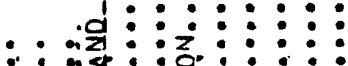

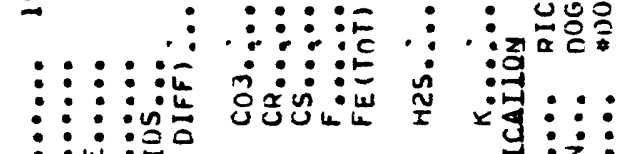

$: \vdots::$ : $:$ :

:

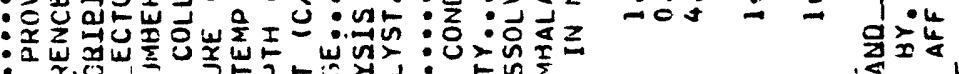

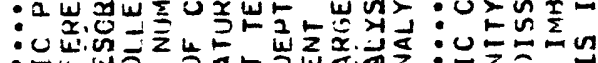

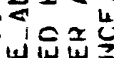

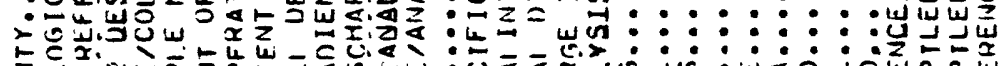

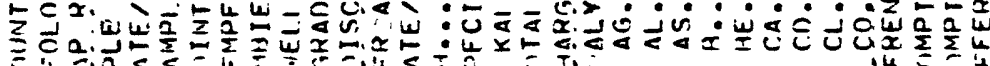

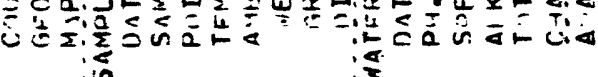
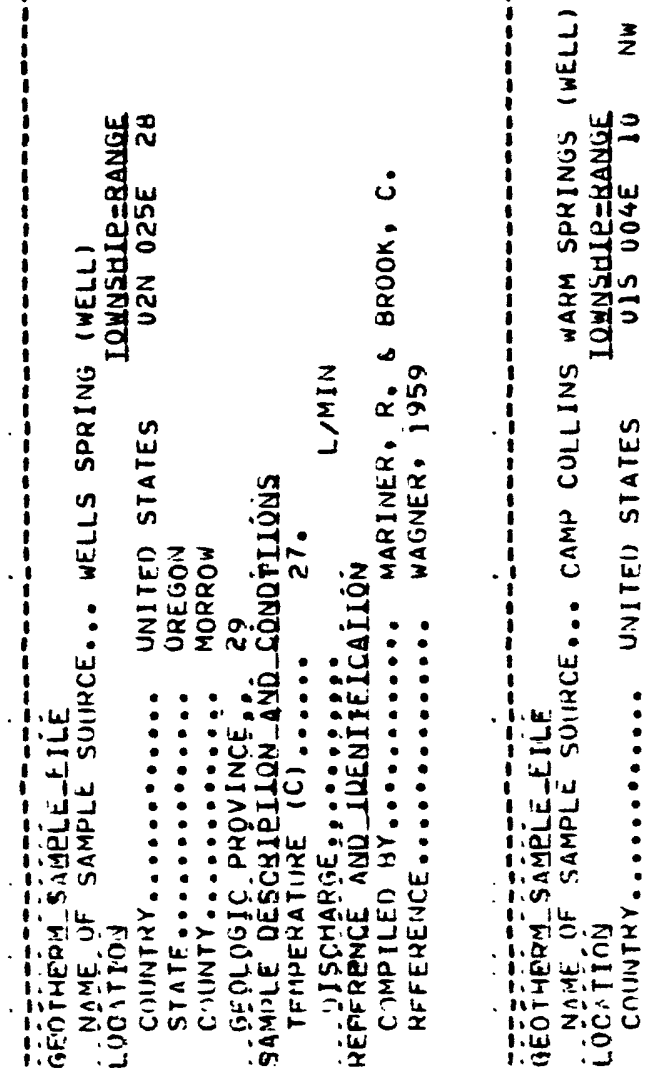


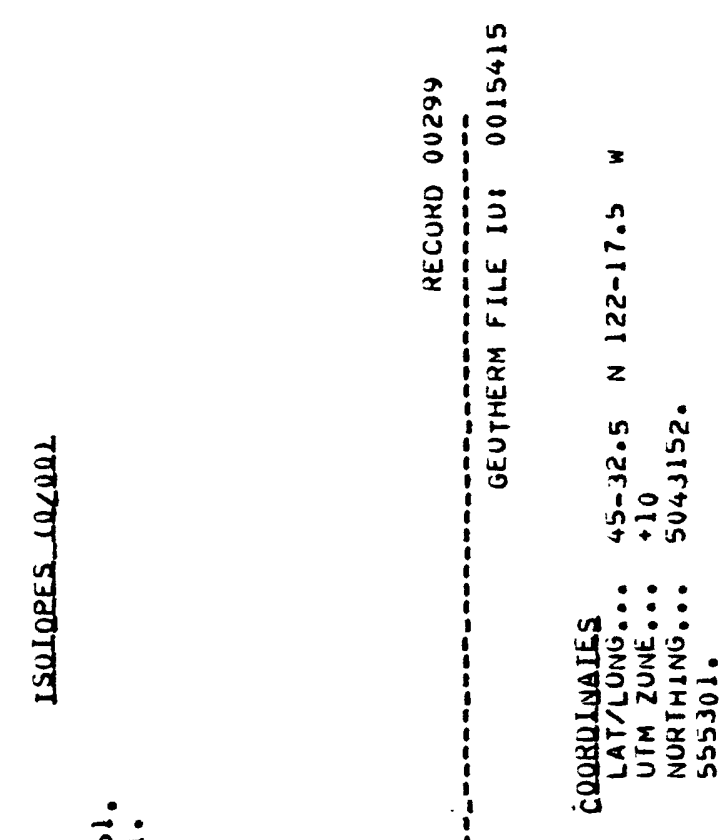

录

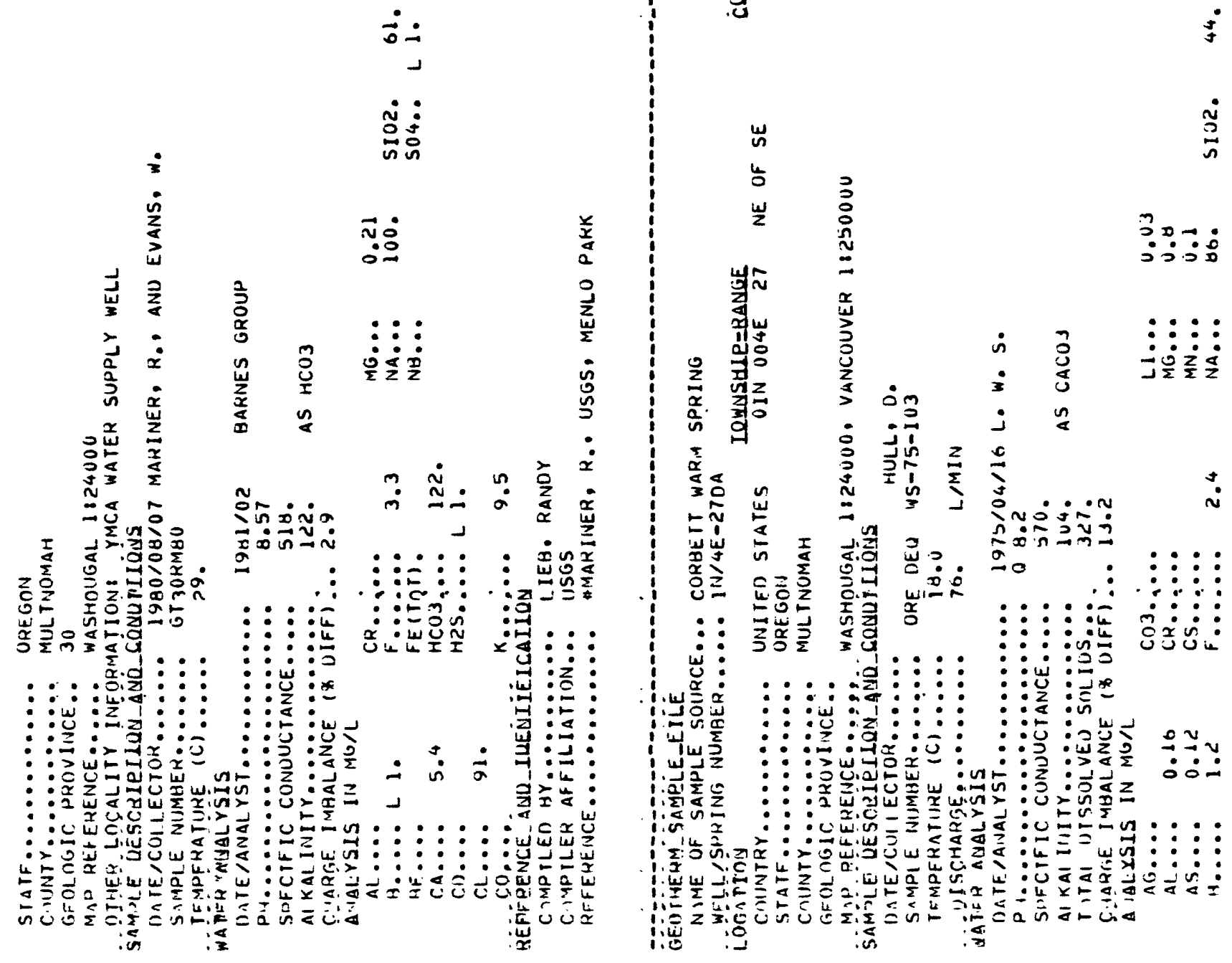




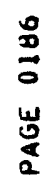
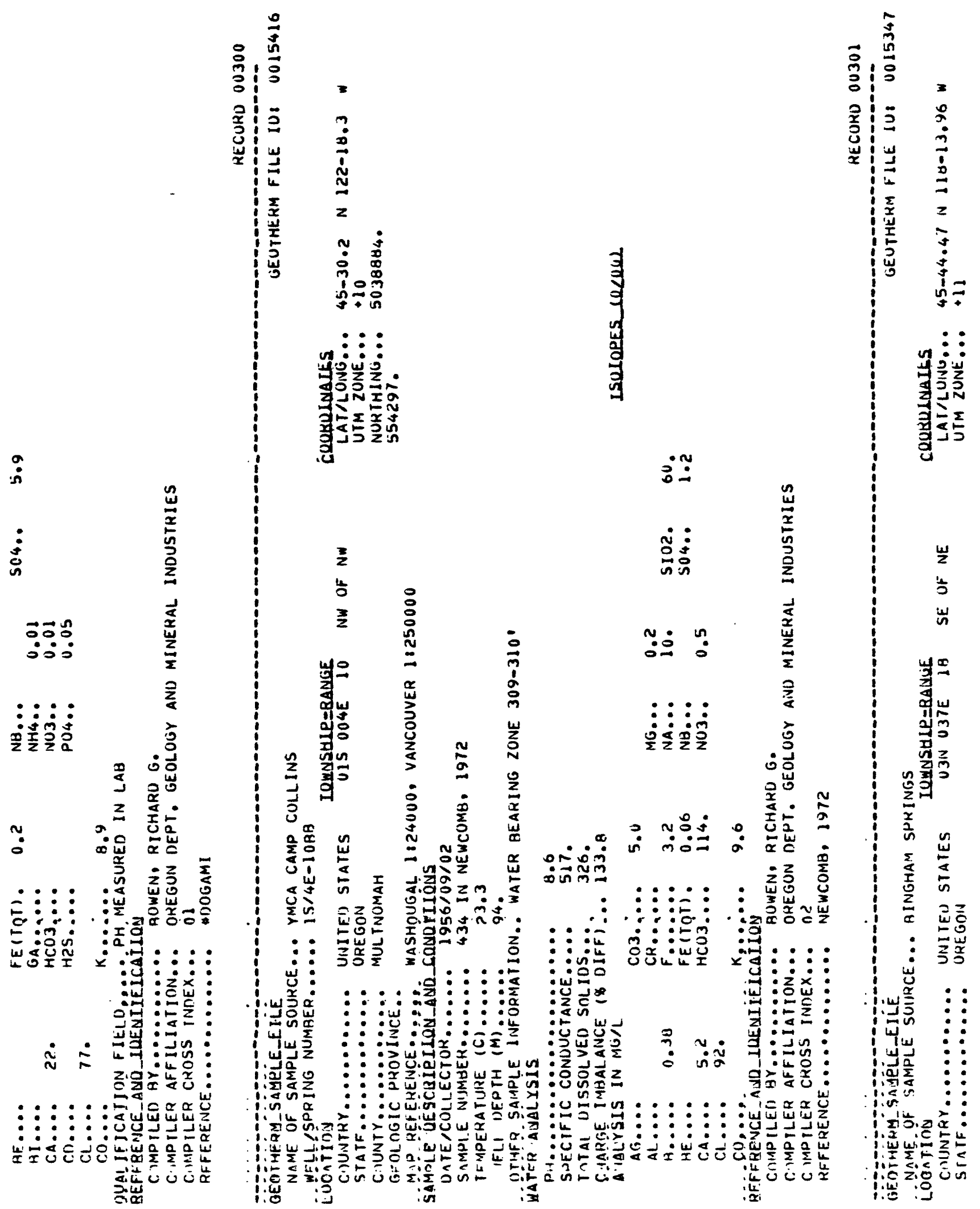
$\frac{\tilde{z}}{\dot{\underline{u}}}$

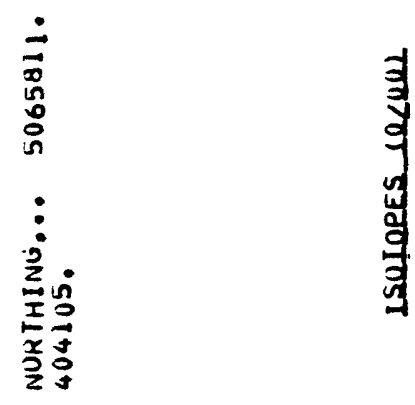

灵

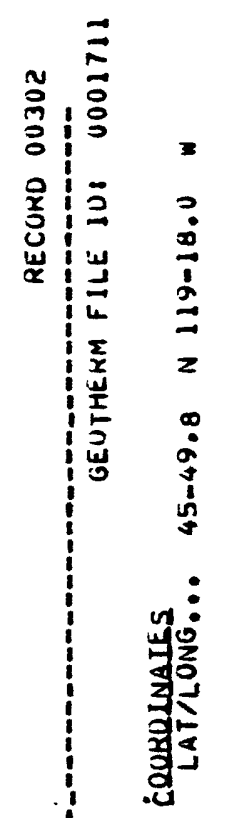

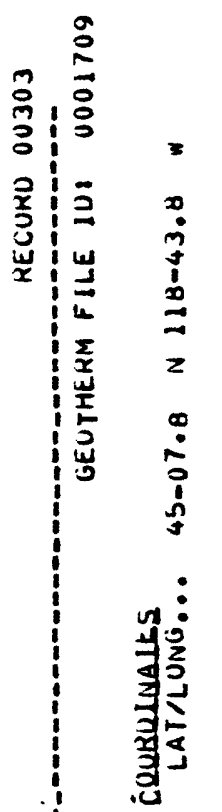

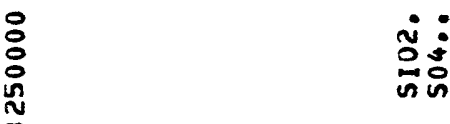

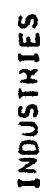

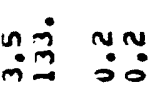

$::::$ श

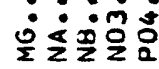

نे

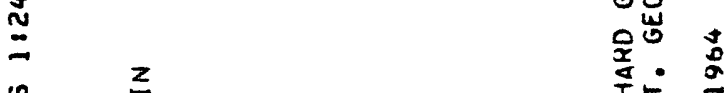

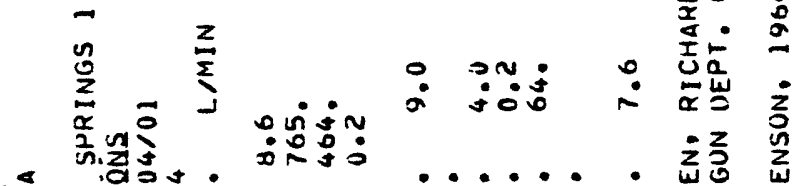

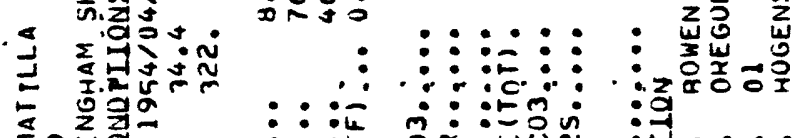

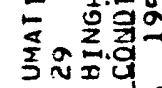

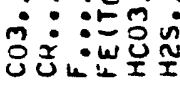

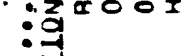

$:::::$ :

$::$ :

光: $: \vdots: \vdots$

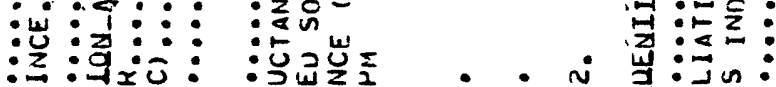

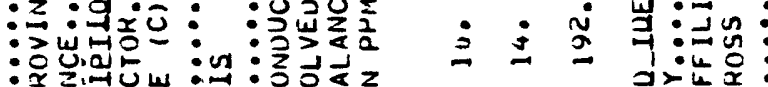

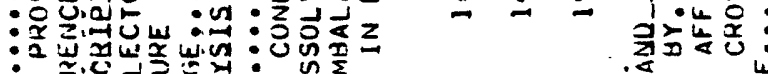
:

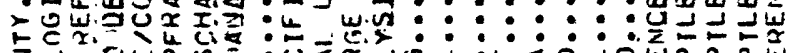

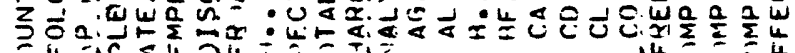

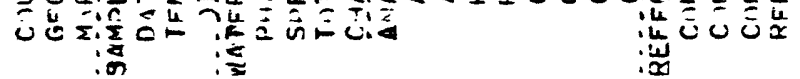
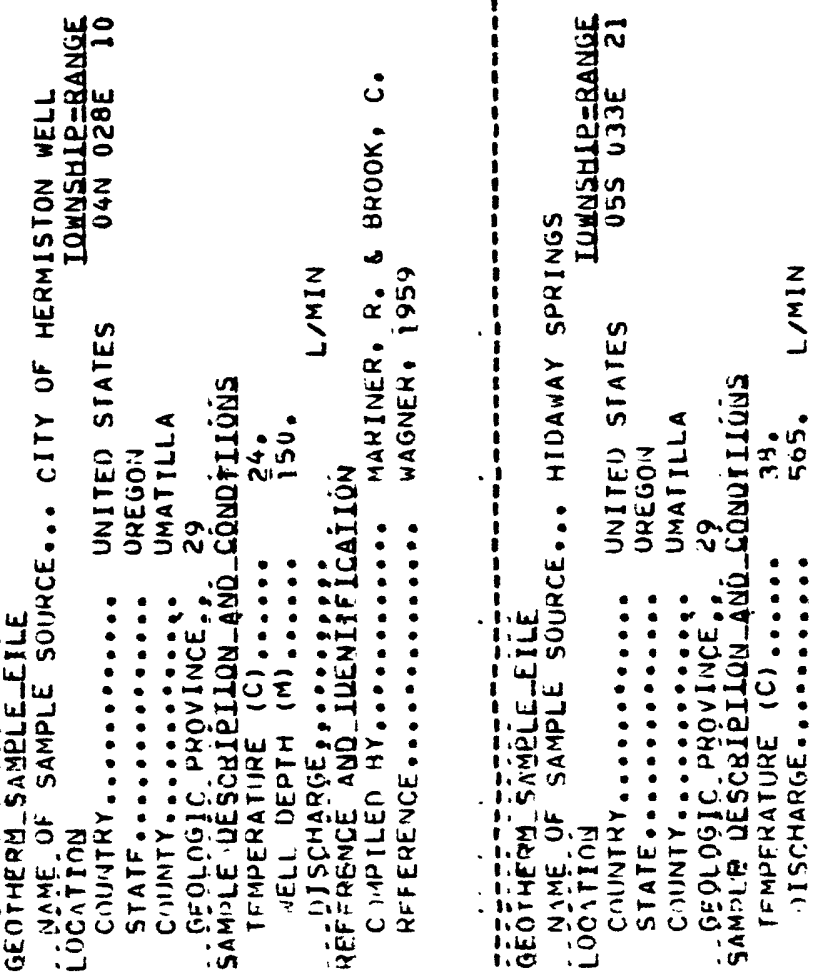
这

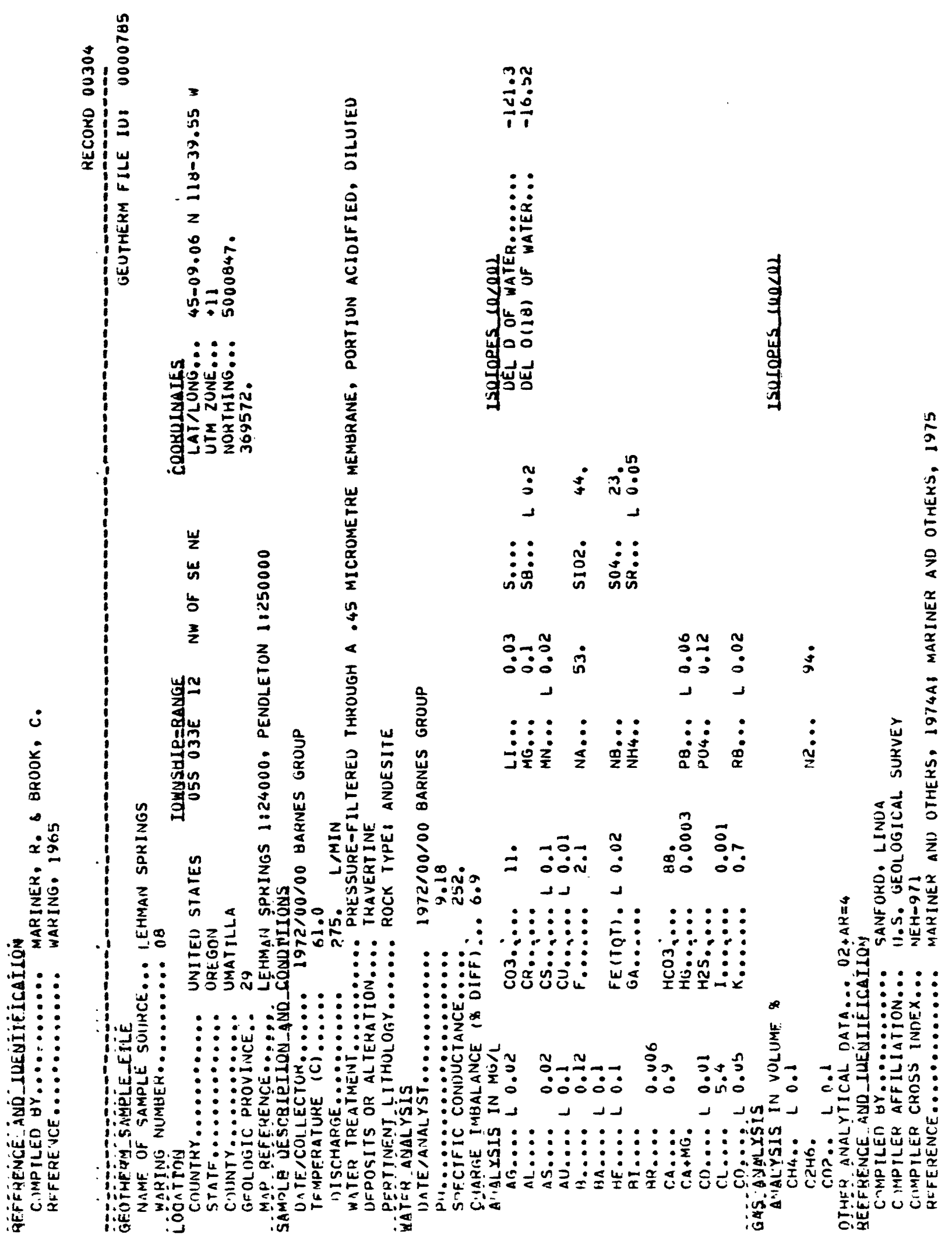


$\frac{\sqrt[3]{2}}{3}$
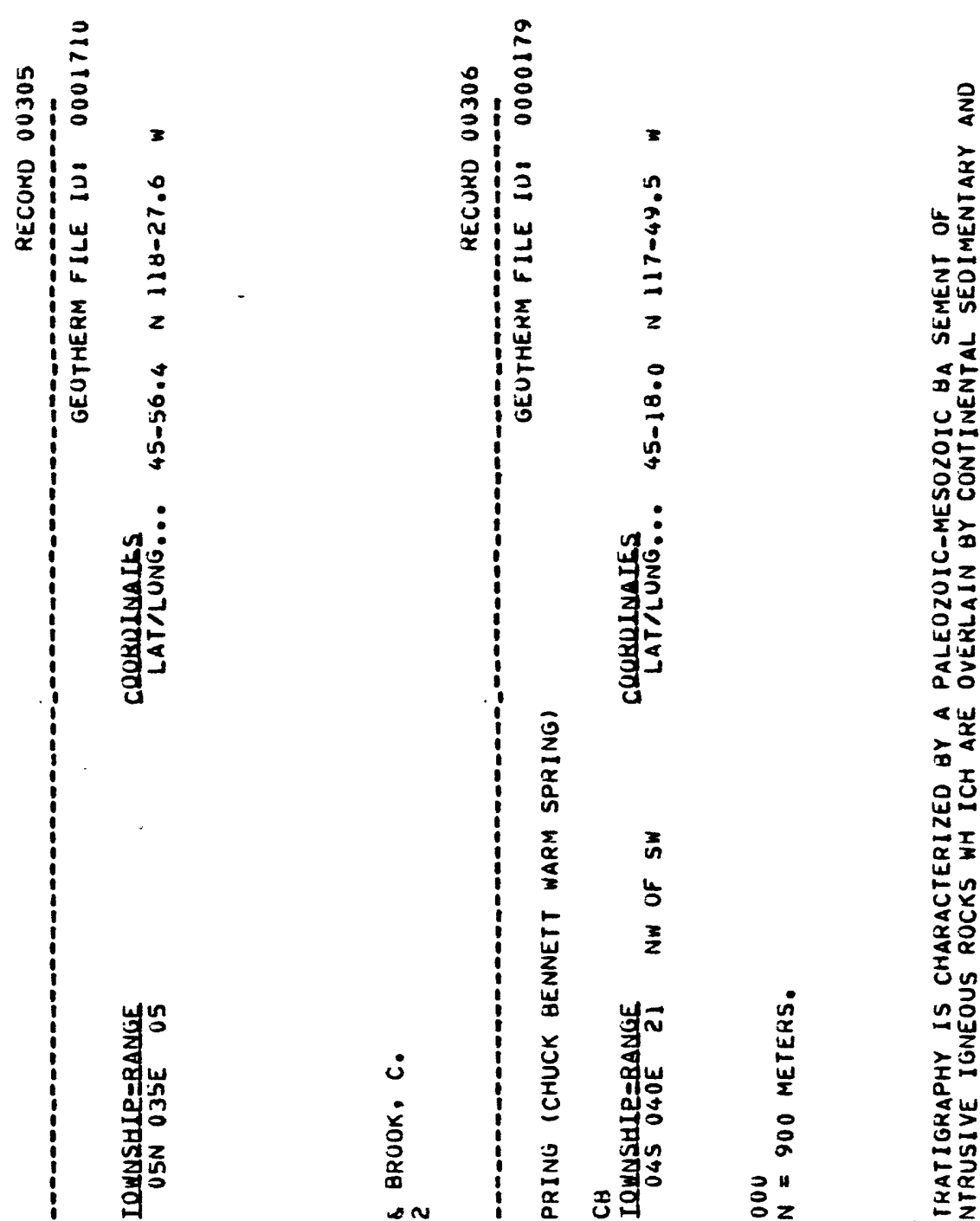

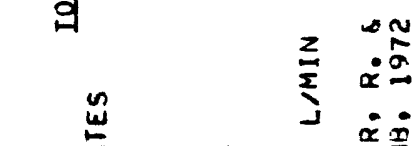

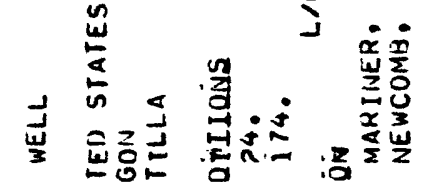

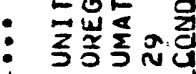

$\dot{8}$

氜

;

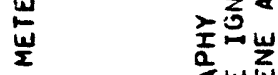

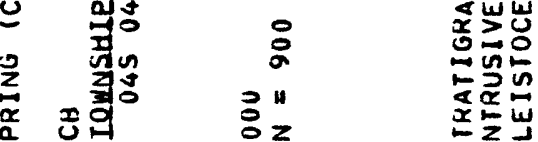

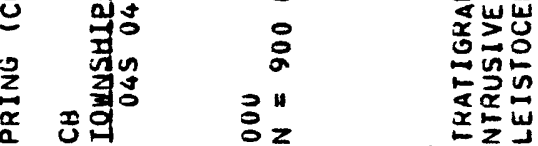

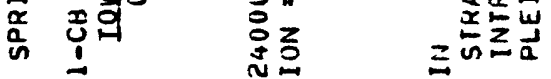

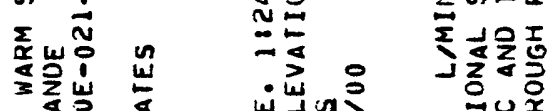

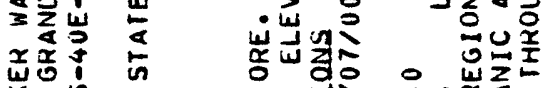

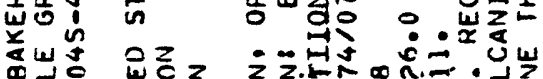

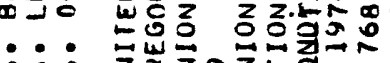

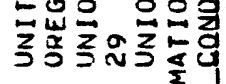

:

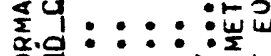

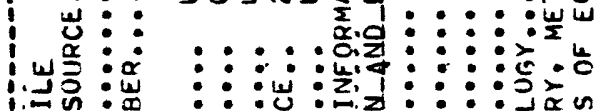

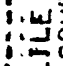

पu

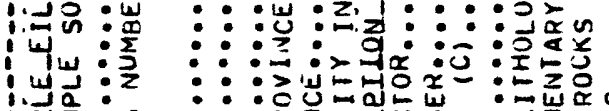

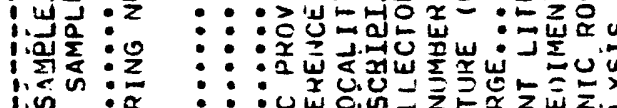

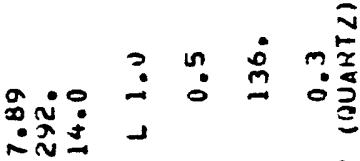

;

䓀

$\underset{⿱}{\&}$

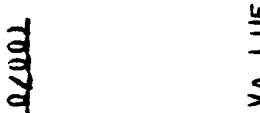

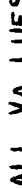

范

今ึ:

竞:

!

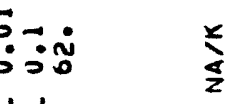

$\vdots: \vdots: \quad$ o

× ฮَّ 事㻤 in 员赑

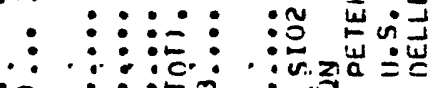

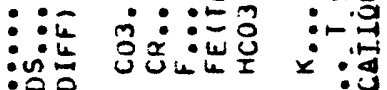

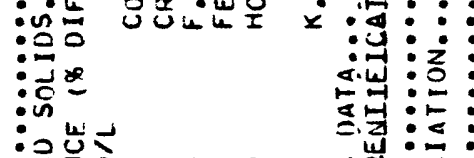

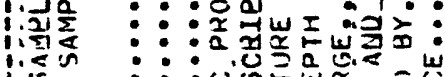

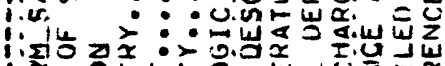
livic: : l.

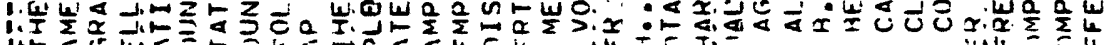
1.

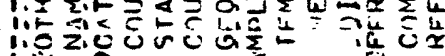

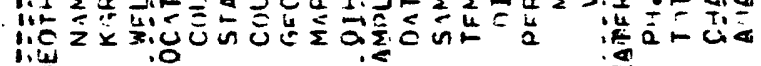


$\frac{2}{3}$
$\frac{a}{0}$
$\frac{a}{a}$

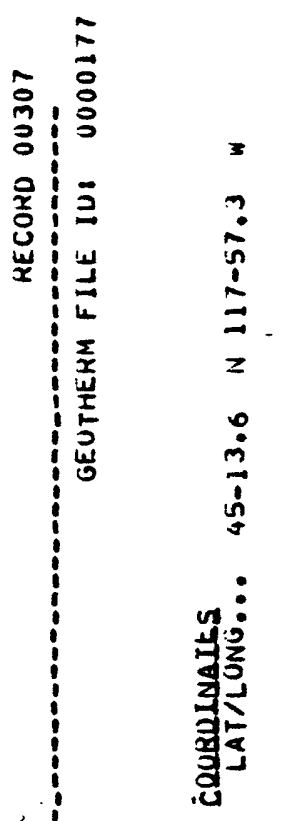

$w$
5

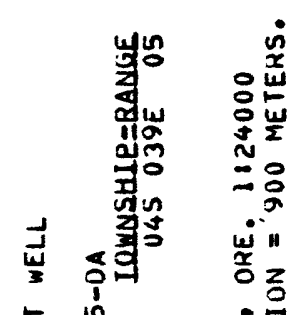

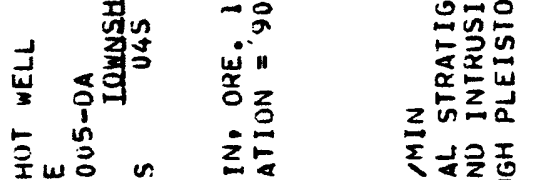

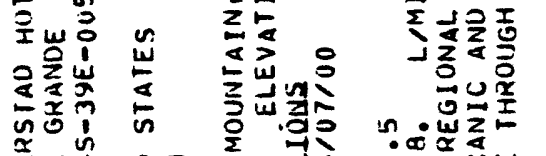

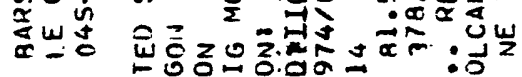

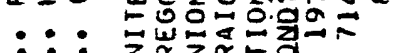

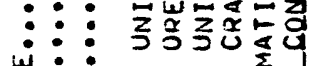

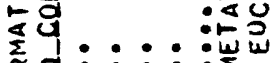

: $:::: \frac{w}{2}$

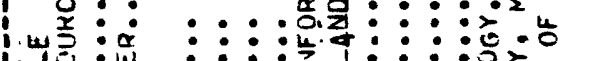

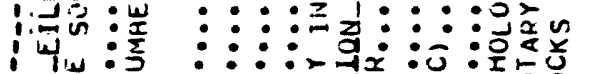

㟧:

濯资: :

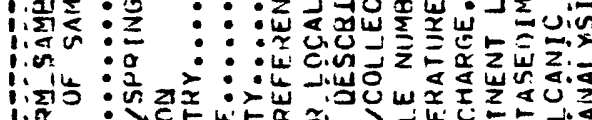

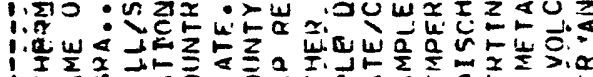

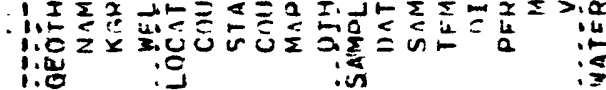

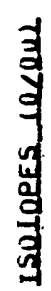

$\stackrel{+}{2} \dot{0}$

ĩ:

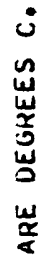

岁

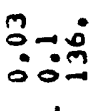

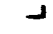

$\vdots: \vdots \vdots$

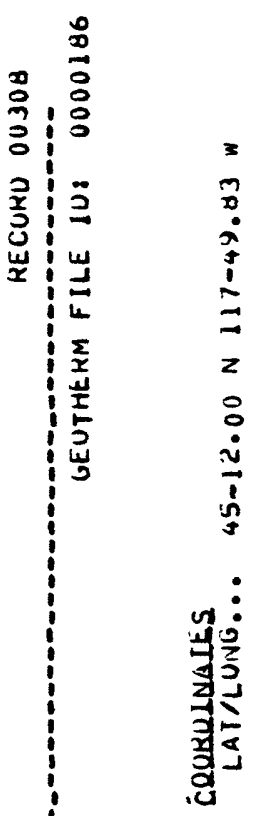

$\frac{12}{z}$

兮

in

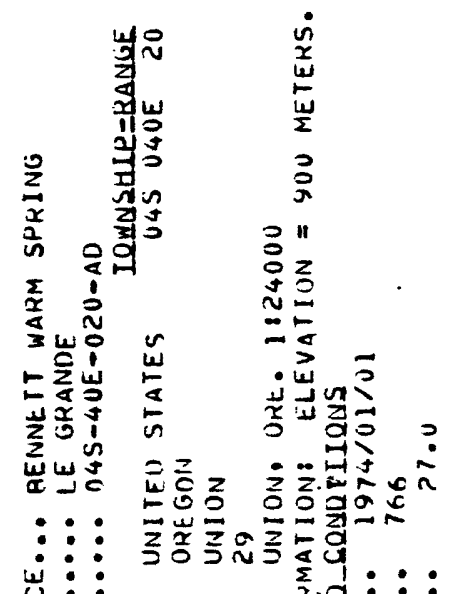

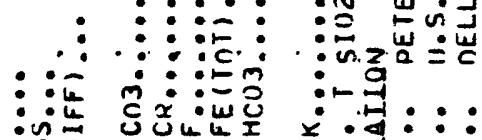

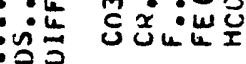

네: $:$

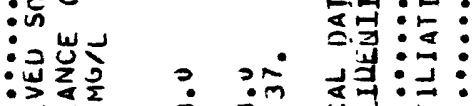
둔단: $:$ : ع.

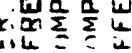

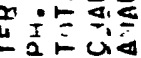

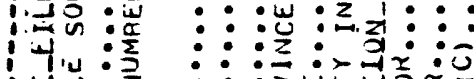

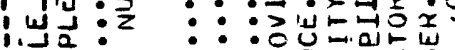

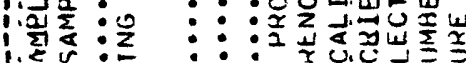

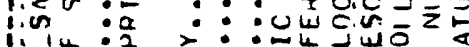

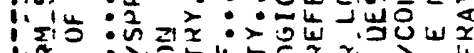

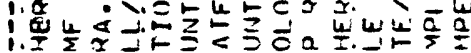

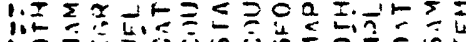

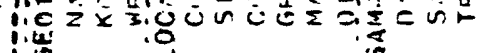


$\vec{a}$
$\overrightarrow{2}$
$\overrightarrow{\underline{c}}$
$a$
$a$ 岁

$<$

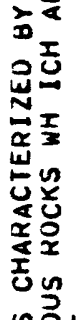

n雚㟧

至或崖

承岂岕

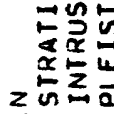

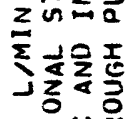

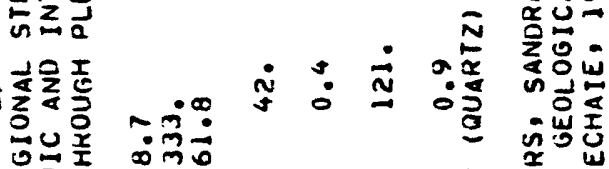

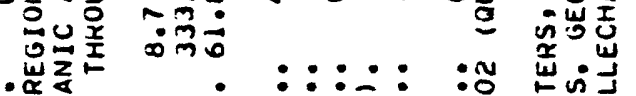

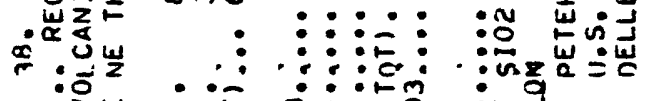
:

:

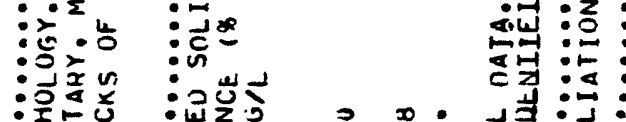

:

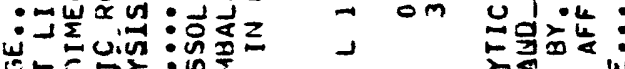

an

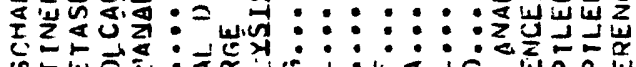

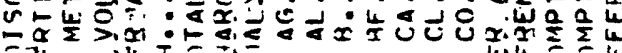

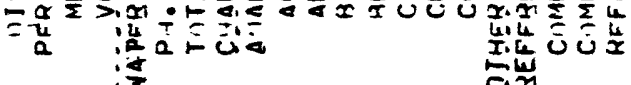
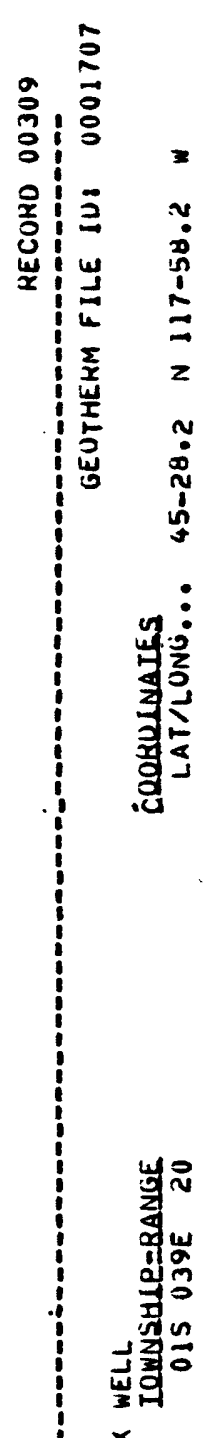

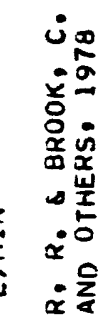

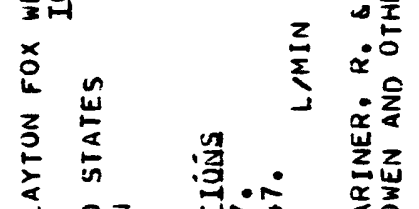

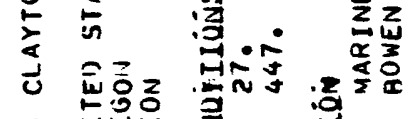

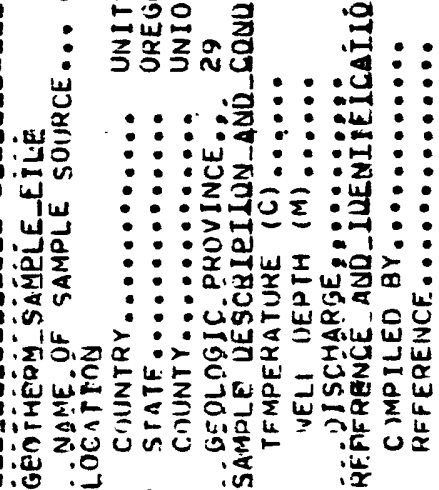

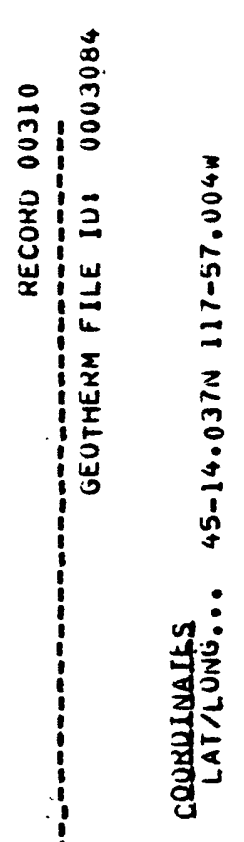

岁

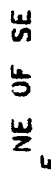

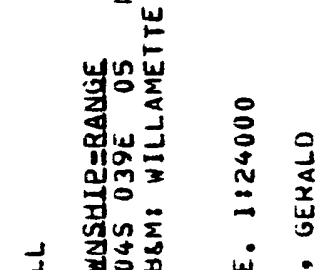

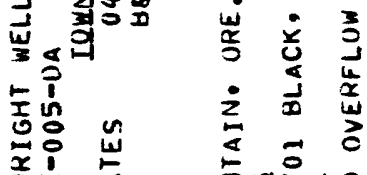

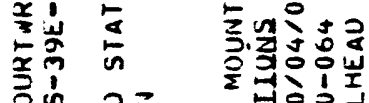

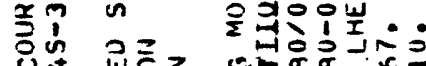

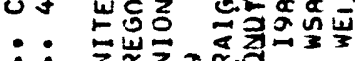

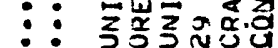

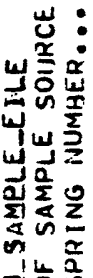

: $: \vdots: \vdots$

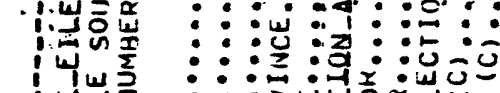

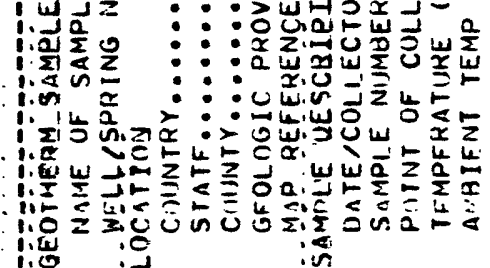



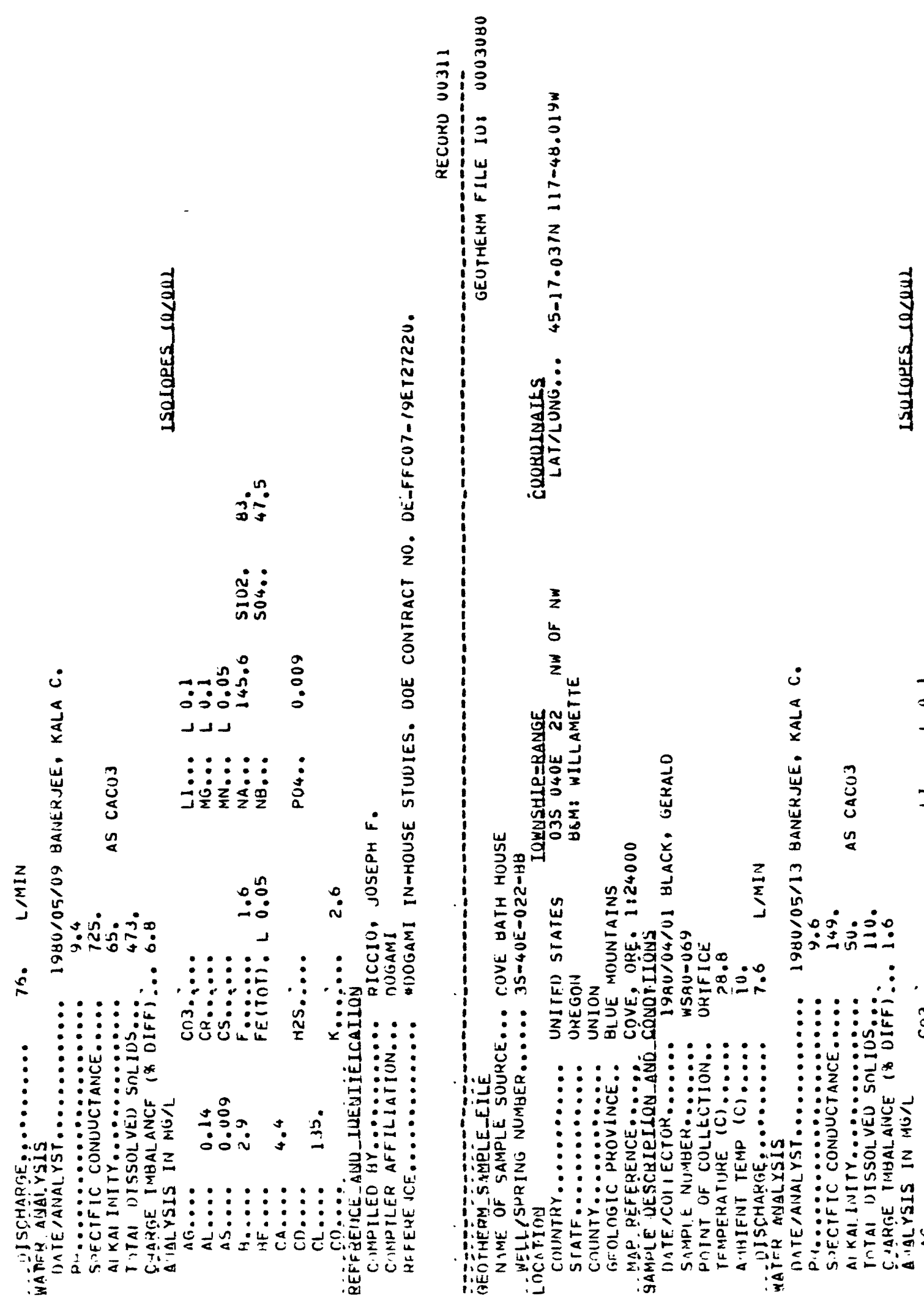
$\frac{\frac{3}{2}}{\frac{3}{3}}$

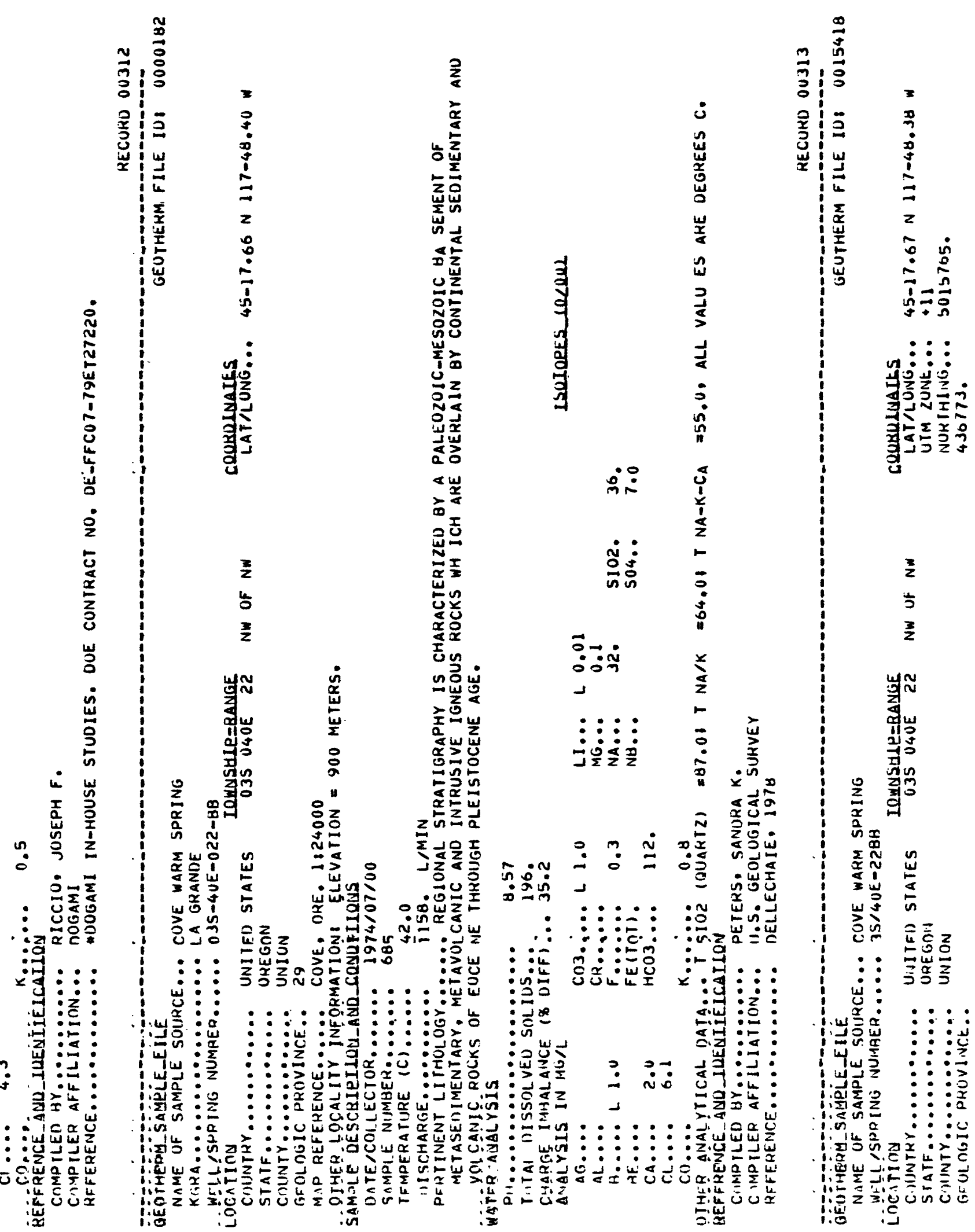


5
$\frac{5}{3}$
4
$\frac{5}{a}$
$a$

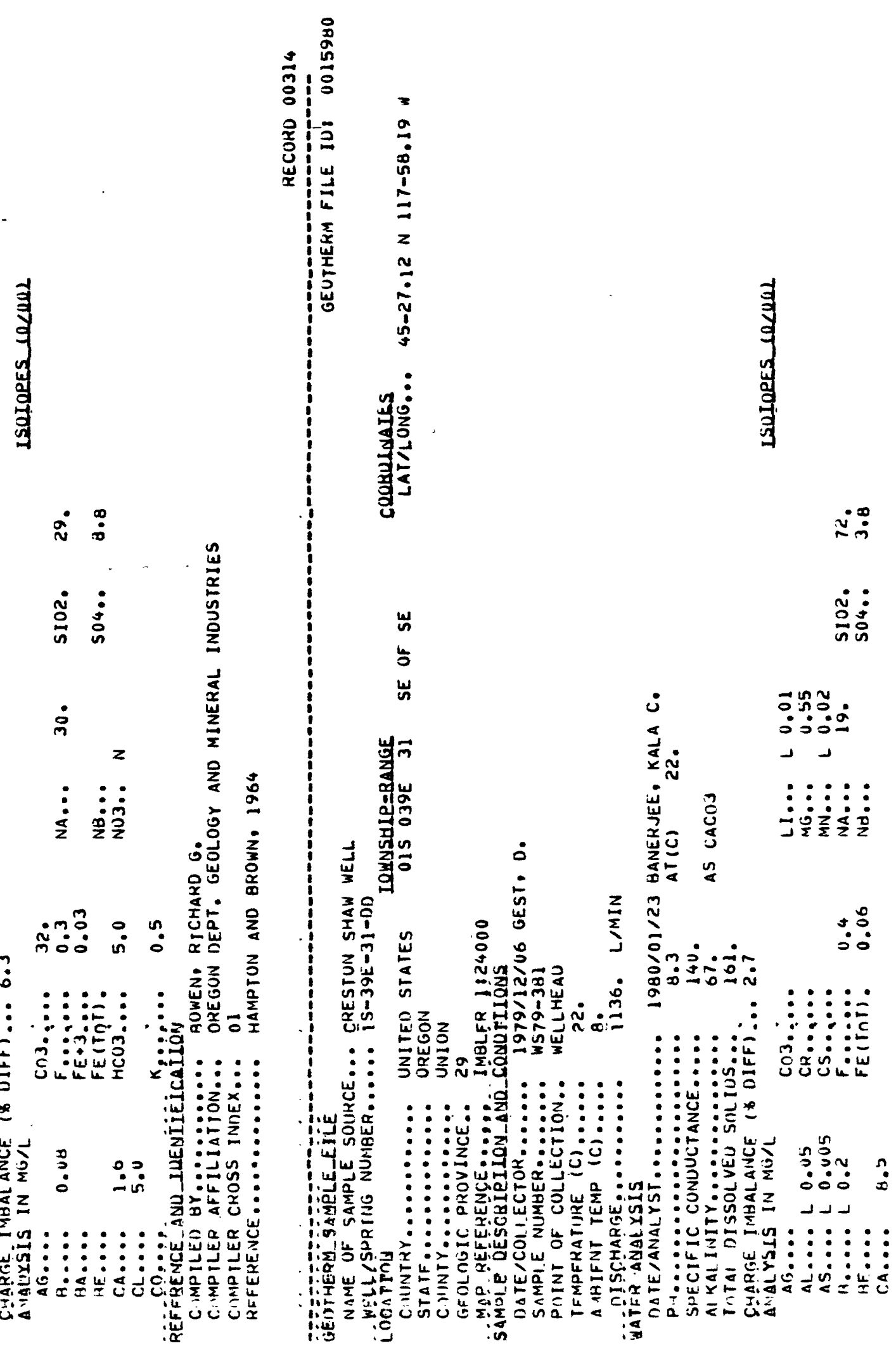




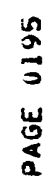

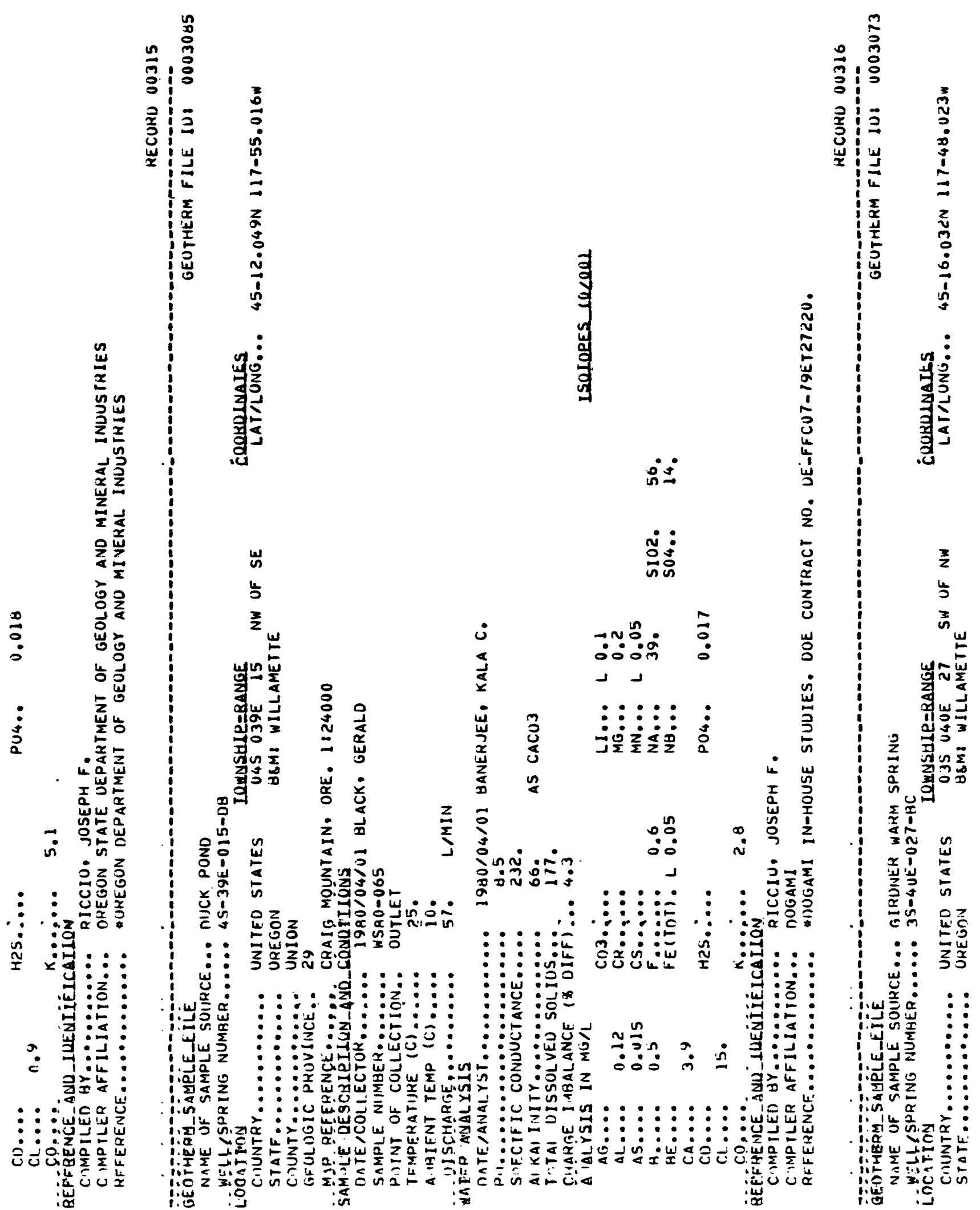




\section{$\stackrel{0}{0}$
0
0
0
$a$}

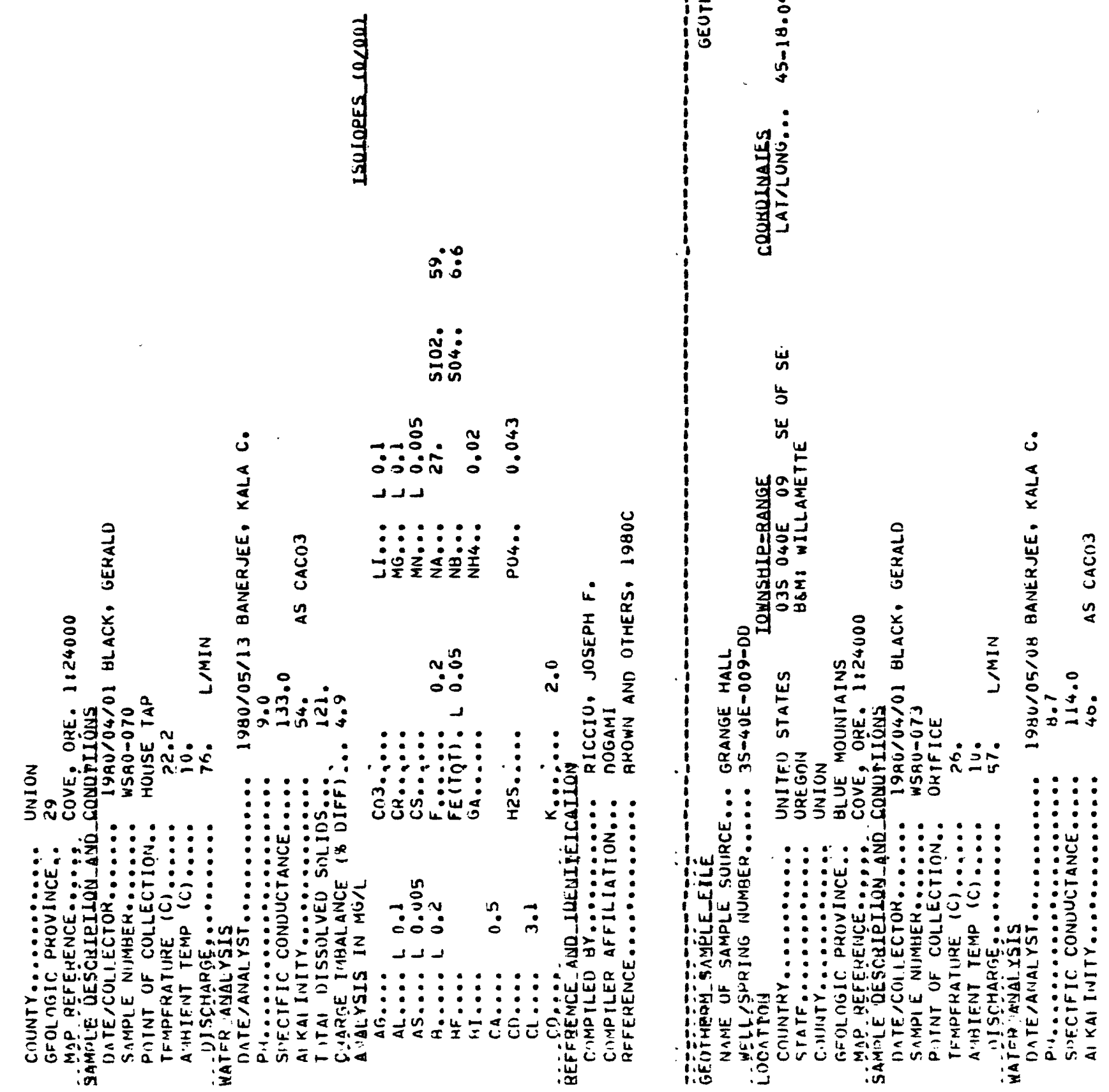


2
$\frac{2}{2}$
$\frac{0}{2}$
2

马ี

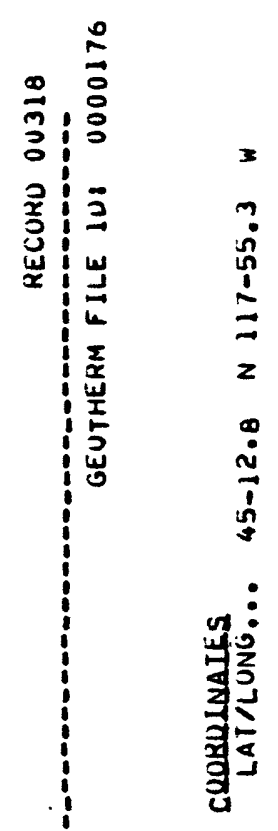

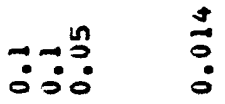

\lrcorner$\perp$

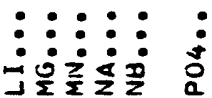

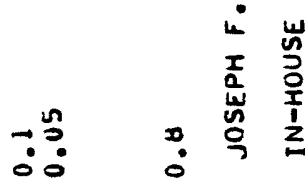

ก่
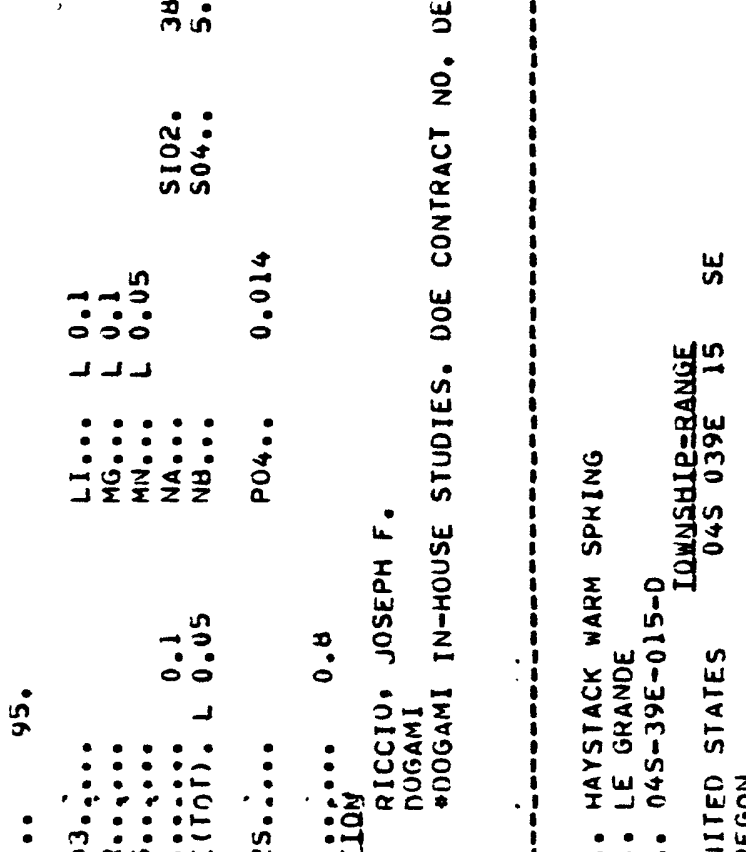

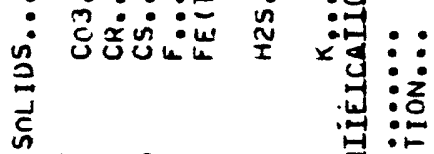

苛

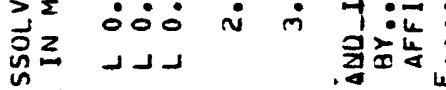

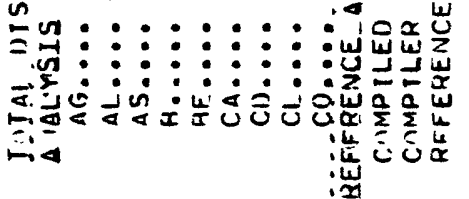<smiles>CCC</smiles>

乩

:

岂

西

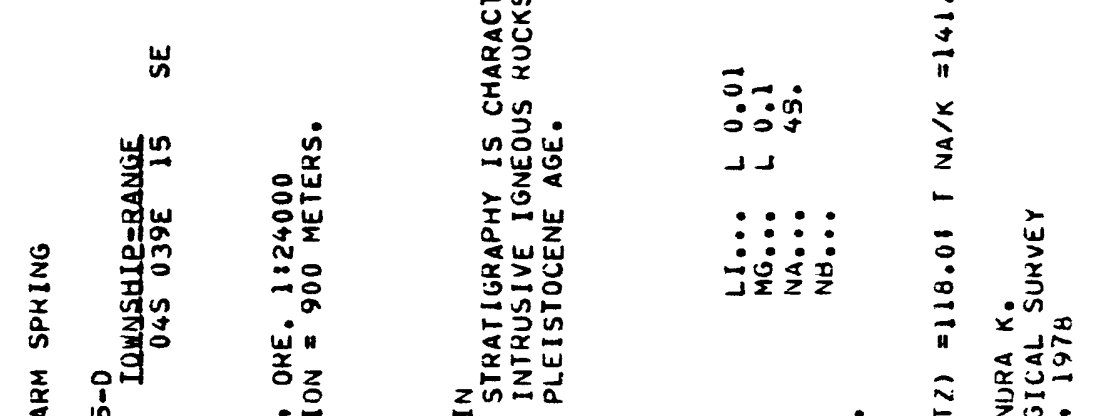

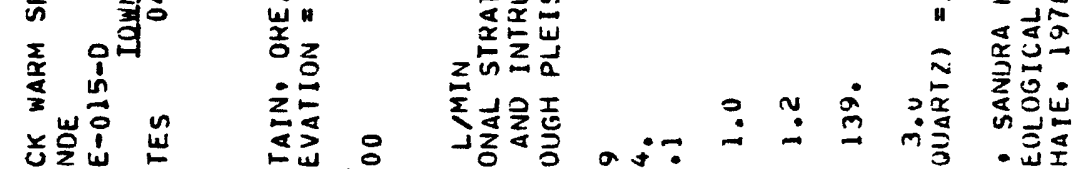

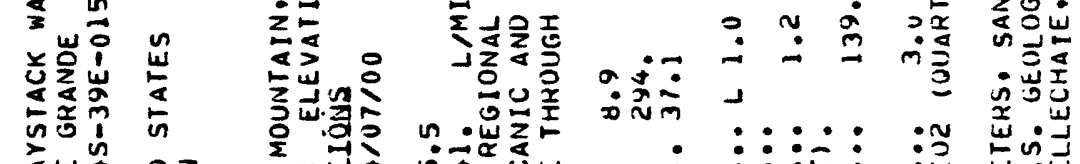

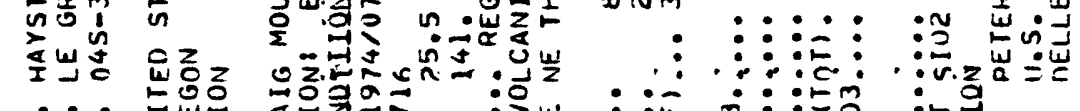

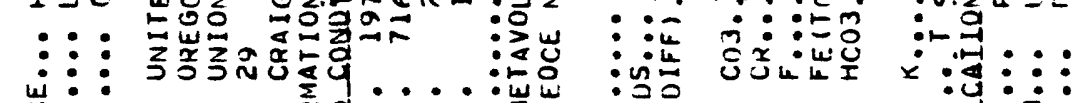

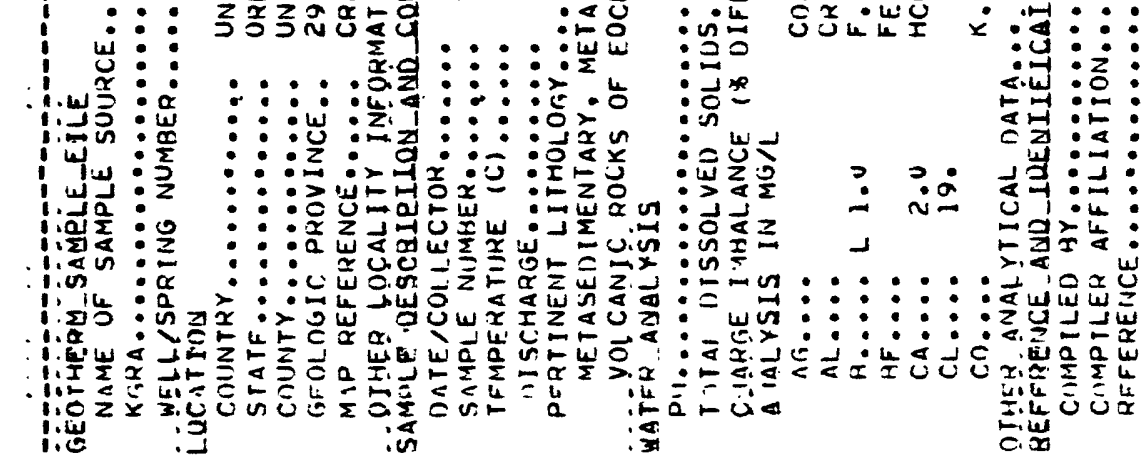



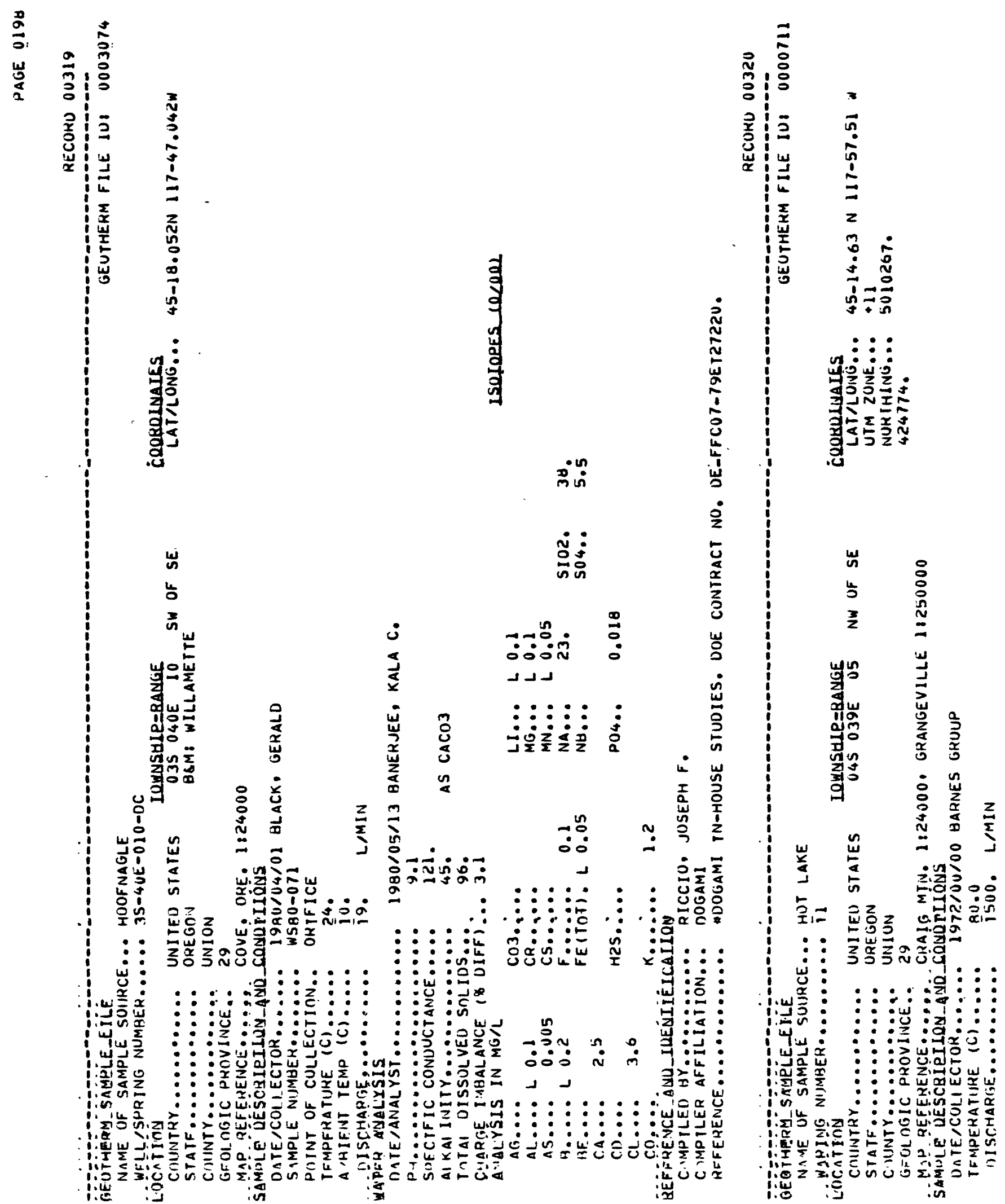


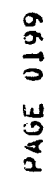

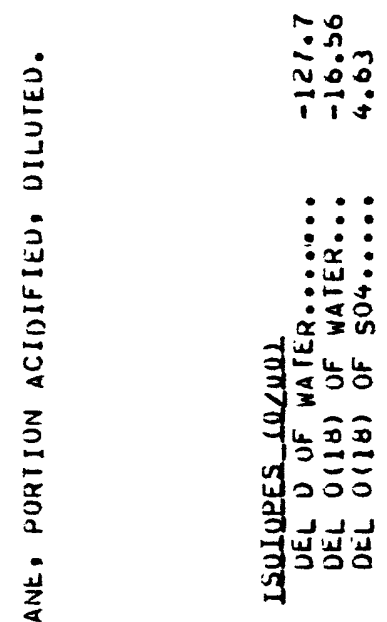

节

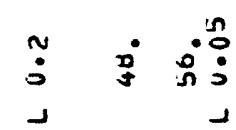

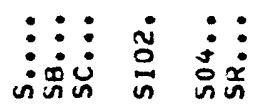

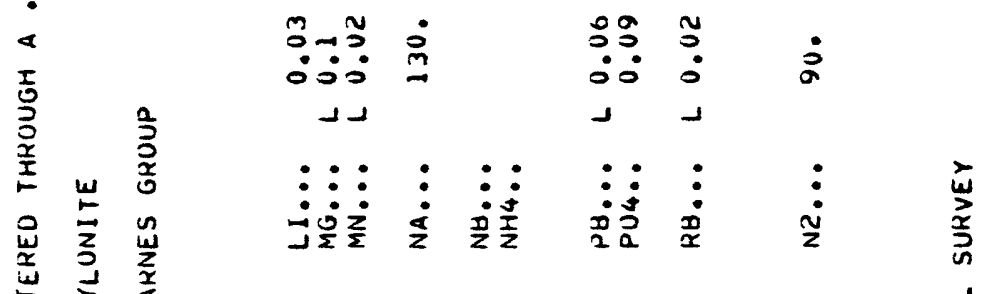

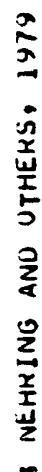

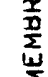

崖

ษ

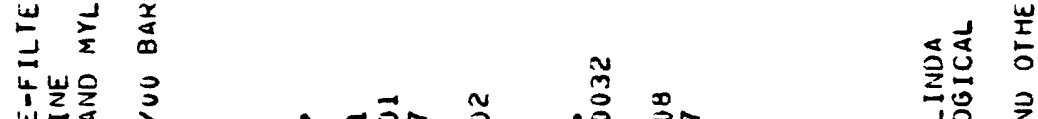

宸造

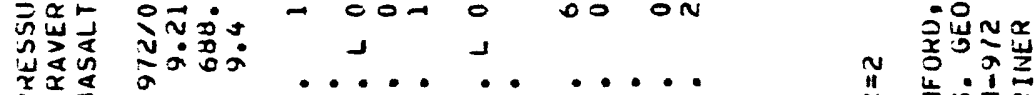

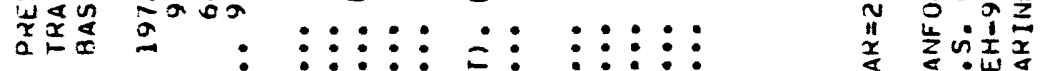

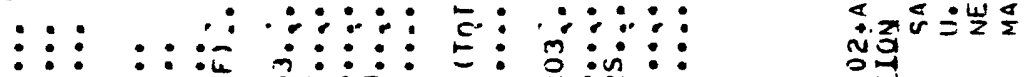

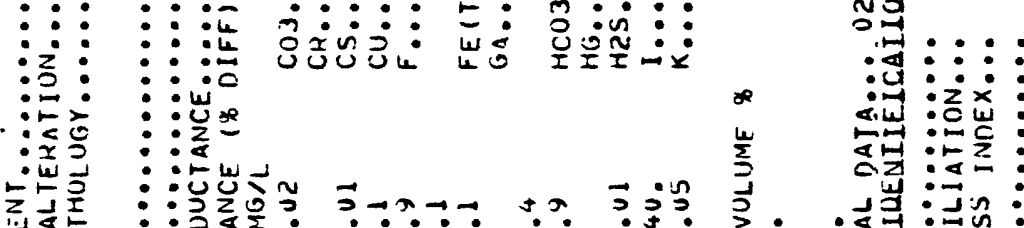

崖

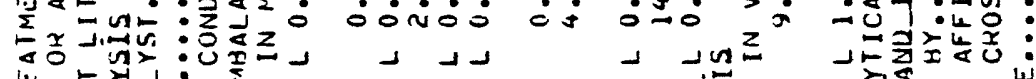

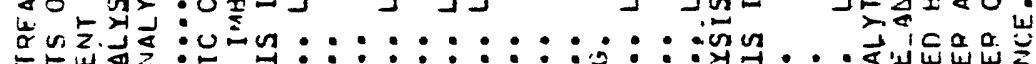

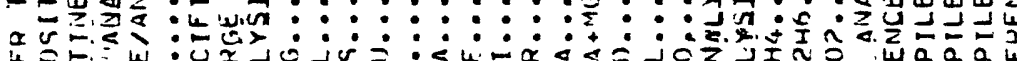

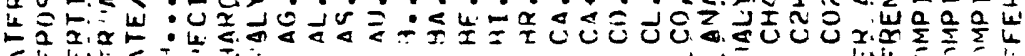

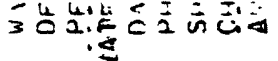

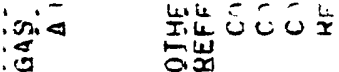
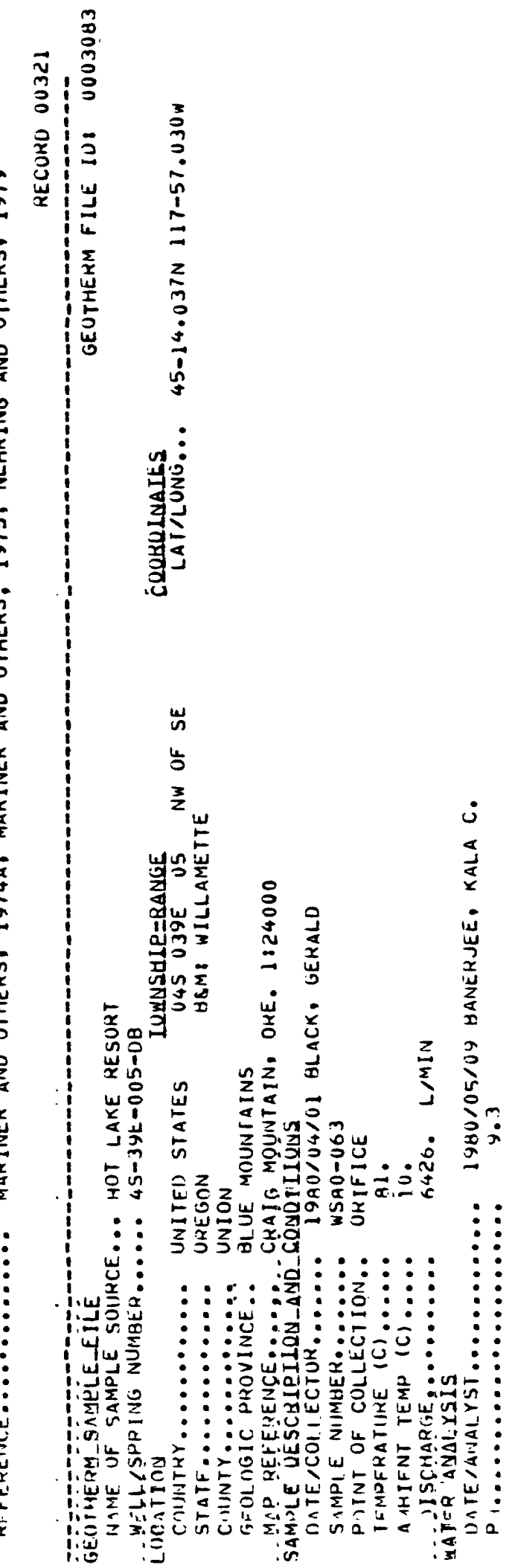


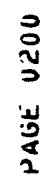

aี

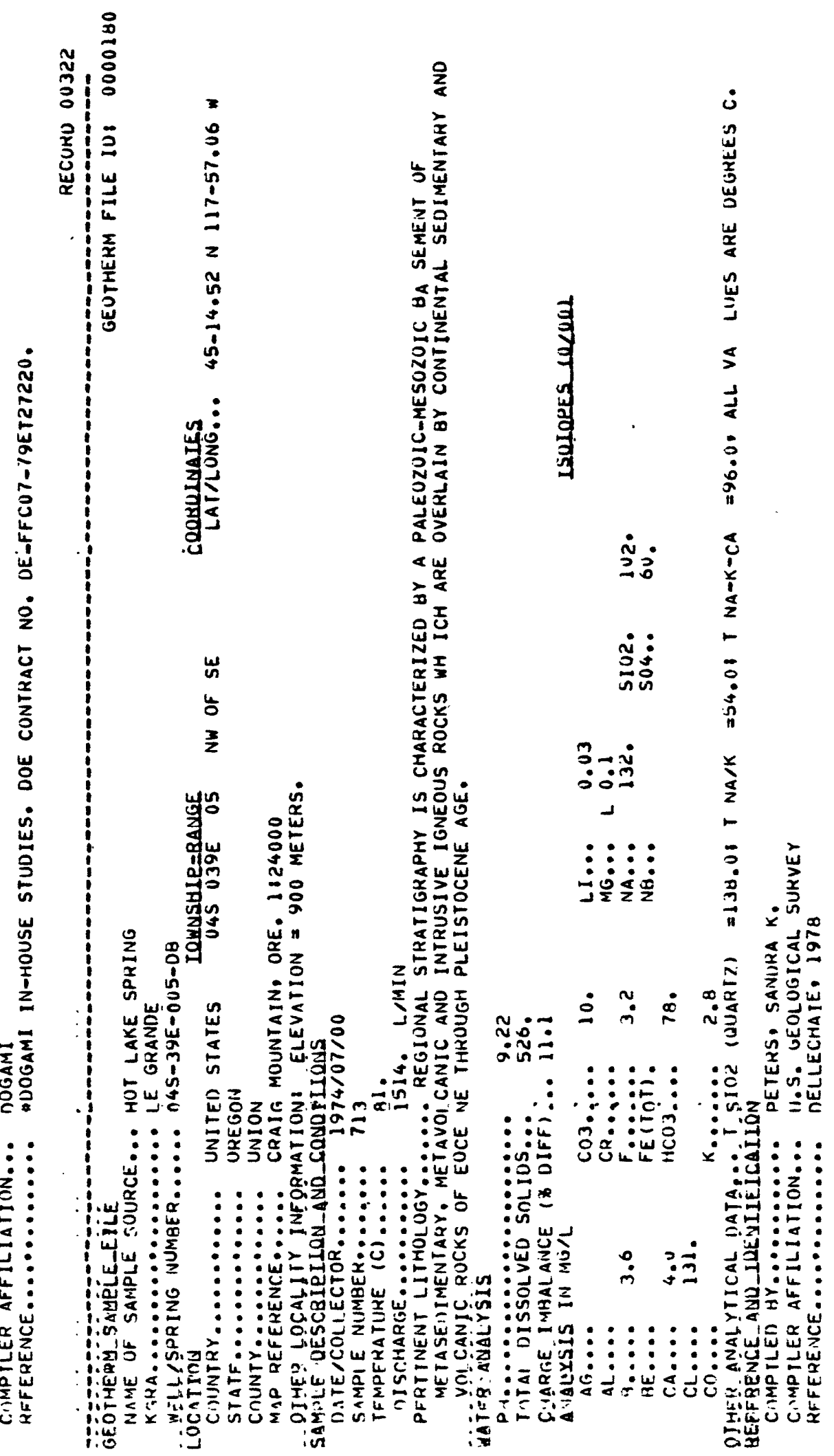


$\frac{2}{3}$
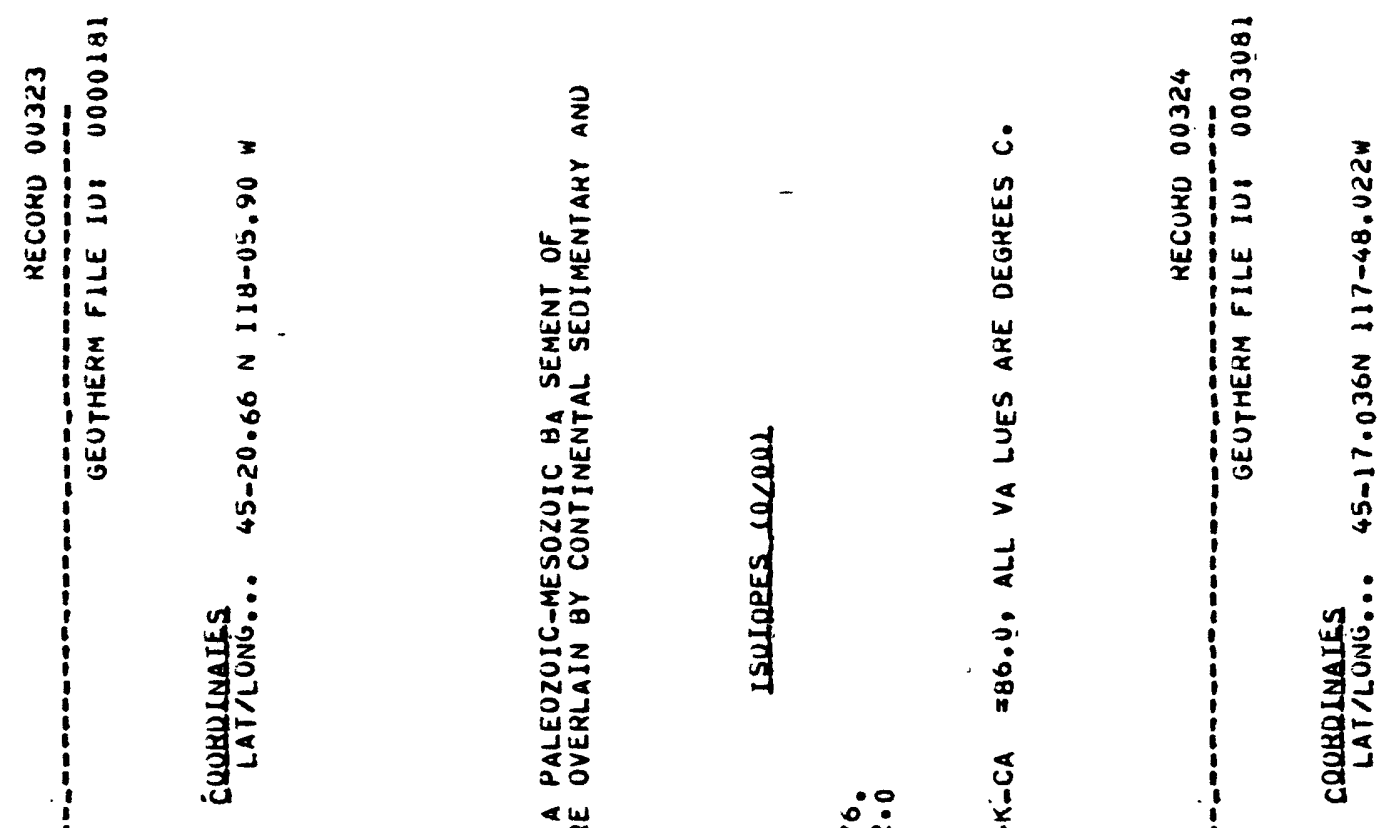

$\omega$
㭊
$\omega$

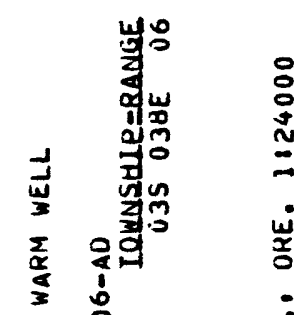

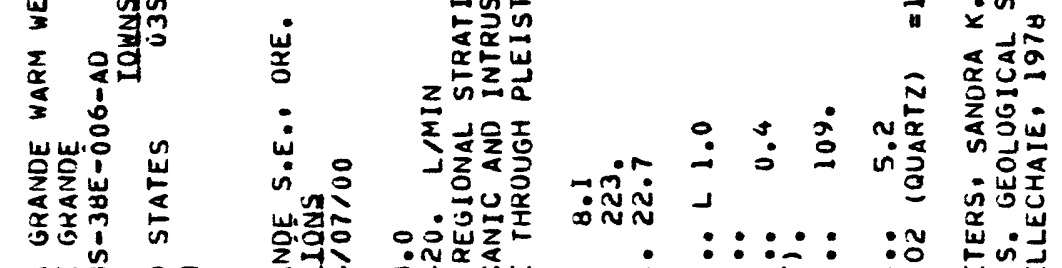

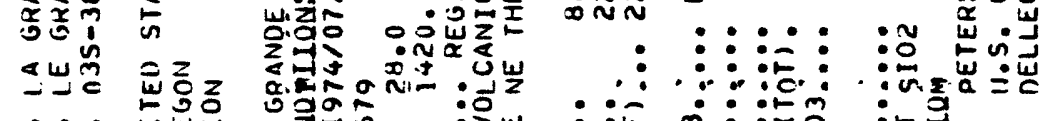

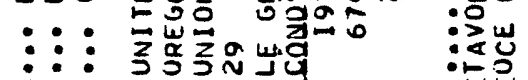

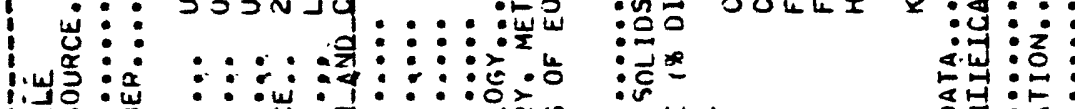
筞

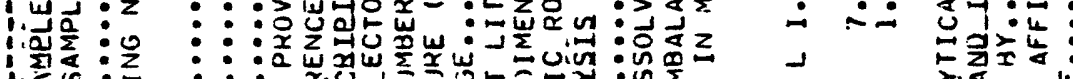

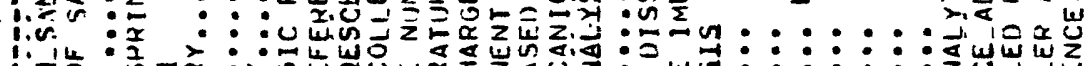
: :

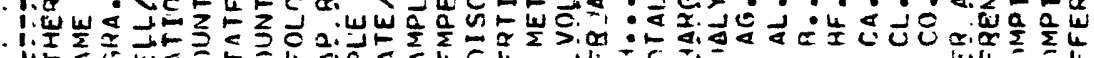

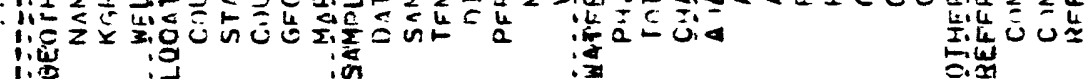

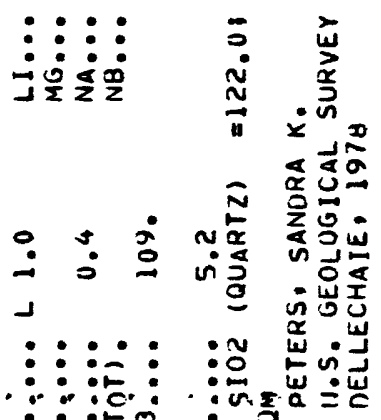
: : :

เ马 


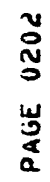

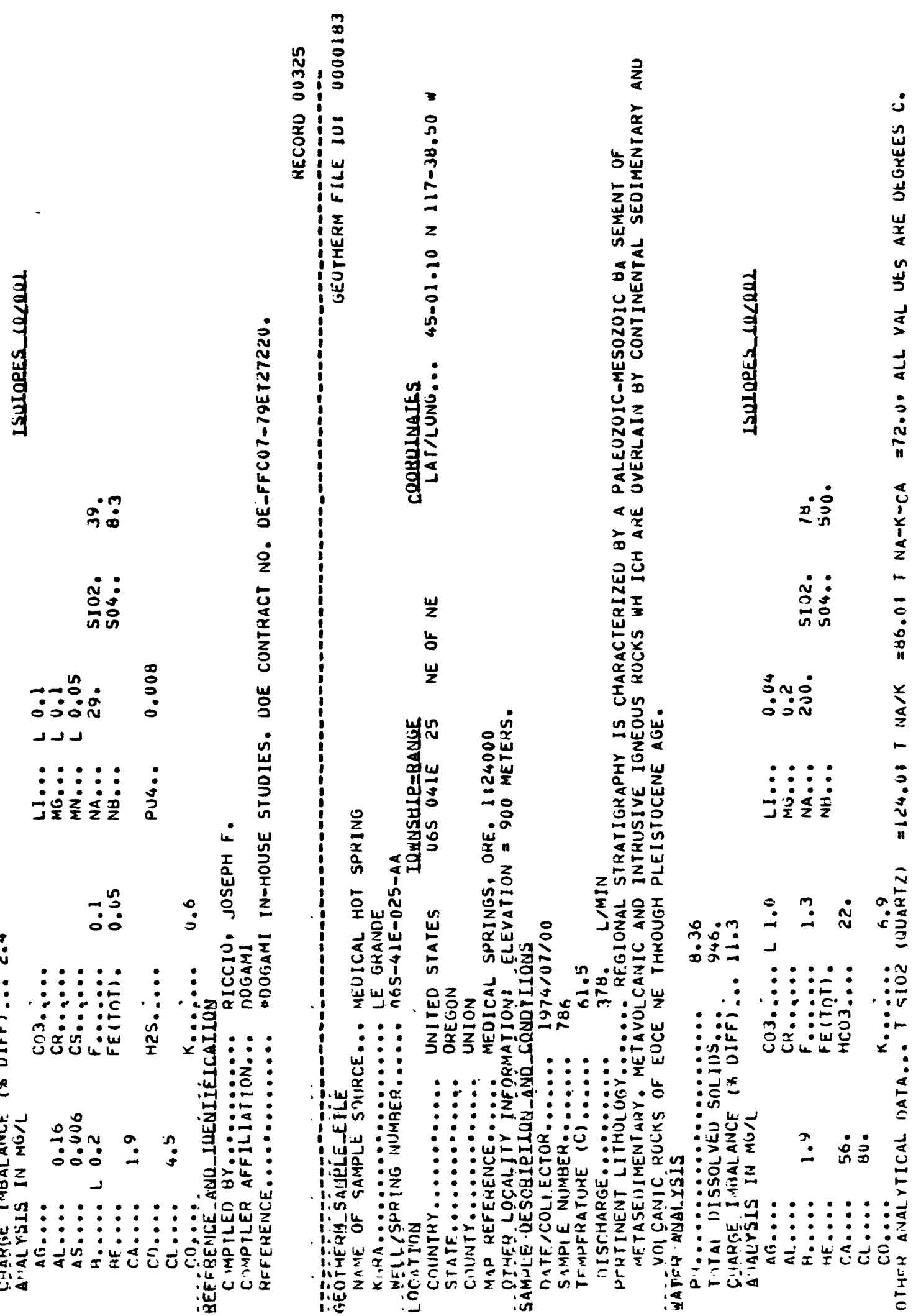




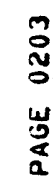

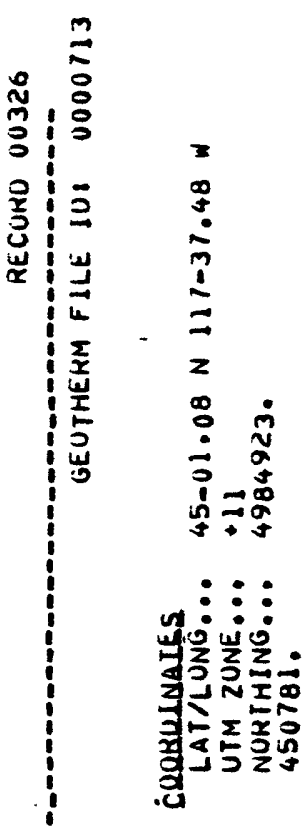

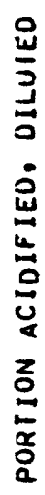

?ุ:
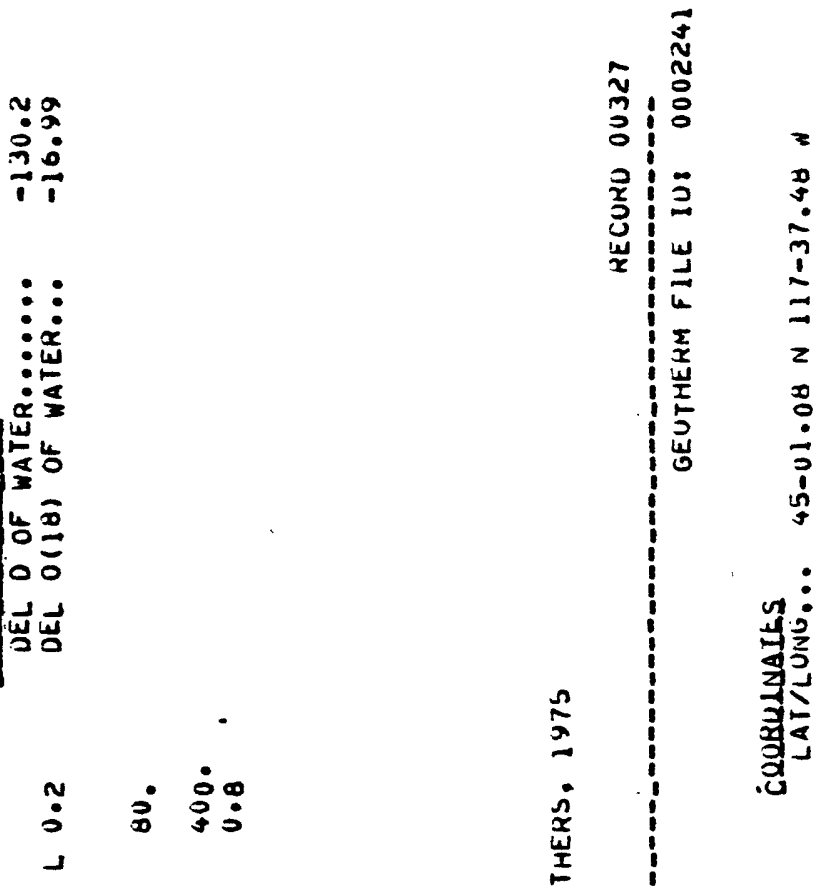

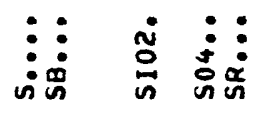
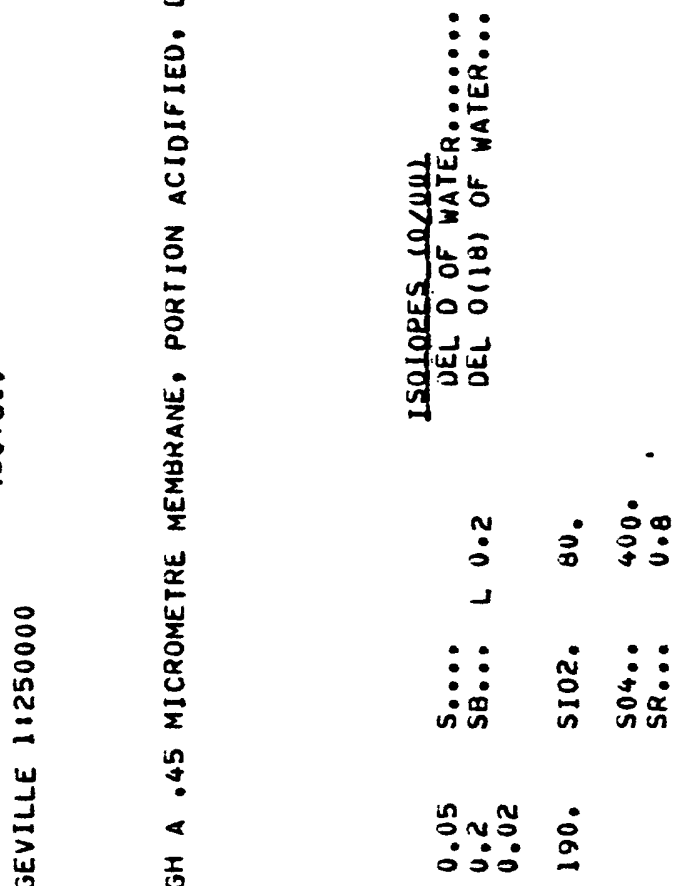

$\stackrel{0}{\circ}:$

岁

乲

荘 !

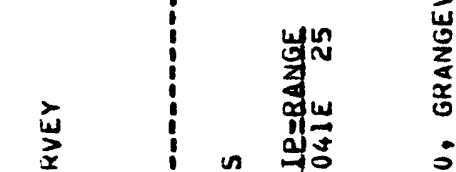

곤

亏ั้

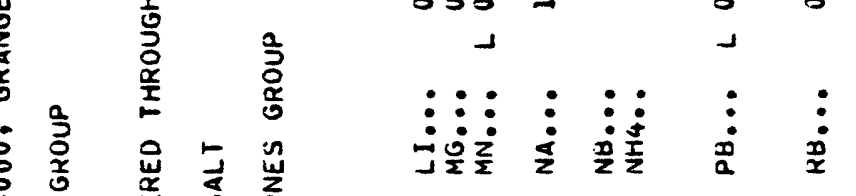

岌

赵运余

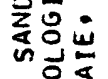

的岁志

至

ก.

:

㟧三

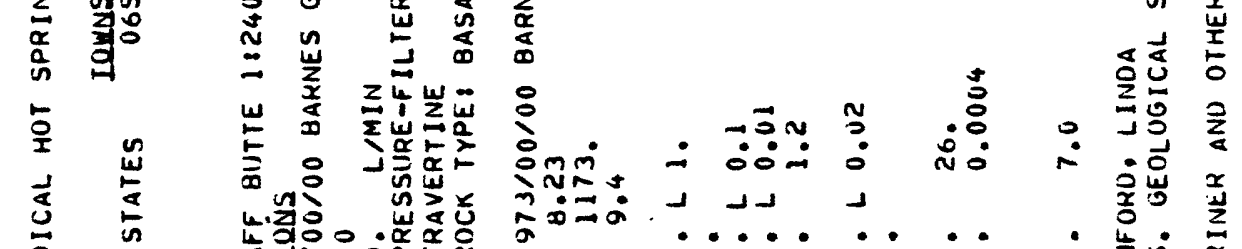

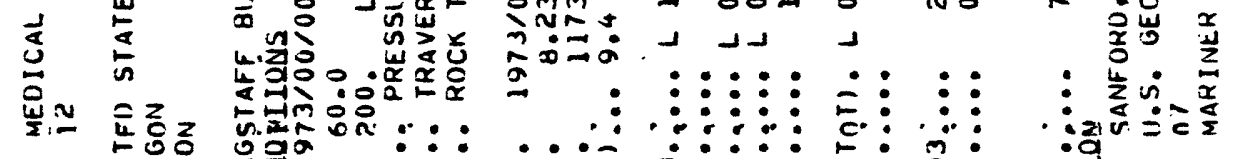

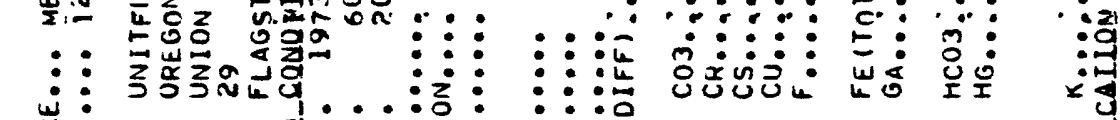

运:

$::::: \div$

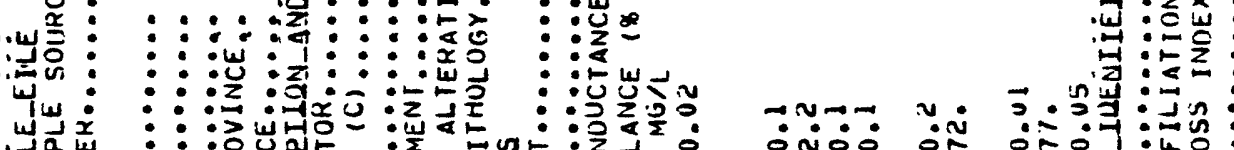

o

:

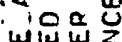




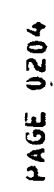

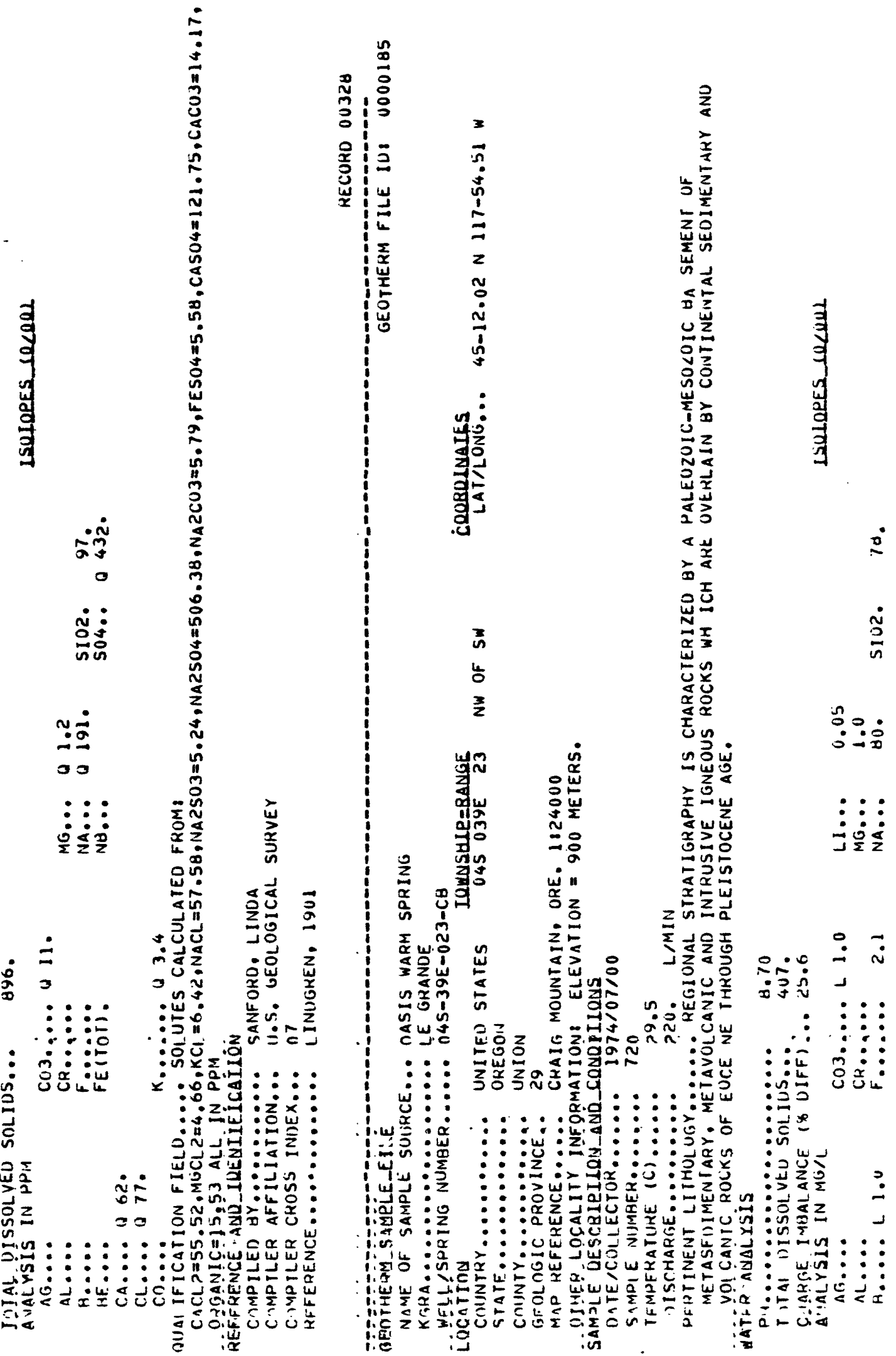




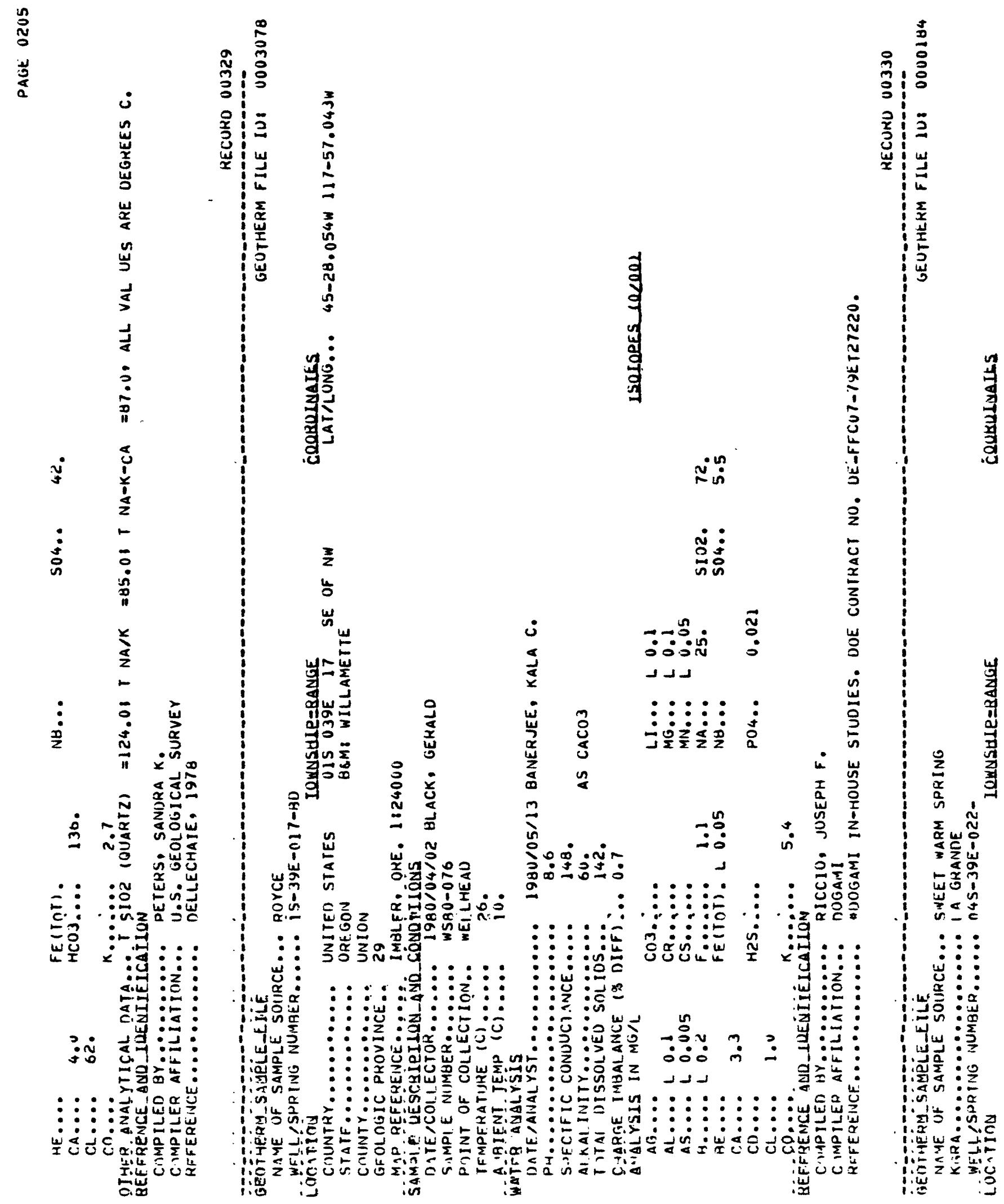



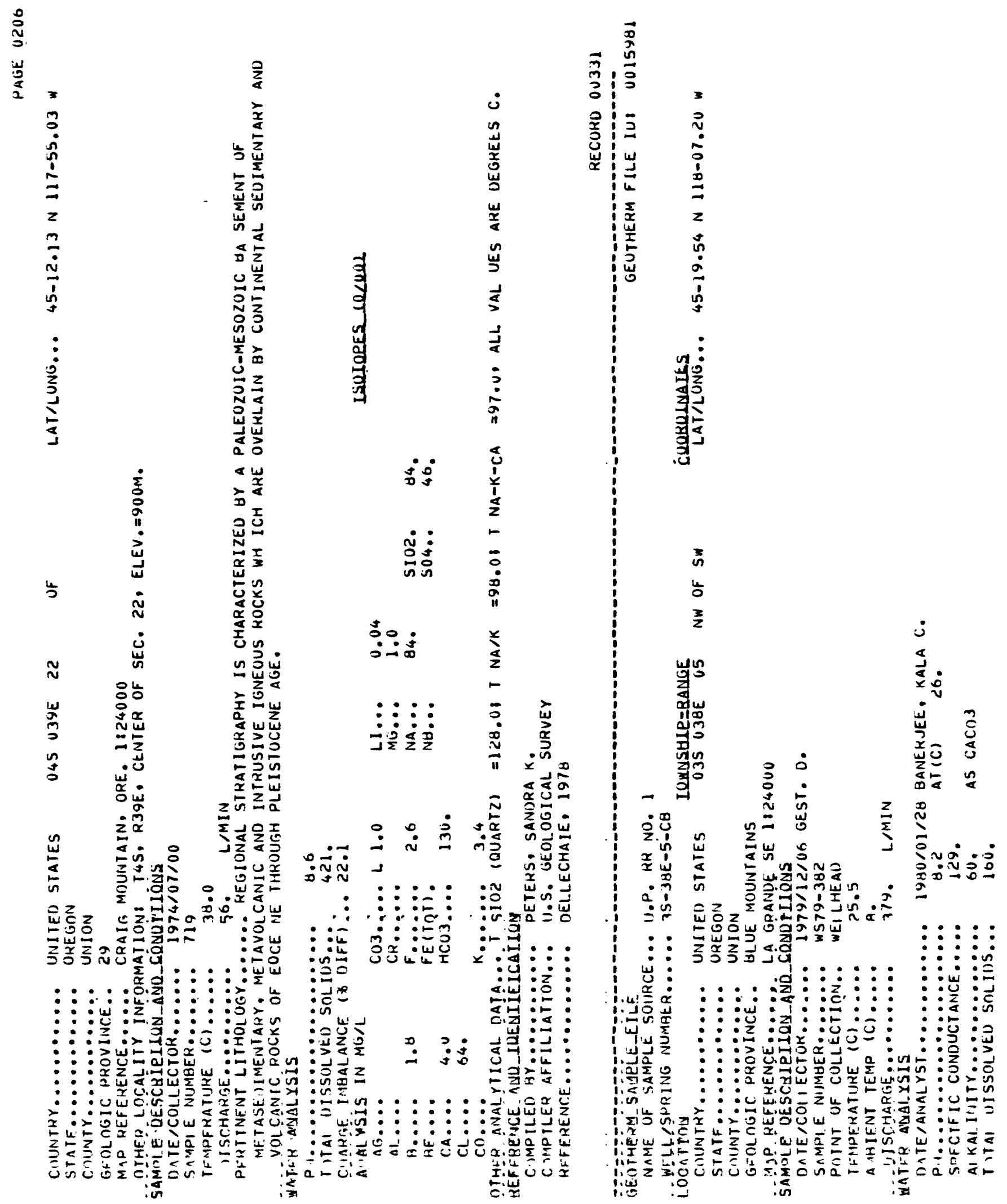


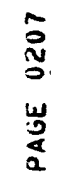

荈
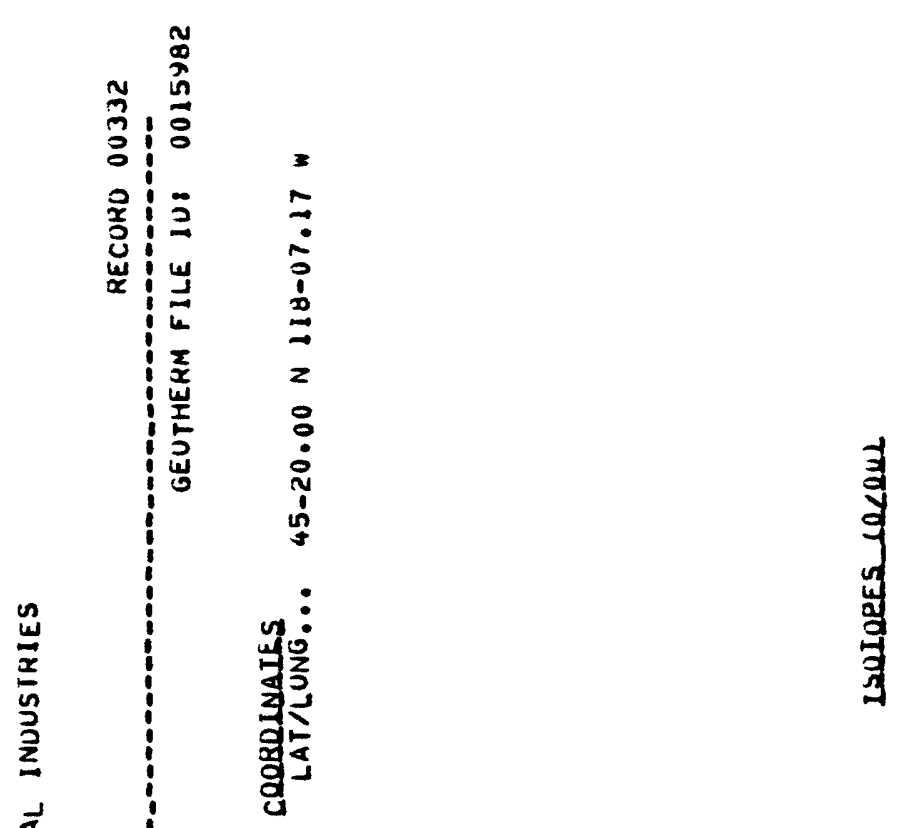

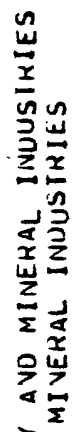

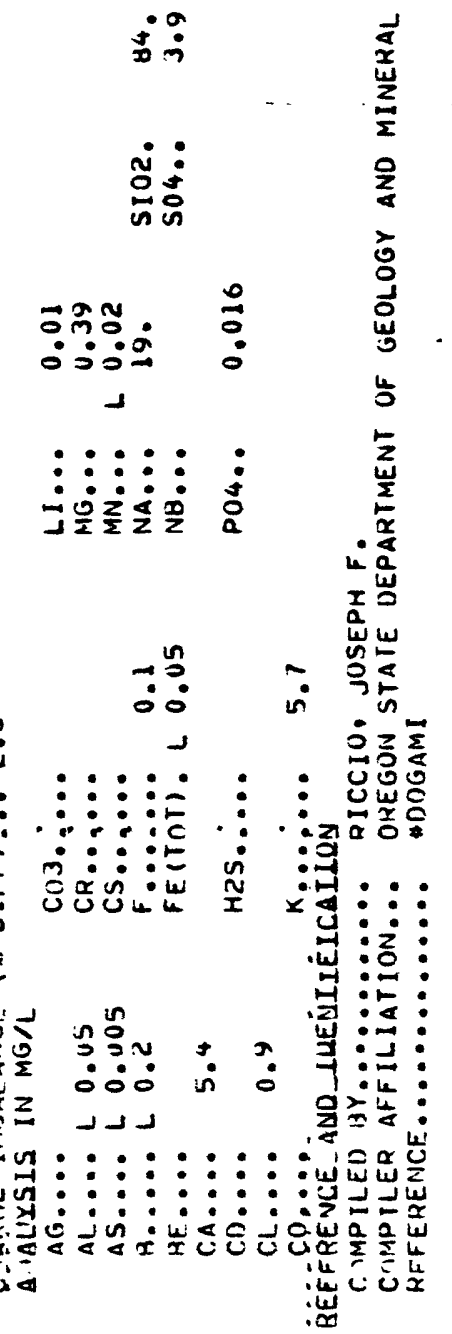

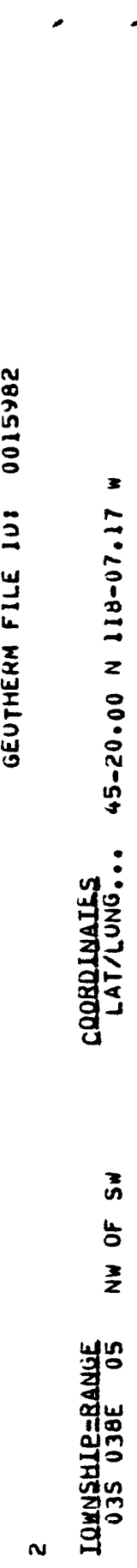

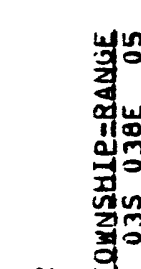

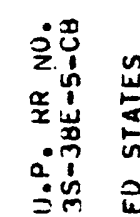

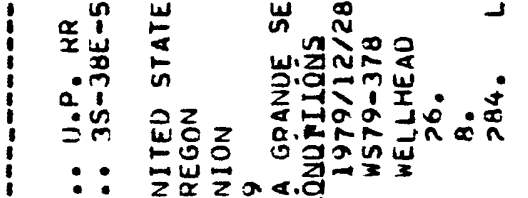

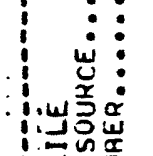

.

y

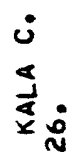

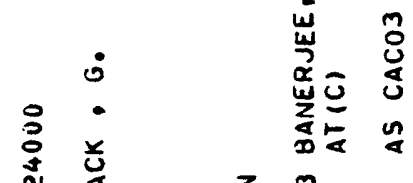

(n)

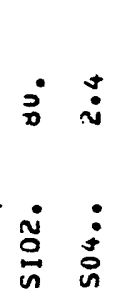

疍

I

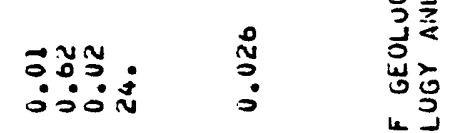

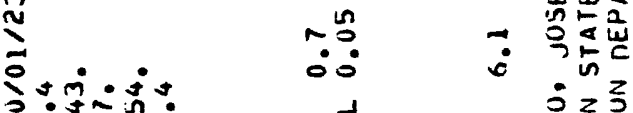

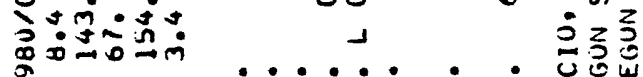
2

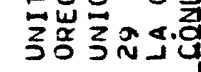

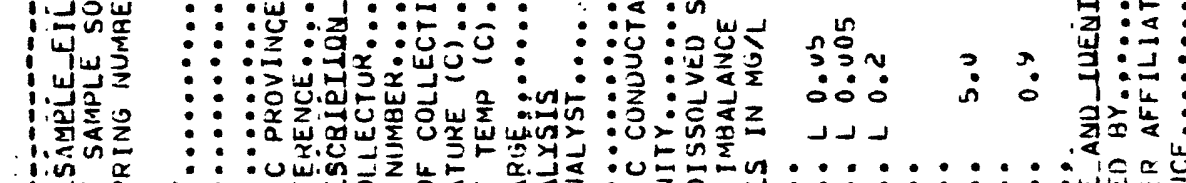

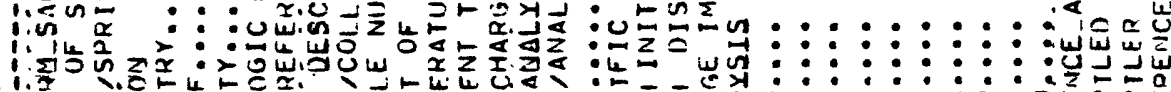

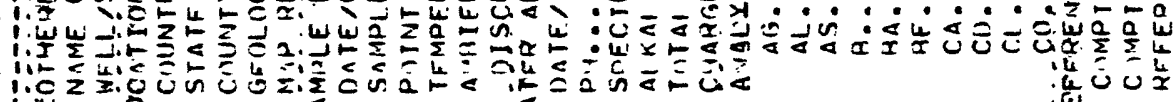

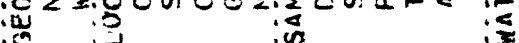



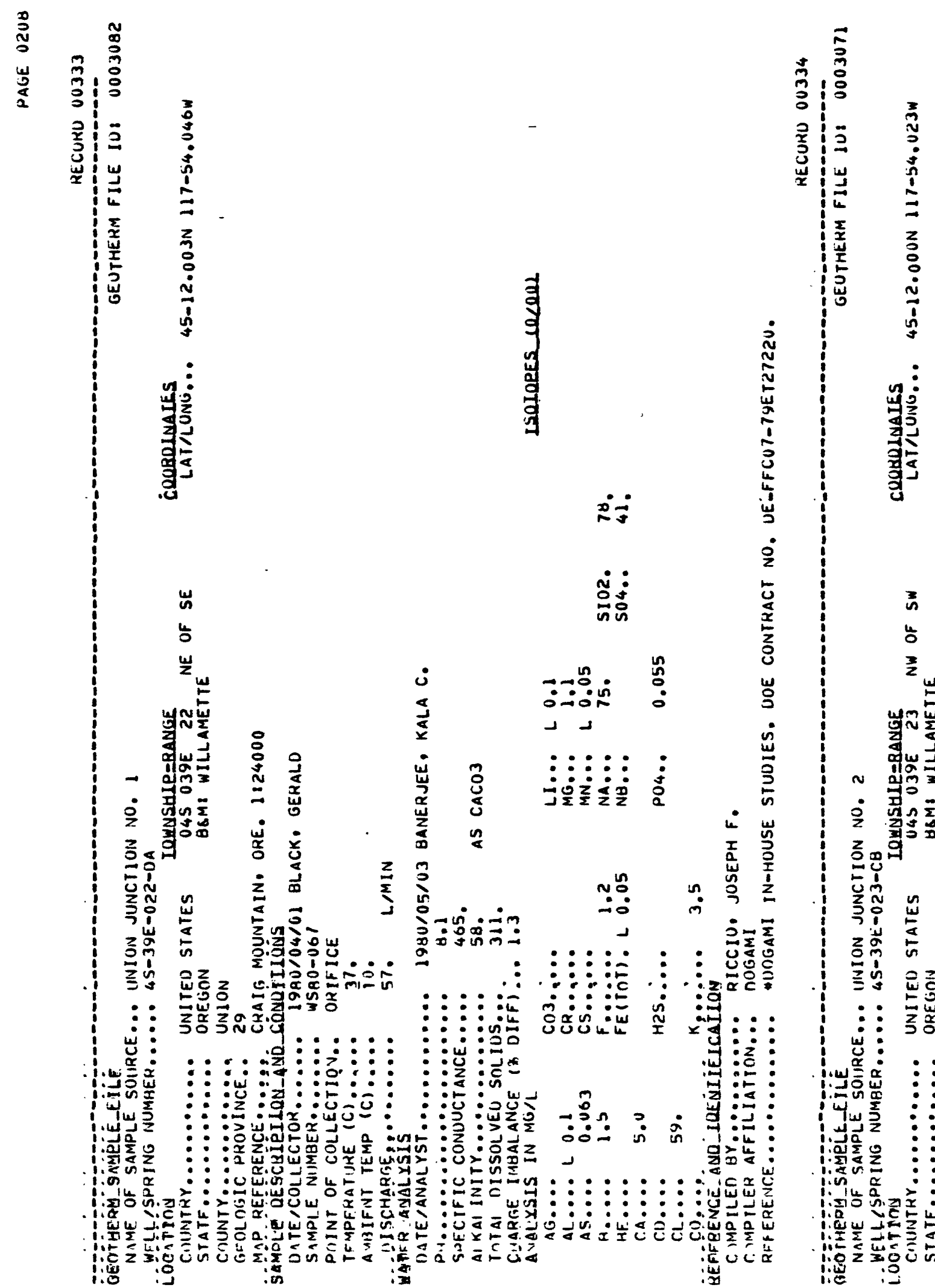


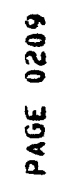
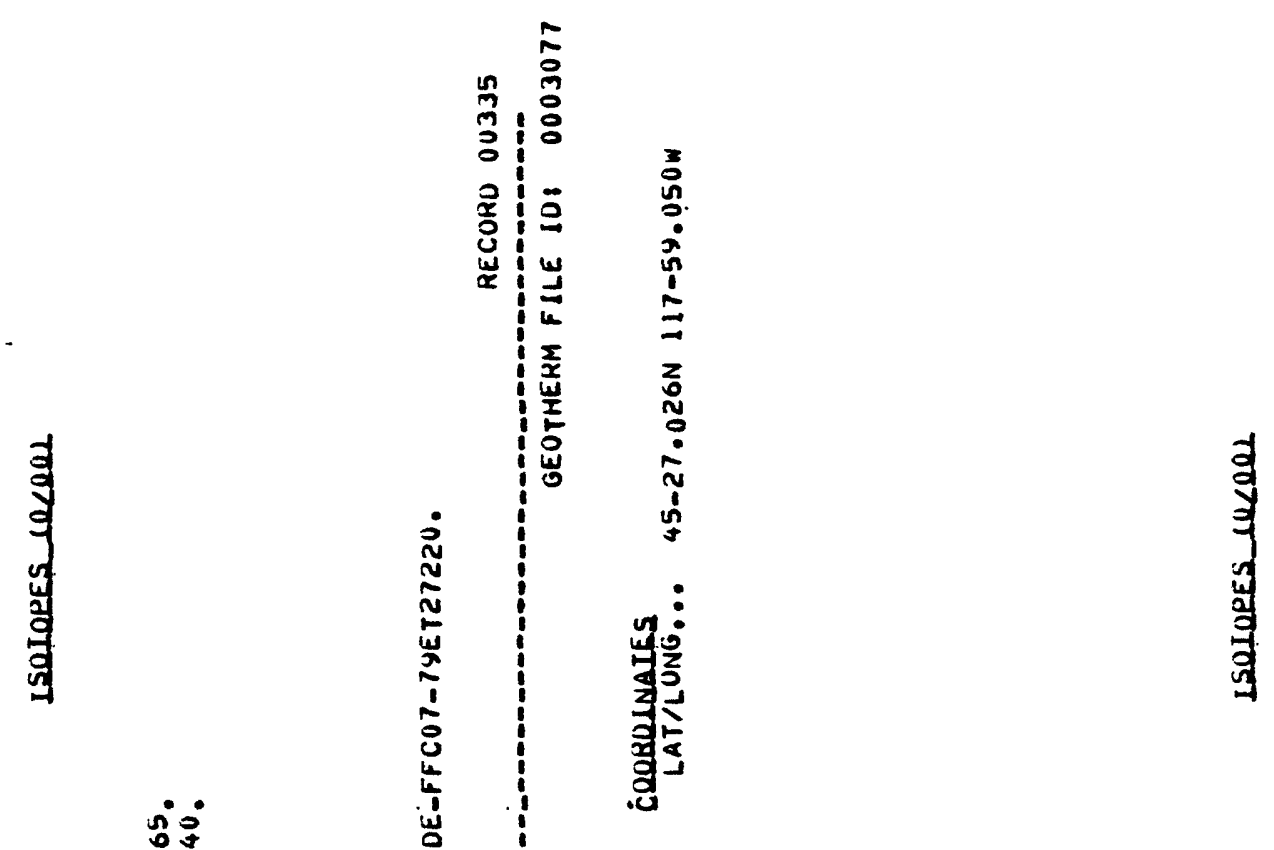

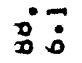

范范

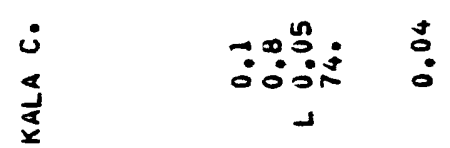

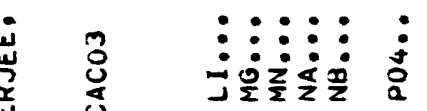

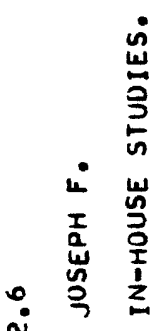

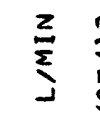

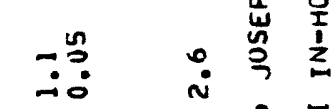
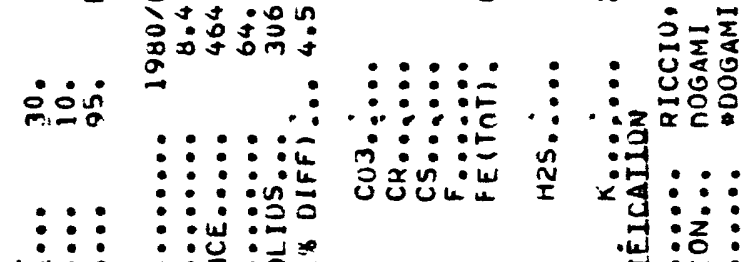

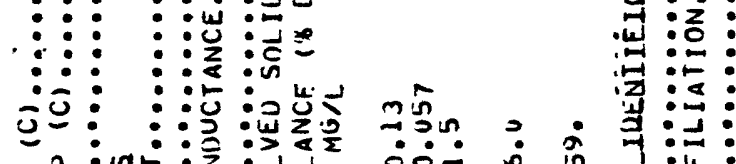

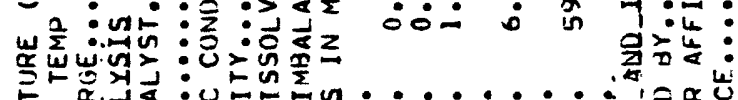

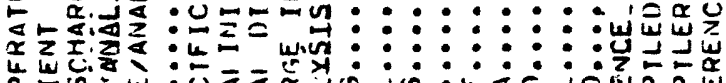

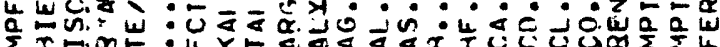

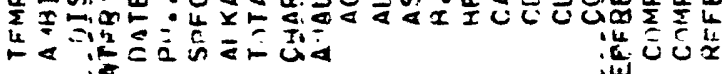
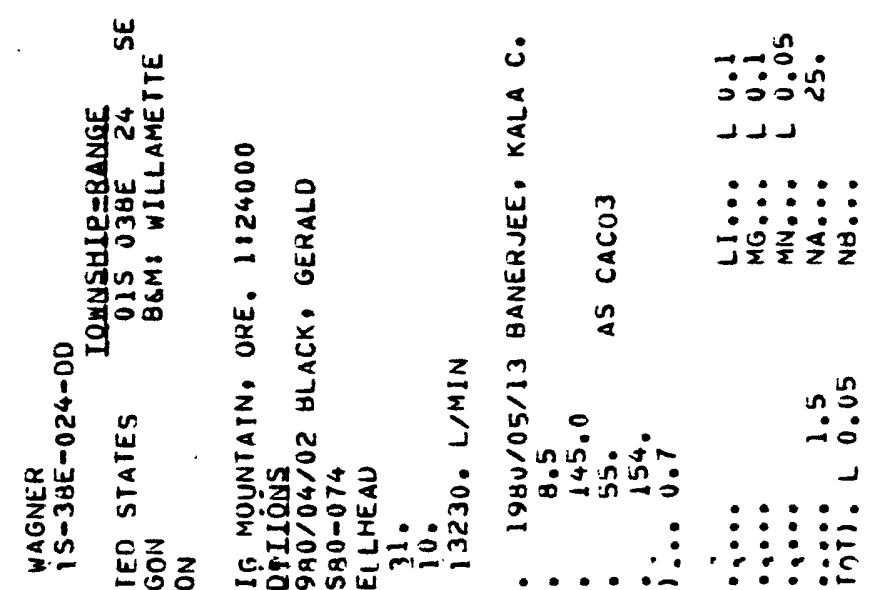

范:

ร

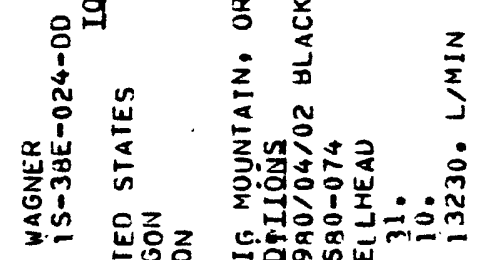

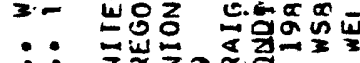

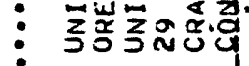

:

:

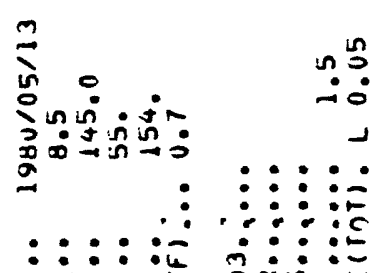

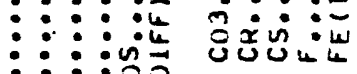

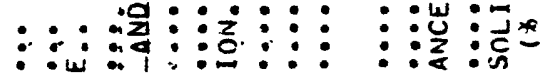

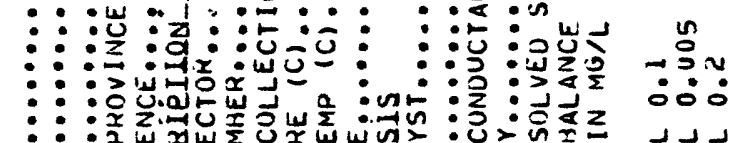

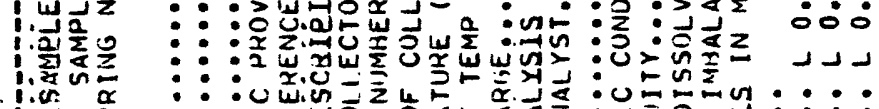
lín

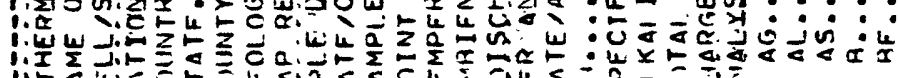

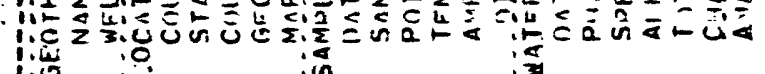




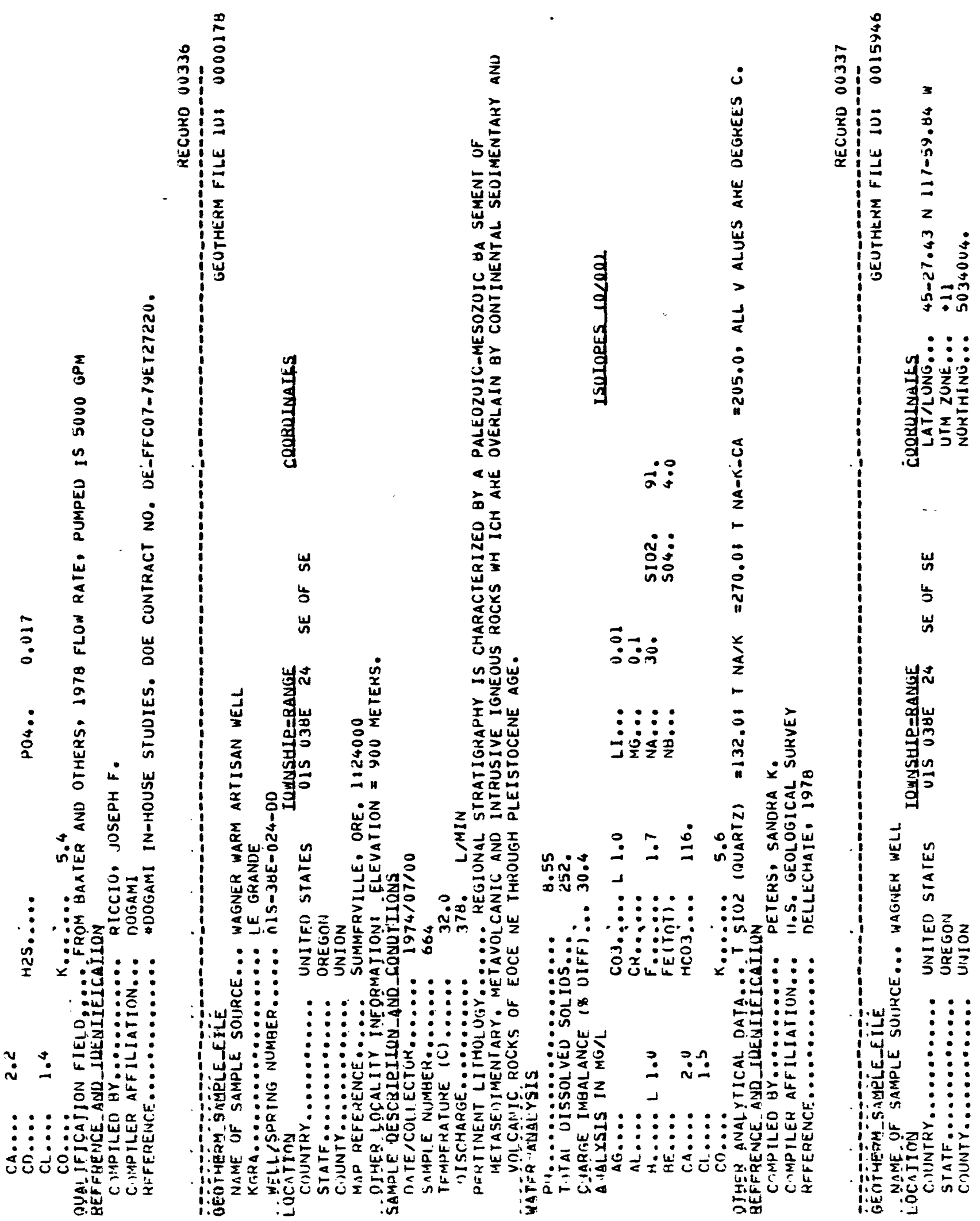




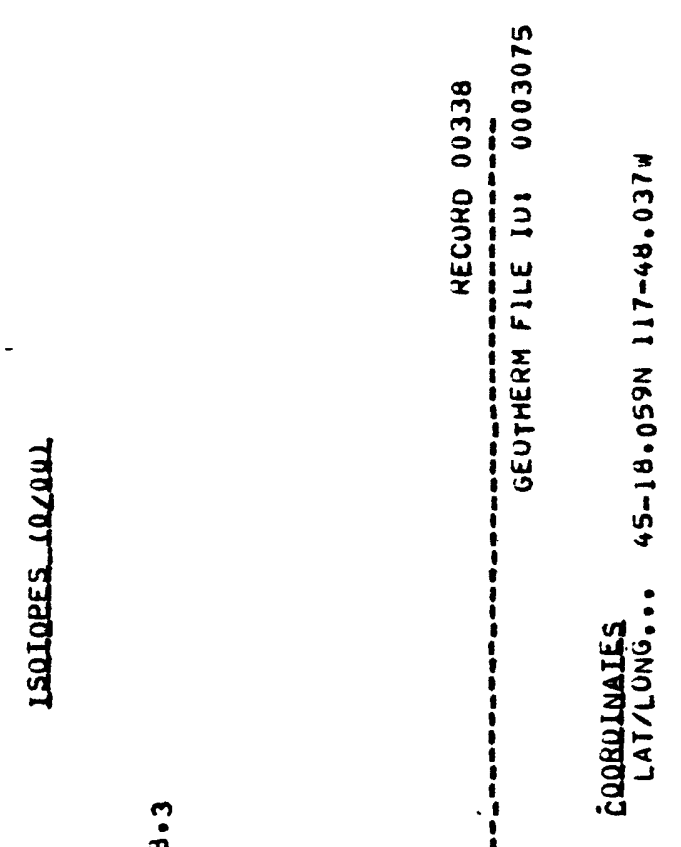

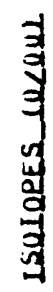

$\underset{\stackrel{N}{\sim}}{\stackrel{\sim}{*}}$

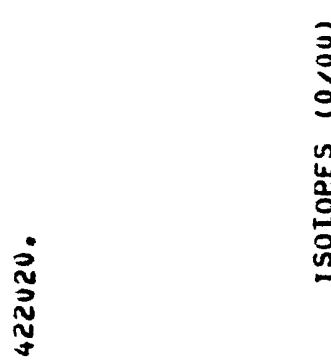

$\stackrel{\infty}{\circ: \infty}$

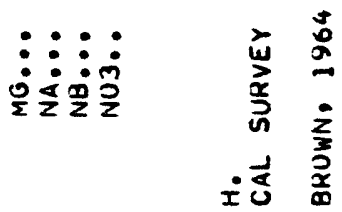

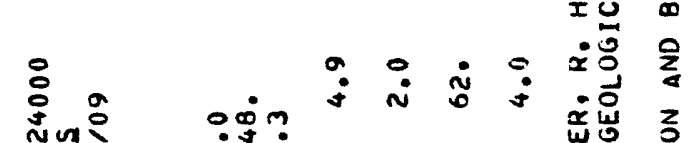

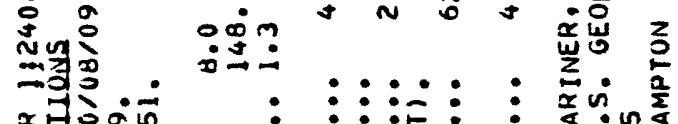

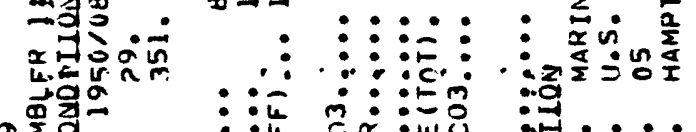
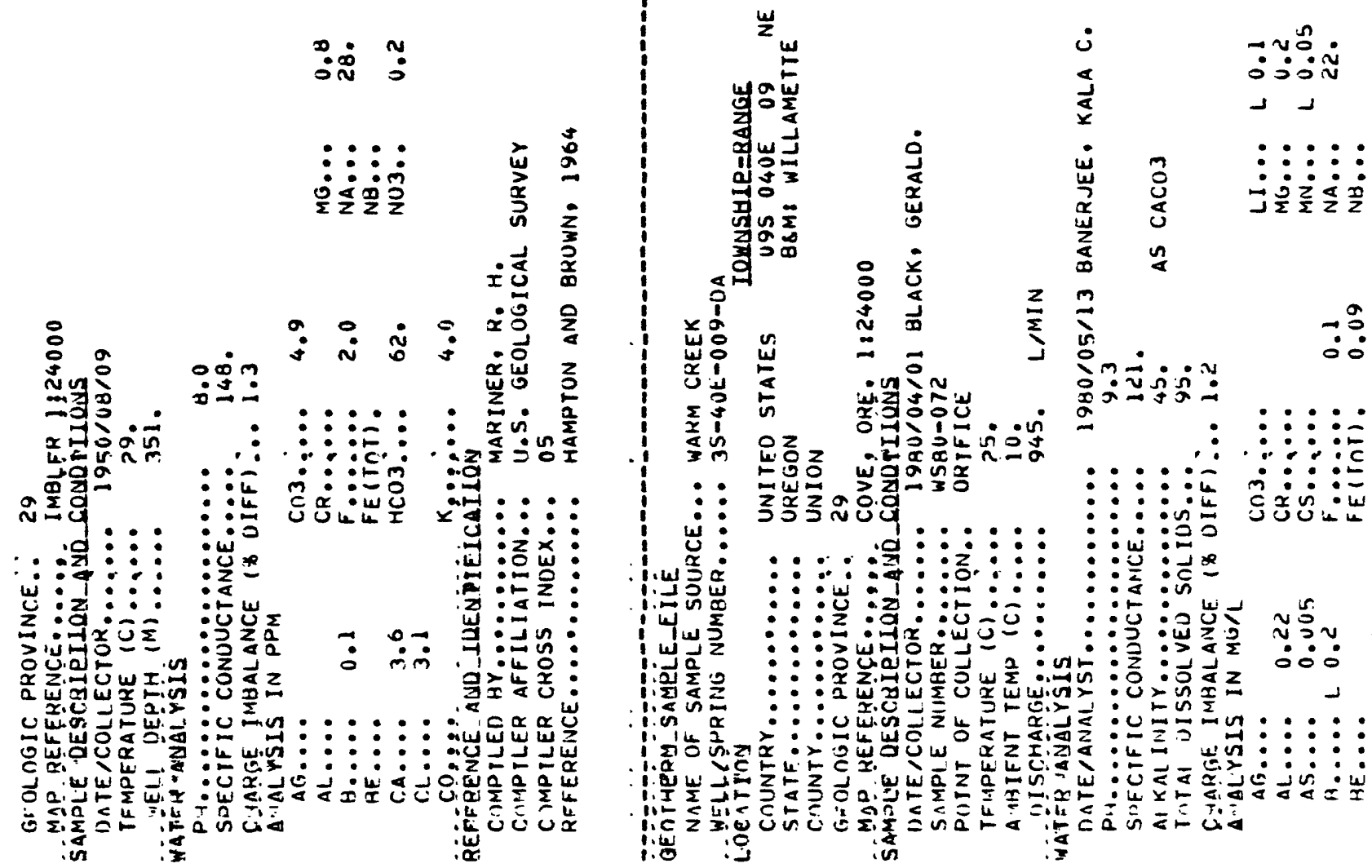

$\omega$
L
$z$

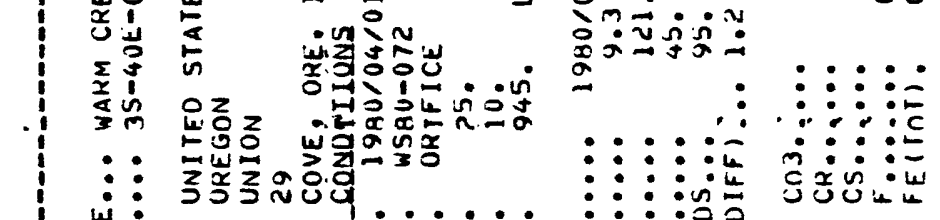

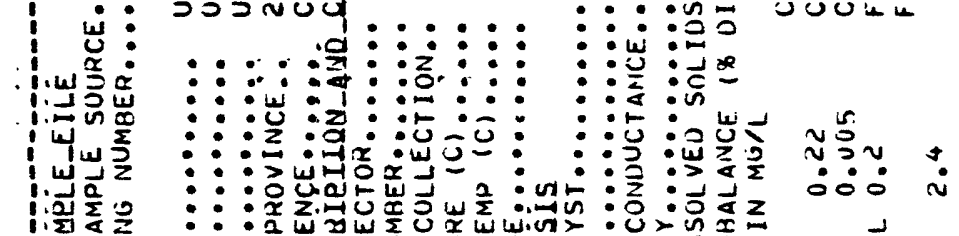

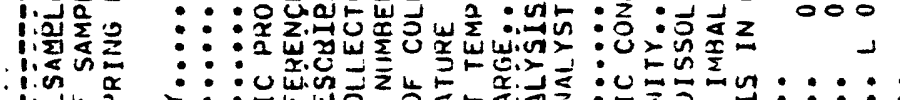

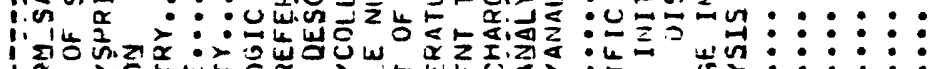

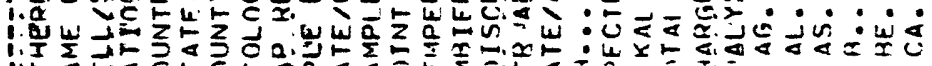

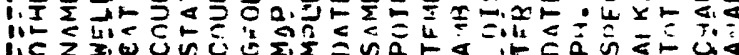

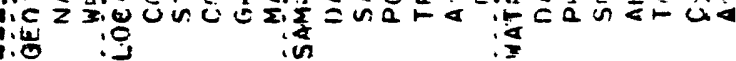




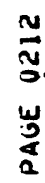

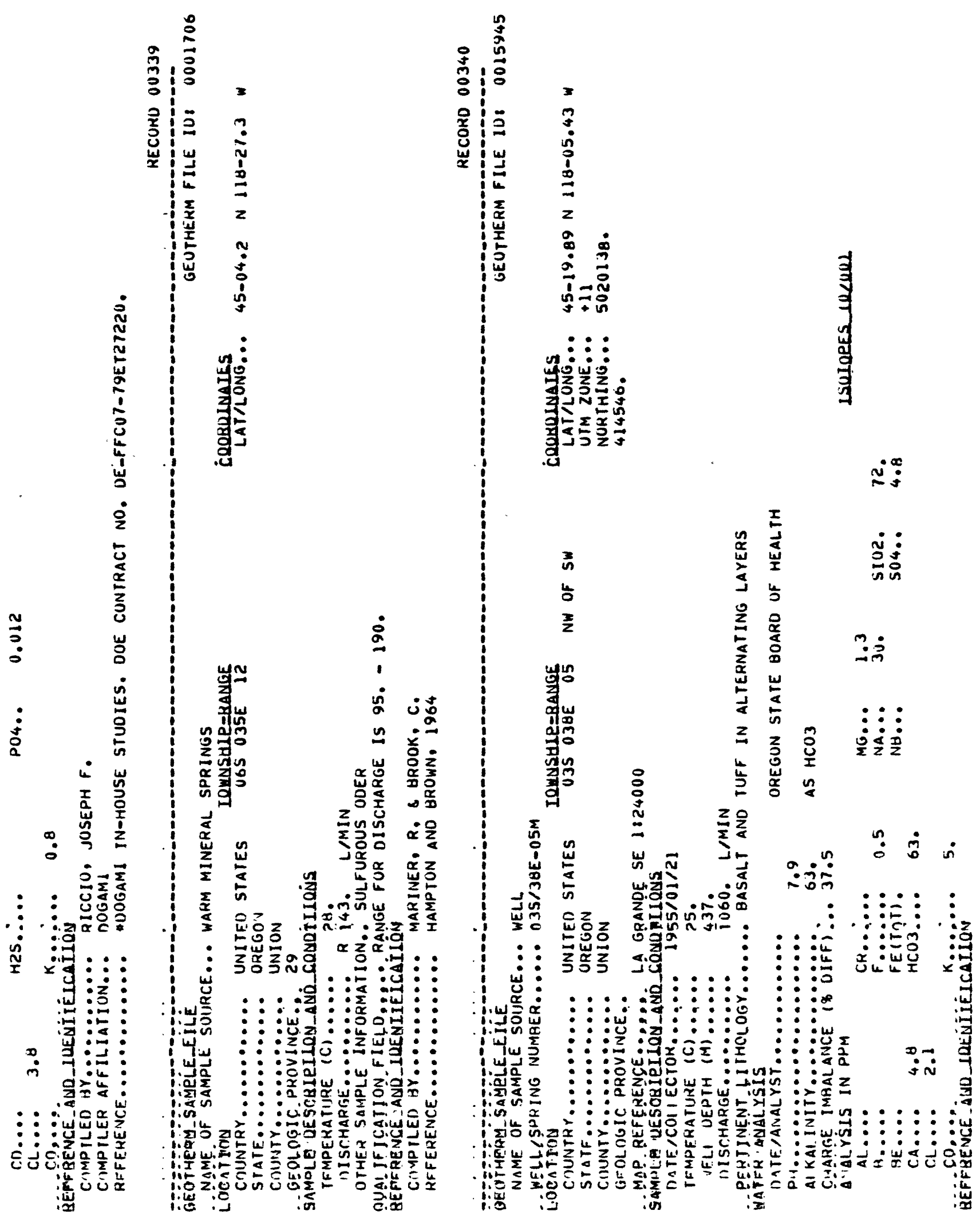




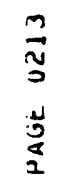

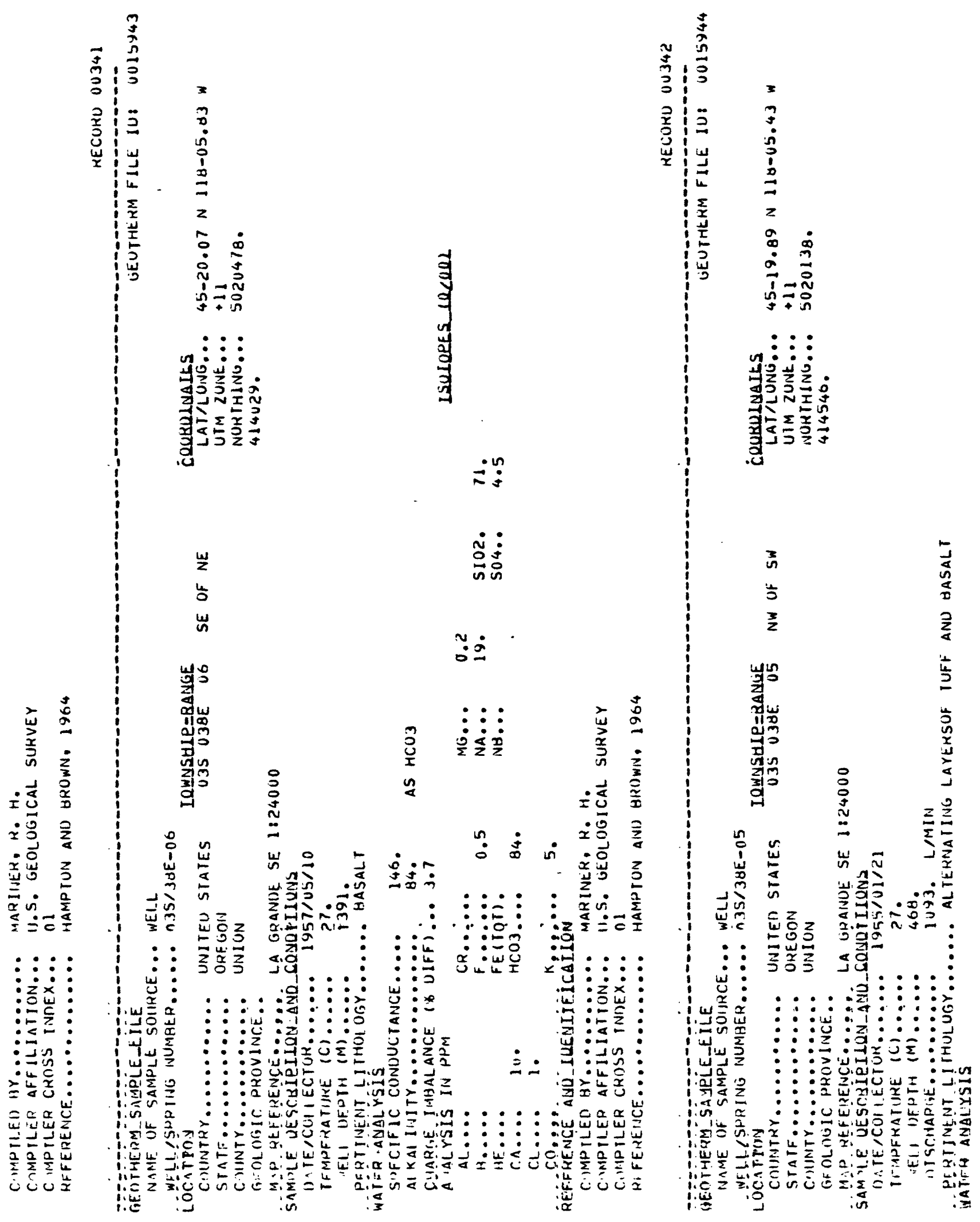




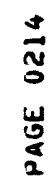

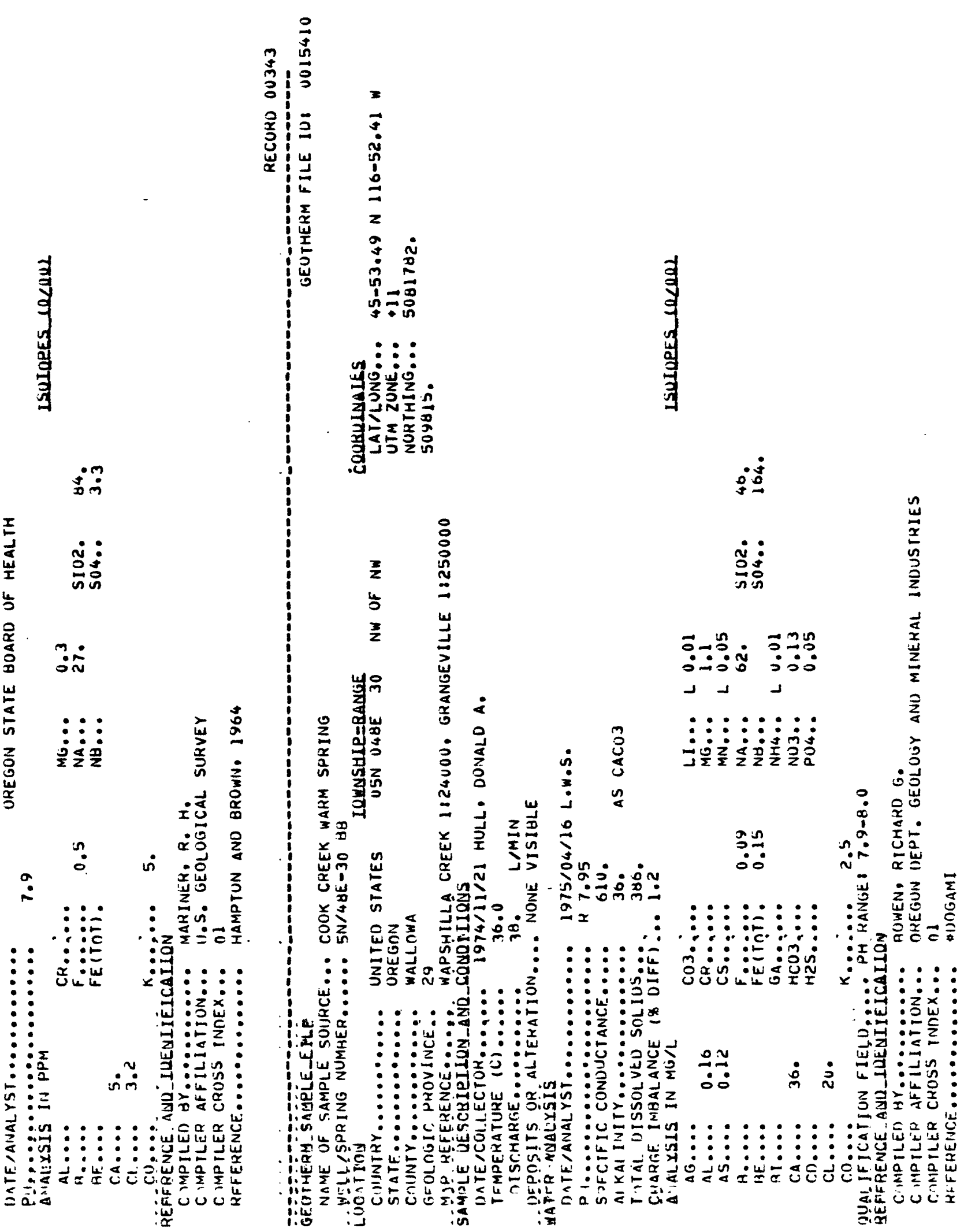



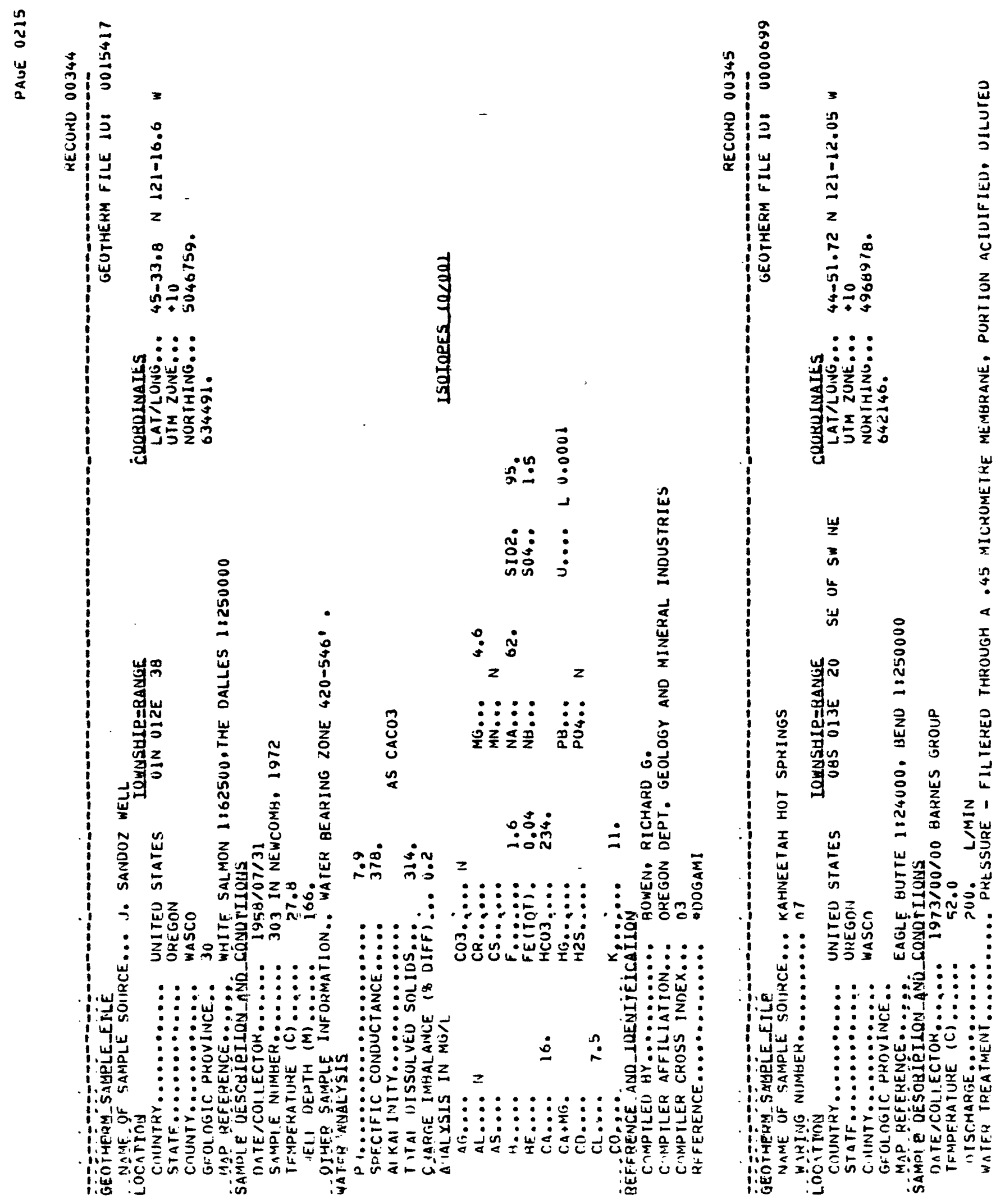


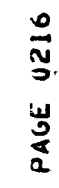
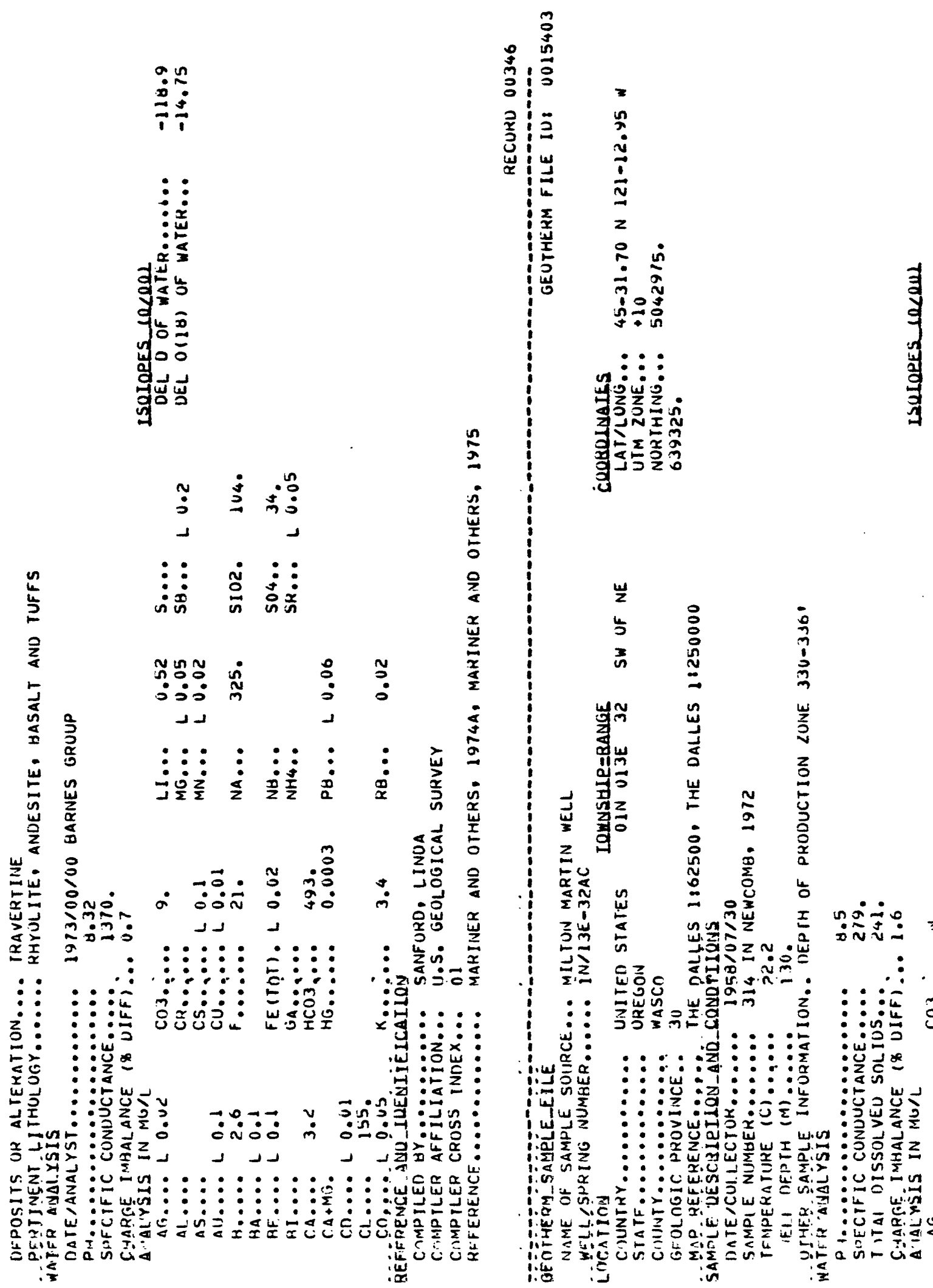


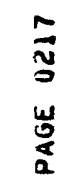

:

: $\quad$ 岂

- $\frac{1}{\frac{d}{2}}$

$2: \quad$ \&

ख⿺辶寸

苟

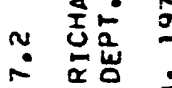

$\vdots \vdots \vdots$ 竎弱

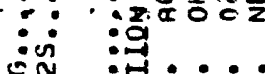

บำ บํํํ: : :

出:安文:

武汇文:

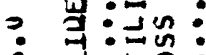

局亩亩:

: : : :

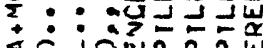

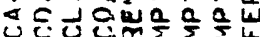

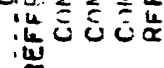




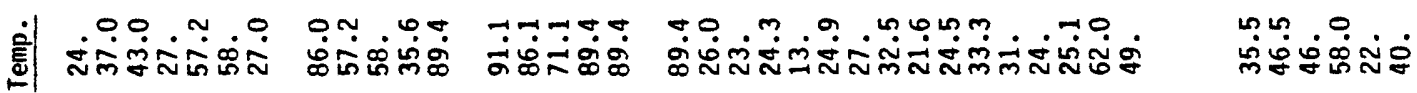

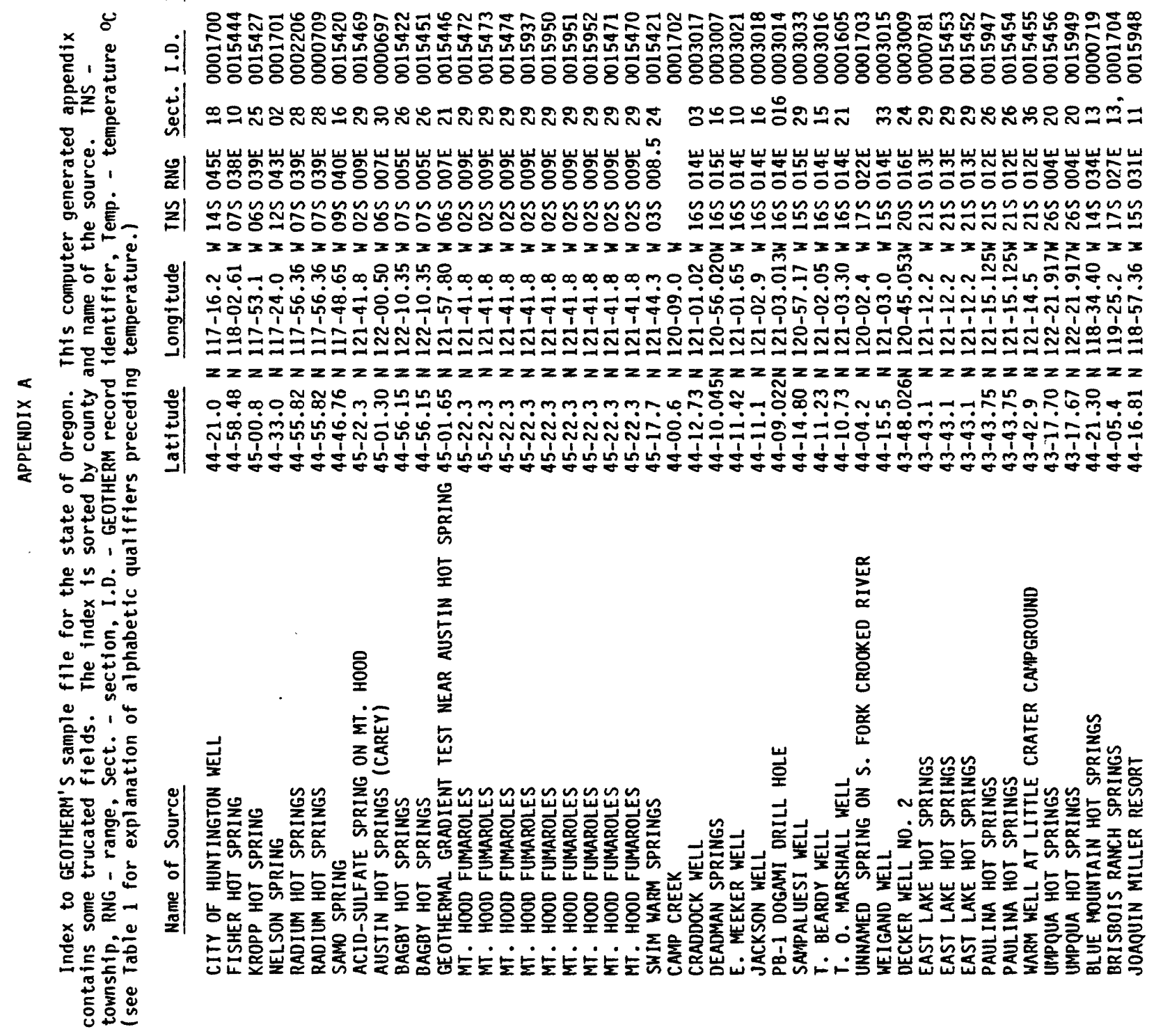

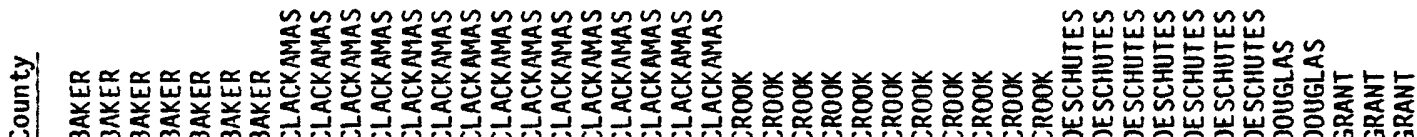


$\stackrel{2}{\text { ก }}$

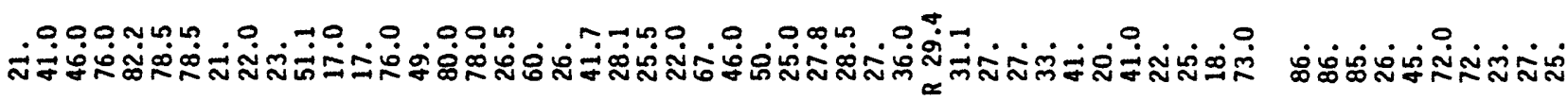

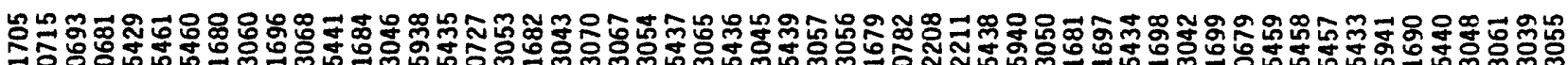

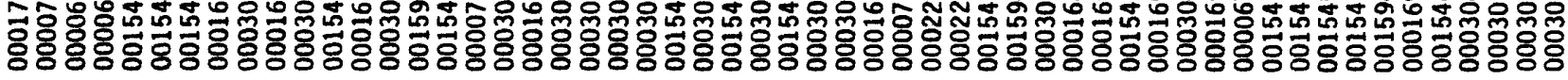

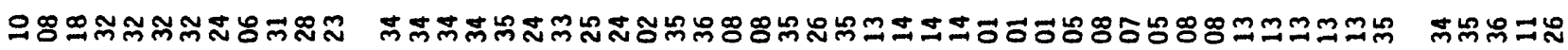

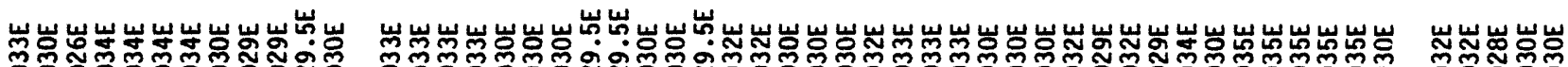

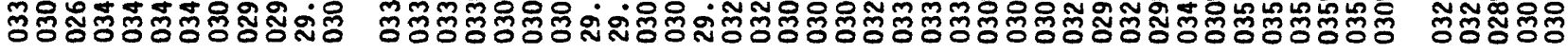

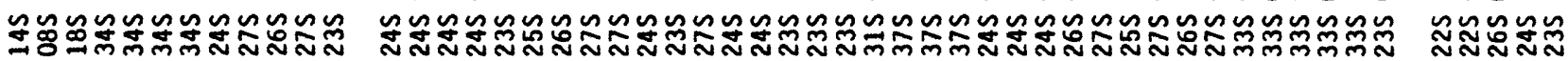

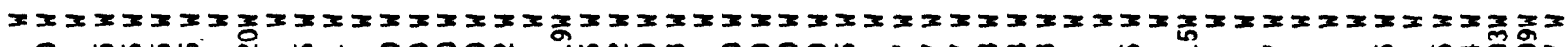

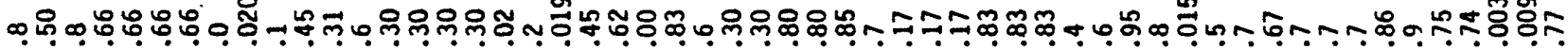

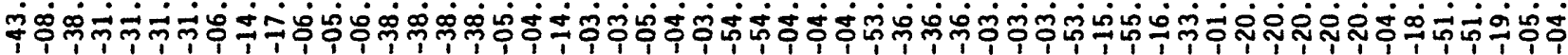

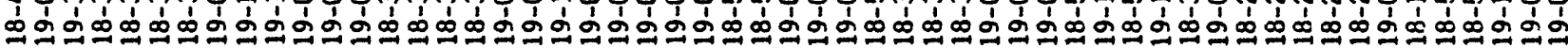

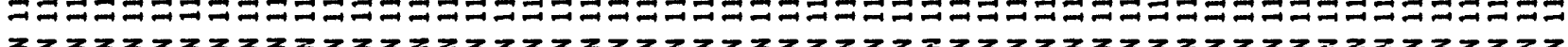

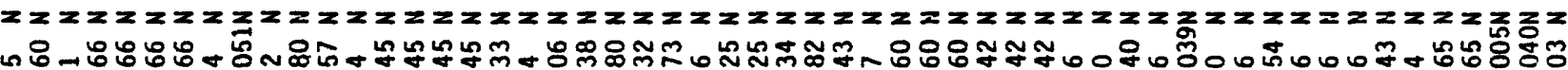
สิن்

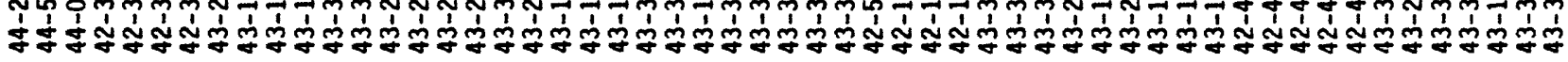

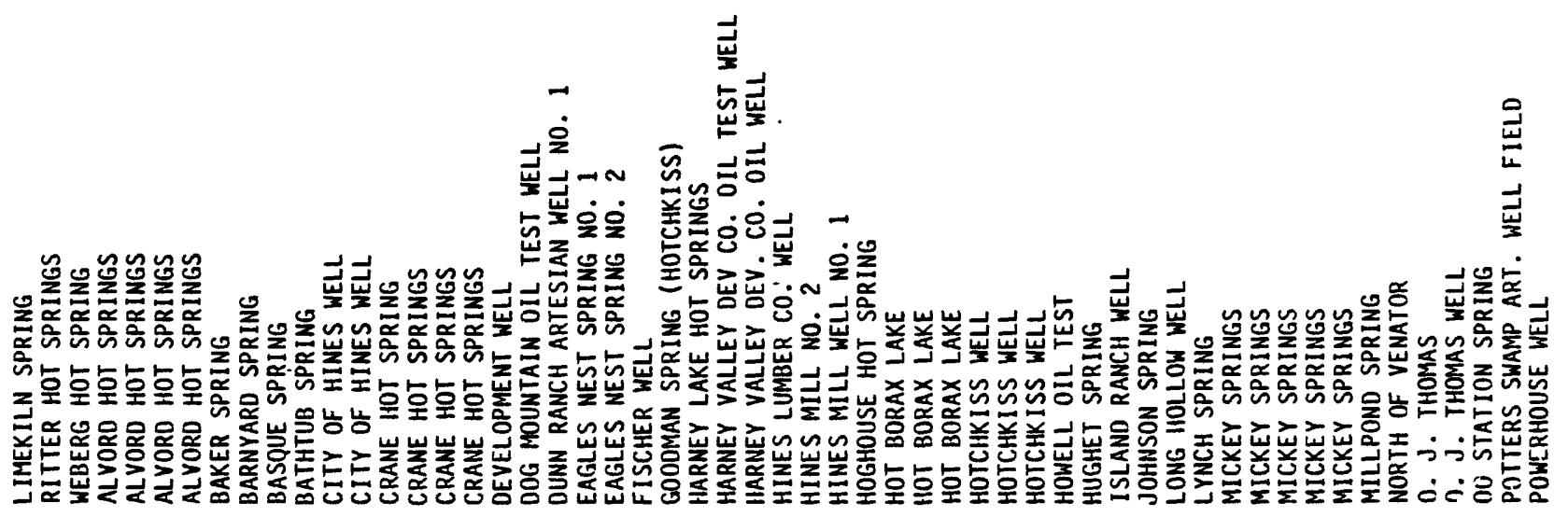


ลิ

ڤ

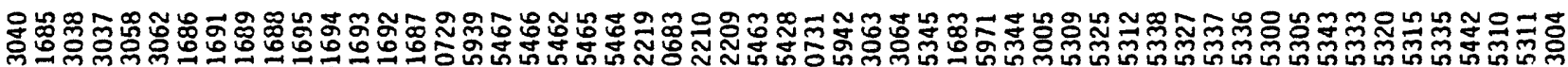

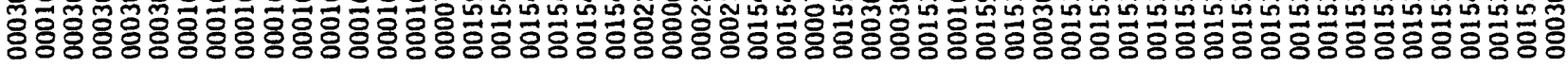

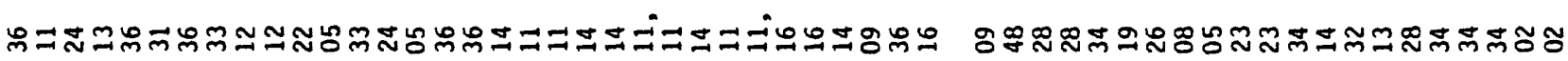

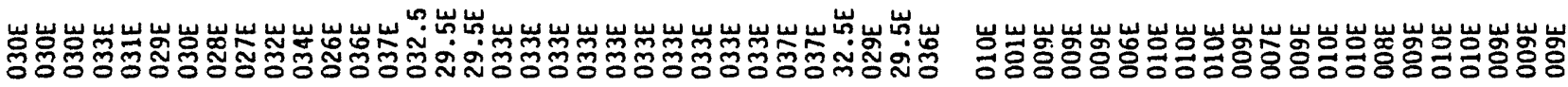

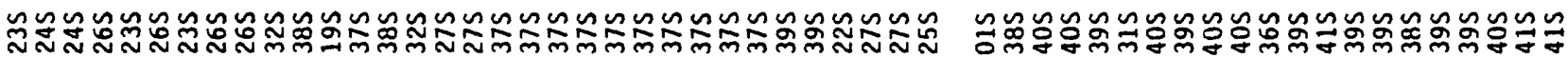

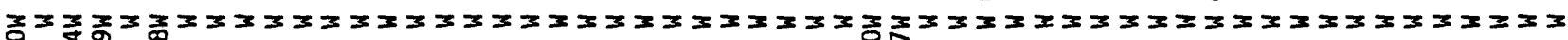

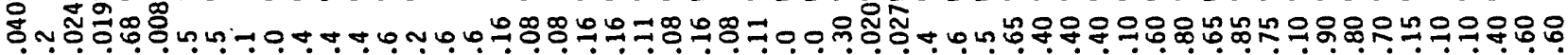
ర்ல்

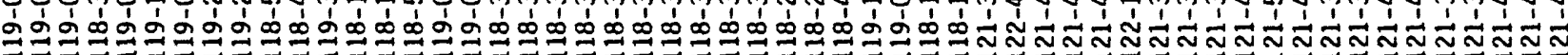

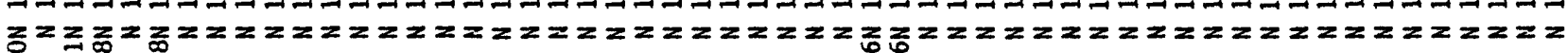

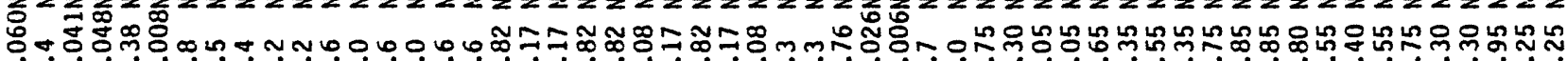
க்

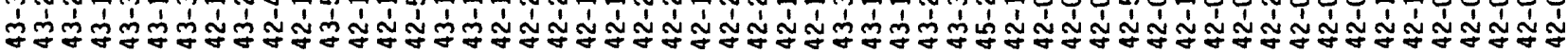

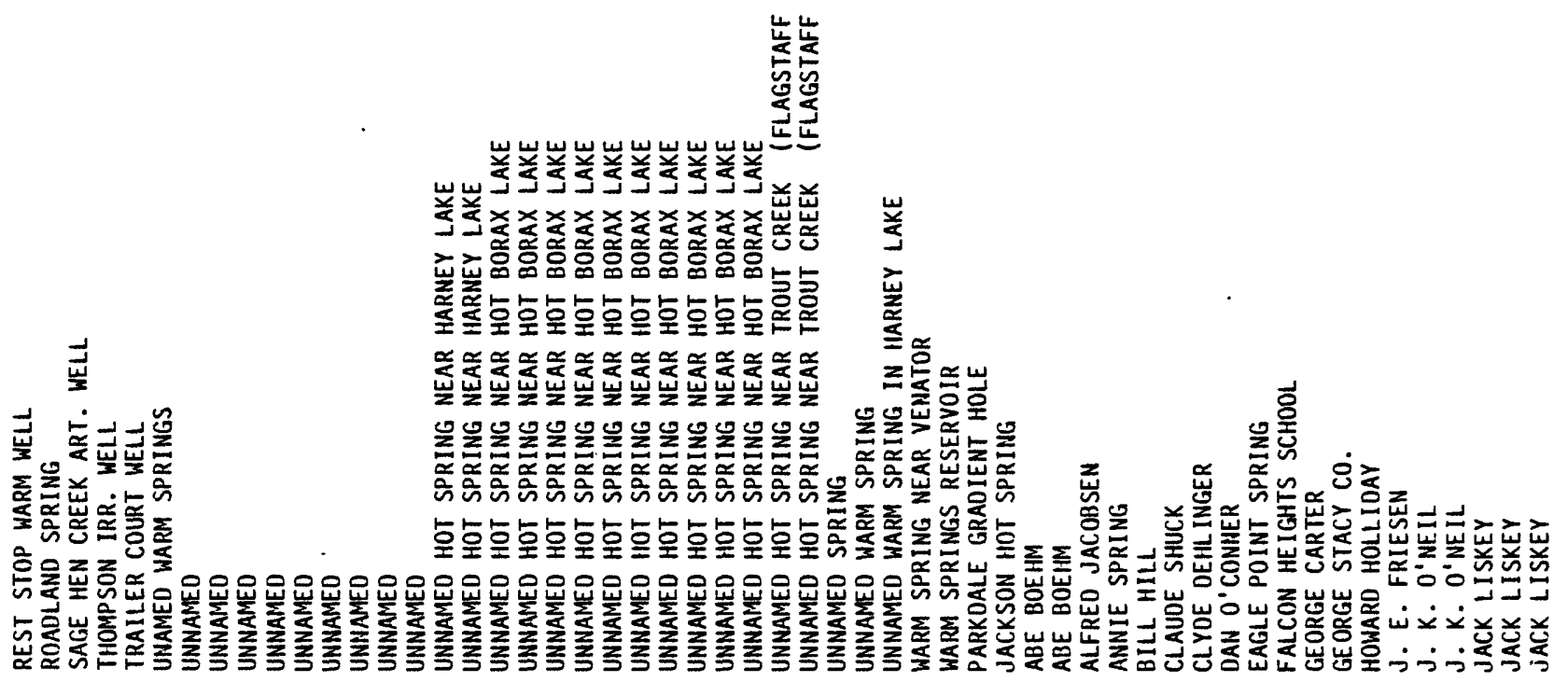


$\overrightarrow{\tilde{N}}$

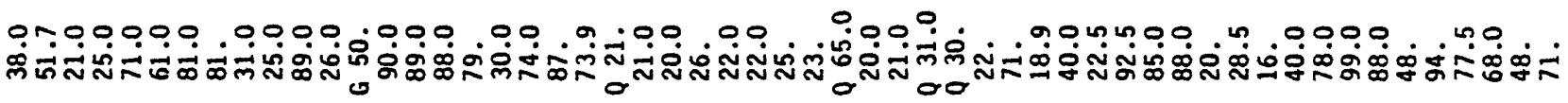

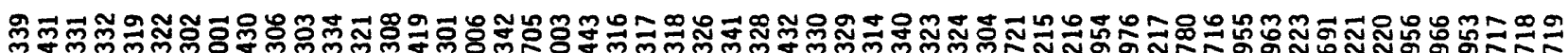

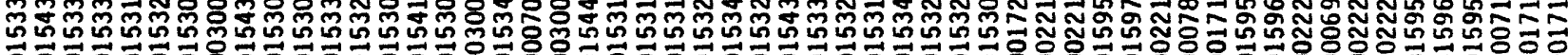

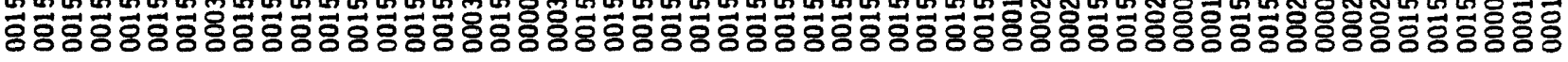

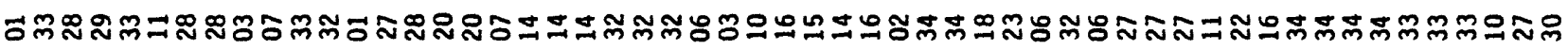

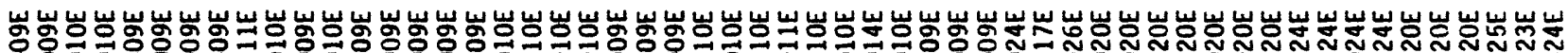

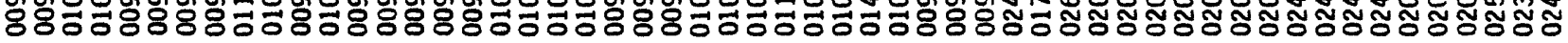

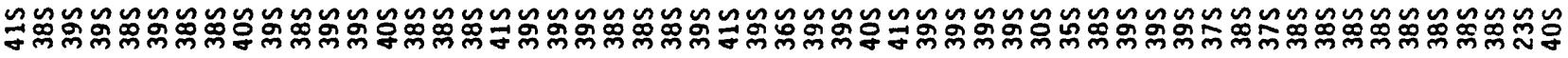

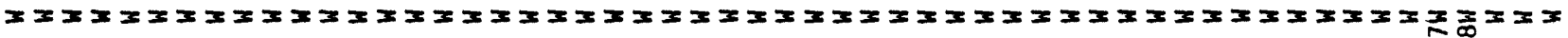

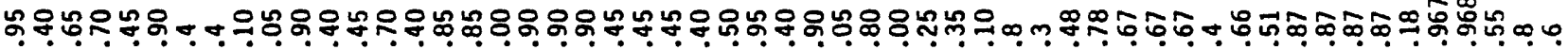

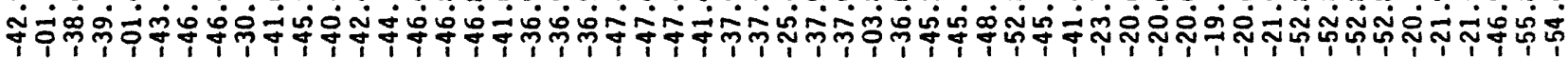

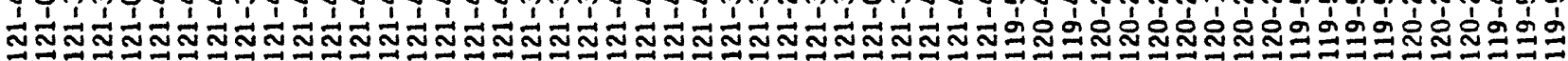
ZZZZZZZZZZZZZZZZZZZZZZZZZZZZZZZZZZZZZZZZZZZZZZZZZZZZZZZZZZZZZ

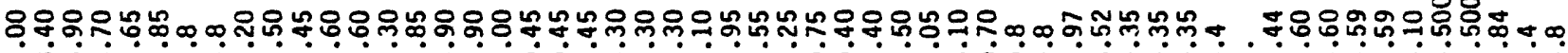
ธิن

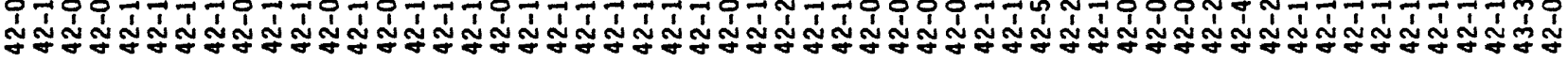

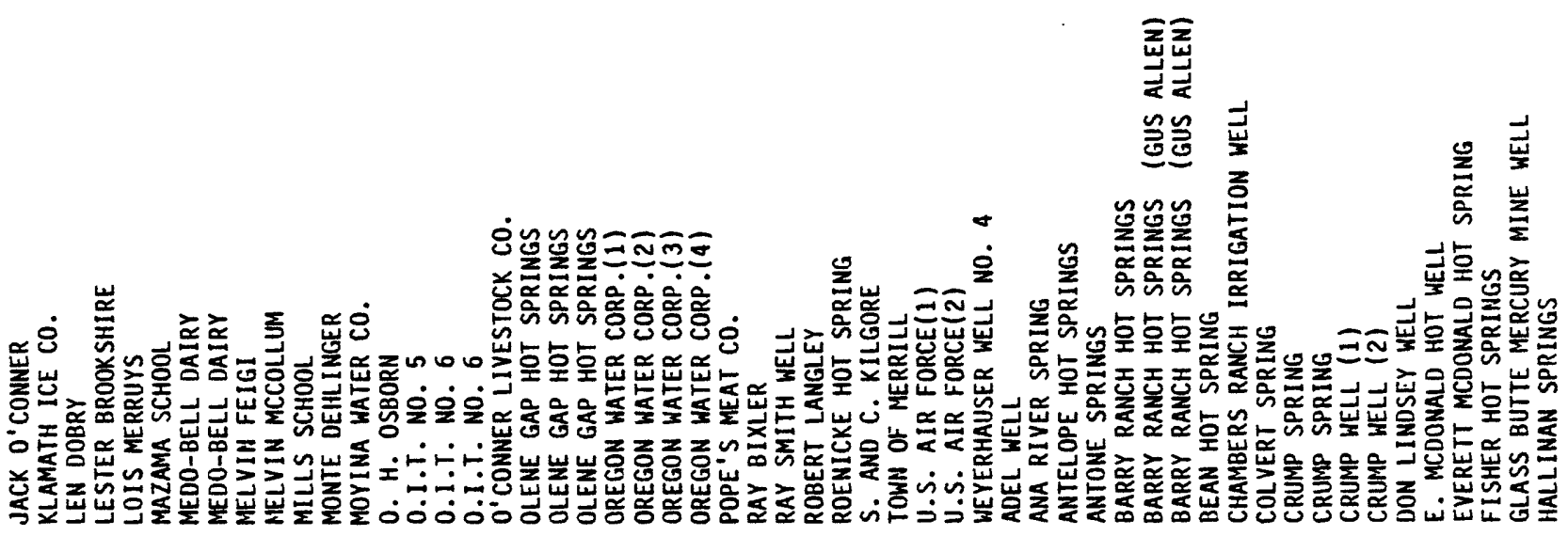




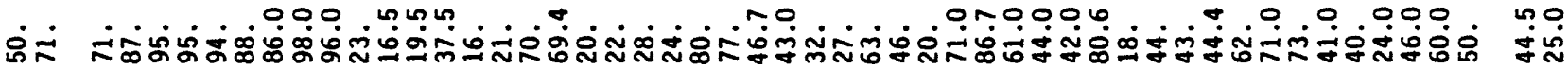

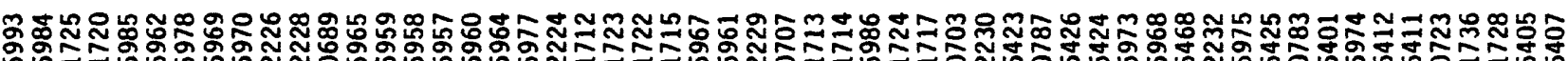

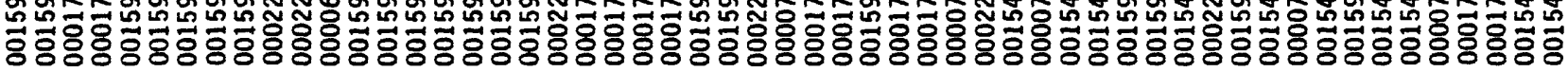

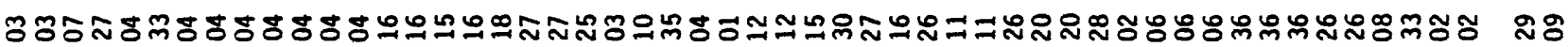

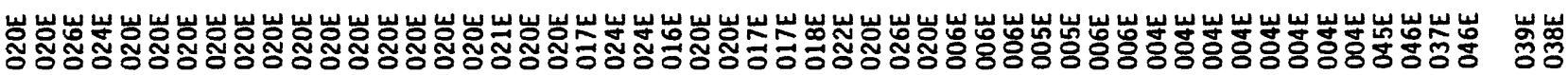

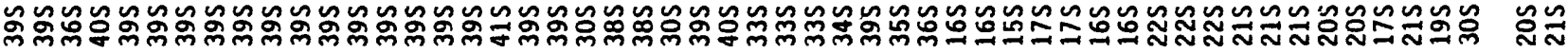

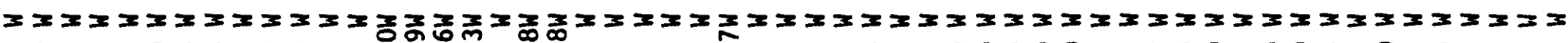

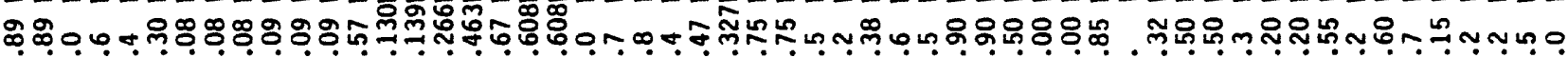
จิจิ์

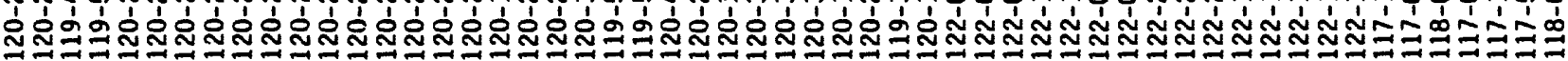

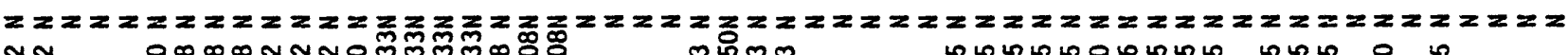

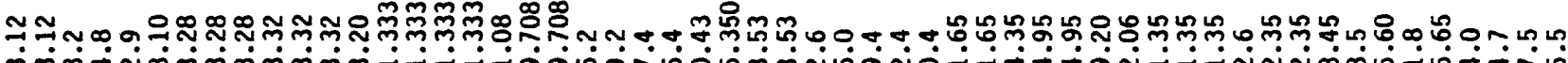
ம்ற்

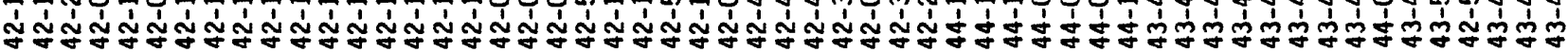

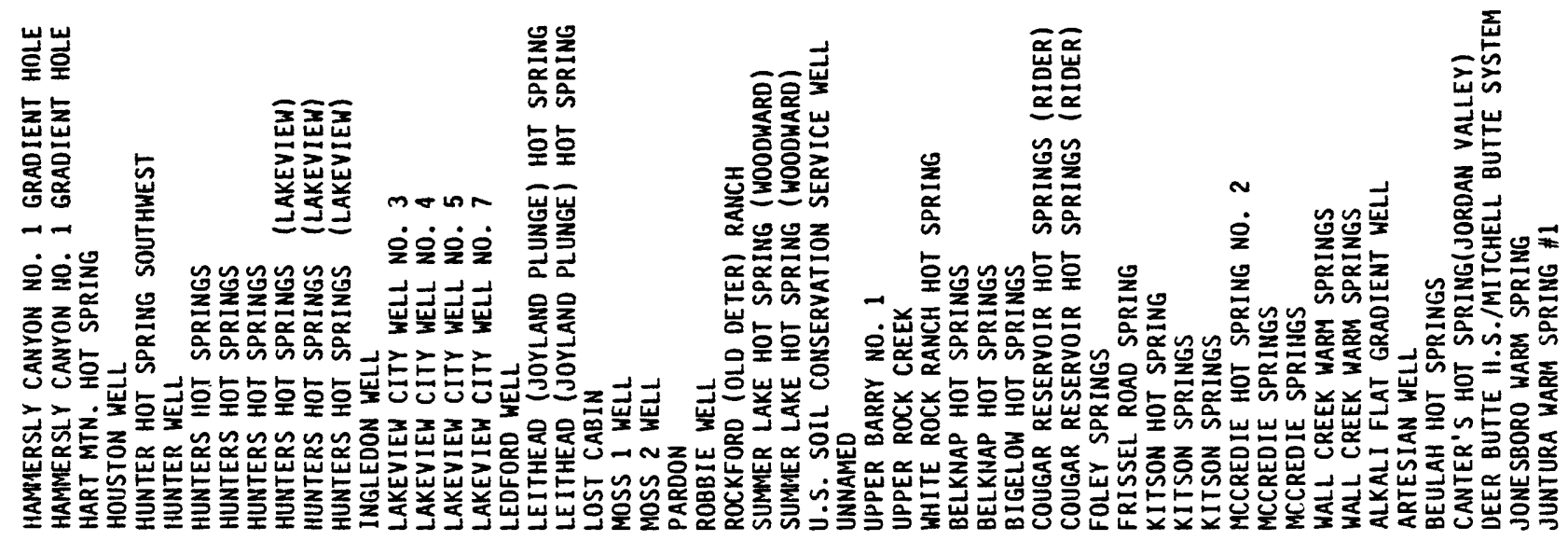

옥윽옥똑 옥

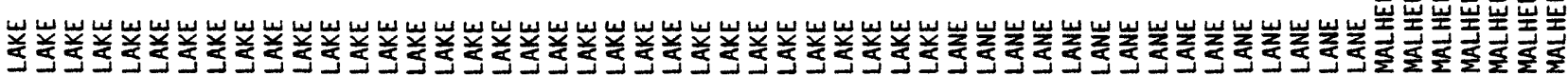


ส

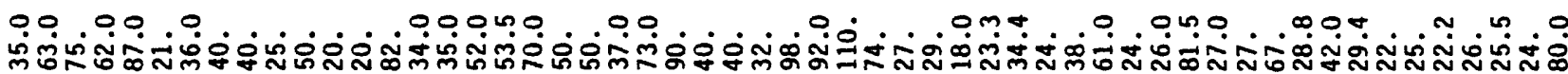

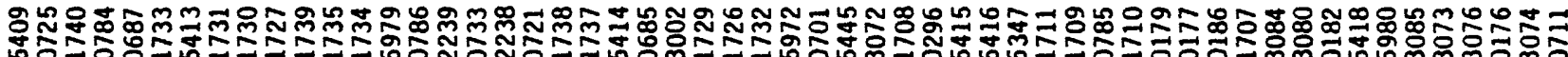

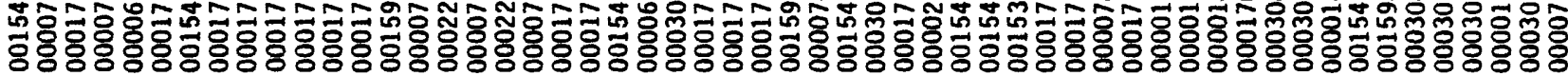

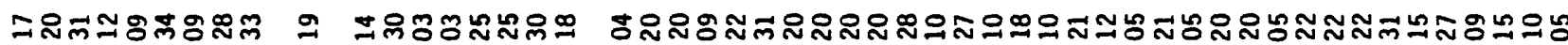

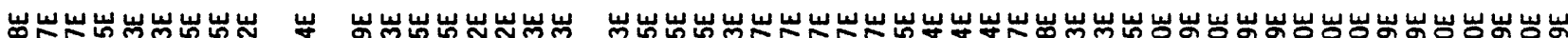

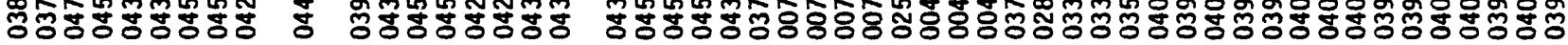

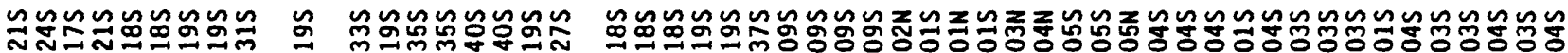

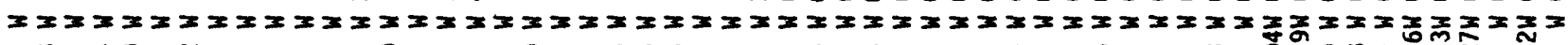

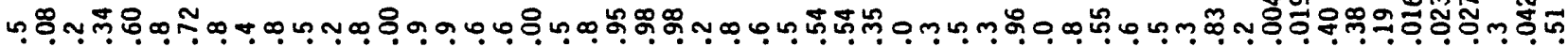

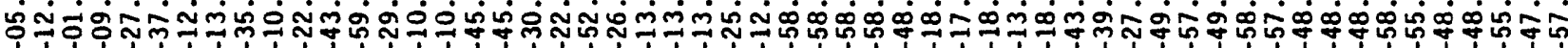

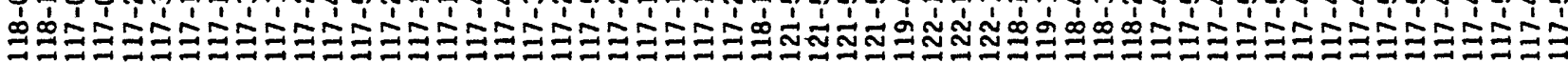

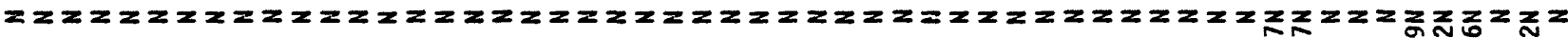

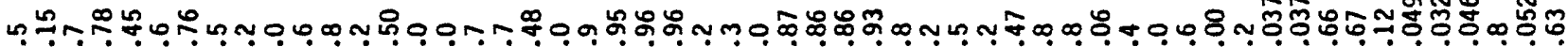

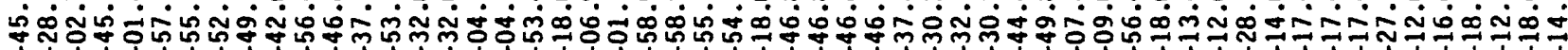

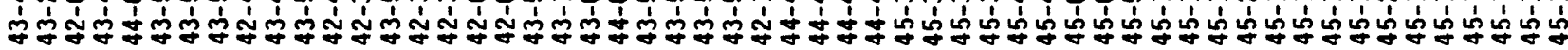

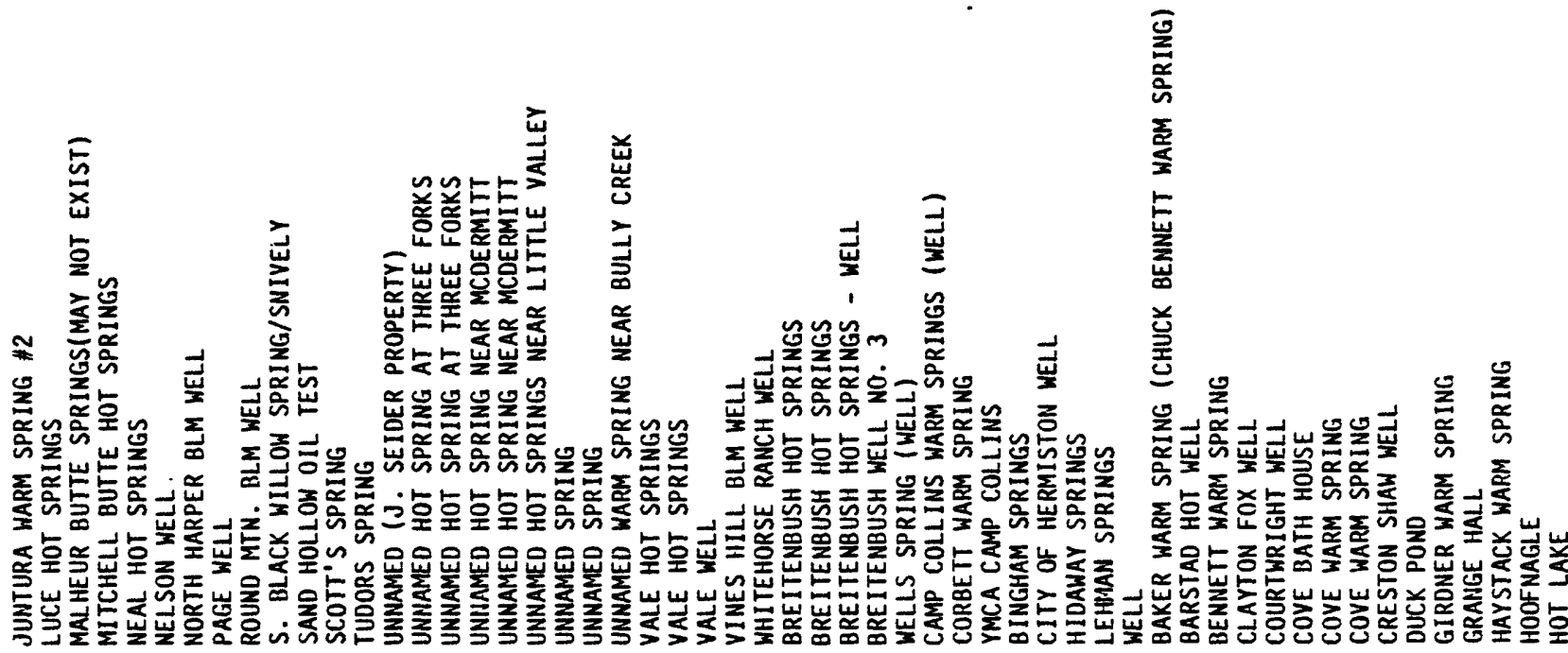

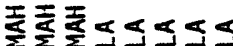

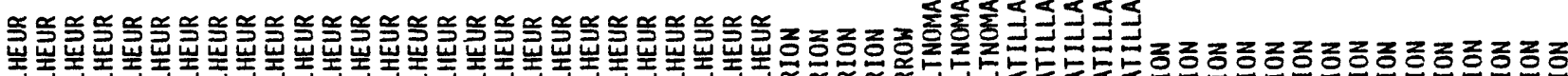

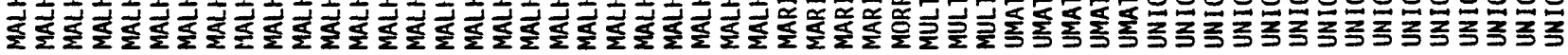




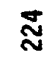

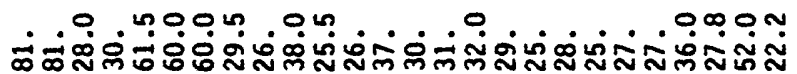

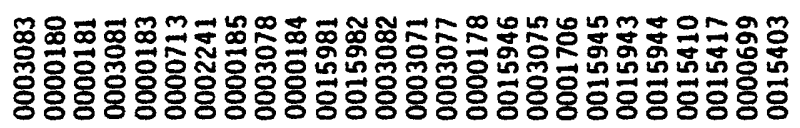

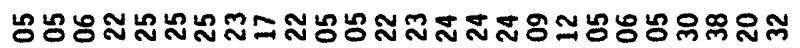

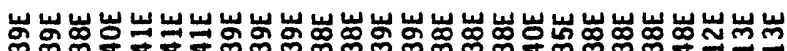

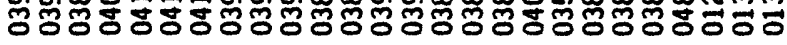

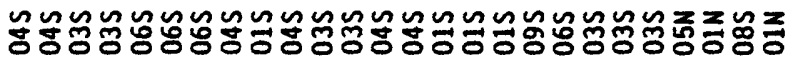

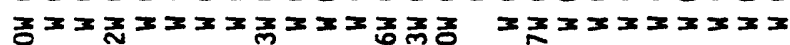

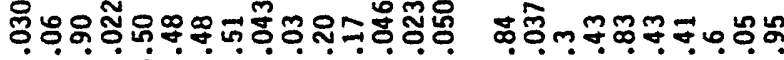
ค่⿻0口⿻上丨

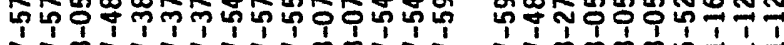

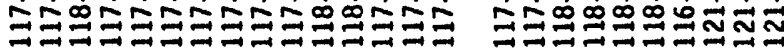

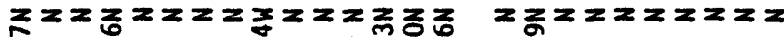

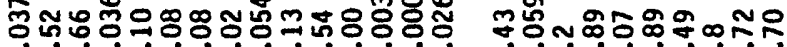

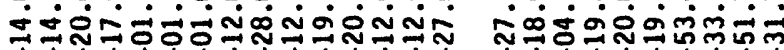

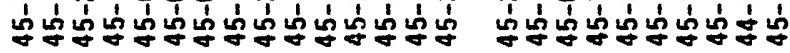

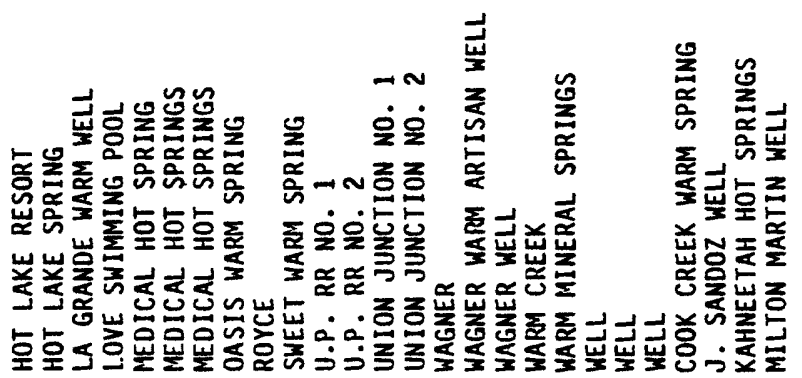

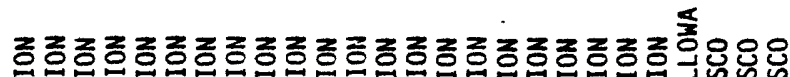

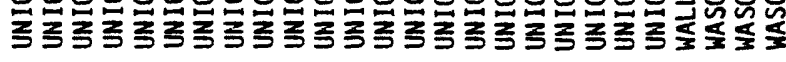




\section{APPENDIX B}

Index to GEOTHERM sample file for the state of Oregon sorted by county, township (TNS), range (RNG), and section (Sect.) Also given are the name of source, GEOTHERM record identifier (I.D.), and temperature (Temp. ${ }^{\circ} \mathrm{C}$ ). See Table 1 for explanation of alphabetic qualifiers preceding temperature.

\begin{tabular}{|c|c|c|c|c|c|c|}
\hline County & TNS & RNG & Sect. & Name of Source & I.D. & Temp \\
\hline BAKER & $06 \mathrm{~S}$ & $039 E$ & 25 & KROPP HOT SPRING & 0015427 & 43.0 \\
\hline BAKER & 075 & $038 E$ & 10 & FISHER HOT SPRING & 0015444 & 37.0 \\
\hline BAKER & 075 & $039 \mathrm{E}$ & 28 & RADIUM HOT SPRINGS & 0002206 & 57.2 \\
\hline BAKER & 075 & $039 E$ & 28 & RADIUM HOT SPRINGS & 0000709 & 58. \\
\hline BAKER & 095 & O4OE & 16 & SAMO SPRING & 0015420 & 27.0 \\
\hline BAKER & $12 S$ & $043 \mathrm{E}$ & 02 & NELSON SPRING & 0001701 & 27 . \\
\hline BAKER & $14 S$ & $045 E$ & 18 & CITY OF HUNTINGTON WELL & 0001700 & 24 . \\
\hline CLACKAMAS & 025 & $009 E$ & 29 & ACID-SULFATE SPRING ON MT. HOOD & 0015469 & \\
\hline CLACKAMAS & 025 & $009 E$ & 29 & MT. HOOD FUMAROLES & 0015473 & \\
\hline CLACKAMAS & 025 & $009 E$ & 29 & MT. HOOD FUMAROLES & 0015470 & 89.4 \\
\hline CLACKAMA & 025 & OO9E & 29 & MT. HOOD FUMAROLES & 0015952 & 89.4 \\
\hline CLACKAMA & $02 S$ & OO9E & 29 & MT. HOOD FUMAROLES & 0015471 & \\
\hline CLACKAMAS & 025 & OO9E & 29 & MT. HOOD FUMAROLES & 0015472 & 89.4 \\
\hline CLACKAMAS & 025 & OO9E & 29 & MT. HOOD FUMAROLES & 0015950 & 71.1 \\
\hline CLACKAMA & 025 & OO9E & 29 & MT. HOOD FUMAROLES & 0015937 & 86.1 \\
\hline CLACKAMA & 025 & $009 E$ & 29 & MT. HOOD FUMAROLES & 0015951 & 89.4 \\
\hline CLACKAMA & 025 & OO9E & 29 & MT. HOOD FUMAROLES & 0015474 & 91.1 \\
\hline CLACKAMA & 035 & 008.5 & 24 & SWIM WARM SPRINGS & 0015421 & 26.0 \\
\hline CLACKAMA & $06 \mathrm{~S}$ & 007E & 21 & GEOTHERMAL GRADIENT TEST NEAR AUSTIN HOT & 0015446 & 35.6 \\
\hline CLACKAMA & $06 \mathrm{~S}$ & $007 \bar{E}$ & 30 & AUSTIN HOT SPRINGS (CAREY) & 0000697 & 86.0 \\
\hline CLACKAMA & 07 & $005 E$ & 26 & BAGBY HOT SPRINGS & 0015422 & 57.2 \\
\hline CLACKAMA & 07 & $005 E$ & 26 & BAGBY HOT SPRINGS & 451 & 58 \\
\hline CROOK & & & & CAMP CREEK & 00 & 23. \\
\hline CROOK & $15 S$ & $014 E$ & 33 & WEIGAND WELL & 0003015 & 24. \\
\hline CROOK & $15 S$ & $015 \mathrm{E}$ & 29 & SAMPALUESI WELL & 0003033 & 21.6 \\
\hline CROOK & $16 \mathrm{~S}$ & $014 \mathrm{E}$ & 016 & PB-1 DOGAMI DRILL HOLE & 0003014 & 32.5 \\
\hline CROOK & $16 \mathrm{~S}$ & $014 E$ & 03 & CRADDOCK WELL & 0003017 & 24.3 \\
\hline CROOK & $16 \mathrm{~S}$ & $014 \bar{E}$ & 10 & E. MEEKER WELL & 0003021 & 24.9 \\
\hline CROOK & $16 \mathrm{~S}$ & $014 \mathrm{E}$ & 15 & T. BEARDY WELL & 0003016 & 24.5 \\
\hline CROOK & $16 \mathrm{~S}$ & $014 E$ & 16 & JACKSON WELL & 3018 & 27 . \\
\hline$C R$ & 16 & $014 \bar{E}$ & 21 & T. 0. MARSHALL WELL & 00 & 33.3 \\
\hline CROOK & 16 & $015 \mathrm{E}$ & 16 & DEADMAN SPRINGS & 3007 & 13. \\
\hline CROOK & 17 & $022 E$ & & UNNAMED SPRING ON S. FORK CROOKED RIVER & 0001703 & \\
\hline DESCHUTES & $20 S$ & $016 \mathrm{E}$ & 24 & DECKER WELL NO. 2 & 0003009 & 25.1 \\
\hline CHUTES & 21 & $012 E$ & 26 & PAULINA HOT SPRINGS & 5947 & \\
\hline HUTES & 21 & $012 E$ & 26 & PAULINA HOT SPRINGS & 454 & \\
\hline CHUTES & 21 & $012 \mathrm{E}$ & 36 & WARM WELL AT LITTLE CRATER CAMPGROUND & 455 & 35.5 \\
\hline & 2 & & 29 & EAST LAKE HOT SPRINGS & 00 & 62.0 \\
\hline CHUTE & 2 & $013 E$ & 29 & EAST LAKE HOT SPRINGS & 00 & 49. \\
\hline DESCHUTES & 2 & & 29 & EAST LAKE HOT SPRINGS & 00 & \\
\hline DOUGLAS & 26 & $004 E$ & 20 & UMPQUA HOT SPRINGS & 0015949 & 46. \\
\hline DOUGLAS & 26 & $004 E$ & 20 & UMPQUA HOT SPRINGS & 0015456 & 46.5 \\
\hline & 08 & O30E & 08 & RITTER HOT SPRINGS & 0000715 & 41.0 \\
\hline & 14 & & 10 & LIMEKILN SPRING & 0001705 & 21 . \\
\hline & 14 & $034 \mathrm{E}$ & 13 & BLUE MOUNTAIN HOT & 0000719 & 58.0 \\
\hline GRANT & 15 & O31E & 11 & JOAQUIN MILLER RESORT & 0015948 & 40. \\
\hline
\end{tabular}




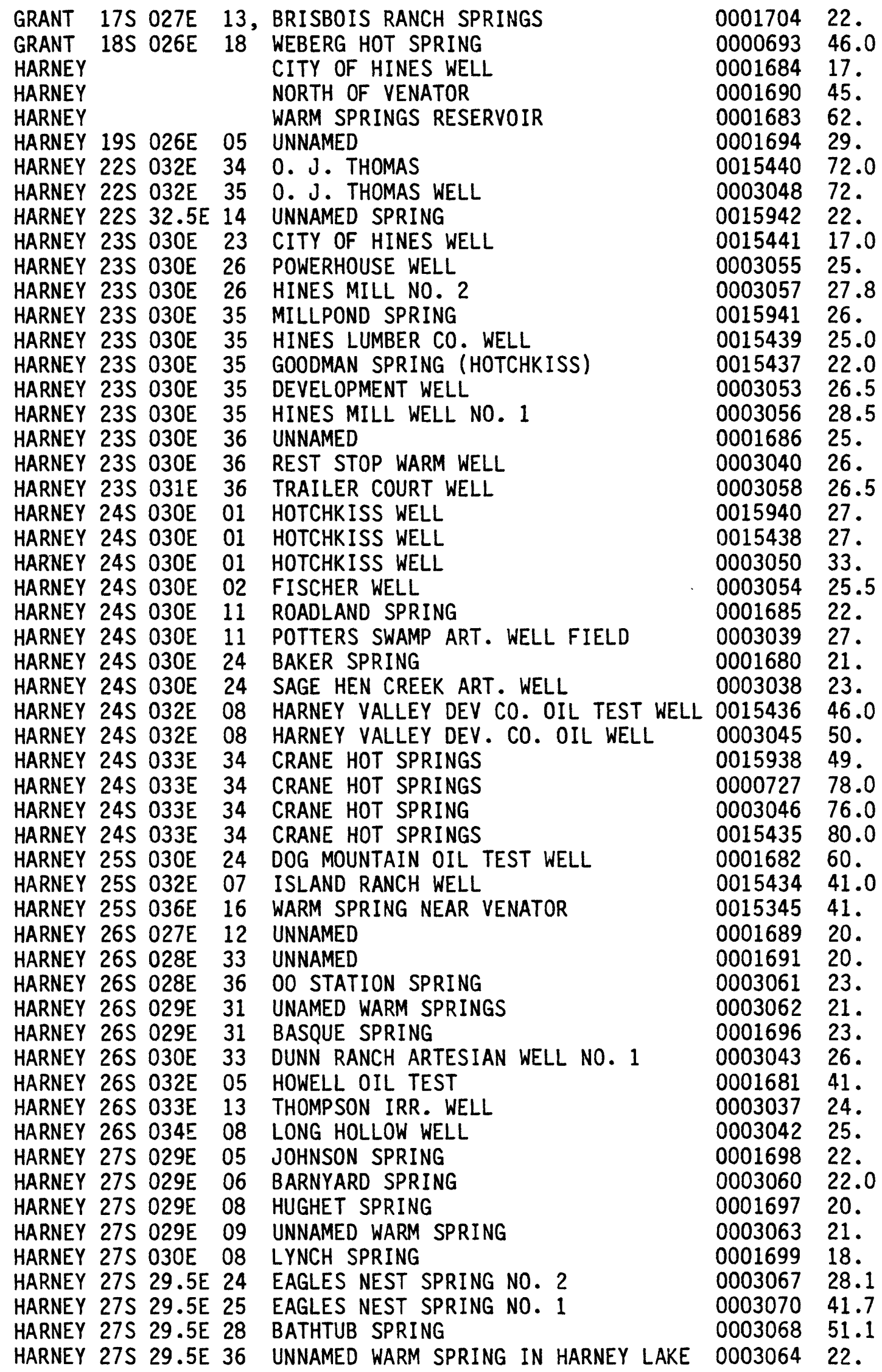




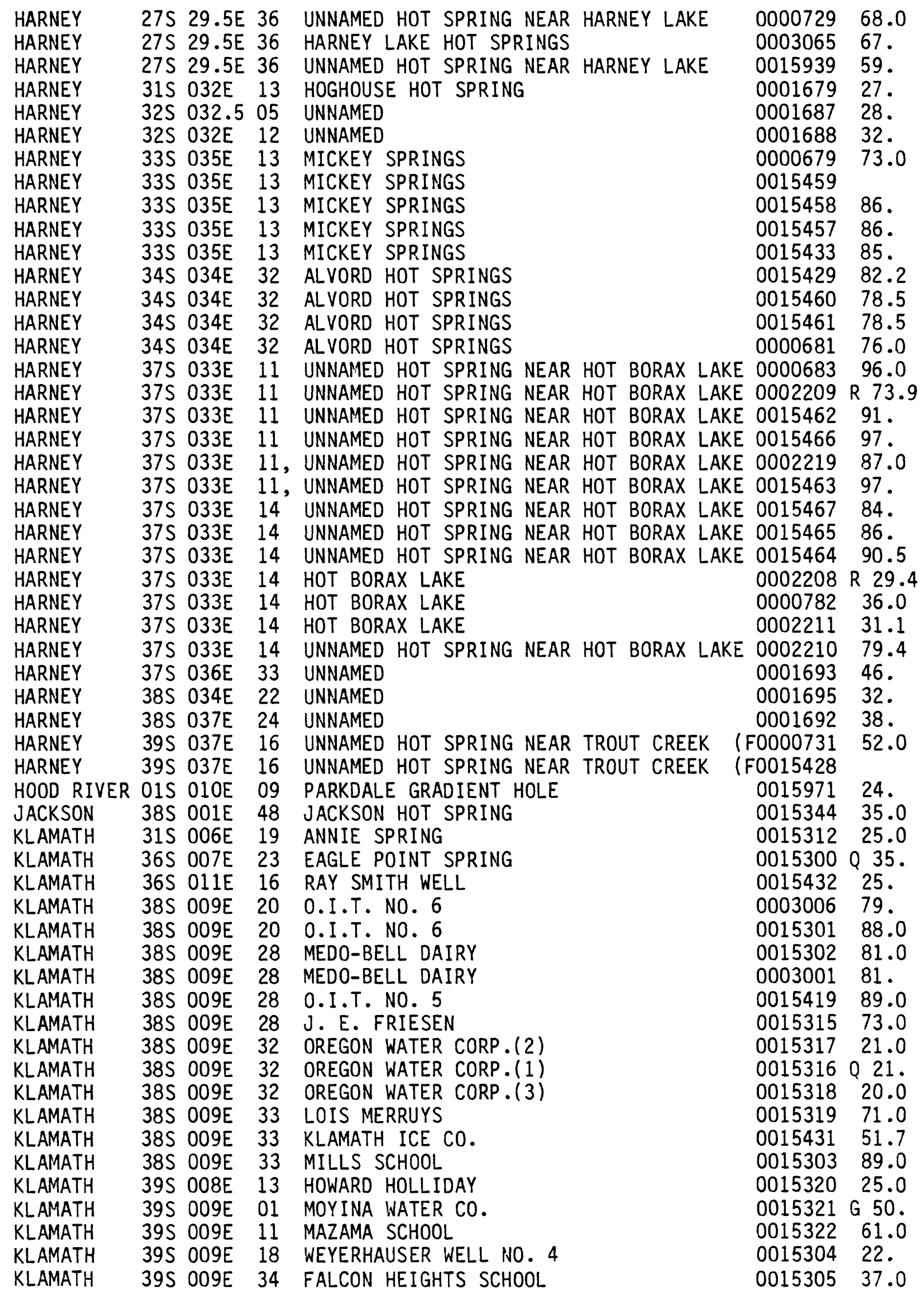


KLAMATH 39S OO9E KLAMATH 39S O09E KLAMATH 39S O09E KLAMATH 39S O1OE KLAMATH 39S O1OE KLAMATH 39S O10E KLAMATH 39S O1OE KLAMATH 39S O10E KLAMATH 39S O10E KLAMATH 39S O10E KLAMATH 39S O1OE KLAMATH 39S O10E KLAMATH 39S O10E KLAMATH 39S O10E KLAMATH 39S O10E KLAMATH 39S OIOE KLAMATH 39S O10E KLAMATH 39S O10E KLAMATH 4OS OO9E KLAMATH 40S O09E KLAMATH 4OS O09E KLAMATH 40S 009E KLAMATH 40S 009E KLAMATH 40S OIOE KLAMATH 4OS O1OE KLAMATH 40S 011E KLAMATH 40S 014E KLAMATH 41S O09E KLAMATH $41 S$ OO9E KLAMATH 41S OO9E KLAMATH 41S O1OE KLAMATH 41S O1OE KLAMATH 41S 010E KLAMATH 41S 010E LAKE 23S 023E LAKE 30S 016E LAKE $30 S$ O17E LAKE 3OS 017E LAKE 33S 017E LAKE 33S O17E LAKE 33S 018E LAKE $34 S 022 E$ LAKE $35 S$ O26E LAKE $35 S$ 026E LAKE 36S O20E LAKE 36S 026E LAKE 37S O20E LAKE 37S O20E LAKE 38S O20E LAKE 38S O20E LAKE 38S O20E LAKE 38S O20E LAKE 38S O20E LAKE 38S O24E LAKE 38S O24E
34

34

34

06

07

08

10

14

14

14

14

15

28

29

32

32

34

34

23

27

28

28

34

05

26

03

16

01

02

02

02

03

07

14

27

35

06

25

12

12

15

30

16

32

26

07

11

16

06

22

33

33

33

03

10
ALFRED JACOBSEN

U.S. AIR FORCE(1)

U.S. AIR FORCE(2)

OREGON WATER CORP. (4)

MELVIN MCCOLLUM

CLAUDE SHUCK

RAY BIXLER

ROENICKE HOT SPRING

OLENE GAP HOT SPRINGS

OLENE GAP HOT SPRINGS

OLENE GAP HOT SPRINGS

ROBERT LANGLEY

LEN DOBRY

LESTER BROOKSHIRE

GEORGE STACY CO.

MONTE DEHLINGER

J. K. O'NEIL

J. K. O'NEIL

DAN O'CONNER

O. H. OSBORN

ABE BOEHM

ABE BOEHM

JACK LISKEY

CLYDE DEHLINGER

BILL HILL

MELVIN FEIGI

S. AND C. KILGORE

JACK O'CONNER

JACK LISKEY

JACK LISKEY

TOWN OF MERRILL

POPE'S MEAT CO.

O'CONNER LIVESTOCK CO.

GEORGE CARTER

GLASS BUTTE MERCURY MINE WELL

PARDON

ANA RIVER SPRING

LOST CABIN

SUMMER LAKE HOT SPRING (WOODWARD)

SUMMER LAKE HOT SPRING (WOODWARD)

U.S. SOIL CONSERVATION SERVICE WELL

UNNAMED

UPPER ROCK CREEK

ANTELOPE HOT SPRINGS

WHITE ROCK RANCH HOT SPRING

HART MTN. HOT SPRING

BEAN HOT SPRING

COLVERT SPRING

ANTONE SPRINGS

CHAMBERS RANCH IRRIGATION WELL

EVERETT MCDONALD HOT SPRING

DON LINDSEY WELL

E. MCDONALD HOT WELL

MOSS 1 WELL

MOSS 2 WELL
$0015325 \quad 30.0$

0015323 Q 31.0

0015324 Q 30.

001532626.

$0015306 \quad 25.0$

001532724 .

$0015328 \quad 22.0$

0015329 Q 65.0

000300387.

$0000705 \quad 74.0$

$0015443 \quad 73.9$

001533023.

001533121.0

$0015332 \quad 25.0$

$0015333 \quad 25.0$

$0015334 \quad 26.0$

0015442

$0015335 \quad 23.0$

$0015336 \quad 24.0$

$0015308 \quad 90.0$

001530925.0

000300525.

$0015310 \quad 93.0$

$0015337 \quad 24.0$

$0015338 \quad 20.0$

$0015430 \quad 31.0$

$0015314 \quad 20.0$

$0015339 \quad 38.0$

001531122.0

000300425.

$0015340 \quad 21.0$

$0015341 \quad 22.0$

$0015342 \quad 30.0$

001534322.

000171848 .

000171524.

$0002215 \quad 18.9$

000171220.

$0002229 \quad 46.7$

$0000707 \quad 43.0$

000171332 .

000171427.

000172446.

$0002216 \quad 40.0$

000171720 .

0001725

000171620.

001596316.

$0015954 \quad 22.5$

$0015955 \quad 28.5$

$0015953 \quad 77.5$

001595648 .

001596694.

000172322.

000172228. 


\begin{tabular}{|c|c|c|c|c|c|}
\hline 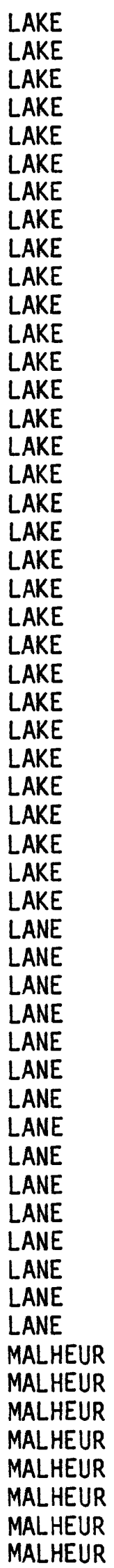 & 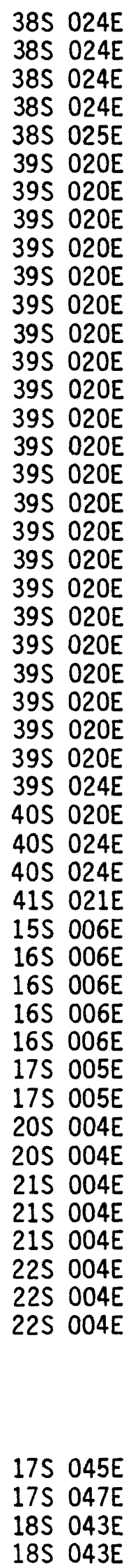 & $\begin{array}{l}04 \\
04 \\
04 \\
04 \\
04 \\
04 \\
04 \\
04 \\
15 \\
16 \\
16 \\
16 \\
27 \\
27 \\
27 \\
27 \\
27 \\
27 \\
33 \\
23 \\
01 \\
27 \\
30 \\
18 \\
26 \\
02 \\
11 \\
11 \\
28 \\
20 \\
20 \\
26 \\
26 \\
36 \\
36 \\
36\end{array}$ & $\begin{array}{l}\text { CRUMP SPRING } \\
\text { CRUMP SPRING } \\
\text { CRUMP WELL (2) } \\
\text { CRUMP WELL (1) } \\
\text { FISHER HOT SPRINGS } \\
\text { HAMMERSLY CANYON NO. } 1 \text { GRADIENT HOLE } \\
\text { HAMMERSLY CANYON NO. } 1 \text { GRADIENT HOLE } \\
\text { HUNTERS HOT SPRINGS } \\
\text { HUNTER HOT SPRING SOUTHWEST } \\
\text { ROBBIE WELL } \\
\text { HUNTERS HOT SPRINGS } \\
\text { HUNTERS HOT SPRINGS } \\
\text { HUNTERS HOT SPRINGS (LAKEVIEW) } \\
\text { HUNTERS HOT SPRINGS (LAKEVIEW) } \\
\text { HUNTERS HOT SPRINGS (LAKEVIEW) } \\
\text { INGLEDON WELL } \\
\text { LAKEVIEW CITY WELL NO. } 5 \\
\text { LAKEVIEW CITY WELL NO. } 7 \\
\text { LAKEVIEW CITY WELL NO. } 4 \\
\text { LAKEVIEW CITY WELL NO. } 3 \\
\text { BARRY RANCH HOT SPRINGS (GUS ALLEN) } \\
\text { LEITHEAD (JOYLAND PLUNGE) HOT SPRING } \\
\text { UPPER BARRY NO. I } \\
\text { BARRY RANCH HOT SPRINGS } \\
\text { LEITHEAD (JOYLAND PLUNGE) HOT SPRING } \\
\text { BARRY RANCH HOT SPRINGS (GUS ALLEN) } \\
\text { HUNTER WELL } \\
\text { ADEL WELL } \\
\text { ROCKFORD (OLD DETER) RANCH } \\
\text { HOUSTON WELL } \\
\text { HALLINAN SPRINGS } \\
\text { LEDFORD WELL } \\
\text { BIGELOW HOT SPRINGS } \\
\text { FRISSEL ROAD SPRING } \\
\text { BELKNAP HOT SPRINGS } \\
\text { BELKNAP HOT SPRINGS } \\
\text { FOLEY SPRINGS } \\
\text { COUGAR RESERVOIR HOT SPRINGS (RIDER) } \\
\text { COUGAR RESERVOIR HOT SPRINGS (RIDER) } \\
\text { WALL CREEK WARM SPRINGS } \\
\text { WALL CREEK WARM SPRINGS } \\
\text { MCCREDIE SPRINGS } \\
\text { MCCREDIE SPRINGS } \\
\text { MCCREDIE HOT SPRING NO. } 2 \\
\text { KITSON SPRINGS } \\
\text { KITSON HOT SPRING } \\
\text { KITSON SPRINGS. } \\
\text { UNNAMED SPRING } \\
\text { SCOTT'S SPRING } \\
\text { DEER BUTTE H.S./MITCHELL BUTTE SYSTEN } \\
\text { S. BLACK WILLOW SPRING/SNIVELY } \\
\text { ALKALI FLAT GRADIENT WELL } \\
\text { MALHEUR BUTTE SPRINGS(MAY NOT EXIST) } \\
\text { UNNAMED WARM SPRING NEAR BULLY CREEK } \\
\text { NEAL HOT SPRINGS }\end{array}$ & $\begin{array}{l}84 \\
78 \\
85 \\
67 \\
70 \\
69 \\
26 \\
28 \\
39 \\
55\end{array}$ & \\
\hline
\end{tabular}




\begin{tabular}{|c|c|c|c|c|c|}
\hline MALHEUR & $18 S 043 E$ & 34 & NELSON WELL & 0001733 & 21 . \\
\hline MALHEUR & $18 S 045 E$ & 20 & VALE HOT SPRINGS & 0003002 & 90 . \\
\hline ALHEUR & $18 S 045 E$ & 20 & VALE HOT SPRINGS & 0000685 & 73.0 \\
\hline ALHEUR & 19S 037E & 02 & BEULAH HOT SPRINGS & 0000723 & 60.0 \\
\hline HEUR & 19S 043E & 22 & VINES HILL BLM WELL & 0001726 & 40 . \\
\hline ALHEUR & $19 S 043 E$ & 30 & UNNAMED ( $\mathrm{J}$. SEIDER PROPERTY) & 0015979 & 82. \\
\hline ALHEUR & $19 S 043 E$ & 30 & UNNAMED HOT SPRINGS NEAR LITTLE VALLEY & 0000721 & 70.0 \\
\hline MALHEUR & 19S $044 E$ & 19 & SAND HOLLOW OIL TEST & 0001739 & 50. \\
\hline ALHEUR & 19S 045E & 09 & VALE WELL & 0001729 & 40. \\
\hline ALHEUR & $19 S 045 E$ & 09 & NORTH HARPER BLM WELL & 0015413 & 36.0 \\
\hline ALHEUR & $19 S 045 E$ & 28 & PAGE WELL & 0001731 & 40. \\
\hline ALHEUR & $20 S 039 E$ & 29 & JONESBORO WARM SPRING & 0015405 & 44.5 \\
\hline ALHEUR & $21 S 038 E$ & 09 & JUNTURA WARM SPRING \#1 & 0015407 & 25.0 \\
\hline MALHEUR & $21 S 038 E$ & 17 & JUNTURA WARM SPRING \#2 & 0015409 & 35.0 \\
\hline ALHEUR & $21 S 045 E$ & 12 & MITCHELL BUTTE HOT SPRINGS & 0000784 & 62.0 \\
\hline ALHEUR & $21 S 046 E$ & 33 & ARTESIAN WELL & 0015411 & 46.0 \\
\hline ALHEUR & $24 S \quad 037 E$ & 20 & LUCE HOT SPRINGS & 0000725 & 63.0 \\
\hline ALHEUR & $27 S 043 E$ & 18 & UNNAMED SPRING & 0001738 & \\
\hline ALHEUR & $30 S 046 E$ & 02 & CANTER'S HOT SPRING(JORDAN VALLEY) & 0001736 & 50 . \\
\hline ALHEUR & $31 S 042 E$ & 33 & ROUND MTN. BLM WELL & 00 & 40. \\
\hline MALH & $33 S 039 E$ & 14 & TUDORS SPRING & 00 & 20. \\
\hline MALH & $35 S 045 E$ & 03 & UNNAMED HOT SPRING AT THREE FORKS & 00 & 34.0 \\
\hline MALH & $35 S 045 E$ & 03 & UNNAMED HOT SPRING AT THREE FORKS & 00 & 35.0 \\
\hline MALH & $37 S \quad 037 E$ & 31 & WHITEHORSE RANCH WELL & 00 & \\
\hline ALH & $40 S 042 E$ & 25 & UNNAMED HOT SPRING NEAR MCDERMITT & 00 & 53.5 \\
\hline ALH & $40 S 042 E$ & 25 & UNNAMED HOT SPRING NEAR MCDERMITT & 00 & 52.0 \\
\hline ARI & O9S 007E & 20 & BREITENBUSH HOT SPRINGS & & 92.0 \\
\hline MARI & O9S 007E & 20 & BREITENBUSH HOT SPRINGS - WELL & & 110. \\
\hline ARION & 09S 007E & 20 & BREITENBUSH WELL N0. 3 & 00 & 74. \\
\hline MARION & O9S 007E & 20 & BREITENBUSH HOT SPRINGS & & 98. \\
\hline MORROW & $02 \mathrm{~N} 025 \mathrm{E}$ & 28 & WELLS SPRING (WELL) & & 27. \\
\hline MULTNOMAH & $01 N 004 E$ & 27 & CORBETT WARM SPRING & & 18.0 \\
\hline MAH & $01 S$ 004E & 10 & YMCA CAMP COLLINS & & 23.3 \\
\hline MAH & 01S 004E & 10 & CAMP COLLINS WARM SPRINGS (WELL) & & 29. \\
\hline UMATILLA & 03N $037 E$ & 18 & BINGHAM SPRINGS & & 34.4 \\
\hline UMATILLA & 04N 028E & 10 & CITY OF HERMISTON WELL & & 24 \\
\hline UMATILLA & $05 \mathrm{~N} 035 \mathrm{E}$ & 05 & WELL & & 24. \\
\hline UMATILLA & 05S 033E & 12 & LEHMAN SPRINGS & & 61.0 \\
\hline UMATILLA & 05S 033E & 21 & HIDAWAY SPRINGS & & 38. \\
\hline UNIC & 01S 038E & 24 & WAGNER & & 31. \\
\hline UNION & $015038 E$ & 24 & WAGNER WARM ARTISAN WELL & & 32.0 \\
\hline UNI & 01S 038E & 24 & WAGNER WELL & & \\
\hline UNION & $015039 E$ & 17 & ROYCE & & \\
\hline UNION & O39E & 20 & CLAYTON FOX WELL & & 27. \\
\hline UNION & O39E & 31 & CRESTON SHAW WELL & & 22. \\
\hline UNION & O38E & 05 & U.P. RR NO. 2 & & \\
\hline UNION & $038 E$ & 05 & U.P. RR NO. 1 & & \\
\hline UNION & O3S 038E & 05 & WELL & & \\
\hline UNION & $038 E$ & 05 & WELL & & \\
\hline UNION & $038 E$ & 06 & WELL & 943 & \\
\hline UNION & $038 E$ & 06 & LA GRANDE WARM WELL & 00 & \\
\hline UNION & O40E & 09 & GRANGE HALL & & \\
\hline UNION & O40E & 10 & HOOFNAGLE & & \\
\hline UNION & O4OE & 22 & LOVE SWIMMING & 0003081 & \\
\hline UNION & O4OE & 22 & COVE WARM SPRING & 0000182 & \\
\hline
\end{tabular}




\begin{tabular}{|c|c|c|c|c|c|}
\hline $\mathrm{N}$ & O3S O40E & 22 & COVE BATH HOUSE & 80 & 28.8 \\
\hline & O3S O40E & 22 & COVE WARM SPRING & 8 & $?$ \\
\hline ION & $\begin{array}{l}\text { O3S } 040 E \\
\text { O4S } 039 E\end{array}$ & $\begin{array}{l}27 \\
05\end{array}$ & $\begin{array}{l}\text { GIRDNER WARM SPRING } \\
\text { HOT LAKE RESORT }\end{array}$ & 0003073 & $2 i$ \\
\hline $\mathrm{N}$ & O4S 039E & 05 & COURTWRIGHT WELL & & \\
\hline N & $4 S 039 E$ & 05 & HOT LAKE SPRING & 30 & \\
\hline & $\mathrm{SO}_{3}$ & 05 & HOT LAKE & & \\
\hline N & $4 S 03$ & 05 & BARSTAD HOT WELL & & \\
\hline & & 15 & WARM SPRING & & \\
\hline N & 04503 & 15 & DUCK $P$ & 35 & \\
\hline & 0 & 22 & ICTION N0. 1 & & \\
\hline I I & 0450 & 22 & SWEET WARM & & \\
\hline & 0 & 23 & OASIS $w$ & & \\
\hline N & $045 \mathrm{C}$ & 23 & UNION JUNCTI & & \\
\hline & & 20 & BENNETT WARM & & \\
\hline & & 21 & $\begin{array}{l}\text { BAKER WARM SPRING } \\
\text { (CHUCK BENNETT WARM SPRING) }\end{array}$ & & \\
\hline & & 12 & WARM MINERAL SPRINGS & & \\
\hline & 06 & 25 & MEL & & \\
\hline & & 25 & MEI & & \\
\hline & 0650 & 25 & L HOT SI & & \\
\hline & 0950 & 09 & CREEK & & \\
\hline OWA & $05 \mathrm{~N} 0$ & 30 & EEK WARM & & \\
\hline & & 20 & J. SANDOZ WELL & & \\
\hline & & & N MARTIN $h$ & & \\
\hline & & & KAH & & \\
\hline
\end{tabular}




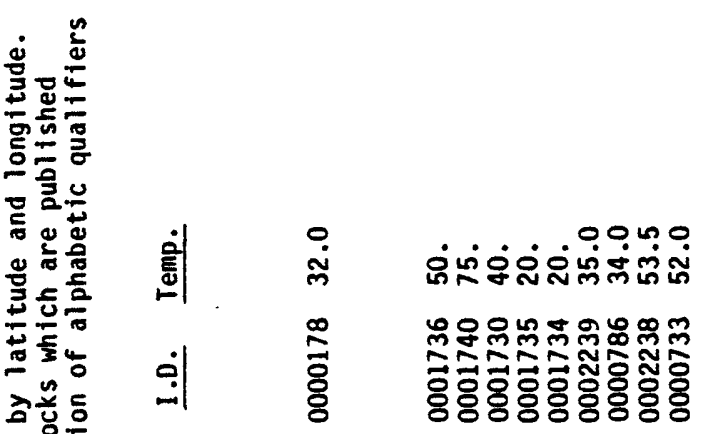

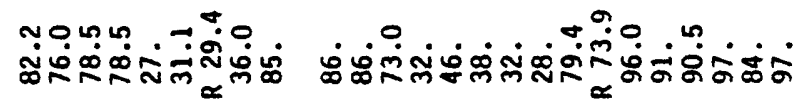

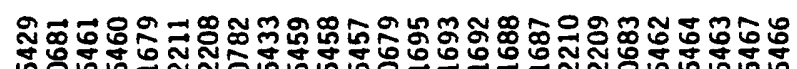

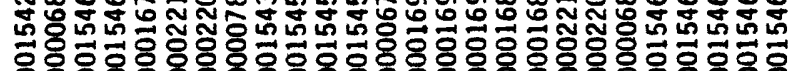

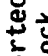

㐫点

峞希安

号产震 山㟧㟧 岸岸记

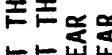
比㸚

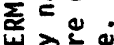

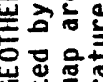

放客

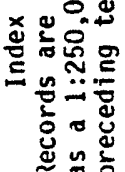

을을울을

究 중

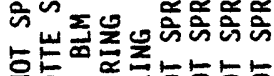

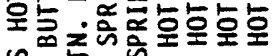
ํㅗㄴ

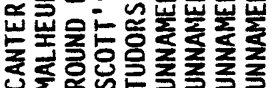
x>13xy3x

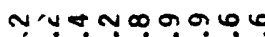
ป二ニニニ二ニ 2Z2Z21212

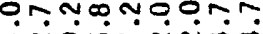
ธิ์ซษ์

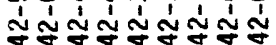
훙해융뭉ㅇㅇㅇㅢ

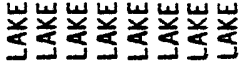

xखx

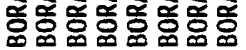

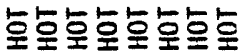

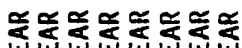

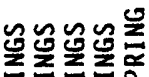

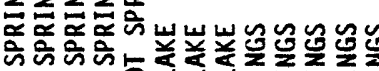

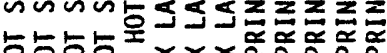

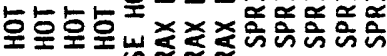

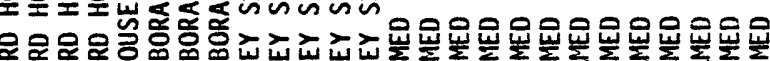

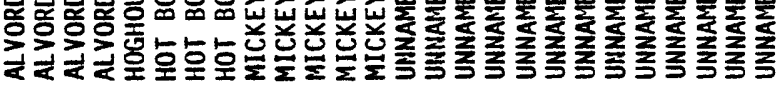

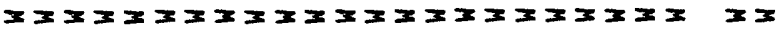

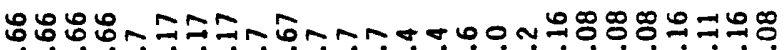

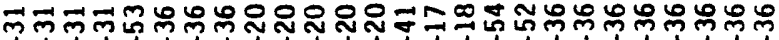

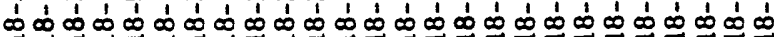

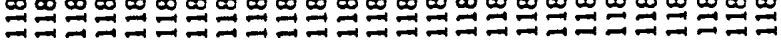

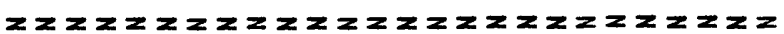

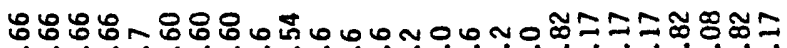

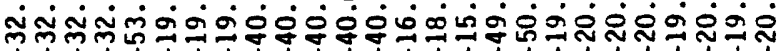

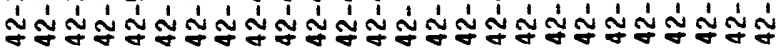




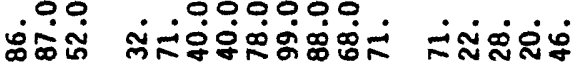

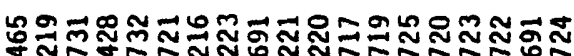
รัธิ์

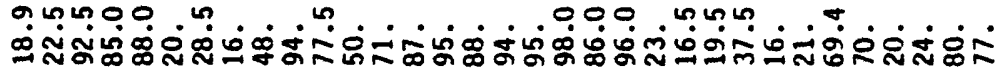

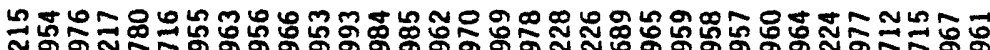

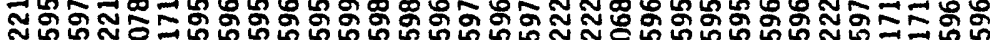

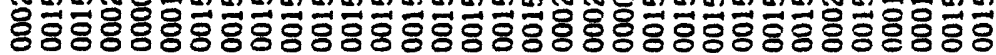

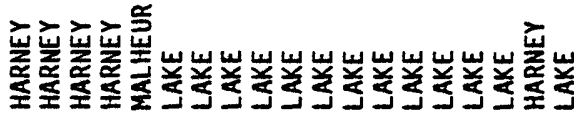

蒙范

或点

崖

美总

도웅ㅎㅀ

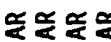

岩岩岸崖

일일

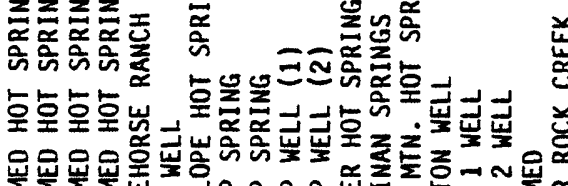

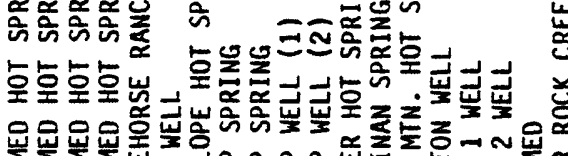

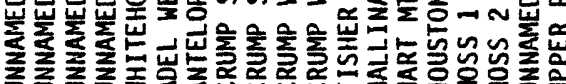

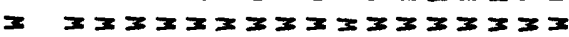

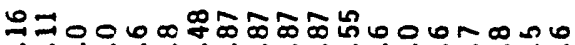

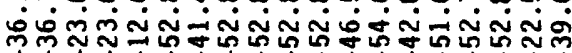

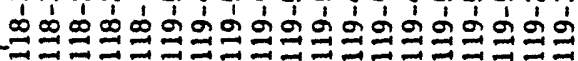
Z2ZZZZZZZZZZZZZZZZZ

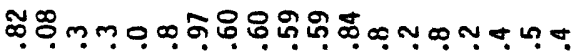
จำ

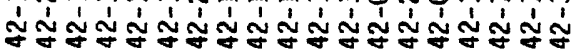

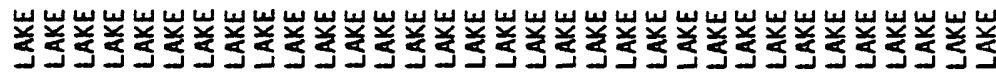

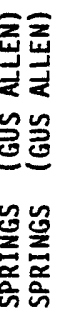

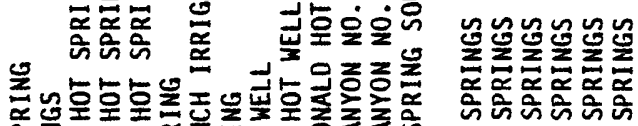

봉뽀홍

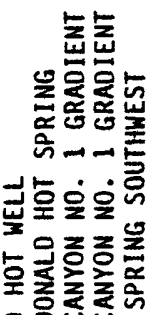

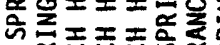

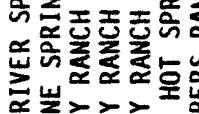

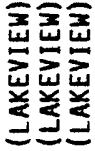

窟

$m+\infty$

غं율욜

工十廾 过

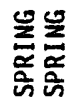

오홍

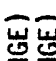

产产

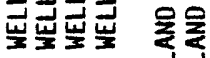

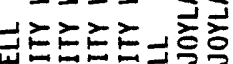

崖

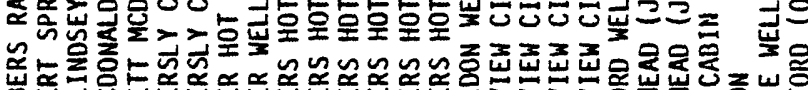

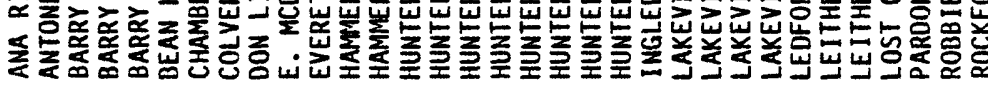

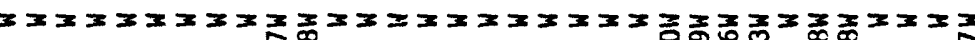

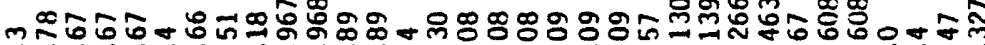

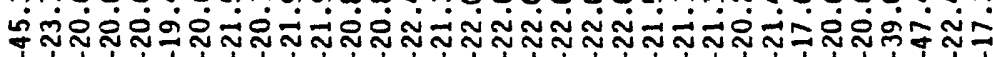

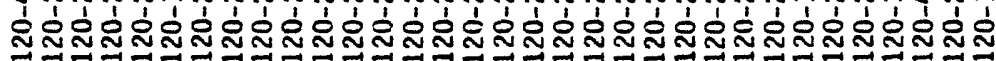

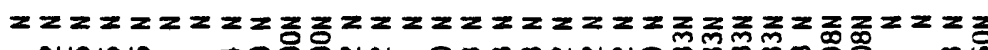

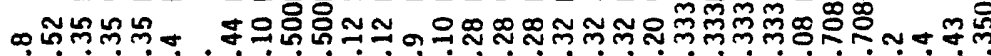

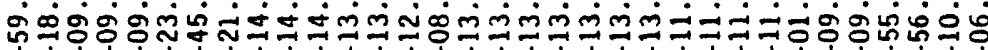

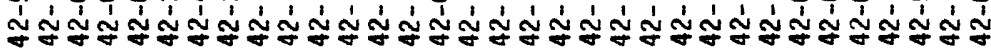




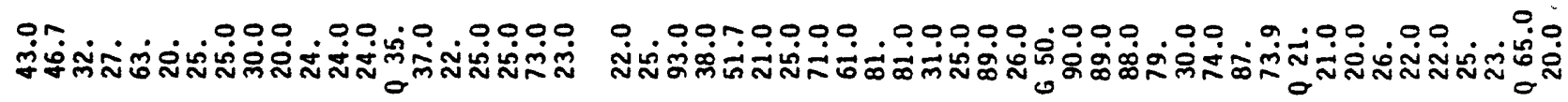

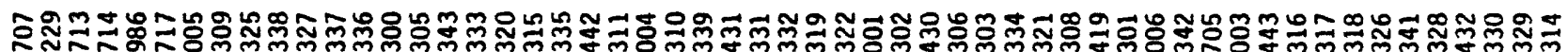

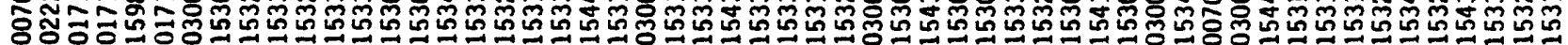

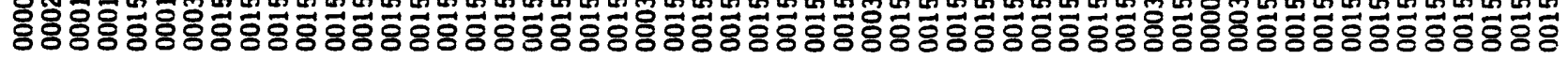

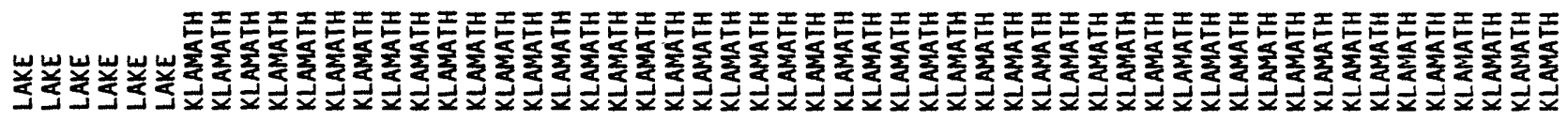

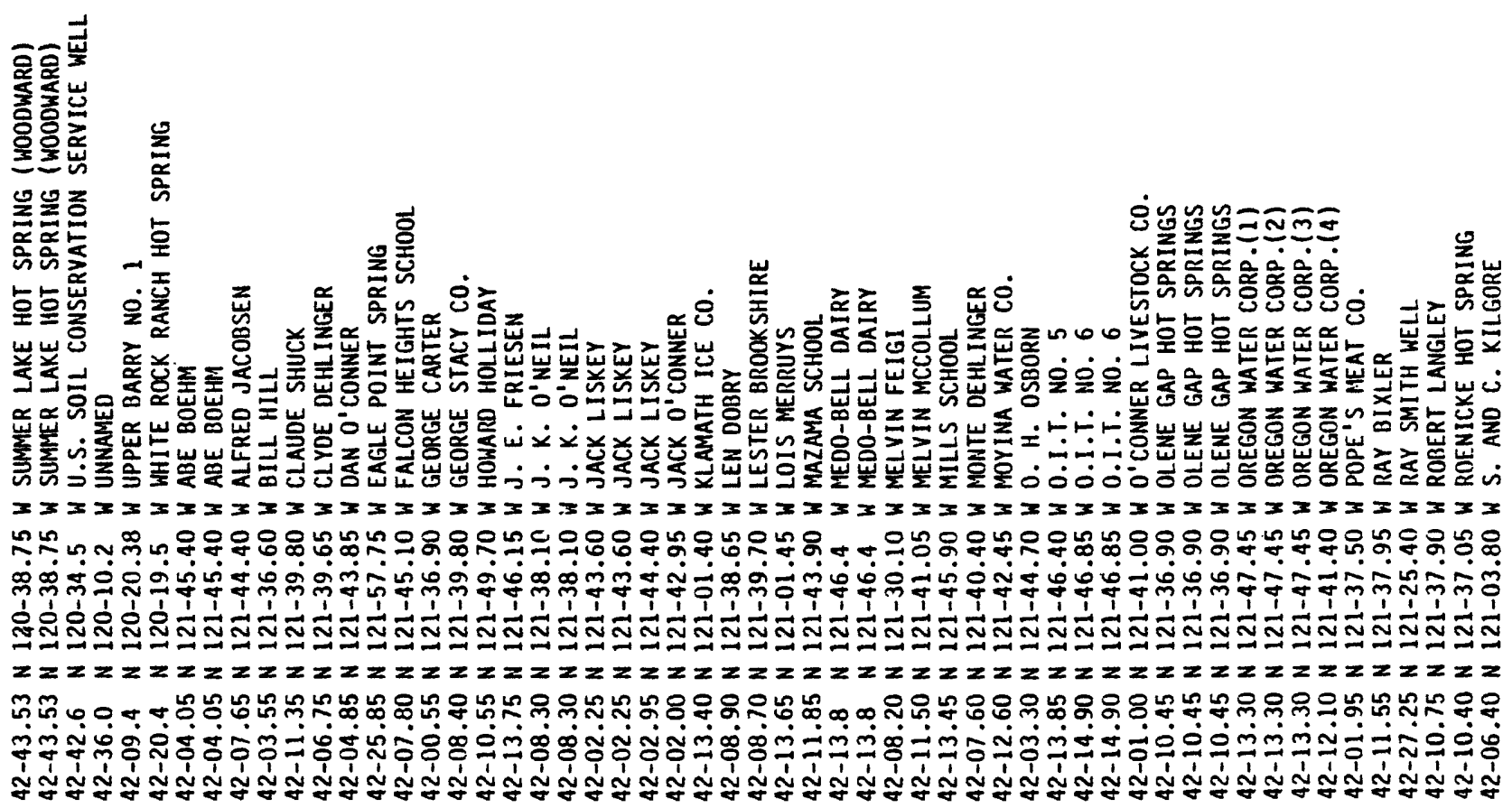


$\stackrel{\sim}{\sim}$

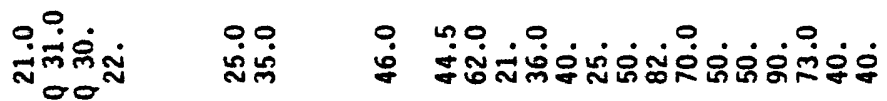

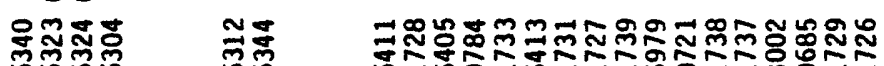

ㅎํㅇㅎㅎ일

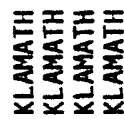

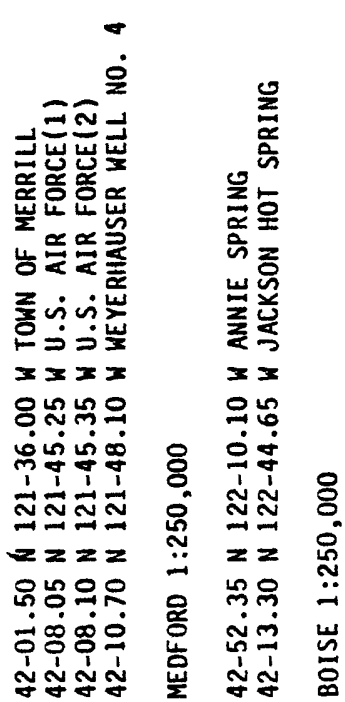

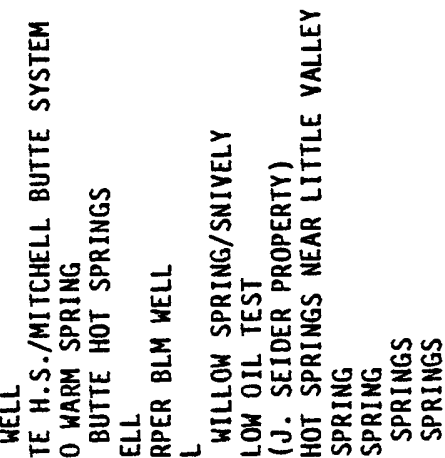

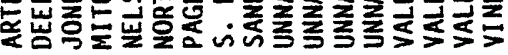

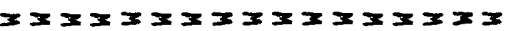
ก ก ம் 군

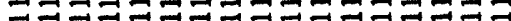
$z=2 z z z=2 z=2 z=2 z$

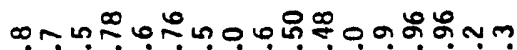

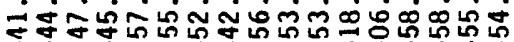

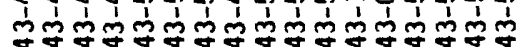

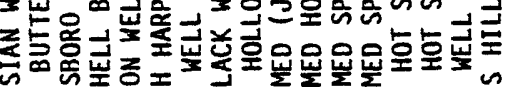

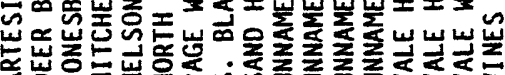

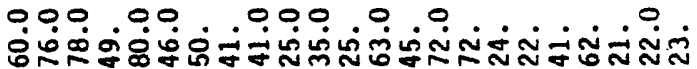

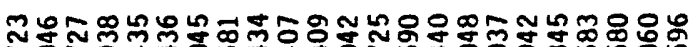
ㅇํㅇ응

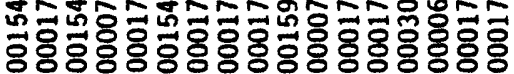

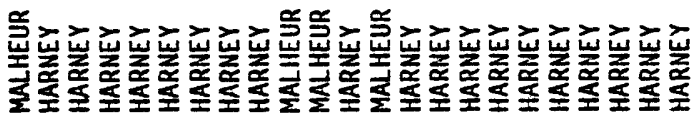


iั

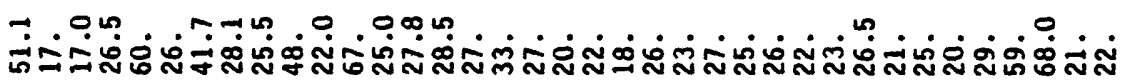

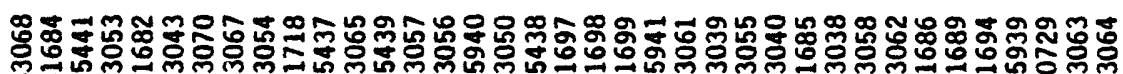

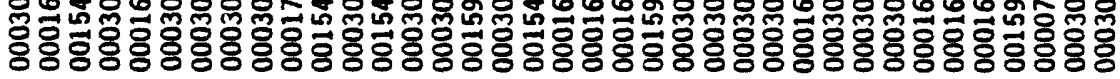

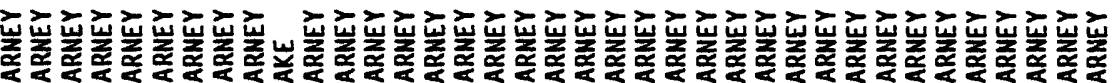

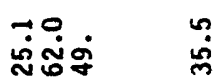

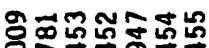

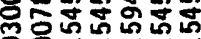
응융형횽ㅎㅇ융용

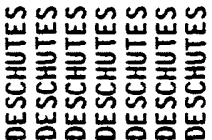

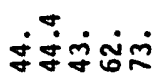

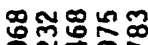

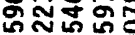
형융횽ㅇㅇ

崖崖崖崖

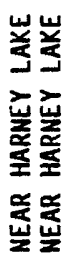

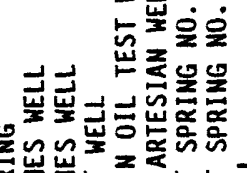

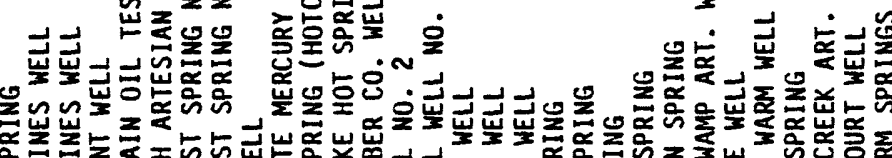

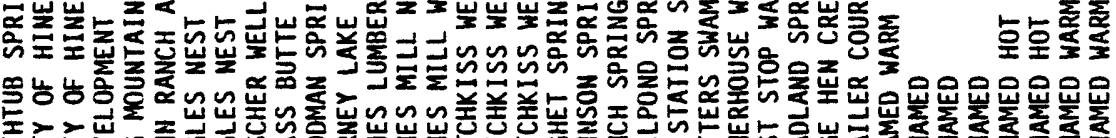

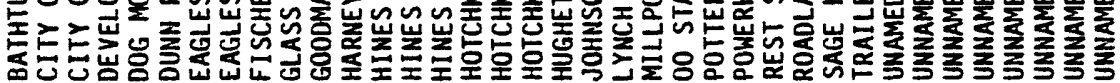

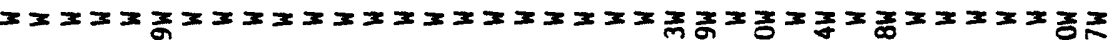

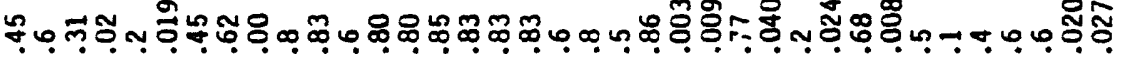

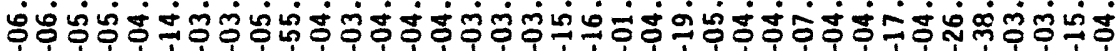

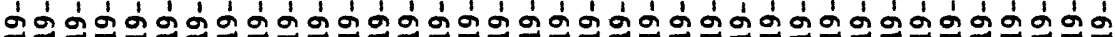

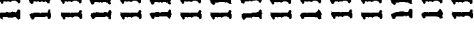

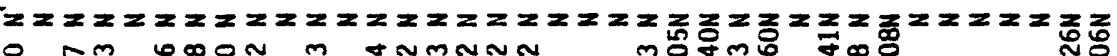

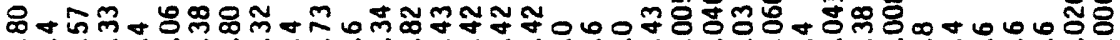

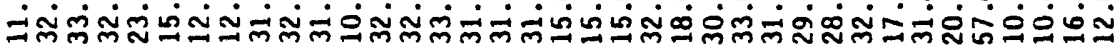

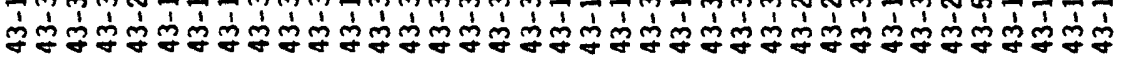

吕

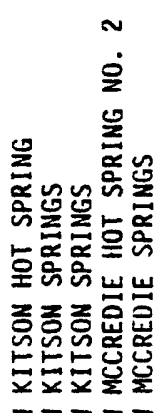

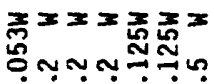
ம்ற்

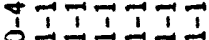

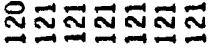
$z=2 z=2$ 今ั กิ-月-

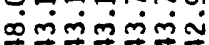

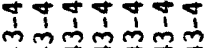


กิ

\begin{tabular}{|c|c|c|c|c|}
\hline : & 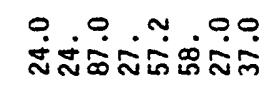 & 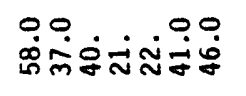 & 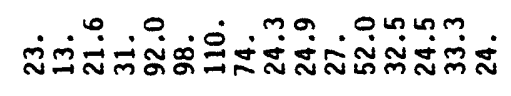 & 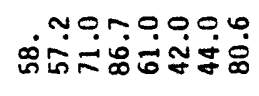 \\
\hline ת & 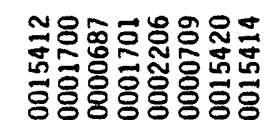 & 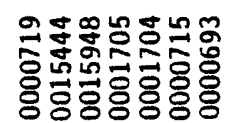 & 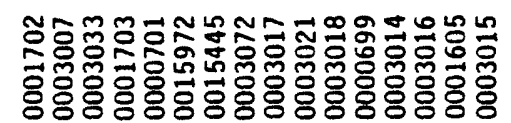 & \\
\hline
\end{tabular}

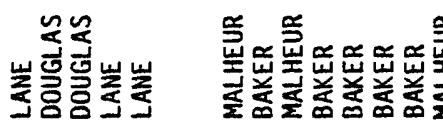

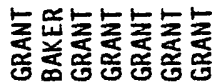

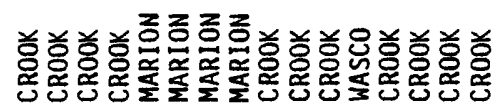

乐舟产

这崖崖崖崖崖崖

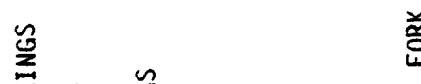

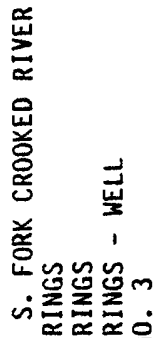

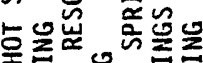

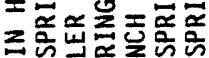

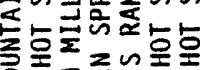

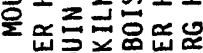

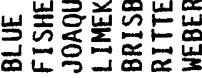

$x \geq 3 x \geq \geq 3$

$\geq \geq \geq 3 \geq x=$

ดักำด

ต่งล่

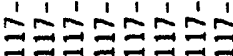

$z=2 z=2 z$

ำㅇำㄸำ

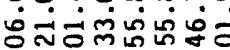

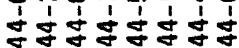

ㅎำ

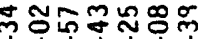

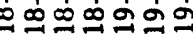

コニコココ

2222222

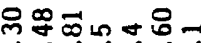

तิळ0ี่ำ

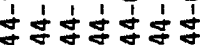

z迢的的这

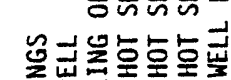

乌롤

总吉状

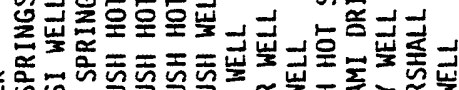

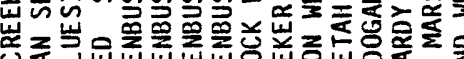

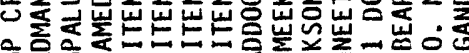

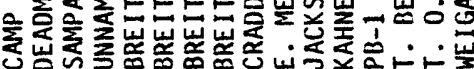

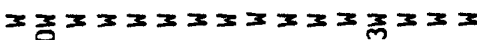

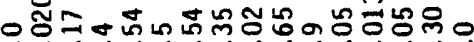
คํ் on

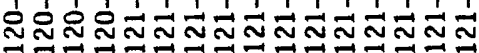
ZZZZZZZZZZZZZZZZ

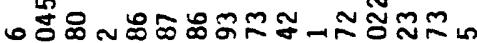

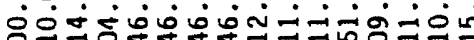

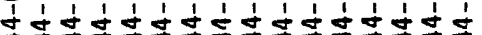

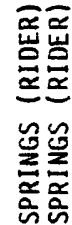

幺ิ원

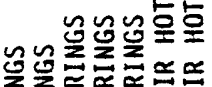
究的的送遂

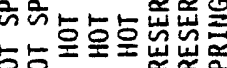

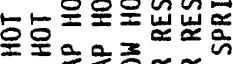

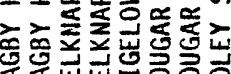

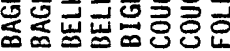
x $x \geq x \geq x \geq$ 뉼융요융요

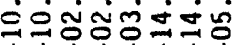

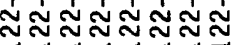
zZZZZZZ

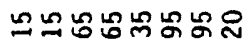

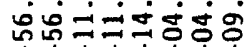

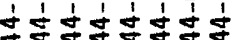


สึ

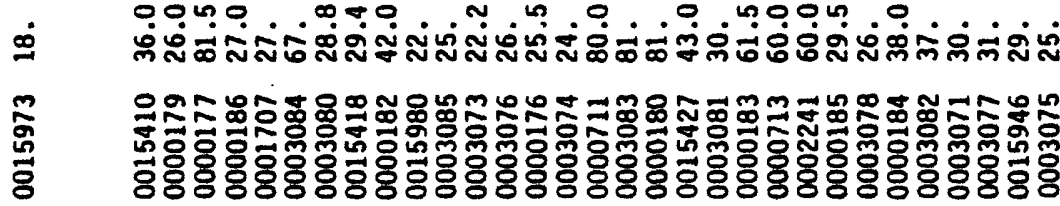

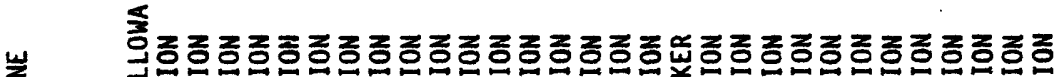

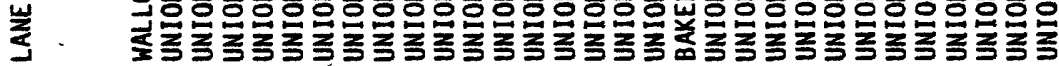

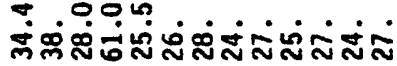

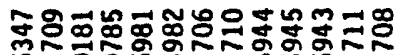

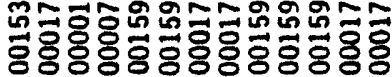

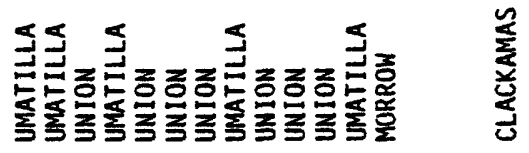

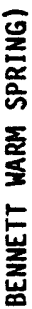

을

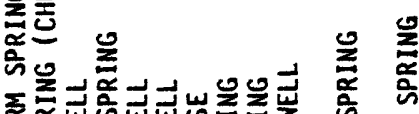

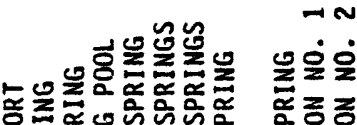

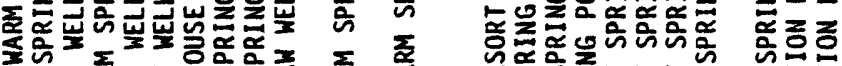

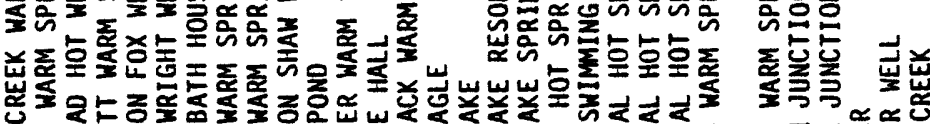

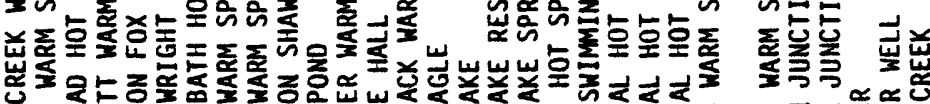

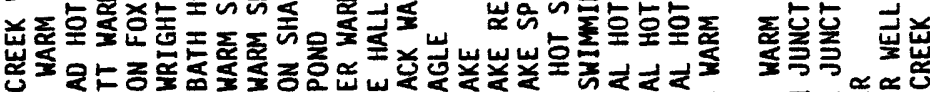

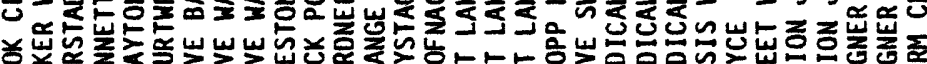

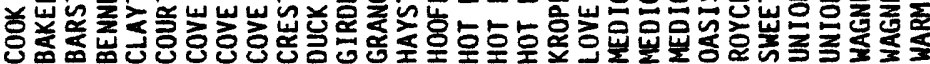

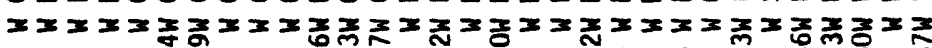

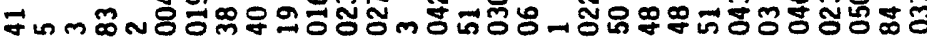
तें

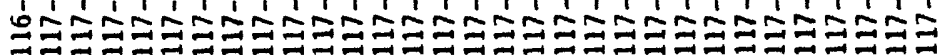

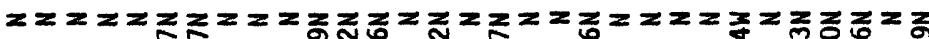

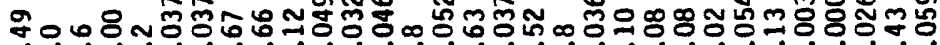
نळ

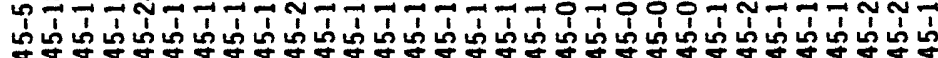




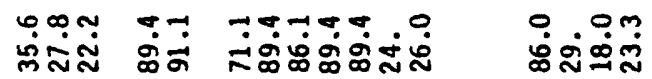

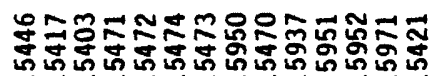

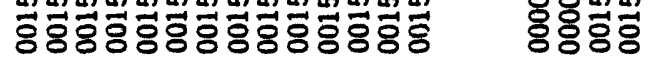

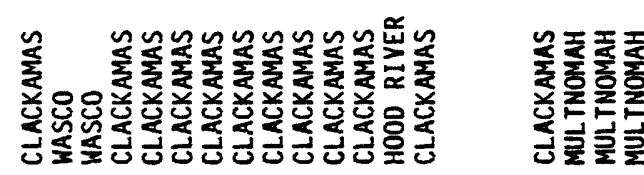

萦

울

䓪

号

点

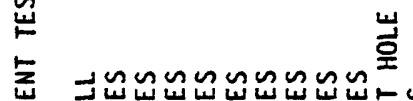

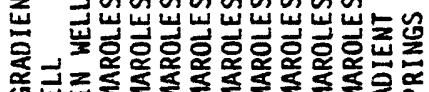

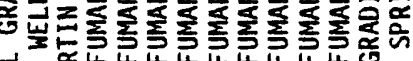

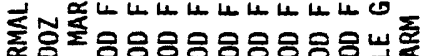

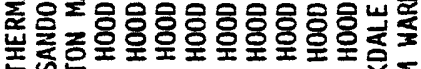

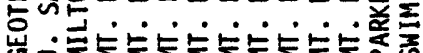

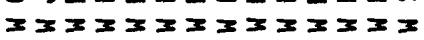
ర్దు

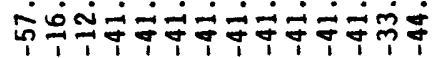

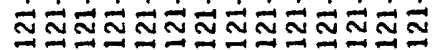
$\geq \geq \geq \geq Z \geq Z \geq Z \geq Z Z \geq Z$

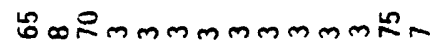
ك́

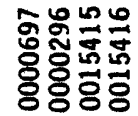

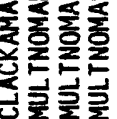

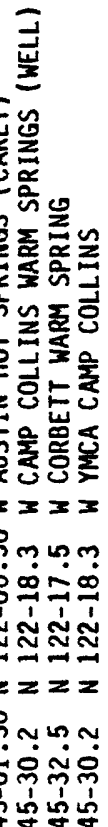




\section{APPENDIX D}

Sources for the records in the GEOTHERM sample file for Oregon. Each reference is proceeded by its abbreviated form (called CODE) used in the sample file (Table 1). Entries in this computer-generated appendix are sorted by CODE.

CODE = AYERS AND CRESWELL, 1951

AYRES, F. D., AND CRESWELL, A. E., 1951, THE MOUNT HOOD FUMAROLES: MAZAMA, V. 33, NO. 13, P. 33-40.

CODE = BOWEN AND OTHERS, 1978

BOWEN, R. G., PETERSON, N. V., RICCIO, J. F., 1978, LOW- TO INTERMEDIATE-TEMPERATURE THERMAL SPRINGS AND WELLS IN OREGON: OREGON DEPARTMENT OF GEOLOGY AND MINERAL INDUSTRIES, GEOLOGICAL MAP SERIES, GMS-10.

CODE = BROWN AND OTHERS, 1980A

BROWN, D. E., MCLEAN, G. D., AND BLACK, G. L., 1980A, PRELIMINARY GEOLOGY AND GEOTHERMAL RESOURCE POTENTIAL OF THE SOUTHERN HARNEY BASIN OREGON: OREGON DEPT. OF GEOLOGY AND MINERAL INDUSTRIES OPEN-FILE REPT. 0-80-7, $90 \mathrm{P}$.

CODE = BROWN AND OTHERS, 1980B

BROWN, D. E., MCLEAN, G. D., AND BLACK, G. L., 1980B, PRELIMINARY GEOLOGY AND GEOTHERMAL RESOURCE POTENTIAL OF THE NORTHERN HARNEY BASIN OREGON: OREGON DEPT. OF GEOLOGY AND MINERAL INDUSTRIES OPEN-FILE REPT. 0-80-6, $52 \mathrm{P}$.

CODE $=$ BROWN AND OTHERS, 1980C

BROWN, D. E., BLACK, G. L., AND MCLEAN, G. D., 1980C, PRELIMINARY GEOLOGY AND GEOTHERMAL RESOURCE POTENTIAL OF THE CRAIG MOUNTAINCOVE AREA, OREGON: OREGON DEPT. OF GEOLOGY AND MINERAL INDUSTRIES OPEN-FILE REPT. 0-80-4, 21 P.

INDUSTRIES OPEN-FILE REPT. 0-80-9.

CODE = DELLECHAIE, 1978

DELLECHAIE, F. 1978, A GEOLOGICAL AND HYDRO-GEOCHEMICAL STUDY OF THE LA GRANDE AREA, UNION COUNTY, OREGON: GEOTHERMAL RESOURCES COUNCIL, TRANSACTIONS, VOL. 2, JULY, 1978, P. 145-148.

CODE = GONTHIER AND OTHERS, 1977

GONTHIER, J. B., COLLINS, C. A., AND ANDERSON, D. B., 1977, GROUND WATER DATA FOR THE DREWSEY RESOURCE AREA, HARNEY AND MALHEUR COUNTIES, OREGON: U. S. GEOLOGICAL SURVEY OPEN-FILE REPORT 77-741, $28 \mathrm{P}$. 
CODE = HAMPTON AND BROWN, 1964

HAMPTON, E. R., AND BROWN, S. G., 1964, GEOLOGY AND GROUND-WATER RESOURCES OF THE UPPER GRANDE RONDE RIVER BASIN, UNION COUNTY, OREGON: U. S. GEOLOGICAL SURVEY WATER-SUPPLY PAPER 1597, 99 P.

CODE $=$ HOGENSON, 1964

HOGENSON, G. M., 1964, GEOLOGY AND GROUND WATER OF THE UMATILLA RIVER BASIN, OREGON: U. S. GEOLOGICAL SURVEY WATER-SUPPLY PAPER 1620, $162 P$.

CODE = LEONARD AND HARRIS, 1974

LEONARD, A. R., AND HARRIS, A. B., 1974, GROUND WATER IN SELECTED AREAS IN THE KLAMATH BASIN, OREGON: OREGON STATE GROUND-WATER REPORT NO. 21, 104 P.

CODE = LEONARD, 1970

LEONARD, A. R., 1970, GROUND-WATER RESOURCES IN HARNEY VALLEY, HARNEY COUNTY, OREGON: OREGON STATE GROUND-WATER REPORT NO. 16, 85 P.

CODE = LINDGREN, 1901

LINDGREN, WALDEMAR, 1901, THE GOLD BELT OF THE BLUE MOUNTAINS OF OREGON: U. S. GEOLOGICAL SURVEY 2ND ANN. REPT., PT. 2, P. 551-776.

CODE $=$ MADISON, 1966

MADISON, R. J., 1966, WATER-QUALITY DATA IN THE WILLAMETTE BASIN, OREGON, 1910-64: U. S. GEOLOGICAL SURVEY BASIC-DATA RELEASE, 40 $P$.

CODE = MARINER AND OTHERS, 1974A

MARINER, R. H., RAPP, J. B., WILLEY, L. M., AND PRESSER, T. S., 1974A, CHEMICAL COMPOSITION AND ESTIMATED MINIMIM THERMAL RESERVOIR TEMPERATURES OF SELECTED HOT SPRINGS IN OREGON: U. S. GEOLOGICAL SURVEY OPEN-FILE REPORT, $32 \mathrm{P}$.

CODE = MARINER AND OTHERS, 1975

MARINER, R. H., PRESSER, T. S., RAPP, J. B., AND WILLEY, L. M., 1975, THE MINOR AND TRACE ELEMENTS, GAS, AND ISOTOPE COMPOSITIONS OF THE PRINCIPAL HOT SPRINGS OF NEVADA AND OREGON: U. S. GEOLOGICAL SURVEY OPEN-FILE REPORT, 27 P. 
CODE = MARINER AND OTHERS, 1980

MARINER, R. H., SWANSON, J. R., ORRIS, G. J., PRESSER, T. S., AND EVANS, W. C., 1980, CHEMICAL AND ISOTOPIC DATA FOR WATER FROM THERMAL SPRINGS AND WELLS OF OREGON: U. S. GEOLOGICAL SURVEY OPEN-FILE REPORT $80-737,50 \mathrm{P}$.

CODE = NEHRING AND OTHERS, 1979

NEHRING, N. L., MARINER, R. H., WHITE, L. D., HUEBNER, M. A., ROBERTS, E. D., HARMON, KAREN, BOWEN, P. A., AND TANNER, LANE, 1979, SULFATE GEOTHERMOMETRY OF THERMAL WATERS IN THE WESTERN UNITED STATES: U.S. GEOLOGICAL SURVEY OPEN-FILE REPORT 79-1135, 12 P.

CODE = NEWCOMB AND HART, 1958

NEWCOMB, R. C., AND HART, D. H., 1958, PRELIMINARY REPORT ON THE GROUND-WATER RESOURCES OF THE KLAMATH RIVER BASIN, OREGON: U. S. GEOLOGICAL SURVEY OPEN-FILE REPORT 466,248 P.

CODE = NEWCOMB, 1972

NEWCOMB, R. C., 1972, QUALITY OF THE GROUND WATER IN BASALT OF THE COLUMBIA RIVER GROUP, WASHINGTON, OREGON, AND IDAHO: U. S. GEOLOGICAL SURVEY WATER-SUPPLY PAPER 1999N, 71 P.

CODE $=$ PETERSON AND GROH, 1967

PETERSON, N. V., AND GROH, E. A., 1967, GEOTHERMAL POTENTIAL OF THE KLAMATH FALLS AREA, OREGON - A PRELIMINARY STUDY: THE ORE-BIN, V. 29 , NO. 11, P. 209-231.

CODE $=$ PHILLIPS, 1936

PHILLIPS, K. N., 1936, A CHEMICAL STUDY OF THE FUMAROLES OF MOUNT HOOD: MAZAMA, V. 18 , N0. 12, P. 44-46

CODE = PIPER AND OTHERS, 1939

PIPER, A. M., ROBINSON, T. W., AND PARK, C. F., JR., 1939, GEOLOGY AND GROUND-WATER RESOURCES OF THE HARNEY BASIN, OREGON: U. $S$. GEOLOGICAL SURVEY WATER-SUPPLY PAPER 841,190 P.

CODE = PRICE, 1967

PRICE, DON, 1967, GROUND-WATER RECONNAISSANCE IN THE BURNT RIVER VALLEY AREA, OREGON: U. S. GEOIOOGICAL SURVEY WATER-SUPPLY PAPER $1839-I, 127$ P.

CODE $=$ ROBISON, 1972

ROBISON, J. H., 1972, AVAILABILITY AND QUALITY OF GROUND WATER IN THE ASHLAND QUADRANGLE, JACKSON COUNTY, OREGON: U. S. GEOLOGICAL

SURVEY HYDROLOGIC INVESTIGATIONS ATLAS HA-421. 